United States

Department of the Interior

Geological Survey

COMPILATION OF HYDROLOGIC DATA,

HELMAND RIVER VALCEY, AFGHANISTAN

THROUGH SEPTEMBER 1960

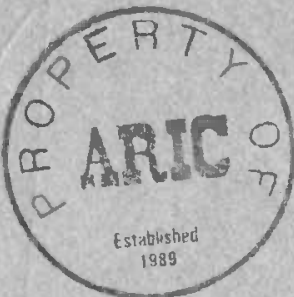

Prepared by the United States Geological Survey

in cooperation with

the Helmand Valley Authorlty of the Royel Government of Afghanistan

$$
\text { and }
$$

the United States Agency for International Development

Mission to Afghanistan

Kabul, Afghanistan

March 1964

Open File Report 
COMPLATION OF HYDROLOGIC DATA,

HELMAND RIVER VALLEY, AFGHANISTAN

THROUGH SEPTEMBER 1960

Prepared by

ROBERT H. BRIGHAM,

UNITED STATES GEOLOGICAL SURVEY

serving as

TECENICAL ADVISOR TO HELMAND VAJLEY AUTHORTTY, ROYAL GOVERIMENT OF AFGHANISTAN, under ausplees of the

UN ITED STATES AGENCY FOR INTERNATIONAL DEVELOPMENT
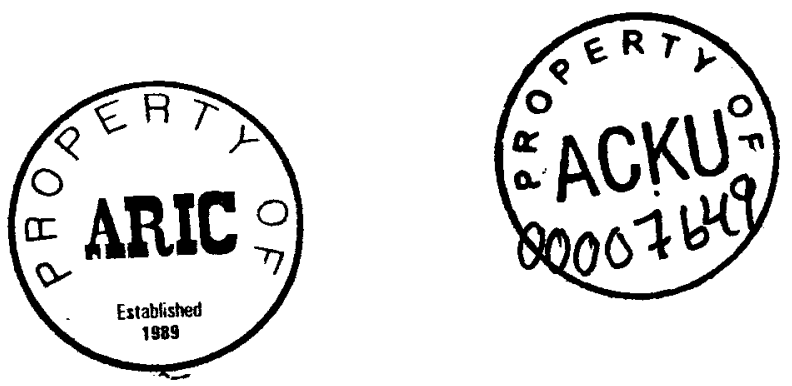

Kabul, Afghanistan

March 1964 


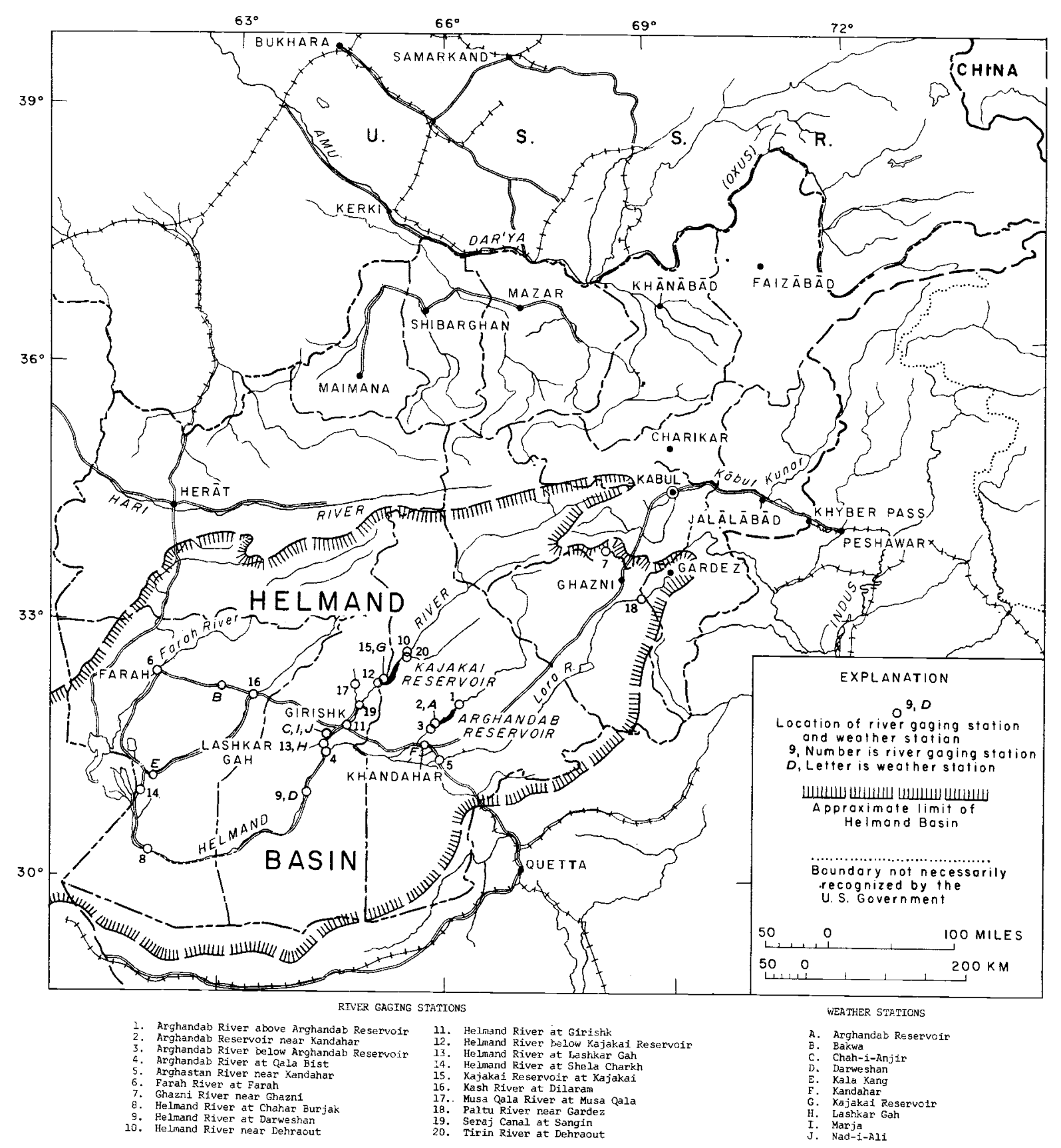

Figure 1.- Map of Afghantstan showing hydrologic stations in the Heimand River Bagin. 


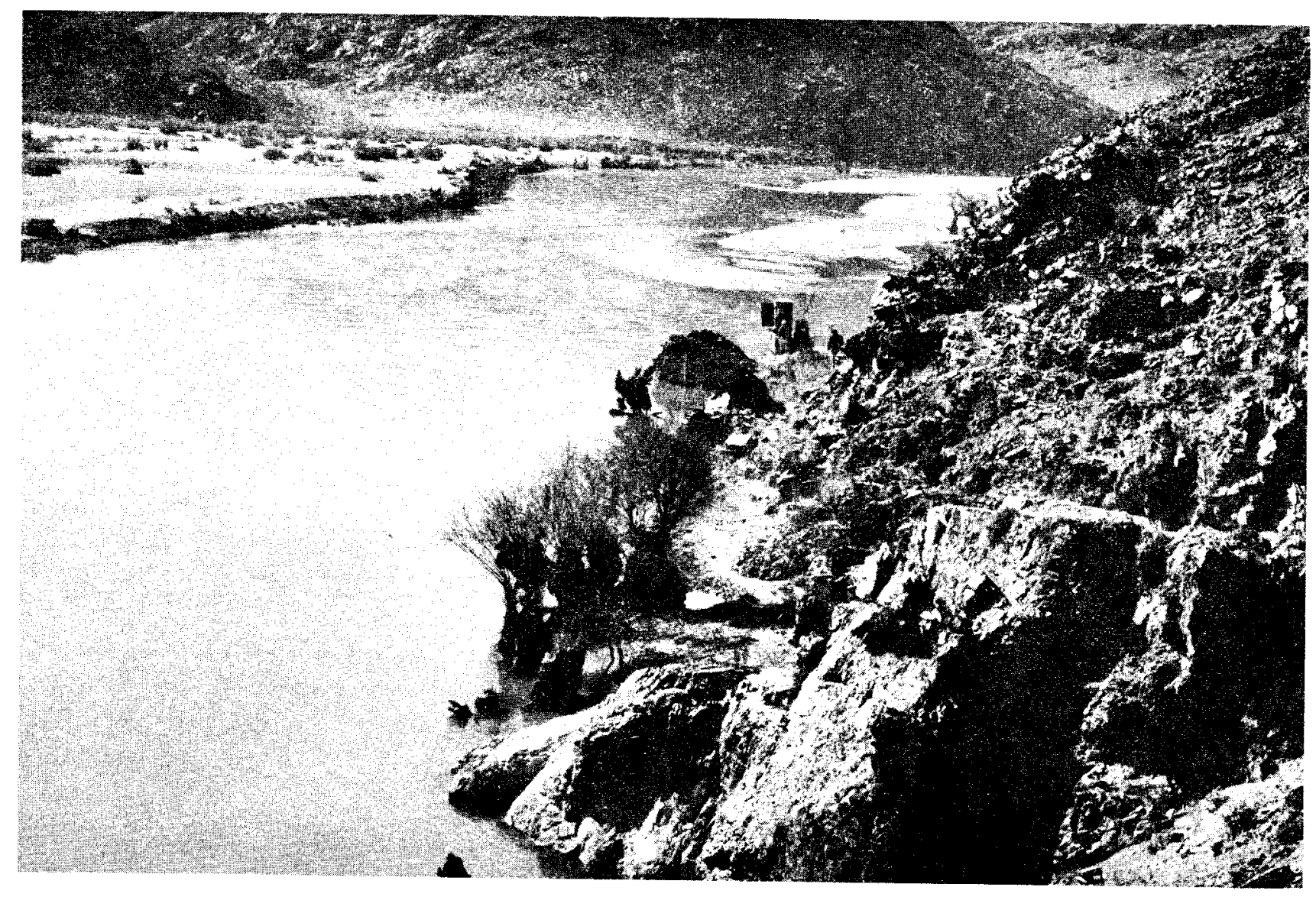

Frontispiece.--Arghandab River above Arghandab Reservoir, Afghanistan showing river gaging station. 
ROYAS COITRNMENT OF AFGHANISTAN

Helmand Valley Authority of Afghanistan

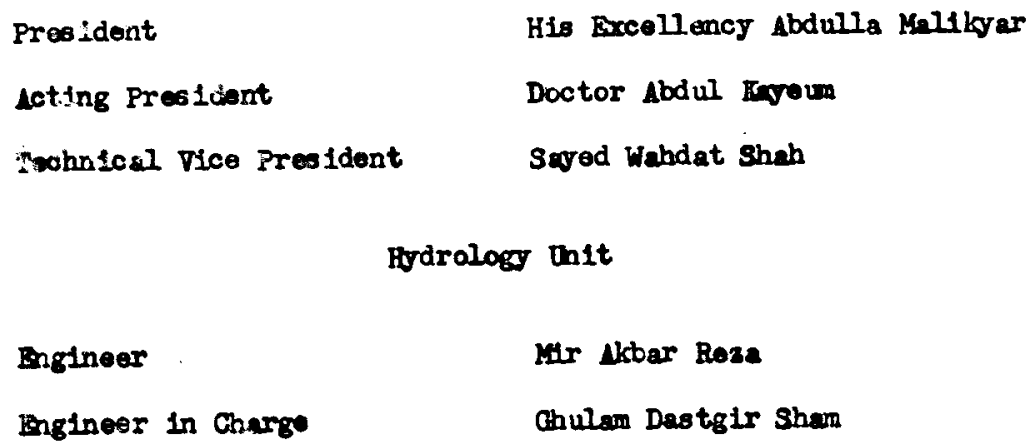

ONITED STATAS OF AMERICA

Ageney for International Dovelopment

Gological surveg

Bobert H. Brighem

Technical advisor 
Introduction. . . . . . . . . . . . . . . . . 1

Administration ..................... 1

History . . . . . . . . . . . . . . . . . 1

Scope of work ....... . . . . . . . . . . 1

Downstream order . . . . . . . . . . . . . . . . . 1

Explanation of data ................... 2

Accuracy of fleld data and computed results . . . . . 3

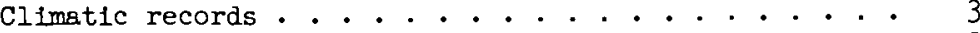

Definition of terms . . . . . . . . . . . . . . 3

Convenient equivalents . . . . . . . . . . . . . 6

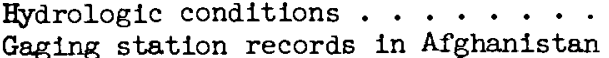

Helmand River above Kajaka1 Reservolr near Dehraout. . . II

Tirin River at Dehraout .............. 21

Kajakai Reservolr at Kajaka1 . . . . . . . . . 31

Helmand R1ver below Kajaka1 Dam. . . . . . . . . . 34

Seraj Canal at Sangin .............. 48

Musa Qala River at Musa Qala . . . . . . . . . 57

Helmand River near Girishk . . . . . . . . . 67

Helmand River at Lashkar Gah ... . . . . . . 72

Arghandab River above Arghandab Reservoir near Kandahar. 79

Arghandab Reservolr near Kandahar. . . . . . . . 89

Arghandab River below Arghandab Dam. . . . . . . 93

Ghazn1 River near Ghaznt.............. 107

Paltu River near Gardez. . . . . . . . . . . . . . 113

Arghastan River near Kandahar. . . . . . . . . 119

Arghandab River near Kala Bist . . . . . . . . 125

Helmand River at Darweshan . . . . . . . . . . 139

Helmand River at Chahar Burjak . . . . . . . . . 144

Helmand River at Shela Charkh. . . ....... 158

Kash River near Dilaram . . . . . . . . . . . 161

Farah River near Farah . . . . . . . . . . 170

Miscellaneous discharge measurements . . . . . . 179 Records of climate

Arghandab Reservolr near Kandahar. . . . . . . . . 184

Bakwa near Farah ....... 191

Chah-1-Anj1r . . . . . . . . . . . . . . 194

Darweshan. . . . . . . . .... 198

Kala Kang. . . . . . . . . . . . . . 201

Kandahar..................... . . . 206

Kajaka1 Reservo1r. . . . . . . . ..... 216

Lashkar Gah. . . . . . . . . . . . . 220

Marja. .................... . 226

$\mathrm{Nad-1-Al1................} \mathrm{.} \mathrm{.} 230$

Snow Surveys. ................... 235 
Front1splece.--Arghandab River above Arghandab Reservolr

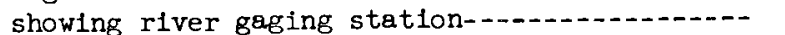

Figure 1.--Map of Afghanistan showling hydrologic stations in the Helmand River Basin-..... I

2.--Monthly minimum, median, and maximum discharges from Kajaka1 and Arghandab Reservoirs for

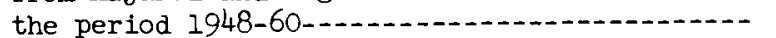

3.--Helmand R1ver above Kajaka1 Reservoir near Dehraout showing current meter discharge measurement being made by wading-...........

4.--Tirin River at Dehraout showing water-stage recorder shelter and well, cableway, and control-_.

5.--Operational pattern of Kajaka1 Reservoir for the calendar year period 1953-60................

6.--Arghandab River above Arghandab Reservolr near Kandahar showling water-stage recorder shelter and well, and staff gage-_...

7.--Operational pattern of Arghandab Reservoir for the calendar year period 1952-60_.............

8.--Arghandab River below Arghandab Dam showing cableway and control-.....

9.--Arghastan R1ver near Kandahar showing waterstage recorder well and shelter-............ 11 \& 


\section{INTRODUCTION}

The flodrolog section of Helmand Valloy Authority presents in this report records of stream flows, miscellaneous discharge measuremente and other pertinent hydrologic data collected from atroam gaging

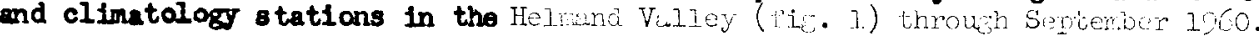

\section{LOMONISTRUTION}

The report has been propared by R. H. Brighem, U. S. Geological Surrey essigned es Technical

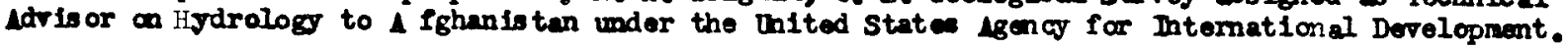

The cooperative program of the bydrologs section is under sayed Wahdat shah, Technical vice

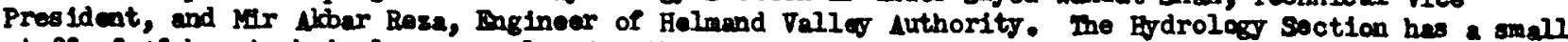
-taff of Ifghan toohnical perscomel under the supervision of Civil Engineer Chulam Destgir Sham. Tho Hodrelogs Section collocte flold data and alyses, and computes the records with the assistance and direction of the Tochnical Natrisor.

\section{HISTORI}

The activity of collecting, proceseing, computing and analyzing of the stream-flow data and weathor records at most iportent oites in the Helmand River Valleg was etarted wen the Horrison-mudseaIfghenisten, Incorporated begen carrying out its contracts with the Royel Gorernmont of Afghenis ten in 1947. Subsequently, as the derelopment program between the United States and If ghanistan took pleoe, Foreign Lid Projoct in 1952, under Point IV; and later, successively as Tochniael cooporation pdednietra-

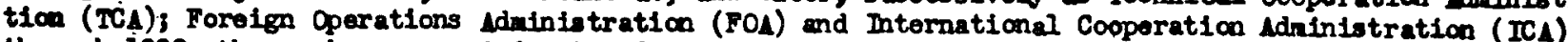
through 1960, the work was carried out and extended by the Hudrology section of Helmand Valley luthorito asisted by technicel advis ors from the Water Resources Division of the Onited states boological survey.

\section{SCOPE OF WORE}

This report includes the dally discherges oollooted at gering stations in the belmend River watershed botween oct. 1, 1947 and Sept. 30, 1960. Wocelleneove discharge monsurements at maln cenal diversion points and at some other looations are included.

Supplementing the run-off data, the significant recorde of procipitation, evaporation, temperatures, humidity and wind velocity colloeted in the area are included. Those at Indehar date back to 1940.

\section{DOWISTREAM ORDER}

In this report, the gaging stations are given in dowstream order, that 10 , from hoadwater downstrem on the main stem. Stations on a tributery entering above a main atem station are giren before that station. If a tributary enters botween two main $\rightarrow$ tem stations, it is listed between then. 
The base data collected at gaging stations consist of records of 8 tage and measurements of discharge. In adition, observations of factors affecting the atege-discharge relation, weather rocords, and othor information are used to supplement base data in determining the daily flow. The records of stage are obtained either from direct readings on a nanrecording gage or fram a wateratage recorder that gives a continuous recard of fluctuations. Wesurements of discharge are made with a current meter by the general methode adopted by the United States ceological Survey on the basis of experience in stream gaging 8 ince 1888. These methods are described in Water-Supply Paper 888 and are also outlined in standard textbooks co the measurement of atream discharge.

Rating tables giving the discharge for any stage are prepared from tage-discharge relation curves defined by discharge measurements. If extensions to the rating curves are necessary to define the extrenes of discharge, they are made on the basis of indirect moasurements of peak discharge (such as slopearea or contracted-opening measurements, computation of flow over dams or weire, and by other methods), velocity-area studies and logarithmic plotting. The application of the daily mean gage height to those rating tables gives the daily mean discharge, from which the monthly and yearly mean discharges are computed. If the stage-discharge relation is subject to change because of frequent or continual ohange in the physical features that form the control, the datly moan discharge is determined by the shiftingcontrol method, in wich oorrection factors besed on individual discharge measurements and notes by engineers and observers are used in applying the gage heights to the rating tables.

Winters in the Helmend River Basin are relatively mild so that ice effect on the stage-discharge relation can be neglected.

The data herein presented generally comprise description of the station, a table showing the daily discharge and manthly and yearly discharge of the strean. Records are published by the water year which begins on october 1 and ends on September 30 .

The description of the station gives the locaticn, drainage area, records available, type of gage, average discharge, extremes of discharge, and general remarks. The locaticn of the gaging atatica and drainage area are obtained from the most accurate maps available. Inder "Gage" are given the type of gage currently in use and the detum of the present gage above mean sea lovel. Inder "Average discharge" is given the average discharge for the number of years indicated. It is given only for stations having five or more complete years of record. Inder "Bxtremes" are given the maximum discharge and gage height; the minimum discharge if there is little or no regulation; the minimum datly discharge if there is extensive regulation; and the minimun gage height (unless it is of no importance). Unless otherwise qualified, the maximum discharge correspands to the crest stage obtained by use of a water $\rightarrow$ tage recorder, or a nonrecording gage read at the time of the crest. Information pertaining to the accuracy of the records and conditions which affect the natural flow at the gaging station is given under "Remarke."

In the table of daily discharge, the figures for the maximum day and the minimun day for each month are underlined. If the figure is repeated it is underlined on the first day of its occurrence.

In the monthly summary below the datly table, the line head "Total" gives the sum of the daily figures; it is the total cfs-days for the month. The line headed "Mean" gives the average flow in cubic feet per second during the month. Discharge for the month is expressed in acre-feet (line headed "Ac-ft").

In the yearly summary below the monthly summary, the figures of maximum are the maximum daily discharges, not the manentary discharges when the water was at crest stage. Ifkewise, the minimuse in this sumary are the minimum daily disch⿲丶丶㇒一母 
Footnotes to the table of daily discharge indicate periods when discharge was camputed or estimated by unusual cr special methods during periods of no gage-helght record or by effects that reduce the degree of accuracy of the records. Days on wich discharge measurements were made are indicated by asterisk and footnote.

For the gaging stations on the reservoirs the data presented comprise a description of the station and a table of month-and contents in acre-feet.

At some gaging stations water temperature has been taken at the time a discharge measurement is made but they are not published in this report.

Discharge measurements at miscellaneous sites, particularly at canal diversice points are given after the stream gaging records.

\section{ACCURACT OF FIED DATA AND CORPUTHD RASULTS}

The accuracy of atream flow data depends primarily on (1) the stability of the stage-discharge relation or if the control is unstable, the frequency of discharge measurements, ond (2) the accuracy of observations of stage, measurements of discharge, and interpretation of records.

The station desoription states the degree of accuracy of the records. "Bxcellent" indicates that, in general, the error in the dally records is belleved to be less than 5 percent; "good" less then 10 percent; "fair" less than 15 percent; and "poor" probably more than 15 percent. The records of monthly and jearly mean discharge and rumoff are, in geweral, more nearly accurate than the daily records.

Mischarge at scane atation, as indicated by the monthly mean, may vary widely fram natural runoff, oring to diversion, consuption, regulation by storage, increase or decrease in evaporation due to artificial causes or to other factors. For these reasons figures of cubic feet per second per square mile and rumaff in inches are not publisbed for these records.

Butensive irrigation is carried on both above and below the gaging stations in the Helmand River Besin of Ifghenistan; therefare, the discharge recorded is that occurring after the present irrigatian denonds have been mot upstream.

\section{CLIMATIC RECORDS}

Becords of temperature, rainfall, evaporation, relative hunidity and wind velocity have been collected at a number of locktion in the Helmand Valies.

The records collected at each site are introduced with a description giving the location and trpe of equipent at the station and a sumery of the data collected.

\section{DEF INITION OF TERM}

The bydrologic terms dafined below are considered to be the most comon cnes being used in comection with a stidy of surface waters. Mrgradations the naterial proces causing the increase or rise of river bed levels at specifie discharges
of a chanel at a given site.

Mluriel rivers one which flows in alluvium formed by depceits of sediment it carries. Such sediment exopt the wash land, in eimilar to that found in its bed. 
Area, drainage: The horizontal projection of the area from which a river or other bodies of water receives surface men-off originating from precipitation. It is also called drainage, watershed, catchinent area or river basin.

Backwater: Water held back by some obstruction such as a dam, control features or body of water.

Banks The eleveted land which confines the water to the natural channel in its normal course of flow.

Bank, right or left; That bank on right or left as the river is viewed when facing dawstream; by convention.

Bankull stagei It is the elevation of the water in the stream when it begins to overflow its natural bants.

Base Flow: The sustained flow of stream resulting from outflow of ground water and from drainage of lakes and swempe.

Besin, river: seme as drainage area.

Bench marks The point whose elevation relative to a given datum is $\mathrm{known}$ and is used as a reference in determining other elevations.

Bluffes High bank of a river within which its width has ranged within hiatoric times.

Chamel, instable: The channel is said to be unstable when there are considerable changes during a year or Irom year to year, caused by natural accretion and scour of the stream bed.

Control, The feature damstream from a gage which determines the relation of stage to discharge. It may be a gravel bar, a chanel constriction, a bend in the river, a long rach of channel, or an artificial structure.

Control section: It is the section in the chamel of a stream where the flow is at oritical depth, hydraulic conditions above such section being wholly dependent upon characteristics of such control section and entirely independent of hodraulic conditions below the section.

Cantrel, shifting: When the control feature downstrean from a gage does not dotermine a stable stagem discharge relation at the gage because of frequent or continued changes in the physical featurea that form the control, such control is considered shifting.

Curve, areas In stream gaging, particularly, an area curve is a graph in witich cross-sectional areas of a stream at a gaging section are plotted against corresponding water-surface elevations.

Curve, velocity, In stream gaging, particularly, a velocity curve is a graph on which mean velocities of atream at a gaging section. are plotted against the corresponding water surface elevations.

Curve, vertical velocity: A vertical velocity curve is a graph of velocities against depth at a point in a strean.

Datim plares It is an agreed horizantal plane from which elovaticns are measured or to which they are referred. (gage datum, sea level datim, etc.)

Flasky strean: A stream on which run-off fror rainfall occurs rapidly causing quick rising and falling Ilood peaks. 
Gage, starf: A graduated scale ch a ataff, flank, or metal plate mourted on fust, plor, wall abutmant, etc. by which the elevation of the water suriace can be read.

Oajing station: A gaging station is a solected site un a river, oquiped and operated tc obtaln data co stroan flow.

Eydrograph, a graph showling elevations, discharbe, velocity, etc. of a flowing etreal with respoct to time.

Hydrology, The science dealing with the occurrence of water on the earth, 1ts prcperties, transformation and novement. Bspecially its course from frocipltaticn on the land and its movements tourd the sea and ultimate return to the atmosphere.

Man soa level, (MLI) Blevation of the surface of the sea if no tido-producing forces acted upon it. It is determined from average radings of soa level at oqul intervals of time over a long period.

Rum-orf, Thet portion of the total procipitatior an an area that finds 1to way into the stroans as ourface flow.

Rum-off coefficlent: The ratie betwoen depth of rin-off to the depth of procipitation producing the runorl over a drainage aroa.

Run-off depth: The total run-off fram a given drainage aroa divided by the area. Bniressed in unite of depth comparable to frocipitation.

Bun-off, acrefret; The voluse of water needed to cover one acre one foot deope

Run-off, hectere motar: The roluwe of water needed to cover one liectare ane sater desp.

Bun-of, mean anrual, The annual ylold of the drainage tasin.

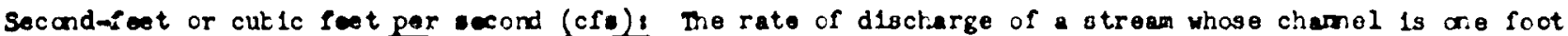
oquare and has mean valocity of one fcot per oecand.

Second-liters or literg per second: The rate of discharge of a streax whose chamel is one square decizoter and wose mean velocity 13 one decimeter per secand.

Second-root dag: The volume of water represented by a discharie of ane eoccnd foot for 24 heure.

Strea gaging: The measurement of the velocity and crose-oectional area of a strex in orjer to determine the rate of discharge.

Water tage recorder: 1 derloe for recording water elevations.

Water shed, Sano as drainace erea.

Tield of dralnaze basin: The mear. amual run-orf. 


\title{
CONVENIENT EQUTVATERTS
}

The following are the most commonly used equivalents of masurements in length, surface, volume and hydraulies in various computations. They include equivalents of English units in metric and vice versa.

\section{Length}

\author{
1 inch $=2.54$ centimeters \\ 1 foot $=12$ inches $=0.3048$ meter \\ 1 yard $=3$ feet $=0.9144$ meter \\ $1 \mathrm{mile}=5280$ feet $=1.60985 \mathrm{kjl}$ cmeters \\ 1 millimeter $=0.0394$ inch \\ 1 centimeter $=0.394$ inch \\ 1 meter $=100$ centimeters $=39.57$ inches $=3.2808$ feet
}

\section{Surface}

1 square inch $=0.006944$ square foot $=6.45163$ square centimetero

1 square foot $=144$ square inches $=0.092906$ aquare meter

1 acre $=43,560$ square feet $=0.404687$ hectare

1 square mile $=27,878,400$ square feet $=640$ acres $=259$ hectares

1 square millimeter $=0.00155$ square inch

1 square centimeter $=0.155$ square inch

1 square meter $=.0001$ hectare $=0.000001$ square kllometer $=1,550$ square inches $=10.7639$ square feet

1 hectare $=10,000$ squire meters $=2.47$ acres $=107,636.8$ square feet

1 square kilometer $=1,000,000$ square meters $=247.1$ acres $=10,763,680$ square feet $=0.3861$ square mile

\section{Volume}

1 cubic inch $=0.0005787$ cubic foot $=16.3872$ cubic centimeters

1 cuble foot $=1,728$ cubic inches $=7.4805$ U. S. gellons $=28.317$ 11ters $=0.028317$ cubic meter

1 cubic $\mathrm{J}$ ard $=27$ cubic feet $=0.76456$ cubic moter

1 acre-foot $=43,560$ cubic feet $=1,235.49$ cubic meters

1 cubic centineter $=0.0810 \mathrm{R}$ cubic inch

1 liter $=1$ cubic decimeter $=0.0353$ cubic foot $=61.022$ cubic inches

1 hectoliter $=0.151$ cublc yend $=3.531$ cubic feet $=26.42$ gallons

1 cubic meter $=1000$ liters $=1.308$ cubic yards $=35.315$ cubic feet

1 liter $=0.2642 \mathrm{U}$. S. gallan

1 hectoliter $=26.42 \mathrm{U} . \mathrm{S} . \mathrm{gall} \mathrm{cms}$ 


\section{Bydraulios}

I J. S. gallon of water weighs 8.34 pounds avoirdupois

1 cublc foot of water weighs 62.5 pounds avoirdupois

I second-foot $=7.48$ U. S. gellans per second $=448.8 \mathrm{U}$. S. gallons per minute $=26,929.9 \mathrm{U}$. 5. gall cms per hour $=646,317 \mathrm{U}$. S. gellons per day

1 second-foot $=86,400$ cubic feet per dag

1 second-f oot $=0.9917$ acre-inch per hour $=1.983471$ acre-feet per day

1 second-f oot $=0.028517$ cubic moter per second $=2,446.58$ cubic meter per dos

1 million $U$. S. gallons per day $=1.55$ second $-f$ set $=3.07$ acre-feet per day $=2.629$ cubic meters per minute

1 second-foot falling 8.81 feet $=1$ horsepower

1 second-foot falling 10 feet $=1.135$ horsepower

1 second-foot felling 11 feet $=1$ horsepower $80 \%$ efficiency

1 second-foot for 365 days will cover 1 square mile 1.1512 feet or 13.5744 inches deep

1 inch deep on 1 square mile $=2,323,200$ cubic feet $=0.0737$ second-foot for 365 days

1 second liter $=0.0353$ second-foot

1 second liter $=0.2642$ U. S. gall on per second $=22,826$ gallons per day

1 second liter for 365 days $=31,536,000$ liters

1 cuble meter per second $=35.315$ second-feet

1 cubic meter per minute $=0.5886$ secard-foot $=4.403$ U. S. gallans per second $=1.1674$ acre-feet

1 cublc mete

1 cubic meter of water weighs 2,200 pounds avoirdupois

1 meter per second $=3.28$ feet per second

1 moter per secand $=2.2304 \mathrm{miles}$ per hour $=3.60 \mathrm{kilometers}$ per day

1 foot per second $=0.6818$ miles per hour 
Monthly and annual discharges from the Arghandab and Kajakai Reservoirs as computed from records of discharge for stations below the reservoirs adjusted for changes in reservoir contents (changes include evaporation losses) showing the monthly and annual minimum, median, and maximum discharges, for the period $194 \overline{8}-60$.

Monthly and annual discharges, in thousands of acre-feet, of Helmand River below Kajakai Dam, Afghanistan

\begin{tabular}{|c|c|c|c|c|c|c|c|c|c|c|c|c|c|}
\hline $\begin{array}{l}\text { Water } \\
\text { Year }\end{array}$ & Oct. & Nov. & Dec. & Jan. & Feb. & Mar. & Apr. & May & June & July & Aug. & Sept. & Annual \\
\hline 1948 & 90.0 & 113.8 & 134.1 & 130.9 & 146.6 & 599.6 & $1,269.0$ & 823.2 & 271.1 & 134.5 & 84.3 & 89.1 & $3,885.0$ \\
\hline 1949 & 120.5 & 143.3 & $\overline{155.7}$ & $\overline{156.8}$ & 223.8 & 715.3 & $1,832.0$ & $1,022.0$ & 373.9 & 194.3 & $1 \overline{35.1}$ & $1 \overline{20.8}$ & $5,194.0$ \\
\hline 1950 & 156.4 & 176.2 & 187.5 & m222.9 & 226.7 & 483.4 & $n], 024.0$ & $1,703.0$ & 607.1 & $224 \cdot 3$ & 125.4 & 119.1 & $5,256.0$ \\
\hline 1951 & 152.0 & 160.2 & 164.7 & 170.7 & $177 \cdot 3$ & 672.5 & $1,337.0$ & $1,997.0$ & 762.0 & 285.3 & 154.3 & 138.1 & 171.0 \\
\hline 1952 & 157.0 & 179.3 & $\operatorname{mr} 88.8$ & 197.0 & 300.7 & 753.6 & $1,367.0$ & m919. 5 & 347.8 & 175.4 & 116.1 & 125.1 & $4,827.0$ \\
\hline 1953 & 160.1 & 176.1 & 181.3 & 175.5 & 139.4 & 310.4 & 414.0 & 469.9 & 455.8 & 425.6 & 419.7 & 283.4 & $3,611.0$ \\
\hline 1954 & 243.4 & $\operatorname{mig} 96$ & 150.6 & 183.5 & 340.0 & 386.4 & 881.1 & $1,395.0$ & 544.6 & 263.4 & 330.2 & $\operatorname{m272.4}$ & $m 5,187.0$ \\
\hline 1955 & 246.0 & 245.5 & 297.1 & 408.2 & 351.2 & 293.2 & 325.3 & 423.5 & 376.8 & 272.5 & $\mathrm{~m} 307.8$ & 284.0 & $3,831.0$ \\
\hline 1956 & 278.8 & 269.8 & 285.3 & 269.7 & 251.3 & 313.4 & $1,918.0$ & $1, \overline{083.0}$ & 309.1 & 388.2 & 466.3 & 376.5 & $6,209.0$ \\
\hline 1957 & $\mathrm{~m} 234.2$ & 280.2 & 290.9 & 374.6 & 311.3 & $\mathrm{~m} 4 \mathrm{l} 8.1$ & $2,070.0$ & $2,572.0$ & $1,126.0$ & 515.8 & 380.6 & 419.1 & $8,993.0$ \\
\hline 1958 & 448.8 & 212.9 & 402.6 & 447.9 & 399.0 & 459.9 & 837.0 & 866.4 & 510.9 & 477.6 & 468.1 & 425.0 & $5,956.0$ \\
\hline 1959 & 406.8 & 287.9 & 256.0 & $\overline{255.5}$ & $m 229.6$ & 307.2 & 778.9 & 815.2 & $\mathrm{~m} 491.7$ & 465.4 & 437.8 & 343.9 & $5,076.0$ \\
\hline 1960 & 263.6 & $\overline{221.0}$ & 336.7 & 276.2 & 213.3 & 232.7 & 210.2 & $880 \cdot 3$ & \begin{tabular}{|l}
555.8 \\
\end{tabular} & $\operatorname{me} 7^{8} .7$ & 269.3 & 253.0 & $3,990.8$ \\
\hline
\end{tabular}

Monthly and annual discharges, in thousands of acre-feet, of Arghandab River below Arghandab Dam, Afghanistan

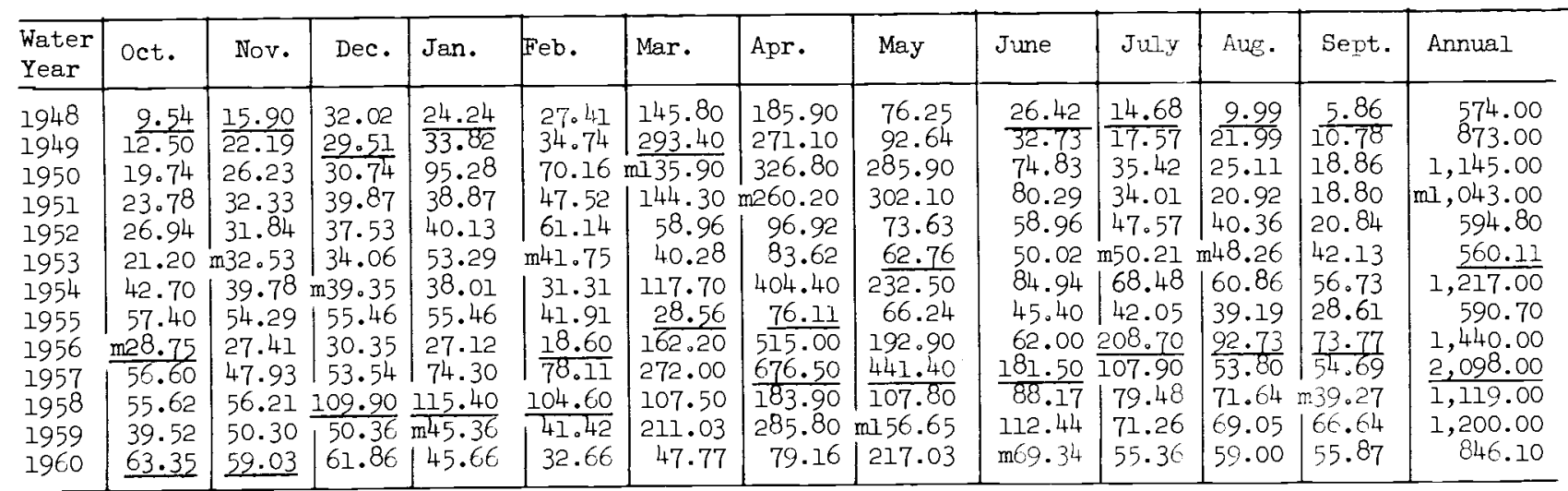




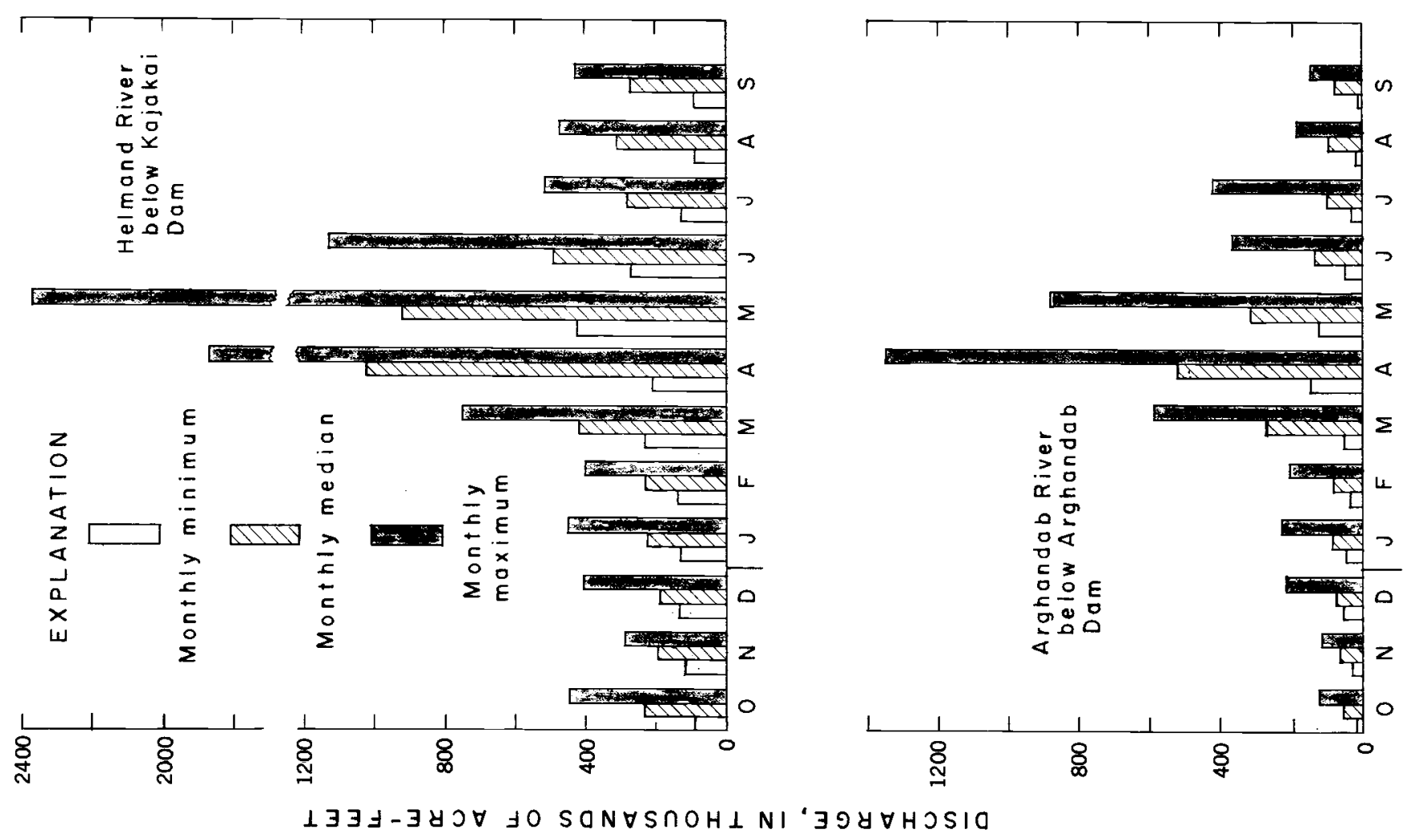

F1gure 2.--Monthiy minimum, median, and maximum discharges from the Kajaka1 and Arghandab Reservoirs for the perlod 1948-60. 


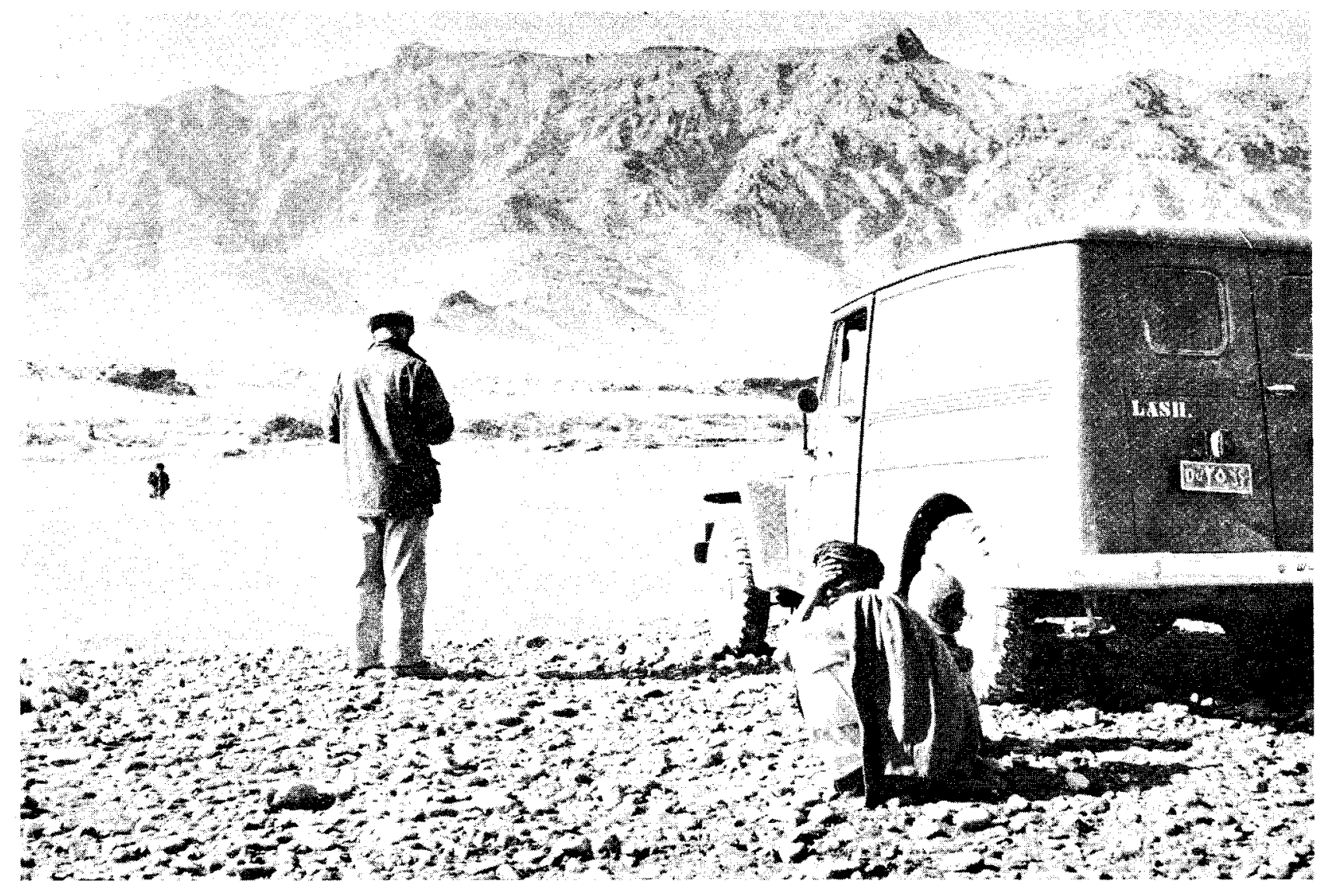

Figure 3.--Helmand River above Kajakai Reservoir near Dehraout showing current meter discharge measurement being made by wading. 


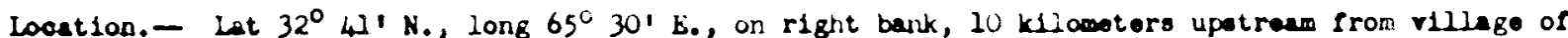
Dohraout, 15 kiloneters upetream from Tirin River, 70 kilamoters upstream from Kajakei Dam, and 120 kdlamoters north of kandahar.

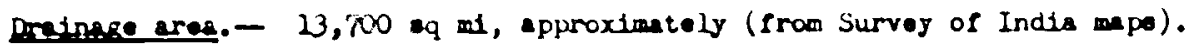

Becorde available.- April 1951 to jeptomber 1952 (partial gage holghts only), October 1952 to Soptember 1960 .

Gane- - Water-tage recorder. Nlitude of gage is 1,100 moters (from Survey of India mape). Prior to Nov, 16, 1952, staff gage st site 2 kllometers upetream at differert datum.

Arasage digcharge.- 9 years $6,935 \mathrm{ea},(5,021,000$ ecro-rt per year).

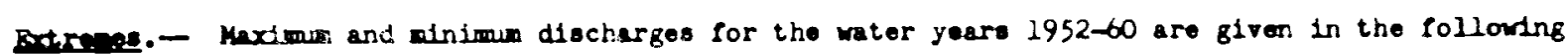
table:

\begin{tabular}{|c|c|c|c|c|c|c|}
\hline \multirow[b]{2}{*}{ Water yoar } & & \multicolumn{2}{|c|}{ Mexian } & \multirow[b]{2}{*}{ Date } & \multicolumn{2}{|c|}{ Mandam } \\
\hline & & $\begin{array}{c}\text { Cage helght } \\
\text { (eotere) }\end{array}$ & $\begin{array}{c}\text { Diacharge } \\
\text { (cfe) }\end{array}$ & & $\begin{array}{l}\text { Gege holght } \\
\text { (netere) }\end{array}$ & $\begin{array}{c}\text { Docharge } \\
\text { (cfa) }\end{array}$ \\
\hline $\begin{array}{l}1953 \\
1954 \\
1955 \\
1956 \\
1957 \\
1958 \\
1959 \\
1960\end{array}$ & $\begin{array}{l}\text { Mar. } 7,1953 \\
\text { Mar. } 30,1954 \\
\text { Mar. 15, } 1955 \\
\text { Mpr. 2, } 1956 \\
\text { Mag } 3,1957 \\
\text { Mpr. 27, } 1958 \\
\text { Lpr. 11, } 1959 \\
\text { Mag } 3,1960\end{array}$ & $\begin{array}{c}2.29 \\
2.70 \\
3.015 \\
3.265 \\
- \\
- \\
2.50 \\
2.85\end{array}$ & $\begin{array}{r}24,500 \\
30,400 \\
40,700 \\
48,100 \\
266,200 \\
226,000 \\
24,400 \\
33,600\end{array}$ & $\begin{array}{l}\text { July 22, } 1953 \\
\text { Jan. 23, } 1954 \\
\text { Sopt. 3-7, } 1955 \\
\text { Oct. 2, } 1955 \\
\text { Oct. 1, } 1956 \\
\text { Aug. 20, } 1958 \\
\text { Sopt. 28, } 1959 \\
\text { Sopt. 1, } 1960\end{array}$ & $\begin{array}{l}.46 \\
.575 \\
.58 \\
.635 \\
.60 \\
.96 \\
.85 \\
.65\end{array}$ & $\begin{array}{l}1,620 \\
1,950 \\
1,540 \\
1,800 \\
2,330 \\
2,300 \\
2,180 \\
1,870\end{array}$ \\
\hline
\end{tabular}

a Man delis decharge.

Brerte.- Rucords good except those for periods of no gege helght rocord, which are fair. Many ll dirersions for irrigation above station. 
HETMLNE RIVER BASIV

Helmand River above Kajakai Reservoir, near Dehraout, Afghanistan

Discharge, in cubic feet per second, water year Octcber 1952 to September 1953

\begin{tabular}{|c|c|c|c|c|c|c|c|c|c|c|c|c|}
\hline $\mathrm{Day}$ & Oct. & Nov $_{0}$ & Dec. & Jan. & Feb. & Mar。 & Apr. & May & June & July & Aug. & Sept. \\
\hline $\begin{array}{l}1 \\
2 \\
3 \\
4 \\
5\end{array}$ & $\begin{array}{l}\frac{2,200}{2,200} \\
2,200 \\
2,200 \\
2,250\end{array}$ & $\begin{array}{l}\frac{2,650}{2,650} \\
2,650 \\
2,650 \\
2,650\end{array}$ & $\begin{array}{l}2,700 \\
2,700 \\
2,670 \\
2,670 \\
2,670\end{array}$ & $\begin{array}{l}2,670 \\
2,600 \\
2,440 \\
2,270 \\
2,240\end{array}$ & $\begin{array}{l}\frac{2,240}{2,440} \\
2,600 \\
2,740 \\
2,840\end{array}$ & $\begin{array}{l}\frac{6,230}{6,750} \\
7,160 \\
7,270 \\
7,710\end{array}$ & $\begin{array}{l}10,800 \\
10,600 \\
10,500 \\
10,600 \\
11,000\end{array}$ & $\begin{array}{l}\frac{15,400}{15,100} \\
15,000 \\
14,800 \\
14,400\end{array}$ & $\begin{array}{r}8,990 \\
9,720 \\
13,000 \\
12,600 \\
10,900\end{array}$ & $\begin{array}{l}\frac{2,670}{2,670} \\
2,670 \\
2,640 \\
2,510\end{array}$ & $\begin{array}{r}1,860 \\
1,860 \\
1,880 \\
* 1,880 \\
1,880\end{array}$ & $\begin{array}{l}1,790 \\
1,790 \\
1,790 \\
1,840 \\
1,820\end{array}$ \\
\hline $\begin{array}{r}6 \\
7 \\
8 \\
9 \\
10\end{array}$ & $\begin{array}{l}2,250 \\
2,250 \\
2,300 \\
2,300 \\
2,300\end{array}$ & $\begin{array}{l}2,650 \\
2,650 \\
2,650 \\
2,650 \\
2,650\end{array}$ & $\begin{array}{r}2,670 \\
2,700 \\
* 2,700 \\
2,640 \\
2,560\end{array}$ & $\begin{array}{l}2,210 \\
2,210 \\
2,240 \\
2,300 \\
2,470\end{array}$ & $\begin{array}{l}2,740 \\
2,740 \\
2,870 \\
3,220 \\
4,630\end{array}$ & $\begin{array}{l}11,400 \\
\frac{21,900}{19,400} \\
14,400 \\
12,300\end{array}$ & $\begin{array}{l}12,400 \\
u_{4}, 000 \\
u_{4}, 200 \\
15,000 \\
16,600\end{array}$ & $\begin{array}{l}13,900 \\
13,700 \\
13,700 \\
13,400 \\
13,000\end{array}$ & $\begin{array}{l}9,480 \\
8,500 \\
7,940 \\
7,270 \\
6,850\end{array}$ & $\begin{array}{l}2,500 \\
2,500 \\
2,530 \\
2,500 \\
2,470\end{array}$ & $\begin{array}{l}1,840 \\
1,840 \\
1,840 \\
1,860 \\
1,860\end{array}$ & $\begin{array}{r}* 1,790 \\
1,790 \\
1,820 \\
1,820 \\
1,830\end{array}$ \\
\hline $\begin{array}{l}11 \\
12 \\
13 \\
14 \\
15\end{array}$ & $\begin{array}{l}2,350 \\
2,400 \\
2,400 \\
2,450 \\
2,450\end{array}$ & $\begin{array}{l}2,650 \\
2,650 \\
2,650 \\
2,650 \\
2,650\end{array}$ & $\begin{array}{l}2,560 \\
2,560 \\
2,550 \\
2,600 \\
2,600\end{array}$ & $\begin{array}{l}2,530 \\
2,560 \\
2,640 \\
2,700 \\
2,670\end{array}$ & $\begin{array}{l}3,580 \\
4,800 \\
4,270 \\
5,110 \\
4,670\end{array}$ & $\begin{array}{r}11,400 \\
10,500 \\
* 9,720 \\
9,230 \\
9,600\end{array}$ & $\begin{array}{l}16,600 \\
18,300 \\
\frac{21,500}{19,600} \\
18,000\end{array}$ & $\begin{array}{r}13,000 \\
12,400 \\
12,100 \\
* 11,700 \\
11,400\end{array}$ & $\begin{array}{l}6,640 \\
6,640 \\
6,540 \\
6,430 \\
6,430\end{array}$ & $\begin{array}{l}2,470 \\
2,330 \\
2,270 \\
1,970 \\
1,840\end{array}$ & $\begin{array}{l}1,860 \\
1,930 \\
1,900 \\
1,860 \\
1,860\end{array}$ & $\begin{array}{l}1,840 \\
1,840 \\
1,850 \\
1,860 \\
1,870\end{array}$ \\
\hline $\begin{array}{l}16 \\
17 \\
18 \\
19 \\
20\end{array}$ & $\begin{array}{l}2,450 \\
2,450 \\
2,500 \\
2,500 \\
2,550\end{array}$ & $\begin{array}{l}2,650 \\
2,650 \\
2,810 \\
2,840 \\
2,870 \\
\end{array}$ & $\begin{array}{l}2,640 \\
2,600 \\
2,700 \\
2,740 \\
2,700\end{array}$ & $\begin{array}{l}2,600 \\
2,470 \\
2,330 \\
2,270 \\
2,360\end{array}$ & $\begin{array}{l}4,270 \\
3,990 \\
3,720 \\
3,540 \\
3,510\end{array}$ & $\begin{array}{l}9,600 \\
8,870 \\
8,390 \\
8,270 \\
8,270\end{array}$ & $\begin{array}{l}17,100 \\
16,800 \\
16,800 \\
16,600 \\
15,400\end{array}$ & $\begin{array}{r}11,200 \\
11,000 \\
10,800 \\
10,200 \\
9,600\end{array}$ & $\begin{array}{r}6,330 \\
5,850 \\
5,750 \\
5,660 \\
* 5,470\end{array}$ & $\begin{array}{l}1,750 \\
1,750 \\
1,710 \\
1,790 \\
1,660\end{array}$ & $\begin{array}{l}1,880 \\
1,930 \\
1,900 \\
1,880 \\
1,840\end{array}$ & $\begin{array}{l}1,880 \\
1,880 \\
1,890 \\
1,900 \\
1,900\end{array}$ \\
\hline $\begin{array}{l}21 \\
22 \\
23 \\
24 \\
25\end{array}$ & $\begin{array}{l}2,600 \\
2,600 \\
2,600 \\
2,600 \\
2,600\end{array}$ & $\begin{array}{l}2,740 \\
2,740 \\
2,700 \\
2,700 \\
2,700\end{array}$ & $\begin{array}{l}2,600 \\
2,440 \\
2,330 \\
2,360 \\
2,470\end{array}$ & $\begin{array}{l}2,330 \\
2,270 \\
2,330 \\
2,400 \\
2,1,40\end{array}$ & $\begin{array}{l}3,510 \\
3,720 \\
3,920 \\
3,950 \\
3,920\end{array}$ & $\begin{array}{r}8,500 \\
9,110 \\
12,400 \\
20,000 \\
19,000\end{array}$ & $\begin{array}{l}14,800 \\
1_{4}, 700 \\
14,700 \\
15,000 \\
15,100\end{array}$ & $\begin{array}{l}9,230 \\
8,870 \\
8,500 \\
8,050 \\
7,820 \\
\end{array}$ & $\begin{array}{l}5,660 \\
4,340 \\
3,510 \\
3,290 \\
3,180\end{array}$ & $\begin{array}{l}1,770 \\
1,680 \\
1,840 \\
1,950 \\
1,880\end{array}$ & $\begin{array}{l}1,820 \\
1,790 \\
1,840 \\
1,900 \\
1,880\end{array}$ & $\begin{array}{l}1,930 \\
1,930 \\
1,900 \\
1,900 \\
1,930\end{array}$ \\
\hline $\begin{array}{l}26 \\
27 \\
28 \\
29 \\
30 \\
31\end{array}$ & $\begin{array}{l}2,600 \\
2,600 \\
2,650 \\
2,650 \\
2,650 \\
2,650\end{array}$ & $\begin{array}{c}2,700 \\
2,670 \\
2,670 \\
2,670 \\
2,670 \\
-\end{array}$ & $\begin{array}{l}2,400 \\
2,470 \\
2,470 \\
2,400 \\
2,440 \\
2,600\end{array}$ & $\begin{array}{r}2,530 \\
* 2,740 \\
2,560 \\
2,400 \\
2,300 \\
2,180 \\
\end{array}$ & $\begin{array}{c}4,070 \\
4,850 \\
5,370 \\
- \\
- \\
-\end{array}$ & $\begin{array}{l}16,800 \\
15,100 \\
13,600 \\
12,400 \\
12,300 \\
11,000\end{array}$ & $\begin{array}{c}15,600 \\
* 15,800 \\
15,900 \\
15,900 \\
15,900 \\
-\end{array}$ & $\begin{array}{r}9,230 \\
12,200 \\
13,000 \\
11,400 \\
10,400 \\
9,720\end{array}$ & $\begin{array}{l}3,150 \\
2,870 \\
2,700 \\
2,670 \\
\frac{2,640}{-}\end{array}$ & $\begin{array}{l}1,880 \\
1,900 \\
1,880 \\
1,900 \\
1,930 \\
1,900\end{array}$ & $\begin{array}{l}1,880 \\
1,880 \\
1,860 \\
1,790 \\
1,790 \\
1,790\end{array}$ & $\begin{array}{l}1,930 \\
1,930 \\
1,930 \\
1,970 \\
1,970 \\
\end{array}$ \\
\hline $\begin{array}{l}\text { Total } \\
\text { Mean } \\
\text { Ac-ft }\end{array}$ & $\begin{array}{r}75,750 \\
2,444 \\
150,200\end{array}$ & $\begin{array}{r}80,530 \\
2,684 \\
159,700\end{array}$ & $\begin{array}{r}79,920 \\
2,578 \\
158,500\end{array}$ & $\begin{array}{r}75,260 \\
2,428 \\
148,300\end{array}$ & $\begin{array}{r}103,830 \\
5,708 \\
205,900\end{array}$ & $\begin{array}{r}358,580 \\
11,570 \\
711,200\end{array}$ & $\begin{array}{r}455,800 \\
15,190 \\
904,100\end{array}$ & $\begin{array}{r}364,220 \\
11,750 \\
722,400\end{array}$ & $\begin{array}{r}191,000 \\
6,367 \\
378,800\end{array}$ & $\begin{array}{r}65,910 \\
2,126 \\
130,700\end{array}$ & $\begin{array}{r}57,660 \\
1,860 \\
114,400\end{array}$ & $\begin{array}{r}56,110 \\
1,870 \\
111,300 \\
\end{array}$ \\
\hline
\end{tabular}

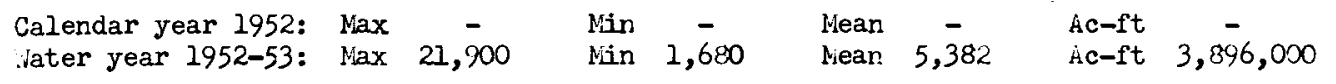

* Discharge measurement made or this day.

liote.--lio gage-height record Oct. 1 to liov. 17, sept. 10-18; discharge interpolated. 
HEIWLUD RIVIP BASIN

Helmand River above Kajakai Reservoir, near Dehraout, Afghanistan

Discharge, in cubic feet per secord, water year October 1953 to jeptember 1954

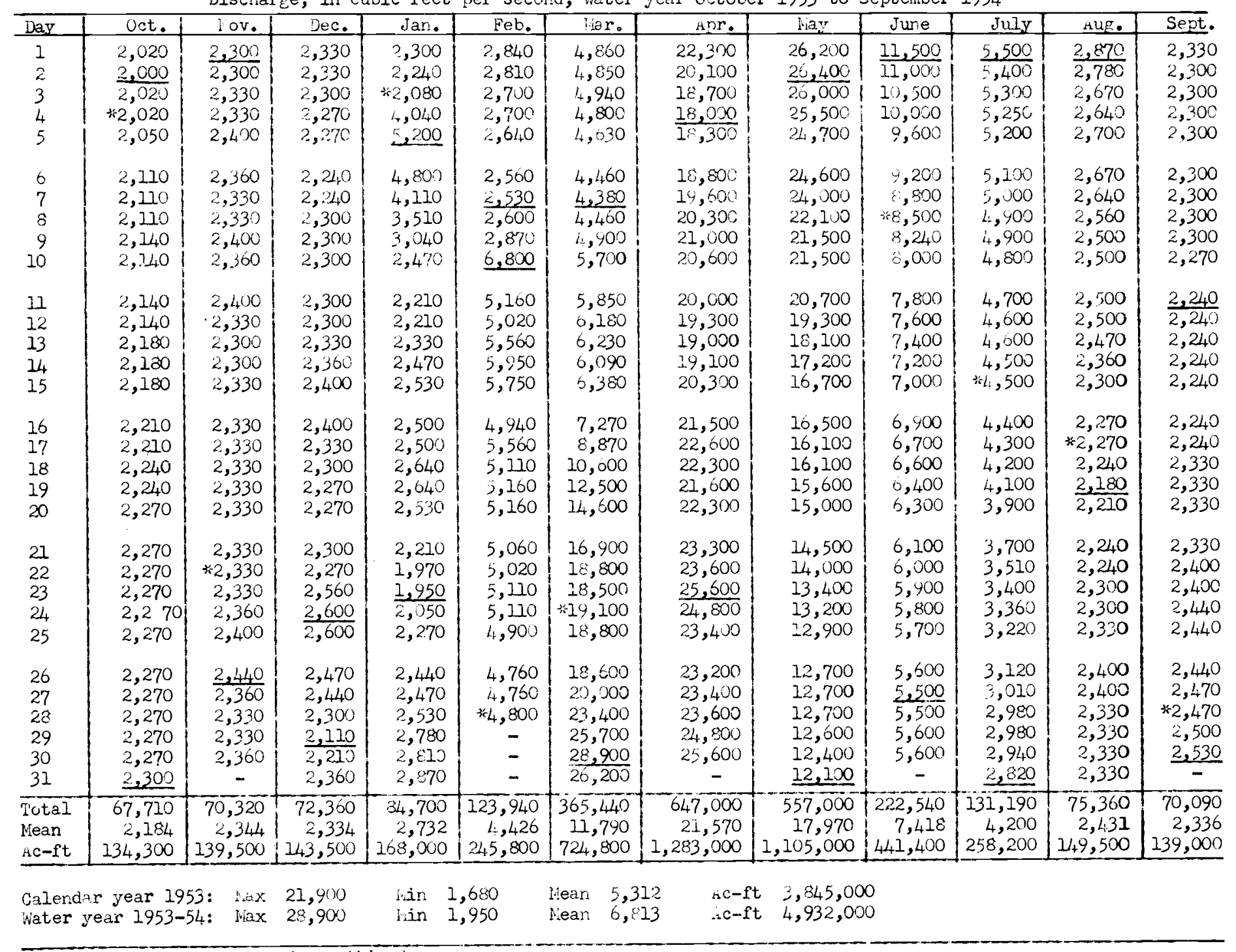

* Discharge measurement made on this day.

liote.--No gace-height record June 10 to Iuly 2l; dischisge interpolated. 
HeImenc River above rajakai Reservoir, near Dehraout, figharistan

Discherge, in cubic feet per secord, leter y ar catober 1054 to jeptenber 1955

\begin{tabular}{|c|c|c|c|c|c|c|c|c|c|c|c|c|}
\hline Day & Oct. & lov. & Dec. & $J E_{2} r_{2}$ & arb. & limer. & spr. & $\mathrm{H} 2 \mathrm{r}$ & June & July & $4 \mathrm{~s}^{\circ}$ & Sept. \\
\hline $\begin{array}{l}1 \\
2 \\
3 \\
4 \\
5\end{array}$ & $\begin{array}{l}2,540 \\
2,540 \\
2,510 \\
2,540 \\
2,600\end{array}$ & $\begin{array}{l}\frac{2,50}{2,850} \\
2,850 \\
2,850 \\
2,850\end{array}$ & $\begin{array}{r}\times 2,930 \\
3,000 \\
3,000 \\
3,0010 \\
3,100\end{array}$ & $\begin{array}{l}\frac{2,290}{2,290} \\
2,430 \\
2,540 \\
2,540\end{array}$ & $\begin{array}{r}2,570 \\
2,540 \\
* 2,680 \\
2,607 \\
2,050\end{array}$ & $\begin{array}{r}\frac{2,680}{* 2,500} \\
2,710 \\
2,710 \\
2,710\end{array}$ & $\begin{array}{r}10,200 \\
9,920 \\
+9,850 \\
10,200 \\
1 ;, 400\end{array}$ & $\begin{array}{l}13,000 \\
12,800 \\
12,700 \\
12,600 \\
16,800\end{array}$ & $\begin{array}{r}9,310 \\
+8,910 \\
9,850 \\
9,040 \\
8,910\end{array}$ & $\begin{array}{l}\frac{3,870}{3,800} \\
3,000 \\
3,800 \\
3,590\end{array}$ & $\begin{array}{l}2,070 \\
2,070 \\
\frac{2,100}{2,020} \\
1,920\end{array}$ & $\begin{array}{l}1,550 \\
1,560 \\
1,540 \\
1,540 \\
1,540\end{array}$ \\
\hline $\begin{array}{r}6 \\
7 \\
8 \\
9 \\
10\end{array}$ & $\begin{array}{l}2,620 \\
2,650 \\
2,620 \\
2,650 \\
2,650\end{array}$ & $\begin{array}{l}2,400 \\
2,900 \\
2,900 \\
2,900 \\
2,900\end{array}$ & $\begin{array}{l}3,000 \\
3,000 \\
3,000 \\
3,000 \\
3,000\end{array}$ & $\begin{array}{l}2,600 \\
2,510 \\
2,600 \\
2,570 \\
2,480\end{array}$ & $\begin{array}{l}2,620 \\
2,600 \\
2,570 \\
2,540 \\
2,540\end{array}$ & $\begin{array}{l}2,810 \\
2,840 \\
2,900 \\
3,030 \\
3,490\end{array}$ & $\begin{array}{l}10,300 \\
10,500 \\
10,500 \\
20,300 \\
10,800\end{array}$ & $\begin{array}{l}20,500 \\
\frac{21,200}{18,600} \\
16,400 \\
16,000\end{array}$ & $\begin{array}{l}8,670 \\
8,320 \\
8,200 \\
8,080 \\
8,080\end{array}$ & $\begin{array}{l}3,450 \\
3,380 \\
3,310 \\
3,280 \\
3,250\end{array}$ & $\begin{array}{l}1,830 \\
1,780 \\
1,730 \\
1,730 \\
1,700\end{array}$ & $\begin{array}{l}1,540 \\
1,540 \\
1,550 \\
1,550 \\
1,560\end{array}$ \\
\hline $\begin{array}{l}11 \\
12 \\
13 \\
14 \\
15\end{array}$ & $\begin{array}{l}2,650 \\
2,650 \\
2,680 \\
2,680 \\
2,710\end{array}$ & $\begin{array}{l}2,900 \\
2,900 \\
2,900 \\
2,900 \\
2,900\end{array}$ & $\begin{array}{l}2,900 \\
2,900 \\
2,900 \\
2,800 \\
2,700\end{array}$ & $\begin{array}{r}2,400 \\
2,430 \\
* 2,540 \\
2,430 \\
2,340\end{array}$ & $\begin{array}{l}2,480 \\
2,480 \\
2,400 \\
2,430 \\
2,460\end{array}$ & $\begin{array}{r}4,210 \\
5,820 \\
6,120 \\
19,000 \\
38,700 \\
\end{array}$ & $\begin{array}{l}11,400 \\
11,600 \\
13,200 \\
11,500 \\
10,200\end{array}$ & $\begin{array}{l}15,200 \\
14,700 \\
13,600 \\
12,700 \\
12,200\end{array}$ & $\begin{array}{l}7,960 \\
7,960 \\
7,720 \\
7,510 \\
7,180\end{array}$ & $\begin{array}{l}3,200 \\
3,100 \\
3,000 \\
2,900 \\
2,800\end{array}$ & $\begin{array}{l}1,660 \\
1,660 \\
1,610 \\
1,590 \\
1,610\end{array}$ & $\begin{array}{l}1,580 \\
1,600 \\
1,600 \\
1,600 \\
1,600\end{array}$ \\
\hline $\begin{array}{l}16 \\
17 \\
18 \\
19 \\
20\end{array}$ & $\begin{array}{l}2,740 \\
2,740 \\
2,740 \\
2,740 \\
2,780\end{array}$ & $\begin{array}{l}\frac{2,950}{2,950} \\
2,950 \\
2,950 \\
2,950\end{array}$ & $\begin{array}{l}2,600 \\
2,500 \\
2,500 \\
2,430 \\
2,320\end{array}$ & $\begin{array}{l}2,370 \\
2,510 \\
2,510 \\
2,540 \\
2,570\end{array}$ & $\begin{array}{l}2,510 \\
2,540 \\
2,540 \\
2,570 \\
2,650\end{array}$ & $\begin{array}{r}27,600 \\
16,000 \\
12,200 \\
10,200 \\
9,240\end{array}$ & $\begin{array}{l}9,450 \\
8,910 \\
8,550 \\
8,320 \\
8,320\end{array}$ & $\begin{array}{l}11,800 \\
11,400 \\
11,200 \\
11,200 \\
11,300\end{array}$ & $\begin{array}{l}6,860 \\
6,360 \\
6,160 \\
5,800 \\
5,500\end{array}$ & $\begin{array}{l}2,700 \\
2,550 \\
2,450 \\
2,340 \\
2,230\end{array}$ & $\begin{array}{l}1,610 \\
1,610 \\
1,610 \\
1,630 \\
1,700\end{array}$ & $\begin{array}{l}1,650 \\
1,650 \\
1,700 \\
1,700 \\
1,700\end{array}$ \\
\hline $\begin{array}{l}21 \\
22 \\
23 \\
24 \\
25\end{array}$ & $\begin{array}{l}2,810 \\
2,810 \\
2,810 \\
2,810 \\
2,810\end{array}$ & $\begin{array}{l}2,950 \\
2,950 \\
2,950 \\
2,950 \\
2,950\end{array}$ & $\begin{array}{l}2,290 \\
2,340 \\
2,460 \\
2,510 \\
2,570\end{array}$ & $\begin{array}{l}2,540 \\
2,600 \\
2,620 \\
2,600 \\
2,540\end{array}$ & $\begin{array}{l}2,650 \\
2,650 \\
2,680 \\
2,710 \\
2,710\end{array}$ & $\begin{array}{l}9,040 \\
8,790 \\
8,380 \\
8,030 \\
7,960\end{array}$ & $\begin{array}{r}8,430 \\
8,550 \\
8,790 \\
9,310 \\
10,100\end{array}$ & $\begin{array}{l}11,600 \\
11,800 \\
12,100 \\
12,400 \\
12,700\end{array}$ & $\begin{array}{l}5,300 \\
5,100 \\
4,900 \\
4,700 \\
4,600\end{array}$ & $\begin{array}{l}2,230 \\
2,230 \\
2,180 \\
2,180 \\
2,180\end{array}$ & $\begin{array}{r}1,700 \\
1,700 \\
1,680 \\
1,590 \\
* 1,590\end{array}$ & $\begin{array}{l}1,750 \\
1,750 \\
1,800 \\
1,800 \\
1,800\end{array}$ \\
\hline $\begin{array}{l}26 \\
27 \\
28 \\
29 \\
30 \\
31\end{array}$ & $\begin{array}{l}2,810 \\
2,810 \\
2,810 \\
2,840 \\
\frac{2,850}{2,850}\end{array}$ & $\begin{array}{c}2,950 \\
2,950 \\
2,950 \\
2,950 \\
2,950 \\
-\end{array}$ & $\begin{array}{l}2,510 \\
2,510 \\
2,570 \\
2,480 \\
2,340 \\
2,200 \\
\end{array}$ & $\begin{array}{l}2,430 \\
2,290 \\
2,340 \\
2,540 \\
2,620 \\
2,570\end{array}$ & $\begin{array}{c}2,680 \\
2,710 \\
2,710 \\
-\end{array}$ & $\begin{array}{r}7,960 \\
8,080 \\
8,080 \\
11,100 \\
12,000 \\
11,200\end{array}$ & $\begin{array}{c}10,800 \\
12,100 \\
12,600 \\
\kappa 12,900 \\
13,000 \\
-\end{array}$ & $\begin{array}{r}13,100 \\
12,700 \\
11,300 \\
10,700 \\
10,200 \\
9,710\end{array}$ & $\begin{array}{r}4,500 \\
4,400 \\
4,200 \\
4,100 \\
* 4,010 \\
\end{array}$ & $\begin{array}{r}* 2,120 \\
2,150 \\
2,120 \\
2,120 \\
2,070 \\
2,070\end{array}$ & $\begin{array}{l}1,590 \\
1,560 \\
1,560 \\
1,590 \\
1,590 \\
1,590\end{array}$ & $\begin{array}{c}1,850 \\
1,850 \\
1,900 \\
1,900 \\
1,900 \\
-\end{array}$ \\
\hline $\begin{array}{l}\text { Total } \\
\text { Jean } \\
\text { Ac-ft }\end{array}$ & $\begin{array}{r}84,050 \\
2,711 \\
166,700 \\
\end{array}$ & $\begin{array}{r}87,500 \\
2,917 \\
173,600 \\
\end{array}$ & $\begin{array}{r}33,360 \\
2,689 \\
165,300 \\
\end{array}$ & $\begin{array}{r}77,090 \\
2,487 \\
152,900 \\
\end{array}$ & $\begin{array}{r}72,470 \\
2,588 \\
143,700 \\
\end{array}$ & $\begin{array}{r}278,310 \\
8,978 \\
552,000 \\
\end{array}$ & $\begin{array}{r}314,500 \\
10,480 \\
623,800\end{array}$ & $\begin{array}{r}414,210 \\
13,360 \\
821,600\end{array}$ & $\begin{array}{r}201,190 \\
6,706 \\
399,100 \\
\end{array}$ & $\begin{array}{r}86,450 \\
2,789 \\
171,500 \\
\end{array}$ & $\begin{array}{r}52,980 \\
1,709 \\
105,100\end{array}$ & $\begin{array}{r}50,160 \\
1,672 \\
99,490\end{array}$ \\
\hline
\end{tabular}

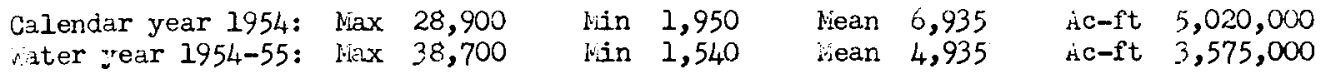

* Discharge measurement made on this day.

lote.-No gage-height record Oct. 30 to Lec. 1\%, Jure 19-29, July 10-18, jept. 7-30; discharge interpolated or

estinated. 
DLLUAD RIVIP BASIN

Helrand River above Kojakai Seservoir, rear Dehraout, ffghenisten

Discharge, in cubic feet per second, water we jober 1955 to sentenber 1956

\begin{tabular}{|c|c|c|c|c|c|c|c|c|c|c|c|c|}
\hline Day & vet. & $\mathrm{NOV}_{4}$ & Dec. & $\tan$ & Feb. & Mar. & Anr. & $\sqrt{13}=$ & June & July & Aug. & $3 e D^{t}$. \\
\hline 1 & 1,900 & $\underline{2,260}$ & 2,150 & 2,740 & 2,620 & 4,980 & 25,300 & 24,100 & 7,300 & 3,620 & 3,510 & 2,240 \\
\hline 2 & $* \overline{1}, 920$ & 2,260 & $* 1,120$ & 2,780 & 2,680 & 4,800 & 39,000 & 23,200 & 7,100 & 3,480 & 3,150 & 2,270 \\
\hline 3 & 1,920 & 2,260 & $\overrightarrow{2,120}$ & $\therefore, 840$ & 2,680 & $5,51,0$ & 43,800 & 22,600 & 6,800 & 3,440 & 2,870 & 2,300 \\
\hline 4 & 1,920 & 2,260 & 2,150 & 2,870 & 2,680 & 8,300 & 36,500 & 21,900 & 6,000 & 3,320 & 2,670 & 2,300 \\
\hline 5 & 1,940 & 2,260 & 2,180 & 2,040 & 2,620 & 13,800 & 33,500 & 21,300 & $6,4,00$ & 3,290 & 2,530 & 2,300 \\
\hline 6 & 1,940 & 2,260 & 2,180 & 2,810 & 2,600 & 18,100 & 32,500 & 20,500 & 6,200 & 3,220 & 2,360 & $?, 300$ \\
\hline 7 & 1,970 & 2,260 & 2,200 & 2,620 & 3,570 & $* 15,000$ & 31,600 & 19,800 & 6,000 & 3,180 & 2,330 & 2,330 \\
\hline 8 & 1,970 & 2,230 & 2,260 & 2,510 & 2,570 & 11,900 & 31,900 & 19,000 & 5,800 & 3,120 & 2,360 & 2,270 \\
\hline 9 & 2,020 & 2,230 & 2,260 & 2,510 & 2,570 & 10,600 & 30,300 & $* 18,200$ & 5,600 & 3,080 & 2,270 & 2,300 \\
\hline 10 & 2,040 & 2,230 & 2,230 & 2,600 & 2,600 & 10,500 & 29,000 & 18,100 & 5,400 & 3,010 & 2,240 & 2,300 \\
\hline 11 & 2,040 & 2,230 & 2,260 & 2,680 & 2,650 & 10,700 & 29,200 & 17,600 & 5,200 & 2,940 & $\therefore, 210$ & 2,300 \\
\hline 12 & 2,040 & 2,200 & 2,290 & 2,780 & 2,710 & $1], 200$ & 30,700 & 17,000 & 5,100 & 2,870 & 2,180 & 2,270 \\
\hline 10 & 2,040 & 2,230 & 2,320 & 2,740 & 2,810 & 11,000 & 32,800 & 16,600 & 5,000 & 2,810 & $\overline{2,180}$ & 2,270 \\
\hline 14 & 2,070 & 2,180 & 2,340 & 2,710 & 2,870 & 10,600 & 34,400 & 15,800 & 4,900 & 2,740 & 2,180 & 2,300 \\
\hline 15 & 2,100 & 2,180 & 2,400 & 2,780 & 2,870 & 10,500 & 34,900 & 15,000 & 4,800 & 2,700 & 2,210 & 2,270 \\
\hline 16 & 2,150 & 2,200 & 2,400 & 2,960 & 2,680 &, 380 & 35, & 14,200 & 4,800 & 2,870 & 2,210 & 2,300 \\
\hline 17 & 2,180 & 2,200 & 2,400 & 3,420 & 2,680 & 9,450 & 35,900 & 13,500 & 4,700 & 7,920 & 2,210 & 2,240 \\
\hline 18 & 2,180 & 2,200 & 2,400 & 3.840 & 2,600 & 9,450 & 36,100 & 13,000 & 4,600 & 6,750 & 2,210 & 2,240 \\
\hline 19 & 2,180 & 2,200 & 2,430 & 3,420 & 2,650 & 12,100 & 38,000 & 12,400 & 4,500 & 6,230 & 2,210 & 2,240 \\
\hline 20 & 2,150 & 2,180 & 2,460 & 3,150 & 2,740 & 18,200 & 37,000 & 11,900 & 4,500 & 5,750 & 2,210 & 2,270 \\
\hline 21 & 2,150 & 2,150 & 2,480 & 2,900 & 2,810 & 15,8 & 38,000 & 11,400 & 4,400 & 5,560 & 2,210 & 2,270 \\
\hline 22 & 2,150 & $\overline{2,150}$ & 2,480 & 2,810 & 2,840 & 14,100 & 35,400 & 11,000 & 4,300 & 5,470 & 2,210 & 2,270 \\
\hline 23 & 2,150 & 2,150 & 2,480 & 2,810 & 2,900 & 13,000 & 33,000 & 10,800 & 4,200 & 5,320 & 2,210 & 2,270 \\
\hline 24 & 2,150 & 2,150 & 2,460 & 2,810 & 2,960 & 13,200 & 31,800 & 10,500 & 4,100 & 13,300 & 2,210 & 2,270 \\
\hline 25 & 2,200 & 2,180 & 2,510 & 2,740 & 4,090 & 13,200 & 31,100 & 10,000 & 4,000 & $\overline{12,100}$ & 2,240 & 2,270 \\
\hline 26 & 2,230 & 2,180 & 2,540 & 2,740 & 6,710 & 13,300 & 29,400 & 9,500 & 4,000 & 10,800 & 2,240 & 2,270 \\
\hline 27 & 2,260 & 2,180 & 2,570 & 2,780 & 7,610 & 13,200 & 27,200 & 4,000 & 3,900 & 12,400 & 2,210 & 2,300 \\
\hline 28 & $\overline{2,260}$ & 2,180 & 2,570 & 2,780 & 6,160 & 13,100 & 26,300 & 8,700 & 3,800 & 11,700 & 2,210 & 2,300 \\
\hline 29 & 2,260 & 2,180 & 2,800 & $* 2,710$ & 5,380 & $* 13,800$ & 25,900 & 8,200 & 3,800 & 7,710 & 2,240 & 2,330 \\
\hline 30 & 2,230 & 2,180 & $\approx 2,620$ & 2,620 & - & 17,100 & 25,200 & 8,000 & $* 3,720$ & 6,330 & $\because 2,210$ & $* 2,330$ \\
\hline 31 & $* 2,260$ & - & 2,680 & 2,600 & - & 20,500 & - & 7,600 & & $* 3,950$ & 2,240 & \\
\hline Total & 64,970 & 65,190 & 73,540 & 87,900 & 94,910 & 376,480 & 981,100 & 460,400 & 151,520 & 170,980 & 73,090 & 68,490 \\
\hline lwean & 2,096 & 2,206 & 2,372 & 2,838 & 3,273 & 12,140 & 32,700 & 14,850 & 5,051 & 5,515 & 2,358 & 2,283 \\
\hline $\mathrm{Ac}-\mathrm{ft}$ & 128,900 & 131,300 & 145,900 & 174,300 & 288,300 & 746,700 & $1,946,000$ & 913,200 & 300,500 & 339,100 & 145,000 & 135,800 \\
\hline
\end{tabular}

Calendar year 1955: lax 38,700 livin 1,540 liean 4,800 Ac-ft 3,475,000 iater year 1955-56: hex 43,800 lin 1,900 liean 7,294 Ac-ft 5,295,000

* Discriarge measurerient made or this day.

licte.-lo gage-leight record apr. 19-21, ilay 24 to Jure 29; discharge computed on basis of change in conterts anc release frota Kajakai lteservir. 
HELMAND RIVEP. BASIN

Helmand River above Kajakai leservoir, near Dehraout, Af thanisten

Discharge, in cubic feet per second, water jear October 1956 to September 1957

\begin{tabular}{|c|c|c|c|c|c|c|c|c|c|c|c|c|}
\hline Day & Oct. & Nov. & Dec, 1 & $\operatorname{Jan} \cdot 1$ & Feb. 1 & Mar. & Apr. & Miarr & June & July & Aug. & Sept. \\
\hline $\begin{array}{l}1 \\
2 \\
3 \\
4 \\
5\end{array}$ & $\begin{array}{l}2,300 \\
2,400 \\
2,470 \\
2,560 \\
2,530\end{array}$ & $\begin{array}{r}3,000 \\
3,050 \\
3,000 \\
* 2,980 \\
2,900\end{array}$ & $\begin{array}{r}2,640 \\
2,600 \\
2,600 \\
* 2,530 \\
2,500\end{array}$ & $\begin{array}{l}2,670 \\
2,700 \\
2,700 \\
2,670 \\
2,640\end{array}$ & $\begin{array}{l}3,580 \\
4,230 \\
4,340 \\
4,340 \\
4,340\end{array}$ & $\begin{array}{l}\frac{4,900}{5,160} \\
5,800 \\
6,090 \\
6,230\end{array}$ & $\begin{array}{l}\frac{24,200}{24,200} \\
24,200 \\
42,200 \\
55,000\end{array}$ & $\begin{array}{l}44,200 \\
59,200 \\
66,200 \\
63,200 \\
56,200\end{array}$ & $\begin{array}{l}\frac{29,200}{28,200} \\
27,200 \\
25,200 \\
23,200\end{array}$ & $\begin{array}{l}\frac{8,800}{9,500} \\
8,300 \\
8,200 \\
8,100\end{array}$ & $\begin{array}{l}4,000 \\
3,800 \\
3,700 \\
3,700 \\
3,500\end{array}$ & $\begin{array}{l}\frac{2,600}{2,600} \\
2,600 \\
2,600 \\
2,600\end{array}$ \\
\hline $\begin{array}{r}6 \\
7 \\
8 \\
9 \\
10\end{array}$ & $\begin{array}{l}2,530 \\
2,530 \\
2,560 \\
2,560 \\
2,600\end{array}$ & $\begin{array}{l}2,900 \\
2,870 \\
2,840 \\
2,840 \\
2,810\end{array}$ & $\begin{array}{l}2,500 \\
2,500 \\
2,500 \\
2,530 \\
2,500\end{array}$ & $\begin{array}{l}2,560 \\
2,400 \\
2,360 \\
2,560 \\
2,670\end{array}$ & $\begin{array}{r}4,190 \\
* 3,960 \\
4,230 \\
3,800 \\
3,540\end{array}$ & $\begin{array}{r}6,590 \\
7,220 \\
7,880 \\
9,230 \\
10,300\end{array}$ & $\begin{array}{l}42,500 \\
39,200 \\
36,200 \\
35,200 \\
34,200\end{array}$ & $\begin{array}{l}50,200 \\
47,200 \\
46,200 \\
46,200 \\
48,200\end{array}$ & $\begin{array}{l}22,200 \\
21,200 \\
21,200 \\
20,200 \\
20,200\end{array}$ & $\begin{array}{l}7,900 \\
7,700 \\
7,500 \\
7,300 \\
7,200\end{array}$ & $\begin{array}{l}3,500 \\
3,400 \\
3,400 \\
3,400 \\
3,300\end{array}$ & $\begin{array}{l}2,600 \\
2,600 \\
2,600 \\
2,600 \\
2,600\end{array}$ \\
\hline $\begin{array}{l}17 \\
12 \\
13 \\
14 \\
15\end{array}$ & $\begin{array}{l}2,600 \\
2,640 \\
2,620 \\
2,620 \\
2,620\end{array}$ & $\begin{array}{l}2,810 \\
2,810 \\
2,810 \\
2,780 \\
2,780\end{array}$ & $\begin{array}{l}2,560 \\
2,600 \\
2,600 \\
2,560 \\
2,560\end{array}$ & $\begin{array}{l}2,500 \\
2,470 \\
2,900 \\
2,900 \\
2,870\end{array}$ & $\begin{array}{l}3,540 \\
3,540 \\
3,510 \\
4,030 \\
4,030\end{array}$ & $\begin{array}{r}9,980 \\
9,290 \\
* 8,990 \\
9,170 \\
10,100\end{array}$ & $\begin{array}{l}38,200 \\
41,200 \\
44,200 \\
49,200 \\
49,200\end{array}$ & $\begin{array}{l}50,200 \\
47,200 \\
44,200 \\
41,200 \\
38,200\end{array}$ & $\begin{array}{l}19,200 \\
18,200 \\
17,200 \\
16,200 \\
16,200\end{array}$ & $\begin{array}{l}7,000 \\
6,800 \\
6,600 \\
6,400 \\
6,200\end{array}$ & $\begin{array}{l}3,400 \\
3,400 \\
3,400 \\
3,400 \\
3,400\end{array}$ & $\begin{array}{l}2,600 \\
2,600 \\
2,600 \\
2,600 \\
2,600\end{array}$ \\
\hline $\begin{array}{l}16 \\
17 \\
18 \\
19 \\
20\end{array}$ & $\begin{array}{l}2,620 \\
2,620 \\
2,620 \\
2,620 \\
2,620\end{array}$ & $\begin{array}{l}2,740 \\
2,740 \\
2,740 \\
2,700 \\
2,700\end{array}$ & $\begin{array}{l}2,530 \\
2,360 \\
2,400 \\
2,530 \\
2,560\end{array}$ & $\begin{array}{l}2,870 \\
2,870 \\
2,740 \\
2,780 \\
2,780\end{array}$ & $\begin{array}{l}4,030 \\
4,110 \\
4,110 \\
4,340 \\
4,340\end{array}$ & $\begin{array}{l}11,200 \\
37,000 \\
55,000 \\
34,200 \\
27,300\end{array}$ & $\begin{array}{l}46,200 \\
43,200 \\
42,200 \\
43,200 \\
44,200\end{array}$ & $\begin{array}{l}38,200 \\
38,200 \\
30,200 \\
35,200 \\
35,200\end{array}$ & $\begin{array}{l}15,200 \\
15,200 \\
14,200 \\
13,200 \\
13,200\end{array}$ & $\begin{array}{l}6,000 \\
5,800 \\
5,800 \\
5,600 \\
5,400\end{array}$ & $\begin{array}{l}3,200 \\
3,200 \\
3,200 \\
3,200 \\
3,000\end{array}$ & $\begin{array}{l}2,600 \\
2,600 \\
2,600 \\
2,600 \\
2,600\end{array}$ \\
\hline $\begin{array}{l}21 \\
22 \\
23 \\
24 \\
25\end{array}$ & $\begin{array}{l}2,620 \\
2,620 \\
2,620 \\
2,620 \\
2,620\end{array}$ & $\begin{array}{l}2,670 \\
2,670 \\
2,670 \\
2,640 \\
2,640\end{array}$ & $\begin{array}{r}2,560 \\
2,530 \\
2,560 \\
* 2,600 \\
2,700 \\
\end{array}$ & $\begin{array}{l}2,980 \\
3,180 \\
3,400 \\
4,430 \\
5,050 \\
\end{array}$ & $\begin{array}{l}4,270 \\
4,230 \\
4,190 \\
3,880 \\
3,880\end{array}$ & $\begin{array}{l}24,400 \\
23,400 \\
20,400 \\
19,400 \\
19,500\end{array}$ & $\begin{array}{l}44,200 \\
44,200 \\
44,200 \\
40,200 \\
38,200\end{array}$ & $\begin{array}{l}34,200 \\
34,200 \\
33,200 \\
31,200 \\
30,200\end{array}$ & $\begin{array}{l}12,200 \\
12,200 \\
11,200 \\
11,200 \\
11,200\end{array}$ & $\begin{array}{l}5,400 \\
5,200 \\
5,000 \\
5,000 \\
4,800\end{array}$ & $\begin{array}{l}3,000 \\
2,900 \\
2,900 \\
2,800 \\
2,800\end{array}$ & $\begin{array}{l}\frac{2,700}{2,700} \\
2,700 \\
2,700 \\
2,700\end{array}$ \\
\hline $\begin{array}{l}26 \\
27 \\
28 \\
29 \\
30 \\
31\end{array}$ & $\begin{array}{l}2,610 \\
2,610 \\
2,700 \\
2,700 \\
2,730 \\
2,880 \\
\end{array}$ & $\begin{array}{l}2,640 \\
2,640 \\
2,640 \\
2,640 \\
2,640\end{array}$ & $\begin{array}{l}2,670 \\
2,330 \\
2,210 \\
2,210 \\
2,400 \\
2,560\end{array}$ & $\begin{array}{l}5,750 \\
4,760 \\
4,340 \\
3,720 \\
3,400 \\
3,440\end{array}$ & $\begin{array}{l}4,1.10 \\
4,720 \\
4,850 \\
\end{array}$ & $\begin{array}{l}18,500 \\
21,500 \\
29,400 \\
28,100 \\
26,100 \\
26,200\end{array}$ & $\begin{array}{l}38,200 \\
39,200 \\
37,200 \\
38,200 \\
37,200\end{array}$ & $\begin{array}{l}\frac{28,200}{28,200} \\
28,200 \\
28,200 \\
28,200 \\
29,200\end{array}$ & $\begin{array}{r}10,200 \\
9,200 \\
9,200 \\
9,200 \\
9,200\end{array}$ & $\begin{array}{l}4,600 \\
4,500 \\
4,400 \\
4,400 \\
4,200 \\
4,000 \\
\end{array}$ & $\begin{array}{l}2,700 \\
2,700 \\
2,500 \\
2,600 \\
2,600 \\
2,600\end{array}$ & $\begin{array}{l}2,700 \\
2,700 \\
2,700 \\
2,700 \\
2,700\end{array}$ \\
\hline $\begin{array}{l}\text { Total } \\
\text { Miean } \\
\text { Ac-ft }\end{array}$ & $\begin{array}{r}80,630 \\
2,601 \\
159,900 \\
\end{array}$ & $\begin{array}{r}83,290 \\
2,776 \\
165,200 \\
\end{array}$ & $\begin{array}{r}77,990 \\
2,516 \\
154,700 \\
\end{array}$ & $\begin{array}{r}101,060 \\
3,260 \\
300,400 \\
\end{array}$ & $\begin{array}{r}11_{4}, 200 \\
4,081 \\
226,500 \\
\end{array}$ & $\begin{array}{r}638,530 \\
17,370 \\
1,068,000 \\
\end{array}$ & $\begin{array}{r}1,199,100 \\
39,970 \\
2,372,000 \\
\end{array}$ & $\begin{array}{r}1, ? 64,200 \\
40,790 \\
2,500,000 \\
\end{array}$ & $\begin{array}{r}501,000 \\
16,700 \\
993,700 \\
\end{array}$ & $\begin{array}{r}192,700 \\
6,216 \\
382,200 \\
\end{array}$ & $\begin{array}{r}98,700 \\
3,1 \% 4 \\
195,800 \\
\end{array}$ & $\begin{array}{r}79,000 \\
2,633 \\
156,700 \\
\end{array}$ \\
\hline fate & & Fiax & $\begin{array}{l}43,800 \\
66,200\end{array}$ & $\begin{array}{ll}\operatorname{Min} & 2 \\
\min & 2\end{array}$ & $\begin{array}{l}80 \\
10\end{array}$ & $\begin{array}{r}7,396 \\
\text { n } \\
11,860\end{array}$ & $\begin{array}{l}A c-f t \\
i c-f t\end{array}$ & $\begin{array}{l}5,369,000 \\
8,589,000\end{array}$ & & & & \\
\hline
\end{tabular}

* Discharge measurement mide on this day.

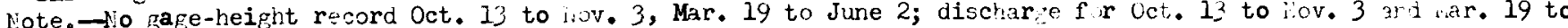

sent. 30 conputed on basis of cranje in contents and relesse from kajakai Reservoir. 
Helmand River above Kajakai Reservoir, rear Dehraout, Hf hanistan

Dischare, in cubic feet ner seconc, water year October 1957 to septenber 1958

\begin{tabular}{|c|c|c|c|c|c|c|c|c|c|c|c|c|}
\hline Day & Oct. & Nov. & Dec. & Jar. & Feb. & $\mathrm{Nar}$ & Apr. & May & June & July & 슬. & Sept. \\
\hline $\begin{array}{l}1 \\
2 \\
3 \\
4 \\
5\end{array}$ & $\begin{array}{l}\frac{2,740}{2,740} \\
2,91,0 \\
2,940 \\
3,080\end{array}$ & $\begin{array}{l}3,880 \\
4,030 \\
4,030 \\
3,990 \\
3,950\end{array}$ & $\begin{array}{l}4,600 \\
5,000 \\
5,800 \\
6,000 \\
5,600\end{array}$ & $\begin{array}{l}4,400 \\
4,300 \\
4,300 \\
4,230 \\
4,110\end{array}$ & $\begin{array}{l}5,700 \\
6,800 \\
0,180 \\
0,000 \\
5,750\end{array}$ & $\begin{array}{l}5,750 \\
5,850 \\
5,800 \\
5,660 \\
5,560\end{array}$ & $\begin{array}{l}17,100 \\
17,600 \\
17,200 \\
\frac{16,800}{17,400}\end{array}$ & $\begin{array}{l}\frac{19,700}{19,000} \\
18,400 \\
17,800 \\
17,200\end{array}$ & $\begin{array}{l}8,560 \\
8,330 \\
8,270 \\
8,270 \\
8,270\end{array}$ & $\begin{array}{l}\frac{l_{1}, 100}{t_{2}, 000} \\
3,900 \\
3,860 \\
3,890\end{array}$ & $\begin{array}{l}\frac{2,660}{2,640} \\
2,600 \\
2,560 \\
2,540\end{array}$ & $\begin{array}{l}\frac{2,360}{2,360} \\
2,380 \\
2,380 \\
2,360\end{array}$ \\
\hline $\begin{array}{r}6 \\
7 \\
8 \\
9 \\
10\end{array}$ & $\begin{array}{l}3,150 \\
3,150 \\
3,220 \\
3,220 \\
3,220\end{array}$ & $\begin{array}{l}4,170 \\
4,420 \\
4,340 \\
4,340 \\
4,230\end{array}$ & $\begin{array}{l}5,400 \\
5,280 \\
5,320 \\
5,240 \\
5,560\end{array}$ & $\begin{array}{l}4,150 \\
4,300 \\
4,460 \\
4,670 \\
5,020\end{array}$ & $\begin{array}{l}5,700 \\
5,660 \\
5,560 \\
5,520 \\
5,280\end{array}$ & $\begin{array}{r}5,520 \\
5,700 \\
6,040 \\
7,270 \\
10,600\end{array}$ & $\begin{array}{l}18,100 \\
18,800 \\
19,300 \\
19,300 \\
18,800\end{array}$ & $\begin{array}{l}16,600 \\
16,200 \\
15,300 \\
14,700 \\
13,600\end{array}$ & $\begin{array}{l}8,100 \\
8,050 \\
7,800 \\
7,600 \\
7,300\end{array}$ & $\begin{array}{l}3,830 \\
3,770 \\
3,650 \\
3,650 \\
3,600\end{array}$ & $\begin{array}{l}2,520 \\
2,480 \\
2,460 \\
2,460 \\
2,460\end{array}$ & $\begin{array}{l}2,380 \\
2,380 \\
2,360 \\
2,380 \\
2,380\end{array}$ \\
\hline $\begin{array}{l}11 \\
12 \\
13 \\
14 \\
15\end{array}$ & $\begin{array}{l}3,360 \\
3,360 \\
3,360 \\
3,510 \\
3,510\end{array}$ & $\begin{array}{l}4,150 \\
4,190 \\
4,300 \\
4,340 \\
4,270\end{array}$ & $\begin{array}{l}\frac{6,230}{5,900} \\
5,560 \\
5,200 \\
5,020\end{array}$ & $\begin{array}{l}5,020 \\
5,060 \\
5,060 \\
4,960 \\
4,900\end{array}$ & $\begin{array}{l}5,170 \\
4,980 \\
4,900 \\
4,900 \\
4,900\end{array}$ & $\begin{array}{l}10,700 \\
10,800 \\
10,700 \\
10,600 \\
10,400\end{array}$ & $\begin{array}{l}18,200 \\
18,100 \\
18,200 \\
18,700 \\
19,000\end{array}$ & $\begin{array}{l}13,900 \\
12,900 \\
11,400 \\
12,400 \\
12,400\end{array}$ & $\begin{array}{l}7,200 \\
7,200 \\
7,100 \\
7,000 \\
6,800\end{array}$ & $\begin{array}{l}3,570 \\
3,520 \\
3,710 \\
3,890 \\
3,540\end{array}$ & $\begin{array}{l}2,420 \\
2,420 \\
2,4,40 \\
2,420 \\
2,380\end{array}$ & $\begin{array}{l}2,380 \\
2,400 \\
2,400 \\
2,400 \\
2,400\end{array}$ \\
\hline $\begin{array}{l}16 \\
17 \\
18 \\
19 \\
20\end{array}$ & $\begin{array}{l}3,650 \\
3,680 \\
3,720 \\
3,720 \\
3,720\end{array}$ & $\begin{array}{l}4,190 \\
4,110 \\
4,110 \\
4,190 \\
5,800\end{array}$ & $\begin{array}{l}4,850 \\
4,760 \\
4,670 \\
4,720 \\
4,760\end{array}$ & $\begin{array}{l}4,760 \\
4,670 \\
4,760 \\
5,240 \\
4,900\end{array}$ & $\begin{array}{l}4,900 \\
4,980 \\
4,980 \\
4,980 \\
4,900\end{array}$ & $\begin{array}{l}10,200 \\
10,500 \\
11,400 \\
11,500 \\
11,900\end{array}$ & $\begin{array}{l}19,000 \\
20,000 \\
21,000 \\
21,000 \\
21,000\end{array}$ & $\begin{array}{r}11,900 \\
10,800 \\
10,200 \\
9,850 \\
9,850\end{array}$ & $\begin{array}{l}6,600 \\
6,600 \\
6,600 \\
6,500 \\
6,400\end{array}$ & $\begin{array}{l}3,410 \\
3,340 \\
3,260 \\
3,150 \\
3,080\end{array}$ & $\begin{array}{l}2,400 \\
2,420 \\
2,380 \\
2,400 \\
2,400\end{array}$ & $\begin{array}{l}2,400 \\
2,420 \\
2,440 \\
2,440 \\
2,480\end{array}$ \\
\hline $\begin{array}{l}21 \\
22 \\
23 \\
24 \\
25\end{array}$ & $\begin{array}{l}3,680 \\
3,680 \\
3,720 \\
3,760 \\
3,800\end{array}$ & $\begin{array}{l}5,850 \\
5,060 \\
4,800 \\
5,520 \\
5,900 \\
\end{array}$ & $\begin{array}{l}4,670 \\
4,630 \\
4,670 \\
4,670 \\
4,630\end{array}$ & $\begin{array}{l}\frac{5,280}{5,240} \\
5,020 \\
4,900 \\
5,020\end{array}$ & $\begin{array}{l}4,900 \\
4,900 \\
4,900 \\
5,020 \\
5,370\end{array}$ & $\begin{array}{l}12,500 \\
13,000 \\
13,600 \\
13,900 \\
14,200\end{array}$ & $\begin{array}{l}21,000 \\
22,000 \\
23,000 \\
24,000 \\
24,000\end{array}$ & $\begin{array}{r}9,920 \\
9,980 \\
10,900 \\
11,100 \\
10,400\end{array}$ & $\begin{array}{l}6,200 \\
6,000 \\
5,800 \\
5,600 \\
5,300\end{array}$ & $\begin{array}{l}3,040 \\
2,940 \\
2,880 \\
2,840 \\
2,81,0\end{array}$ & $\begin{array}{l}2,360 \\
2,360 \\
2,340 \\
2,340 \\
2,320\end{array}$ & $\begin{array}{l}2,460 \\
2,440 \\
2,440 \\
2,480 \\
2,480\end{array}$ \\
\hline $\begin{array}{l}26 \\
27\end{array}$ & $\begin{array}{l}3,800 \\
3,800\end{array}$ & $\begin{array}{l}5,660 \\
5,280\end{array}$ & $\begin{array}{l}4,460 \\
4,420 \\
\end{array}$ & $\begin{array}{l}5,200 \\
5,240\end{array}$ & $\begin{array}{l}5,560 \\
5,560\end{array}$ & $\begin{array}{l}15,600 \\
16,700\end{array}$ & $\begin{array}{l}25,000 \\
26,000 \\
\end{array}$ & $\begin{array}{l}9,980 \\
9,720\end{array}$ & $\begin{array}{l}5,000 \\
4,800\end{array}$ & $\begin{array}{l}2,820 \\
2,790\end{array}$ & $\begin{array}{l}2,340 \\
2,320\end{array}$ & $\begin{array}{r}* 2,480 \\
2,500\end{array}$ \\
\hline $\begin{array}{l}28 \\
29 \\
30 \\
31\end{array}$ & $\begin{array}{l}3,800 \\
3,840 \\
3,880 \\
3,840\end{array}$ & $\begin{array}{c}4,940 \\
4,590 \\
4,600 \\
-\end{array}$ & $\begin{array}{l}4,540 \\
4,590 \\
4,070 \\
4,590\end{array}$ & $\begin{array}{l}5,240 \\
5,160 \\
5,020 \\
4,980\end{array}$ & $\begin{array}{c}5,560 \\
- \\
- \\
-\end{array}$ & $\begin{array}{l}19,100 \\
\frac{19,300}{18,000} \\
17,100\end{array}$ & $\begin{array}{c}24,000 \\
21,000 \\
20,300 \\
-\end{array}$ & $\begin{array}{l}9,480 \\
9,290 \\
9,170 \\
8,870\end{array}$ & $\begin{array}{l}4,600 \\
4,400 \\
\frac{4,200}{-}\end{array}$ & $\begin{array}{l}2,760 \\
2,740 \\
2,700 \\
2,700\end{array}$ & $\begin{array}{l}2,320 \\
2,340 \\
2,360 \\
2,340\end{array}$ & $\begin{array}{c}2,500 \\
\frac{2,520}{2,520} \\
-\end{array}$ \\
\hline $\begin{array}{l}\text { Total } \\
\text { Mean } \\
\text { a }=f t\end{array}$ & $\begin{array}{r}107,590 \\
3,471 \\
213,1,00 \\
\end{array}$ & $\begin{array}{r}137,170 \\
1,, 572 \\
272,100 \\
\end{array}$ & $\begin{array}{r}156,010 \\
5,030 \\
309,400 \\
\end{array}$ & $\begin{array}{r}49,650 \\
4,827 \\
296,800 \\
\end{array}$ & $\begin{array}{r}49,450 \\
5,338 \\
290,400 \\
\end{array}$ & $\begin{array}{r}345,450 \\
11,140 \\
685,200 \\
\end{array}$ & $\begin{array}{r}604,900 \\
20,160 \\
1,200,000 \\
\end{array}$ & $\begin{array}{r}392,910 \\
12,670 \\
779,300 \\
\end{array}$ & $\begin{array}{r}200,4,50 \\
6,682 \\
397,600 \\
\end{array}$ & $\begin{array}{r}103,770 \\
3,347 \\
205,800 \\
\end{array}$ & $\begin{array}{r}75,200 \\
2,426 \\
149,200 \\
\end{array}$ & $\begin{array}{r}72,700 \\
2,423 \\
144,200 \\
\end{array}$ \\
\hline $\begin{array}{l}\text { Cale } \\
\text { :ate }\end{array}$ & & $\begin{array}{l}\operatorname{ligx} \\
\text { lwax }\end{array}$ & 26,000 & $\begin{array}{l}\text { jinin } \\
\text { lin }\end{array}$ & $\begin{array}{l}360 \\
320\end{array}$ & Iriean & & $t$ & $\begin{array}{l}.000 \\
.000\end{array}$ & & & \\
\hline
\end{tabular}

* Discharge measurement made on tlis day.

Note. -No gage-height record Nov. 30 to Dec. 6, Apr. 15-29, June 8 to July 3 ; discharge computed on basis of change in coriterts and release fror Kejakai Reservoir. 
HEIMAND RTVER. BASIN

Helmand River above Kajakai Reservoir, near Dehraout, Afghanistan

D1scharge, in cubic feet per second, water year October 1958 to September 1959

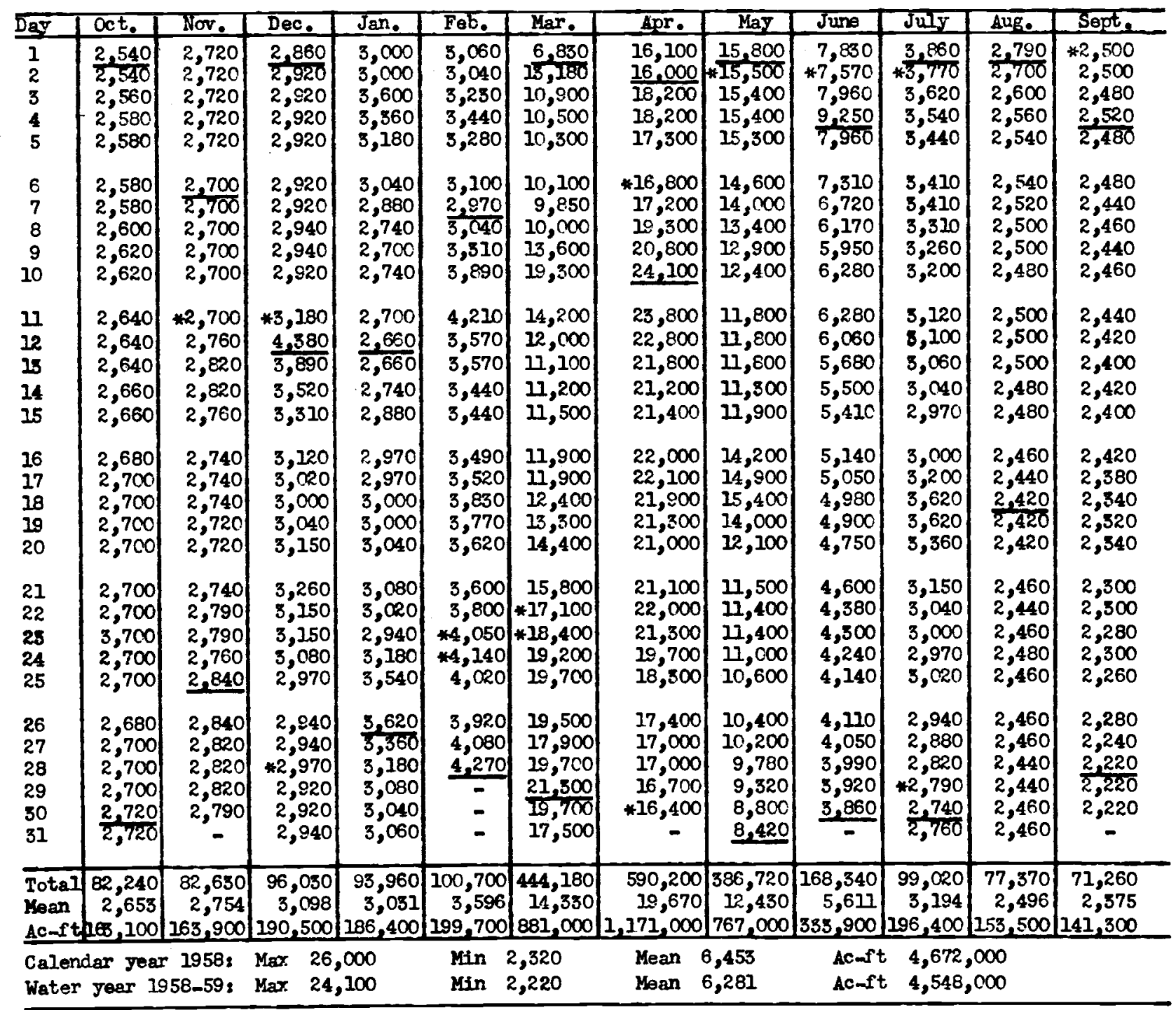

* Discharge measurement made on this day. 
HELMAN RIVER BASIN

Helnand River above Kajakai Heservolr, naar Dehraout, Afghantstan

Dischargo, in cuble feet per eecond, water year October 1959 to September 1960

\begin{tabular}{|c|c|c|c|c|c|c|c|c|c|c|c|c|}
\hline Dar & $\alpha t_{2}$ & Nor. & Dec. & $\operatorname{san}_{4}$ & Fob. & Mar. & $4 x_{0}$ & May & Juns & Jary & sug. & Sopt. \\
\hline $\begin{array}{l}1 \\
2 \\
3 \\
4 \\
5\end{array}$ & $\begin{array}{l}\frac{2,200}{2,200} \\
2,220 \\
2,240 \\
2,240\end{array}$ & $\begin{array}{l}2,520 \\
2,580 \\
2,720 \\
2,880 \\
2,970\end{array}$ & $\begin{array}{r}3,280 \\
4,420 \\
+4,050 \\
3,860 \\
3,830\end{array}$ & $\begin{array}{l}2,940 \\
2,880 \\
2,860 \\
2,860 \\
2,880\end{array}$ & $\begin{array}{l}3,060 \\
3,020 \\
3,040 \\
3,000 \\
2,940 \\
\end{array}$ & $\begin{array}{l}4,220 \\
4,270 \\
4,340 \\
5,140 \\
6,500\end{array}$ & $\begin{array}{r}8,650 \\
8,900 \\
9,180 \\
9,620 \\
* 9,790\end{array}$ & $\begin{array}{l}23,000 \\
25,500 \\
31,400 \\
29,200 \\
24,200\end{array}$ & $\begin{array}{l}\frac{11}{11}, 600 \\
11,500 \\
\frac{11}{11}, 400 \\
4,600\end{array}$ & $\begin{array}{r}4,680 \\
4,600 \\
4,380 \\
4,270 \\
4,240\end{array}$ & $\begin{array}{l}2,540 \\
2,480 \\
2,500 \\
2,440 \\
2,420\end{array}$ & $\begin{array}{l}1,900 \\
1,920 \\
1,940 \\
1,940 \\
1,960\end{array}$ \\
\hline $\begin{array}{r}6 \\
7 \\
8 \\
9 \\
10\end{array}$ & $\begin{array}{r}2,260 \\
* 2,260 \\
2,280 \\
2,260 \\
2,260\end{array}$ & $\begin{array}{l}3,060 \\
3, \infty 60 \\
3,040 \\
3,000 \\
3,000\end{array}$ & $\begin{array}{l}3,800 \\
3,770 \\
3,710 \\
3,740 \\
3,740\end{array}$ & $\begin{array}{l}2,970 \\
5,100 \\
5,120 \\
3,120 \\
3,120\end{array}$ & $\begin{array}{l}3,340 \\
3,120 \\
3,200 \\
3,310 \\
3,360\end{array}$ & $\begin{array}{l}5,860 \\
5,410 \\
5,140 \\
4,900 \\
4,860\end{array}$ & $\begin{array}{r}9,920 \\
10,500 \\
11,900 \\
11,300 \\
11,400\end{array}$ & $\begin{array}{l}21,500 \\
19,200 \\
17,700 \\
17,400 \\
17,900\end{array}$ & $\begin{array}{r}11,200 \\
11,100 \\
10,600 \\
10,400 \\
9,850\end{array}$ & $\begin{array}{l}4,180 \\
4,020 \\
3,890 \\
3,800 \\
3,620\end{array}$ & $\begin{array}{l}2,420 \\
2,380 \\
2,380 \\
2,340 \\
2,300\end{array}$ & $\begin{array}{l}1,940 \\
1,940 \\
1,980 \\
1,980 \\
2,920\end{array}$ \\
\hline$\frac{11}{12}$ & $\begin{array}{l}2,280 \\
2,300\end{array}$ & $\begin{array}{l}2,970 \\
2,970\end{array}$ & $\begin{array}{l}3,710 \\
3,680\end{array}$ & $\begin{array}{l}3,150 \\
3,150\end{array}$ & $\begin{array}{l}3,440 \\
3,490\end{array}$ & $\begin{array}{l}5,140 \\
5,520\end{array}$ & $\begin{array}{l}10,700 \\
10,900\end{array}$ & $\begin{array}{l}18,700 \\
19,800\end{array}$ & $\begin{array}{l}9,550 \\
8,380\end{array}$ & $\begin{array}{l}3,520 \\
3,410\end{array}$ & $\begin{array}{l}2,280 \\
2,220\end{array}$ & $\begin{array}{l}2,040 \\
2,040\end{array}$ \\
\hline 18 & 2,320 & 2,970 & 3,680 & 3,150 & 3,620 & 6,170 & 13,600 & 20,800 & 8,500 & 3,360 & 2,220 & 2,080 \\
\hline $\begin{array}{l}14 \\
15\end{array}$ & $\begin{array}{l}2,540 \\
2,540\end{array}$ & $\begin{array}{l}2,970 \\
2,970\end{array}$ & $\begin{array}{l}3,620 \\
3,620\end{array}$ & $\begin{array}{l}5,120 \\
3,000\end{array}$ & $\begin{array}{l}3,920 \\
4,210\end{array}$ & $\begin{array}{l}7,050 \\
7,700\end{array}$ & $\begin{array}{l}16,400 \\
17,800\end{array}$ & $\begin{array}{l}21,900 \\
22,500\end{array}$ & $\begin{array}{l}7,760 \\
7,510\end{array}$ & $\begin{array}{l}3,310 \\
3,2,30\end{array}$ & $\begin{array}{l}2,200 \\
2,200\end{array}$ & $\begin{array}{l}2,100 \\
2,100\end{array}$ \\
\hline $\begin{array}{l}16 \\
17 \\
18 \\
19 \\
20\end{array}$ & $\begin{array}{l}2,560 \\
2,580 \\
2,400 \\
2,400 \\
2,400\end{array}$ & $\begin{array}{l}2,970 \\
2,970 \\
2,970 \\
2,970 \\
2,940\end{array}$ & $\begin{array}{l}3,600 \\
3,600 \\
3,540 \\
3,540 \\
3,540\end{array}$ & $\begin{array}{l}2,380 \\
2,880 \\
2,970 \\
3,060 \\
3,060\end{array}$ & $\begin{array}{l}3,560 \\
4,450 \\
4,140 \\
3,920 \\
3,560\end{array}$ & $\begin{array}{l}8,160 \\
7,640 \\
6,940 \\
6,660 \\
6,560\end{array}$ & $\begin{array}{l}18,600 \\
22,400 \\
25,000 \\
21,100 \\
19,300\end{array}$ & $\begin{array}{l}23,100 \\
23,700 \\
25,100 \\
22,000 \\
21,100\end{array}$ & $\begin{array}{l}6,830 \\
6,500 \\
6,770 \\
6,000 \\
5,770\end{array}$ & $\begin{array}{l}3,180 \\
3,060 \\
3,060 \\
2,920 \\
2,860\end{array}$ & $\begin{array}{l}2,160 \\
2,140 \\
2,140 \\
2,120 \\
2,120\end{array}$ & $\begin{array}{l}2,120 \\
2,2120 \\
2,120 \\
2,160 \\
2,160\end{array}$ \\
\hline $\begin{array}{l}21 \\
22 \\
23 \\
24 \\
25\end{array}$ & $\begin{array}{l}2,400 \\
2,420 \\
2,440 \\
2,460 \\
2,460\end{array}$ & $\begin{array}{l}2,940 \\
2,940 \\
2,920 \\
2,940 \\
5,000\end{array}$ & $\begin{array}{l}3,540 \\
3,520 \\
3,480 \\
3,440 \\
3,580\end{array}$ & $\begin{array}{l}3,240 \\
2,880 \\
2,820 \\
2,380 \\
2,970\end{array}$ & $\begin{array}{l}3,890 \\
3,990 \\
3,990 \\
3,920 \\
3,990\end{array}$ & $\begin{array}{l}6,500 \\
6,340 \\
6,120 \\
5,900 \\
6,000\end{array}$ & $\begin{array}{l}18,600 \\
19,400 \\
19,500 \\
18,800 \\
17,900\end{array}$ & $\begin{array}{r}20,300 \\
+18,900 \\
17,600 \\
16,400 \\
15,900\end{array}$ & $\begin{array}{l}5,680 \\
5,590 \\
5,640 \\
5,360 \\
5,230\end{array}$ & $\begin{array}{l}2,820 \\
2,790 \\
2,740 \\
2,740 \\
2,740\end{array}$ & $\begin{array}{l}2,100 \\
2,100 \\
2,080 \\
2,080 \\
2,080\end{array}$ & $\begin{array}{l}2,160 \\
2,160 \\
2,180 \\
2,220 \\
2,210\end{array}$ \\
\hline $\begin{array}{l}26 \\
27 \\
28 \\
29 \\
30 \\
31\end{array}$ & $\begin{array}{r}2,480 \\
2,460 \\
2,500 \\
2,580 \\
2,580 \\
2,530 \\
\end{array}$ & $\begin{array}{c}3,060 \\
3,340 \\
3,340 \\
3,360 \\
5,2,20 \\
\ldots\end{array}$ & $\begin{array}{r}5,360 \\
3,310 \\
3,280 \\
3,280 \\
-3,260 \\
3,080 \\
\end{array}$ & $\begin{array}{r}3,000 \\
-3,940 \\
3,040 \\
3,250 \\
3,310 \\
3,150\end{array}$ & $\begin{array}{r}4,110 \\
4,180 \\
4,240 \\
4,210 \\
-\end{array}$ & $\begin{array}{l}7,050 \\
9,180 \\
8,880 \\
8,580 \\
8,580 \\
8,580\end{array}$ & $\begin{array}{r}17,600 \\
+17,600 \\
18,200 \\
19,300 \\
21,100 \\
=\end{array}$ & $\begin{array}{l}15,400 \\
15,100 \\
14,300 \\
13,200 \\
12,200 \\
12,300\end{array}$ & $\begin{array}{l}5,100 \\
5,050 \\
4,980 \\
4,920 \\
4,750 \\
\end{array}$ & $\begin{array}{r}2,740 \\
+2,700 \\
2,640 \\
2,620 \\
2,600 \\
2,560 \\
\end{array}$ & $\begin{array}{l}2,050 \\
2,060 \\
2,000 \\
2,020 \\
1,980 \\
1,900 \\
\end{array}$ & $\begin{array}{l}2,220 \\
2,220 \\
2,240 \\
2,260 \\
2,260 \\
-\end{array}$ \\
\hline $\begin{array}{l}\text { Total } \\
\text { Moan } \\
\text { Ac-Pt }\end{array}$ & $\begin{array}{r}73,240 \\
2,362 \\
145,300 \\
\end{array}$ & $\begin{array}{r}89,840 \\
2,995 \\
178,200 \\
\end{array}$ & $\begin{array}{r}11,240 \\
3,588 \\
220,600 \\
\end{array}$ & $\begin{array}{r}93,530 \\
3,017 \\
185,500 \\
\end{array}$ & $\begin{array}{r}107,220 \\
3,597 \\
212,700 \\
\end{array}$ & $\begin{array}{r}199,680 \\
6,441 \\
396,100 \\
\end{array}$ & $\begin{array}{r}463,950 \\
15,460 \\
920,000 \\
\end{array}$ & $\begin{array}{r}614,500 \\
19,830 \\
1,219,000 \\
\end{array}$ & $\begin{array}{r}236,800 \\
7,869 \\
468,300 \\
\end{array}$ & $\begin{array}{r}105,280 \\
3,532 \\
204,300 \\
\end{array}$ & $\begin{array}{r}68,520 \\
2,210 \\
135,900 \\
\end{array}$ & $\begin{array}{r}62,740 \\
2,091 \\
124,400 \\
\end{array}$ \\
\hline & -200 & & 31,4 & & $\begin{array}{ll}\text { Min } & 2,20 \\
\text { Min } & 1,90\end{array}$ & & $\begin{array}{ll}\text { Mean } & 6,31 \\
\text { Mean } & 6,07\end{array}$ & Ac. & $\begin{array}{l}-f t \\
-f t\end{array}$ & $\begin{array}{l}.000 \\
.000\end{array}$ & & \\
\hline
\end{tabular}

* Discharge measurement made on this day. 


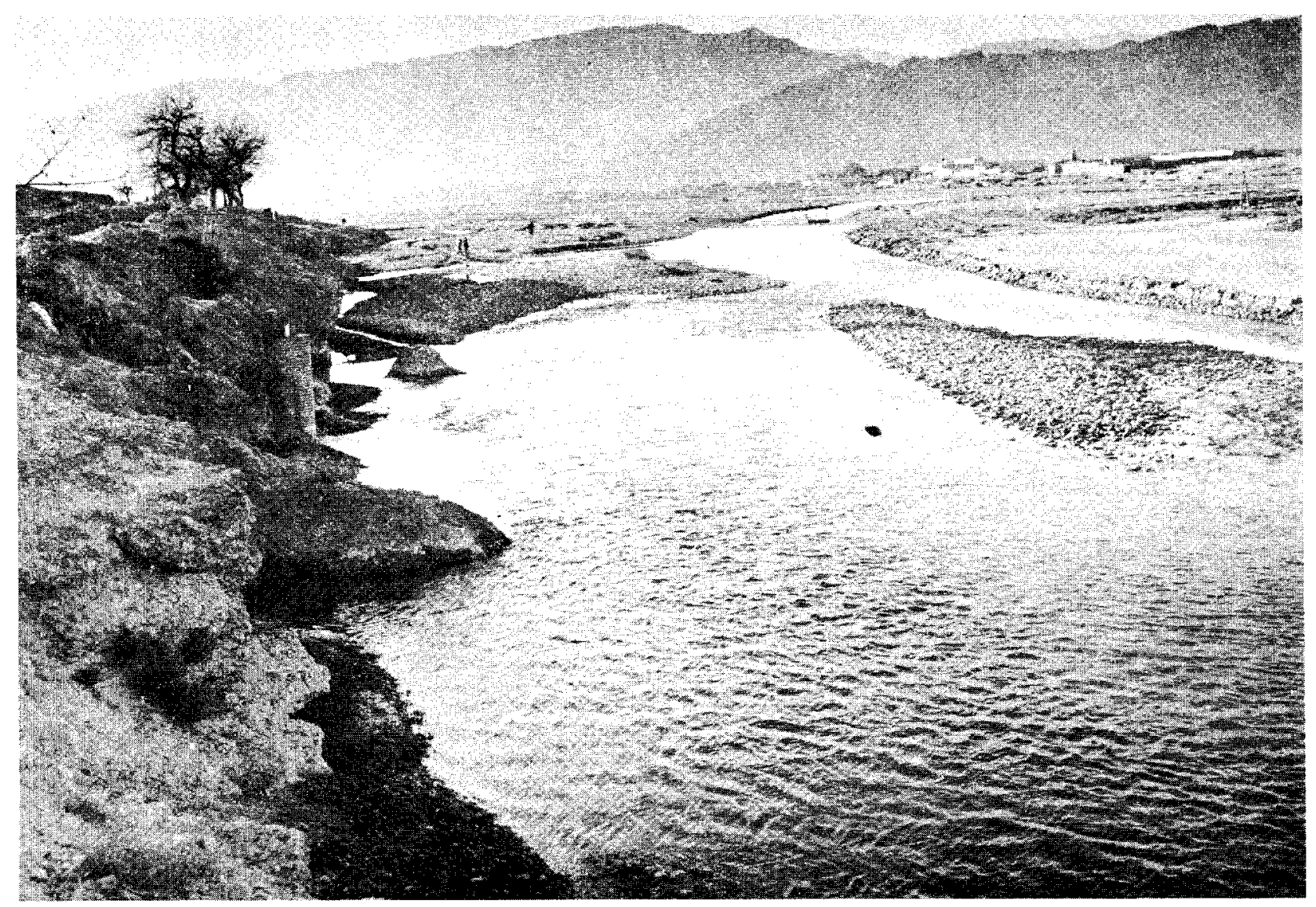

Figure 4.--Tirin River at Dehraout showlng water-stage recorder shelter and well, cableway, and control. 
Tirin hiver at Dehraout, Afghanistan

Location. -Lat $32^{\circ} 401 \mathrm{~N}$, long $65^{\circ} 30 \mathrm{I}$., on left bank at village of Dehraout, 6 kilometers upstream from mouth, about 65 kilometers upstream from Kajakai dam, and 235 kilometers north of Kandahar by road.

Drainage area. $-2,160 \mathrm{sq} \mathrm{mi}$, approxinately.

Records available.-April to June 1951 (gage heights only), March 1952 to September 1960.

Gage. - Water-stage recorder. Altitude of gage is about 1,100 meters (from Survey of India maps).

Average discharge. -8 years, 618 cfs ( 447,400 acre-feet per year).

Extremes.-Maximum and minimum discharge for the 1951-60 water years are given in the following table:

\begin{tabular}{|c|c|c|c|c|c|c|}
\hline \multirow[b]{2}{*}{ water Year } & \multirow[b]{2}{*}{ Date } & \multicolumn{2}{|c|}{ Maximum } & \multirow[b]{2}{*}{ Date } & \multicolumn{2}{|c|}{ Minimum } \\
\hline & & $\begin{array}{c}\text { Gage Height } \\
\text { (meters) }\end{array}$ & $\begin{array}{c}\text { Dis charge } \\
\text { (cis) }\end{array}$ & & $\begin{array}{c}\text { Gage Height } \\
\text { (meters) }\end{array}$ & $\begin{array}{c}\text { Discharge } \\
\text { (cfs) }\end{array}$ \\
\hline $\begin{array}{l}1952 \\
1953 \\
1954 \\
1955 \\
1956 \\
1957 \\
1958 \\
1959 \\
1960\end{array}$ & $\begin{array}{l}\text { Mar. } 27,1952 \\
\text { May } 26,1953 \\
-\bar{M} \\
\text { Mar. } 14,1955 \\
\text { Mar. 20, } 1956 \\
\text { Mar. } 17,1957 \\
\text { Feb. 2, } 1958 \\
\text { Mar. 2, } 1959 \\
\text { Apr. 16, } 1960\end{array}$ & $\begin{array}{l}2.00 \\
1.64 \\
-2 \\
1.19 \\
2.10 \\
2.65 \\
1.70 \\
1.695 \\
1.705\end{array}$ & $\begin{array}{c}3,610 \\
2,480 \\
(a) \\
1,690 \\
6,080 \\
8,800 \\
4,400 \\
4,320 \\
3,390\end{array}$ & $\begin{array}{l}\text { Aug. } 10,18,1953 \\
\text { Oct. } 1,1953 \\
\text { Aug. } 6,7,1955 \\
\text { Oct. } 4,1955 \\
\text { Oct. } 5,1956 \\
\text { Sept. } 24,1958 \\
\text { Aug. } 31,1959 \\
\text { Aug. } 1,1960\end{array}$ & $\begin{array}{l}0.17 \\
-7 \\
- \\
1.07 \\
1.22 \\
0.66 \\
0.725 \\
0.56\end{array}$ & $\begin{array}{c}a \\
b 71 \\
95 \\
77 \\
95 \\
150 \\
250 \\
1140 \\
104\end{array}$ \\
\hline
\end{tabular}

a Not determined.

b Mean daily discharge.

Remarks.--Records good except those f'or periods of no gage-height record, which are fair, and those for 1952 and March-May 1954, which are poor. Many small diversions for irrigation above the station. 
HERLMAND RIVER BASIN

Tirin River at Dehraout, Afghanistan

Discharge, in cubic feet per second, period March to September 1952

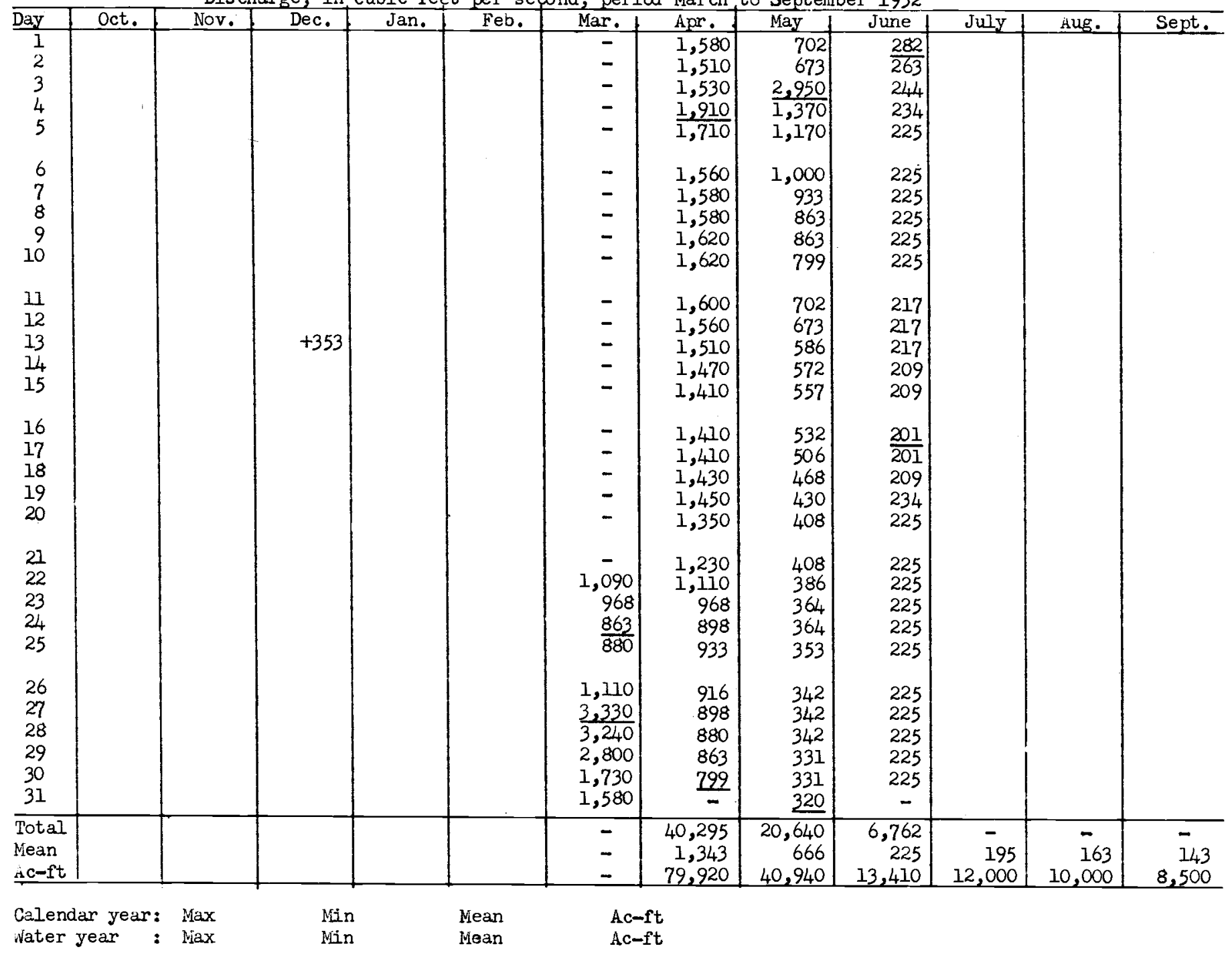

f. Result of discharge measurement.

Note. -No gage-height record Oct. 1 to Dec. 12, Dec. 14 to Mar. 21, July 1 to Sept. 30; discharge for

July-september estivated on basis of recession curve during the period of no precipitation. 
HELILALD RIVET BRSIL

Tirin Hiver at Dehraout, afighanistan

Discharge, in cubic feet per second, water year Octooer 1952 to September 1953

\begin{tabular}{|c|c|c|c|c|c|c|c|c|c|c|c|c|}
\hline$\frac{D a y}{I}$ & $\frac{\text { Oct. }}{130}$ & $\frac{\text { Nov. }}{230}$ & Dec. & Jan. & Feo. & $\operatorname{Mr} r_{0}$ & spr. & viay & June & July & Aug. & Sept. \\
\hline $\begin{array}{l}1 \\
2\end{array}$ & $\frac{130}{130}$ & $\begin{array}{l}230 \\
234\end{array}$ & $\frac{277}{220}$ & 331 & 336 & 799 & 916 & 455 & 234 & 88 & 74 & 116 \\
\hline 2 & 130 & 234 & 282 & 326 & $\overline{342}$ & 863 & 863 & 430 & 560 & 88 & 74 & 88 \\
\hline 3 & 130 & 209 & 287 & 315 & 430 & 898 & 831 & 430 & 380 & 88 & 81 & 84 \\
\hline$\frac{4}{5}$ & 130 & 205 & 287 & 310 & 586 & 907 & 880 & 408 & 301 & 95 & $* 95$ & 84 \\
\hline 5 & 135 & 193 & 292 & 301 & 408 & 959 & 959 & 408 & 24 & 102 & 81 & $88^{\circ}$ \\
\hline 6 & 135 & 193 & 292 & 301 & 424 & 1,190 & 1,000 & 375 & 217 & 102 & & $* 88$ \\
\hline 7 & 140 & 209 & 301 & 301 & 443 & 1,680 & 1,020 & 320 & $\begin{array}{l}2 \perp 1 \\
209\end{array}$ & $\begin{array}{r}102 \\
92\end{array}$ & $7 i_{4}$ & $\begin{array}{r}788 \\
05\end{array}$ \\
\hline 8 & 140 & 209 & 292 & 310 & 4.3 & 1,700 & $1, \infty 0$ & 244 & 160 & $\begin{array}{l}92 \\
88\end{array}$ & 74 & 95 \\
\hline 9 & 145 & 209 & 296 & 301 & 506 & 1,500 & 986 & 230 & 176 & $\begin{array}{l}88 \\
95\end{array}$ & 74 & 95 \\
\hline 10 & 150 & 209 & 296 & 306 & 1,400 & 1,400 & 986 & 230 & 168 & 95 & $\begin{array}{l}74 \\
71\end{array}$ & $\begin{array}{l}98 \\
95\end{array}$ \\
\hline II & 150 & 209 & 301 & 310 & 766 & 1,250 & 1,000 & 221 & 152 & 88 & 7) & 02 \\
\hline 12 & 155 & 201 & 306 & 306 & 891 & 1,150 & 1,090 & 225 & 116 & 88 & $\begin{array}{l}14 \\
87\end{array}$ & $\begin{array}{l}92 \\
88\end{array}$ \\
\hline 13 & 160 & 209 & 301 & 443 & 1,340 & 1,100 & 1,110 & 217 & 109 & 88 & 81 & 88 \\
\hline 14 & 160 & $24 i$ & 301 & 519 & 1,240 & $* 1,040$ & $\overline{1,040}$ & $* 209$ & 176 & 88 & $\begin{array}{l}81 \\
88\end{array}$ & $\begin{array}{l}84 \\
81\end{array}$ \\
\hline 15 & 165 & 21,4 & 301 & $\overline{408}$ & 869 & 986 & 968 & 180 & 123 & 88 & 84 & $\begin{array}{l}01 \\
81\end{array}$ \\
\hline 16 & 170 & 244 & 310 & 364 & 630 & 898 & 907 & 176 & 144 & 87 & 71 & 8) \\
\hline 17 & 175 & 24,4 & 331 & 353 & 572 & 750 & 847 & 172 & 144 & 81 & 74 & $\begin{array}{l}81 \\
78\end{array}$ \\
\hline 18 & 180 & $* 244$ & $\overline{320}$ & 375 & 544 & 750 & 847 & 168 & 120 & 81 & $\begin{array}{l}74 \\
77\end{array}$ & 78 \\
\hline 19 & 180 & 244 & 320 & 380 & 532 & 718 & 815 & 168 & 109 & 74 & 71 & $\frac{14}{87}$ \\
\hline 20 & 180 & 249 & 320 & 364 & 519 & 718 & 782 & 160 & 130 & $\frac{14}{74}$ & $\begin{array}{l}14 \\
81\end{array}$ & $\begin{array}{l}81 \\
81\end{array}$ \\
\hline 21 & 184 & 254 & 310 & 353 & 494 & 750 & 658 & 156 & 106 & 7 & 88 & 8 \\
\hline 22 & $* 184$ & 258 & 310 & 358 & 481 & 750 & 615 & $\frac{164}{164}$ & 102 & $\begin{array}{l}14 \\
88\end{array}$ & 88 & 84 \\
\hline 23 & 184 & 254 & 310 & 353 & 481 & 1,110 & 586 & 172 & 116 & 74 & $\begin{array}{r}73 \\
0\end{array}$ & $\begin{array}{l}88 \\
02\end{array}$ \\
\hline 24 & 184 & 249 & 320 & 358 & 468 & 1,820 & 544 & $\begin{array}{l}176 \\
176\end{array}$ & 109 & 84 & $\frac{100}{102}$ & $\begin{array}{l}42 \\
05\end{array}$ \\
\hline 25 & 176 & 254 & 320 & 364 & 481 & 1,750 & 519 & 193 & 116 & 88 & 102 & $\begin{array}{l}72 \\
95\end{array}$ \\
\hline 26 & 184 & 254 & 310 & 358 & 481 & $1,64,0$ & 494 & 124 & 102 & 88 & & \\
\hline 27 & 188 & 272 & 310 & 331 & 557 & 1,490 & $\approx 455$ & 874 & $\begin{array}{ll}102 \\
81\end{array}$ & 88 & 106 & 95 \\
\hline 28 & 197 & 277 & 315 & $\star 353$ & 630 & 1,310 & 455 & 519 & $\frac{91}{95}$ & 74 & 95 & 95 \\
\hline 29 & 209 & 282 & 331 & 342 & - & 1,170 & 455 & 353 & $\begin{array}{l}72 \\
98\end{array}$ & 81 & 92 & 92 \\
\hline 30 & 215 & 277 & 331 & 331 & - & 1,040 & 455 & 301 & 98 & 81 & 88 & 95 \\
\hline 31 & 225 & - & 326 & 331 & - & 968 & - & 263 & 95 & 81 & 88 & 95 \\
\hline Total & 5.170 & $7, \overline{063}$ & 9,506 & 10,756 & 17.354 & 35.051 & & & - & 81 & 102 & - \\
\hline Wean & 167 & 235 & 307 & 347 & 620 & $\begin{array}{r}35,054 \\
1,131\end{array}$ & 24,083 & 9,021 & 4,932 & 2,655 & 2,618 & 2,678 \\
\hline $\mathrm{Ac}-\mathrm{ft}$ & 10,250 & 14,010 & 18,850 & 21,330 & 34,420 & $\begin{array}{r}1,131 \\
69,530\end{array}$ & $\begin{array}{r}803 \\
47.770\end{array}$ & $\begin{array}{r}291 \\
17.890\end{array}$ & 164 & 85.6 & 84.5 & 89.3 \\
\hline & & & & & & & 48,170 & 1,890 & 9,780 & 2701 &, 190 & 5,310 \\
\hline
\end{tabular}

Calendar year 1952: wiax

ivean -

s. $-f t$

dater year 1952-53: $\operatorname{Max} 1,820$ Min 71 Mean 359 Ac-ft 259,600

* Discharge measurenent made on this day.

Note. - jo gage-height record Oct. 1-21, 34, 31, war. 8-13; dischure itterpolated or estimated on basis

of records for Helnana iver reir Dehriout. 
HETWAN RIVER BASIN

Tirin River at Deriraout, Afghanistan

Discharee, in cubic feet per second, water jear October 1953 to October 1954

\begin{tabular}{|c|c|c|c|c|c|c|c|c|c|c|c|c|}
\hline Day & Oct. & Nov. & Dec. & Jan. & Feb. & Mar. & Apr. & May & June & July & AuE. & Sept. \\
\hline $\begin{array}{l}1 \\
2 \\
3 \\
4 \\
5\end{array}$ & $\begin{array}{r}\frac{95}{98} \\
102 \\
* 109 \\
123\end{array}$ & $\begin{array}{l}168 \\
160 \\
164 \\
164 \\
172\end{array}$ & $\begin{array}{l}\frac{258}{263} \\
263 \\
263 \\
263\end{array}$ & $\begin{array}{r}455 \\
449 \\
455 \\
1,150 \\
3,180 \\
\end{array}$ & $\begin{array}{l}644 \\
644 \\
644 \\
644 \\
644\end{array}$ & $\begin{array}{l}1,500 \\
1,300 \\
1,200 \\
1,100 \\
\frac{1}{1,100}\end{array}$ & $\begin{array}{l}\frac{3,500}{3,100} \\
2,900 \\
2,700 \\
2,600\end{array}$ & $\begin{array}{l}\frac{2,300}{2,200} \\
2,100 \\
2,100 \\
2,000\end{array}$ & $\begin{array}{l}630 \\
650 \\
630 \\
610 \\
590\end{array}$ & $\begin{array}{l}\frac{340}{330} \\
330 \\
320 \\
320\end{array}$ & $\begin{array}{l}\frac{230}{230} \\
230 \\
220 \\
220\end{array}$ & $\begin{array}{l}\frac{180}{170} \\
170 \\
170 \\
170\end{array}$ \\
\hline $\begin{array}{r}6 \\
7 \\
8 \\
9 \\
10\end{array}$ & $\begin{array}{l}137 \\
130 \\
120 \\
127 \\
137\end{array}$ & $\begin{array}{l}180 \\
180 \\
201 \\
197 \\
197\end{array}$ & $\begin{array}{l}268 \\
268 \\
263 \\
272 \\
277\end{array}$ & $\begin{array}{r}* 1,160 \\
766 \\
644 \\
622 \\
519\end{array}$ & $\begin{array}{r}644 \\
637 \\
637 \\
644 \\
2,290\end{array}$ & $\begin{array}{l}1,100 \\
1,100 \\
1,100 \\
1,100 \\
1,200\end{array}$ & $\begin{array}{l}2,600 \\
2,500 \\
2,500 \\
2,500 \\
2,400 \\
\end{array}$ & $\begin{array}{l}2,000 \\
1,900 \\
1,900 \\
1,800 \\
1,800\end{array}$ & $\begin{array}{r}570 \\
550 \\
* 532 \\
510 \\
500\end{array}$ & $\begin{array}{l}310 \\
310 \\
320 \\
300 \\
300\end{array}$ & $\begin{array}{l}220 \\
220 \\
220 \\
220 \\
210\end{array}$ & $\begin{array}{l}\frac{160}{160} \\
160 \\
160 \\
160\end{array}$ \\
\hline $\begin{array}{l}11 \\
12 \\
13 \\
14 \\
15\end{array}$ & $\begin{array}{l}130 \\
130 \\
140 \\
152 \\
168\end{array}$ & $\begin{array}{l}193 \\
193 \\
201 \\
213 \\
217\end{array}$ & $\begin{array}{l}287 \\
292 \\
306 \\
310 \\
315\end{array}$ & $\begin{array}{l}600 \\
750 \\
750 \\
750 \\
750\end{array}$ & $\begin{array}{l}\frac{2,460}{1,800} \\
2,370 \\
1,940 \\
1,840\end{array}$ & $\begin{array}{l}1,200 \\
1,300 \\
1,300 \\
1,300 \\
1,400\end{array}$ & $\begin{array}{l}2,400 \\
2,400 \\
2,400 \\
2,400 \\
2,400\end{array}$ & $\begin{array}{l}1,700 \\
1,700 \\
1,600 \\
1,600 \\
1,500\end{array}$ & $\begin{array}{l}480 \\
470 \\
460 \\
450 \\
440\end{array}$ & $\begin{array}{l}300 \\
290 \\
290 \\
290 \\
280\end{array}$ & $\begin{array}{l}210 \\
210 \\
210 \\
210 \\
210\end{array}$ & $\begin{array}{l}160 \\
160 \\
160 \\
160 \\
160\end{array}$ \\
\hline $\begin{array}{l}16 \\
17 \\
18 \\
19 \\
20\end{array}$ & $\begin{array}{l}176 \\
184 \\
184 \\
172 \\
168\end{array}$ & $\begin{array}{l}217 \\
217 \\
217 \\
221 \\
225\end{array}$ & $\begin{array}{l}306 \\
306 \\
301 \\
306 \\
310\end{array}$ & $\begin{array}{l}700 \\
700 \\
673 \\
666 \\
651\end{array}$ & $\begin{array}{l}1,860 \\
1,910 \\
1,940 \\
1,660 \\
1,560\end{array}$ & $\begin{array}{l}1,400 \\
1,500 \\
1,600 \\
1,800 \\
2,000\end{array}$ & $\begin{array}{l}2,500 \\
2,500 \\
2,600 \\
2,600 \\
2,700\end{array}$ & $\begin{array}{l}1,400 \\
1,300 \\
1,300 \\
1,200 \\
1,200\end{array}$ & $\begin{array}{l}430 \\
420 \\
410 \\
410 \\
400\end{array}$ & $\begin{array}{l}280 \\
280 \\
270 \\
270 \\
270\end{array}$ & $\begin{array}{r}210 \\
* 204 \\
200 \\
200 \\
200\end{array}$ & $\begin{array}{l}160 \\
160 \\
160 \\
160 \\
160\end{array}$ \\
\hline $\begin{array}{l}21 \\
22 \\
23 \\
24 \\
25\end{array}$ & $\begin{array}{l}160 \\
156 \\
152 \\
152 \\
152\end{array}$ & $\begin{array}{r}221 \\
* 225 \\
230 \\
239 \\
244\end{array}$ & $\begin{array}{l}306 \\
320 \\
\frac{802}{644} \\
512\end{array}$ & $\begin{array}{l}644 \\
644 \\
644 \\
637 \\
630\end{array}$ & $\begin{array}{l}1,390 \\
1,410 \\
1,330 \\
1,350 \\
1,350\end{array}$ & $\begin{array}{l}2,200 \\
2,300 \\
2,300 \\
2,200 \\
2,200\end{array}$ & $\begin{array}{l}2,900 \\
3,100 \\
3,300 \\
3,200 \\
3,000\end{array}$ & $\begin{array}{r}1,100 \\
1,100 \\
1,000 \\
950 \\
900\end{array}$ & $\begin{array}{l}390 \\
390 \\
380 \\
370 \\
370\end{array}$ & $\begin{array}{r}270 \\
* 261 \\
260 \\
250 \\
250\end{array}$ & $\begin{array}{l}200 \\
200 \\
190 \\
190 \\
190\end{array}$ & $\begin{array}{l}160 \\
160 \\
160 \\
160 \\
160\end{array}$ \\
\hline $\begin{array}{l}26 \\
27 \\
28 \\
29 \\
30 \\
31\end{array}$ & $\begin{array}{l}144 \\
144 \\
152 \\
160 \\
160 \\
168\end{array}$ & $\begin{array}{l}239 \\
244 \\
249 \\
254 \\
254 \\
-\end{array}$ & $\begin{array}{l}488 \\
461 \\
468 \\
462 \\
462 \\
462\end{array}$ & $\begin{array}{l}630 \\
630 \\
630 \\
658 \\
658 \\
651\end{array}$ & $\begin{array}{c}1,200 \\
* 950 \\
1,000 \\
- \\
- \\
-\end{array}$ & $\begin{array}{l}2,100 \\
2,100 \\
2,300 \\
3,000 \\
5,000 \\
4,000\end{array}$ & $\begin{array}{c}2,800 \\
2,700 \\
2,600 \\
2,500 \\
2,400 \\
-\end{array}$ & $\begin{array}{l}900 \\
850 \\
800 \\
800 \\
750 \\
700 \\
\end{array}$ & $\begin{array}{l}360 \\
360 \\
350 \\
350 \\
340 \\
-\end{array}$ & $\begin{array}{l}250 \\
240 \\
240 \\
240 \\
240 \\
230 \\
\end{array}$ & $\begin{array}{l}190 \\
180 \\
180 \\
180 \\
180 \\
180\end{array}$ & $\begin{array}{c}160 \\
160 \\
* 162 \\
160 \\
160 \\
-\end{array}$ \\
\hline $\begin{array}{l}\text { Total } \\
\text { Mean } \\
\text { Ac-ft }\end{array}$ & $\begin{array}{r}4,482 \\
145 \\
8,890\end{array}$ & $\begin{array}{r}6,296 \\
210 \\
12,490\end{array}$ & $\begin{array}{r}11,104 \\
358 \\
22,020\end{array}$ & $\begin{array}{r}23,446 \\
756 \\
46,500\end{array}$ & $\begin{array}{r}37,392 \\
1,335 \\
74,170\end{array}$ & $\begin{array}{r}56,300 \\
1,816 \\
111,700\end{array}$ & $\begin{array}{r}80,600 \\
2,687 \\
159,900\end{array}$ & $\begin{array}{r}44,450 \\
1,434 \\
88,170\end{array}$ & $\begin{array}{r}13,852 \\
468 \\
27,480\end{array}$ & $\begin{array}{r}8,721 \\
281 \\
17,300\end{array}$ & $\begin{array}{r}6,344 \\
205 \\
12,580 \\
\end{array}$ & $\begin{array}{r}4,862 \\
162 \\
9,640 \\
\end{array}$ \\
\hline
\end{tabular}

Calendar Year 1953: Max 1,820 Min 71 Nean 359 Ac-ft 259,900 Water Year 1953-54: $\operatorname{kax} 5,000$ lin 95 liear \&16 Ac-ft 590,800

*Discharge measurement made on this day.

Note. - No gage-heig.t record Jan. 11-17, $\vec{i} \in b .2,3,26$, Feb. 28 to Sept. 30; discharge for Jan. 1]-17, Feb. 2, 3, 26, interpolated, discharse for $\mathrm{Feb} .26$ to jent. 30 estimated on the basis of 4 discharge neasurements and records for stations on fielmand kiver near Dehraout and Arghandab River above Arghandab reservoir. 
HELMAND RIVER BASIN

Tirin River at Dehraout, Afghanistan

Discharge, in cubic feet per second, water year October 1954 to September 1955

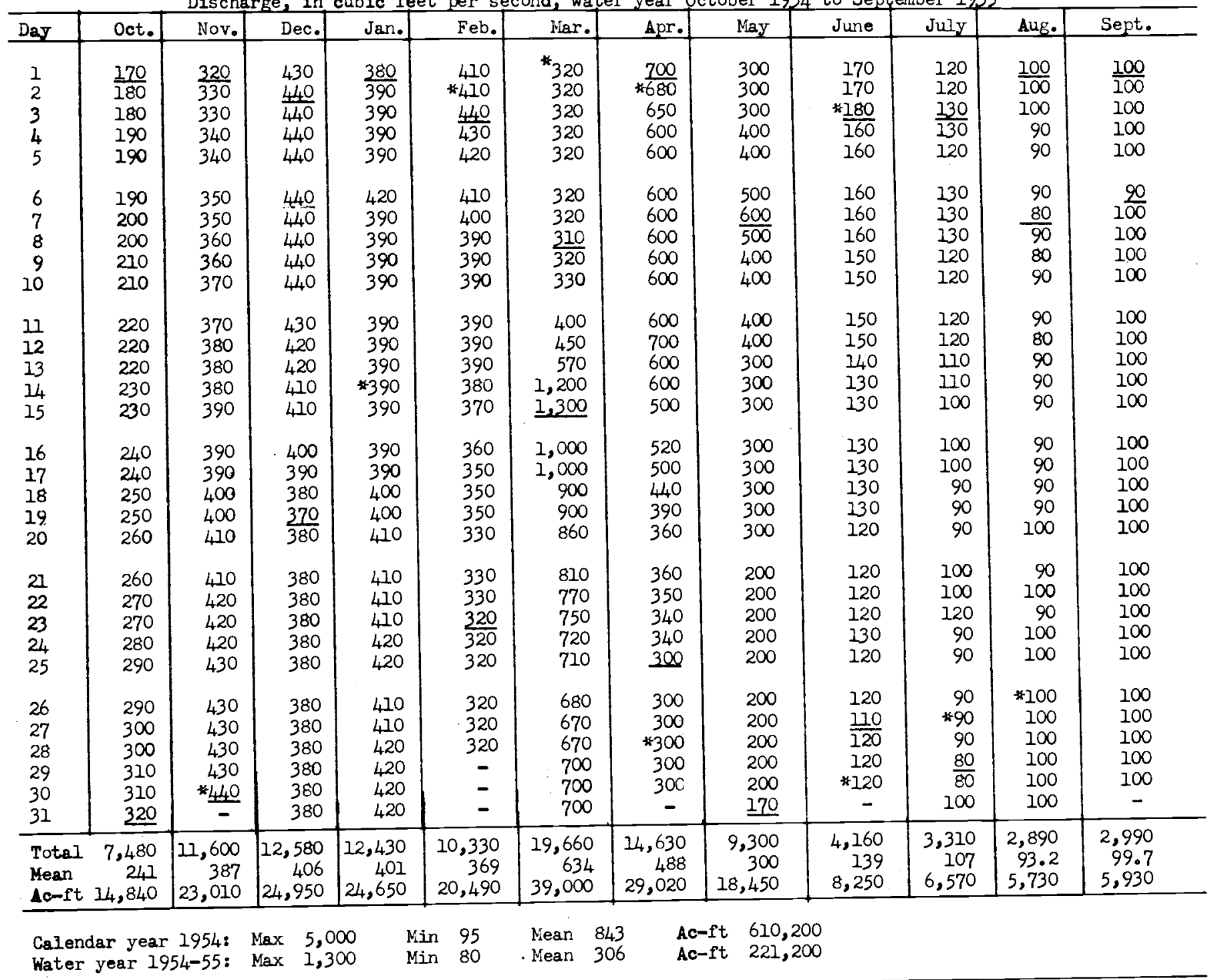

* Discharge measurement made on this day.

Note.

Note.- No ga. 18 and record for Helmand River near Dehraout. 
HETMAND RIVER BASTN

Tirin River at Dehraout, Afghanistan

Discharge, in cubic feet per second, water year October 1955 to September 1956

\begin{tabular}{|c|c|c|c|c|c|c|c|c|c|c|c|c|}
\hline Dar & Oct & Nov & Dec & Jan. & $\mathrm{Feb}$ & Mar. & Apr. & Mey & Jurie & JuIy & Aug. & Sept. \\
\hline $\begin{array}{l}1 \\
2 \\
3 \\
4 \\
5\end{array}$ & $\begin{array}{l}\frac{102}{110} \\
210 \\
106 \\
110\end{array}$ & $\begin{array}{l}162 \\
156 \\
162 \\
168 \\
168\end{array}$ & $\begin{array}{r}210 \\
* 210 \\
222 \\
222 \\
235\end{array}$ & $\begin{array}{l}381 \\
235 \\
210 \\
\frac{324}{343}\end{array}$ & $\begin{array}{l}381 \\
400 \\
400 \\
362 \\
362\end{array}$ & $\begin{array}{r}946 \\
875 \\
970 \\
2,750 \\
4,260\end{array}$ & $\begin{array}{l}4,480 \\
4,680 \\
4,800 \\
4,720 \\
4,600\end{array}$ & $\begin{array}{l}\frac{2,340}{2,280} \\
2,250 \\
2,220 \\
2,040\end{array}$ & $\begin{array}{r}305 \\
288 \\
* 270 \\
235 \\
235\end{array}$ & $\begin{array}{l}270 \\
288 \\
288 \\
288 \\
288\end{array}$ & $\begin{array}{l}500 \\
450 \\
400 \\
370 \\
350\end{array}$ & $\begin{array}{l}\frac{230}{220} \\
220 \\
220 \\
220\end{array}$ \\
\hline $\begin{array}{r}6 \\
7 \\
8 \\
9 \\
10\end{array}$ & $\begin{array}{l}110 \\
110 \\
114 \\
214 \\
128\end{array}$ & $\begin{array}{l}162 \\
168 \\
175 \\
175 \\
175\end{array}$ & $\begin{array}{r}419 \\
1,620 \\
780 \\
628 \\
708\end{array}$ & $\begin{array}{l}362 \\
362 \\
381 \\
400 \\
400\end{array}$ & $\begin{array}{l}362 \\
324 \\
305 \\
343 \\
343\end{array}$ & $\begin{array}{r}* 3,090 \\
2,680 \\
2,280 \\
2,160 \\
2,100\end{array}$ & $\begin{array}{l}4,560 \\
4,100 \\
3,880 \\
3,590 \\
3,520\end{array}$ & $\begin{array}{l}1,940 \\
1,910 \\
1,820 \\
1,730 \\
1,620\end{array}$ & $\begin{array}{l}235 \\
235 \\
270 \\
288 \\
252\end{array}$ & $\begin{array}{l}288 \\
252 \\
252 \\
235 \\
\frac{2305}{30}\end{array}$ & $\begin{array}{l}330 \\
325 \\
315 \\
310 \\
300\end{array}$ & $\begin{array}{l}220 \\
220 \\
210 \\
210 \\
210\end{array}$ \\
\hline $\begin{array}{l}11 \\
12 \\
13 \\
14 \\
15\end{array}$ & $\begin{array}{l}118 \\
123 \\
123 \\
128 \\
133\end{array}$ & $\begin{array}{l}175 \\
168 \\
168 \\
168 \\
175\end{array}$ & $\begin{array}{r}609 \\
590 \\
1,660 \\
1,390 \\
852\end{array}$ & $\begin{array}{l}\frac{419}{419} \\
419 \\
400 \\
400\end{array}$ & $\begin{array}{l}343 \\
362 \\
400 \\
400 \\
362\end{array}$ & $\begin{array}{l}2,160 \\
2,190 \\
2,130 \\
2,100 \\
1,820\end{array}$ & $\begin{array}{l}3,560 \\
3,590 \\
3,800 \\
3,960 \\
3,960\end{array}$ & $\begin{array}{l}1,560 \\
1,470 \\
1,440 \\
1,420 \\
1,330\end{array}$ & $\begin{array}{l}235 \\
235 \\
235 \\
252 \\
252\end{array}$ & $\begin{array}{r}252 \\
324 \\
270 \\
868 \\
1,060\end{array}$ & $\begin{array}{l}290 \\
285 \\
280 \\
275 \\
270\end{array}$ & $\begin{array}{l}210 \\
210 \\
210 \\
210 \\
200\end{array}$ \\
\hline $\begin{array}{l}16 \\
17 \\
18 \\
19 \\
20\end{array}$ & $\begin{array}{l}150 \\
156 \\
156 \\
162 \\
162\end{array}$ & $\begin{array}{l}190 \\
190 \\
190 \\
190 \\
190\end{array}$ & $\begin{array}{l}685 \\
628 \\
571 \\
552 \\
590\end{array}$ & $\begin{array}{l}343 \\
288 \\
305 \\
324 \\
324\end{array}$ & $\begin{array}{l}343 \\
343 \\
343 \\
324 \\
305\end{array}$ & $\begin{array}{l}1,700 \\
1,640 \\
1,670 \\
2,450 \\
4,180\end{array}$ & $\begin{array}{l}3,840 \\
3,730 \\
3,700 \\
3,420 \\
3,420\end{array}$ & $\begin{array}{r}1,360 \\
1,300 \\
1,140 \\
1,020 \\
946\end{array}$ & $\begin{array}{l}252 \\
252 \\
235 \\
235 \\
270\end{array}$ & $\begin{array}{l}1,750 \\
3,040 \\
2,090 \\
1,970 \\
1,500\end{array}$ & $\begin{array}{l}265 \\
260 \\
260 \\
250 \\
250\end{array}$ & $\begin{array}{l}200 \\
200 \\
200 \\
200 \\
200\end{array}$ \\
\hline $\begin{array}{l}21 \\
22 \\
23 \\
24 \\
25\end{array}$ & $\begin{array}{l}168 \\
162 \\
168 \\
168 \\
168\end{array}$ & $\begin{array}{l}210 \\
200 \\
200 \\
210 \\
210\end{array}$ & $\begin{array}{l}571 \\
533 \\
514 \\
476 \\
476\end{array}$ & $\begin{array}{l}362 \\
362 \\
381 \\
381 \\
400\end{array}$ & $\begin{array}{l}324 \\
324 \\
362 \\
362 \\
968\end{array}$ & $\begin{array}{l}3,160 \\
3,200 \\
2,750 \\
2,820 \\
2,680\end{array}$ & $\begin{array}{l}3,380 \\
3,300 \\
3,120 \\
3,060 \\
2,950\end{array}$ & $\begin{array}{l}922 \\
875 \\
828 \\
732 \\
666\end{array}$ & $\begin{array}{l}\frac{210}{210} \\
210 \\
210 \\
210\end{array}$ & $\begin{array}{r}1,210 \\
970 \\
970 \\
732 \\
1,220\end{array}$ & $\begin{array}{l}250 \\
240 \\
240 \\
240 \\
240\end{array}$ & $\begin{array}{l}200 \\
190 \\
190 \\
190 \\
190\end{array}$ \\
\hline $\begin{array}{l}26 \\
27 \\
28 \\
29 \\
30 \\
31\end{array}$ & $\begin{array}{r}175 \\
175 \\
175 \\
182 \\
* 190 \\
190\end{array}$ & $\begin{array}{l}210 \\
210 \\
\frac{222}{222} \\
210 \\
-\end{array}$ & $\begin{array}{r}476 \\
438 \\
419 \\
400 \\
* 419 \\
419\end{array}$ & $\begin{array}{r}400 \\
381 \\
362 \\
* 381 \\
381 \\
381\end{array}$ & $\begin{array}{c}\frac{2,220}{1,730} \\
1,330 \\
1,060 \\
=\end{array}$ & $\begin{array}{l}2,540 \\
2,480 \\
2,510 \\
3,020 \\
3,700 \\
3,960\end{array}$ & $\begin{array}{c}2,880 \\
2,820 \\
2,640 \\
2,580 \\
\frac{2,440}{-}\end{array}$ & $\begin{array}{l}571 \\
533 \\
476 \\
400 \\
324 \\
305 \\
\end{array}$ & $\begin{array}{r}210 \\
235 \\
252 \\
+324 \\
305 \\
-\end{array}$ & $\begin{array}{r}1,160 \\
1,390 \\
1,500 \\
1,300 \\
720 \\
580\end{array}$ & $\begin{array}{r}240 \\
240 \\
230 \\
230 \\
* 230 \\
230\end{array}$ & $\begin{array}{c}190 \\
190 \\
180 \\
* 180 \\
180 \\
-\end{array}$ \\
\hline $\begin{array}{l}\text { Total } \\
\text { Mean } \\
\text { le-ft }\end{array}$ & $\begin{array}{r}4,436 \\
143 \\
8,799 \\
\end{array}$ & $\begin{array}{r}5,579 \\
186 \\
11,070 \\
\end{array}$ & $\begin{array}{r}18,522 \\
597 \\
36,740 \\
\end{array}$ & $\begin{array}{r}11,211 \\
362 \\
22,21,0 \\
\end{array}$ & $\begin{array}{r}15,787 \\
544 \\
31,310 \\
\end{array}$ & $\begin{array}{r}76,971 \\
2,583 \\
152,700 \\
\end{array}$ & $\begin{array}{r}109,080 \\
3,636 \\
216,400 \\
\end{array}$ & $\begin{array}{r}39,768 \\
1,283 \\
78,880 \\
\end{array}$ & $\begin{array}{r}7,442 \\
248 \\
14,760 \\
\end{array}$ & $\begin{array}{r}27,530 \\
888 \\
54,600 \\
\end{array}$ & $\begin{array}{r}8,945 \\
289 \\
17,740 \\
\end{array}$ & $\begin{array}{r}6,100 \\
203 \\
12,100 \\
\end{array}$ \\
\hline $\begin{array}{l}\text { Caler } \\
\text { Wate: }\end{array}$ & $x$ & $6:$ & $\begin{array}{ll}\operatorname{ax} & 1,6 \\
\operatorname{ax} & 4,8\end{array}$ & & $\begin{array}{r}80 \\
102\end{array}$ & $\begin{array}{l}\text { Mean } \\
\text { Mean }\end{array}$ & $\begin{array}{l}280 \\
905\end{array}$ & $\begin{array}{l}-f t \\
-f t\end{array}$ & $\begin{array}{l}700 \\
300\end{array}$ & & & \\
\hline
\end{tabular}

* Discharge measurement made on this day.

Note - No gage-heights July 20 to Sept. 30; discharge estimated on basis of two discharge measurements and

records for Helmand River near Delraout. 
HETMAND RIVER BASIN

Tirin River at Dehraout, Afghanistan

Discharge, in cubic feet per second, water year October 1956 to September 1957

\begin{tabular}{|c|c|c|c|c|c|c|c|c|c|c|c|c|}
\hline Day & Oct. & Nove & Dec. & Jan. & Feb. & Mar. & Apr. & May & June & July & Aug. & Sept. \\
\hline $\begin{array}{l}1 \\
2 \\
3 \\
4 \\
5\end{array}$ & $\begin{array}{l}\frac{162}{168} \\
162 \\
162 \\
162\end{array}$ & $\begin{array}{r}400 \\
362 \\
343 \\
* 324 \\
330 \\
1\end{array}$ & $\begin{array}{r}370 \\
370 \\
370 \\
* 381 \\
381\end{array}$ & $\begin{array}{l}\frac{419}{419} \\
419 \\
419 \\
419\end{array}$ & $\begin{array}{l}732 \\
828 \\
780 \\
756 \\
732\end{array}$ & $\begin{array}{l}685 \\
732 \\
780 \\
804 \\
804\end{array}$ & $\begin{array}{r}800 \\
800 \\
800 \\
800 \\
2,000\end{array}$ & $\begin{array}{c}\frac{750}{750} \\
800 \\
1\end{array}$ & $\begin{array}{c}800 \\
1\end{array}$ & $\begin{array}{c}800 \\
1\end{array}$ & 700 & $\begin{array}{c}550 \\
1\end{array}$ \\
\hline $\begin{array}{r}6 \\
7 \\
8 \\
9 \\
10\end{array}$ & $\begin{array}{l}162 \\
162 \\
162 \\
162 \\
168\end{array}$ & & $\begin{array}{l}381 \\
381 \\
381 \\
381 \\
381\end{array}$ & $\begin{array}{l}419 \\
457 \\
476 \\
457 \\
419\end{array}$ & $\begin{array}{l}\frac{875}{828} \\
732 \\
708 \\
708\end{array}$ & $\begin{array}{r}828 \\
922 \\
1,020 \\
1,270 \\
1,300\end{array}$ & $\begin{array}{r}\frac{4,500}{1,800} \\
800 \\
800 \\
800\end{array}$ & & & & 700 & $\left.*\right|_{550} ^{*}$ \\
\hline $\begin{array}{l}11 \\
12 \\
13 \\
14 \\
15\end{array}$ & $\begin{array}{l}175 \\
182 \\
175 \\
182 \\
182\end{array}$ & $\begin{array}{c}330 \\
320 \\
1\end{array}$ & $\begin{array}{l}381 \\
381 \\
381 \\
381 \\
381\end{array}$ & $\begin{array}{l}419 \\
476 \\
552 \\
533 \\
495\end{array}$ & $\begin{array}{l}685 \\
685 \\
685 \\
666 \\
666\end{array}$ & $\begin{array}{r}1,190 \\
1,110 \\
* 1,160 \\
1,190 \\
1,220\end{array}$ & $\begin{array}{l}800 \\
800 \\
800 \\
800 \\
800\end{array}$ & & & 800 & 650 & 500 \\
\hline $\begin{array}{l}16 \\
17 \\
18 \\
19 \\
20\end{array}$ & $\begin{array}{l}175 \\
182 \\
175 \\
182 \\
175\end{array}$ & & $\begin{array}{l}381 \\
381 \\
381 \\
381 \\
400\end{array}$ & $\begin{array}{l}476 \\
495 \\
514 \\
514 \\
533\end{array}$ & $\begin{array}{l}685 \\
685 \\
708 \\
685 \\
666\end{array}$ & $\begin{array}{r}1,270 \\
4,580 \\
3,380 \\
800 \\
700\end{array}$ & $\begin{array}{r}800 \\
800 \\
800 \\
1,000 \\
850\end{array}$ & & & 750 & $1_{650}$ & \\
\hline $\begin{array}{l}21 \\
22 \\
23 \\
24 \\
25\end{array}$ & $\begin{array}{l}182 \\
182 \\
182 \\
182 \\
182\end{array}$ & & $\begin{array}{r}400 \\
400 \\
* 419 \\
419 \\
419\end{array}$ & $\begin{array}{r}533 \\
533 \\
533 \\
647 \\
2,410 \\
\end{array}$ & $\begin{array}{l}666 \\
666 \\
666 \\
647 \\
647\end{array}$ & $\begin{array}{l}600 \\
571 \\
552 \\
571 \\
533 \\
\end{array}$ & $\begin{array}{l}800 \\
750 \\
750 \\
750 \\
750\end{array}$ & & & & 1 & 100 \\
\hline $\begin{array}{l}26 \\
27 \\
28 \\
29 \\
30 \\
31\end{array}$ & $\begin{array}{l}190 \\
190 \\
200 \\
200 \\
270 \\
615 \\
\end{array}$ & $\begin{array}{c}1 \\
320 \\
350 \\
350 \\
-\end{array}$ & $\begin{array}{l}400 \\
400 \\
400 \\
381 \\
381 \\
400\end{array}$ & $\begin{array}{r}1,220 \\
970 \\
852 \\
780 \\
732 \\
685\end{array}$ & $\begin{array}{c}647 \\
647 \\
685 \\
- \\
- \\
-\end{array}$ & $\begin{array}{l}533 \\
533 \\
552 \\
875 \\
875 \\
828\end{array}$ & $\begin{array}{l}750 \\
750 \\
750 \\
750 \\
750 \\
-\end{array}$ & 800 & $\begin{array}{l}100 \\
-\end{array}$ & $\left.\right|_{750}$ & $\frac{1}{600}$ & $\left.\right|_{400}$ \\
\hline $\begin{array}{l}\text { Total } \\
\text { Mean } \\
\text { Ac-ft } \\
\end{array}$ & $\begin{array}{r}5,992 \\
193 \\
11,880 \\
\end{array}$ & $\begin{array}{r}9,939 \\
331 \\
19,710 \\
\end{array}$ & $\begin{array}{r}12,025 \\
388 \\
23,850 \\
\end{array}$ & $\begin{array}{r}19,225 \\
620 \\
38,130 \\
\end{array}$ & $\begin{array}{r}19,766 \\
706 \\
39,210 \\
\end{array}$ & $\begin{array}{r}32,768 \\
1,057 \\
64,990 \\
\end{array}$ & $\begin{array}{r}29,700 \\
990 \\
58,910 \\
\end{array}$ & $\begin{array}{r}24,700 \\
797 \\
48,990 \\
\end{array}$ & $\begin{array}{r}24,000 \\
800 \\
47,600 \\
\end{array}$ & $\begin{array}{r}24,000 \\
774 \\
47,600 \\
\end{array}$ & $\begin{array}{r}20,100 \\
681 \\
39,870 \\
\end{array}$ & $\begin{array}{r}15,000 \\
500 \\
29,750 \\
\end{array}$ \\
\hline $\begin{array}{l}\text { Calen } \\
\text { iater }\end{array}$ & r 195 & $\begin{array}{l}\operatorname{Max} \\
\operatorname{Max}\end{array}$ & $\begin{array}{l}4,800 \\
4,580\end{array}$ & $\begin{array}{l}\text { Min } \\
\text { Win }\end{array}$ & $\begin{array}{l}\mathrm{Me} \\
\mathrm{lie}\end{array}$ & $\begin{array}{ll}\text { an } & 631 \\
\text { an } & 650\end{array}$ & $\begin{array}{l}A c-f t \\
A C-f t\end{array}$ & $\begin{array}{l}656,100 \\
470,500\end{array}$ & & & & \\
\hline
\end{tabular}

* Discharje measurement made on this day.

Note--Doubtful or no gage-heights Kov. 5 to Dec. 3, liar. 18-21, jur. 1 to jept. 30; discharge t'stimated 作 Reservoir. 
HELMAND RIVRR BASTN

Tirin River at Dehraout, LFhanistan

Discharge, in cuble feet per second, water year October 1957 to September 1958

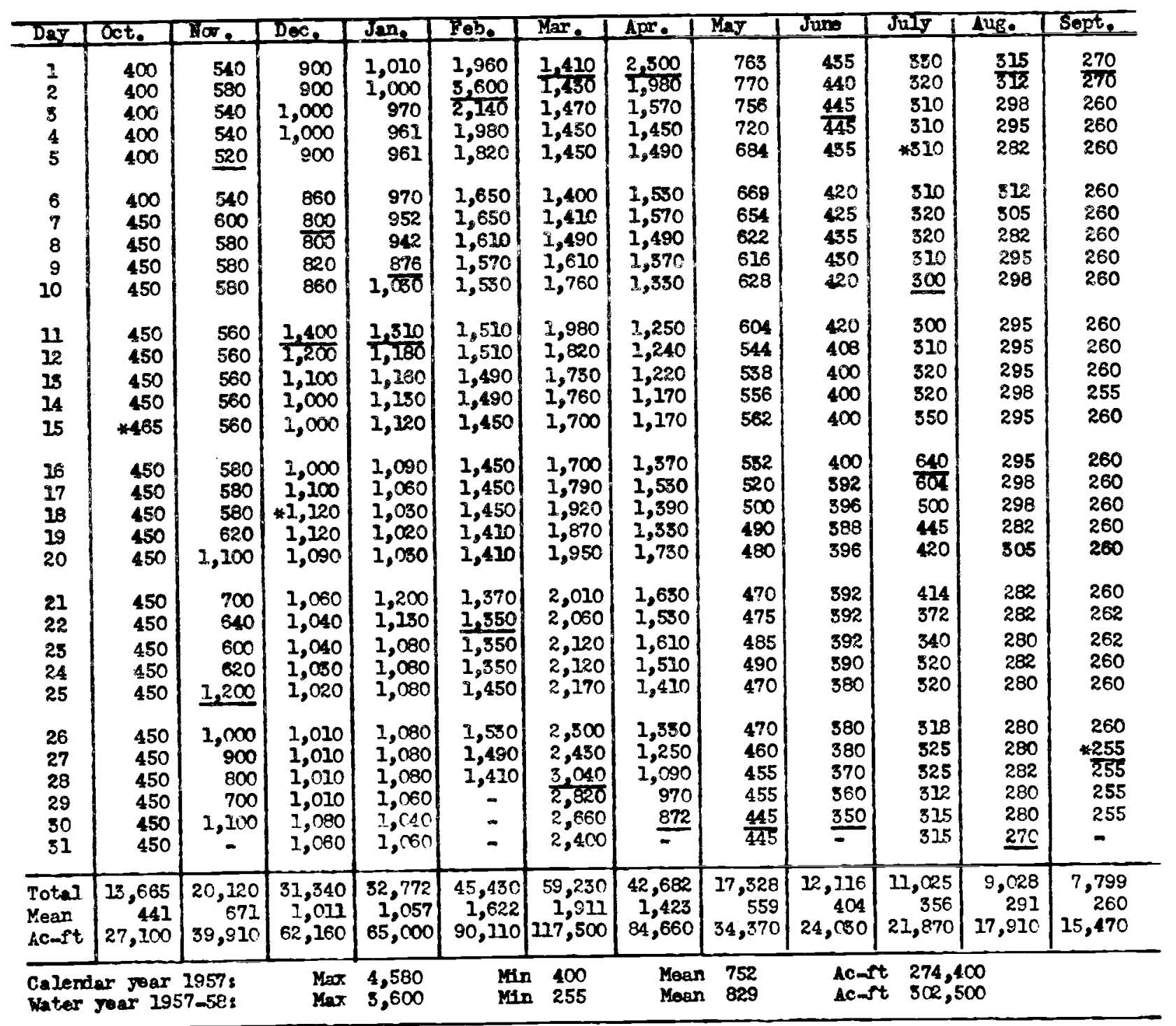

* Discharge measurement rade on this day.

Note. Dect. I to Dec. 17, June 24 to July 15, Aug. 26, 27, Aug. 29 to Sept. 4, Sept. 6m.3, 16.10; discharge estimated on basis of Helmand River near Dehraout and change in contents and release for Kajakai Reservoir. 
HEINGAND HIVER BASTN

Tirin River at Dehraout, Afghanistan

Discharge, in cubic feet per second, water year October 1958 to September 1959

\begin{tabular}{|c|c|c|c|c|c|c|c|c|c|c|c|c|}
\hline Day & Oct. & Nove & Dec. & Jan. & Feb. & Mar. & Apr. & lay & June & July & AuE. & Sept. \\
\hline $\begin{array}{l}1 \\
2 \\
3 \\
4 \\
5\end{array}$ & $\frac{260}{1}$ & $\prod_{280}^{280}$ & $\begin{array}{l}\frac{380}{384} \\
388 \\
388 \\
392\end{array}$ & $\begin{array}{l}580 \\
580 \\
770 \\
684 \\
610\end{array}$ & $\begin{array}{l}\frac{580}{580} \\
616 \\
610 \\
592\end{array}$ & $\begin{array}{l}1,350 \\
3,080 \\
1,760 \\
1,700 \\
1,650\end{array}$ & $\begin{array}{l}1,860 \\
2,020 \\
2,500 \\
2,260 \\
2,260\end{array}$ & $\begin{array}{l}882 \\
820 \\
820 \\
820 \\
820\end{array}$ & $\begin{array}{r}310 \\
310 \\
* 304 \\
317 \\
317\end{array}$ & $\begin{array}{r}246 \\
+246 \\
242 \\
280 \\
256\end{array}$ & $\begin{array}{l}193 \\
188 \\
188 \\
198 \\
198\end{array}$ & $\begin{array}{l}160 \\
156 \\
160 \\
156 \\
160\end{array}$ \\
\hline $\begin{array}{r}6 \\
7 \\
8 \\
9 \\
10\end{array}$ & & $\left.\right|_{290} ^{290}$ & $\begin{array}{r}396 \\
400 \\
412 \\
420 \\
* 430\end{array}$ & $\begin{array}{l}592 \\
586 \\
580 \\
574 \\
568\end{array}$ & $\begin{array}{l}580 \\
580 \\
580 \\
622 \\
727\end{array}$ & $\begin{array}{l}1,490 \\
1,450 \\
1,410 \\
1,700 \\
2,800\end{array}$ & $\begin{array}{l}2,220 \\
2,160 \\
2,190 \\
2,160 \\
2,160\end{array}$ & $\begin{array}{l}775 \\
730 \\
718 \\
696 \\
639\end{array}$ & $\begin{array}{l}304 \\
304 \\
285 \\
285 \\
285\end{array}$ & $\begin{array}{l}256 \\
261 \\
246 \\
246 \\
242\end{array}$ & $\begin{array}{l}188 \\
164 \\
156 \\
169 \\
169\end{array}$ & $\begin{array}{l}160 \\
164 \\
164 \\
169 \\
164\end{array}$ \\
\hline $\begin{array}{l}11 \\
12 \\
13 \\
14 \\
15\end{array}$ & & $\begin{array}{l}300 \\
310 \\
320 \\
320 \\
320\end{array}$ & $\begin{array}{l}505 \\
742 \\
568 \\
550 \\
544\end{array}$ & $\begin{array}{l}568 \\
574 \\
568 \\
562 \\
568\end{array}$ & $\begin{array}{l}705 \\
647 \\
634 \\
634 \\
622\end{array}$ & $\begin{array}{l}1,700 \\
1,400 \\
1,200 \\
1,100 \\
\frac{1,100}{1,10}\end{array}$ & $\begin{array}{l}2,190 \\
2,160 \\
2,050 \\
2,050 \\
2,050\end{array}$ & $\begin{array}{r}628 \\
606 \\
586 \\
586 \\
1,190\end{array}$ & $\begin{array}{l}285 \\
275 \\
266 \\
266 \\
275\end{array}$ & $\begin{array}{l}242 \\
237 \\
242 \\
232 \\
227\end{array}$ & $\begin{array}{l}169 \\
169 \\
169 \\
169 \\
169\end{array}$ & $\begin{array}{l}169 \\
164 \\
169 \\
169 \\
164\end{array}$ \\
\hline $\begin{array}{l}16 \\
17 \\
18 \\
19 \\
20\end{array}$ & $\left.\right|_{260}$ & $\begin{array}{l}325 \\
325 \\
330 \\
328 \\
338\end{array}$ & $\begin{array}{l}544 \\
556 \\
568 \\
568 \\
598\end{array}$ & $\begin{array}{l}568 \\
562 \\
568 \\
556 \\
550\end{array}$ & $\begin{array}{l}628 \\
634 \\
698 \\
705 \\
662\end{array}$ & $\begin{array}{l}1,100 \\
1,150 \\
1,200 \\
1,300 \\
1,550\end{array}$ & $\begin{array}{l}2,050 \\
2,020 \\
1,990 \\
1,910 \\
1,860\end{array}$ & $\begin{array}{r}1,100 \\
1,450 \\
1,260 \\
1,040 \\
882\end{array}$ & $\begin{array}{l}266 \\
266 \\
266 \\
266 \\
256\end{array}$ & $\begin{array}{l}266 \\
304 \\
275 \\
270 \\
251\end{array}$ & $\begin{array}{l}169 \\
169 \\
178 \\
169 \\
169\end{array}$ & $\begin{array}{l}169 \\
169 \\
178 \\
178 \\
178\end{array}$ \\
\hline $\begin{array}{l}21 \\
22 \\
23 \\
24 \\
25\end{array}$ & 270 & $\begin{array}{l}338 \\
344 \\
348 \\
352 \\
356\end{array}$ & $\begin{array}{l}\frac{628}{616} \\
610 \\
592 \\
586\end{array}$ & $\begin{array}{l}\frac{544}{544} \\
544 \\
\frac{807}{720}\end{array}$ & $\begin{array}{r}654 \\
698 \\
* 712 \\
691 \\
684\end{array}$ & $\begin{array}{l}1,700 \\
1,800 \\
1,830 \\
1,880 \\
1,990\end{array}$ & $\begin{array}{l}1,910 \\
2,020 \\
2,470 \\
1,800 \\
1,560\end{array}$ & $\begin{array}{l}730 \\
628 \\
578 \\
530 \\
514\end{array}$ & $\begin{array}{l}256 \\
246 \\
256 \\
256 \\
246 \\
\end{array}$ & $\begin{array}{l}208 \\
213 \\
213 \\
208 \\
203\end{array}$ & $\begin{array}{l}169 \\
169 \\
174 \\
169 \\
174\end{array}$ & $\begin{array}{l}178 \\
178 \\
178 \\
178 \\
178\end{array}$ \\
\hline $\begin{array}{l}26 \\
27 \\
28 \\
29 \\
30 \\
31 \\
\end{array}$ & $1_{270}$ & $\begin{array}{l}360 \\
364 \\
372 \\
372 \\
380 \\
-\end{array}$ & $\begin{array}{r}586 \\
580 \\
* 580 \\
586 \\
580 \\
580 \\
\end{array}$ & $\begin{array}{l}628 \\
610 \\
604 \\
604 \\
598 \\
592 \\
\end{array}$ & $\begin{array}{c}698 \\
742 \\
727 \\
- \\
- \\
- \\
\end{array}$ & $\begin{array}{l}1,990 \\
1,940 \\
2,050 \\
2,050 \\
2,100 \\
1,960 \\
\end{array}$ & $\begin{array}{c}1,470 \\
1,340 \\
1,220 \\
1,120 \\
944 \\
- \\
\end{array}$ & $\begin{array}{l}434 \\
434 \\
369 \\
324 \\
324 \\
324\end{array}$ & $\begin{array}{l}246 \\
256 \\
256 \\
256 \\
246 \\
- \\
\end{array}$ & $\begin{array}{r}193 \\
198 \\
193 \\
* 193 \\
188 \\
188 \\
\end{array}$ & $\begin{array}{l}169 \\
169 \\
156 \\
152 \\
\frac{152}{152} \\
+152 \\
\end{array}$ & $\begin{array}{l}183 \\
188 \\
193 \\
193 \\
\frac{203}{-}\end{array}$ \\
\hline $\begin{array}{l}\text { Total } \\
\text { Mean }\end{array}$ & $\begin{array}{r}8,170 \\
264 \\
6,200\end{array}$ & $\begin{array}{r}9,652 \\
322 \\
19,340\end{array}$ & $\begin{array}{r}16,257 \\
524 \\
32,250\end{array}$ & $\begin{array}{r}18,563 \\
599 \\
36,820\end{array}$ & $\begin{array}{r}18,21,2 \\
652 \\
36,180\end{array}$ & $\begin{array}{r}52,480 \\
1,693 \\
104,100 \\
\end{array}$ & $\begin{array}{r}57,974 \\
1,932 \\
115,000\end{array}$ & $\begin{array}{r}22,217 \\
717 \\
44,070\end{array}$ & $\begin{array}{r}8,228 \\
274 \\
16,320 \\
\end{array}$ & $\begin{array}{r}7,265 \\
234 \\
14,410 \\
\end{array}$ & $\begin{array}{r}5,315 \\
171 \\
10,540 \\
\end{array}$ & $\begin{array}{r}5,170 \\
172 \\
10,250 \\
\end{array}$ \\
\hline
\end{tabular}

$\begin{array}{llllllll}\text { Caiendar year 1958: } & \text { Max } & 3,600 & \text { Min } 255 & \text { Mean } 744 & \text { Ac-ft } 538,500 \\ \text { Water year 1958-59: } & \text { Max } 3,080 & \text { Min } 152 & \text { Mean } 629 & \text { Ac-ft } 455,300\end{array}$

* Discharge measurement made on this day.

Note.- No gage-heights Oct. I to Nov. Il and Mar.9-22; discharge estimated on basis of recorded range in stage Mar. 9-22 and record for Helmand River at Dehraout. 
HEIMAND RIVER BASIN

Tirin River at Dehraout, Afghanistan

Discharge, in cuble feet per second, water year October 1959 to September 1960

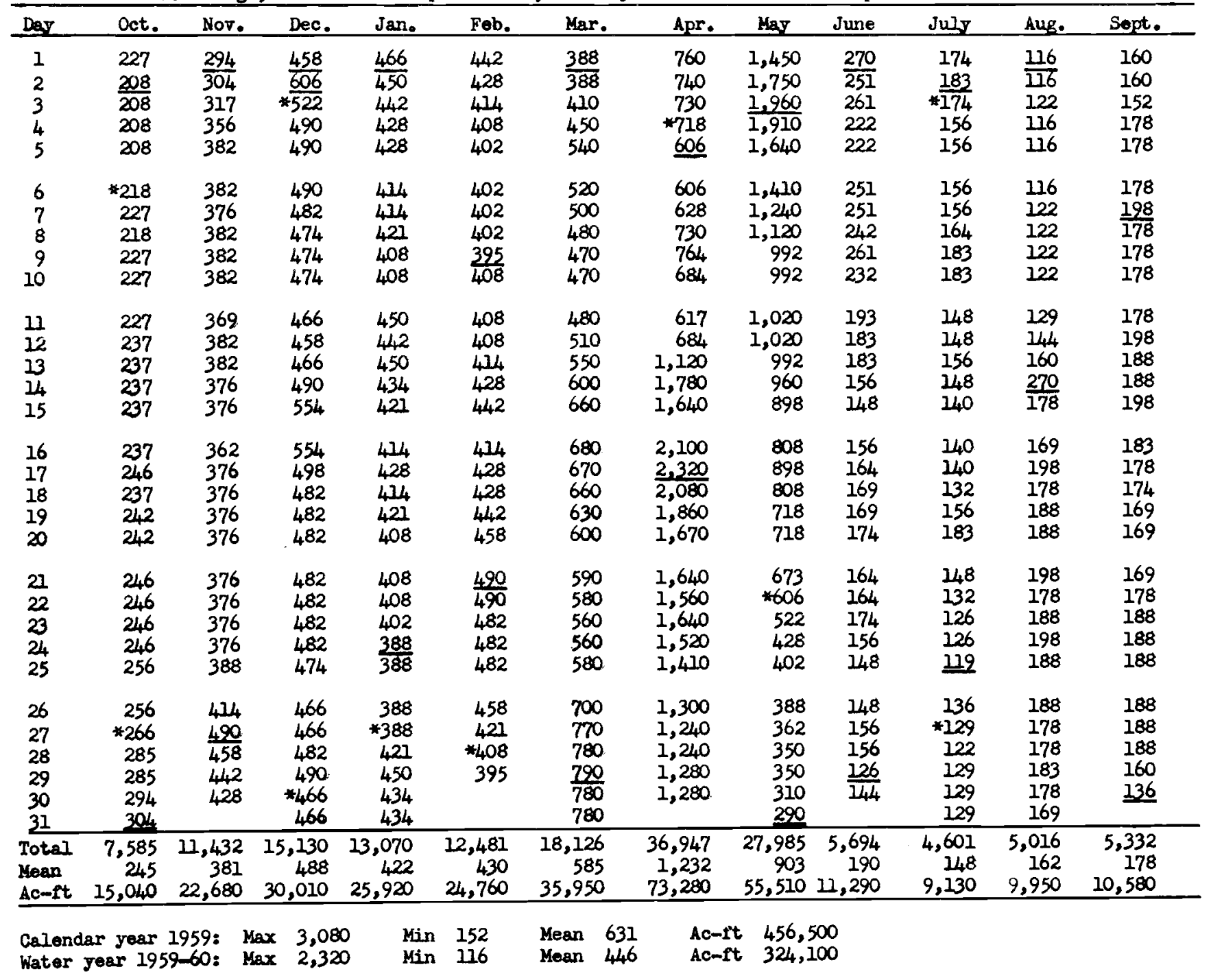

* Discharge messurement made on this day.

Note. - No gage-heights Kar. 31 to Apr. 3; discharge estimated on basis of record for helmand River near Dehraout. 
HETNAND RIVER BASIN

\section{Kajakai Reservoir at Kajakai, Afghanistan}

Location.--Lat $32^{\circ} 19^{\prime} \mathrm{N} \%$, long $65^{\circ} \mathrm{O} 7^{\prime} \mathrm{E}$. on gate control tower near left end of Kajakai dam on the Helmand River, about 3 kilometers northeast of village of Kajakai, about 40 kilometers upstream from the Musa Qala River, about 75 kilometers nor theast of Girishk, and about 125 kilometers upstream from Arghandab River. Drainage area.--16,300 sq mi, approxinately (from Survey of India maps). Records available.--January 1953 to September 1960.

Gage.-Water-stage recorder. Datum of gage is at nean sea level (from Morrison-rinudsen Afghanistan surveys

based on survey of India datum). Prior to $\mathrm{Nar} 23,1953$, records are from levels to water surface.

Extremes.-Maximum and minimum contents for the water years 1953-60 are given in the following table:

\begin{tabular}{|c|c|c|c|c|c|c|}
\hline \multirow[b]{2}{*}{ Water year } & \multirow[b]{2}{*}{ Date } & \multicolumn{2}{|c|}{ Laximum } & \multirow[b]{2}{*}{ Date } & \multicolumn{2}{|c|}{ Minimum } \\
\hline & & $\begin{array}{l}\text { Elevation } \\
\text { Leters }\end{array}$ & $\begin{array}{l}\text { Contents } \\
\text { Acre-ft }\end{array}$ & & $\begin{array}{l}\text { Elevation } \\
\text { meters }\end{array}$ & $\begin{array}{l}\text { Contents } \\
\text { Acre-ft }\end{array}$ \\
\hline $\begin{array}{l}1953 \\
1954 \\
1955 \\
1956 \\
1957 \\
1958 \\
1959 \\
1960\end{array}$ & $\begin{array}{l}\text { June } 8 \\
\text { May } 3 \\
\text { June } 26 \\
\text { Apr. } 22 \\
\text { May } 4 \\
\text { Apr. } 27 \\
\text { Apr. } 23 \\
\text { May } 19\end{array}$ & $\begin{array}{l}1031.56 \\
1035.83 \\
1034.29 \\
1036.67 \\
1037.505 \\
1035.37 \\
1035.25 \\
1035.54 \\
\end{array}$ & $\begin{array}{l}1,381,900 \\
1,639,300 \\
1,543,000 \\
1,692,900 \\
1,747,300 \\
1,610,300 \\
1,602,800 \\
1,621,000\end{array}$ & $\begin{array}{l}\text { Dec. } 21 \\
\text { Mar. } 10 \\
\text { Feb. } 25 \\
\text { Feb. } 25 \\
\text { Sept. } 30 \\
\text { Jan. } 24 \\
\text { Feb.11-14 }\end{array}$ & $\begin{array}{l}1004.24 \\
1008.24 \\
1014.05 \\
1011.64 \\
1014.25 \\
1002.77 \\
999.82 \\
\end{array}$ & $\begin{array}{l}347,300 \\
442,200 \\
612,600 \\
537,200 \\
619,200 \\
316,600 \\
262,900\end{array}$ \\
\hline
\end{tabular}

Remarks.-Reservoir is formed by earth-fill dam; storage began Jan. 28, 1953; dam completed November 1953. Present capacity, 1,495,000 acre-ft between 965.0 meters, center-line of irrigation outlet, and 1033.50 meters, crest of ungated spillway, above sea level. Elevation of top of dam is 1050.0 meters; capacity with future gated spillway, 2,300,000 acre-ft. No dead storage (future power plant installation will govern). Records herein represent total contents. Reservoir is used for irrigation of up to 600,000 acres in the Helmand River Valley and for a future installed power capacity of about 120,000 KW. Reservoir release is through three hollow-jet valves backed up by three 84 -inch roto-valves and vertical lift gates. Naximum valve release is $8,400 \mathrm{cfs}$ at 1050 meters pond stage and 7,660 cfs at 1033.5 meters pond stage; maximum spillway design capacity is $350,000 \mathrm{cfs}$. 
HEIVIND RIVIR BASTN

Kajakai Reservoir at Kajakai, Afghanistan

Contents, in thousands of acre-feet, on last day of month, of Kajakai Reservoir at Kajakai, Afghanistan

\begin{tabular}{|c|c|c|c|c|c|c|c|c|c|c|c|c|}
\hline Year & Oct. & Nov. & Dec. & $\operatorname{Jan}$ & Feb. & Mar. & Apr. & May & June & July & Aug. & Sept. \\
\hline $\begin{array}{l}1953 \\
1954 \\
1955 \\
1956 \\
1957 \\
1958 \\
1959 \\
1960\end{array}$ & $\begin{array}{r}409.8 \\
1,021.5 \\
903.9 \\
941.3 \\
819.1 \\
415.2 \\
452.6\end{array}$ & $\begin{array}{l}371.1 \\
968.8 \\
787.1 \\
845.8 \\
943.4 \\
350.8 \\
437.8\end{array}$ & $\begin{array}{l}394.2 \\
863.2 \\
727.4 \\
738.2 \\
936.1 \\
346.0 \\
334.6\end{array}$ & $\begin{array}{r}6.3 \\
421.5 \\
654.5 \\
666.1 \\
625.2 \\
846.0 \\
327.9 \\
269.8\end{array}$ & $\begin{array}{l}122.7 \\
415.7 \\
482.5 \\
645.0 \\
567.5 \\
835.6 \\
354.3 \\
289.6\end{array}$ & $\begin{array}{r}583.5 \\
891.3 \\
820.6 \\
1,295.1 \\
1,317.0 \\
1,173.8 \\
1,085.5 \\
474.2\end{array}$ & $\begin{array}{l}1,097.8 \\
1,633.6 \\
1,138.6 \\
1,633.6 \\
1,671.1 \\
1,601.5 \\
1,577.1 \\
1,184.5\end{array}$ & $\begin{array}{l}1,345.5 \\
1,551.0 \\
1,532.4 \\
1,525.0 \\
1,631.1 \\
1,522.0 \\
1,525.0 \\
1,576.5\end{array}$ & $\begin{array}{l}1,273.6 \\
1,460.9 \\
1,540.5 \\
1,535.5 \\
1,533.1 \\
1,423.2 \\
1,375.1 \\
1,519.0\end{array}$ & $\begin{array}{r}991.7 \\
1,420.3 \\
1,442.4 \\
1,548.2 \\
1,437.7 \\
1,186.5 \\
1,076.6 \\
1,456.9\end{array}$ & $\begin{array}{r}677.3 \\
1,233.7 \\
1,233.7 \\
1,224.7 \\
1,290.2 \\
877.4 \\
758.6 \\
1,333.2\end{array}$ & $\begin{array}{r}507.8 \\
1,093.8 \\
1,049.8 \\
987.3 \\
1,037.2 \\
619.2 \\
550.2 \\
1,195.5\end{array}$ \\
\hline
\end{tabular}




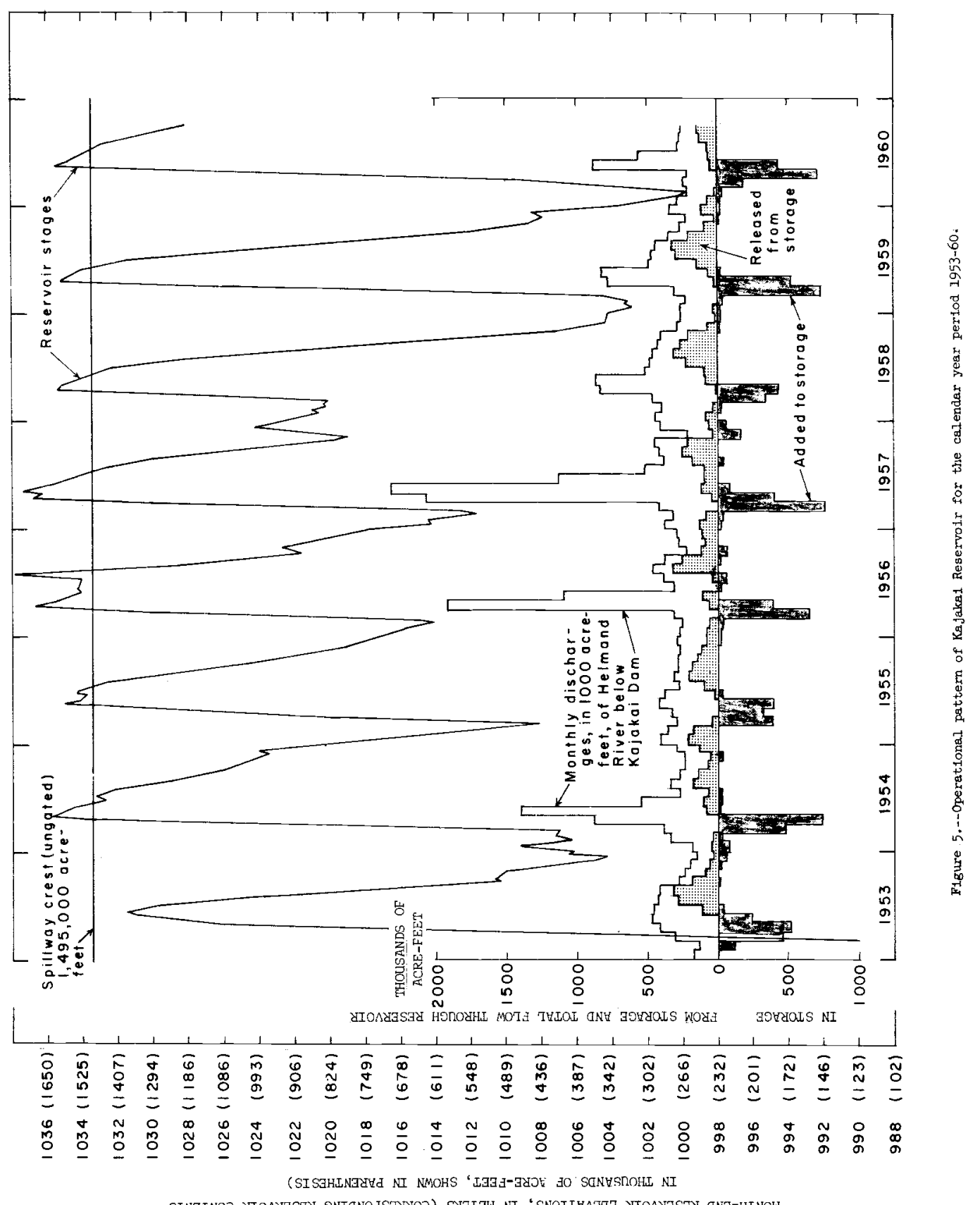


HEIMIAND RIVER BASTN

Helmand River below Kajakal Dam, Afghanistan

Location.-Lat $32^{\circ} 19^{\prime} \mathrm{N}$, long $65^{\circ} 06^{\prime} \mathrm{E}$, on left bank 2 kilometers downstream from Kajakai Dam, about 100 kilameters northwest of Kandahar, and about 123 kilometers upstream from Arghandab River.

Drainage are a. - 16,300 sq $\mathrm{mi}$, approximately (from Survey of India maps).

Rocords avilable.-October 1947 to September 1960.

Gege. - Water-stage recorder. Datum of gage is 690.169 meters above mean sea level (from Morrison-Knudsen

Afgharistan survey based on Survey of India datum).

Average discharge.-13 years, 7,369 cfs $(5,336,000$ acre-ft per year) adjusted for storage.

Bxtrames.-Yadmim and minimim discharges for the water years 1948-60 are given in the following table:

\begin{tabular}{|c|c|c|c|c|c|c|}
\hline \multirow[b]{2}{*}{ Water Year } & \multirow[b]{2}{*}{ Date } & \multicolumn{2}{|c|}{ Madimum } & \multirow[b]{2}{*}{ Date } & \multicolumn{2}{|c|}{ Minjimm } \\
\hline & & $\begin{array}{l}\text { Gage Height } \\
\text { (meters) }\end{array}$ & $\begin{array}{l}\text { Discharge } \\
\text { (cfs) }\end{array}$ & & $\begin{array}{l}\text { Gage Height } \\
\text { (meters) }\end{array}$ & $\begin{array}{l}\text { Discharge } \\
\text { (cfs) }\end{array}$ \\
\hline $\begin{array}{l}1948 \\
1949 \\
1950 \\
1951 \\
1952 \\
1953 \\
1954 \\
1955 \\
1956 \\
1957 \\
1958 \\
1959 \\
1960 \\
\end{array}$ & $\begin{array}{l}\text { Mar. } 8,1948 \\
\text { Apr. } 26 \text { or } 27,1949 \\
\text { May } 11,1950 \\
\text { May } 7,1951 \\
\text { Apr. 20, } 1952 \\
\text { May 28-30, 1953 } \\
\text { May 3, 1954 } \\
\text { May } 30 \text { to June } 2,1955 \\
\text { Apr. 21, } 1956 \\
\text { May } 5,1957 \\
\text { Apr. 27, } 1958 \\
\text { Apr. 23, } 1959 \\
\text { Mev 18, } 1960\end{array}$ & $\begin{array}{r}2.76 \\
83.79 \\
3.10 \\
3.70 \\
2.65 \\
1.32 \\
2.84 \\
1.47 \\
- \\
- \\
2.26 \\
2.11 \\
2.15 \\
\end{array}$ & $\begin{array}{r}31,000 \\
50,100 \\
37,300 \\
48,400 \\
29,000 \\
7,810 \\
32,200 \\
9,300 \\
44,500 \\
59,800 \\
24,900 \\
22,000 \\
23,400 \\
\end{array}$ & $\begin{array}{l}\text { Oct. } 1,1947 \\
\text { Oct. 1-6, 11, } 1948 \\
\text { Nany days } \\
\text { Oct. 1-3, 1950 } \\
\text { Aug. 16-25, 1952 } \\
\text { liar.25, Sept. } 29,1953 \\
\text { Nov.12,13, 1953 } \\
\text { Dec.5-8, 1954 } \\
\text { Sept.5-8, 1956 } \\
\text { Feb.26,27, } 1957 \\
\text { Nov.7-14, 1957 } \\
\text { Nov. 18-25, 1958 } \\
\text { Sept.22,23,27, 1960 }\end{array}$ & $\begin{array}{l}- \\
- \\
= \\
= \\
-0.60 \\
= \\
- \\
= \\
- \\
\\
.675 \\
.49\end{array}$ & $\begin{array}{r}1,100 \\
1,810 \\
1,980 \\
2,000 \\
1,810 \\
40 \\
15 \\
5 \\
1,200 \\
5 \\
5 \\
3,820 \\
2,360 \\
\end{array}$ \\
\hline
\end{tabular}

a From floodmark

Remarks.-Records good except those for water years 1950,1951 , those below 300 cfs and those for periods of no gage-height record, which are fair. Flow regulated by Ka jakai Reservoir since Jan. $28,1953$. 
HETWHD ZIVHA B.SSIN

Helukand iiver below Kujukai Dm, af thanistan

Discharge, in cubic feet per seccnd, water.jear Octoper 1947 to sejteriber 1948

\begin{tabular}{|c|c|c|c|c|c|c|c|c|c|c|c|c|}
\hline Devir & Oct. 1 & Nov. 1 & Dec。 & $\operatorname{van}_{0}$ & res. & is & $\ldots$ & {$[\because x y$} & iune & Juli & ilug. & Sept. \\
\hline 1 & 1,100 & 1,750 & 2,070 & 1,940 & 2,400 & 3,060 & 13,700 & 23,100 & 6,090 & 3,190 & 1,610 & 1,260 \\
\hline 2 & 1,150 & 1,750 & 2,070 & 1,900 & 2,500 & 3,140 & $\overline{1,500}$ &.$\overline{21,500}$ & 0,490 &,- 080 & 1,570 & 1,200 \\
\hline 3 & 1,150 & 1,750 & 2,070 & 2,070 & 2,500 & 3,370 & 16,900 & a.21,000 & 6,200 & 43,000 & 1,540 & 1,260 \\
\hline 4 & 1,200 & 1,750 & 2,070 & 2,120 & 2,400 & 4,700 & 18,100 & 221,000 & 6,000 & 22,900 & 1,500 & 1,300 \\
\hline 5 & 1,200 & 1,750 & 2,400 & 2,070 & 2,350 & 5,910 & 16,000 & $=20,500$ & 5,910 & 22,800 & 1,500 & 1,330 \\
\hline 6 & 1,200 & 1,800 & 2,600 & 2,070 & 2,210 & 6,310 & 14,500 & 220,000 & 5,720 & $a 2,700$ & $1,4,60$ & 1,330 \\
\hline 7 & 1,250 & 1,800 & 2,450 & 2,070 & 2,120 & 17,700 & 14,500 & $a 19,000$ & 5,630 & 12,600 & 1,430 & 1,330 \\
\hline 8 & 1,250 & 1,800 & 2,400 & 2,120 & $\overline{2,120}$ & 24,400 & $* 16,400$ & al 8,000 & 5,460 & 22,500 & 1,460 & 1,330 \\
\hline 9 & 1,250 & 1,800 & 2,450 & 2,210 & 2,160 & 14,609 & 20,200 & $=17,500$ & 5,280 & $=2,500$ & 1,460 & 1,360 \\
\hline 10 & 1,300 & 1,850 & 2,400 & 2,210 & 2,300 & 11,900 & 20,700 & $=17,000$ & 5,190 & 22,400 & 1,460 & 1,306 \\
\hline 11 & 1,350 & 1,850 & 2,300 & 2,160 & 2,350 & 10,900 & 20,200 & al 6,000 & 4,940 & 22,300 & 1,460 & 1,360 \\
\hline 12 & 1,350 & 1,350 & 2,210 & 2,070 & 2,350 & 9,590 & 18,100 & 15,000 & 4,700 & $=2,300$ & 1,460 & 1,360 \\
\hline 13 & 1,400 & 1,900 & 2,120 & 1,980 & $2,4,0$ & 8,650 & 17,700 & a14, 000 & 4,620 & $a 2,200$ & 1,430 & 1,360 \\
\hline 14 & 1,400 & 1,950 & 2,120 & 1,940 & 2,400 & 8,090 & 17,900 & 213,000 & 4,540 & a2,200 & 1,400 & 1,400 \\
\hline 15 & 1,450 & 1,950 & 2,160 & 1,940 & $2,+00$ & 7,420 & 18,500 & $\alpha 12,500$ & 4,460 & 32,100 & 1,330 & 1,460 \\
\hline 16 & 1,450 & 2,000 & 2,120 & 1,980 & $2,4,00$ & 7,000 & 19,700 & 212,000 & 4,310 & 22,100 & 1,200 & 1,500 \\
\hline 17 & 1,500 & 2,000 & 2,120 & 1,980 & 2,400 & $\approx 7,000$ & 22,700 & 11,100 & 4,170 & 32,0010 & 1,260 & 1,500 \\
\hline 18 & 1,500 & 2,000 & 2,070 & 1,980 & 2,500 & 7,420 & 26,600 & 10,800 & 4,100 & $=1,900$ & 1,260 & 1,540 \\
\hline 19 & 1,550 & 2,000 & 2,020 & 2,020 & 2,550 & 8,880 & 28,800 & 10,300 & 3,950 & 21,900 & 1,300 & $1,54 \mathrm{C}$ \\
\hline 20 & 1,600 & 2,020 & 2,070 & 1,980 & 2,700 & 9,940 & 29,200 & 10,100 & 3,750 & 1,850 & 1,300 & 1,570 \\
\hline 21 & 1,600 & 1,980 & 2,020 & $* 2,020$ & 2,810 & 10,200 & 28,100 & 9,820 & 3,680 & 1,890 & 1,300 & 1,650 \\
\hline 22 & 1,600 & 1,980 & 2,070 & 2,020 & 2,810 & 10,300 & 25,300 & 9,590 & 3,620 & 1,810 & 1,300 & 1,690 \\
\hline 23 & 1,600 & 1,980 & 2,160 & 2,070 & 2,760 & 10,200 & 24,400 & 9,350 & 3,490 & 1,810 & 1,300 & 1,730 \\
\hline 24 & 1,650 & 1,980 & 2,160 & 2,210 & $* 2,650$ & 10,600 & 24,400 & 9,000 & 3,430 & 1,770 & 1,260 & 1,770 \\
\hline 25 & 1,650 & 2,020 & 2,160 & 2,210 & 2,700 & 10,400 & a25, 000 & 8,650 & 3,430 & 1,770 & 1,230 & 1,770 \\
\hline 26 & 1,650 & 2,020 & 2,120 & 2,210 & $3,0<0$ & 10,900 & a25,500 & 8,310 & 3,430 & 1,730 & 1,260 & 1,690 \\
\hline 27 & 1,700 & 2,020 & 2,160 & 2,260 & 3,250 & 11,300 & a 26,000 & 7,860 & $\because 3,370$ & 1,730 & 1,260 & 1,690 \\
\hline 28 & $\overline{1,700}$ & 2,020 & 2,160 & 2,860 & 3,250 & 11,700 & a26,000 & 7,640 & 3,370 & 1,730 & 1,260 & al, 700 \\
\hline 29 & 1,700 & 2,020 & 2,160 & $\overline{2,450}$ & 3,140 & 12,400 & 25,500 & 7,420 & 3,370 & $\therefore 1,730$ & $* 1,300$ & al, 750 \\
\hline 30 & 1,700 & 2,020 & 2,120 & 2,400 & - & 11,900 & 24,600 & 7,100 & 3,310 & 1,690 & 1,300 & al, 750 \\
\hline 31 & 1,700 & - & 2,020 & $2, i_{4} \mathrm{CO}$ & - & $12,400^{\prime}$ & - & 6,900 & - & 1,650 & 1,260 & - \\
\hline $\begin{array}{l}\text { Total } \\
\text { iean } \\
\text { ic-ft }\end{array}$ & $\begin{array}{r}44,850 \\
1,4,47 \\
88,960\end{array}$ & $\begin{array}{r}57,360 \\
1,912 \\
13,800\end{array}$ & $\begin{array}{r}67,600 \\
2,181 \\
134,100\end{array}$ & $\begin{array}{r}66,000 \\
2,129 \\
130,900\end{array}$ & $\begin{array}{r}73,900 \\
2,548 \\
146,600 \\
\end{array}$ & $\begin{array}{r}302,300 \\
9,752 \\
599,6001\end{array}$ & $\begin{array}{r}640,000 \\
21,330 \\
1,269,000 \\
\end{array}$ & $\begin{array}{r}415,0,0 \\
13,390 \\
823,200\end{array}$ & $\begin{array}{r}136,690 \\
4,556 \\
271,100 \\
\end{array}$ & $\begin{array}{r}67,820 \\
2,188 \\
134,500 \\
\end{array}$ & $\begin{array}{r}2,520 \\
1,372 \\
84,340\end{array}$ & $\begin{array}{r}4,900 \\
1,497 \\
89,050\end{array}$ \\
\hline
\end{tabular}

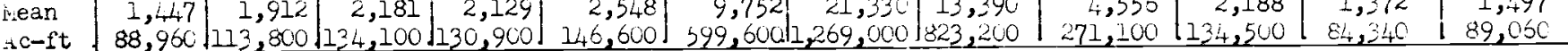

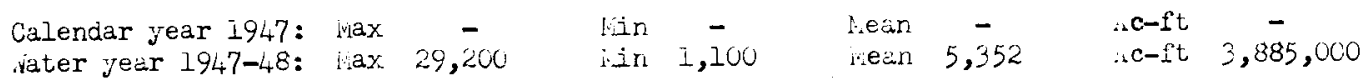

* Dischare ueasurernent riage on tris diy.

a io gate-lieizht record; aischar estinted. 
HELMAND RIVER BASIN

Helmand River below Kajakai Dam, Afghanistan

Discharge, in cubic feet per second, water year October 1948 to september 1949

\begin{tabular}{|c|c|c|c|c|c|c|c|c|c|c|c|c|}
\hline Day & Oct. & Nov. & Dec. & Jan. & Feb. & Mar. & Apr. & May & June & July & Aug. & Sept. \\
\hline 1 & 1,810 & $\frac{22,300}{32,300}$ & 2,400 & 2,650 & 3,300 & 3,880 & 20,000 & $\mathrm{a} 27,000$ & 8,850 & $\frac{42460}{300}$ & 2,400 & 2,020 \\
\hline $\begin{array}{l}2 \\
3\end{array}$ & al, 810 & $\begin{array}{l}22,30 y \\
22,350\end{array}$ & 2,400 & $\begin{array}{l}2,600 \\
2,500\end{array}$ & $\frac{2,020}{5.370}$ & 3,950 & 22,000 & 225,000 & $\begin{array}{l}8,540 \\
8,310\end{array}$ & 4,390 & 2,400 & 2,020 \\
\hline 3 & $\begin{array}{l}\text { all, } 10 \\
\text { a1, } 810\end{array}$ & $\begin{array}{l}a 2,354 \\
22,350\end{array}$ & $\begin{array}{l}2,430 \\
2,500\end{array}$ & $\begin{array}{l}2,500 \\
2,400\end{array}$ & & 4,100 & & $\mathrm{a} 23,000$ & 8,310 & 4,310 & 2,350 & 2,020 \\
\hline $\begin{array}{l}4 \\
5\end{array}$ & al, 810 & $a 2,350$ & $\begin{array}{r}2,500 \\
\mathrm{a} 2,500\end{array}$ & $\begin{array}{l}2,400 \\
2,260\end{array}$ & $\begin{array}{l}4,390 \\
3,880\end{array}$ & $\begin{array}{l}4,310 \\
4,460\end{array}$ & $\begin{array}{l}25,300 \\
25,100\end{array}$ & $\begin{array}{l}22,000 \\
22,000\end{array}$ & $\begin{array}{l}8,090 \\
7,860\end{array}$ & $\begin{array}{r}4,2,40 \\
a 4,100\end{array}$ & $\begin{array}{l}2,350 \\
2,350\end{array}$ & $\begin{array}{l}2,020 \\
2,020\end{array}$ \\
\hline 6 & al, 810 & a2, 400 & 22,500 & 2,160 & 3,620 & 4,460 & 25,500 & 21,600 & 7,640 & a3,900 & 2,350 & 1,980 \\
\hline 7 & al, 850 & a2, 400 & $\mathrm{a} 2,500$ & 2,120 & 3,370 & 4,540 & 26,800 & 21,500 & 7,530 & a3,700 & 2,500 & 2,020 \\
\hline 8 & al, 850 & 22,400 & $\mathrm{a} 2,500$ & 2,160 & $\begin{array}{r}* \\
*\end{array}$ & 4,700 & 26,600 & 21,300 & 7,420 & $a 3,550$ & $* 2,450$ & 2,020 \\
\hline 9 & al, 850 & $\mathrm{a} 2,400$ & 22,500 & 2,210 & 3,140 & 9,620 & 29,200 & 20,900 & 7,210 & $* 3,430$ & 2,450 & 2,020 \\
\hline 10 & $* 1,850$ & 22,450 & a2, 500 & 2,300 & 3,250 & 29,400 & 38,200 & 20,400 & 7,000 & 3,370 & 2,400 & 2,020 \\
\hline 11 & 1,810 & a2, 450 & a2, 600 & 2,350 & 3,310 & 22,500 & a 30,000 & 19,700 & 6,900 & 3,310 & 2,300 & $* 2,020$ \\
\hline 12 & $2], 850$ & a2, 450 & $a 2,600$ & 2,400 & 3,370 & 13,700 & $\mathrm{a} 25,000$ & 19,300 & 6,590 & 3,190 & 2,260 & 1,980 \\
\hline 13 & al, 850 & $\mathrm{a} 2,450$ & 22,600 & 2,300 & 3,370 & 12,000 & $* 21,400$ & 18,100 & 6,390 & 3,140 & 2,210 & 1,980 \\
\hline 14 & al,, 900 & a2, 450 & $a 2,600$ & 2,300 & 3,370 & 11,600 & $* 33,300$ & 17,000 & 6,200 & 3,080 & 2,160 & 1,980 \\
\hline 15 & al,, 900 & a2, 400 & 22,600 & 2,450 & 3,310 & 11,500 & a 32,000 & 15,900 & 6,000 & 3,020 & 2,160 & 1,980 \\
\hline 16 & al,, 900 & $\mathrm{a} 2,400$ & 22,600 & 2,450 & 3,310 & 11,200 & a 31,000 & 15,200 & 5,910 & 2,970 & 2,120 & 1,980 \\
\hline 17 & al, 950 & 22,400 & 22,600 & 2,500 & 3,310 & 10,800 & a 30,000 & $u_{4}, 800$ & 5,720 & 2,920 & 2,120 & 1,980 \\
\hline 18 & al, 950 & a2, 400 & $a 2,650$ & 2,500 & 3,310 & 10,400 & a 30,000 & $u_{4}, 500$ & 5,630 & 2,920 & 2,120 & 2,020 \\
\hline 19 & $a 2,000$ & 22,450 & $a \overline{2,650}$ & 2,550 & 3,310 & 10,100 & a 31,000 & $u_{4}, 000$ & 5,540 & 2,860 & 2,120 & 2,020 \\
\hline 20 & $a 2,000$ & a2, 450 & 2,650 & 2,550 & 3,430 & 10,600 & a32,000 & 13,600 & 5,460 & 2,810 & 2,070 & 2,020 \\
\hline 21 & 22,000 & a2, 450 & 2,600 & 2,600 & 3,560 & 11,500 & 32,500 & 13,000 & 5,370 & 2,760 & 2,070 & 2,020 \\
\hline 22 & a2, 050 & a2, 450 & 2,550 & 2,760 & 4,460 & 11,700 & 33,100 & 12,400 & 5,280 & 2,700 & 2,070 & 2,020 \\
\hline 23 & a2, 050 & $a 2,450$ & 2,600 & 2,810 & 5,910 & 12,600 & a 34,000 & 12,400 & 5,190 & 2,650 & 2,070 & 2,020 \\
\hline 24 & 22,050 & $\mathrm{a} 2,450$ & 2,600 & 3,020 & 5,370 & 13,100 & a35,000 & $* 12,400$ & 5,110 & 2,600 & 2,070 & 2,020 \\
\hline 25 & 22,100 & $\mathrm{a} 2,450$ & 2,450 & 3,250 & 4,860 & 12,800 & a 37,000 & 12,600 & 5,020 & 2,600 & 2,070 & 2,070 \\
\hline 26 & a2,100 & a2, 400 & 2,350 & 3,020 & 4,460 & 12,700 & $\mathrm{a} / 40,000$ & 13,000 & 4,940 & 2,550 & 2,020 & 2,070 \\
\hline 27 & 22,150 & $\mathrm{a} 2,400$ & $\overline{2,450}$ & 2,810 & 4,170 & 13,100 & 45,000 & 11,900 & 4,860 & 2,550 & 2,020 & 2,120 \\
\hline 28 & $\mathrm{a} 2,150$ & $* 2,400$ & 2,550 & 2,810 & 4,100 & 14,600 & $a \sqrt{40,000}$ & 10,900 & 4,780 & 2,500 & 2,020 & 2,120 \\
\hline 29 & $a 2,200$ & 2,400 & 2,450 & 2,760 & - & 18,600 & a 35,000 & 10,200 & 4,620 & 2,500 & 2,020 & 2,160 \\
\hline 30 & $* a 2,250$ & 2,400 & 2,450 & 2,700 & - & 21,600 & a 30,000 & 9,700 & 4,540 & 2,450 & 2,020 & $\overline{2,160}$ \\
\hline 31 & 2,260 & , & 2,550 & 2,810 & - & 20,500 & - & 9,230 & & 2,450 & 2,020 & - \\
\hline Total & 60,730 & 72,250 & 78,500 & 79,060 & 112,820 & 360,620 & 923,600 & 515,030 & 188,530 & 97,980 & 68,110 & 60,900 \\
\hline Kean & 1,959 & 2,408 & 2,532 & 2,550 & 4,029 & 11,630 & 30,790 & 16,610 & 6,284 & 3,161 & 2,197 & 2,030 \\
\hline $\mathrm{s}-\mathrm{ft}$ & 120,500 & 143,300 & 155,700 & 156,800 & 223,800 & 715,300 & $1,832,000$ & $1,022,000$ & 373,900 & 194,300 & 135,100 & 120,800 \\
\hline
\end{tabular}

Calendar year 1948: $\operatorname{Max} 29,200$ Min 1,100. iviean 5,466 ac-ft 3,968,000 water year 1948-49: Max 45,000 Min 1,810 Mean 7,173 Ac-ft 5,194,000

* Discharge measurement miade on this day.

a No gage-height record; discharge estinated on basis of discharge measurelients, weather records, t'lood marks and recession curves. 


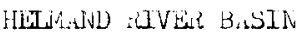

Heliknd river velor najaki Dam, afinistan

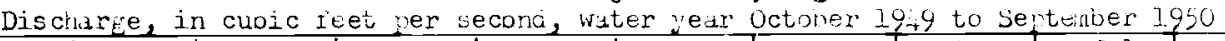

\begin{tabular}{|c|c|c|c|c|c|c|c|c|c|c|c|c|}
\hline Dav & Oct. & Nov. 1 & Dec. & Jin. & reo. & in: $: r_{0} \mid$ & $\therefore r_{0}$ & $1.2 y$ & c line & July &. $\operatorname{sug}$ & Sect. \\
\hline 1 & 2,120 & 2,810 & 3,140 & 3,020 & 4,620 & 5,110 & 13,400 & 23,100 & 17,200 &, \pm 940 & $\approx, 450$ & 21,980 \\
\hline 2 & 2,100 & $\overrightarrow{2}, 860$ & 3,140 & 3,020 & 4,020 & 5,400 & 15,900 & 25,900 & $\overline{16,000}$ & 4,800 & 2,300 & $3 \longdiv { I , 9 3 0 }$ \\
\hline 3 & 2,210 & 2,860 & 3,080 & 3,020 & 3,080 & 5,030 & 15,900 & 26,000 & 15,200 & 4,780 & 2,260 & 21,930 \\
\hline 4 & 2,200 & 2,920 & 2,970 & 2,920 & 3,490 & 5,630 & $\because 24,600$ & 26,600 & 14,600 & 4,620 & 2,210 & al, 980 \\
\hline 5 & 2,300 & 2,920 & 2,970 & 2,700 & 3,490 & 5,540 & 14,200 & 29,000 & 14,000 &,+ 390 & 2,160 & 31,930 \\
\hline 6 & 2,400 & 2,920 & 2,970 & 2,600 & 3,750 & 5,460 & 13,900 & 32,000 & 13,600 & 4,310 & 2,120 & 21,980 \\
\hline 7 & 2,450 & 2,920 & 2,970 & 2,600 & 4,230 & 5,630 & 13,400 & 31,000 & 13,300 & 4,240 & 2,070 & 31,930 \\
\hline 8 & 2,450 & 2,920 & 2,970 & 2,650 & 4,780 & 5,910 & 13,600 & 30,800 & 13,300 & 4,100 & 2,020 & $a 1,980$ \\
\hline 9 & 2,500 & 2,920 & 3,080 & 2,810 & 4,780 & 6,290 & $13,7 \cup 0$ & 30,700 & 12,600 &, 950 & 2,020 & 11,930 \\
\hline 10 & 2,550 & 2,920 & 3,190 & 2,970 & 4,700 & 0,590 & $1 / 4,200$ & 32,900 & 12,100 & 3,880 & 2,020 & $x 1,980$ \\
\hline 11 & 2,550 & 2,970 & 3,250 & 3,020 & 4,460 & 7,860 & 15,200 & 30,200 & 11,900 & 3,830 & 1,980 & al, 980 \\
\hline 12 & 2,500 & 2,970 & 3,310 & 3,140 & 4,230 & 8,850 & 17,400 & 34,400 & 11,500 & 3,820 & $\therefore \bar{I}, 980$ & 1,980 \\
\hline 13 & 2,500 & 2,970 & 3,310 & 3,250 & 4,020 & 3,540 & 19,300 & 33,300 & 10,900 & 3,820 & dl, 980 & 1,980 \\
\hline 14 & 2,500 & 2,970 & 3,250 & 3,250 & 4,100 & 8,310 & 19,500 & 31,000 & 10,300 & 3,750 & 21,980 & 1,980 \\
\hline 15 & 2,500 & 2,970 & 3,190 & 3,250 & 4,100 & 7,980 & 20,000 & 30,700 & 10,100 & 3,680 & $\vdots 1,980$ & $2, \cup 20$ \\
\hline 16 & 2,550 & 2,970 & $3,1,0$ & 3,250 & 4,020 & 7,750 & 21,100 & 30,500 & $a y, 700$ & 3,020 & al, 980 & 2,020 \\
\hline 17 & 2,550 & 2,920 & 3,140 & 3,190 & 3,950 & 7,850 & 19,700 & $3 I, 400$ & $\therefore 9,400$ & 3,560 & $\therefore 1,980$ & 2,020 \\
\hline 18 & 2,000 & 2,920 & 3,140 & 3,190 & 3,880 & 7,860 & 19,300 & 31,400 & 39,000 & 3,490 & 31,980 & 2,020 \\
\hline 19 & 2,600 & 2,920 & 3,080 & 3,190 & 3,750 & 7,640 & 18,900 & 30,800 & 38,600 & 3,370 & al, 980 & 2,020 \\
\hline 20 & 2,600 & 2,920 & 3,080 & 3,140 &, 750 & 8,770 & 18,300 & 30,500 & 38,300 & 3,310 & $: 1,980$ & 2,020 \\
\hline 21 & 2,650 & 2,970 & 3,020 & 3,080 & 3,820 & 10,600 & 17,000 & 29,000 & 28,000 & 3,250 & $\therefore I, 950$ & 2,020 \\
\hline 22 & 2,650 & 2,970 & 3,020 & 2,970 & 3,820 & 11,700 & 18,100 & 27,000 & $a 7,000$ & 3,250 & $3.1,980$ & 2,020 \\
\hline 23 & 2,650 & 2,970 & 3,020 & 2,810 & 3,820 & $\overline{10,900}$ & $18,1 \cup 0$ & 25,500 & $\mathrm{a} 7,30 \mathrm{O}$ & 3,190 & al, 980 & 2,020 \\
\hline 24 & 2,700 & 2,970 & 2,020 & 2,700 & 3,820 & 10,200 & 15,900 & 24,600 & 20,900 & $\because, 140$ & 21,980 & 2,020 \\
\hline 25 & 2,700 & 2,970 & 2,970 & 2,760 & 3,880 & 9,590 & 15,400 & 25,300 & 36,500 & 3,080 & $a 1,980$ & 2,020 \\
\hline 26 & 2,760 & 2,970 & 2,920 & 3,850 & 4,100 & 9,230 & 16,400 & 21,600 & 16,200 & 3,020 & 21,980 & 2,620 \\
\hline 27 & 2,760 & 3,020 & 2,700 & 5,820 & 4,460 & 8,770 & 18,100 & 20,400 & 25,400 & 2,970 & 31,900 & 2,020 \\
\hline 28 & 2,760 & 3,140 & 2,700 & 6,240 & 4,780 & 8,310 & 19,800 & 20,400 & $a 5,600$ & 2,850 & il, & 2,120 \\
\hline 29 & 2,760 & 3,190 & 2,810 & 12,100 & - & 3,310 & 22,200 & 19,700 & 5,370 & 2,700 & $=1,980$ & 2,020 \\
\hline 30 & 2,810 &, 190 & 2,920 & 6,490 & - & 8,650 & 24,00 & 15,900 & 5,110 & 2,650 & 11,980 & 2,020 \\
\hline 3 & 2,810 & - & 3,020 & 5,370 & - & 8,770 & $\rightarrow$ & 18,300 & - & 2,550 & 1,980 & - \\
\hline $\begin{array}{l}\text { Totat } \\
\text { Wean }\end{array}$ & $\begin{array}{r}78,800 \\
2,544 \\
156,400\end{array}$ & $\begin{array}{r}83,830 \\
2,961 \\
176,200\end{array}$ & $\begin{array}{r}9 i, 550 \\
3,650 \\
187,500\end{array}$ & $\begin{array}{r}112,400 \\
3,626 \\
222,900\end{array}$ & $\begin{array}{r}114,300 \\
4,082 \\
226,700\end{array}$ & $\begin{array}{r}243,730 \\
7,852 \\
483,400\end{array}$ & $\begin{array}{r}516,500 \\
17,220 \\
1,024,000\end{array}$ & $\begin{array}{r}858,700 \\
27,700 \\
1,703,000\end{array}$ & $\begin{array}{r}306,080 \\
10,200 \\
607,100\end{array}$ & $\begin{array}{r}113,90 \\
3,048 \\
224,800\end{array}$ & $\left|\begin{array}{r}63,210 \\
2,039 \\
125,400\end{array}\right|$ & $\begin{array}{r}60,040 \\
2,101 \\
119,100\end{array}$ \\
\hline
\end{tabular}

\begin{tabular}{|c|c|c|c|c|c|c|}
\hline $\begin{array}{l}\text { Calendar year 1949: } \\
\text { wter Year 19l.9-50: }\end{array}$ & $\begin{array}{l}10 x \text { 45, 060 } \\
\ln 20 \times 3 \%, 200\end{array}$ & Win & $\begin{array}{l}1,980 \\
1,980\end{array}$ & $\begin{array}{l}\text { We:n } \\
\text { de: esn }\end{array}$ & $\begin{array}{l}7,312 \\
7,261\end{array}$ & $\begin{array}{l}. . c-f t \\
. c-f t\end{array}$ \\
\hline
\end{tabular}

* Discharde jesurenent hure on taic dive

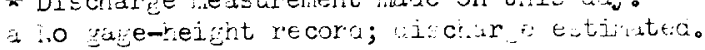




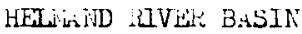

Helranc hiver below Kajukai Dan, afibunistion

Discharge, in cuivic feet per second, water year October 1950 to sertenber 1951

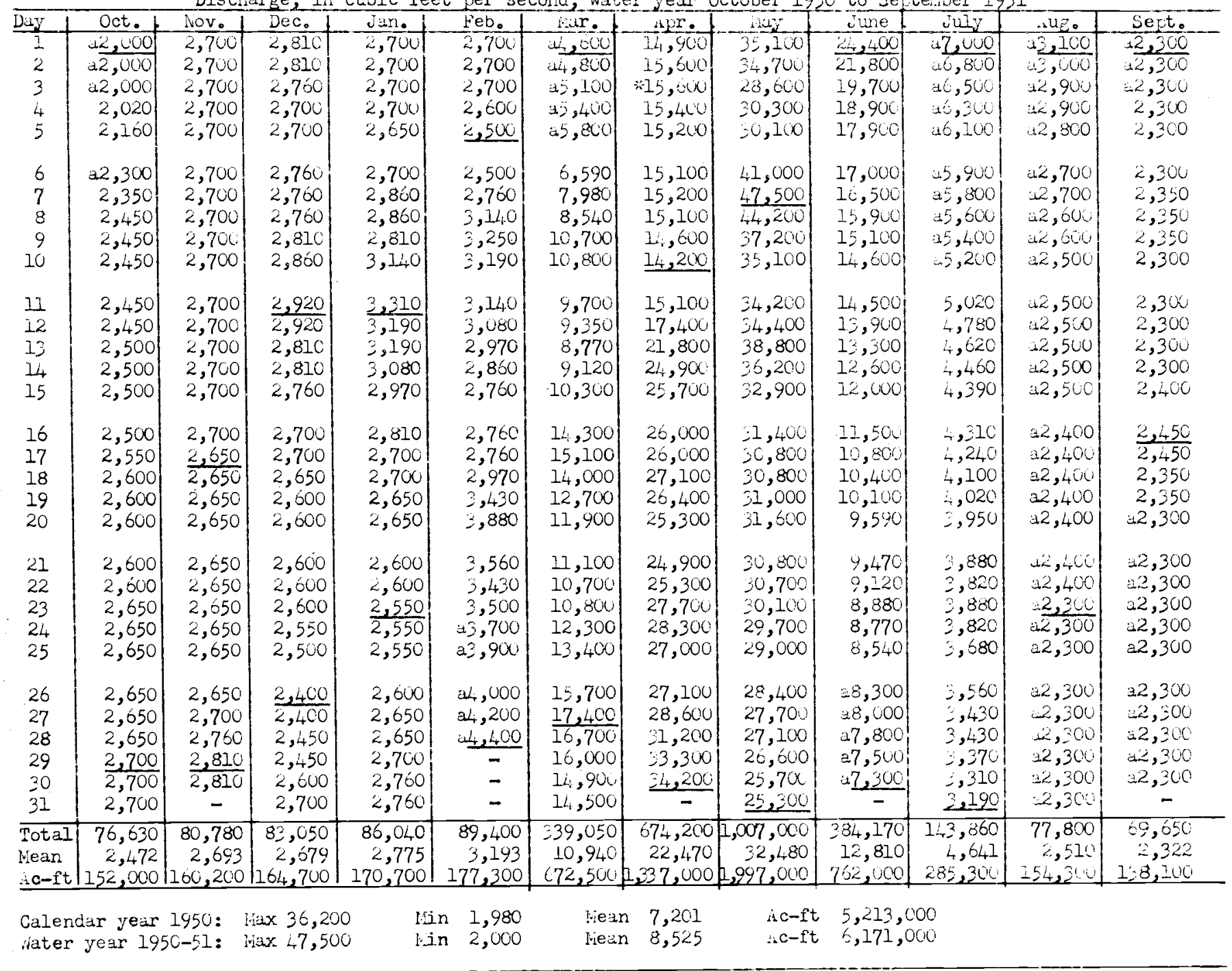

* Discriarge messurement mude on tinis dayo

a lo gage-height record; discrurge estimted. 


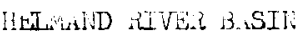

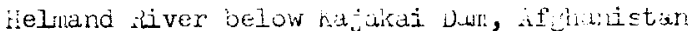

Discharge, in cucic feet jer secona, witer veir Octoper icil to jefteriber 1952

\begin{tabular}{|c|c|c|c|c|c|c|c|c|c|c|c|c|}
\hline Day & oct. & Wov. & Dec. & din. I & ieb. & $\therefore 3 r$. & $-4 r$ & ivity & June & duly & $\therefore$ UE。 & Secto \\
\hline 1 & 2,300 & $32+30$ & 3,080 & 2,970 & 0,680 & 5,010 & $13,1 \cup 0$ & 19,80 & 9,940 & $\because, 620$ & 2,160 & 1,850 \\
\hline 2 & $\approx 2,300$ & 3,250 & 3,080 & 3,140 & 3,020 & 5,820 & 17,800 & 19,500 & 9,940 &, 230 & 2,670 & $\overline{1,940}$ \\
\hline 3 & 2,300 & $\therefore 190$ & 3,080 & 3,140 & 3,560 & 5,820 & 17,900 & $22,7 \mathrm{C}$ & 9,590 & 3,10 & 2,040 & $I, 980$ \\
\hline 4 & 2,350 & 3,140 & 3,080 & $\because, 1 ; C$ & 3,490 & 6,06 & 19,700 & 23,500 & 9,090 & 3,190 & 1.980 & 2,020 \\
\hline 5 & 2,350 & 3,140 & $, 0,080$ & 3,190 & 2,30 & 7,760 & 21,100 & 20,900 & 7,750 & 3,080 & $1,9,0$ & 2,020 \\
\hline 6 & 2,350 & -3080 & 3,080 & 3,310 & 3,430 & 9,350 & 20,700 & 19,100 & 7,640 & 3,020 & 1,940 & 1,980 \\
\hline 7 & 2,350 & $3,08 \mathrm{u}$ & 3,140 & 3,370 & $3,4+90$ & 9,000 & 20,500 & 18,400 & 7,320 & $\therefore, 0,00$ & 1,340 & 1,940 \\
\hline 8 & 2,350 & 3,620 & 3,140 & 3,370 & 3,430 & 8,430 & 21,300 & 18,300 & 6,590 & 2,970 & 3,940 & 1,940 \\
\hline 9 & 2,400 & 3,020 & 3,140 & 3,310 & $3,+30$ & 7,540 & $22,40 x$ & $18,1 \cup 0$ & 6,290 & 2,970 & 1,900 & $2,9+0$ \\
\hline 10 & $2,4,00$ & 3,020 & $\therefore, 1,0$ & $\therefore, 250$ & 3,490 & $7,21$. & $23,1,0$ & 28,500 & 6,100 & $2,97 \cup$ & 1,940 & $=, 980$ \\
\hline II & 2,600 & 2,020 & 3,140 & 3,310 & 3,490 & 7,210 & 23,800 & 18,300 & 5,910 & 2,920 & $2,9 i_{r} 0$ & 2,020 \\
\hline 12 & 2,450 & 2,970 & 3,140 & 3,950 & $=, 750$ & 7,530 & $2+, 200$ & 16,900 & 5,720 & 2,920 & 1,890 & 2,070 \\
\hline 13 & 2,450 & $\mathbf{2}, 970$ & 3,140 & 3,620 & 4,020 & 10,300 & $2 i_{+}, 200$ & 15,900 & $5,5,0$ & 2,020 & 2,850 & 2,160 \\
\hline$u_{4}$ & 2,450 & 2,920 & 3,140 & 3,620 & 8,700 & $1_{i_{i}}, 800$ & $2 \xi, 800$ & 15,600 & 5,620 & 2,800 & 2,850 & 2,160 \\
\hline 25 & 2,550 & 2,920 & 2,140 & 2,490 & 13,300 & $1 ;, 300$ & 24,000 & $1 /, 900$ & $\because 5,540$ & 2,810 & 1,950 & 2,160 \\
\hline 16 & 2,600 & 2,920 & $3, U_{4} 0$ & 2,250 & 9,700 & 13,100 & $2 i_{i}, 600$ & 14,200 & $5,-60$ & 2,700 & $1,8: 0$ & $\approx 210$ \\
\hline 17 & 2,550 & 2,920 & 3,140 & 2,600 & 7,640 & $12,+00$ & 25,500 & 13,000 & $=, 280$ & 2,810 & $\overline{1,813}$ & $\overline{2,210}$ \\
\hline 18 & 2,550 & 2,920 & $3,11,0$ & 2,550 & 6,590 & 11,200 & 27,000 & 12,400 & 5,020 & 2,970 & $* 1,510$ & 2,210 \\
\hline 19 & 2,550 & 2,920 & 3,140 & 2,500 & 5,910 & 10,900 & 28,100 & 12,100 & 4,940 & 3,080 & 1,810 & 2,210 \\
\hline 20 & 2,550 & 2,920 & $3,1,0$ & 2,810 & 5,450 & 11,500 & 25,500 & 11,900 & 4,780 & 2,970 & 1,810 & 2,210 \\
\hline 21 & 2,550 & 2,920 & $3,1 / 40$ & $\because 1 ; 0$ & 5,280 & 12,400 & $\because 7,000$ & $1.7,500$ & 4,620 & 2,860 & $I, 810$ & $* 2,160$ \\
\hline 22 & 2,500 & 2,920 &, $1_{\tau} \mathrm{O}$ & 3,190 & 5,140 & 14,500 & 24,700 & $11,3 \cup 0$ & 4,540 & 2,700 & 1,810 & 2,160 \\
\hline 23 & 2,550 & 2,920 & $3,1,0$ & 3,140 & 5,140 & 13,900 & 23,500 & $11,2,0$ &,- 450 & 2,700 & 1,810 & $\therefore 160$ \\
\hline 24 & 2,550 & 2,920 & 3,140 & 3,140 & $5,1,0$ & 12,300 & 22,900 & 11,360 & 4,390 & 2,700 & 1,810 & 2,160 \\
\hline 25 & 2,550 & 2,920 &, 146 & 3,220 & $5,14 \mathrm{C}$ & 12,800 & 22,700 & 11,200 & $\therefore, 21+0$ & 2,650 & 1,810 & 2,160 \\
\hline 26 & 2,500 & 2,970 & 2,970 & 2,920 & $5,1.40$ & $1 /+, 300$ & 22,500 & 10,800 & $i, 170$ & 2,550 & $I, 850$ & 2,210 \\
\hline 27 & 2,500 & 3,020 & 2,810 & 2,850 & 5,190 & 22,200 & 22,700 & $10,700:$ & 4,100 & 2,450 & 1,850 & $\therefore, 210$ \\
\hline 28 & 2,700 & 3,020 & 2,760 & 2,970 & 5,726 & 27,000 & 22,700 & 20,500 & 4,020 & 2,400 & 1,850 & $\therefore, 210$ \\
\hline 29 & 3,080 & 3,020 & 2,810 & $=190$ & 6,100 & $2 t_{t}, 000$ & 23,100 & $\pm 0,300$ & , sess & 2,20 & 1,290 & 2,210 \\
\hline 30 & 3,370 & 2,020 & 2,850 & 3,750 & - & 20,700 & $2 l, 100$ & 10,200 & 680 & 2,260 & $1,8=0$ & 2,210 \\
\hline 31 &, 560 & - & 2,860 & 2,820 & & 18,600 & - & $10,10 \mathrm{~s}$ & - & 22270 & 1,000 & - \\
\hline & & 360 & $1+1 \times 28$ & 97,200 & 1,810 & $1,3,0$ & $\begin{array}{l}8,592 \\
6,650\end{array}$ & & $x-1$ & & & \\
\hline
\end{tabular}

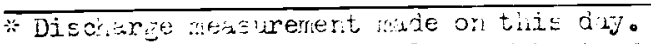

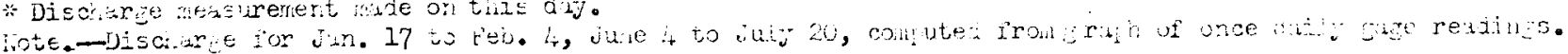


HELMAND RIVER BASIN

Helmand River below Kajakai Dam, Afghanistan

Discharge, in cubic feet per second, water year October 1952 to September 1953

\begin{tabular}{|c|c|c|c|c|c|c|c|c|c|c|c|c|}
\hline Day & Oct. & Nov。 & Dec. & Jan. & Feb. & Mar. & Apr. & May & June & July & Aug. & Sept. \\
\hline $\begin{array}{l}1 \\
2 \\
3 \\
4 \\
5\end{array}$ & $\begin{array}{l}\frac{2,320}{2,320} \\
2,320 \\
2,370 \\
2,370\end{array}$ & $\begin{array}{l}\frac{2,910}{2,910} \\
2,910 \\
2,910 \\
2,910\end{array}$ & $\begin{array}{r}3,010 \\
3,010 \\
3,010 \\
3,010 \\
23,010\end{array}$ & $\begin{array}{r}2,960 \\
2,960 \\
2,910 \\
2,750 \\
* 2,650\end{array}$ & $\begin{array}{r}2,270 \\
* 2,270 \\
2,270 \\
2,320 \\
2,410\end{array}$ & $\begin{array}{r}* 4,620 \\
4,690 \\
4,690 \\
4,750 \\
4,610\end{array}$ & $\begin{array}{r}6,430 \\
6,430 \\
6,510 \\
* 6,510 \\
6,590\end{array}$ & $\begin{array}{l}7,490 \\
7,490 \\
7,490 \\
7,490 \\
7,490\end{array}$ & $\begin{array}{l}7,700 \\
7,700 \\
7,700 \\
7,700 \\
7,700\end{array}$ & $\begin{array}{r}\frac{7,590}{* 5,010} \\
\frac{2,960}{2,960} \\
6,000\end{array}$ & $\begin{array}{r}7,090 \\
* 7,090 \\
7,090 \\
6,990 \\
6,990\end{array}$ & $\begin{array}{r}6,510 \\
6,510 \\
* 6,510 \\
6,510 \\
6,510\end{array}$ \\
\hline $\begin{array}{r}6 \\
7 \\
8 \\
9 \\
10\end{array}$ & $\begin{array}{l}2,370 \\
2,410 \\
2,410 \\
2,460 \\
2,460\end{array}$ & $\begin{array}{l}2,910 \\
2,910 \\
2,910 \\
2,910 \\
2,910\end{array}$ & $\begin{array}{l}3,010 \\
3,010 \\
3,010 \\
3,010 \\
2,960\end{array}$ & $\begin{array}{l}2,610 \\
2,560 \\
2,560 \\
2,560 \\
2,700\end{array}$ & $\begin{array}{l}2,460 \\
2,460 \\
2,560 \\
2,560 \\
2,650\end{array}$ & $\begin{array}{l}4,880 \\
4,940 \\
5,210 \\
5,350 \\
5,420\end{array}$ & $\begin{array}{l}6,590 \\
6,590 \\
6,510 \\
6,690 \\
6,790\end{array}$ & $\begin{array}{l}7,490 \\
7,590 \\
7,590 \\
2,590 \\
7,590\end{array}$ & $\begin{array}{l}7,700 \\
7,700 \\
7,700 \\
7,700 \\
7,700\end{array}$ & $\begin{array}{l}7,490 \\
7,490 \\
7,490 \\
7,490 \\
7,490\end{array}$ & $\begin{array}{l}6,990 \\
6,990 \\
6,890 \\
6,890 \\
6,890\end{array}$ & $\begin{array}{l}6,430 \\
6,430 \\
6,430 \\
6,430 \\
6,340\end{array}$ \\
\hline $\begin{array}{l}17 \\
12 \\
13 \\
14 \\
15\end{array}$ & $\begin{array}{l}2,460 \\
2,510 \\
2,560 \\
2,560 \\
2,560\end{array}$ & $\begin{array}{l}2,910 \\
2,910 \\
2,960 \\
2,960 \\
2,910\end{array}$ & $\begin{array}{r}2,960 \\
\mathrm{a} 2,960 \\
2,960 \\
2,960 \\
2,960\end{array}$ & $\begin{array}{l}2,850 \\
2,910 \\
2,960 \\
3,220 \\
3,220\end{array}$ & $\begin{array}{l}2,210 \\
1,700 \\
1,660 \\
1,660 \\
1,620 \\
\end{array}$ & $\begin{array}{l}5,490 \\
5,560 \\
5,560 \\
5,630 \\
5,630\end{array}$ & $\begin{array}{l}6,790 \\
6,890 \\
6,690 \\
6,600 \\
6,890\end{array}$ & $\begin{array}{l}7,590 \\
7,590 \\
7,590 \\
7,590 \\
7,700\end{array}$ & $\begin{array}{l}7,700 \\
7,700 \\
7,700 \\
7,700 \\
7,700\end{array}$ & $\begin{array}{l}7,490 \\
7,390 \\
7,390 \\
7,390 \\
7,390\end{array}$ & $\begin{array}{l}6,890 \\
6,890 \\
6,890 \\
6,890 \\
6,890\end{array}$ & $\begin{array}{l}6,340 \\
6,340 \\
6,260 \\
4,810 \\
4,070\end{array}$ \\
\hline $\begin{array}{l}16 \\
17 \\
18 \\
19 \\
20\end{array}$ & $\begin{array}{l}2,560 \\
2,610 \\
2,650 \\
2,650 \\
2,700\end{array}$ & $\begin{array}{r}* 2,910 \\
2,910 \\
3,060 \\
\frac{3,110}{3,060}\end{array}$ & $\begin{array}{r}3,010 \\
3,010 \\
3,060 \\
\mathrm{a} 3,100 \\
3,110 \\
\end{array}$ & $\begin{array}{r}a 3,100 \\
3,010 \\
2,910 \\
2,850 \\
2,750\end{array}$ & $\begin{array}{l}1,620 \\
1,660 \\
1,660 \\
1,660 \\
2,180\end{array}$ & $\begin{array}{l}5,700 \\
5,770 \\
5,770 \\
5,770 \\
5,770\end{array}$ & $\begin{array}{l}6,990 \\
7,090 \\
7,190 \\
7,190 \\
7,190\end{array}$ & $\begin{array}{l}7,700 \\
7,700 \\
7,700 \\
7,700 \\
7,700\end{array}$ & $\begin{array}{l}7,700 \\
7,700 \\
7,700 \\
7,700 \\
2,590 \\
\end{array}$ & $\begin{array}{l}7,390 \\
7,290 \\
7,290 \\
7,290 \\
7,290\end{array}$ & $\begin{array}{l}6,890 \\
6,790 \\
6,790 \\
6,790 \\
6,790\end{array}$ & $\begin{array}{l}4,070 \\
4,070 \\
4,070 \\
4,070 \\
4,070\end{array}$ \\
\hline $\begin{array}{l}21 \\
22 \\
23 \\
24 \\
25\end{array}$ & $\begin{array}{l}2,700 \\
2,750 \\
2,750 \\
2,800 \\
2,800\end{array}$ & $\begin{array}{l}3,010 \\
3,010 \\
3,010 \\
3,010 \\
3,010\end{array}$ & $\begin{array}{r}3,060 \\
* 2,910 \\
2,800 \\
2,750 \\
2,800\end{array}$ & $\begin{array}{r}2,960 \\
2,850 \\
\mathrm{a} 2,850 \\
2,850 \\
2,910\end{array}$ & $\begin{array}{r}2,750 \\
* 2,750 \\
3,420 \\
1,090 \\
4,560\end{array}$ & $\begin{array}{r}5,770 \\
5,850 \\
5,850 \\
3,540 \\
40\end{array}$ & $\begin{array}{l}7,290 \\
7,290 \\
7,290 \\
7,390 \\
\frac{7,390}{7}\end{array}$ & $\begin{array}{l}7,700 \\
7,700 \\
7,700 \\
7,700 \\
7,700\end{array}$ & $\begin{array}{l}7,590 \\
7,590 \\
7,590 \\
7,590 \\
7,590\end{array}$ & $\begin{array}{l}7,290 \\
7,290 \\
7,290 \\
7,190 \\
7,190\end{array}$ & $\begin{array}{l}6,790 \\
6,690 \\
6,690 \\
6,690 \\
6,690\end{array}$ & $\begin{array}{l}4,070 \\
4,070 \\
4,070 \\
4,070 \\
4,070\end{array}$ \\
\hline $\begin{array}{l}26 \\
27 \\
28 \\
29 \\
30 \\
31\end{array}$ & $\begin{array}{r}2,800 \\
2,850 \\
2,910 \\
* 2,910 \\
2,910 \\
2,910\end{array}$ & $\begin{array}{c}3,010 \\
3,010 \\
3,010 \\
2,960 \\
2,960 \\
-\end{array}$ & $\begin{array}{l}2,800 \\
2,800 \\
2,850 \\
2,850 \\
2,800 \\
2,850\end{array}$ & $\begin{array}{r}2,960 \\
3,170 \\
3,220 \\
3,010 \\
2,500 \\
2,220 \\
\end{array}$ & $\begin{array}{c}\frac{4,620}{4,620} \\
4,620 \\
= \\
= \\
=\end{array}$ & $\begin{array}{r}260 \\
3,720 \\
6,260 \\
6,340 \\
6,430 \\
6,430 \\
\end{array}$ & $\begin{array}{c}* 7,390 \\
7,390 \\
7,390 \\
7,390 \\
7,390 \\
- \\
\end{array}$ & $\begin{array}{r}7,700 \\
7,700 \\
* 7,810 \\
7,810 \\
7,810 \\
7,700 \\
\end{array}$ & $\begin{array}{c}7,590 \\
7,590 \\
7,590 \\
7,590 \\
7,590 \\
-\end{array}$ & $\begin{array}{l}7,190 \\
7,190 \\
7,090 \\
7,090 \\
7,090 \\
7,090 \\
\end{array}$ & $\begin{array}{l}6,590 \\
0,590 \\
6,590 \\
6,590 \\
6,590 \\
6,590\end{array}$ & $\begin{array}{c}4,070 \\
4,070 \\
1,360 \\
205 \\
- \\
\end{array}$ \\
\hline $\begin{array}{l}\text { Total } \\
\text { Mean } \\
\text { Ac-ft }\end{array}$ & $\begin{array}{r}80,720 \\
2,604 \\
160,100\end{array}$ & $\begin{array}{r}88,800 \\
2,960 \\
176,100\end{array}$ & $\begin{array}{r}91,410 \\
2,949 \\
161,300\end{array}$ & $\begin{array}{r}88,500 \\
2,855 \\
175,500\end{array}$ & $\begin{array}{r}70,290 \\
2,510 \\
139,400\end{array}$ & $\begin{array}{r}156,500 \\
5,048 \\
310,400\end{array}$ & $\begin{array}{r}208,750 \\
6,958 \\
44_{4}, 000 \\
\end{array}$ & $\begin{array}{r}236,890 \\
7,642 \\
469,900 \\
\end{array}$ & $\begin{array}{r}229,790 \\
7,660 \\
455,800 \\
\end{array}$ & $\begin{array}{r}214,560 \\
6,921 \\
425,600 \\
\end{array}$ & $\begin{array}{r}211,590 \\
6,825 \\
419,700\end{array}$ & $\begin{array}{r}142,375 \\
4,762 \\
283,400\end{array}$ \\
\hline
\end{tabular}

$\begin{array}{lrrrrrrr}\text { Calendar year 1952: } & \text { Max } 28,600 & \text { Min 1,810 } & \text { Mean 6,640 } & \text { Ac-ft } 1,820,000 \\ \text { Water year 1952-53: } & \text { Max } 7,810 & \text { Min } & 40 & \text { Mean 4,988 } & \text { Ac-ft } 3,611,000\end{array}$

* Discharge measurament made on this day.

a No gage-heizht record; discharge estimated.

Note. - Discharge regulated by Kajakai Dasn after Jan. 28. 
HEIMIND RIVER BASIN

Helmand River below Kajakai Dan, Afghanistan

Discharge, in cubic feet per second, water year October 1953 to September 1954

\begin{tabular}{|c|c|c|c|c|c|c|c|c|c|c|c|c|}
\hline Dar & oct. & Nov. & Dec. & Jan. & Feb. & Mar. & Apr. & May & June & July & Aug. & Sept. \\
\hline $\begin{array}{l}1 \\
2 \\
3 \\
4 \\
5\end{array}$ & $\begin{array}{r}4,040 \\
* 4,010 \\
4,010 \\
4,010 \\
4,010\end{array}$ & $\begin{array}{r}3,890 \\
* 3,890 \\
3,890 \\
3,860 \\
3,830\end{array}$ & $\begin{array}{r}3,920 \\
3,920 \\
2,210 \\
245 \\
* 22 \\
\end{array}$ & $\begin{array}{r}1,980 \\
1,200 \\
a 620 \\
1, \overline{950} \\
1,480\end{array}$ & $\begin{array}{r}26,020 \\
* 6,100 \\
5,770 \\
5,700 \\
6,100\end{array}$ & $\begin{array}{l}a 6,100 \\
* 6,260 \\
a 6,100 \\
a 6,100 \\
a 6,100\end{array}$ & $\begin{array}{r}a 7,190 \\
* 7,390 \\
7,440 \\
7,490 \\
7,540\end{array}$ & $\begin{array}{l}31,600 \\
32,000 \\
32,200 \\
32,000 \\
31,600\end{array}$ & $\begin{array}{l}\frac{13,500}{13,200} \\
13,000 \\
12,700 \\
12,300\end{array}$ & $\begin{array}{r}* a 830 \\
2820 \\
1,340 \\
4,940 \\
4,780\end{array}$ & $\begin{array}{l}* 5,420 \\
5,420 \\
5,350 \\
5,350 \\
5,350\end{array}$ & $\begin{array}{l}5,350 \\
5,350 \\
5,350 \\
5,350 \\
5,350\end{array}$ \\
\hline $\begin{array}{r}6 \\
7 \\
8 \\
9 \\
10\end{array}$ & $\begin{array}{l}4,010 \\
4,010 \\
4,010 \\
4,010 \\
3,980\end{array}$ & $\begin{array}{l}3,830 \\
3,830 \\
3,800 \\
3,720 \\
2,500\end{array}$ & $\begin{array}{r}465 \\
4,070 \\
4,070 \\
4,070 \\
4,070\end{array}$ & $\begin{array}{r}1,320 \\
2,020 \\
2,020 \\
a 2,000 \\
a 1,500\end{array}$ & $\begin{array}{l}6,020 \\
6,020 \\
6,020 \\
6,020 \\
6,260\end{array}$ & $\begin{array}{l}a 6,100 \\
a 6,100 \\
a 6,100 \\
a 6,100 \\
86,100\end{array}$ & $\begin{array}{l}7,590 \\
7,640 \\
7,700 \\
7,760 \\
7,810\end{array}$ & $\begin{array}{l}31,000 \\
30,600 \\
29,700 \\
28,600 \\
27,600\end{array}$ & $\begin{array}{l}12,100 \\
11,700 \\
11,300 \\
11,000 \\
10,400\end{array}$ & $\begin{array}{l}4,620 \\
4,400 \\
4,370 \\
4,370 \\
4,370\end{array}$ & $\begin{array}{l}5,350 \\
5,280 \\
5,350 \\
5,350 \\
5,350\end{array}$ & $\begin{array}{l}5,350 \\
4,540 \\
4,250 \\
4,250 \\
4,250\end{array}$ \\
\hline $\begin{array}{l}11 \\
12 \\
13 \\
14 \\
15\end{array}$ & $\begin{array}{l}3,980 \\
3,980 \\
3,950 \\
3,950 \\
3,950\end{array}$ & $\begin{array}{r}* 40 \\
015 \\
a 15 \\
1,170 \\
3,660\end{array}$ & $\begin{array}{l}4,070 \\
4,070 \\
4,070 \\
4,070 \\
4,070\end{array}$ & $\begin{array}{l}a 1,500 \\
a 1,500 \\
a 1,500 \\
a 2,000 \\
a 2,000\end{array}$ & $\begin{array}{l}6,100 \\
6,100 \\
6,180 \\
6,180 \\
6,180\end{array}$ & $\begin{array}{l}a 6,100 \\
a 6,100 \\
a 6,100 \\
a 6,100 \\
a 6,100\end{array}$ & $\begin{array}{l}7,810 \\
7,860 \\
7,910 \\
7,960 \\
7,960\end{array}$ & $\begin{array}{l}27,000 \\
26,000 \\
24,600 \\
23,400 \\
22,200\end{array}$ & $\begin{array}{r}10,200 \\
9,700 \\
9,360 \\
8,900 \\
8,560\end{array}$ & $\begin{array}{l}4,370 \\
4,370 \\
4,370 \\
4,370 \\
4,370\end{array}$ & $\begin{array}{l}5,350 \\
5,420 \\
5,420 \\
5,350 \\
5,350\end{array}$ & $\begin{array}{l}4,250 \\
4,250 \\
4,370 \\
4,630 \\
4,780\end{array}$ \\
\hline $\begin{array}{l}16 \\
17 \\
18 \\
19 \\
20\end{array}$ & $\begin{array}{l}3,950 \\
3,950 \\
3,950 \\
3,950 \\
3,950\end{array}$ & $\begin{array}{l}3,660 \\
3,660 \\
3,660 \\
3,660 \\
3,660\end{array}$ & $\begin{array}{l}4,070 \\
4,070 \\
4,070 \\
4,010 \\
4,010\end{array}$ & $\begin{array}{l}a 2,000 \\
a 2,000 \\
a 2,000 \\
a 4,100 \\
a 4,100\end{array}$ & $\begin{array}{l}6,180 \\
6,180 \\
6,180 \\
6,180 \\
6,180\end{array}$ & $\begin{array}{l}a 6,100 \\
a 6,100 \\
a 6,180 \\
a 6,180 \\
a 6,180\end{array}$ & $\begin{array}{r}8,020 \\
8,080 \\
3,350 \\
11,000 \\
*]_{4}, 900\end{array}$ & $\begin{array}{l}21,400 \\
20,600 \\
20,000 \\
19,500 \\
18,900\end{array}$ & $\begin{array}{r}8,240 \\
8,020 \\
7,910 \\
* 7,700 \\
7,700\end{array}$ & $\begin{array}{l}4,370 \\
4,370 \\
4,370 \\
4,370 \\
4,370\end{array}$ & $\begin{array}{l}5,350 \\
5,350 \\
5,420 \\
5,350 \\
5,350\end{array}$ & $\begin{array}{l}4,780 \\
4,780 \\
4,750 \\
4,750 \\
4,750\end{array}$ \\
\hline $\begin{array}{l}21 \\
22 \\
23 \\
24 \\
25\end{array}$ & $\begin{array}{l}3,9.50 \\
3,920 \\
3,920 \\
3,920 \\
3,920\end{array}$ & $\begin{array}{l}3,660 \\
3,640 \\
3,740 \\
\frac{3,950}{3,950}\end{array}$ & $\begin{array}{r}2,670 \\
840 \\
235 \\
235 \\
235\end{array}$ & $\begin{array}{l}\mathrm{a}_{4}, 100 \\
\mathrm{a}_{4}, 100 \\
\mathrm{a} 4,100 \\
\mathrm{a} 4,100 \\
\mathrm{a} 4,100\end{array}$ & $\begin{array}{l}6,180 \\
6,180 \\
6,180 \\
6,180 \\
6,340\end{array}$ & $\begin{array}{l}a 6,260 \\
a 6,340 \\
a 6,340 \\
a 6,430 \\
a 6,510\end{array}$ & $\begin{array}{r}* 18,600 \\
22,200 \\
26,600 \\
* 29,000 \\
* 30,000\end{array}$ & $\begin{array}{l}18,200 \\
17,600 \\
17,100 \\
16,400 \\
16,000\end{array}$ & $\begin{array}{l}7,700 \\
7,700 \\
7,700 \\
7,700 \\
7,590\end{array}$ & $\begin{array}{l}4,370 \\
4,370 \\
4,370 \\
4,370 \\
4,490\end{array}$ & $\begin{array}{l}5,350 \\
5,420 \\
5,350 \\
5,420 \\
5,420\end{array}$ & $\begin{array}{l}4,690 \\
4,750 \\
4,750 \\
4,750 \\
4,300\end{array}$ \\
\hline $\begin{array}{l}26 \\
27 \\
28 \\
29 \\
30 \\
31\end{array}$ & $\begin{array}{l}3,920 \\
3,920 \\
3,890 \\
3,890 \\
3,890 \\
3,890\end{array}$ & $\begin{array}{c}3,950 \\
3,920 \\
3,920 \\
3,920 \\
3,920 \\
-\end{array}$ & $\begin{array}{r}235 \\
030 \\
a 30 \\
1,600 \\
1,980 \\
1,980 \\
\end{array}$ & $\begin{array}{l}a 4,100 \\
a 5,000 \\
a 6,020 \\
a 6,020 \\
26,020 \\
a 6,020\end{array}$ & $\begin{array}{c}6,180 \\
6,180 \\
6,340 \\
= \\
= \\
=\end{array}$ & $\begin{array}{l}a 6,590 \\
a 6,590 \\
a 6,690 \\
a 6,790 \\
a 6,890 \\
a 6,990\end{array}$ & $\begin{array}{c}30,000 \\
30,000 \\
30,400 \\
30,800 \\
\frac{31,200}{-}\end{array}$ & $\begin{array}{r}15,500 \\
15,100 \\
14,800 \\
11,400 \\
14,000 \\
* 13,800 \\
\end{array}$ & $\begin{array}{c}7,590 \\
7,590 \\
7,700 \\
5,000 \\
828 \\
-\end{array}$ & $\begin{array}{l}5,140 \\
5,490 \\
5,420 \\
5,420 \\
5,420 \\
5,420\end{array}$ & $\begin{array}{l}5,420 \\
5,420 \\
5,350 \\
5,350 \\
5,350 \\
5,350 \\
\end{array}$ & $\begin{array}{r}3,720 \\
3,720 \\
* 3,720 \\
3,610 \\
3,830 \\
-\end{array}$ \\
\hline $\begin{array}{l}\text { Total } \\
\text { Mean } \\
\text { Ac-ft } \\
\end{array}$ & $\begin{array}{r}122,690 \\
3,958 \\
243,400\end{array}$ & $\begin{array}{r}98,810 \\
3,294 \\
196,000 \\
\end{array}$ & $\begin{array}{r}75,912 \\
2,449 \\
150,600 \\
\end{array}$ & $\begin{array}{r}92,490 \\
2,984 \\
183,500 \\
\end{array}$ & $\begin{array}{r}171,430 \\
6,122 \\
340,000\end{array}$ & $\begin{array}{r}194,820 \\
6,285 \\
386,400\end{array}$ & $\begin{array}{r}444,200 \\
4,810 \\
881,100\end{array}$ & $\begin{array}{r}703,400 \\
22,690 \\
1,395,000 \\
\end{array}$ & $\begin{array}{r}274,588 \\
9,153 \\
544,600 \\
\end{array}$ & $\begin{array}{r}132,820 \\
4,285 \\
263,400\end{array}$ & $\begin{array}{r}166,480 \\
5,370 \\
330,200\end{array}$ & $\begin{array}{r}137,320 \\
4,577 \\
272,400 \\
\end{array}$ \\
\hline $\begin{array}{l}\text { Cal } \\
\text { Wat }\end{array}$ & I 195 & $\begin{array}{l}\operatorname{Max} \\
\operatorname{Max}\end{array}$ & $\begin{array}{r}7,810 \\
32,200\end{array}$ & $\begin{array}{l}\text { Min } \\
\text { Min }\end{array}$ & $\begin{array}{l}\text { Mear } \\
\text { Mear }\end{array}$ & $\begin{array}{l}5,088 \\
7,164\end{array}$ & $\begin{array}{l}A c-f t \\
A c-f t\end{array}$ & $\begin{array}{l}3,684,000 \\
5,187,000\end{array}$ & & & & \\
\hline
\end{tabular}

* Discharge measurement made on this day.

a No gage-height record; discharge estimated on basis of reservoir releases. 
HELMAND RIVER BHSIN

Helmand River below Kajakai Dam, Afghanistan

Discharge, in cubic feet per second, water year October 1954 to Sept ember 1955

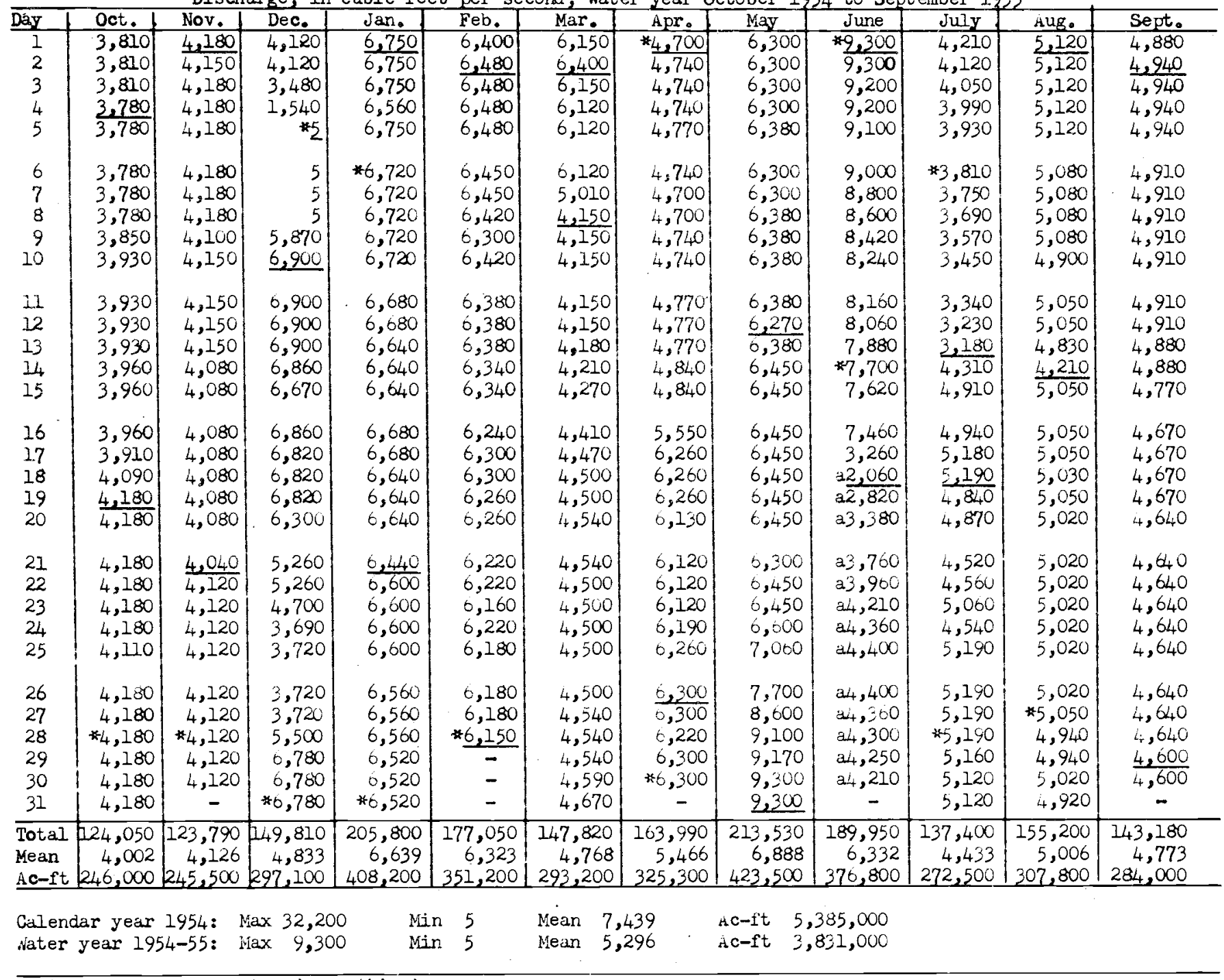

* Discharge neasurement made on this day.

a No gage-height record; dischar ce computed from spillway overflow.

wote.-Gates closed and river stage below Dec.5-8 intake; discharge estinited. 
HETMAND RIVER BASIN

Helmand River below Kajakai Dam, afghanistan

Discharge, in cubic feet per second, water year October 1955 to jeptember 1956

\begin{tabular}{|c|c|c|c|c|c|c|c|c|c|c|c|c|}
\hline Day & Oct. & $\mathrm{Nov}_{9}$ & Dec. & Jan. & $\mathrm{Feb}_{0}$ & Mar. & Apr. & Miay & Jurie & Julz & Aug. & Sept. \\
\hline $\begin{array}{l}1 \\
2 \\
3 \\
4 \\
5\end{array}$ & $\begin{array}{r}4,600 \\
4,600 \\
4,600 \\
* 4,600 \\
4,600\end{array}$ & $\begin{array}{l}4,450 \\
4,450 \\
4,450 \\
4,450 \\
4,450\end{array}$ & $\begin{array}{r}4,670 \\
4,670 \\
* 4,670 \\
4,670 \\
4,670\end{array}$ & $\begin{array}{r}* 4,560 \\
4,600 \\
4,180 \\
\frac{4,990}{4,640}\end{array}$ & $\begin{array}{l}4,420 \\
4,420 \\
4,420 \\
4,420 \\
4,450\end{array}$ & $\begin{array}{l}4,520 \\
4,520 \\
4,560 \\
4,670 \\
4,670\end{array}$ & $\begin{array}{r}7,770 \\
7,820 \\
7,910 \\
10,100 \\
17,700\end{array}$ & $\begin{array}{l}\frac{28,900}{27,900} \\
27,000 \\
26,000 \\
25,000\end{array}$ & $\begin{array}{r}7,280 \\
4,810 \\
* 5,380 \\
5,680 \\
6,010\end{array}$ & $\begin{array}{l}4,010 \\
3,920 \\
3,720 \\
3,470 \\
3,500\end{array}$ & $\begin{array}{r}\frac{10,600}{7,440} \\
6,730 \\
7,460 \\
8,240\end{array}$ & $\begin{array}{r}7,260 \\
* 7,260 \\
7,260 \\
a 5,200 \\
a 1,200 \\
\end{array}$ \\
\hline $\begin{array}{r}6 \\
7 \\
8 \\
9 \\
10\end{array}$ & $\begin{array}{l}4,600 \\
4,560 \\
4,560 \\
4,560 \\
4,560\end{array}$ & $\begin{array}{l}4,450 \\
4,450 \\
4,450 \\
4,480 \\
4,480\end{array}$ & $\begin{array}{l}4,640 \\
4,640 \\
4,640 \\
4,600 \\
\frac{4,600}{4}\end{array}$ & $\begin{array}{l}4,240 \\
4,240 \\
4,240 \\
4,240 \\
4,210\end{array}$ & $\begin{array}{l}4,450 \\
4,450 \\
4,450 \\
4,340 \\
4,280\end{array}$ & $\begin{array}{l}4,670 \\
4,700 \\
4,740 \\
4,740 \\
4,780\end{array}$ & $\begin{array}{r}* 24,200 \\
* 27,700 \\
32,100 \\
34,200 \\
* 34,600\end{array}$ & $\begin{array}{l}23,900 \\
22,800 \\
21,800 \\
21,000 \\
20,300\end{array}$ & $\begin{array}{l}6,180 \\
6,220 \\
6,180 \\
6,100 \\
5,930\end{array}$ & $\begin{array}{r}* 3,500 \\
3,500 \\
3,500 \\
3,470 \\
3,440\end{array}$ & $\begin{array}{l}7,720 \\
7,680 \\
7,680 \\
7,680 \\
7,630\end{array}$ & $\begin{array}{r}a 1,200 \\
a 1,200 \\
a l, 200 \\
a 5,100 \\
7,440\end{array}$ \\
\hline $\begin{array}{l}17 \\
12 \\
13 \\
14 \\
15\end{array}$ & $\begin{array}{l}4,560 \\
4,560 \\
4,520 \\
4,420 \\
4,560\end{array}$ & $\begin{array}{l}4,480 \\
4,480 \\
4,480 \\
4,480 \\
4,480\end{array}$ & $\begin{array}{l}4,600 \\
4,600 \\
4,700 \\
4,670 \\
4,670\end{array}$ & $\begin{array}{l}4,210 \\
4,210 \\
4,140 \\
4,450 \\
4,450\end{array}$ & $\begin{array}{l}4,280 \\
4,280 \\
4,310 \\
4,280 \\
4,280\end{array}$ & $\begin{array}{l}4,810 \\
4,810 \\
4,840 \\
4,880 \\
4,880\end{array}$ & $\begin{array}{l}34,400 \\
34,800 \\
35,700 \\
37,200 \\
38,800\end{array}$ & $\begin{array}{r}19,800 \\
19,100 \\
18,700 \\
* 18,000 \\
17,600\end{array}$ & $\begin{array}{l}5,720 \\
5,680 \\
5,600 \\
5,570 \\
5,180\end{array}$ & $\begin{array}{l}3,410 \\
3,350 \\
3,320 \\
\frac{3,300}{3,470}\end{array}$ & $\begin{array}{l}7,630 \\
7,630 \\
7,580 \\
7,580 \\
7,530\end{array}$ & $\begin{array}{l}7,440 \\
7,400 \\
7,400 \\
7,350 \\
7,350\end{array}$ \\
\hline $\begin{array}{l}16 \\
17 \\
18 \\
19 \\
20\end{array}$ & $\begin{array}{l}4,560 \\
4,560 \\
4,560 \\
4,560 \\
4,560\end{array}$ & $\begin{array}{l}4,480 \\
4,480 \\
4,450 \\
4,280 \\
4.670\end{array}$ & $\begin{array}{l}4,670 \\
4,670 \\
4,670 \\
4,670 \\
4,670\end{array}$ & $\begin{array}{l}4,450 \\
4,450 \\
4,450 \\
4,450 \\
4,420\end{array}$ & $\begin{array}{l}4,280 \\
4,280 \\
4,280 \\
4,310 \\
4,310\end{array}$ & $\begin{array}{l}4,920 \\
4,920 \\
4,920 \\
5,060 \\
5,030\end{array}$ & $\begin{array}{r}40,000 \\
a 41,700 \\
a 42,600 \\
a 43,600 \\
a 44,100\end{array}$ & $\begin{array}{l}16,900 \\
16,400 \\
15,700 \\
15,100 \\
14,300\end{array}$ & $\begin{array}{l}4,840 \\
4,880 \\
4,880 \\
4,840 \\
4,780\end{array}$ & $\begin{array}{l}3,950 \\
4,740 \\
7,120 \\
8,340 \\
8,790\end{array}$ & $\begin{array}{l}7,530 \\
7,480 \\
7,480 \\
7,480 \\
7,480\end{array}$ & $\begin{array}{l}7,350 \\
7,300 \\
7,300 \\
7,300 \\
7,260\end{array}$ \\
\hline $\begin{array}{l}21 \\
22 \\
23 \\
24 \\
25\end{array}$ & $\begin{array}{l}4,520 \\
4,520 \\
4,520 \\
4,480 \\
4,480\end{array}$ & $\begin{array}{l}4,670 \\
4,670 \\
4,670 \\
4,670 \\
4,670\end{array}$ & $\begin{array}{l}4,640 \\
4,640 \\
4,640 \\
4,640 \\
4,600\end{array}$ & $\begin{array}{r}4,420 \\
4,420 \\
4,380 \\
4,380 \\
44,380\end{array}$ & $\begin{array}{l}4,310 \\
4,310 \\
4,310 \\
4,380 \\
4,450\end{array}$ & $\begin{array}{l}5,060 \\
5,060 \\
4,920 \\
5,100 \\
5,140\end{array}$ & $\begin{array}{r}a 44,500 \\
a 44,400 \\
a 42,600 \\
38,800 \\
37,100\end{array}$ & $\begin{array}{l}13,700 \\
13,200 \\
12,800 \\
12,300 \\
12,000\end{array}$ & $\begin{array}{l}4,700 \\
4,670 \\
4,640 \\
4,520 \\
4,450\end{array}$ & $\begin{array}{l}7,770 \\
7,170 \\
6,640 \\
7,260 \\
9,270\end{array}$ & $\begin{array}{l}7,440 \\
7,440 \\
7,440 \\
7,400 \\
7,400\end{array}$ & $\begin{array}{l}7,260 \\
7,260 \\
7,220 \\
7,220 \\
7,220\end{array}$ \\
\hline $\begin{array}{l}26 \\
27 \\
28 \\
29 \\
30 \\
31\end{array}$ & $\begin{array}{r}4,480 \\
4,480 \\
4,450 \\
* 4,450 \\
4,450 \\
4,450\end{array}$ & $\begin{array}{c}4,670 \\
4,670 \\
4,670 \\
4,670 \\
4,670 \\
-\end{array}$ & $\begin{array}{l}4,600 \\
4,600 \\
4,600 \\
4,600 \\
4,600 \\
4,600\end{array}$ & $\begin{array}{l}4,380 \\
4,380 \\
4,380 \\
4,200 \\
4,420 \\
4,420\end{array}$ & $\begin{array}{r}4,480 \\
4,520 \\
* 4,420 \\
4,520\end{array}$ & $\begin{array}{r}5,140 \\
5,180 \\
* 5,180 \\
6,180 \\
7,680 \\
7,720 \\
\end{array}$ & $\begin{array}{l}35,500 \\
34,000 \\
32,100 \\
31,000 \\
29,900\end{array}$ & $\begin{array}{r}11,700 \\
11,400 \\
11,000 \\
10,800 \\
* 10,600 \\
10,300 \\
\end{array}$ & $\begin{array}{r}4,380 \\
4,340 \\
* 4,340 \\
4,040 \\
4,040\end{array}$ & $\begin{array}{r}9,680 \\
10,100 \\
10,400 \\
* 12,500 \\
\frac{15,600}{13,500}\end{array}$ & $\begin{array}{l}7,350 \\
7,350 \\
7,300 \\
7,300 \\
7,300 \\
7,120\end{array}$ & $\begin{array}{l}7,220 \\
7,220 \\
7,170 \\
7,120 \\
7,120\end{array}$ \\
\hline $\begin{array}{l}\text { Total } \\
\text { Mean } \\
\text { Ac-ft }\end{array}$ & $\begin{array}{r}140,540 \\
4,534 \\
278,800\end{array}$ & $\begin{array}{r}136,020 \\
4,534 \\
269,800\end{array}$ & $\begin{array}{r}143,820 \\
4,639 \\
285,300 \\
\end{array}$ & $\begin{array}{r}135,980 \\
4,386 \\
269,700 \\
\end{array}$ & $\begin{array}{r}126,690 \\
4,369 \\
251,300 \\
\end{array}$ & $\begin{array}{r}158,000 \\
5,097 \\
313,400 \\
\end{array}$ & $\begin{array}{r}966,900 \\
32,230 \\
1,918,000 \\
\end{array}$ & $\begin{array}{r}546,000 \\
17,610 \\
1,083,000\end{array}$ & $\begin{array}{r}155,860 \\
5,195 \\
309,100 \\
\end{array}$ & $\begin{array}{r}195,710 \\
6,313 \\
388,200\end{array}$ & $\begin{array}{r}235,100 \\
7,584 \\
466,300\end{array}$ & $\begin{array}{r}169,800 \\
6,327 \\
376,500 \\
\end{array}$ \\
\hline & & $\begin{array}{l}\text { Max } \\
\text { Max }\end{array}$ & & Min & & $\begin{array}{ll}\text { an } & 5 \\
\text { an } & 8\end{array}$ & $\begin{array}{l}A c-f t \\
A c-f t\end{array}$ & $6,209,0$ & & & & \\
\hline
\end{tabular}

* Dischare measurement made on this day.

a No gage-height record; discharge computed from gate and spillway discharge. 
HELWIND RIVER BASIN

Helnand River below hajakai Dam, Af ghanistan

Dischargè in cubic feet per second, water year October 1956 to Septeuber 1957

\begin{tabular}{|c|c|c|c|c|c|c|c|c|c|c|c|c|}
\hline Day & oct. & Nov. & Dec. 1 & $\operatorname{Jan} .1$ & Feo. 1 & Mar. 1 & spr. & $\mathrm{Nuy}$ & June & Iuly & Aug. & Sept. \\
\hline 1 & $* 7,120$ & 4,740 & 4,670 & 5,720 & 6,510 & 6,420 & 7,580 & 39,800 & 29,300 & 10,800 & 7,500 & 7,300 \\
\hline 2 & 7,120 & 4,740 & $* 4,670$ & 5,680 & 6,470 & 6,470 & 7,630 & 44,200 & 29,300 & 10,500 & 7,450 & 7,300 \\
\hline 3 & 7,120 & 4,740 & 4,070 & 5,000 & 0,470 & 0,470 & 7,030 & 54,800 & 29,200 & 10,000 & 7,450 & 7,300 \\
\hline 4 & 7,080 & 4,740 & $\therefore, 070$ & 0,140 & 0,470 & 0,470 & 7,820 & 59,500 & 27,600 & 10,000 & 7,400 & $\approx 7,300$ \\
\hline 5 & 7,080 & 4,740 & 4,070 & 5,720 & 0,420 & 6,510 & 11,300 & 59,800 & $* 25,800$ & 9,840 & 7,400 & 7,300 \\
\hline 6 & 7,080 & 4,740 & 4,640 & 5,680 & 6,380 & 0,510 & 22,300 & 56,800 & 25,000 & 9,500 & 7,350 & 7,300 \\
\hline 7 & 7,040 & 4,740 & 4,640 & 5,680 & 0,380 & 6,470 & 30,100 & 53,000 & 24,200 & 9,340 & 7,350 & 7,300 \\
\hline 8 & 7,040 & 4,740 & 4,040 & 5,680 & 0,380 & 0,470 & 33,100 & 50,400 & 23,300 & 9,120 & 7,350 & 7,300 \\
\hline 9 & 0,460 & 4,740 & 4,600 & 5,680 & 6,340 & 0,140 & 36,000 & 48,800 & 22,400 & 9,010 & 7,350 & 7,350 \\
\hline 10 & 7,000 & 4,700 & 4,600 & 5,040 & 6,300 & 0,470 & 36,000 & 48,400 & 22,300 & 8,850 & 7,350 & 7,350 \\
\hline 11 & 7,000 & 4,700 & 4,000 & 5,040 & 0,300 & $c, 550$ & 36,200 & 49,100 & 21,600 & 8,700 & 7,350 & 7,350 \\
\hline 12 & 4,080 & 4,700 & 4,6010 & 5,720 & 5,300 & 0,550 & 38,000 & 49,300 & 21,000 & 8,550 & 7,300 & 7,300 \\
\hline 13 & 1,580 & 4,700 & 4,070 & 5,720 & $t, 260$ & 6,550 & 39,800 & 48,000 & 20,200 & 8,400 & 4,500 & 7,300 \\
\hline 14 & $* 1,580$ & 4,700 & 4,040 & 5,640 & 0,260 & 6,550 & 42,200 & 45,800 & 19,300 & 8,300 & 1,180 & 7,400 \\
\hline 15 & 1,580 & 4,700 & 4,640 & 5,640 & 6,220 & 0,510 & 45,600 & 43,400 & 18,700 & 8,150 & $\overline{1,180}$ & 7,400 \\
\hline 16 & 1,580 & 4,700 & 4,040 & 5,640 & 6,220 & 0,470 & 46,600 & 41,500 & 17,900 & 7,950 & $* 1,180$ & 7,400 \\
\hline 17 & 1,300 & 4,740 & 4,640 & 5,930 & 6,220 & 6,730 & $\overline{46,000}$ & 40,100 & 17,100 & 7,800 & 1,180 & 7,400 \\
\hline 18 & 1,800 & 4,740 & 4,6010 & 6,550 & 0,220 & 0,040 & 44,800 & 39,000 & 10,500 & 7,750 & 1,180 & 7,400 \\
\hline 19 & 1,800 & 4,740 & 4,000 & 0,000 & 0,220 & 0,730 & $\therefore, 200$ & 32,200 & 15,500 & 7,680 & 4,290 & 7,400 \\
\hline 20 & 1,800 & 4,500 & 4,600 & 6,600 & 6,180 & 0,910 & 44,400 & 37,400 & 14,900 & 7,600 & 7,400 & 7,450 \\
\hline 21 & 1,800 & 4,740 & 4,000 & 0,550 & $c, 140$ & 7,120 & $4,4,400$ & 36,500 & 14,500 & 7,000 & 7,400 & 7,450 \\
\hline 22 & 1,800 & 4,740 & 4,600 & 0,550 & 0,140 & 7,170 & 44,600 & 35,900 & 14,000 & 0,930 & 7,400 & 7,450 \\
\hline 23 & 1,030 & 4,740 & 4,000 & 0,550 & 0,140 & 7,170 & 44,200 & 35,500 & 13,600 & $\overline{7,500}$ & 7,400 & 7,400 \\
\hline 24 & $\overline{1,030}$ & 4,700 & 4,600 & 0,040 & 6,140 & 7,220 & 43,800 & 34,300 & 13,200 & 7,550 & 7,400 & 7,450 \\
\hline 25 & 1,030 & 4,700 & 4,000 & $\overline{6,600}$ & 2,180 & 7,260 & 42,600 & 33,400 & 12,800 & 7,550 & 7,400 & 7,400 \\
\hline 26 & 1,030 & 4,700 & 4,600 & 0,550 & 2 & 7,260 & 41,000 & 31,800 & 12,400 & 7,550 & 7,400 & 7,500 \\
\hline 27 & 1,030 & 4,070 & 4,000 & 6,550 & $\overline{5}$ & 7,300 & 40,600 & 30,500 & 12,000 & 7,500 & 7,400 & 7,450 \\
\hline 28 & $* 1,810$ & 4,070 & 4,000 & 0,340 & $\because, 690$ & 7,350 & $\star 37,5 \cup 0$ & 29,700 & 11,600 & 7,500 & 7,350 & 7,450 \\
\hline 29 & 3,820 & 4,070 & $* 5,720$ & $* 0,550$ & - & 7,400 & $* 7,800$ & 29,300 & 11,200 & 7,500 & 7,350 & $47,-50$ \\
\hline 30 & 4,740 & $\therefore, 670$ & 5,720 & $0,5,0$ & - & $* 7,440$ & 39,800 & 29,200 & $\because 1,000$ & 7,500 & 7,350 & 7,400 \\
\hline 31 & 4,740 & - & 5,720 & 0,470 & - & 7.530 & & 29,200 & - & $* 7,500$ & 7,350 & - \\
\hline Total & 118,100 & 141,280 & 146,660 & 188,880 & 156,960 & 210,810 & $1, \overline{043,560}$ & $1,296,600$ & 507,600 & 260,030 & 191,890 & 221,300 \\
\hline Nean & 3,810 & 4,709 & 4,731 & 0,093 & 5,606 & 5,800 & 34,790 & 41,830 & $18,9: 20$ & 8,388 & $0,1,00$ & 7,377 \\
\hline$i c-f t$ & 234,200 & 280,200 & 290,900 & $37 i, 000$ & 311,200 & $418,100 t$ & & $0,572,0(0)$ & $h, 2=0,00)^{3}$ & 512,800 & 30,0001 & 419,100 \\
\hline $\mathrm{Ca}$ & & & Ka & Min & 1,030 & liein & 8,514 & $\Delta c-f t$ & $0,181,00$ & & & \\
\hline & & & 800 & lin & 5 & & $12,+50$ & $\Delta c-f t$ & $890=1010$ & & & \\
\hline
\end{tabular}

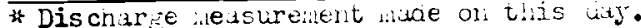

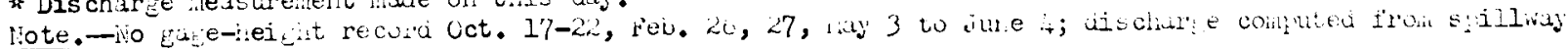

and valve rutinte. 


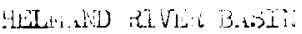

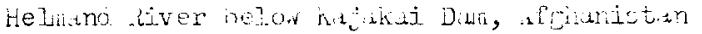

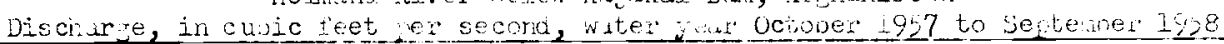

\begin{tabular}{|c|c|c|c|c|c|c|c|c|c|c|c|c|}
\hline Disy & oct. 1 & Nov. 1 & Dec. 1 & jun. & reo. & ilur. & wr. & hing & Jurie & .uly & $\therefore u g_{0}$ & Gept. \\
\hline 1 & 7,140 & 7,150 & +2720 & $* 7,500$ & 7,200 & 7,260 & 7,950 & 22,500 & 2,420 & $\because 3,200$ & 1,850 & $7,4,00$ \\
\hline 2 & 7,400 & $* 7,150$ & 4,720 & 7,500 & 7,150 & 7,200 & 8,000 & 21,000 & 9,700 & 8,200 & $* 7,800$ & 7,350 \\
\hline 3 & 7,400 & 7,150 & $\therefore, 800$ & 7,500 & 7,150 & $\because 7,250$ & 8,050 & 20,700 & 9,590 & 8,200 & 7,300 & 7,350 \\
\hline 4 & 7,450 & 7,200 & $\because, 800$ & 7,500 & 7,150 & 7,250 & 8,100 & 19,800 & 9,150 & $8,7.50$ & 7,800 & 7,300 \\
\hline 5 & 7,450 & 7,200 & 4,800 & 7,50 & 7,150 & 7,250 & 8,100 & $* 18,600$ & 9,320 & 3,150 & 7,300 & 7,300 \\
\hline 6 & 7,450 & 3,600 & 4,840 & 7,450 & 7,150 & 7,250 & 8,150 & 18,300 & 9,150 & 8,150 & 7,800 & 7,300 \\
\hline 7 & $7,+50$ & 5 & 4,960 & 7,450 & 7,150 & 7,100 & 8,150 & 17,400 & 9,040 & 8,150 & 7,750 & 7,300 \\
\hline 8 & 7,450 & 5 & 4,920 & 7,450 & 7,150 & 7,250 & 8,200 & 16,800 & 8,880 & $3,+00$ & 7,750 & 7,300 \\
\hline 9 & 7,450 & 5 & 4,920 & 7,450 & 7,150 & 7,250 & 8,200 & $x_{16}, 000$ & 6,760 & $4,0,0$ & 7,700 & 7,300 \\
\hline 10 & 7,450 & 5 & $\therefore, 920$ & 7,450 & 7,150 & 7,250 & 8,200 & 15,200 & 8,660 & 8,100 & 7,700 & 7,200 \\
\hline 17 & 7,300 & 5 & 0,040 & 7,450 & 7,200 & 7,250 & $3,2 \cup 0$ & 14,900 & 8,500 & 8,100 & 7,650 & 7,200 \\
\hline 12 & 7,300 & 5 & 7,400 & 7,400 & 7,200 & 7,350 & 3,200 & $1 i_{+}, t_{+} 00$ & 8,400 & 8,050 & 7,650 & 7,200 \\
\hline 13 & 7,300 & 5 & 7,400 & $7,4,00$ & 7,200 & 7,350 & 8,200 & 13,800 & 8,350 & 8,050 & 7,650 & 7,200 \\
\hline 14 & 7,450 & 5 & 7,400 & 7,400 & 7,200 & 7,350 & 8,300 & 15,600 & 0,300 & 8,050 & 7,600 & 7,200 \\
\hline 15 & 7,300 & 380 & $7,4 \omega$ & $7,4,0$ & 7,200 & 7,400 & 8,700 & 13,300 & 8,250 & 8,100 & 7,600 & 7,100 \\
\hline 16 & 7,300 & 750 & 7,400 & 7,050 & $7,20 x$ & 7,400 & 10,200 & 12,800 & $\because 8,250$ & 8,100 & 7,600 & 7,100 \\
\hline 17 & 7,300 & 4,750 & 7,400 & 7,250 & 7,200 & 7,400 & 12,800 & 12,400 & 8,250 & $8,10 u$ & 7,550 & 7,100 \\
\hline 18 & 7,300 & 4,750 & 7,400 & 7,200 & 7,200 & 7,400 & 15,000 & 12,000 & 8,250 & $8,1 \cup 0$ & 7,550 & 7,100 \\
\hline 19 & 7,300 & 4,750 & 7,400 & 7,200 & 7,200 & 7,550 & 16,400 & 11,600 & 3,250 & 8,050 & 7,550 & 7,100 \\
\hline 20 & 7,250 & 4,770 & 7,350 & 7,200 & 7,200 & 7,550 & 17,800 & 11,400 & 3,250 & 8,050 & 7,550 & 7,000 \\
\hline 21 & 7,300 & 4,770 & 7,300 & 6,950 & 7,200 & 7,600 & 19,500 & 11,300 & 8,250 & $8,050 !$ & 7,550 & 7,000 \\
\hline 22 & 7,250 & 4,780 & 7,350 & $\overline{7,150}$ & 7,200 & 7,000 & 20,400 & 12,400 & 8,250 & $3,000^{\prime}$ & $7,5,0$ & 7,000 \\
\hline 23 & 7,200 & 4,780 & 7,450 & 7,150 & 7,200 & 7,650 & 21,200 & 11,40 & 8,250 & $7,950 !$ & 7,500 & 7,0001 \\
\hline 24 & 7,200 & 4,790 & 7,450 & 7,100 & 7,200 & 7,650 & 22,200 & $1.1,300$ & 8,250 & 7,9501 & 7,500 & 6,800 \\
\hline 25 & 6,950 & 4,790 & 7,450 & 7,100 & 7,200 & 7,300 & 23,200 & 11,200 & 8,250 & 7,950 & 7,500 & 7,050 \\
\hline 26 & 7,200 & 4,800 & 7,500 & 7,100 & 7,200 & 7,800 & $2 t, 100$ & 11,000 & 8,250 & 7,950 & $7,5 a$ & 7,050 \\
\hline 27 & 7,200 & 4,800 & 7,500 & 7,100 & $7,20 x$ & 7,850 & 24,800 & 10,800 & 0,200 & 7,950 & 7,450 & $* 7,000$ \\
\hline 28 & 7,200 & $*_{4}, 760$ & 7,500 & 7,100 & 7,200 & 7,850 & $\overline{2 l_{i}, 800}$ & 10,70 & 8,200 & 7,900 & 7,450 & 7,000 \\
\hline 29 & 7,200 & 4,720 & 7,500 & 7,100 & - & 7,900 & $2 i_{4}, 000$ & 10,500 & 8,200 & 7,900 & 7,450 & 7,000 \\
\hline 30 & 7,200 & 4,720 & 7,500 & 37,100 & - & 2,950 & 23,200 & 10,200 & 8,200 & 7,850 & 7,450 & 3,950 \\
\hline 31 & 7,200 & - & 7,500 & 7,150 & - & $* 7,950$ & - & $\because 9,920$ & $2-1$ & 7,850 & $\therefore 7,400$ & - \\
\hline Tot 31 & 226,290 & 107,350 & 202,990 & 225,800 & 201,150 & 231,050 & 422,000 & 426,820 & 257,580 & 240,8 & $2=6,000$ & $21 i_{+},<50$ \\
\hline Hean & 7,300 & 3,578 & 6,548 & 7,284 & 7,184 & $7,1+79$ & $11_{t}, 070$ & 14,090 & 8,586 & 7,758 & 7,0 & 7,142 \\
\hline$s c-f t$ & 48,800 & $k 12,900$ & 1402,600 & 117,900 & $399,(100)$ & 454,900 & $8 ; 7,000$ & $8,4,4 x$ & 510,9001 & $.77,0001$ & 408,100 & $1,25,000$ \\
\hline & & & & & & & & & & & & \\
\hline & & & & & 5 & $e n$ & & $-1 t$ &, 600 & & & \\
\hline
\end{tabular}

* Discharge newsurenent nade on tij. $\mathrm{sij}$.

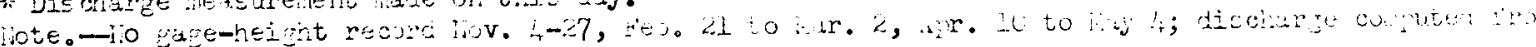

srifide aric valve ritings. 
HETMAND RIVER BASIN

Helmand River below Kajakai Dam, ifghanistan Discharge, in cubic feet per second, water year Qctober 1958 to September 1959

\begin{tabular}{|c|c|c|c|c|c|c|c|c|c|c|c|c|}
\hline Day & Oct. & Nov. & Dec. & Jan. & Feb. & Mar. & Apr. & May & June & July & Aug. & Sept。 \\
\hline $\begin{array}{l}1 \\
2 \\
3 \\
4 \\
5\end{array}$ & $\begin{array}{r}6,950 \\
6,900 \\
* 6,900 \\
6,900 \\
6,900\end{array}$ & $\begin{array}{l}6,420 \\
6,420 \\
6,420 \\
6,380 \\
6,380\end{array}$ & $\begin{array}{r}4,160 \\
4,160 \\
4,160 \\
4,160 \\
* 4,160\end{array}$ & $\begin{array}{l}\frac{4,120}{4,160} \\
4,160 \\
4,160 \\
4,160\end{array}$ & $\begin{array}{r}* 4,160 \\
4,160 \\
4,160 \\
4,160 \\
4,160\end{array}$ & $\begin{array}{r}4,320 \\
* 4,200 \\
4,240 \\
4,280 \\
4,320\end{array}$ & $\begin{array}{r}* 7,400 \\
7,400 \\
7,450 \\
7,500 \\
7,500\end{array}$ & $\begin{array}{l}17,900 \\
16,800 \\
16,300 \\
15,700 \\
15,500\end{array}$ & $\begin{array}{r}* 9,810 \\
9,640 \\
9,540 \\
9,480 \\
9,480\end{array}$ & $\begin{array}{r}7,700 \\
* 7,700 \\
7,750 \\
7,700 \\
7,700\end{array}$ & $\begin{array}{l}7,350 \\
7,350 \\
7,350 \\
7,300 \\
7,300\end{array}$ & $\begin{array}{l}6,900 \\
6,900 \\
6,850 \\
6,850 \\
6,850\end{array}$ \\
\hline $\begin{array}{r}6 \\
7 \\
8 \\
9 \\
10\end{array}$ & $\begin{array}{l}6,850 \\
6,850 \\
6,800 \\
6,800 \\
6,800\end{array}$ & $\begin{array}{l}6,380 \\
6,330 \\
6,330 \\
6,330 \\
6,280\end{array}$ & $\begin{array}{l}4,160 \\
4,160 \\
4,160 \\
4,120 \\
4,160\end{array}$ & $\begin{array}{r}4,160 \\
4,160 \\
4,160 \\
* 4,160 \\
4,160\end{array}$ & $\begin{array}{l}4,120 \\
4,120 \\
4,120 \\
4,160 \\
4,200 \\
\end{array}$ & $\begin{array}{r}4,360 \\
4,360 \\
4,400 \\
4,480 \\
* 4,520\end{array}$ & $\begin{array}{l}7,600 \\
7,600 \\
7,650 \\
7,650 \\
7,500\end{array}$ & $\begin{array}{l}15,300 \\
15,100 \\
14,600 \\
14,200 \\
13,700\end{array}$ & $\begin{array}{l}9,370 \\
9,150 \\
8,930 \\
8,710 \\
7,850\end{array}$ & $\begin{array}{l}7,700 \\
7,700 \\
7,700 \\
7,700 \\
7,650\end{array}$ & $\begin{array}{l}7,300 \\
7,250 \\
7,200 \\
7,200 \\
7,200\end{array}$ & $\begin{array}{l}6,800 \\
6,420 \\
5,970 \\
6,060 \\
6,060\end{array}$ \\
\hline $\begin{array}{l}11 \\
12 \\
13 \\
14 \\
15\end{array}$ & $\begin{array}{l}6,800 \\
6,800 \\
6,750 \\
6,750 \\
6,700\end{array}$ & $\begin{array}{l}6,280 \\
4,600 \\
3,860 \\
3,820 \\
3,820\end{array}$ & $\begin{array}{l}4,160 \\
4,160 \\
4,240 \\
4,200 \\
4,160\end{array}$ & $\begin{array}{l}4,160 \\
4,160 \\
4,160 \\
4,160 \\
4,160\end{array}$ & $\begin{array}{l}4,160 \\
4,160 \\
4,160 \\
4,200 \\
4,200\end{array}$ & $\begin{array}{r}* 4,560 \\
4,600 \\
4,640 \\
4,640 \\
4,640\end{array}$ & $\begin{array}{l}7,750 \\
7,800 \\
7,850 \\
7,900 \\
8,350\end{array}$ & $\begin{array}{l}13,400 \\
13,000 \\
12,600 \\
12,300 \\
12,100\end{array}$ & $\begin{array}{l}8,150 \\
8,000 \\
7,900 \\
7,850 \\
7,800\end{array}$ & $\begin{array}{l}7,650 \\
7,650 \\
7,600 \\
7,600 \\
7,600\end{array}$ & $\begin{array}{l}7,150 \\
7,150 \\
7,100 \\
7,100 \\
7,100\end{array}$ & $\begin{array}{l}6,060 \\
6,020 \\
6,020 \\
6,020 \\
6,020\end{array}$ \\
\hline $\begin{array}{l}16 \\
17 \\
18 \\
19 \\
20\end{array}$ & $\begin{array}{l}6,700 \\
6,700 \\
6,650 \\
5,200 \\
5,200\end{array}$ & $\begin{array}{l}3,860 \\
3,860 \\
3,820 \\
3,820 \\
3,820\end{array}$ & $\begin{array}{l}4,160 \\
4,160 \\
4,160 \\
4,160 \\
4,160\end{array}$ & $\begin{array}{l}4,160 \\
4,160 \\
4,160 \\
4,160 \\
4,160\end{array}$ & $\begin{array}{l}4,200 \\
4,160 \\
4,200 \\
4,120 \\
4,120\end{array}$ & $\begin{array}{l}4,640 \\
4,640 \\
4,660 \\
4,680 \\
4,690\end{array}$ & $\begin{array}{l}10,700 \\
13,400 \\
16,000 \\
18,000 \\
19,400\end{array}$ & $\begin{array}{l}12,000 \\
12,600 \\
13,400 \\
13,800 \\
13,700\end{array}$ & $\begin{array}{l}7,800 \\
7,800 \\
7,800 \\
7,750 \\
7,750\end{array}$ & $\begin{array}{l}7,550 \\
7,550 \\
7,550 \\
7,550 \\
7,160 \\
\end{array}$ & $\begin{array}{l}7,100 \\
7,100 \\
7,100 \\
7,100 \\
7,100\end{array}$ & $\begin{array}{l}5,970 \\
5,970 \\
5,970 \\
5,970 \\
5,920\end{array}$ \\
\hline $\begin{array}{l}21 . \\
22 \\
23 \\
24 \\
25\end{array}$ & $\begin{array}{l}6,600 \\
6,600 \\
6,600 \\
6,600 \\
6,700\end{array}$ & $\begin{array}{l}3,820 \\
3,820 \\
3,820 \\
3,820 \\
3,820\end{array}$ & $\begin{array}{l}4,160 \\
4,160 \\
4,160 \\
4,160 \\
4,200\end{array}$ & $\begin{array}{l}4,160 \\
4,120 \\
4,120 \\
4,160 \\
4,160\end{array}$ & $\begin{array}{l}4,000 \\
4,040 \\
4,080 \\
4,120 \\
4,080\end{array}$ & $\begin{array}{l}4,700 \\
4,750 \\
4,800 \\
4,820 \\
4,850\end{array}$ & $\begin{array}{l}20,500 \\
21,200 \\
\frac{22,000}{21,700} \\
21,100\end{array}$ & $\begin{array}{l}13,400 \\
13,000 \\
12,700 \\
12,400 \\
12,000\end{array}$ & $\begin{array}{l}7,750 \\
7,750 \\
7,750 \\
7,750 \\
7,750\end{array}$ & $\begin{array}{l}7,550 \\
7,500 \\
7,500 \\
7,500 \\
7,500\end{array}$ & $\begin{array}{l}7,100 \\
7,050 \\
7,050 \\
7,000 \\
7,000\end{array}$ & $\begin{array}{l}5,920 \\
5,920 \\
5,250 \\
4,400 \\
4,400\end{array}$ \\
\hline $\begin{array}{l}26 \\
27 \\
28 \\
29 \\
30 \\
31\end{array}$ & $\begin{array}{r}6,600 \\
6,550 \\
6,500 \\
6,500 \\
* 6,460 \\
6,460\end{array}$ & $\begin{array}{c}4,000 \\
4,240 \\
4,200 \\
4,160 \\
4,240 \\
-\end{array}$ & $\begin{array}{l}4,160 \\
4,160 \\
4,160 \\
4,160 \\
4,160 \\
4,120\end{array}$ & $\begin{array}{l}4,160 \\
4,160 \\
4,160 \\
4,120 \\
4,160 \\
4,160\end{array}$ & $\begin{array}{c}4,080 \\
4,080 \\
4,080 \\
- \\
-\end{array}$ & $\begin{array}{l}4,880 \\
6,430 \\
7,500 \\
7,450 \\
7,450 \\
7,400\end{array}$ & $\begin{array}{c}20,300 \\
19,100 \\
18,500 \\
18,000 \\
* 17,900 \\
-\end{array}$ & $\begin{array}{r}11,600 \\
11,400 \\
10,800 \\
10,200 \\
\frac{9,700}{9,810}\end{array}$ & $\begin{array}{c}7,750 \\
7,750 \\
7,700 \\
7,700 \\
7,700 \\
-\end{array}$ & $\begin{array}{r}7,500 \\
7,500 \\
7,450 \\
7,450 \\
7,400 \\
\times 7,350\end{array}$ & $\begin{array}{r}7,000 \\
6,950 \\
6,950 \\
6,900 \\
6,900 \\
* 6,900\end{array}$ & $\begin{array}{l}4,400 \\
4,400 \\
4,400 \\
4,360 \\
\frac{4,320}{-}\end{array}$ \\
\hline $\begin{array}{l}\text { Total } \\
\text { Mean } \\
\text { ac-ft } \\
\end{array}$ & $\begin{array}{r}205,070 \\
6,615 \\
406,800\end{array}$ & $\begin{array}{r}145,170 \\
4,839 \\
287,900 \\
\end{array}$ & $\begin{array}{r}129,040 \\
4,163 \\
256,000\end{array}$ & $\begin{array}{r}128,800 \\
4,155 \\
255,500 \\
\end{array}$ & $\begin{array}{r}115,760 \\
4,134 \\
229,600 \\
\end{array}$ & $\begin{array}{r}154,900 \\
4,997 \\
307,200 \\
\end{array}$ & $\begin{array}{r}392,700 \\
13,090 \\
778,900\end{array}$ & $\begin{array}{r}411,010 \\
13,260 \\
815,200\end{array}$ & $\begin{array}{r}247,910 \\
8,264 \\
491,700 \\
\end{array}$ & $\begin{array}{r}234,660 \\
7,570 \\
465,400 \\
\end{array}$ & $\begin{array}{r}220,700 \\
7,119 \\
427,800 \\
\end{array}$ & $\begin{array}{r}173,370 \\
5,779 \\
343,900 \\
\end{array}$ \\
\hline $\begin{array}{l}\text { Cale } \\
\text { wate }\end{array}$ & & & $\operatorname{Max} 22$, & I & $\begin{array}{l}3,400 \\
3,820\end{array}$ & $\begin{array}{l}\text { Mean } \\
\text { Mean }\end{array}$ & $\begin{array}{l}8,070 \\
7,011\end{array}$ & $\begin{array}{l}A C-f \\
A C-f\end{array}$ & 5,076 & $\begin{array}{l}000 \\
000\end{array}$ & & \\
\hline
\end{tabular}

* Discharge measurement made on this day.

Note.-No gage-height recora Oct. 19-29, har. 15-31, Way 18-31; dischare comiuted from recorded range instage ano valve ratings. 
HEIMAND RIVER BASIN

Helmand River below Kajaka1 Dam, Lfghanistan

Discharge, in cubic feet per second, water year October 1959 to September 1960

\begin{tabular}{|c|c|c|c|c|c|c|c|c|c|c|c|c|}
\hline Day & oct. & Nov. & Dec. & Jan. & reb. & Mar. & spr. & May & June & July & Aug. & Sept. \\
\hline $\begin{array}{l}1 \\
2 \\
3 \\
4 \\
5\end{array}$ & $\begin{array}{r}4,320 \\
4,320 \\
4,320 \\
* 4,280 \\
4,280\end{array}$ & $\begin{array}{l}4,320 \\
4,320 \\
4,320 \\
4,320 \\
4,320\end{array}$ & $\begin{array}{l}\frac{3,460}{3,500} \\
3,500 \\
3,500 \\
3,500\end{array}$ & $\begin{array}{l}6,060 \\
6,020 \\
6,020 \\
5,970 \\
6,020\end{array}$ & $\begin{array}{l}\frac{3,940}{3,720} \\
3,720 \\
3,720 \\
3,710\end{array}$ & $\begin{array}{l}3,720 \\
3,720 \\
3,720 \\
3,720 \\
3,720\end{array}$ & $\begin{array}{l}2,420 \\
2,420 \\
2,420 \\
2,420 \\
2,420\end{array}$ & $\begin{array}{l}\frac{4,280}{4,280} \\
4,370 \\
4,370 \\
4,320\end{array}$ & $\begin{array}{l}13,700 \\
13,100 \\
12,800 \\
12,600 \\
12,300\end{array}$ & $\begin{array}{l}\frac{5,700}{5,560} \\
5,480 \\
5,340 \\
5,250\end{array}$ & $\begin{array}{r}4,110 \\
4,110 \\
4,110 \\
* 4,110 \\
4,110\end{array}$ & $\begin{array}{l}\frac{4,480}{4,480} \\
4,480 \\
4,480 \\
4,480\end{array}$ \\
\hline $\begin{array}{r}6 \\
7 \\
8 \\
9 \\
10\end{array}$ & $\begin{array}{l}4,280 \\
4,280 \\
4,280 \\
4,280 \\
4,280\end{array}$ & $\begin{array}{r}4,320 \\
4,320 \\
* 4,320 \\
* 3,900 \\
3,460 \\
\end{array}$ & $\begin{array}{r}3,500 \\
3,540 \\
3,540 \\
3,540 \\
* 6,060\end{array}$ & $\begin{array}{r}* 5,970 \\
5,970 \\
5,920 \\
5,160 \\
3,940\end{array}$ & $\begin{array}{l}3,710 \\
3,710 \\
3,700 \\
3,700 \\
3,690\end{array}$ & $\begin{array}{l}3,720 \\
3,720 \\
3,720 \\
3,720 \\
3,720\end{array}$ & $\begin{array}{l}2,420 \\
2,420 \\
3,140 \\
3,540 \\
3,580\end{array}$ & $\begin{array}{r}4,320 \\
4,370 \\
5,480 \\
7,950 \\
10,400\end{array}$ & $\begin{array}{l}12,000 \\
11,900 \\
11,600 \\
11,400 \\
11,200\end{array}$ & $\begin{array}{l}5,160 \\
5,070 \\
4,980 \\
4,890 \\
4,800\end{array}$ & $\begin{array}{l}4,160 \\
4,160 \\
4,110 \\
4,110 \\
4,110\end{array}$ & $\begin{array}{r}* 4,460 \\
4,240 \\
4,240 \\
4,240 \\
4,240\end{array}$ \\
\hline $\begin{array}{l}11 \\
12 \\
15 \\
14 \\
15\end{array}$ & $\begin{array}{l}4,280 \\
4,280 \\
4,230 \\
4,280 \\
4,240\end{array}$ & $\begin{array}{l}3,500 \\
3,500 \\
3,500 \\
3,460 \\
3,460\end{array}$ & $\begin{array}{l}6,420 \\
6,420 \\
6,420 \\
6,420 \\
6,420\end{array}$ & $\begin{array}{l}3,940 \\
3,940 \\
3,940 \\
3,940 \\
3,940\end{array}$ & $\begin{array}{l}3,690 \\
3,680 \\
3,680 \\
3,680 \\
3,680\end{array}$ & $\begin{array}{l}3,700 \\
3,700 \\
5,700 \\
3,700 \\
3,700\end{array}$ & $\begin{array}{l}3,580 \\
3,620 \\
3,620 \\
3,700 \\
3,740\end{array}$ & $\begin{array}{l}12,500 \\
14,700 \\
16,500 \\
18,300 \\
20,000\end{array}$ & $\begin{array}{r}10,300 \\
10,500 \\
10,200 \\
9,360 \\
9,590\end{array}$ & $\begin{array}{l}4,700 \\
4,600 \\
4,500 \\
4,400 \\
4,300\end{array}$ & $\begin{array}{l}4,110 \\
4,360 \\
4,560 \\
4,560 \\
4,560\end{array}$ & $\begin{array}{l}4,240 \\
4,240 \\
4,240 \\
4,240 \\
4,240\end{array}$ \\
\hline $\begin{array}{l}16 \\
17 \\
18 \\
19 \\
20\end{array}$ & $\begin{array}{l}4,240 \\
4,240 \\
4,240 \\
4,240 \\
4,240\end{array}$ & $\begin{array}{l}3,500 \\
3,500 \\
3,500 \\
3,500 \\
3,460\end{array}$ & $\begin{array}{l}6,330 \\
6,330 \\
6,330 \\
6,330 \\
6,330\end{array}$ & $\begin{array}{l}3,940 \\
3,940 \\
3,940 \\
3,940 \\
3,900\end{array}$ & $\begin{array}{l}3,690 \\
3,690 \\
3,690 \\
3,690 \\
3,690\end{array}$ & $\begin{array}{l}3,700 \\
3,700 \\
3,700 \\
3,700 \\
3,700\end{array}$ & $\begin{array}{l}3,900 \\
3,860 \\
3,360 \\
3,900 \\
3,940\end{array}$ & $\begin{array}{l}21,300 \\
22,200 \\
23,000 \\
23,000 \\
22,900\end{array}$ & $\begin{array}{l}9,150 \\
8,820 \\
8,200 \\
7,900 \\
7,600\end{array}$ & $\begin{array}{l}4,200 \\
4,160 \\
4,110 \\
4,110 \\
4,110\end{array}$ & $\begin{array}{l}4,560 \\
4,560 \\
4,560 \\
4,560 \\
4,560\end{array}$ & $\begin{array}{l}4,240 \\
4,240 \\
4,240 \\
4,240 \\
4,240\end{array}$ \\
\hline $\begin{array}{l}21 \\
22 \\
23 \\
24 \\
25\end{array}$ & $\begin{array}{l}4,230 \\
4,280 \\
4,280 \\
4,280 \\
4,280\end{array}$ & $\begin{array}{l}3,460 \\
3,460 \\
3,460 \\
3,460 \\
3,460\end{array}$ & $\begin{array}{l}6,330 \\
6,240 \\
6,240 \\
6,240 \\
6,240\end{array}$ & $\begin{array}{l}3,900 \\
3,900 \\
3,900 \\
3,900 \\
3,900\end{array}$ & $\begin{array}{l}3,700 \\
3,700 \\
3,700 \\
3,700 \\
3,700\end{array}$ & $\begin{array}{r}4,200 \\
4,200 \\
4,200 \\
4,200 \\
* 4,200\end{array}$ & $\begin{array}{l}3,980 \\
3,980 \\
4,030 \\
4,070 \\
4,110\end{array}$ & $\begin{array}{l}22,300 \\
21,600 \\
20,700 \\
19,600 \\
18,300\end{array}$ & $\begin{array}{l}7,300 \\
7,100 \\
6,950 \\
6,750 \\
6,600\end{array}$ & $\begin{array}{l}4,110 \\
4,110 \\
4,110 \\
4,210 \\
4,110\end{array}$ & $\begin{array}{l}4,560 \\
4.560 \\
4,560 \\
4,560 \\
4,480\end{array}$ & $\begin{array}{l}4,200 \\
4,160 \\
4,200 \\
4,110 \\
4,110\end{array}$ \\
\hline $\begin{array}{l}26 \\
27 \\
28 \\
29 \\
30 \\
31\end{array}$ & $\begin{array}{l}4,280 \\
4,280 \\
4,320 \\
4,420 \\
4,370 \\
4,370\end{array}$ & $\begin{array}{c}3,460 \\
3,460 \\
3,460 \\
3,460 \\
3,460 \\
-\end{array}$ & $\begin{array}{l}6,240 \\
6,240 \\
6,200 \\
6,150 \\
6,150 \\
6,100\end{array}$ & $\begin{array}{r}3,900 \\
3,860 \\
3,900 \\
* 3,900 \\
3,900 \\
3,900\end{array}$ & $\begin{array}{c}3,720 \\
3,720 \\
3,720 \\
3,720 \\
- \\
-\end{array}$ & $\begin{array}{l}4,200 \\
4,240 \\
3,940 \\
3,380 \\
3,380 \\
2,960\end{array}$ & $\begin{array}{r}4,110 \\
4,160 \\
4,160 \\
* 4,200 \\
4,240 \\
\end{array}$ & $\begin{array}{r}16,800 \\
16,200 \\
15,800 \\
15,100 \\
+14,700 \\
14,200\end{array}$ & $\begin{array}{l}6,330 \\
6,200 \\
6,060 \\
5,920 \\
5,790 \\
-\end{array}$ & $\begin{array}{l}4,210 \\
4,070 \\
4,070 \\
4,070 \\
4,210 \\
4,110\end{array}$ & $\begin{array}{l}4,480 \\
4,480 \\
4,480 \\
4,480 \\
4,480 \\
4,480\end{array}$ & $\begin{array}{r}4,110 \\
4,110 \\
4,110 \\
4,110 \\
* 4,110 \\
\ldots\end{array}$ \\
\hline $\begin{array}{l}\text { Total } \\
\text { Mean } \\
\text { loaft }\end{array}$ & $\begin{array}{r}132,920 \\
4,288 \\
263,600\end{array}$ & $\begin{array}{r}11,400 \\
3,713 \\
221,000\end{array}$ & $\begin{array}{r}169,760 \\
5,476 \\
336,700 \\
\end{array}$ & $\begin{array}{r}139,270 \\
4,493 \\
276,200 \\
\end{array}$ & $\begin{array}{r}107,560 \\
3,709 \\
215,300 \\
\end{array}$ & $\begin{array}{r}17,300 \\
3,784 \\
232,700 \\
\end{array}$ & $\begin{array}{r}105,960 \\
3,532 \\
210,200\end{array}$ & $\begin{array}{r}443,340 \\
14,320 \\
880,300 \\
\end{array}$ & $\begin{array}{r}280,220 \\
9,341 \\
555,800 \\
\end{array}$ & $\begin{array}{r}140,510 \\
4,533 \\
278,700 \\
\end{array}$ & $\begin{array}{r}155,750 \\
4,379 \\
269,300 \\
\end{array}$ & $\begin{array}{r}127,550 \\
4,252 \\
253,000 \\
\end{array}$ \\
\hline $\begin{array}{l}\mathrm{Ca} \\
\text { Wa }\end{array}$ & & & 23 & & Min & $\begin{array}{l}60 \\
20\end{array}$ & $\begin{array}{l}\text { lean } \\
\text { lean }\end{array}$ & $\begin{array}{l}6,852 \\
5,497\end{array}$ & 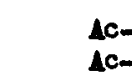 & et & $\begin{array}{l}, 000 \\
, 800\end{array}$ & \\
\hline
\end{tabular}

* Discharge measurement made on this day.

Note.-No gage-helghts Dec. 11-27, Fob. 2 to Jan. 24, July 6-17, Lug. 12 to Sept. 5, Sept. 7-20; discharge computed from range lines and reservoir release. 
HETMARD RIVER BASIN

Seraj Canal at Sangin, Afghanistan

Iocation.-Lat $32^{\circ} 031$ N., Iong $64^{\circ} 50^{\prime}$ E., on left bank about 100 meters upat ream from office of the Governor of Sangin district in village of Sangin, about 500 meters downstream frcm canal inlet and control gate structure on HeImand River, 35 kd loneters northeast of Girishk, and about $40 \mathrm{kllometers}$ dometrom Kajakes dam on the Helmand River.

Beoard arellable.-October 1952 to September 1960.

Cege.-Staff gage read twice daily.

Trerage dis charge -8 years, 254 cfs (183,900 acre-ft per year)

ixtremes. - Medmum daily discharges for the water years 1953-60 are given in the following table:

\begin{tabular}{|c|c|c|c|}
\hline Water Ioar & Date & $\begin{array}{l}\text { Marimam } \\
\text { Gage Height } \\
\text { (Eeters) }\end{array}$ & $\begin{array}{l}\text { Discharge } \\
\text { (cfs) }\end{array}$ \\
\hline $\begin{array}{l}1953 \\
1954 \\
1955 \\
1956 \\
1957 \\
1958 \\
1959 \\
1960\end{array}$ & $\begin{array}{l}\text { Kay 1, 1953 } \\
\text { Apr. 21, 1954 } \\
\text { Dec. 25, 26, 1954 } \\
\text { Apr. 13, 14, } 1956 \\
\text { Kar. 19, 1957 } \\
\text { Dec. 15, 1957 } \\
\text { Har. 3, 1959 } \\
\text { Apr. 15, } 1960\end{array}$ & $\begin{array}{l}1.68 \\
1.80 \\
2.00 \\
2.00 \\
1.34 \\
1.74 \\
1.44\end{array}$ & $\begin{array}{l}445 \\
593 \\
596 \\
622 \\
612 \\
397 \\
519 \\
328\end{array}$ \\
\hline
\end{tabular}

Remaks.-Becords good exoupt those for periods of no gage-height record, which are poor. Water 1s diverted from left bank of Helmand River for irrigation of about 24,000 acres extending from Sangin to Iakchal and southwesterly towand Kala Bist. 
HETMAND RTVER BASIN

Ceraj Canal at Sangin, Afghanistan

Discharge, in cubic feet per second, wate: year October 1952 to September 1953

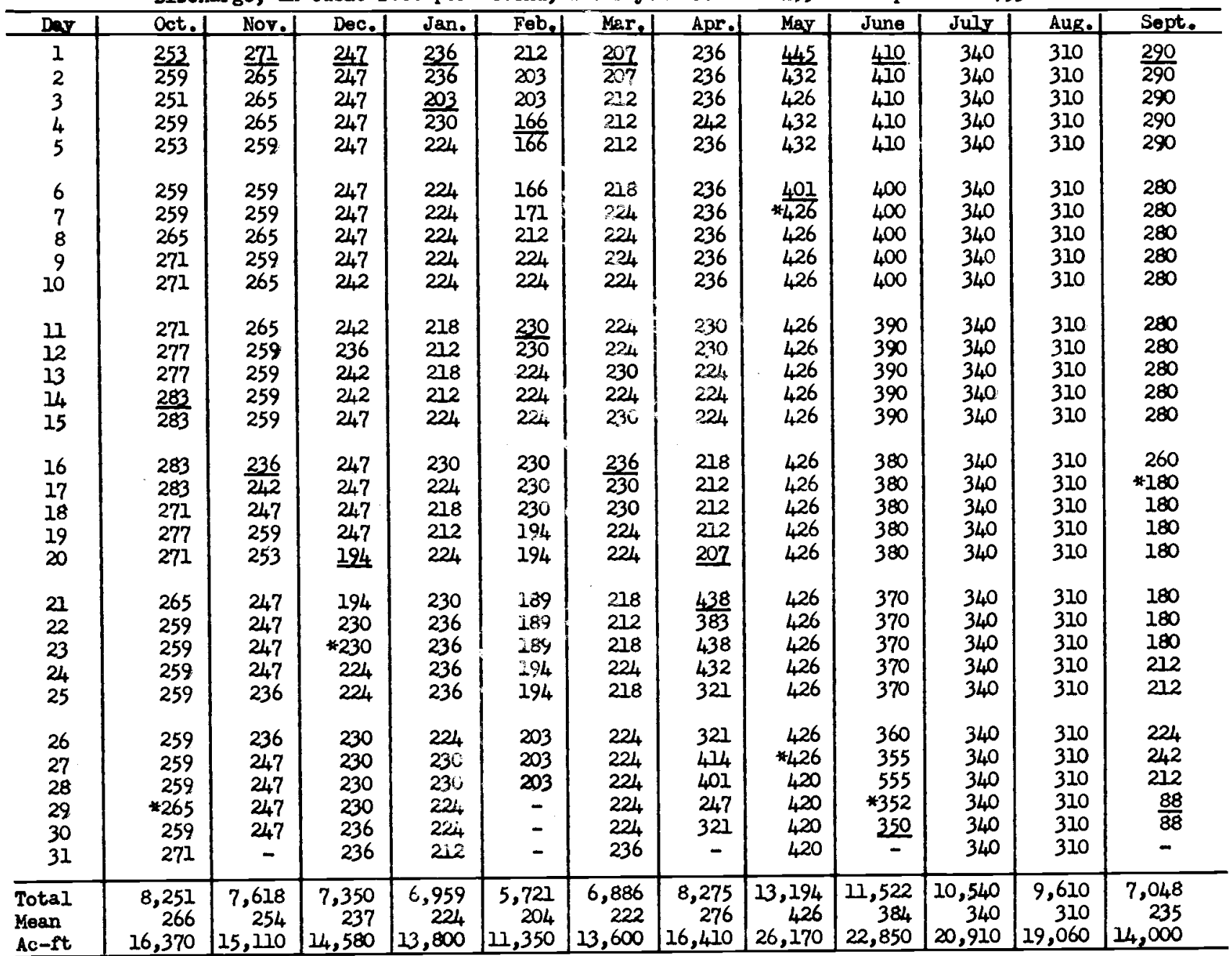

Calendar year 1952: Max - Min - Mean - Ac-ft -

Water year 1952-53: $\operatorname{Max} 445$ Min 88 Mean 281 Ac-ft 204,300

* Discharge measurement made on this day.

Note.- No gage-height record May 8-26, May 28 to June 28, June 30 to Sept. 16, Sept. 18-25, discharge

interpolated or computed on basis of llelmand River records. 
HEIMAND RIVER BASIN

Seraj Canal at Sangin, Afghanistan

Discharge, in cubic feet per second, water year October 1953 to September 1954

\begin{tabular}{|c|c|c|c|c|c|c|c|c|c|c|c|c|}
\hline Dar & Oct, & Nov. & Dec, & Jan. & Feb. & Mar. & Apr. & May & June & Julr & Aug. & Sept. \\
\hline $\begin{array}{l}1 \\
2 \\
3 \\
4 \\
5\end{array}$ & $\begin{array}{l}\frac{146}{259} \\
283 \\
\frac{321}{321}\end{array}$ & $\begin{array}{r}253 \\
* 271 \\
296 \\
308 \\
296\end{array}$ & $\begin{array}{r}296 \\
296 \\
296 \\
100 \\
10 \\
\end{array}$ & $\begin{array}{l}\frac{106}{106} \\
110 \\
110 \\
110\end{array}$ & $\begin{array}{l}200 \\
200 \\
200 \\
200 \\
200\end{array}$ & $\begin{array}{l}166 \\
166 \\
162 \\
166 \\
162\end{array}$ & $\begin{array}{l}445 \\
395 \\
358 \\
358 \\
345\end{array}$ & $\begin{array}{l}345 \\
358 \\
370 \\
333 \\
333\end{array}$ & $\begin{array}{l}271 \\
236 \\
212 \\
203 \\
194\end{array}$ & $\begin{array}{r}1.0 \\
10 \\
50 \\
118 \\
118\end{array}$ & $\begin{array}{r}* 171 \\
170 \\
170 \\
170 \\
339\end{array}$ & $\begin{array}{l}\frac{327}{321} \\
321 \\
308 \\
308\end{array}$ \\
\hline $\begin{array}{r}6 \\
7 \\
8 \\
9 \\
10\end{array}$ & $\begin{array}{l}265 \\
247 \\
236 \\
277 \\
259\end{array}$ & $\begin{array}{l}308 \\
308 \\
308 \\
308 \\
302\end{array}$ & $\begin{array}{l}100 \\
271 \\
296 \\
\frac{302}{302}\end{array}$ & $\begin{array}{l}110 \\
110 \\
110 \\
110 \\
110\end{array}$ & $\begin{array}{l}200 \\
200 \\
200 \\
200 \\
200\end{array}$ & $\begin{array}{l}162 \\
158 \\
158 \\
184 \\
166\end{array}$ & $\begin{array}{l}333 \\
321 \\
321 \\
314 \\
314\end{array}$ & $\begin{array}{l}345 \\
432 \\
407 \\
474 \\
358\end{array}$ & $\begin{array}{l}184 \\
175 \\
166 \\
146 \\
133\end{array}$ & $\begin{array}{l}125 \\
194 \\
333 \\
407 \\
438\end{array}$ & $\begin{array}{r}383 \\
420 \\
236 \\
\frac{10}{10}\end{array}$ & $\begin{array}{l}308 \\
308 \\
308 \\
308 \\
302\end{array}$ \\
\hline $\begin{array}{l}11 \\
12 \\
13 \\
14 \\
15\end{array}$ & $\begin{array}{l}271 \\
283 \\
271 \\
283 \\
259\end{array}$ & $\begin{array}{r}\frac{12}{12} \\
12 \\
100 \\
247\end{array}$ & $\begin{array}{l}296 \\
302 \\
302 \\
302 \\
296\end{array}$ & $\begin{array}{l}110 \\
110 \\
110 \\
110 \\
110\end{array}$ & $\begin{array}{l}200 \\
200 \\
200 \\
200 \\
200\end{array}$ & $\begin{array}{l}166 \\
184 \\
189 \\
224 \\
158\end{array}$ & $\begin{array}{l}333 \\
426 \\
426 \\
420 \\
420\end{array}$ & $\begin{array}{l}358 \\
333 \\
321 \\
296 \\
283\end{array}$ & $\begin{array}{r}121 \\
114 \\
103 \\
95 \\
95\end{array}$ & $\begin{array}{l}443 \\
457 \\
463 \\
463 \\
457\end{array}$ & $\begin{array}{l}407 \\
407 \\
401 \\
401 \\
395\end{array}$ & $\begin{array}{l}302 \\
302 \\
302 \\
290 \\
283\end{array}$ \\
\hline $\begin{array}{l}16 \\
17 \\
18 \\
19 \\
20\end{array}$ & $\begin{array}{l}259 \\
259 \\
259 \\
259 \\
259\end{array}$ & $\begin{array}{l}271 \\
271 \\
271 \\
271 \\
271\end{array}$ & $\begin{array}{l}296 \\
296 \\
296 \\
296 \\
296\end{array}$ & $\begin{array}{l}110 \\
110 \\
110 \\
110 \\
110\end{array}$ & $\begin{array}{l}200 \\
333 \\
308 \\
236 \\
203\end{array}$ & $\begin{array}{l}175 \\
175 \\
175 \\
175 \\
175\end{array}$ & $\begin{array}{l}407 \\
414 \\
414 \\
450 \\
500\end{array}$ & $\begin{array}{l}364 \\
407 \\
414 \\
395 \\
383\end{array}$ & $\begin{array}{l}92 \\
88 \\
88 \\
88 \\
88\end{array}$ & $\begin{array}{l}463 \\
463 \\
457 \\
457 \\
451\end{array}$ & $\begin{array}{l}395 \\
389 \\
383 \\
383 \\
370\end{array}$ & $\begin{array}{l}277 \\
277 \\
277 \\
271 \\
277\end{array}$ \\
\hline $\begin{array}{l}21 \\
22 \\
23 \\
24 \\
25\end{array}$ & $\begin{array}{l}259 \\
259 \\
259 \\
247 \\
247\end{array}$ & $\begin{array}{l}271 \\
271 \\
271 \\
296 \\
296\end{array}$ & $\begin{array}{r}290 \\
100 \\
10 \\
10 \\
10\end{array}$ & $\begin{array}{l}110 \\
110 \\
110 \\
110 \\
110\end{array}$ & $\begin{array}{l}198 \\
189 \\
184 \\
180 \\
166 \\
\end{array}$ & $\begin{array}{l}166 \\
166 \\
171 \\
162 \\
166\end{array}$ & $\begin{array}{r}* 593 \\
401 \\
327 \\
327 \\
333\end{array}$ & $\begin{array}{l}370 \\
358 \\
352 \\
333 \\
327\end{array}$ & $\begin{array}{l}283 \\
333 \\
352 \\
\frac{352}{345}\end{array}$ & $\begin{array}{l}445 \\
438 \\
426 \\
426 \\
426\end{array}$ & $\begin{array}{l}370 \\
370 \\
370 \\
358 \\
364\end{array}$ & $\begin{array}{l}271 \\
271 \\
277 \\
290 \\
290\end{array}$ \\
\hline $\begin{array}{l}26 \\
27 \\
28 \\
29 \\
30 \\
31\end{array}$ & $\begin{array}{l}247 \\
242 \\
242 \\
247 \\
236 \\
242\end{array}$ & $\begin{array}{c}302 \\
296 \\
296 \\
302 \\
296 \\
-\end{array}$ & $\begin{array}{r}10 \\
10 \\
10 \\
50 \\
103 \\
103\end{array}$ & $\begin{array}{l}\frac{150}{150} \\
150 \\
150 \\
150 \\
150\end{array}$ & $\begin{array}{c}175 \\
166 \\
166 \\
- \\
- \\
-\end{array}$ & $\begin{array}{l}162 \\
158 \\
158 \\
158 \\
562 \\
469\end{array}$ & $\begin{array}{c}327 \\
308 \\
302 \\
\frac{302}{302} \\
321 \\
-\end{array}$ & $\begin{array}{l}327 \\
308 \\
290 \\
203 \\
\frac{198}{296}\end{array}$ & $\begin{array}{c}345 \\
339 \\
100 \\
\frac{10}{10} \\
-\end{array}$ & $\begin{array}{r}420 \\
383 \\
321 \\
259 \\
166 \\
50\end{array}$ & $\begin{array}{r}358 \\
345 \\
345 \\
* 376 \\
339 \\
333\end{array}$ & $\begin{array}{r}162 \\
154 \\
* 150 \\
154 \\
162 \\
-\end{array}$ \\
\hline $\begin{array}{l}\text { Total } \\
\text { Mean } \\
\text { Ac-ft }\end{array}$ & $\begin{array}{r}8,003 \\
258 \\
15,870\end{array}$ & $\begin{array}{r}7,593 \\
253 \\
15,060\end{array}$ & $\begin{array}{r}5,953 \\
192 \\
11,810\end{array}$ & $\begin{array}{r}3,842 \\
124 \\
7,620 \\
\end{array}$ & $\begin{array}{r}5,704 \\
204 \\
11,310\end{array}$ & $\begin{array}{r}5,944 \\
192 \\
11,790 \\
\end{array}$ & $\begin{array}{r}11,255 \\
375 \\
22,320\end{array}$ & $\begin{array}{r}10,611 \\
342 \\
21,050\end{array}$ & $\begin{array}{r}5,361 \\
179 \\
10,630 \\
\end{array}$ & $\begin{array}{r}10,137 \\
327 \\
20,110\end{array}$ & $\begin{array}{r}9,938 \\
321 \\
19,710\end{array}$ & $\begin{array}{r}8,158 \\
272 \\
16,180\end{array}$ \\
\hline $\begin{array}{l}\text { Calend: } \\
\text { Water J }\end{array}$ & $\begin{array}{l}\text { ar } 195 \\
1953=5\end{array}$ & $\begin{array}{l}\text { Max } \\
\text { Max }\end{array}$ & $\begin{array}{l}445 \\
593\end{array}$ & $\begin{array}{ll}\text { Min } 10 \\
\text { Min } 10\end{array}$ & $\begin{array}{l}\text { Mean } \\
\text { Mean }\end{array}$ & $\begin{array}{l}278 \\
253\end{array}$ & $\begin{array}{l}A c-f t \\
A c-f t\end{array}$ & $\begin{array}{l}201,00 \\
183,50\end{array}$ & & & & \\
\hline
\end{tabular}

* Discharge measurement made on this day.

Nete. - No gage-hejghts Nov. 12-14, Dec. 22-29, Jan. 3 to Feb. 16, June 28 to July 3; discharge computed on basis of discharge measurements, canal operation or interpolated. 
HEIMAND RIVER GASIN

Seraj Canal at Sangin, Afghanistan

Discharge, in cubic feet per second, water year October 1954 to September 1955

\begin{tabular}{|c|c|c|c|c|c|c|c|c|c|c|c|c|}
\hline Dar & Octef & Nov. & Dec. & Jan. & Feb. & Mare & Apr. & May & June & Julv & Aug. & Septe \\
\hline $\begin{array}{l}1 \\
2 \\
3 \\
4 \\
5\end{array}$ & $\begin{array}{l}\frac{241}{284} \\
347 \\
347 \\
347\end{array}$ & $\begin{array}{l}347 \\
347 \\
347 \\
347 \\
347\end{array}$ & $\begin{array}{l}305 \\
29,8 \\
319 \\
340 \\
100\end{array}$ & $\begin{array}{l}253 \\
259 \\
253 \\
259 \\
259\end{array}$ & $\begin{array}{l}\frac{229}{229} \\
229 \\
229 \\
229\end{array}$ & $\begin{array}{l}\frac{90}{20} \\
\text { a) } 20 \\
2160 \\
\text { a) } 60\end{array}$ & $\begin{array}{l}421_{4} \\
421_{4} \\
42 d_{4} \\
421_{4} \\
409\end{array}$ & $\begin{array}{l}490 \\
490 \\
490 \\
490 \\
490\end{array}$ & $\begin{array}{l}\frac{474}{474} \\
474 \\
474 \\
466\end{array}$ & $\begin{array}{l}* 312 \\
8310 \\
8310 \\
2310 \\
2300\end{array}$ & $\begin{array}{l}\frac{277}{277} \\
277 \\
284 \\
284\end{array}$ & $\begin{array}{l}\frac{340}{362} \\
362 \\
362 \\
362\end{array}$ \\
\hline $\begin{array}{r}6 \\
7 \\
8 \\
9 \\
10\end{array}$ & $\begin{array}{l}347 \\
355 \\
362 \\
362 \\
370\end{array}$ & $\begin{array}{l}347 \\
347 \\
355 \\
355 \\
347\end{array}$ & $\begin{array}{r}0 \\
0 \\
0 \\
241 \\
474\end{array}$ & $\begin{array}{l}\frac{265}{265} \\
259 \\
253 \\
253\end{array}$ & $\begin{array}{l}229 \\
223 \\
223 \\
217 \\
217\end{array}$ & $\begin{array}{l}2220 \\
2220 \\
2280 \\
2280 \\
2350\end{array}$ & $\begin{array}{l}\frac{401}{441} \\
490 \\
490 \\
490\end{array}$ & $\begin{array}{l}490 \\
490 \\
490 \\
490 \\
490\end{array}$ & $\begin{array}{l}474 \\
441 \\
424 \\
424 \\
424\end{array}$ & $\begin{array}{r}2300 \\
8290 \\
2290 \\
a 280 \\
277\end{array}$ & $\begin{array}{l}291 \\
305 \\
305 \\
305 \\
305\end{array}$ & $\begin{array}{l}362 \\
362 \\
362 \\
362 \\
362\end{array}$ \\
\hline $\begin{array}{l}12 \\
12 \\
13 \\
14 \\
15\end{array}$ & $\begin{array}{l}370 \\
370 \\
362 \\
378 \\
\frac{370}{370}\end{array}$ & $\begin{array}{l}347 \\
355 \\
355 \\
355 \\
355\end{array}$ & $\begin{array}{l}474 \\
441 \\
441 \\
441 \\
424\end{array}$ & $\begin{array}{l}253 \\
253 \\
253 \\
247 \\
247\end{array}$ & $\begin{array}{l}217 \\
212 \\
212 \\
212 \\
202\end{array}$ & $\begin{array}{r}a 350 \\
a / 10 \\
409 \\
340 \\
370\end{array}$ & $\begin{array}{r}490 \\
499 \\
482 \\
* 466 \\
474\end{array}$ & $\begin{array}{l}490 \\
490 \\
490 \\
490 \\
490\end{array}$ & $\begin{array}{r}441 \\
441 \\
424 \\
* 416 \\
409\end{array}$ & $\begin{array}{l}\frac{271}{277} \\
277 \\
277 \\
284\end{array}$ & $\begin{array}{l}305 \\
305 \\
305 \\
298 \\
298\end{array}$ & $\begin{array}{l}362 \\
362 \\
362 \\
362 \\
362\end{array}$ \\
\hline $\begin{array}{l}16 \\
17 \\
18 \\
19 \\
20\end{array}$ & $\begin{array}{l}370 \\
362 \\
362 \\
347 \\
347\end{array}$ & $\begin{array}{l}\frac{362}{362} \\
362 \\
362 \\
347\end{array}$ & $\begin{array}{l}424 \\
409 \\
393 \\
167 \\
154\end{array}$ & $\begin{array}{l}247 \\
247 \\
247 \\
247 \\
241\end{array}$ & $\begin{array}{l}197 \\
192 \\
187 \\
162 \\
159\end{array}$ & $\begin{array}{l}449 \\
291 \\
235 \\
217 \\
207\end{array}$ & $\begin{array}{l}490 \\
578 \\
524 \\
482 \\
490\end{array}$ & $\begin{array}{l}490 \\
466 \\
466 \\
457 \\
466\end{array}$ & $\begin{array}{r}393 \\
362 \\
355 \\
a 360 \\
a 360\end{array}$ & $\begin{array}{l}298 \\
326 \\
333 \\
347 \\
401\end{array}$ & $\begin{array}{l}298 \\
291 \\
284 \\
291 \\
291\end{array}$ & $\begin{array}{l}362 \\
362 \\
362 \\
362 \\
362\end{array}$ \\
\hline $\begin{array}{l}21 \\
22 \\
23 \\
24 \\
25\end{array}$ & $\begin{array}{l}347 \\
347 \\
347 \\
347 \\
347\end{array}$ & $\begin{array}{l}305 \\
305 \\
305 \\
305 \\
305\end{array}$ & $\begin{array}{l}141 \\
138 \\
138 \\
347 \\
596\end{array}$ & $\begin{array}{l}241 \\
241 \\
241 \\
241 \\
241\end{array}$ & $\begin{array}{r}154 \\
150 \\
138 \\
56 \\
\frac{60}{60}\end{array}$ & $\begin{array}{l}207 \\
333 \\
490 \\
490 \\
490\end{array}$ & $\begin{array}{l}490 \\
490 \\
490 \\
490 \\
490\end{array}$ & $\begin{array}{l}466 \\
466 \\
466 \\
474 \\
457\end{array}$ & $\begin{array}{l}a 350 \\
a 350 \\
a 340 \\
a 340 \\
a 330\end{array}$ & $\begin{array}{l}\frac{424}{424} \\
424 \\
424 \\
401\end{array}$ & $\begin{array}{l}298 \\
305 \\
305 \\
305 \\
305\end{array}$ & $\begin{array}{l}362 \\
362 \\
362 \\
362 \\
362\end{array}$ \\
\hline $\begin{array}{l}26 \\
27 \\
28 \\
29 \\
30 \\
31\end{array}$ & $\begin{array}{r}333 \\
333 \\
* 347 \\
347 \\
347 \\
347\end{array}$ & $\begin{array}{r}305 \\
298 \\
* 305 \\
\frac{291}{298} \\
-\end{array}$ & $\begin{array}{r}5 \% 6 \\
424 \\
277 \\
259 \\
* 217 \\
253\end{array}$ & $\begin{array}{r}235 \\
235 \\
235 \\
235 \\
* 229 \\
229\end{array}$ & $\begin{array}{r}60 \\
60 \\
* 64 \\
- \\
- \\
-\end{array}$ & $\begin{array}{l}432 \\
432 \\
441 \\
552 \\
424 \\
424\end{array}$ & $\begin{array}{l}490 \\
490 \\
490 \\
490 \\
490 \\
-\end{array}$ & $\begin{array}{l}457 \\
457 \\
466 \\
474 \\
474 \\
474\end{array}$ & $\begin{array}{c}8330 \\
2330 \\
8330 \\
8320 \\
320 \\
-\end{array}$ & $\begin{array}{l}401 \\
370 \\
340 \\
333 \\
305 \\
277\end{array}$ & $\begin{array}{r}305 \\
* 347 \\
319 \\
326 \\
333 \\
333\end{array}$ & $\begin{array}{r}452 \\
452 \\
2430 \\
2430 \\
2430 \\
-\end{array}$ \\
\hline $\begin{array}{l}\text { Total } \\
\text { Mean } \\
\text { Ac-ft }\end{array}$ & $\begin{array}{r}10,789 \\
348 \\
21,400\end{array}$ & $\begin{array}{r}10,070 \\
336 \\
19,970 \\
\end{array}$ & $\begin{array}{r}9,255 \\
295 \\
18,660\end{array}$ & $\begin{array}{r}7,651 \\
247 \\
15,180 \\
\end{array}$ & $\begin{array}{r}4,948 \\
177 \\
9,810\end{array}$ & $\begin{array}{r}9,993 \\
322 \\
19,820 \\
\end{array}$ & $\begin{array}{r}14,292 \\
476 \\
28,350\end{array}$ & $\begin{array}{r}14,826 \\
478 \\
29,400\end{array}$ & $\begin{array}{r}11,850 \\
395 \\
23,500\end{array}$ & $\begin{array}{r}10,196 \\
329 \\
20,220\end{array}$ & $\begin{array}{r}9,357 \\
302 \\
18,560\end{array}$ & $\begin{array}{r}11,182 \\
383 \\
22,180\end{array}$ \\
\hline
\end{tabular}

Calendar year 1954: Max 596 Min 0 Mean 277 Ac-ft 200,300 Water year 1954-55: Max 596 Min 0 Mean 34l Ac-ft 246,800

* Discharge measurement made on this daj.

* Discharge measurement meb. 25-27, Mar. 1-12, June 19-30, Julj 2-9, Sept. 28m30; discharge computed

on basis of discharge measurements and interpolated gage heights. 
HETMATD RIVER BASTII

Saraf Cmal at Sangin, Afghantstan

Discharge In cubto feet por second, water year october 1955 to September 1956

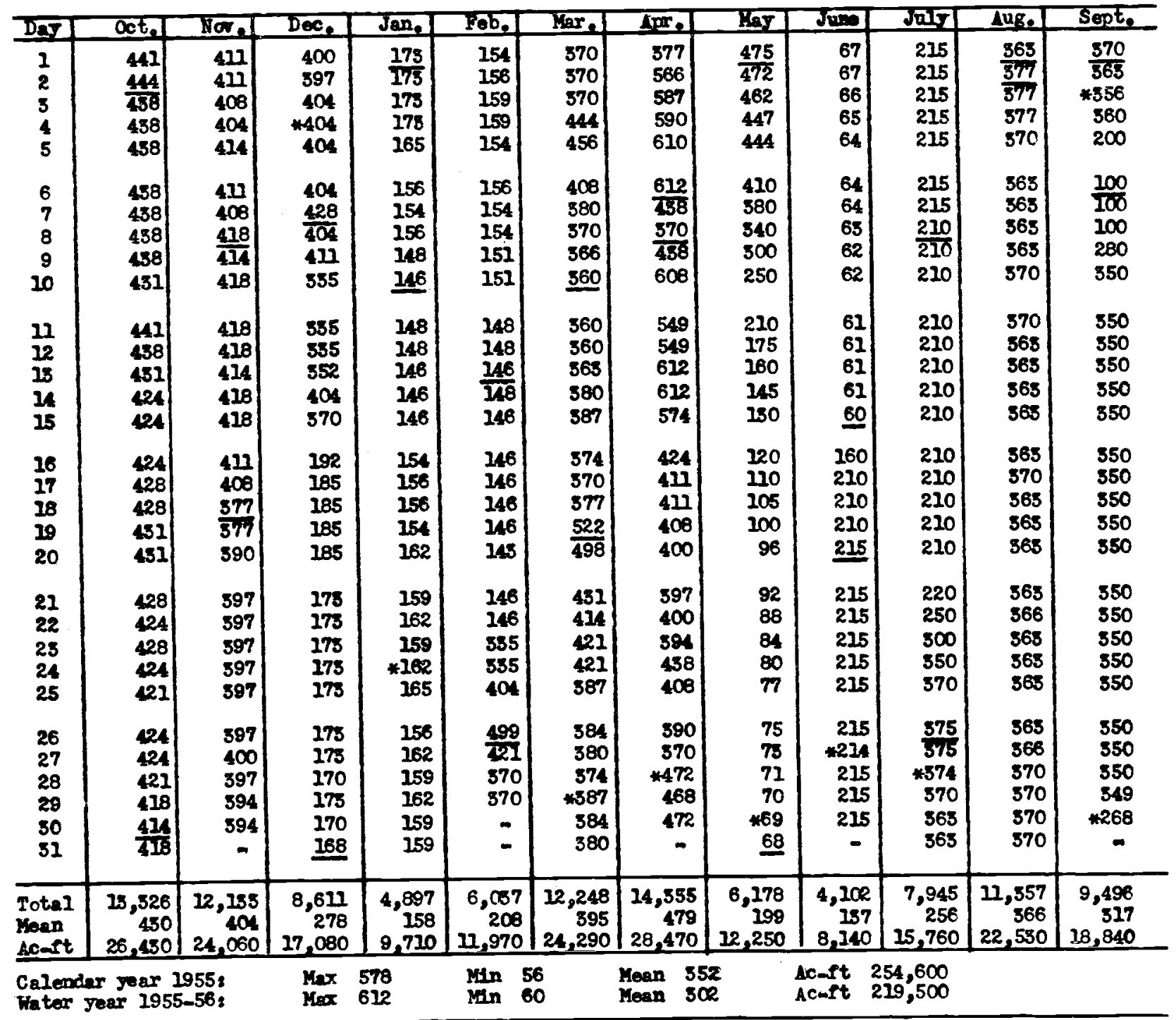

* Dischare measurement made on this day.

Note.-No gage-helghts May 6 to July 29, Sept. 5-28; discharge estimated on basis of discharge measurements and records for Helmand River belom Kajakai Reservoir. 
HEIMAND RIVER BASIN

Seraf Crnal at Sangin, Afghanistan

Discharge, in cubic feet per second, water year October 1956 to September 1957

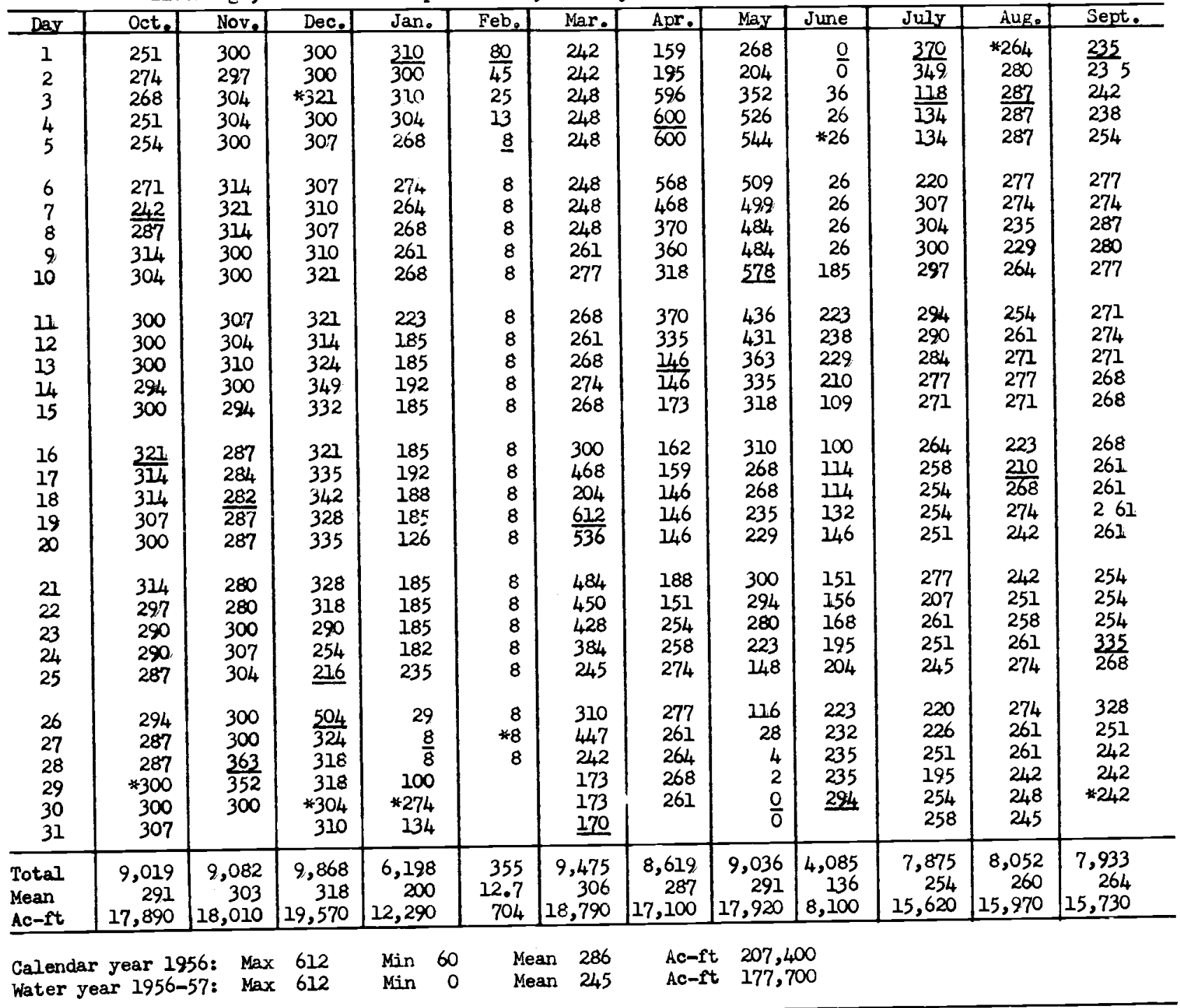

\section{* Discharge measurement mede on this day}

* Discharge measurement ma records for Helmand River below Kajakai Reservoir. 
HEIMAND RIVER BASIN

Sera Canal at Sangin, Afghanistan

Discharge, in cubic feet per second, water year October 1957 to september 1958

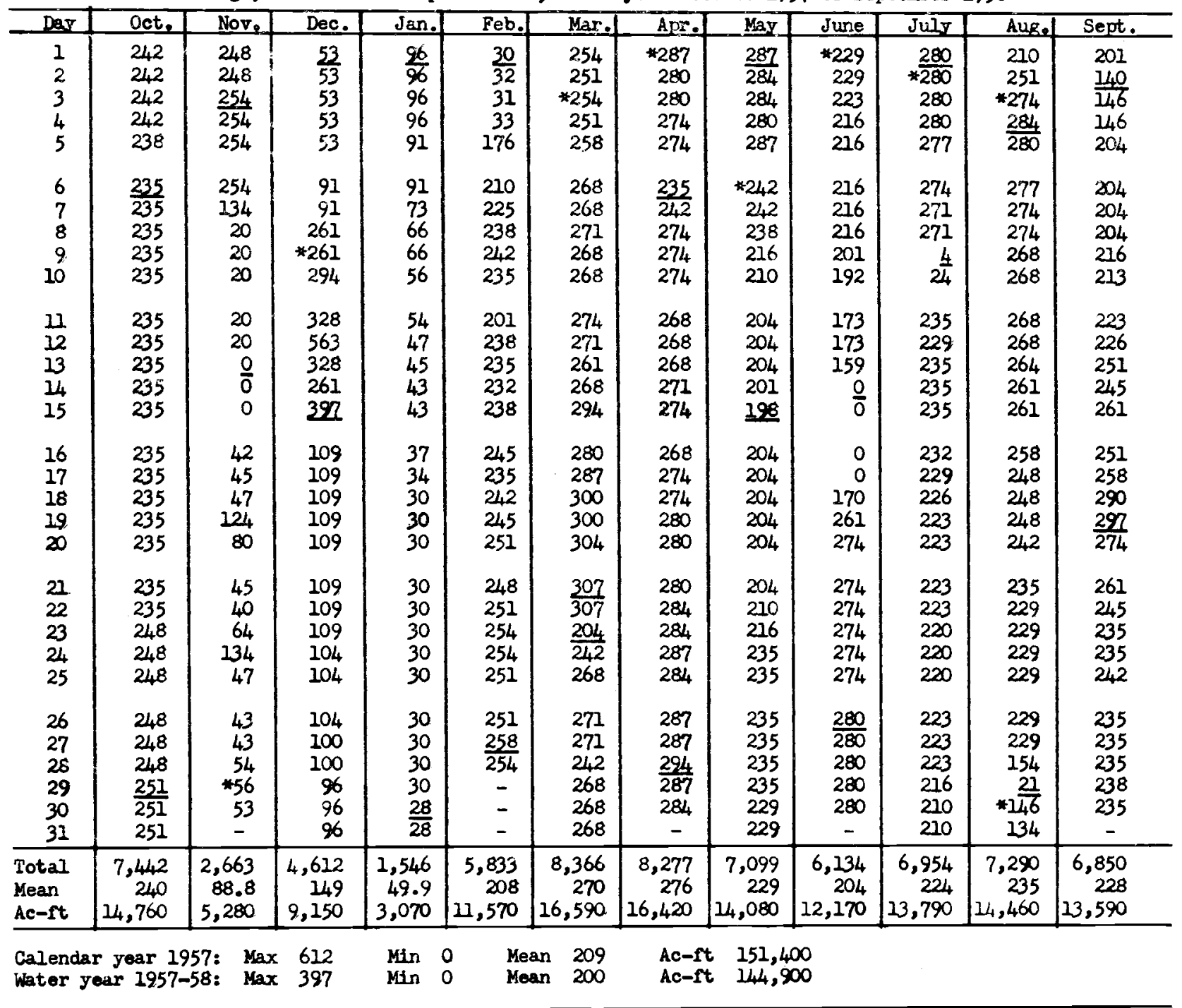

* Discharge measurement made on this day. 
HEIMAND RIVER BASIN

Seraj Canal at Sangin, Afghanistan

Discharge, in cubic feet per second, water year October 1958 to September 1959

\begin{tabular}{|c|c|c|c|c|c|c|c|c|c|c|c|c|}
\hline Dar & Oct. & Nove & Dec. & Jan. & Feb. & Mar. & Apr. & May & June & July & Aug. & Sept. \\
\hline $\begin{array}{l}1 \\
2 \\
3 \\
4 \\
5\end{array}$ & $\begin{array}{l}235 \\
238 \\
232 \\
235 \\
235\end{array}$ & $\begin{array}{l}\frac{238}{229} \\
226 \\
238 \\
235\end{array}$ & $\begin{array}{l}\frac{232}{198} \\
223 \\
204 \\
198\end{array}$ & $\begin{array}{l}129 \\
151 \\
168 \\
168 \\
173 \\
\end{array}$ & $\begin{array}{l}140 \\
134 \\
134 \\
132 \\
129\end{array}$ & $\begin{array}{l}173 \\
213 \\
519 \\
1146 \\
146\end{array}$ & $\begin{array}{l}\frac{162}{216} \\
226 \\
245 \\
254\end{array}$ & $\begin{array}{l}287 \\
274 \\
274 \\
280 \\
280\end{array}$ & $\begin{array}{l}\frac{280}{277} \\
274 \\
274 \\
268\end{array}$ & $\begin{array}{l}271 \\
264 \\
261 \\
268 \\
268\end{array}$ & $\begin{array}{l}\frac{216}{223} \\
223 \\
242 \\
245\end{array}$ & $\begin{array}{l}268 \\
277 \\
287 \\
287 \\
280\end{array}$ \\
\hline $\begin{array}{r}6 \\
7 \\
8 \\
9 \\
10\end{array}$ & $\begin{array}{l}232 \\
226 \\
223 \\
223 \\
220\end{array}$ & $\begin{array}{l}235 \\
232 \\
229 \\
229 \\
223\end{array}$ & $\begin{array}{r}* 91 \\
91 \\
86 \\
86 \\
82\end{array}$ & $\begin{array}{l}173 . \\
173 \\
162 \\
162 \\
162\end{array}$ & $\begin{array}{r}114 \\
96 \\
96 \\
145 \\
146\end{array}$ & $\begin{array}{l}134 \\
129 \\
129 \\
185 \\
118\end{array}$ & $\begin{array}{l}268 \\
268 \\
261 \\
251 \\
254\end{array}$ & $\begin{array}{l}280 \\
274 \\
268 \\
268 \\
268\end{array}$ & $\begin{array}{l}268 \\
268 \\
268 \\
268 \\
280\end{array}$ & $\begin{array}{l}268 \\
274 \\
268 \\
268 \\
274\end{array}$ & $\begin{array}{l}254 \\
248 \\
242 \\
242 \\
242\end{array}$ & $\begin{array}{l}280 \\
261 \\
261 \\
274 \\
274\end{array}$ \\
\hline $\begin{array}{l}11 \\
12 \\
13 \\
14 \\
15\end{array}$ & $\begin{array}{l}216 \\
210 \\
210 \\
210 \\
210\end{array}$ & $\begin{array}{r}223 \\
223 \\
226 \\
151 \\
52\end{array}$ & $\begin{array}{r}104 \\
129 \\
185 \\
356 \\
50\end{array}$ & $\begin{array}{l}156 \\
156 \\
156 \\
162 \\
140\end{array}$ & $\begin{array}{l}146 \\
146 \\
146 \\
146 \\
151\end{array}$ & $\begin{array}{r}118 \\
114 \\
109 \\
129 \\
36\end{array}$ & $\begin{array}{l}251 \\
248 \\
251 \\
268 \\
280\end{array}$ & $\begin{array}{l}264 \\
261 \\
268 \\
271 \\
274\end{array}$ & $\begin{array}{l}280 \\
261 \\
\frac{254}{254} \\
254\end{array}$ & $\begin{array}{l}274 \\
274 \\
280 \\
280 \\
280\end{array}$ & $\begin{array}{l}251 \\
261 \\
261 \\
254 \\
251\end{array}$ & $\begin{array}{l}274 \\
287 \\
287 \\
300 \\
314\end{array}$ \\
\hline $\begin{array}{l}16 \\
17 \\
18 \\
19 \\
20\end{array}$ & $\begin{array}{l}210 \\
207 \\
204 \\
204 \\
204\end{array}$ & $\begin{array}{r}13 \\
7 \\
0 \\
0 \\
0\end{array}$ & $\begin{array}{l}56 \\
78 \\
47 \\
82 \\
82\end{array}$ & $\begin{array}{l}146 \\
146 \\
129 \\
\frac{118}{129}\end{array}$ & $\begin{array}{l}156 \\
162 \\
165 \\
\overline{156} \\
134\end{array}$ & $\begin{array}{r}\frac{18}{40} \\
40 \\
148 \\
173\end{array}$ & $\begin{array}{l}280 \\
268 \\
284 \\
294 \\
294\end{array}$ & $\begin{array}{l}274 \\
274 \\
268 \\
268 \\
274\end{array}$ & $\begin{array}{l}254 \\
254 \\
254 \\
261 \\
261\end{array}$ & $\begin{array}{l}\frac{287}{287} \\
287 \\
287 \\
287\end{array}$ & $\begin{array}{l}274 \\
274 \\
274 \\
274 \\
280 \\
\end{array}$ & $\begin{array}{l}314 \\
307 \\
300 \\
294 \\
287\end{array}$ \\
\hline $\begin{array}{l}21 \\
22 \\
23 \\
24 \\
25\end{array}$ & $\begin{array}{l}\frac{132}{258} \\
\frac{204}{201} \\
204\end{array}$ & $\begin{array}{l}0 \\
0 \\
0 \\
0 \\
0\end{array}$ & $\begin{array}{l}82 \\
78 \\
78 \\
73 \\
78\end{array}$ & $\begin{array}{l}129 \\
124 \\
124 \\
124 \\
129\end{array}$ & $\begin{array}{r}129 \\
114 \\
82 \\
37 \\
30\end{array}$ & $\begin{array}{l}185 \\
185 \\
185 \\
192 \\
198\end{array}$ & $\begin{array}{l}300 \\
304 \\
300 \\
307 \\
\frac{307}{307}\end{array}$ & $\begin{array}{l}274 \\
277 \\
284 \\
287 \\
287\end{array}$ & $\begin{array}{l}261 \\
268 \\
268 \\
268 \\
271\end{array}$ & $\begin{array}{l}287 \\
287 \\
287 \\
287 \\
287\end{array}$ & $\begin{array}{l}280 \\
280 \\
280 \\
280 \\
280\end{array}$ & $\begin{array}{l}287 \\
287 \\
280 \\
280 \\
277\end{array}$ \\
\hline $\begin{array}{l}26 \\
27 \\
28 \\
29 \\
30 \\
31\end{array}$ & $\begin{array}{r}198 \\
198 \\
198 \\
204 \\
* 213 \\
235\end{array}$ & $\begin{array}{l}0 \\
0 \\
0 \\
0 \\
0 \\
-\end{array}$ & $\begin{array}{l}78 \\
78 \\
78 \\
78 \\
76 \\
73\end{array}$ & $\begin{array}{r}129 \\
129 \\
129 \\
134 \\
140 \\
* 140\end{array}$ & $\begin{array}{r}\frac{20}{20} \\
* 140 \\
- \\
- \\
-\end{array}$ & $\begin{array}{r}198 \\
201 \\
195 \\
198 \\
192 \\
* 198\end{array}$ & $\begin{array}{r}304 \\
294 \\
294 \\
* 294 \\
294 \\
-\end{array}$ & $\begin{array}{l}287 \\
287 \\
\frac{294}{294} \\
294 \\
294\end{array}$ & $\begin{array}{c}268 \\
268 \\
271 \\
268 \\
268 \\
-\end{array}$ & $\begin{array}{l}287 \\
280 \\
280 \\
280 \\
280 \\
280\end{array}$ & $\begin{array}{r}280 \\
280 \\
280 \\
277 \\
* 274 \\
274\end{array}$ & $\begin{array}{r}274 \\
245 \\
70 \\
35 \\
36 \\
-\end{array}$ \\
\hline $\begin{array}{l}\text { Total } \\
\text { Mean } \\
\text { Ac-It }\end{array}$ & $\begin{array}{r}6,629 \\
214 \\
13,150\end{array}$ & $\begin{array}{r}3,209 \\
107 \\
6,360\end{array}$ & $\begin{array}{r}3,510 \\
113 \\
6,960\end{array}$ & $\begin{array}{r}4,490 \\
145 \\
8,910\end{array}$ & $\begin{array}{r}3,347 \\
120 \\
6,640\end{array}$ & $\begin{array}{r}4,944 \\
159 \\
9,810\end{array}$ & $\begin{array}{r}8,121 \\
271 \\
16,110\end{array}$ & $\begin{array}{r}8,608 \\
278 \\
17,070\end{array}$ & $\begin{array}{r}7,991 \\
266 \\
15,850\end{array}$ & $\begin{array}{r}8,629 \\
278 \\
17,120\end{array}$ & $\begin{array}{r}8,096 \\
261 \\
16,060\end{array}$ & $\begin{array}{r}7,784 \\
259 \\
15,440\end{array}$ \\
\hline
\end{tabular}

Calendar year 1958: Max 307 Min 0 Mean 196 Ac-ft 142,200 Water year 1958-59: Max 519 Min O Mean 206 Ac-ft 149,500

* Discharge measurement made on this day. 
HETMAND RIVER BASIN

Seraj Canal at Sangin, Afghanistan

Discharge, in cubic feet per second, water year October 1959 to September 1960

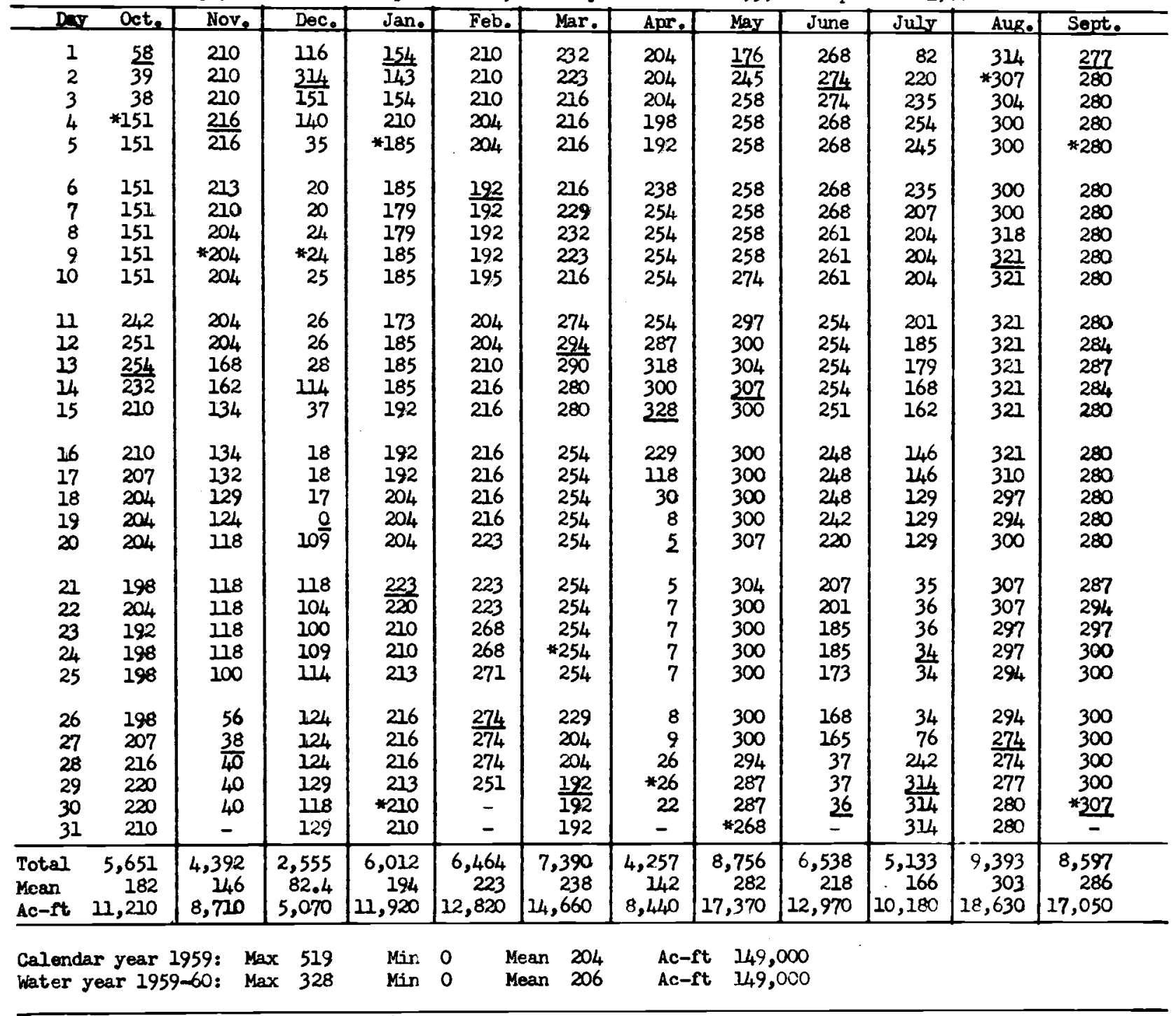

* Discharge measurement made on this day. 
HETLAND RIVER BASTN

Lusa Qala River at Musa Qala, Afghanistan

Lecation.--Lat $32^{\circ} 201 \mathrm{~N}$, long $64^{\circ} 46^{\circ} \mathrm{E}$, on left bank at south end of village of Musa Q2ala, about 26 lilometers upotream from Helmand River, and 60 kilometers north of Girishk.

Drainage ares. $-1,450 \mathrm{sq} \mathrm{mil}$, approxinately.

Aecords available.-April 1952 to September 1960.

Gare- Staff gage read twice daily. Altitude of gage is about 970 meters (from comparison with Helmand River below Kajakai Dam elevation 960 meters).

Average discharge.-8 years, 416 cfs $(301,200$ acre-ft per year).

Extremes.-Xaximum and minimum discharges for the water years 1953-60 are given in the following table.

\begin{tabular}{|c|c|c|c|c|c|}
\hline \multirow{2}{*}{$\begin{array}{l}\text { Water } \\
\text { Iear }\end{array}$} & \multicolumn{3}{|c|}{ Yacimum } & \multicolumn{2}{|l|}{ Kinimm Daily } \\
\hline & Date & $\begin{array}{c}\text { Gage Height } \\
\text { (matera) }\end{array}$ & $\begin{array}{c}\text { Discharge } \\
\text { (efr) }\end{array}$ & Dater & $\begin{array}{c}\text { Discharge } \\
\text { (afe) }\end{array}$ \\
\hline $\begin{array}{l}1953 \\
1954 \\
1955 \\
1956 \\
1957 \\
1958 \\
1959 \\
1960\end{array}$ & $\begin{array}{l}\text { Feb. 14, } 1953 \\
\text { Apr. 5, } 1954 \\
\text { Jar. 14, } 1955 \\
\text { Dec. 13, } 1955 \\
\text { Yar. 17, } 1957 \\
\text { Dec. 11, } 1957 \\
\text { Kar. 3, } 1959 \\
\text { Apr. 18, } 1960\end{array}$ & $\begin{array}{c}-\overline{1.56} \\
2.53 \\
1.78 \\
3.60 \\
- \\
2.93\end{array}$ & $\begin{array}{r}* 6,620 \\
7,600 \\
38,000 \\
16,200 \\
871,000 \\
* 2,450 \\
* 3,500 \\
* 4,500\end{array}$ & $\begin{array}{l}\text { Aug. } 25 \text { to Sept. } 30,1953 \\
\text { Aug. } 20-22,24-28,1954 \\
\text { Sept. } 21-27,1955 \\
\text { Nov. } 16-30,1955 \\
\text { Oct. } 1-10,1956 \\
\text { Aug. } 5,1958 \\
\text { Nov. 24, } 1958 \\
\text { Oct. } 1,2,1959\end{array}$ & $\begin{array}{r}0 \\
2 \\
2 \\
2 \\
9 \\
6 \\
4 \\
20\end{array}$ \\
\hline
\end{tabular}

* Mean daily.

- Slopo-area measureant.

Remarks.-Records poor prior to Kay 1954, fair thereafter.

Kany ans diversions for irrigation upstream and downtream. 
HMYAND RIVER BASD

Musa Qula River at Musa Qala, Afghanistan

Discharge, in cubio feet per second, period April to September 1952

\begin{tabular}{|c|c|c|c|c|c|c|c|c|c|c|c|c|}
\hline Date & oct. & Nor. & Dec. & Jan. & Peb. & Yar. & Apr. & May & June & July & Aug. & Sept. \\
\hline $\begin{array}{l}1 \\
2 \\
3 \\
4 \\
5\end{array}$ & & & & & & & $\begin{array}{l}- \\
- \\
- \\
-\end{array}$ & $\begin{array}{l}108 \\
100 \\
195 \\
\frac{243}{195}\end{array}$ & $\begin{array}{l}\frac{34}{34} \\
27 \\
27 \\
21\end{array}$ & $\begin{array}{r}10 \\
8 \\
8 \\
8 \\
12 \\
\end{array}$ & $\begin{array}{l}\frac{8}{8} \\
8 \\
6 \\
6\end{array}$ & $\begin{array}{l}\frac{1}{1} \\
\frac{1}{1} \\
1\end{array}$ \\
\hline $\begin{array}{r}6 \\
7 \\
8 \\
9 \\
10\end{array}$ & & & & & & & $\begin{array}{l}= \\
= \\
=\end{array}$ & $\begin{array}{r}173 \\
132 \\
116 \\
100 \\
91\end{array}$ & $\begin{array}{l}21 \\
21 \\
21 \\
18 \\
18\end{array}$ & $\begin{array}{r}12 \\
12 \\
10 \\
8 \\
8\end{array}$ & $\begin{array}{l}6 \\
4 \\
2 \\
2 \\
2\end{array}$ & $\begin{array}{l}1 \\
1 \\
1 \\
1 \\
1\end{array}$ \\
\hline $\begin{array}{l}11 \\
12 \\
13 \\
14 \\
15\end{array}$ & & & & & & & $\begin{array}{l}= \\
= \\
=\end{array}$ & $\begin{array}{l}83 \\
68 \\
54 \\
43 \\
39\end{array}$ & $\begin{array}{l}18 \\
16 \\
16 \\
16 \\
16\end{array}$ & $\begin{array}{l}8 \\
8 \\
8 \\
8 \\
8\end{array}$ & $\begin{array}{l}2 \\
2 \\
2 \\
2 \\
2\end{array}$ & $\begin{array}{l}1 \\
1 \\
2 \\
1 \\
1\end{array}$ \\
\hline $\begin{array}{l}16 \\
17 \\
18 \\
19 \\
20\end{array}$ & & & & & & & $\begin{array}{l}= \\
=\end{array}$ & $\begin{array}{l}34 \\
34 \\
39 \\
39 \\
34\end{array}$ & $\begin{array}{l}14 \\
14 \\
14 \\
12 \\
12\end{array}$ & $\begin{array}{l}8 \\
8 \\
8 \\
8 \\
8\end{array}$ & $\begin{array}{l}2 \\
2 \\
2 \\
2 \\
1\end{array}$ & $\begin{array}{l}1 \\
2 \\
2 \\
2 \\
2\end{array}$ \\
\hline $\begin{array}{l}22 \\
22 \\
23 \\
24 \\
25\end{array}$ & & & & & & & $\begin{array}{l}219 \\
219 \\
195 \\
195 \\
173\end{array}$ & $\begin{array}{l}34 \\
34 \\
32 \\
34 \\
34\end{array}$ & $\begin{array}{l}12 \\
12 \\
12 \\
12 \\
12\end{array}$ & $\begin{array}{l}8 \\
8 \\
8 \\
8 \\
8\end{array}$ & $\begin{array}{l}1 \\
1 \\
1 \\
1 \\
0\end{array}$ & $\begin{array}{l}2 \\
2 \\
2 \\
4 \\
4\end{array}$ \\
\hline $\begin{array}{l}26 \\
27 \\
28 \\
29 \\
30 \\
31\end{array}$ & & & & & & & $\begin{array}{c}151 \\
110 \\
132 \\
124 \\
116 \\
-\end{array}$ & $\begin{array}{l}31 \\
31 \\
34 \\
39 \\
34 \\
34\end{array}$ & $\begin{array}{l}12 \\
12 \\
12 \\
12 \\
10 \\
-\end{array}$ & $\begin{array}{l}8 \\
8 \\
8 \\
8 \\
8 \\
8\end{array}$ & $\begin{array}{l}0 \\
0 \\
0 \\
0 \\
0 \\
0\end{array}$ & $\begin{array}{l}3 \\
3 \\
2 \\
2 \\
2 \\
-\end{array}$ \\
\hline $\begin{array}{l}\text { Total } \\
\text { Moan } \\
\text { Acent }\end{array}$ & & & & & & & $\begin{array}{l}- \\
-\end{array}$ & $\begin{array}{r}2,290 \\
73.9 \\
4,540\end{array}$ & $\begin{array}{r}508 \\
16.9 \\
1,010\end{array}$ & $\begin{array}{l}264 \\
8.5 \\
512\end{array}$ & $\begin{array}{r}75 \\
2.4 \\
148\end{array}$ & $\begin{array}{r}51 \\
1.7 \\
101\end{array}$ \\
\hline $\begin{array}{l}\text { Calenda } \\
\text { Water y }\end{array}$ & & $:$ & $\begin{array}{l}\text { Max } \\
\text { Max }\end{array}$ & & Min & & $\begin{array}{l}\text { Moan } \\
\text { Kean }\end{array}$ & & $\begin{array}{l}c-r t \\
c-f t\end{array}$ & & & \\
\hline
\end{tabular}


HEIMAND RIVER BASIN

Musa Qala River at Yusa Qala, Afghanistan

Discharge, in cubic feet per second, water year October 1952 to September 1953

\begin{tabular}{|c|c|c|c|c|c|c|c|c|c|c|c|c|}
\hline Day & Oct. & Nor. & Dec. & Jan. & Feb. & Mar. & Apr & May & June & July & Aug. & Sept. \\
\hline $\begin{array}{l}1 \\
2 \\
3 \\
4 \\
5\end{array}$ & $\begin{array}{l}\frac{2}{2} \\
2 \\
2 \\
2\end{array}$ & $\begin{array}{l}\frac{2}{2} \\
2 \\
2 \\
2\end{array}$ & $\begin{array}{l}\frac{6}{6} \\
6 \\
6 \\
6\end{array}$ & $\begin{array}{r}\frac{8}{8} \\
8 \\
8 \\
10\end{array}$ & $\begin{array}{l}\frac{12}{12} \\
16 \\
27 \\
43\end{array}$ & $\begin{array}{l}\frac{132}{151} \\
162 \\
219 \\
243\end{array}$ & $\begin{array}{l}243 \\
219 \\
195 \\
184 \\
173\end{array}$ & $\begin{array}{l}34 \\
34 \\
27 \\
27 \\
27\end{array}$ & $\begin{array}{l}116 \\
162 \\
243 \\
315 \\
402\end{array}$ & $\begin{array}{l}\frac{16}{16} \\
16 \\
16 \\
16\end{array}$ & $\begin{array}{l}\frac{8}{6} \\
6 \\
6 \\
6\end{array}$ & $\begin{array}{l}\frac{0}{1} \\
1 \\
1 \\
1\end{array}$ \\
\hline $\begin{array}{r}6 \\
7 \\
8 \\
9 \\
10\end{array}$ & $\begin{array}{l}2 \\
2 \\
4 \\
8 \\
8\end{array}$ & $\begin{array}{l}2 \\
2 \\
2 \\
2 \\
2\end{array}$ & $\begin{array}{l}6 \\
6 \\
6 \\
8 \\
8\end{array}$ & $\begin{array}{l}12 \\
12 \\
12 \\
12 \\
16\end{array}$ & $\begin{array}{r}83 \\
116 \\
184 \\
267 \\
640\end{array}$ & $\begin{array}{l}279 \\
344 \\
431 \\
402 \\
373\end{array}$ & $\begin{array}{l}151 \\
132 \\
116 \\
195 \\
373\end{array}$ & $\begin{array}{l}27 \\
27 \\
27 \\
\frac{21}{21}\end{array}$ & $\begin{array}{l}\frac{446}{350} \\
267 \\
200 \\
116\end{array}$ & $\begin{array}{l}16 \\
16 \\
16 \\
16 \\
16\end{array}$ & $\begin{array}{l}6 \\
6 \\
6 \\
6 \\
6\end{array}$ & $\begin{array}{l}1 \\
1 \\
2 \\
2 \\
2\end{array}$ \\
\hline $\begin{array}{l}11 \\
12 \\
13 \\
14 \\
15\end{array}$ & $\begin{array}{l}2 \\
2 \\
2 \\
2 \\
2\end{array}$ & $\begin{array}{l}2 \\
2 \\
2 \\
2 \\
2\end{array}$ & $\begin{array}{l}8 \\
7 \\
8 \\
8 \\
8\end{array}$ & $\begin{array}{l}16 \\
16 \\
21 \\
\frac{27}{21}\end{array}$ & $\begin{array}{r}1,400 \\
2,460 \\
3,210 \\
6,620 \\
d 2,000\end{array}$ & $\begin{array}{l}344 \\
315 \\
291 \\
267 \\
243\end{array}$ & $\begin{array}{r}762 \\
1,400 \\
2,130 \\
2,810 \\
3,830 \\
\end{array}$ & $\begin{array}{l}21 \\
21 \\
21 \\
21 \\
21\end{array}$ & $\begin{array}{r}100 \\
83 \\
68 \\
54 \\
43\end{array}$ & $\begin{array}{l}16 \\
16 \\
16 \\
16 \\
16\end{array}$ & $\begin{array}{r}6 \\
4 \\
4 \\
4 \\
* 4\end{array}$ & $\begin{array}{l}2 \\
2 \\
2 \\
\frac{4}{2}\end{array}$ \\
\hline $\begin{array}{l}16 \\
17 \\
18 \\
19 \\
20\end{array}$ & $\begin{array}{l}2 \\
2 \\
2 \\
2 \\
2\end{array}$ & $\begin{array}{l}2 \\
2 \\
2 \\
2 \\
2\end{array}$ & $\begin{array}{l}\frac{12}{12} \\
12 \\
12 \\
12\end{array}$ & $\begin{array}{l}16 \\
14 \\
14 \\
12 \\
12\end{array}$ & $\begin{array}{r}\mathrm{dl}, 000 \\
\mathrm{~d} 500 \\
\mathrm{~d} 300 \\
219 \\
195\end{array}$ & $\begin{array}{l}219 \\
195 \\
195 \\
195 \\
193\end{array}$ & $\begin{array}{r}1,000 \\
d 500 \\
267 \\
d 200 \\
116\end{array}$ & $\begin{array}{l}21 \\
21 \\
21 \\
21 \\
21\end{array}$ & $\begin{array}{l}34 \\
27 \\
21 \\
21 \\
21\end{array}$ & $\begin{array}{l}16 \\
16 \\
16 \\
16 \\
16\end{array}$ & $\begin{array}{l}4 \\
4 \\
2 \\
2 \\
2\end{array}$ & $\begin{array}{l}3 \\
3 \\
3 \\
3 \\
3\end{array}$ \\
\hline $\begin{array}{l}21 \\
22 \\
23 \\
24 \\
25\end{array}$ & $\begin{array}{l}2 \\
2 \\
2 \\
2 \\
3\end{array}$ & $\begin{array}{l}2 \\
2 \\
2 \\
2 \\
2\end{array}$ & $\begin{array}{r}12 \\
12 \\
12 \\
12 \\
8\end{array}$ & $\begin{array}{l}12 \\
12 \\
12 \\
12 \\
12\end{array}$ & $\begin{array}{l}173 \\
173 \\
151 \\
151 \\
151\end{array}$ & $\begin{array}{l}151 \\
132 \\
195 \\
243 \\
315\end{array}$ & $\begin{array}{r}100 \\
83 \\
68 \\
54 \\
54\end{array}$ & $\begin{array}{l}21 \\
21 \\
21 \\
21 \\
27\end{array}$ & $\begin{array}{l}21 \\
21 \\
16 \\
16 \\
16\end{array}$ & $\begin{array}{l}16 \\
16 \\
16 \\
16 \\
16\end{array}$ & $\begin{array}{l}1 \\
1 \\
1 \\
1 \\
0\end{array}$ & $\begin{array}{l}3 \\
3 \\
3 \\
3 \\
3\end{array}$ \\
\hline $\begin{array}{l}26 \\
27 \\
28 \\
29 \\
30 \\
31 \\
\end{array}$ & $\begin{array}{l}4 \\
4 \\
3 \\
2 \\
2 \\
2\end{array}$ & $\begin{array}{l}2 \\
4 \\
4 \\
5 \\
5 \\
-\end{array}$ & $\begin{array}{r}8 \\
8 \\
8 \\
8 \\
7 \\
10\end{array}$ & $\begin{array}{r}8 \\
8 \\
8 \\
8 \\
8 \\
12\end{array}$ & $\begin{array}{l}83 \\
83 \\
83 \\
- \\
- \\
- \\
\end{array}$ & $\begin{array}{l}431 \\
416 \\
344 \\
315 \\
291 \\
267\end{array}$ & $\begin{array}{l}43 \\
43 \\
43 \\
34 \\
34 \\
- \\
\end{array}$ & $\begin{array}{r}34 \\
43 \\
54 \\
68 \\
83 \\
100 \\
\end{array}$ & $\begin{array}{l}16 \\
16 \\
16 \\
16 \\
16 \\
-\end{array}$ & $\begin{array}{r}12 \\
12 \\
12 \\
12 \\
8 \\
8\end{array}$ & $\begin{array}{l}0 \\
0 \\
0 \\
0 \\
0 \\
0\end{array}$ & $\begin{array}{l}3 \\
3 \\
3 \\
3 \\
3 \\
-\end{array}$ \\
\hline $\begin{array}{l}\text { Total } \\
\text { Mean } \\
\text { Ac-ft } \\
\end{array}$ & $\begin{array}{r}82 \\
2.6 \\
162 \\
\end{array}$ & $\begin{array}{r}70 \\
2.3 \\
139 \\
\end{array}$ & $\begin{array}{l}268 \\
8.6 \\
532 \\
\end{array}$ & $\begin{array}{c}387 \\
12.5 \\
768\end{array}$ & $\begin{array}{r}20,352 \\
727 \\
40,370 \\
\end{array}$ & $\begin{array}{r}8,275 \\
265 \\
16,400 \\
\end{array}$ & $\begin{array}{r}15,552 \\
518 \\
30,850 \\
\end{array}$ & $\begin{array}{r}975 \\
31.4 \\
1,930 \\
\end{array}$ & $\begin{array}{r}3,259 \\
109 \\
6,460\end{array}$ & $\begin{array}{r}464 \\
15.0 \\
920\end{array}$ & $\begin{array}{l}102 \\
3.3 \\
202 \\
\end{array}$ & $\begin{array}{r}69 \\
2.3 \\
137 \\
\end{array}$ \\
\hline $\begin{array}{l}\text { Calen } \\
\text { Water }\end{array}$ & year & & $\begin{array}{l}\text { Max } \\
\operatorname{Max}\end{array}$ & $\overline{6} 20$ & $\operatorname{Min}_{\text {Min }} 0$ & $\begin{array}{l}\text { Mean } \\
\text { Mean }\end{array}$ & & $\begin{array}{l}c-f t \\
c-f t\end{array}$ & $\overline{870}$ & & & \\
\hline
\end{tabular}

* Discharge measurement made on this day.

d Doubtful gage-height record; discharge estimated on basis of recession curve or interpolated.

Note. -No gage-height record Sept. 16-30; discharge eatimated on basis of known low water conditions. 
HOMAND RIVER BASIN

Musa Qala River at Musa Qala, Afghanistan

Discharge, in cubic feet per second, water yoar October 1953 to September 1954

\begin{tabular}{|c|c|c|c|c|c|c|c|c|c|c|c|c|}
\hline Dar & oet. & Nov. & Dec. & Jan. & Feb. & Mar. & Apr. & May & June & July & Aug. & Sept。 \\
\hline $\begin{array}{l}1 \\
2 \\
3 \\
4 \\
5\end{array}$ & $\begin{array}{l}5 \\
5 \\
5 \\
5 \\
5\end{array}$ & $\begin{array}{l}10 \\
10 \\
10 \\
10 \\
10\end{array}$ & $\begin{array}{l}\frac{20}{20} \\
20 \\
20 \\
20\end{array}$ & $\begin{array}{l}\frac{500}{291} \\
231 \\
184 \\
151\end{array}$ & $\begin{array}{l}\frac{34}{34} \\
34 \\
34 \\
34\end{array}$ & $\begin{array}{l}303 \\
255 \\
243 \\
219 \\
195\end{array}$ & $\begin{array}{r}460 \\
1,400 \\
1,270 \\
5,170 \\
* 5,880 \\
\end{array}$ & $\begin{array}{r}400 \\
* 373 \\
358 \\
315 \\
303\end{array}$ & $\begin{array}{l}75 \\
75 \\
75 \\
75 \\
68\end{array}$ & $\begin{array}{l}\frac{18}{18} \\
18 \\
18 \\
18\end{array}$ & $\begin{array}{r}* 3 \\
3 \\
3 \\
3 \\
3\end{array}$ & $\begin{array}{l}\frac{2}{2} \\
2 \\
2 \\
2\end{array}$ \\
\hline $\begin{array}{r}6 \\
7 \\
8 \\
9 \\
10\end{array}$ & $\begin{array}{l}5 \\
5 \\
5 \\
5 \\
5\end{array}$ & $\begin{array}{l}10 \\
10 \\
10 \\
10 \\
10\end{array}$ & $\begin{array}{l}20 \\
20 \\
20 \\
20 \\
20\end{array}$ & $\begin{array}{r}108 \\
21 \\
43 \\
34 \\
31\end{array}$ & $\begin{array}{r}34 \\
34 \\
291 \\
2,650 \\
3,920 \\
\end{array}$ & $\begin{array}{l}184 \\
184 \\
184 \\
\frac{173}{416}\end{array}$ & $\begin{array}{l}1,670 \\
4,840 \\
5,170 \\
3,370 \\
2,730\end{array}$ & $\begin{array}{r}966 \\
830 \\
* 431 \\
402 \\
373\end{array}$ & $\begin{array}{l}60 \\
60 \\
60 \\
60 \\
60\end{array}$ & $\begin{array}{l}18 \\
18 \\
18 \\
18 \\
18\end{array}$ & $\begin{array}{l}\frac{2}{2} \\
3 \\
3 \\
3\end{array}$ & $\begin{array}{l}2 \\
2 \\
2 \\
2 \\
2\end{array}$ \\
\hline $\begin{array}{l}11 \\
12 \\
13 \\
14 \\
15\end{array}$ & $\begin{array}{l}5 \\
5 \\
5 \\
5 \\
5\end{array}$ & $\begin{array}{l}10 \\
10 \\
10 \\
10 \\
10\end{array}$ & $\begin{array}{l}30 \\
30 \\
30 \\
30 \\
30\end{array}$ & $\begin{array}{l}\frac{27}{27} \\
27 \\
27 \\
27\end{array}$ & $\begin{array}{l}2,460 \\
1,450 \\
1,560 \\
1,140 \\
1,220\end{array}$ & $\begin{array}{l}303 \\
279 \\
279 \\
279 \\
303\end{array}$ & $\begin{array}{r}2,810 \\
2,060 \\
1,140 \\
600 \\
358\end{array}$ & $\begin{array}{l}358 \\
315 \\
303 \\
279 \\
243\end{array}$ & $\begin{array}{r}60 \\
60 \\
49 \\
39 \\
* 24\end{array}$ & $\begin{array}{l}215 \\
210 \\
* 4 \\
24 \\
24\end{array}$ & $\begin{array}{l}3 \\
3 \\
3 \\
3 \\
3\end{array}$ & $\begin{array}{r}2 \\
2 \\
* 3 \\
2 \\
2\end{array}$ \\
\hline $\begin{array}{l}16 \\
17 \\
18 \\
19 \\
20\end{array}$ & $\begin{array}{l}5 \\
5 \\
5 \\
5 \\
5\end{array}$ & $\begin{array}{l}10 \\
10 \\
10 \\
10 \\
10\end{array}$ & $\begin{array}{l}30 \\
30 \\
30 \\
30 \\
30\end{array}$ & $\begin{array}{l}27 \\
27 \\
27 \\
27 \\
27\end{array}$ & $\begin{array}{r}2,060 \\
966 \\
500 \\
426 \\
388\end{array}$ & $\begin{array}{l}358 \\
402 \\
267 \\
267 \\
402\end{array}$ & $\begin{array}{l}315 \\
279 \\
243 \\
207 \\
195\end{array}$ & $\begin{array}{l}267 \\
243 \\
231 \\
207 \\
173\end{array}$ & $\begin{array}{l}21 \\
18 \\
18 \\
\frac{14}{18}\end{array}$ & $\begin{array}{l}24 \\
24 \\
24 \\
24 \\
24\end{array}$ & $\begin{array}{l}3 \\
3 \\
4 \\
3 \\
2\end{array}$ & $\begin{array}{l}2 \\
2 \\
2 \\
2 \\
2\end{array}$ \\
\hline $\begin{array}{l}21 \\
22 \\
23 \\
24 \\
25\end{array}$ & $\begin{array}{l}5 \\
5 \\
5 \\
5 \\
5\end{array}$ & $\begin{array}{l}10 \\
10 \\
10 \\
10 \\
10\end{array}$ & $\begin{array}{l}40 \\
40 \\
40 \\
40 \\
40\end{array}$ & $\begin{array}{l}27 \\
27 \\
27 \\
27 \\
27\end{array}$ & $\begin{array}{l}373 \\
330 \\
330 \\
330 \\
303\end{array}$ & $\begin{array}{r}640 \\
694 \\
460 \\
2400 \\
2450\end{array}$ & $\begin{array}{r}373 \\
1,220 \\
500 \\
358 \\
279\end{array}$ & $\begin{array}{l}162 \\
151 \\
132 \\
124 \\
108\end{array}$ & $\begin{array}{l}18 \\
18 \\
18 \\
18 \\
18\end{array}$ & $\begin{array}{l}a 4 \\
24 \\
a 4 \\
a 4 \\
24\end{array}$ & $\begin{array}{r}2 \\
2 \\
* 3 \\
2 \\
2\end{array}$ & $\begin{array}{l}3 \\
2 \\
2 \\
3 \\
3\end{array}$ \\
\hline $\begin{array}{l}26 \\
27 \\
28 \\
29 \\
30 \\
31\end{array}$ & $\begin{array}{l}5 \\
5 \\
5 \\
5 \\
5 \\
5\end{array}$ & $\begin{array}{r}10 \\
10 \\
10 \\
10 \\
10 \\
-\end{array}$ & $\begin{array}{r}40 \\
40 \\
100 \\
200 \\
\frac{402}{330}\end{array}$ & $\begin{array}{l}27 \\
27 \\
68 \\
83 \\
43 \\
34\end{array}$ & $\begin{array}{c}303 \\
267 \\
303 \\
= \\
- \\
-\end{array}$ & $\begin{array}{r}a 500 \\
8480 \\
431 \\
388 \\
358 \\
291\end{array}$ & $\begin{array}{r}207 \\
440 \\
358 \\
388 \\
\text { a400 } \\
-\end{array}$ & $\begin{array}{r}108 \\
100 \\
91 \\
83 \\
83 \\
15\end{array}$ & $\begin{array}{l}18 \\
18 \\
18 \\
18 \\
18 \\
-\end{array}$ & $\begin{array}{l}a / 4 \\
a 4 \\
24 \\
24 \\
24 \\
24\end{array}$ & $\begin{array}{l}2 \\
2 \\
2 \\
3 \\
5 \\
\frac{5}{3}\end{array}$ & $\begin{array}{l}2 \\
2 \\
2 \\
3 \\
3 \\
-\end{array}$ \\
\hline $\begin{array}{l}\text { Total } \\
\text { Mean } \\
\text { Ac-ft }\end{array}$ & $\begin{array}{r}155 \\
5 \\
307\end{array}$ & $\begin{array}{r}300 \\
10 \\
595\end{array}$ & $\begin{array}{r}1,812 \\
58.5 \\
3,590\end{array}$ & $\begin{array}{r}2,281 \\
73.6 \\
4,520\end{array}$ & $\begin{array}{r}21,798 \\
778 \\
43,210\end{array}$ & $\begin{array}{r}10,587 \\
342 \\
21,000\end{array}$ & $\begin{array}{r}44,390 \\
1,480 \\
88,050\end{array}$ & $\begin{array}{r}8,587 \\
277 \\
17,030\end{array}$ & $\begin{array}{r}1,169 \\
38.9 \\
2,320\end{array}$ & $\begin{array}{l}281 \\
9.0 \\
557\end{array}$ & $\begin{array}{r}86 \\
2.8 \\
171\end{array}$ & $\begin{array}{r}66 \\
2.2 \\
131\end{array}$ \\
\hline
\end{tabular}

Calendar year 1953: Max 6,620 Min 0 Mean 14l Acm 102,000 Water year 1954 : Kax 5,680 Min 2 Mean 251 Ac-ft 161,600

* Discharge measurement made on this day.

a No gage-height record; discharge interpolated.

a No gage ho conditions at the station. 
HITMAND RIVER BASIN

Musa Oala Rivor at Musa Oala, Afghanistan

Discharge, in cubic feet per second, water year October 1954 to September 1955

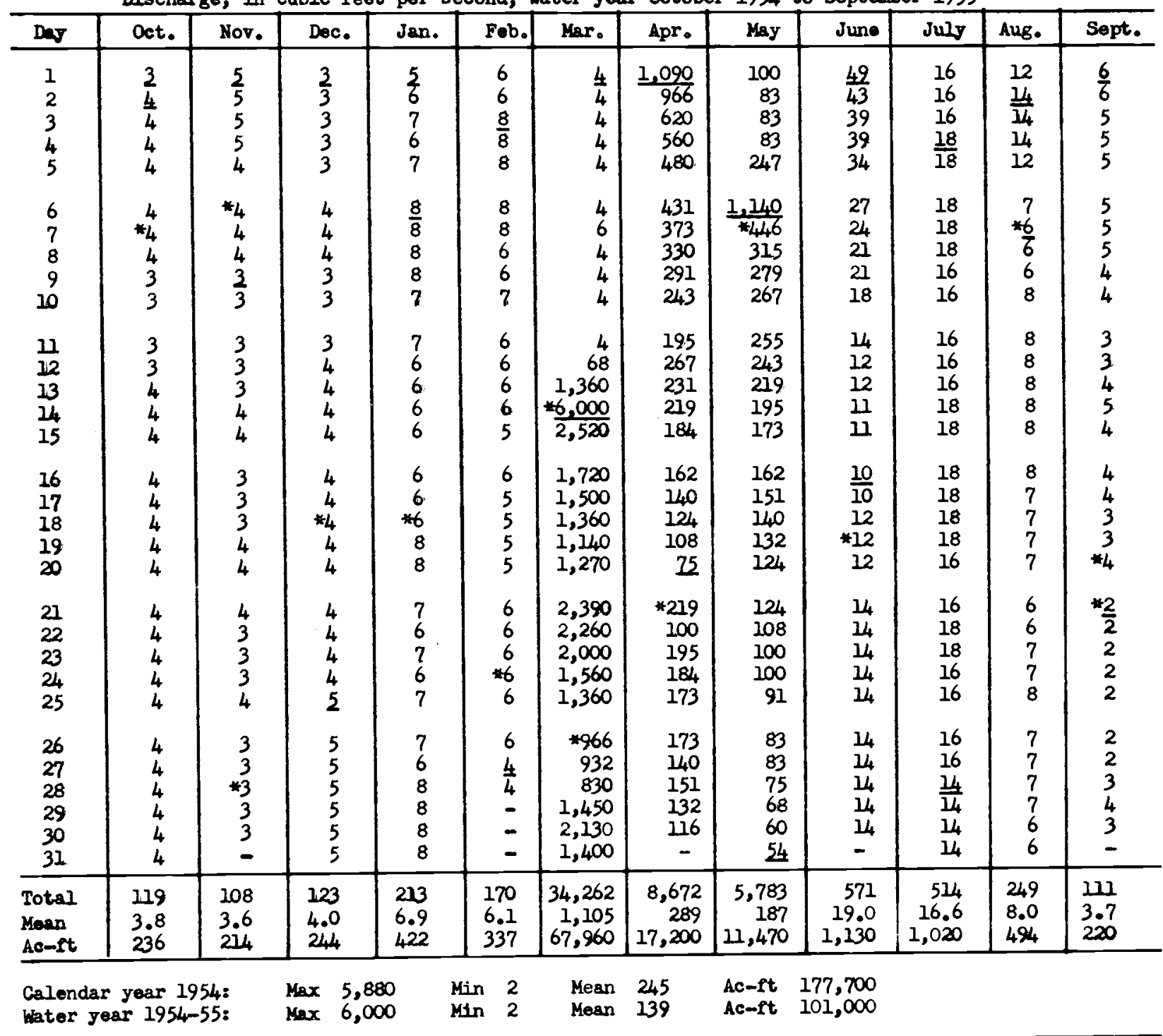

* Discharge measurament made on this day.

Note.-Gage-height from graph of gage readings Mar. $14,15,20,21,29,30$, May 5-7. 
HELMAND RIVER BASTN

Musa Qala River at Musa Q2la, Afghantstan

Discharge, in cubic feet per second, water year October 1955 to September 1956

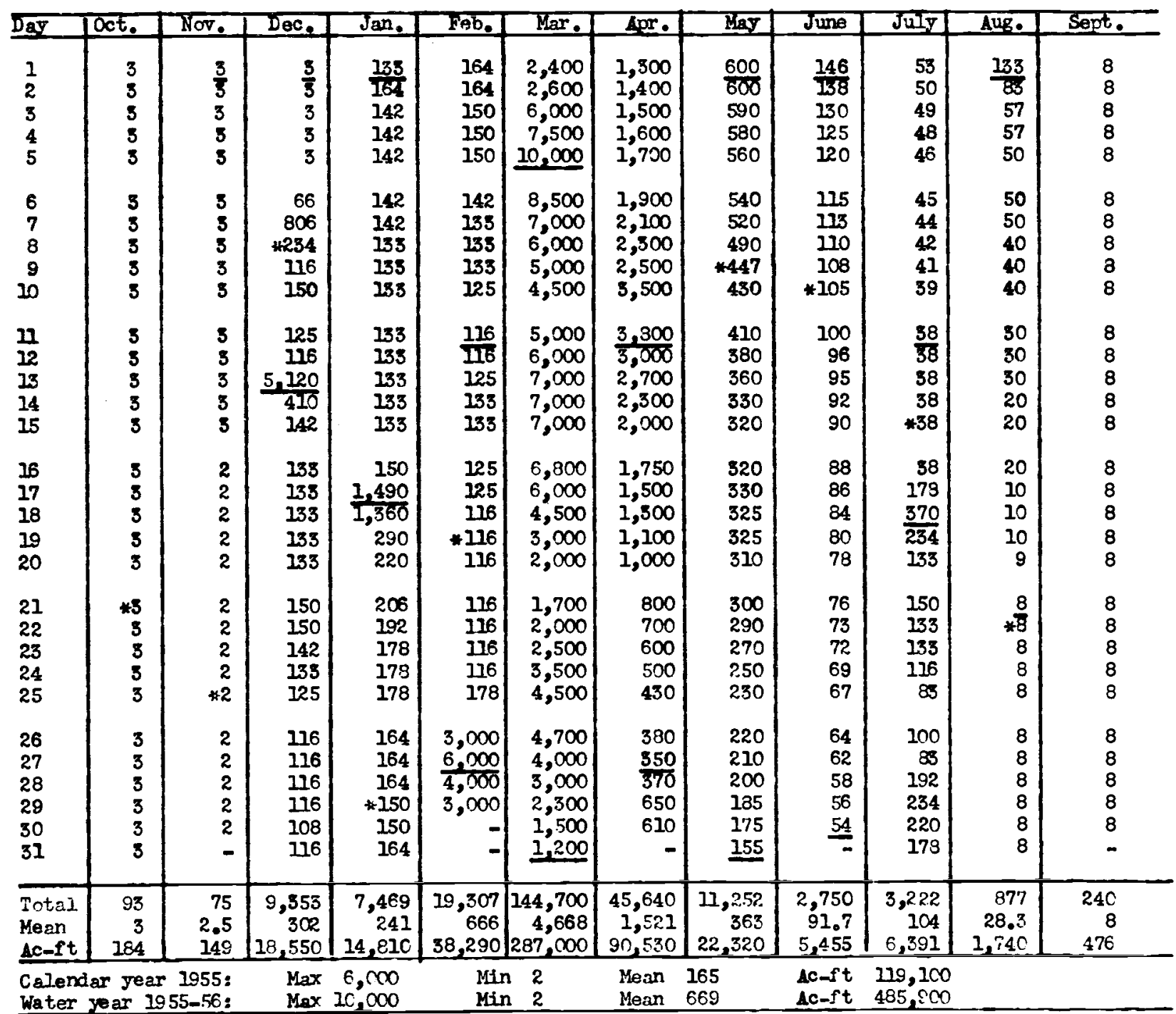

* Dischiarge measuremert made on this day.

Note.-No gage-heights Mar. 5, 1I, 17-21, 24, 30, Apr. 11 to July 14, Aus. 4 to Sept. 30; discharge

computed on basis of field visits and recoris $f a r$ Kash River at Dilaran. 
HEIMAND RIVER BASIN

Musa Qala River at Musa Qala, Afghanistan

Discharge, in cublc feet per second, water year October 1956 to September 1957

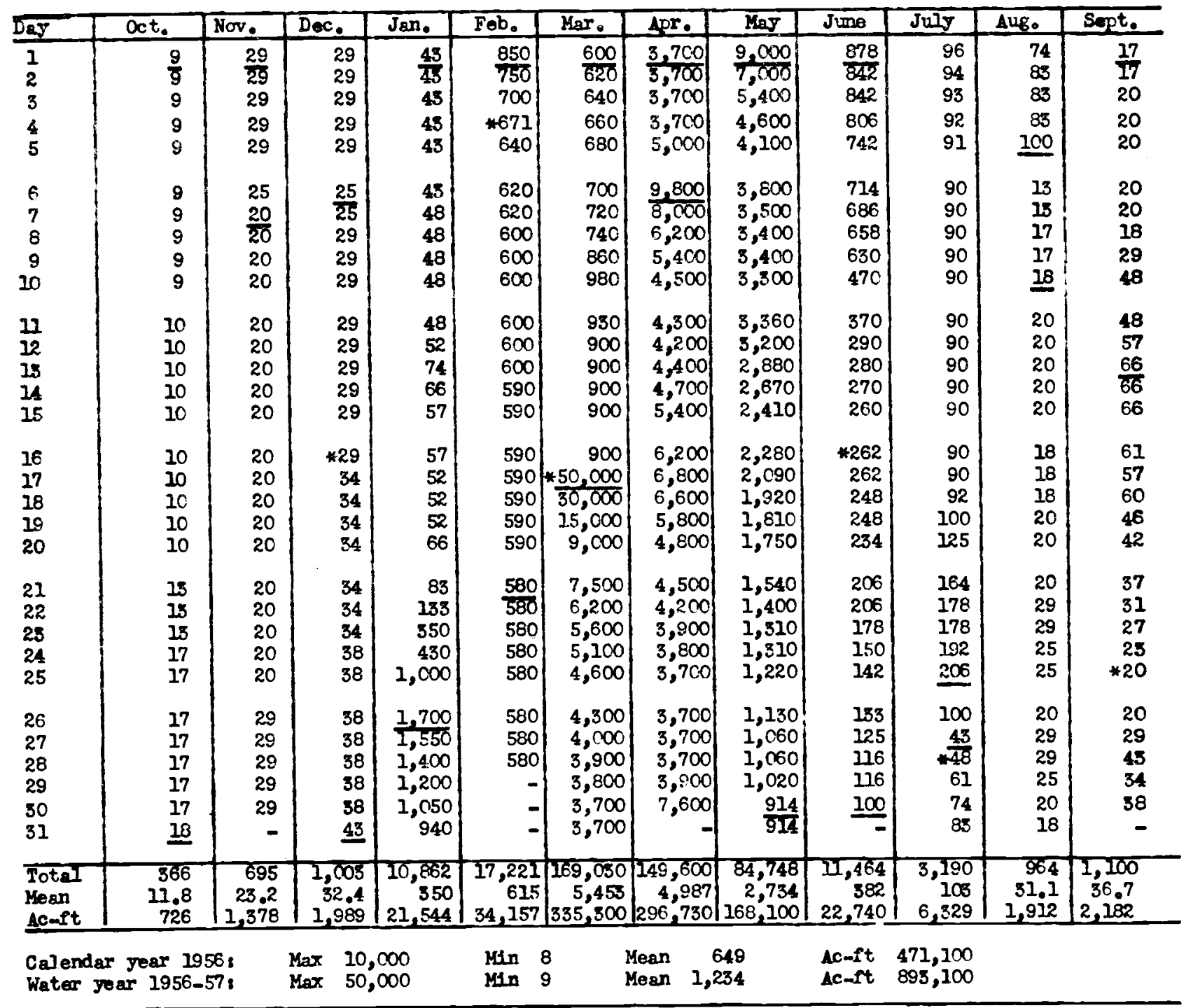

Wischarge measurement mede on this day.

Hote. No gage-heights oct. 120, Jan. 25 to May 10, June 13-15, July 120, Sept. 18-24; discharge computed on basis of field visits and records for Kash River at Dilaram. 
HETMAND RIVER BASIN

Musa Qala River at Musa Qala, Afghanistan

Discharge, in cubic feet per second, water year October 1957 to September 1958

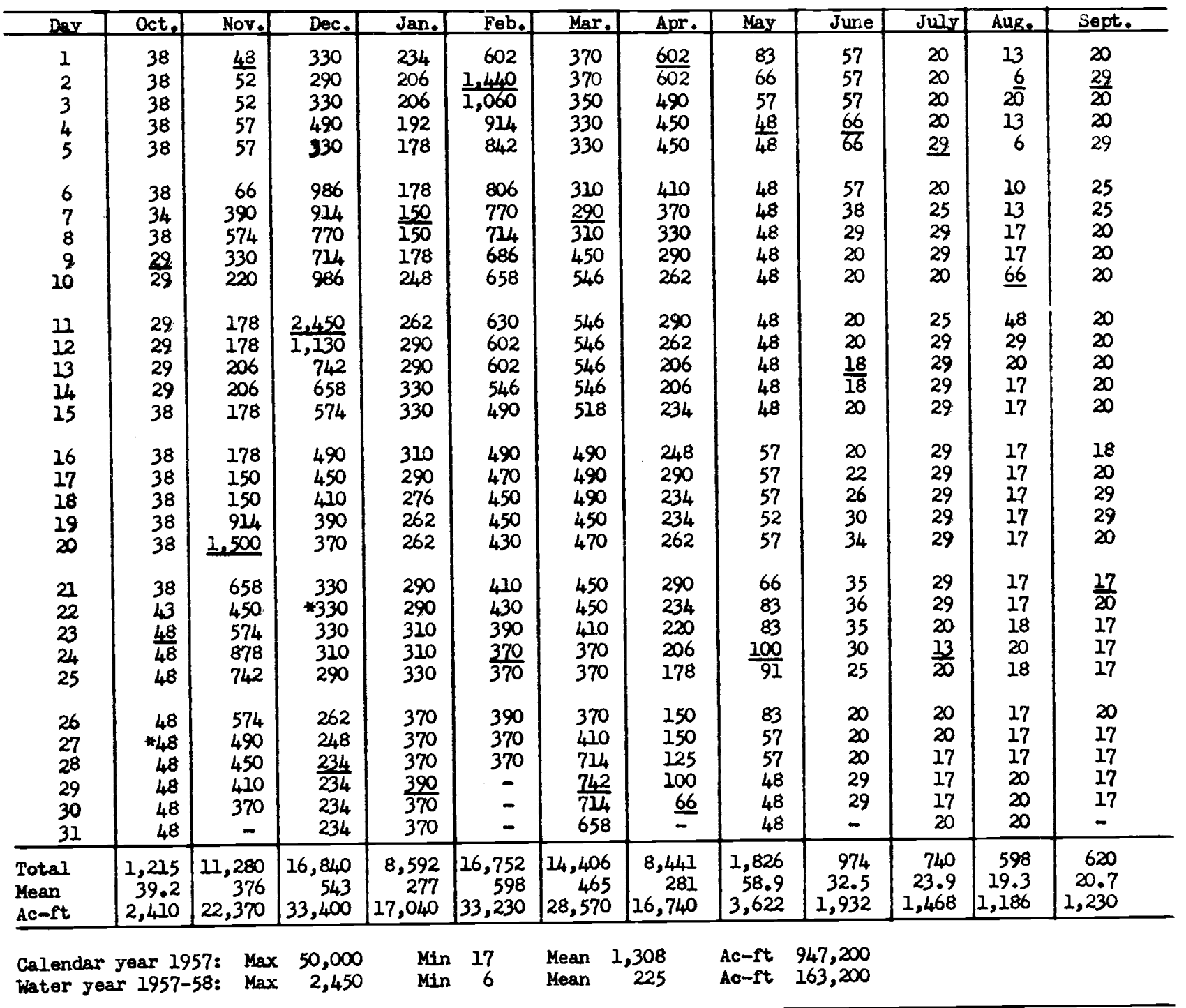

* Discharge measurement made on this day.

Note. - No gage-heights Nov, 20, Dec. 11, June 17-24; discharge computed on basis of field visits and

records for Kash Biver at Dilaram. 
HEIMAND RIVER BASIN

Musa Qala River at Musa Qala, Afghanistan

Discharge, in cubic feet per second, water year October 1958 to September 1959

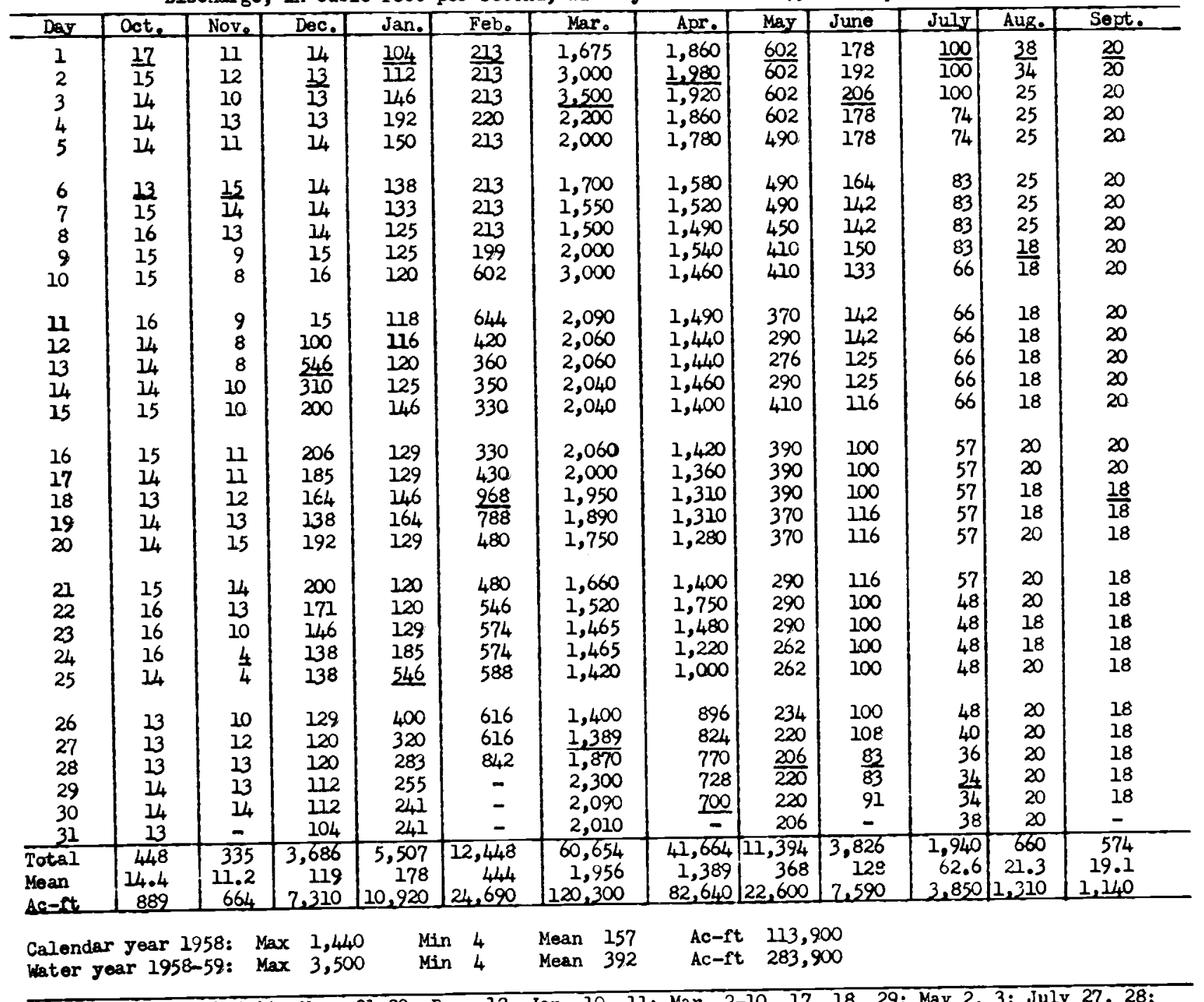

Nete.- Ho gage-hoights Novo $21-23$; Dec. 12 ; Jan. 10,11 ; Mar. $2-10,17,18,29 ;$ May
discharge computed on basis of field visits and records for Kash River at Dilaram. 
HEIMAND RIVER BASIN

Musa Qala River at Musa Qala, Afghanistan

Disoharge, in cubic feet per second, water year October 1959 to Sept ember 1960

\begin{tabular}{|c|c|c|c|c|c|c|c|c|c|c|c|c|}
\hline Dar & Oct. & $\mathrm{Nov}_{2}$ & Dec, & Jan. & Feb. & Mar, & Apr. & $\mathrm{Mar}$ & June & July & Aug. & Sept. \\
\hline $\begin{array}{l}1 \\
2 \\
3 \\
4 \\
5\end{array}$ & $\begin{array}{l}\frac{20}{20} \\
25 \\
25 \\
25\end{array}$ & $\begin{array}{l}29 \\
29 \\
34 \\
34 \\
29\end{array}$ & $\begin{array}{r}\frac{38}{3,000} \\
270 \\
276 \\
255\end{array}$ & $\begin{array}{l}\frac{164}{164} \\
164 \\
164 \\
164\end{array}$ & $\begin{array}{l}164 \\
164 \\
164 \\
164 \\
164\end{array}$ & $\begin{array}{l}164 \\
150 \\
146 \\
\frac{142}{142}\end{array}$ & $\begin{array}{r}1,080 \\
914 \\
770 \\
658 \\
1,040\end{array}$ & $\begin{array}{l}\frac{969}{878} \\
824 \\
757 \\
714\end{array}$ & $\begin{array}{l}157 \\
157 \\
164 \\
150 \\
142\end{array}$ & $\begin{array}{l}\frac{116}{108} \\
104 \\
100 \\
100\end{array}$ & $\begin{array}{l}59 \\
61 \\
\frac{59}{59} \\
57 \\
52\end{array}$ & $\begin{array}{l}43 \\
38 \\
38 \\
38 \\
43\end{array}$ \\
\hline $\begin{array}{r}6 \\
7 \\
8 \\
9 \\
10\end{array}$ & $\begin{array}{l}25 \\
25 \\
25 \\
25 \\
25\end{array}$ & $\begin{array}{l}\frac{25}{27} \\
29 \\
29 \\
29\end{array}$ & $\begin{array}{l}248 \\
227 \\
206 \\
186 \\
178\end{array}$ & $\begin{array}{l}164 \\
157 \\
150 \\
150 \\
150\end{array}$ & $\begin{array}{l}164 \\
164 \\
164 \\
164 \\
164\end{array}$ & $\begin{array}{l}150 \\
164 \\
157 \\
150 \\
146\end{array}$ & $\begin{array}{l}968 \\
842 \\
842 \\
806 \\
770\end{array}$ & $\begin{array}{l}672 \\
644 \\
602 \\
480 \\
440\end{array}$ & $\begin{array}{l}142 \\
138 \\
133 \\
125 \\
120\end{array}$ & $\begin{array}{l}96 \\
94 \\
92 \\
91 \\
95\end{array}$ & $\begin{array}{l}54 \\
50 \\
48 \\
46 \\
45\end{array}$ & $\begin{array}{l}43 \\
46 \\
48 \\
48 \\
48\end{array}$ \\
\hline $\begin{array}{l}\frac{11}{12} \\
13 \\
14 \\
15\end{array}$ & $\begin{array}{l}25 \\
25 \\
25 \\
25 \\
25\end{array}$ & $\begin{array}{l}27 \\
25 \\
25 \\
25 \\
29\end{array}$ & $\begin{array}{l}178 \\
171 \\
164 \\
164 \\
164\end{array}$ & $\begin{array}{l}150 \\
150 \\
150 \\
150 \\
150\end{array}$ & $\begin{array}{l}164 \\
164 \\
164 \\
164 \\
164\end{array}$ & $\begin{array}{r}1,2 \\
248 \\
1,080 \\
932 \\
860\end{array}$ & $\begin{array}{r}729 \\
686 \\
686 \\
878 \\
1,090\end{array}$ & $\begin{array}{l}400 \\
380 \\
350 \\
300 \\
290\end{array}$ & $\begin{array}{l}120 \\
120 \\
120 \\
120 \\
120\end{array}$ & $\begin{array}{r}95 \\
100 \\
100 \\
87 \\
87\end{array}$ & $\begin{array}{l}43 \\
40 \\
36 \\
36 \\
36\end{array}$ & $\begin{array}{l}48 \\
43 \\
43 \\
48 \\
48\end{array}$ \\
\hline $\begin{array}{l}16 \\
17 \\
18 \\
19 \\
20\end{array}$ & $\begin{array}{l}25 \\
25 \\
25 \\
25 \\
25\end{array}$ & $\begin{array}{l}29 \\
29 \\
29 \\
29 \\
29\end{array}$ & $\begin{array}{l}192 \\
185 \\
178 \\
178 \\
178\end{array}$ & $\begin{array}{l}150 \\
150 \\
150 \\
150 \\
150\end{array}$ & $\begin{array}{l}164 \\
164 \\
164 \\
164 \\
164\end{array}$ & $\begin{array}{l}630 \\
490 \\
490 \\
470 \\
460\end{array}$ & $\begin{array}{l}1,520 \\
2,380 \\
4,500 \\
\frac{3,400}{2,800}\end{array}$ & $\begin{array}{l}283 \\
276 \\
269 \\
262 \\
255\end{array}$ & $\begin{array}{l}116 \\
116 \\
112 \\
112 \\
108\end{array}$ & $\begin{array}{l}87 \\
78 \\
78 \\
74 \\
74\end{array}$ & $\begin{array}{l}36 \\
38 \\
46 \\
46 \\
52\end{array}$ & $\begin{array}{l}50 \\
52 \\
50 \\
52 \\
52\end{array}$ \\
\hline $\begin{array}{l}21 \\
22 \\
23 \\
24 \\
25\end{array}$ & $\begin{array}{l}25 \\
25 \\
25 \\
27 \\
29 \\
\end{array}$ & $\begin{array}{l}29 \\
29 \\
29 \\
29 \\
34\end{array}$ & $\begin{array}{l}178 \\
178 \\
178 \\
178 \\
178\end{array}$ & $\begin{array}{l}150 \\
150 \\
150 \\
150 \\
150\end{array}$ & $\begin{array}{l}164 \\
164 \\
164 \\
164 \\
164\end{array}$ & $\begin{array}{l}450 \\
450 \\
440 \\
420 \\
370\end{array}$ & $\begin{array}{l}2,700 \\
2,480 \\
2,540 \\
2,800 \\
1,720\end{array}$ & $\begin{array}{l}241 \\
241 \\
220 \\
206 \\
199\end{array}$ & $\begin{array}{l}104 \\
100 \\
157 \\
150 \\
146\end{array}$ & $\begin{array}{l}78 \\
70 \\
70 \\
70 \\
66\end{array}$ & $\begin{array}{l}50 \\
50 \\
48 \\
48 \\
48\end{array}$ & $\begin{array}{l}50 \\
50 \\
48 \\
52 \\
52\end{array}$ \\
\hline $\begin{array}{l}26 \\
27 \\
28 \\
29 \\
30 \\
31 \\
\end{array}$ & $\begin{array}{l}29 \\
29 \\
29 \\
29 \\
29 \\
29 \\
\end{array}$ & $\begin{array}{l}36 \\
\frac{38}{38} \\
38 \\
38 \\
-\end{array}$ & $\begin{array}{l}178 \\
178 \\
178 \\
164 \\
164 \\
164\end{array}$ & $\begin{array}{l}150 \\
150 \\
150 \\
150 \\
157 \\
164\end{array}$ & $\begin{array}{c}* 164 \\
164 \\
164 \\
164 \\
- \\
-\end{array}$ & $\begin{array}{r}420 \\
1,520 \\
1,220 \\
1,150 \\
1,090 \\
1,080\end{array}$ & $\begin{array}{c}1,610 \\
1,490 \\
1,220 \\
1,080 \\
1,040 \\
-\end{array}$ & $\begin{array}{l}178 \\
171 \\
164 \\
157 \\
150 \\
150\end{array}$ & $\begin{array}{l}142 \\
142 \\
133 \\
129 \\
120\end{array}$ & $\begin{array}{l}61 \\
59 \\
57 \\
57 \\
52 \\
54\end{array}$ & $\begin{array}{l}50 \\
50 \\
46 \\
48 \\
43 \\
46 \\
\end{array}$ & $\begin{array}{l}48 \\
52 \\
52 \\
57 \\
59\end{array}$ \\
\hline $\begin{array}{l}\text { Total } \\
\text { Mean } \\
\text { Ac-ft } \\
\end{array}$ & $\begin{array}{r}795 \\
25.6 \\
1.577 \\
\end{array}$ & $\begin{array}{r}908 \\
30.3 \\
1,801 \\
\end{array}$ & $\begin{array}{r}8,551 \\
276 \\
16,980 \\
\end{array}$ & $\begin{array}{r}4,762 \\
154 \\
9,445 \\
\end{array}$ & $\begin{array}{r}4,756 \\
164 \\
9,433\end{array}$ & $\begin{array}{r}15,923 \\
514 \\
31,580\end{array}$ & $\begin{array}{r}46,039 \\
1,535 \\
91,320\end{array}$ & $\begin{array}{r}12,121 \\
391 \\
24,040\end{array}$ & $\begin{array}{r}3,915 \\
130 \\
7,765 \\
\end{array}$ & $\begin{array}{r}2,550 \\
82.2 \\
5,058 \\
\end{array}$ & $\begin{array}{r}1,469 \\
47.4 \\
2,914\end{array}$ & $\begin{array}{r}1,439 \\
48 \\
2,854 \\
\end{array}$ \\
\hline $\begin{array}{l}\text { Cale } \\
\text { Wate }\end{array}$ & 700 & & $\begin{array}{l}3,500 \\
4,500\end{array}$ & $\frac{\text { Min }}{\text { Min }}$ & $\begin{array}{l}18 \\
20\end{array}$ & $\begin{array}{l}\text { Mean } \\
\text { Mean }\end{array}$ & & ft & $\begin{array}{l}\infty \\
\infty\end{array}$ & & & \\
\hline
\end{tabular}

* Discharge messurement made on this day.

Nete.- No gage-helghts Dec. 2, 3; Feb. 21-24; July 7-E; discharge estimated. 
HEIMAND RIVER BASIN

Helmand River near Girishk, Afghanistan

Location.-Lat $31^{\circ} 4^{\prime}$, long $64^{\circ} 35^{\prime}$, on left bank, under highway bridge about 2.5 kilometers eatof Girishk and 51 kilometers upstream from Arghandab River.

Drainage area. $-20,300 \mathrm{sq} \mathrm{mi}$ (by MKA from Survey of India Mapsd.

Records available.--July 1946 to March 1949.

Gage.-Water stage recorder. Altitude of gage is 816 meters. Feb. 16 to $\mathrm{Apr} .30$, 1947 at site about 5

kilometers upstream.

Extremes..-1946-47: Maximum discharge during year, 11,200 cfs ( gage height, 28.90 meters) Mar. 22; minimum $336 \mathrm{c}$ fs (gage height 15.85 meters) Aug. 16

1947-48: Maximum discharge during year, 36,000 cfs (gage height, 18.08 meters) Mar. 8; minimum 750 cfs

(gage height, 16.10 meters) 0ct. 1.

Remarks.--Records fair, except those for periods of no gage height which are poor. Niany diversions for irrigation upstream. 
HELMAND RIVTR BASIN

Helmand River near uirishk, Afghanistan

Discharge, in cubic feet per second, water year October 1945 to September 1946

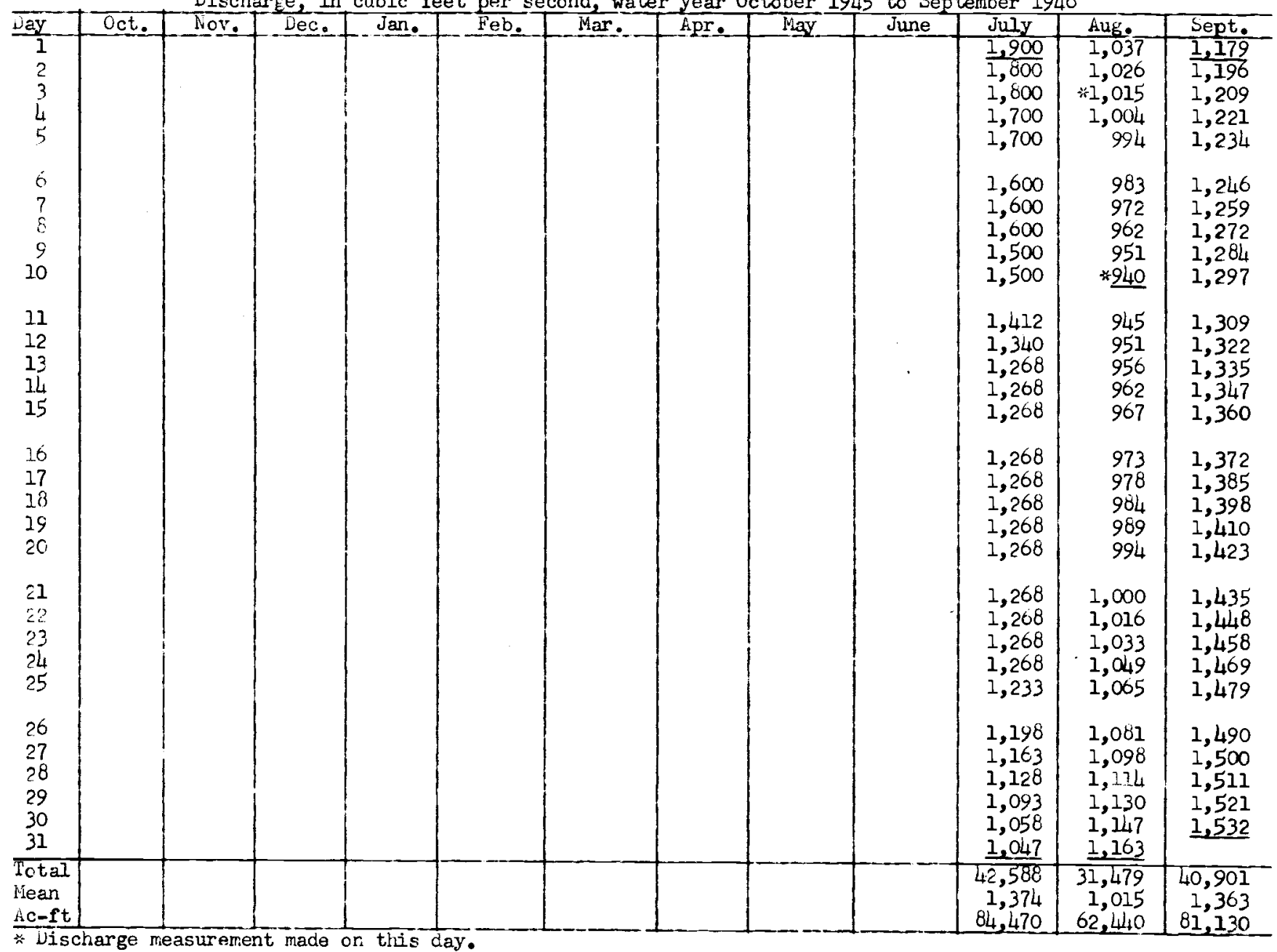

Note.--Only occasional gage..heights; discharge averaged. 
HELMAND RIVER BASIN

Helmand River near Girishk, Afghanistan

Discharge, in cubic feet per second, water year October 1946 to September 1947

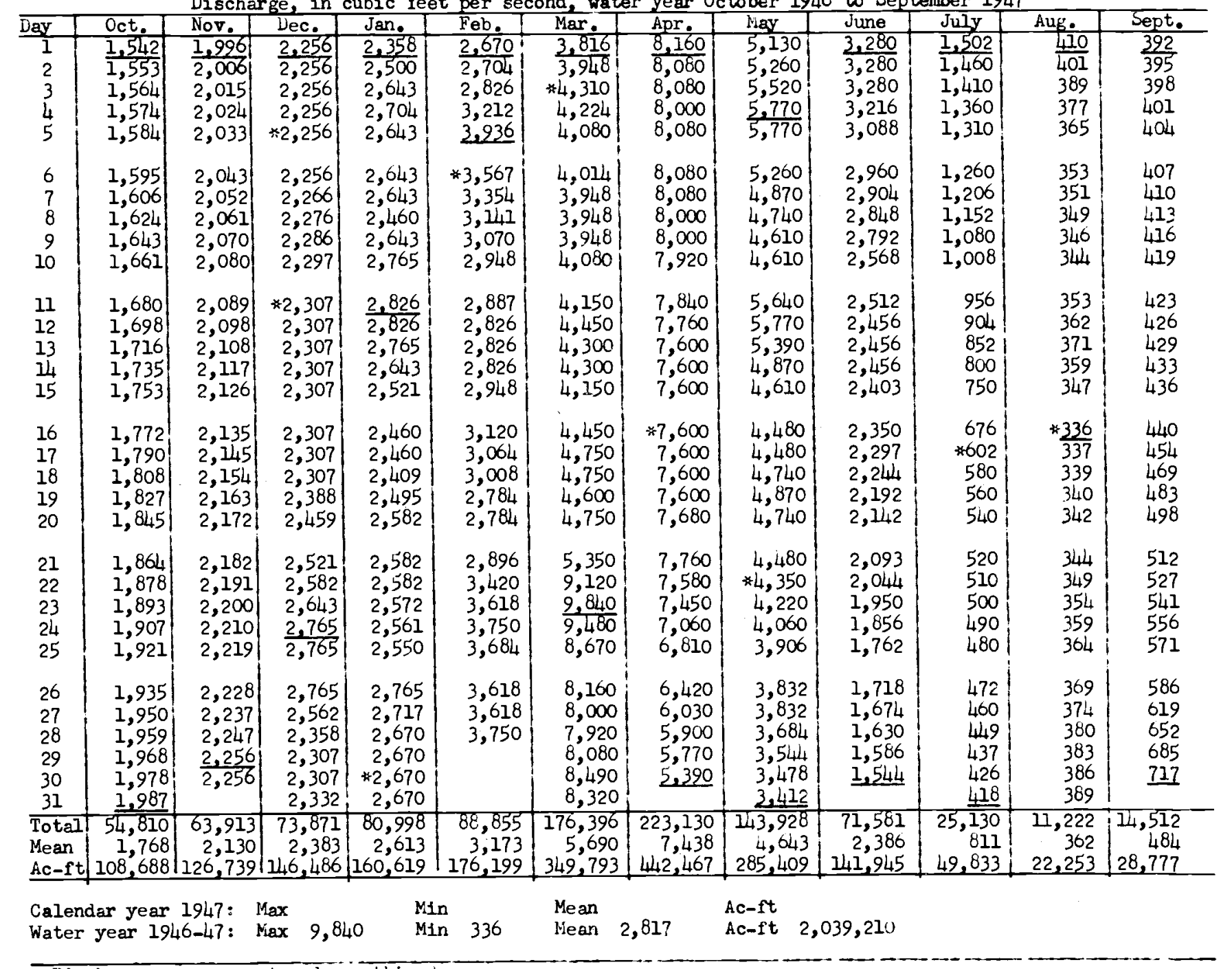

* Discharge measurement made on this day.

Note.--Only occasional gage--heights for Oct. 1 to Vec. 20 and June 15 to ber.t. 30; discharge averaged or estimated on basis of records at Kajakai. 
HELMAND RIVER BASIN

Helmand River near Girishk, Afghanistan

Vischarge, in cubic feet per second, water year October 1947 to September 1948

\begin{tabular}{|c|c|c|c|c|c|c|c|c|c|c|c|c|}
\hline $\bar{D} \mathbf{a y}$ & Oct. & Nov. & Dec. & Jan. & Feb. & Mar. & Apr. & May & June & July & Aug. & Sept. \\
\hline $\begin{array}{l}1 \\
2 \\
3 \\
4 \\
5\end{array}$ & $\begin{array}{l}750 \\
771 \\
792 \\
813 \\
835\end{array}$ & $\begin{array}{l}1,544 \\
1,544 \\
1,544 \\
1,544 \\
1,544\end{array}$ & $\begin{array}{l}\frac{1,900}{1,948} \\
1,996 \\
2,044 \\
2,140\end{array}$ & $\begin{array}{l}2,192 \\
2,140 \\
2,192 \\
2,296 \\
2,296\end{array}$ & $\begin{array}{l}2,736 \\
2,736 \\
2,848 \\
2,792 \\
2,680\end{array}$ & $\begin{array}{l}3,346 \\
3,280 \\
3,478 \\
4,140 \\
5,990\end{array}$ & $\begin{array}{l}12,000 \\
12,600 \\
13,800 \\
17,600 \\
16,800\end{array}$ & $\begin{array}{l}20,600 \\
18,900 \\
18,000 \\
18,000 \\
18,300\end{array}$ & $\begin{array}{l}\frac{5,360}{5,120} \\
5,000 \\
4,880 \\
4,770\end{array}$ & $\begin{array}{l}\frac{2,512}{2,400} \\
2,192 \\
2,140 \\
2,140\end{array}$ & $\begin{array}{l}1,420 \\
1,420 \\
1,460 \\
1,460 \\
1,420\end{array}$ & $\begin{array}{l}840 \\
870 \\
870 \\
870 \\
900\end{array}$ \\
\hline $\begin{array}{r}6 \\
7 \\
8 \\
9 \\
10\end{array}$ & $\begin{array}{l}857 \\
878 \\
900 \\
936 \\
972\end{array}$ & $\begin{array}{l}1,544 \\
1,544 \\
1,544 \\
1,586 \\
1,628\end{array}$ & $\begin{array}{l}2,512 \\
2,624 \\
2,512 \\
2,512 \\
2,456\end{array}$ & $\begin{array}{l}2,244 \\
2,296 \\
2,244 \\
2,296 \\
2,348\end{array}$ & $\begin{array}{l}2,624 \\
2,456 \\
2,348 \\
2,348 \\
2,400\end{array}$ & $\begin{array}{r}6,130 \\
13,200 \\
25,800 \\
20,600 \\
14,000\end{array}$ & $\begin{array}{l}15,100 \\
14,400 \\
14,700 \\
18,000 \\
20,000\end{array}$ & $\begin{array}{l}18,000 \\
17,000 \\
16,300 \\
15,600 \\
15,100\end{array}$ & $\begin{array}{l}4,550 \\
4,4,40 \\
4,330 \\
4,220 \\
4,060\end{array}$ & $\begin{array}{l}2,140 \\
2,092 \\
1,996 \\
1,996 \\
1,996\end{array}$ & $\begin{array}{l}1,380 \\
1,420 \\
1,380 \\
1,340 \\
1,340\end{array}$ & $\begin{array}{l}900 \\
900 \\
900 \\
936 \\
936\end{array}$ \\
\hline $\begin{array}{l}11 \\
12 \\
13 \\
14 \\
15\end{array}$ & $\begin{array}{l}1,008 \\
1,044 \\
1,068 \\
1,092 \\
1,116\end{array}$ & $\begin{array}{l}1,628 \\
1,670 \\
1,670 \\
1,670 \\
1,716\end{array}$ & $\begin{array}{l}2,400 \\
2,348 \\
2,296 \\
2,244 \\
2,192\end{array}$ & $\begin{array}{l}2,348 \\
2,348 \\
2,244 \\
2,140 \\
2,140\end{array}$ & $\begin{array}{l}2,568 \\
2,624 \\
2,624 \\
2,624 \\
2,624\end{array}$ & $\begin{array}{r}12,200 \\
10,400 \\
9,320 \\
8,200 \\
7,750\end{array}$ & $\begin{array}{l}19,400 \\
17,600 \\
16,500 \\
16,000 \\
16,500\end{array}$ & $\begin{array}{l}14,400 \\
13,200 \\
12,200 \\
11,600 \\
11,000\end{array}$ & $\begin{array}{l}3,906 \\
3,832 \\
3,832 \\
3,832 \\
3,758\end{array}$ & $\begin{array}{l}1,948 \\
1,948 \\
1,948 \\
1,716 \\
1,900\end{array}$ & $\begin{array}{l}1,380 \\
1,380 \\
1,340 \\
1,300 \\
1,260\end{array}$ & $\begin{array}{r}972 \\
972 \\
1,008 \\
1,008 \\
1,008\end{array}$ \\
\hline $\begin{array}{l}16 \\
17 \\
18 \\
19 \\
20\end{array}$ & $\begin{array}{l}1,170 \\
1,224 \\
1,243 \\
1,262 \\
1,281\end{array}$ & $\begin{array}{l}1,762 \\
1,808 \\
1,854 \\
1,900 \\
\frac{1}{1,900}\end{array}$ & $\begin{array}{l}2,192 \\
2,192 \\
2,192 \\
2,140 \\
2,044\end{array}$ & $\begin{array}{l}2,192 \\
2,192 \\
2,244 \\
2,244 \\
2,192\end{array}$ & $\begin{array}{l}2,624 \\
2,568 \\
2,624 \\
2,680 \\
2,792\end{array}$ & $\begin{array}{r}* 7,300 \\
7,000 \\
7,150 \\
8,820 \\
10,500\end{array}$ & $\begin{array}{l}17,000 \\
18,900 \\
22,100 \\
26,200 \\
29,400\end{array}$ & $\begin{array}{r}10,500 \\
10,020 \\
9,490 \\
8,980 \\
8,820\end{array}$ & $\begin{array}{l}3,758 \\
3,684 \\
3,610 \\
3,544 \\
3,478\end{array}$ & $\begin{array}{l}1,854 \\
1,854 \\
1,808 \\
1,762 \\
1,808\end{array}$ & $\begin{array}{l}1,260 \\
1,260 \\
1,260 \\
1,224 \\
1,188\end{array}$ & $\begin{array}{l}1,044 \\
1,044 \\
1,044 \\
1,044 \\
1,080\end{array}$ \\
\hline $\begin{array}{l}21 \\
22 \\
23 \\
24 \\
25\end{array}$ & $\begin{array}{l}1,300 \\
1,340 \\
1,380 \\
1,420 \\
1,460\end{array}$ & $\begin{array}{l}1,900 \\
1,854 \\
1,854 \\
1,854 \\
1,854\end{array}$ & $\begin{array}{l}2,044 \\
2,044 \\
2,092 \\
2,192 \\
2,192\end{array}$ & $\begin{array}{r}2,192 \\
* 2,192 \\
2,244 \\
2,296 \\
2,400\end{array}$ & $\begin{array}{r}2,904 \\
3,024 \\
* 2,960 \\
2,904 \\
2,848\end{array}$ & $\begin{array}{l}10,900 \\
10,900 \\
10,500 \\
10,400 \\
10,200\end{array}$ & $\begin{array}{l}28,700 \\
25,800 \\
22,700 \\
22,400 \\
23,400\end{array}$ & $\begin{array}{l}8,500 \\
8,200 \\
8,050 \\
7,900 \\
7,600\end{array}$ & $\begin{array}{l}3,478 \\
3,478 \\
3,412 \\
3,413 \\
3,412\end{array}$ & $\begin{array}{l}1,716 \\
1,716 \\
1,670 \\
1,586 \\
1,762\end{array}$ & $\begin{array}{l}1,152 \\
1,116 \\
1,080 \\
1,044 \\
1,008\end{array}$ & $\begin{array}{l}1,152 \\
1,188 \\
1,224 \\
1,260 \\
1,340\end{array}$ \\
\hline $\begin{array}{l}26 \\
27 \\
28 \\
29 \\
30 \\
31\end{array}$ & $\begin{array}{r}1,502 \\
* 1,502 \\
1,502 \\
1,544 \\
1,544 \\
1,544 \\
\end{array}$ & $\begin{array}{l}1,854 \\
1,900 \\
1,900 \\
1,900 \\
1,900\end{array}$ & $\begin{array}{r}2,192 \\
2,192 \\
2,192 \\
2,192 \\
2,192 \\
2,192 \\
\end{array}$ & $\begin{array}{l}2,456 \\
2,568 \\
3,412 \\
3,544 \\
2,960 \\
2,848\end{array}$ & $\begin{array}{l}2,904 \\
3,152 \\
3,412 \\
\frac{3,346}{3}\end{array}$ & $\begin{array}{l}10,020 \\
10,700 \\
10,500 \\
10,900 \\
11,200 \\
10,700\end{array}$ & $\begin{array}{l}24,500 \\
24,800 \\
24,800 \\
24,500 \\
22,700\end{array}$ & $\begin{array}{l}7,150 \\
6,550 \\
6,270 \\
5,990 \\
5,730 \\
5,600 \\
\end{array}$ & $\begin{array}{l}3,412 \\
3,412 \\
3,412 \\
3,412 \\
2,812\end{array}$ & $\begin{array}{l}1,716 \\
1,380 \\
1,340 \\
1,300 \\
1,260 \\
1,198\end{array}$ & $\begin{array}{l}972 \\
936 \\
900 \\
840 \\
810 \\
840\end{array}$ & $\begin{array}{l}1,340 \\
1,260 \\
1,300 \\
1,260 \\
1,260\end{array}$ \\
\hline $\begin{array}{l}\text { Total } \\
\text { Mean } \\
\text { Ac-ft }\end{array}$ & $\begin{array}{r}36,050 \\
1,163 \\
71,487\end{array}$ & $\begin{array}{r}51,9 \text { II } \\
1,730 \\
102,945\end{array}$ & $\begin{array}{r}68,600 \\
2,213 \\
136,034\end{array}$ & $\begin{array}{r}73,940 \\
2,385 \\
146,623\end{array}$ & $\begin{array}{r}79,774 \\
2,751 \\
158,192\end{array}$ & $\begin{array}{r}305,524 \\
9,856 \\
605,854\end{array}$ & $\begin{array}{r}598,900 \\
19,963 \\
1187,619\end{array}$ & $\begin{array}{r}363,450 \\
11,724 \\
720,721\end{array}$ & $\begin{array}{r}117,316 \\
3,910 \\
232,638 \\
\end{array}$ & $\begin{array}{r}56,794 \\
1,832 \\
112,623 \\
\end{array}$ & $\begin{array}{r}37,590 \\
1,213 \\
74,541 \\
\end{array}$ & $\begin{array}{r}31,730 \\
1,058 \\
62,921 \\
\end{array}$ \\
\hline
\end{tabular}

Calendar year 1947: Max 9,840 Min 336 Mean 2,719 Ac-ft 1,967,760

Water year 1947-48: Max 29,400 Min 750 Mean 4,983 Ac-ft 3,612,200

* Discharge measurement made on this day

Note.--No gage-heights 0ct. 2-7, 9-10, 13-14, 16, 18-20, 30, Nov. 29, 30; discharge averaged. 
HEIMAND RIVLR BASIN

Helmand River near Girishk, Afghanistan

Discharge in cubic feet per second, water year October 1948 to September 1949

\begin{tabular}{|c|c|c|c|c|c|c|c|c|c|c|c|c|}
\hline$\overline{\mathrm{D}}$ ay & $0 \mathrm{ct.1}$ & Nov. & Dec. 1 & Jan. & Feb. & Mar. & Apr. & May & June & July & Aug. & Sept. \\
\hline $\begin{array}{l}1 \\
2 \\
3 \\
4 \\
5\end{array}$ & $\begin{array}{l}1,250 \\
1,300 \\
1,300 \\
1,340 \\
1,340\end{array}$ & $\begin{array}{l}\frac{2,060}{2,120} \\
2,060 \\
2,060 \\
2,060\end{array}$ & $\begin{array}{l}2,280 \\
2,280 \\
2,280 \\
2,360 \\
2,360\end{array}$ & $\begin{array}{l}2,280 \\
2,440 \\
2,440 \\
2,360 \\
2,280\end{array}$ & $\begin{array}{l}2,800 \\
16,000\end{array}$ & $\begin{array}{l}3,760 \\
3,760 \\
3,900 \\
4,300 \\
4,460\end{array}$ & & & & & & \\
\hline $\begin{array}{r}6 \\
7 \\
8 \\
9 \\
10\end{array}$ & $\begin{array}{l}1,340 \\
1,390 \\
1,420 \\
1,500 \\
1,500\end{array}$ & $\begin{array}{l}2,060 \\
2,060 \\
2,120 \\
2,120 \\
2,120\end{array}$ & $\begin{array}{l}\frac{2}{2}, 440 \\
2,360 \\
2,360 \\
2,360\end{array}$ & $\begin{array}{l}2,200 \\
2,120 \\
2,120 \\
2,120 \\
2,200\end{array}$ & $\begin{array}{r}-3,500 \\
3,070 \\
3,070\end{array}$ & $\begin{array}{r}4,460 \\
4,620 \\
6,400 \\
28,900 \\
32,200\end{array}$ & & & & & & \\
\hline $\begin{array}{l}11 \\
12 \\
13 \\
14 \\
15\end{array}$ & $\begin{array}{l}1,420 \\
1,700 \\
1,540 \\
1,590 \\
1,590\end{array}$ & $\begin{array}{l}2,120 \\
2,120 \\
2,120 \\
2,120 \\
2,120\end{array}$ & $\begin{array}{l}2,360 \\
2,360 \\
2,360 \\
2,360 \\
2,360\end{array}$ & $\begin{array}{l}2,280 \\
2,360 \\
2,280 \\
2,280 \\
2,360\end{array}$ & $\begin{array}{l}3,070 \\
3,070 \\
3,170 \\
3,170 \\
3,170\end{array}$ & 20,750 & & & & & & \\
\hline $\begin{array}{l}16 \\
17 \\
18 \\
19 \\
20\end{array}$ & $\begin{array}{l}1,590 \\
1,590 \\
1,590 \\
1,590 \\
1,700\end{array}$ & $\begin{array}{l}2,200 \\
2,200 \\
2,200 \\
2,200 \\
2,200\end{array}$ & $\begin{array}{l}2,360 \\
2,360 \\
2,360 \\
2,360 \\
2,440\end{array}$ & $\begin{array}{l}2,360 \\
2,440 \\
2,440 \\
2,440 \\
2,440\end{array}$ & $\begin{array}{l}3,070 \\
3,070 \\
3,070 \\
3,070 \\
3,170\end{array}$ & & & & & & & \\
\hline $\begin{array}{l}21 \\
22 \\
23 \\
24 \\
25\end{array}$ & $\begin{array}{l}1,750 \\
1,750 \\
1,800 \\
1,800 \\
1,800\end{array}$ & $\begin{array}{l}2,280 \\
2,280 \\
2,280 \\
2,280 \\
2,280\end{array}$ & $\begin{array}{l}2,440 \\
2,440 \\
2,360 \\
2,360 \\
2,360\end{array}$ & $\begin{array}{l}2,520 \\
2,520 \\
2,520 \\
2,520 \\
2,520\end{array}$ & $\begin{array}{l}3,270 \\
3,640 \\
6,560 \\
5,900 \\
4,920\end{array}$ & & & & & & & \\
\hline $\begin{array}{l}26 \\
27 \\
28 \\
29 \\
30 \\
31 \\
\end{array}$ & $\begin{array}{l}1,860 \\
1,750 \\
1,920 \\
1,920 \\
1,920 \\
1,920\end{array}$ & $\begin{array}{l}\frac{2,360}{2,360} \\
2,360 \\
2,200 \\
2,200\end{array}$ & $\begin{array}{l}2,360 \\
2,360 \\
2,280 \\
2,280 \\
2,280 \\
2,280 \\
\end{array}$ & $\begin{array}{r}2,660 \\
2,660 \\
2,660 \\
2,750 \\
2,750 \\
2,750 \\
\end{array}$ & $\begin{array}{l}4,300 \\
4,040 \\
3,760\end{array}$ & & & & & & & \\
\hline $\begin{array}{l}\text { Total } \\
\text { Mean } \\
\text { Ac-ft }\end{array}$ & $\begin{array}{r}49,770 \\
1,605 \\
99,000\end{array}$ & $\begin{array}{r}65,320 \\
2,144 \\
130,000 \\
\end{array}$ & $\begin{array}{r}73,000 \\
2,355 \\
145,000 \\
\end{array}$ & $\begin{array}{r}75,070 \\
2,422 \\
149,000 \\
\end{array}$ & $\begin{array}{r}112,830 \\
4,029 \\
224,000 \\
\end{array}$ & & & & & & & \\
\hline
\end{tabular}

Calendar year 1948: Max 29,400 Min 810 Mean 5,063 Ac-ft 3,675,730

Note.-No gage-heights Feb. 1-8, Mar. 9-11; discharge estimated on basis of records at Kajakal.

Gaging station destroyed by flood Mar. 1l, 1949. 
HELMAND RIVER BASIN

Helmand River at Iashkar Gah, Afghanistan

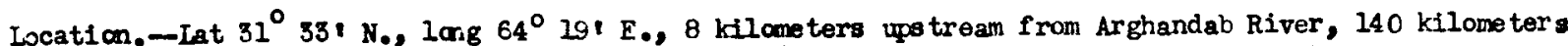
west of Kandahar and 320 kilometers uptrean from Chahar BurJak.

Drainage area.- $-22,300 \mathrm{sq} \mathrm{mi}$, approximately.

Records avallable.--October 1954 to May 1957.

Gage. - - Etaff gage an bridge pile with zero at elevation 764.27 meter mean sea level.

Bxtremes .-Waximum and minimm discharge for the 1954-57 water years are given in the follang tables

\begin{tabular}{|c|c|c|c|c|c|c|}
\hline \multirow[b]{2}{*}{$\begin{array}{l}\text { Water } \\
\text { Year }\end{array}$} & \multicolumn{3}{|c|}{ Maximum } & \multicolumn{3}{|c|}{ Mnimum } \\
\hline & Date & $\begin{array}{l}\text { Gage height } \\
\text { (meters) }\end{array}$ & $\begin{array}{c}\text { Discharg } \\
\text { (cf8) }\end{array}$ & Date & $\begin{array}{l}\text { Gage height } \\
\text { (neters) }\end{array}$ & $\begin{array}{c}\text { Digcharge } \\
\text { (crs) }\end{array}$ \\
\hline $\begin{array}{l}1954 \\
1955 \\
1956 \\
1957\end{array}$ & $\begin{array}{l}\text { Feb. } 10,1954 \\
\text { Mar. } 15,1955 \\
\text { Apr. 9, } 1956 \\
\text { May 5, } 1957\end{array}$ & $\begin{array}{l}3.70 \\
4.58 \\
4.54 \\
4.78\end{array}$ & $\begin{array}{r}29,500 \\
43,600 \\
42,900 \\
249,700\end{array}$ & $\begin{array}{l}\text { Nov. } 14,1953 \\
\text { Dec. 9, } 1954 \\
\text { Sept. 8-10, } 1956 \\
\text { Oct. 29, } 1956\end{array}$ & $\begin{array}{l}1.05 \\
1.055 \\
-.885\end{array}$ & $\begin{array}{r}* 207 \\
2185 \\
21,600 \\
2199\end{array}$ \\
\hline
\end{tabular}

a Mean dafly discharge.

* Dis charge measurement.

Remarks.-Records fair except those for periods of no gage-heights which are poor. 
HELMAND RIVER BASTN

Helmand River at Lashkar Gah, Afgranistan

Discharge, in cubic feet per second, water year october 1953 to september 1954

\begin{tabular}{|c|c|c|c|c|c|c|c|c|c|c|c|c|}
\hline Day & oct. & Nor. & Dec. & Jan. & Feb. & Mar. & Apr. & May & Jume & July & Aug. & Sept. \\
\hline $\begin{array}{l}1 \\
2 \\
3 \\
4 \\
5\end{array}$ & $\begin{array}{l}3,750 \\
5,750 \\
3,750 \\
3,700 \\
3,700\end{array}$ & $\begin{array}{l}\frac{3,600}{3,600} \\
3,600 \\
3,600 \\
3,600\end{array}$ & $\begin{array}{l}3,600 \\
3,600 \\
3,600 \\
3,500 \\
2,000\end{array}$ & $\begin{array}{l}1,800 \\
1,800 \\
1,800 \\
1,900 \\
2,200\end{array}$ & $\begin{array}{l}6,000 \\
\frac{6}{0}, 00 \\
6,100 \\
6,200 \\
6,600\end{array}$ & $\begin{array}{l}6,770 \\
6,870 \\
6,820 \\
6,630 \\
6,530\end{array}$ & $\begin{array}{r}12,600 \\
10,100 \\
9,190 \\
8,700 \\
8,350\end{array}$ & $\begin{array}{l}27,400 \\
27,600 \\
27,700 \\
28,000 \\
28,400 \\
\end{array}$ & $\begin{array}{l}\frac{13,200}{12,600} \\
12,300 \\
11,800 \\
11,300\end{array}$ & $\begin{array}{r}2,970 \\
750 \\
* 596 \\
978 \\
2,010\end{array}$ & $\begin{array}{l}4,360 \\
4,400 \\
4,360 \\
4,300 \\
4,300\end{array}$ & $\begin{array}{l}\frac{4,200}{4,200} \\
4,200 \\
4,200 \\
4,200\end{array}$ \\
\hline $\begin{array}{r}6 \\
7 \\
8 \\
9 \\
10\end{array}$ & $\begin{array}{l}3,700 \\
3,700 \\
3,700 \\
3,700 \\
3,700\end{array}$ & $\begin{array}{l}3,550 \\
3,550 \\
3,550 \\
3,500 \\
3,500\end{array}$ & $\begin{array}{r}250 \\
250 \\
500 \\
3,600 \\
3,700\end{array}$ & $\begin{array}{r}* 7,460 \\
* 3,530 \\
2,600 \\
2,300 \\
2,000\end{array}$ & $\begin{array}{r}7,200 \\
9,000 \\
15,000 \\
20,200 \\
* 18,600\end{array}$ & $\begin{array}{l}6,440 \\
6,390 \\
6,250 \\
6,300 \\
6,440\end{array}$ & $\begin{array}{l}7,930 \\
7,650 \\
7,440 \\
7,300 \\
7,370\end{array}$ & $\begin{array}{l}27,900 \\
27,600 \\
27,700 \\
27,600 \\
27,600\end{array}$ & $\begin{array}{r}11,200 \\
11,000 \\
10,800 \\
10,400 \\
0,950\end{array}$ & $\begin{array}{l}2,890 \\
3,340 \\
3,130 \\
3,130 \\
3,010\end{array}$ & $\begin{array}{l}4,300 \\
4,300 \\
4,300 \\
4,300 \\
4,300\end{array}$ & $\begin{array}{r}4,200 \\
4,200 \\
4,200 \\
* 3,320 \\
3,250\end{array}$ \\
\hline $\begin{array}{l}11 \\
12 \\
13 \\
14 \\
15\end{array}$ & $\begin{array}{l}3,700 \\
3,700 \\
3,700 \\
3,700 \\
3,650\end{array}$ & $\begin{array}{r}3,300 \\
1,500 \\
220 \\
* 207 \\
\frac{250}{250}\end{array}$ & $\begin{array}{l}3,700 \\
3,750 \\
3,750 \\
3,750 \\
3,750\end{array}$ & $\begin{array}{r}1,700 \\
1,500 \\
1,450 \\
1,400 \\
* 1,390\end{array}$ & $\begin{array}{r}17,300 \\
12,200 \\
10,400 \\
10,100 \\
9,750\end{array}$ & $\begin{array}{l}6,440 \\
6,390 \\
6,390 \\
6,340 \\
6,300\end{array}$ & $\begin{array}{l}7,110 \\
7,110 \\
6,920 \\
6,820 \\
6,820\end{array}$ & $\begin{array}{l}26,600 \\
25,300 \\
25,000 \\
24,900 \\
24,500\end{array}$ & $\begin{array}{l}9,890 \\
9,470 \\
9,190 \\
8,770 \\
8,280\end{array}$ & $\begin{array}{r}* 3,130 \\
3,090 \\
3,010 \\
3,010 \\
3,090\end{array}$ & $\begin{array}{l}4,300 \\
4,300 \\
4,300 \\
4,300 \\
4,300\end{array}$ & $\begin{array}{l}3,250 \\
3,250 \\
3,250 \\
3,200 \\
3,200\end{array}$ \\
\hline $\begin{array}{l}16 \\
17 \\
18 \\
19 \\
20\end{array}$ & $\begin{array}{l}3,650 \\
3,650 \\
3,650 \\
3,650 \\
3,650\end{array}$ & $\begin{array}{l}1,100 \\
3,100 \\
3,300 \\
3,350 \\
3,350\end{array}$ & $\begin{array}{l}3,750 \\
3,750 \\
3,750 \\
3,750 \\
3,750\end{array}$ & $\begin{array}{l}1,800 \\
1,850 \\
1,850 \\
1,850 \\
2,400\end{array}$ & $\begin{array}{r}9,400 \\
8,490 \\
10,800 \\
8,840 \\
8,350\end{array}$ & $\begin{array}{l}6,250 \\
6,250 \\
6,160 \\
6,200 \\
6,300\end{array}$ & $\begin{array}{l}8,000 \\
9,050 \\
7,650 \\
8,210 \\
9,680\end{array}$ & $\begin{array}{l}22,800 \\
21,000 \\
19,500 \\
18,800 \\
18,400\end{array}$ & $\begin{array}{r}* 8,070 \\
7,720 \\
7,510 \\
7,370 \\
7,110\end{array}$ & $\begin{array}{l}3,010 \\
3,920 \\
3,260 \\
3,260 \\
3,130\end{array}$ & $\begin{array}{l}4,300 \\
4,300 \\
4,300 \\
4,300 \\
4,300\end{array}$ & $\begin{array}{l}3,300 \\
3,300 \\
3,400 \\
3,500 \\
3,500\end{array}$ \\
\hline $\begin{array}{l}21 \\
22 \\
23 \\
24 \\
25\end{array}$ & $\begin{array}{l}\frac{3,600}{3,600} \\
3,500 \\
3,600 \\
3,600\end{array}$ & $\begin{array}{l}3,350 \\
3,400 \\
3,400 \\
3,400 \\
3,500\end{array}$ & $\begin{array}{r}3,750 \\
3,500 \\
2,000 \\
280 \\
260\end{array}$ & $\begin{array}{l}3,700 \\
3,900 \\
4,100 \\
4,400 \\
4,600\end{array}$ & $\begin{array}{l}7,930 \\
7,720 \\
7,580 \\
7,110 \\
7,010\end{array}$ & $\begin{array}{l}6,400 \\
6,600 \\
6,700 \\
6,820 \\
6,770\end{array}$ & $\begin{array}{r}12,600 \\
* 16,400 \\
* 23,600 \\
26,000 \\
28,300\end{array}$ & $\begin{array}{r}18,000 \\
17,800 \\
17,400 \\
* 15,200 \\
16,800\end{array}$ & $\begin{array}{l}6,920 \\
6,870 \\
6,630 \\
6,630 \\
6,580\end{array}$ & $\begin{array}{r}3,260 \\
3,260 \\
3,260 \\
+3,260 \\
3,170\end{array}$ & $\begin{array}{l}4,300 \\
4,300 \\
4,250 \\
4,250 \\
4,250\end{array}$ & $\begin{array}{l}3,500 \\
3,500 \\
3,500 \\
3,500 \\
3,500\end{array}$ \\
\hline $\begin{array}{l}26 \\
27 \\
28 \\
29 \\
30 \\
31\end{array}$ & $\begin{array}{l}3,600 \\
3,600 \\
3,600 \\
3,600 \\
3,600 \\
3,600\end{array}$ & $\begin{array}{l}3,600 \\
3,600 \\
3,500 \\
3,600 \\
3,600\end{array}$ & $\begin{array}{r}250 \\
240 \\
240 \\
240 \\
240 \\
1,500\end{array}$ & $\begin{array}{r}4,900 \\
5,200 \\
5,500 \\
5,900 \\
6,000 \\
* 6,130\end{array}$ & $\begin{array}{l}6,970 \\
6,770 \\
6,870\end{array}$ & $\begin{array}{r}6,770 \\
6,820 \\
6,360 \\
7,010 \\
21,800 \\
14,300\end{array}$ & $\begin{array}{r}* 28,400 \\
27,900 \\
27,500 \\
27,400 \\
27,300\end{array}$ & $\begin{array}{l}16,000 \\
15,300 \\
15,000 \\
14,800 \\
14,500 \\
14,200 \\
\end{array}$ & $\begin{array}{l}6,340 \\
6,340 \\
6,250 \\
6,060 \\
5,880 \\
\end{array}$ & $\begin{array}{l}3,170 \\
3,420 \\
3,700 \\
4,140 \\
4,360 \\
4,360\end{array}$ & $\begin{array}{l}4,250 \\
4,250 \\
4,250 \\
4,250 \\
4,200 \\
4,200\end{array}$ & $\begin{array}{l}3,300 \\
3,100 \\
3,000 \\
3,000 \\
3,000\end{array}$ \\
\hline $\begin{array}{l}\text { Total } \\
\text { Mean } \\
\text { Ac-ft }\end{array}$ & $\begin{array}{r}113,450 \\
3,660 \\
225,020\end{array}$ & $\begin{array}{r}90,377 \\
3,013 \\
179,260 \\
\end{array}$ & $\begin{array}{r}74,550 \\
2,405 \\
147,870 \\
\end{array}$ & $\begin{array}{r}98,910 \\
3,191 \\
10 \%, 180 \\
\end{array}$ & $\begin{array}{r}264,490 \\
9,446 \\
524,610 \\
\end{array}$ & $\begin{array}{r}225,410 \\
7,271 \\
447,090 \\
\end{array}$ & $\begin{array}{r}409,400 \\
13,650 \\
812,030 \\
\end{array}$ & $\begin{array}{r}680,400 \\
21,950 \\
1,349,550 \\
\end{array}$ & $\begin{array}{r}262,440 \\
3,748 \\
520,540 \\
\end{array}$ & $\begin{array}{r}94,074 \\
3,035 \\
186,590 \\
\end{array}$ & $\begin{array}{r}132,970 \\
4,289 \\
263,740 \\
\end{array}$ & $\begin{array}{r}10,220 \\
3,541 \\
210,680 \\
\end{array}$ \\
\hline \multicolumn{3}{|c|}{ Water year 1953-54: } & $\operatorname{Max}$ & 28,400 & Hin & 207 & Men & 6,994 & $A C-f t$ & 5,06 & & \\
\hline
\end{tabular}

* Discharge measurement made on this daj.

Note.-No gage-heights Oct. 1 to Feb. 8, Jar. 19-23, Apr. 16-24, Aug. 4 to Sept. 30; discharge estinated

on basis of records for stations below Kajakal Reservolr and at Chahar Burjak. 
HEUTAN RIVER BAS DI

Helnmd River at Iashkar Gah, Mfghanistan

Diseherge, in cublc feet per second, water year october 1954 to september 1955

\begin{tabular}{|c|c|c|c|c|c|c|c|c|c|c|c|c|}
\hline 4 & oct. & Ior. & bee. & Jin. & 10.0 & Yxe & 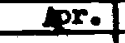 & $7: 2$ & June & suty & 8. & Sept. \\
\hline $\begin{array}{l}1 \\
2 \\
5 \\
4 \\
5\end{array}$ & $\begin{array}{l}2,700 \\
2,700 \\
2,700 \\
2,700 \\
2,700\end{array}$ & $\begin{array}{r}3,260 \\
3,170 \\
5,170 \\
5,170 \\
5,220\end{array}$ & $\begin{array}{l}3,170 \\
3,150 \\
2,780 \\
2,380 \\
1,420\end{array}$ & $\begin{array}{l}6,410 \\
6,300 \\
6,160 \\
6,160 \\
6,200\end{array}$ & $\begin{array}{l}7,650 \\
6,510 \\
6,600 \\
6,920 \\
7,160\end{array}$ & $\begin{array}{l}5,510 \\
4,940 \\
4,660 \\
4,570 \\
4,480\end{array}$ & $\begin{array}{l}4,500 \\
, 500 \\
4,500 \\
4,500 \\
4,500\end{array}$ & $\begin{array}{l}5,200 \\
5,200 \\
5,200 \\
5,200 \\
5,200\end{array}$ & $\begin{array}{l}7,500 \\
7,600 \\
7,600 \\
7,500 \\
7,500\end{array}$ & $\begin{array}{l}3,400 \\
\frac{3,200}{3,300} \\
3,200 \\
3,150\end{array}$ & $\begin{array}{l}3,200 \\
3,250 \\
5,300 \\
5,300 \\
5,350\end{array}$ & $\begin{array}{l}3,550 \\
3,550 \\
3,550 \\
3,550 \\
3,550\end{array}$ \\
\hline $\begin{array}{r}6 \\
7 \\
8 \\
9 \\
10\end{array}$ & $\begin{array}{l}2,700 \\
2,710 \\
2,620 \\
2,630 \\
2,600 \\
\end{array}$ & $\begin{array}{l}5,170 \\
5,220 \\
5,220 \\
5,260 \\
5,220\end{array}$ & $\begin{array}{r}528 \\
272 \\
203 \\
* 185 \\
2,710\end{array}$ & $\begin{array}{l}6,400 \\
6,600 \\
6,800 \\
7,000 \\
7,200\end{array}$ & $\begin{array}{l}6,960 \\
6,960 \\
6,870 \\
6,480 \\
6,820\end{array}$ & $\begin{array}{l}4,560 \\
4,220 \\
4,400 \\
4,220 \\
3,880\end{array}$ & $\begin{array}{l}4,500 \\
4,500 \\
4,500 \\
4,500 \\
4,500\end{array}$ & $\begin{array}{l}5,200 \\
5,200 \\
5,200 \\
5,200 \\
5,200\end{array}$ & $\begin{array}{l}7,200 \\
7,100 \\
7,000 \\
6,800 \\
6,700\end{array}$ & $\begin{array}{l}3,100 \\
3,050 \\
5,000 \\
2,950 \\
2,900\end{array}$ & $\begin{array}{l}3,400 \\
3,400 \\
3,400 \\
3,400 \\
3,400\end{array}$ & $\begin{array}{l}\mathbf{5 5 0} \\
\mathbf{5 5 0} \\
\mathbf{5 5 0} \\
\mathbf{5 5 0} \\
\mathbf{5 5 0}\end{array}$ \\
\hline $\begin{array}{l}11 \\
12 \\
15 \\
14 \\
15\end{array}$ & $\begin{array}{r}2,650 \\
2,670 \\
2,750 \\
2,820 \\
2,850\end{array}$ & $\begin{array}{l}5,090 \\
3,090 \\
3,130 \\
3,170 \\
3,090\end{array}$ & $\begin{array}{l}5,100 \\
5,600 \\
5,700 \\
5,700 \\
5,650\end{array}$ & $\begin{array}{l}7,200 \\
7,200 \\
7,200 \\
7,200 \\
7,200\end{array}$ & $\begin{array}{l}6,620 \\
6,410 \\
6,200 \\
6,500 \\
6,500\end{array}$ & $\begin{array}{r}5,650 \\
5,420 \\
3,580 \\
5,420 \\
29,900 \\
\end{array}$ & $\begin{array}{l}4,500 \\
4,500 \\
4,500 \\
4,500 \\
4,500\end{array}$ & $\begin{array}{l}5,200 \\
5,200 \\
5,200 \\
5,200 \\
5,200\end{array}$ & $\begin{array}{l}6,600 \\
6,400 \\
6,100 \\
6,000 \\
5,900\end{array}$ & $\begin{array}{l}2,900 \\
2,850 \\
2,800 \\
2,800 \\
2,750\end{array}$ & $\begin{array}{l}3,350 \\
3,300 \\
3,500 \\
5,400 \\
3,400\end{array}$ & $\begin{array}{l}550 \\
550 \\
50 \\
550 \\
550\end{array}$ \\
\hline $\begin{array}{l}18 \\
17 \\
18 \\
19 \\
20\end{array}$ & $\begin{array}{l}2,850 \\
2,850 \\
2,890 \\
2,930 \\
5,010\end{array}$ & $\begin{array}{l}3,010 \\
3,010 \\
5,010 \\
3,010 \\
5,090\end{array}$ & $\begin{array}{l}5,840 \\
5,790 \\
5,790 \\
5,790 \\
5,790\end{array}$ & $\begin{array}{l}7,200 \\
7,200 \\
7,500 \\
7,400 \\
7,500\end{array}$ & $\begin{array}{l}6,020 \\
6,930 \\
6,590 \\
6,200 \\
6,020\end{array}$ & $\begin{array}{r}19,800 \\
12,700 \\
10,600 \\
8,490 \\
9,100\end{array}$ & $\begin{array}{l}4,600 \\
4,700 \\
4,900 \\
5,200 \\
5,200\end{array}$ & $\begin{array}{l}5,300 \\
5,300 \\
5,300 \\
5,500 \\
5,500\end{array}$ & $\begin{array}{l}5,800 \\
5,700 \\
5,600 \\
5,500 \\
5,400\end{array}$ & $\begin{array}{l}2,700 \\
2,600 \\
2,500 \\
2,450 \\
\frac{2,550}{2}\end{array}$ & $\begin{array}{r}+380 \\
3,350 \\
5,350 \\
3,400 \\
5,400\end{array}$ & $\begin{array}{l}3,550 \\
3,500 \\
3,500 \\
3,500 \\
3,500\end{array}$ \\
\hline $\begin{array}{l}21 \\
22 \\
25 \\
24 \\
25\end{array}$ & $\begin{array}{l}3,010 \\
3,090 \\
3,090 \\
3,090 \\
5,080\end{array}$ & $\begin{array}{l}3,090 \\
3,090 \\
5,010 \\
3,010 \\
5,010\end{array}$ & $\begin{array}{l}5,970 \\
4,800 \\
4,800 \\
4,400 \\
5,920\end{array}$ & $\begin{array}{l}7,600 \\
7,600 \\
7,600 \\
7,600 \\
7,600\end{array}$ & $\begin{array}{l}5,790 \\
5,740 \\
5,560 \\
5,700 \\
5,700\end{array}$ & $\begin{array}{l}9,960 \\
6,580 \\
6,300 \\
6,160 \\
6,000\end{array}$ & $\begin{array}{r}5,200 \\
5,100 \\
5,000 \\
5,000 \\
5,990\end{array}$ & $\begin{array}{l}5,300 \\
5,300 \\
5,300 \\
5,300 \\
5,300\end{array}$ & $\begin{array}{l}5,300 \\
5,200 \\
4,700 \\
4,000 \\
3,400\end{array}$ & $\begin{array}{l}2,900 \\
3,000 \\
3,100 \\
3,200 \\
3,200\end{array}$ & $\begin{array}{l}3,400 \\
3,400 \\
3,450 \\
3,450 \\
5,500 \\
\end{array}$ & $\begin{array}{l}500 \\
.450 \\
.400 \\
550 \\
.550\end{array}$ \\
\hline $\begin{array}{l}28 \\
27 \\
28 \\
28 \\
50 \\
51\end{array}$ & $\begin{array}{l}3,010 \\
5,090 \\
5,090 \\
3,090 \\
3,090 \\
3,150\end{array}$ & $\begin{array}{r}5,010 \\
*, 150 \\
5,170 \\
5,170 \\
5,170\end{array}$ & $\begin{array}{r}3,750 \\
3,750 \\
3,750 \\
* 5,470 \\
6,060 \\
6,550 \\
\end{array}$ & $\begin{array}{r}7,650 \\
7,650 \\
7,650 \\
* 7,650 \\
7,650 \\
* 7,650\end{array}$ & $\begin{array}{r}5,750 \\
* 5,700 \\
5,740\end{array}$ & $\begin{array}{r}5,840 \\
5,740 \\
5,510 \\
5,510 \\
+4,440 \\
4,400\end{array}$ & $\begin{array}{l}5,000 \\
5,100 \\
5,100 \\
5,100 \\
5,100\end{array}$ & $\begin{array}{l}5,400 \\
6,000 \\
6,500 \\
7,000 \\
7,300 \\
7,400\end{array}$ & $\begin{array}{r}+5,160 \\
3,300 \\
5,400 \\
5,400 \\
5,400\end{array}$ & $\begin{array}{l}3,100 \\
3,000 \\
5,000 \\
3,050 \\
5,100 \\
5,150\end{array}$ & $\begin{array}{l}3,500 \\
3,500 \\
3,500 \\
3,500 \\
3,500 \\
3,500\end{array}$ & $\begin{array}{l}3,400 \\
3,450 \\
3,450 \\
3,500 \\
3,500\end{array}$ \\
\hline $\begin{array}{l}\text { Total } \\
\text { loan } \\
\text { co-ft }\end{array}$ & $\begin{array}{r}89,080 \\
2,874 \\
17,700\end{array}$ & $\begin{array}{r}98,610 \\
3,121 \\
185,750\end{array}$ & $\begin{array}{r}126,729 \\
4,088 \\
251,360\end{array}$ & $\begin{array}{r}221,760 \\
7,154 \\
489,850\end{array}$ & $\begin{array}{r}177,530 \\
6,340 \\
552,130\end{array}$ & $\begin{array}{r}219,450 \\
7,078 \\
455,250\end{array}$ & $\begin{array}{r}142,790 \\
4,760 \\
285,220\end{array}$ & $\begin{array}{r}170,600 \\
5,505 \\
558,380\end{array}$ & $\begin{array}{r}170,560 \\
5,685 \\
538,800\end{array}$ & $\begin{array}{r}92,150 \\
2,975 \\
182,780\end{array}$ & $\begin{array}{r}105,250 \\
5,395 \\
208,720\end{array}$ & $\begin{array}{r}105,150 \\
3,505 \\
208,550\end{array}$ \\
\hline & & & Fin & & & $\begin{array}{l}185 \\
185\end{array}$ & & $\operatorname{an}_{n}$ & & $-f t$ & & \\
\hline
\end{tabular}

* DIscharg meas urewent ande on this dVy.

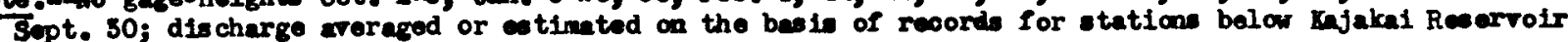
and at Chahar Burjak. 


\section{HELMAND RTVER BASTN}

Helmand River at Lashkar Gah, Afghanistan

Discharge, in cubic feet per second, water year october 1955 to September 1956

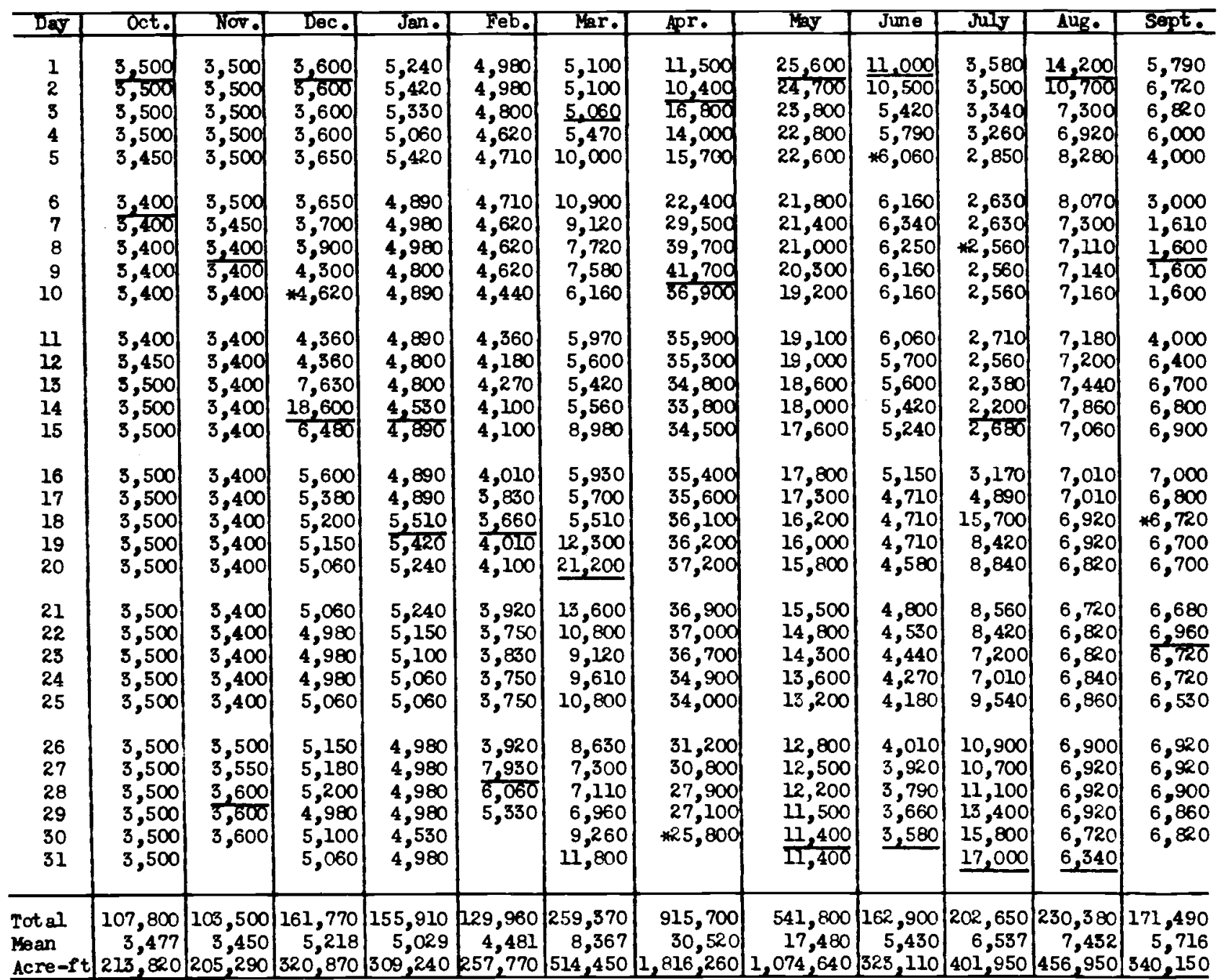

$\begin{array}{lllllll}\text { Calendar Year 1955: } & \text { Max 29,900 } & \text { Min } 3,160 & \text { Mean 4,872 } & \text { Ac-ft 3,527,150 } \\ \text { Water year 1955-56: } & \text { Max 41,700 } & \text { Min } 1,600 & \text { Mean 8,588 } & \text { Ac-ft } 6,234,500\end{array}$

* Discharge measurement made on this day.

Note.-No gage-heights Oct. 1 to Dec. 9, Dec. 27, Jan. 1, Feb. 3, Mar. 23, May 2, 11, 25, 30, June 1, 8, 28, July 13, 15, 29, Aug. 9-11, 24-26, Sept. 4-6, 3-17, 19-20, 28, 29; discharge averaged or est1mated on the

bas is of records for stations below Kajakai Reservoir and at Chahar Burjak. 
HETMAND RIVER BASIN

Helnand River at Lashkar Gah, Mghanistan

Discharge, in cubic feet per second, water year October 1956 to September 1957

\begin{tabular}{|c|c|c|c|c|c|c|c|c|c|c|c|c|}
\hline DEV & oct. & Hor. & Doc. & Jan. & Feb. & Mar. & pro. & My & Jume & JuY & Auge & sept. \\
\hline $\begin{array}{l}1 \\
2 \\
3 \\
4 \\
5\end{array}$ & $\begin{array}{l}7,010 \\
7,060 \\
7,110 \\
7,110 \\
7,060\end{array}$ & $\begin{array}{l}5,600 \\
5,600 \\
5,600 \\
5,600 \\
5,740\end{array}$ & $\begin{array}{l}5,700 \\
5,700 \\
5,700 \\
5,700 \\
5,700\end{array}$ & $\begin{array}{l}8,000 \\
8,000 \\
7,720 \\
8,140 \\
8,200\end{array}$ & $\begin{array}{l}10,500 \\
10,900 \\
11,100 \\
10,400 \\
10,400\end{array}$ & $\begin{array}{l}7,460 \\
8,840 \\
9,120 \\
9,120 \\
9,120\end{array}$ & $\begin{array}{l}16,000 \\
16,000 \\
18,000 \\
26,900 \\
40,900\end{array}$ & $\begin{array}{l}36,000 \\
38,300 \\
42,800 \\
48,700 \\
49,700 \\
\end{array}$ & & & & \\
\hline $\begin{array}{r}6 \\
7 \\
8 \\
9 \\
10\end{array}$ & $\begin{array}{l}7,110 \\
7,200 \\
7,200 \\
7,440 \\
7,440\end{array}$ & $\begin{array}{l}5,740 \\
5,770 \\
5,740 \\
5,700 \\
5,970 \\
\end{array}$ & $\begin{array}{l}5,700 \\
5,790 \\
5,850 \\
5,850 \\
5,850\end{array}$ & $\begin{array}{l}8,200 \\
8,140 \\
8,000 \\
8,000 \\
8,000\end{array}$ & $\begin{array}{l}20,900 \\
11,200 \\
\frac{11}{2}, 200 \\
11,200 \\
11,100\end{array}$ & $\begin{array}{l}9,050 \\
9,050 \\
9,120 \\
9,050 \\
9,120\end{array}$ & $\begin{array}{l}40,400 \\
40,500 \\
42,500 \\
41,700 \\
41,400\end{array}$ & $\begin{array}{l}48,100 \\
45,200 \\
43,000 \\
42,000 \\
41,000\end{array}$ & & & & \\
\hline $\begin{array}{l}11 \\
12 \\
13 \\
14 \\
15\end{array}$ & $\begin{array}{l}7,300 \\
7,300 \\
4,980 \\
2,070 \\
1,900\end{array}$ & $\begin{array}{l}5,790 \\
5,790 \\
5,790 \\
5,790 \\
5,790\end{array}$ & $\begin{array}{l}5,850 \\
5,850 \\
5,850 \\
5,850 \\
5,850\end{array}$ & $\begin{array}{r}8,000 \\
8,000 \\
16,000 \\
10,000 \\
8,630\end{array}$ & $\begin{array}{r}10,400 \\
10,400 \\
9,960 \\
9,960 \\
9,960\end{array}$ & $\begin{array}{l}9,050 \\
9,260 \\
9,680 \\
9,820 \\
9,820\end{array}$ & $\begin{array}{l}40,700 \\
40,200 \\
40,700 \\
42,100 \\
44,100\end{array}$ & $\begin{array}{l}41,000 \\
41,000 \\
41,000 \\
40,000 \\
37,000\end{array}$ & & & & \\
\hline $\begin{array}{l}16 \\
17 \\
18 \\
19 \\
20\end{array}$ & $\begin{array}{r}1,370 \\
1,420 \\
1,370 \\
1,320 \\
610\end{array}$ & $\begin{array}{l}5,790 \\
5,790 \\
5,790 \\
5,790 \\
5,790\end{array}$ & $\begin{array}{l}\mathbf{5}, 850 \\
\mathbf{5}, 850 \\
\mathbf{5}, 850 \\
\mathbf{5}, 850 \\
\mathbf{5 , 8 5 0}\end{array}$ & $\begin{array}{l}8,280 \\
8,280 \\
8,690 \\
9,540 \\
9,380\end{array}$ & $\begin{array}{l}9,960 \\
9,960 \\
9,960 \\
9,960 \\
8,960\end{array}$ & $\begin{array}{r}9,680 \\
1.0,200 \\
49,000 \\
44,000 \\
30,000\end{array}$ & $\begin{array}{l}45,000 \\
45,000 \\
43,700 \\
42,500 \\
42,800\end{array}$ & $\begin{array}{l}35, \infty 00 \\
34,0 \infty 0 \\
32, \infty 00 \\
31, \infty 00 \\
30, \infty 00\end{array}$ & & & & \\
\hline $\begin{array}{l}21 \\
22 \\
23 \\
24 \\
25\end{array}$ & $\begin{array}{r}610 \\
* 98 \\
459 \\
448 \\
426\end{array}$ & $\begin{array}{l}5,790 \\
5,790 \\
5,790 \\
5,790 \\
5,790\end{array}$ & $\begin{array}{l}6,000 \\
6,400 \\
6,600 \\
6,500 \\
6,530\end{array}$ & $\begin{array}{r}9,820 \\
3,780 \\
9,750 \\
11,100 \\
15,2.00\end{array}$ & $\begin{array}{l}9,960 \\
9,400 \\
9,540 \\
9,540 \\
9,540\end{array}$ & $\begin{array}{l}21,200 \\
19,500 \\
17,800 \\
17, \infty 00 \\
16,000\end{array}$ & $\begin{array}{l}42,800 \\
41,200 \\
40,800 \\
40,400 \\
40,000\end{array}$ & $\begin{array}{l}29,000 \\
28,500 \\
28,000 \\
27,500 \\
27,000\end{array}$ & & & & \\
\hline $\begin{array}{l}26 \\
27 \\
28 \\
29 \\
30 \\
31\end{array}$ & $\begin{array}{r}338 \\
316 \\
272 \\
199 \\
2, \frac{150}{150} \\
5,560\end{array}$ & $\begin{array}{l}5,730 \\
5,790 \\
5,790 \\
5,790 \\
5,790\end{array}$ & $\begin{array}{l}6,500 \\
6,500 \\
6,500 \\
7,000 \\
7,600 \\
7,800\end{array}$ & $\begin{array}{l}19,400 \\
16,000 \\
12,600 \\
12,300 \\
11,700 \\
11,100\end{array}$ & $\begin{array}{l}5,790 \\
3,000 \\
1,460 \\
\end{array}$ & $\begin{array}{l}17,000 \\
18,000 \\
20,000 \\
19,200 \\
17,800 \\
16,400\end{array}$ & $\begin{array}{l}38,000 \\
37,000 \\
37,000 \\
36,000 \\
35,000\end{array}$ & $\begin{array}{l}25,000 \\
24,000 \\
22,800 \\
23,000 \\
23,000 \\
23,000\end{array}$ & & & & \\
\hline $\begin{array}{l}\text { Total } \\
\text { Moan } \\
\text { se-ft. }\end{array}$ & $\begin{array}{r}112,635 \\
3,635 \\
223,410 \\
\end{array}$ & $\begin{array}{r}172,850 \\
5,761 \\
342,800 \\
\end{array}$ & $\begin{array}{r}189,970 \\
6,128 \\
876,800 \\
\end{array}$ & $\begin{array}{r}313,900 \\
10,130 \\
622,610 \\
\end{array}$ & $\begin{array}{r}287,610 \\
9,558 \\
530,800 \\
\end{array}$ & $\begin{array}{r}473,460 \\
15,270 \\
939,090 \\
\end{array}$ & $\begin{array}{r}1,139,400 \\
37,980 \\
2,259,970 \\
\end{array}$ & $\begin{array}{r}1,077,600 \\
34,760 \\
2,137,390 \\
\end{array}$ & & & & \\
\hline end & yesx & & $\operatorname{Max}$ & 37,200 & vin & 199 & Hean & & $-f t$ & $=0$ & & \\
\hline
\end{tabular}

* Discharge measurement made an this day.

Hote - Ho gage-hoights Nov. 26, Now. 28 to Dec. 2 , Dec. $8-24,26-31, \operatorname{Jan} .5,6,9-11,18,22,25,27,30$, 31 , Fob. 27, Mar. 18-20, 22, 24-27, 30, ppr. 1-3, Apr. 25 to May 1, 84 27, 29-31; discharge averaged or estimated on basis of records for stations below Kajakai Reservoir and at Darweshan. 
HELAAND RIVER AT LASHKAR GAH, LTOHANISTAN

Fragmentary Rocord

1958

\begin{tabular}{|c|c|c|c|c|c|}
\hline Date & $\begin{array}{c}\text { Gage HeIfht } \\
\text { (weters) }\end{array}$ & $\begin{array}{c}\text { Discharge } \\
\text { (cis) }\end{array}$ & Dato & $\begin{array}{l}\text { Cage holght } \\
\text { (meters) }\end{array}$ & $\begin{array}{c}\text { Discharge } \\
\text { (cfs) }\end{array}$ \\
\hline $\begin{array}{c}\text { Yor. } 20,1957 \\
24 \\
25 \\
26 \\
\text { Dec. } 6 \\
7 \\
* 24 \\
* 26\end{array}$ & $\begin{array}{l}0.885 \\
1.62 \\
1.645 \\
1.62 \\
1.65 \\
1.95 \\
1.89 \\
1.88\end{array}$ & $\begin{array}{r}595 \\
4,620 \\
4,940 \\
4,710 \\
5,550 \\
8,560 \\
7,720 \\
7,580\end{array}$ & $\begin{array}{c}* \text { Dec. } 30,1957 \\
\text { pr. } 17,1958 \\
18 \\
19 \\
20 \\
21 \\
27\end{array}$ & $\begin{array}{l}1.68 \\
2.04 \\
2.10 \\
2.40 \\
2.50 \\
2.62 \\
2.90\end{array}$ & $\begin{array}{l}8,700 \\
10,700 \\
11,500 \\
15,800 \\
17,300 \\
19,100 \\
23,300\end{array}$ \\
\hline
\end{tabular}

* Discharge masurement made on this da

1959

\begin{tabular}{|c|c|c|c|c|c|}
\hline Date & $\begin{array}{l}\text { Gage Heifht } \\
\text { (meters) }\end{array}$ & $\begin{array}{c}\text { Discharge } \\
\text { (cfs) }\end{array}$ & Date & $\begin{array}{l}\text { Gage helght } \\
\text { (meters) }\end{array}$ & $\begin{array}{c}\text { Discharse } \\
\text { (cis) }\end{array}$ \\
\hline $\begin{array}{l}\text { Jan. } 19,1959 \\
20 \\
21 \\
\text { Feb. } 18\end{array}$ & $\begin{array}{l}1.73 \\
1.475 \\
1.425 \\
1.86\end{array}$ & $\begin{array}{l}3,090 \\
1,440 \\
1,220 \\
4,180\end{array}$ & $\begin{array}{l}\text { Feb. } 19,1959 \\
\text { Mar. } 2 \\
-5\end{array}$ & $\begin{array}{l}1.54 \\
4.33 \\
2.85\end{array}$ & $\begin{array}{r}1,780 \\
39,600 \\
16,600\end{array}$ \\
\hline
\end{tabular}

Peak at 8 P.Ko Sar. 24,53 moters about 42,800 cfs 


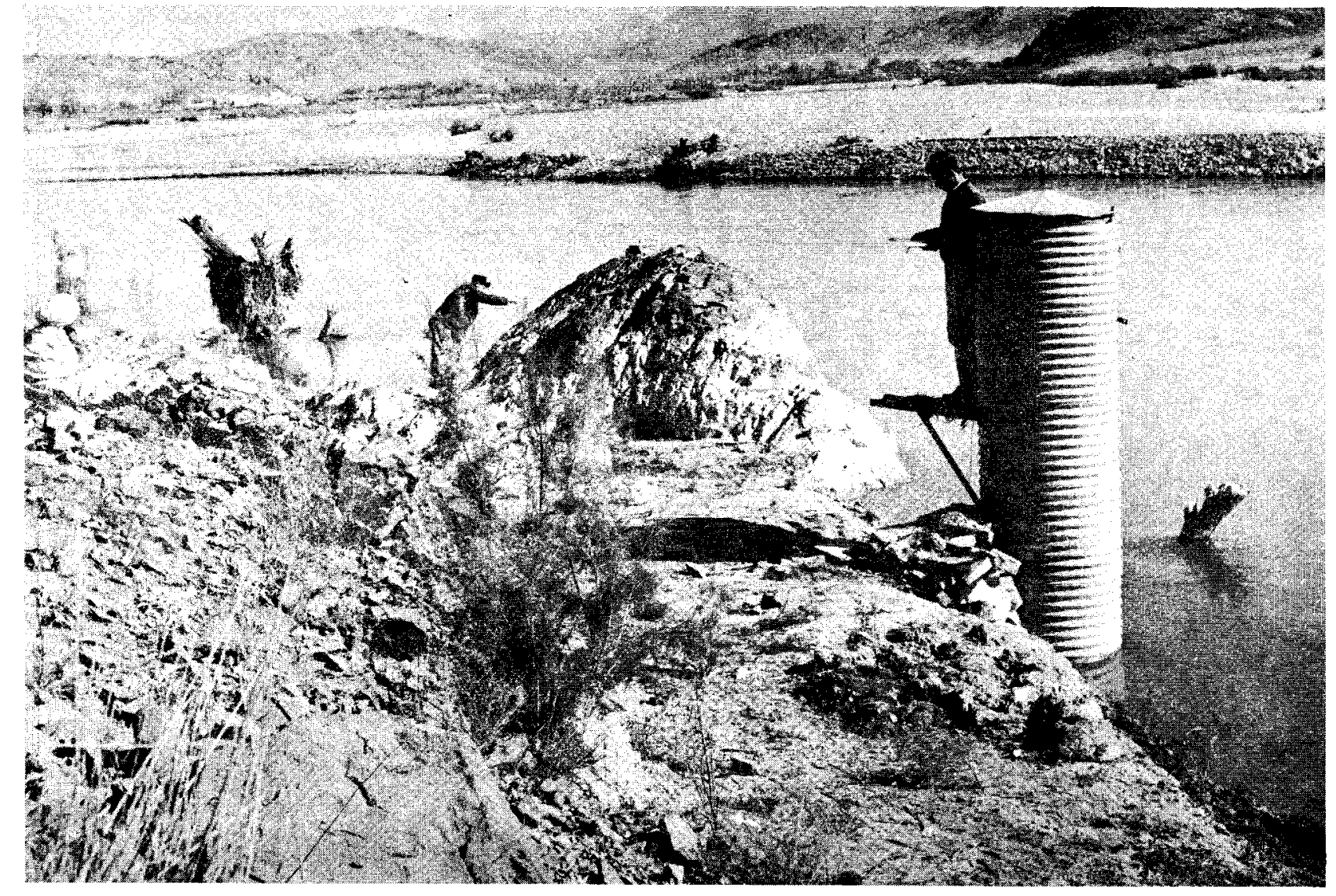

Figure 6.--Arghandab River above Arghandab Reservoir near Kandahar, showing waterstage recorder shelter and well, and staff gage. 


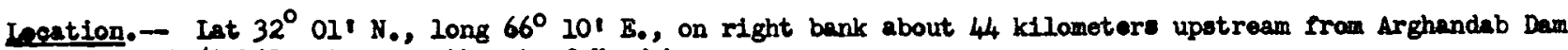
and about 64 kilometers northesst of Kandahar.

Dmainage area.- 6,540 oq mi (from Survey of India maps) of which about 640 sq mi is considered non-contributing. Records araileble. - Karch to September 1951 (gage beights only), October 1951 to September 1960.

Cage.- Wter-tage recorder. Datum of gage is at mean sea level (from MKa surveys from Surver of India

datum). Prior to Jan. 13, 1952, otaff gage at same eite and datum, Jan. 13, 1952 to Aug. 7, 1958 water

tage recorder at site about 1 mile domatrean at datum 14 meters lower.

Averege discharge.- 9 jears, 1,578 cfs $(1,142,000$ acro-ft year $)$.

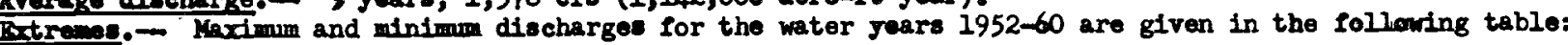

\begin{tabular}{|c|c|c|c|c|c|c|}
\hline \multirow[b]{2}{*}{ What Year } & \multirow[b]{2}{*}{ Date } & \multicolumn{2}{|c|}{ Haxtmom } & \multirow[b]{2}{*}{ Date } & \multicolumn{2}{|c|}{ Minimm } \\
\hline & & $\begin{array}{c}\text { Glevetion } \\
\text { (motere) }\end{array}$ & $\begin{array}{c}\text { Discharge } \\
\text { (cfs) }\end{array}$ & & $\begin{array}{l}\text { Milevation } \\
\text { (metere) }\end{array}$ & $\begin{array}{c}\text { Disehargo } \\
\text { (cfo) }\end{array}$ \\
\hline $\begin{array}{l}1952 \\
1953 \\
1954 \\
1955 \\
1956 \\
1957 \\
1958 \\
1959 \\
1960\end{array}$ & $\begin{array}{l}\text { Feb. 14, } 1952 \\
\text { Feb. 12, } 1953 \\
\text { Mar. } 30,1954 \\
\text { Mar. 15, } 1955 \\
\text { July 28, } 1956 \\
\text { Kar. 17, } 1957 \\
\text { Nov. 19, } 1958 \\
\text { Kar. 2, } 1959 \\
\text { Apr. } 17,1960\end{array}$ & $\begin{array}{l}1115.41 \\
1116.00 \\
1116.10 \\
1115.76 \\
1117.23 \\
1117.54 \\
= \\
1119.71\end{array}$ & $\begin{array}{r}7,170 \\
12,500 \\
14,100 \\
9,860 \\
29,700 \\
235,700 \\
b 9,000 \\
\text { b } 11,900 \\
11,400\end{array}$ & 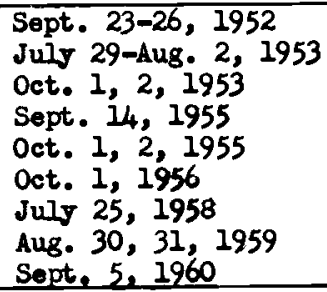 & $\begin{array}{l}1113.71 \\
1113.80 \\
-1114.20 \\
1114.20 \\
= \\
1118.16\end{array}$ & $\begin{array}{r}b 232 \\
71 \\
145 \\
b 64 \\
b 136 \\
480 \\
b 205 \\
b 280 \\
b 267 \\
\end{array}$ \\
\hline
\end{tabular}

a Prom rating curre extended above $12,000 \mathrm{cls}$.

b Kean daily discharge.

$\Lambda$ floodmark, elevation $1,118.0$ moters (about 42,000 efs) is believed to be for $1939 \mathrm{flood}$.

Pemaries. - Records good except those prior to December 1951 and October, November, 1954, July to September 1955; and those for perlods of no gage helght record, which are fair. Manj amall diversions for irrigation above the tation. Water is otored in Axghandab reservoir imadiately downstream (capacity at spillway alevation of 1210.0 meters 388,000 acre-ft) for irrigation of about 150,000 acres in Arghandab valley. 
HEIMAIID RIVWT BASIN

Arghandab River above Äghandab Resrovoir, ${ }^{2}$ gharistan

Discharae, in cubic feet ner second, water year octsher 195

\begin{tabular}{|c|c|c|c|c|c|c|c|c|c|c|c|c|}
\hline Day & Oct. & Nov. & Dec. & Jan. & Feb. & iner. & Apr. & Maz & Juse & July & Aug. & Sept. \\
\hline $\begin{array}{l}1 \\
2 \\
3 \\
4 \\
5\end{array}$ & $\begin{array}{l}\frac{301}{301} \\
301 \\
308 \\
308\end{array}$ & $\begin{array}{l}415 \\
425 \\
435 \\
435 \\
435\end{array}$ & $\begin{array}{l}560 \\
560 \\
575 \\
575 \\
575\end{array}$ & $\begin{array}{l}610 \\
595 \\
595 \\
575 \\
560 \\
\end{array}$ & $\begin{array}{l}860 \\
810 \\
835 \\
835 \\
835\end{array}$ & $\begin{array}{l}1,248 \\
1,210 \\
1,210 \\
1,324 \\
2,410\end{array}$ & $\begin{array}{l}1,050 \\
3,980 \\
3,920 \\
4,110 \\
4,540\end{array}$ & $\begin{array}{l}2,980 \\
2,720 \\
2,915 \\
3,175 \\
2,990\end{array}$ & $\begin{array}{l}* 940 \\
885 \\
860 \\
835 \\
785\end{array}$ & $\begin{array}{l}495 \\
485 \\
475 \\
1.75 \\
465\end{array}$ & $\begin{array}{l}425 \\
4 ! 15 \\
407 \\
399 \\
399\end{array}$ & $\begin{array}{r}361 \\
* 361 \\
368 \\
368 \\
375\end{array}$ \\
\hline $\begin{array}{r}6 \\
7 \\
8 \\
9 \\
10\end{array}$ & $\begin{array}{l}308 \\
308 \\
314 \\
314 \\
314\end{array}$ & $\begin{array}{l}445 \\
445 \\
445 \\
445 \\
445\end{array}$ & $\begin{array}{l}575 \\
575 \\
575 \\
575 \\
575\end{array}$ & $\begin{array}{l}595 \\
630 \\
630 \\
610 \\
595\end{array}$ & $\begin{array}{l}810 \\
810 \\
810 \\
810 \\
810\end{array}$ & $\begin{array}{l}1,998 \\
1,845 \\
1,743 \\
1,641 \\
1,590\end{array}$ & $\begin{array}{l}4,540 \\
1,480 \\
4,480 \\
4,1,800 \\
4,480\end{array}$ & $\begin{array}{l}2,850 \\
2,534 \\
2,348 \\
2,286 \\
2,286\end{array}$ & $\begin{array}{l}760 \\
720 \\
700 \\
700 \\
680\end{array}$ & $\begin{array}{r}455 \\
* 445 \\
445 \\
435 \\
435\end{array}$ & $\begin{array}{r}391 \\
* 358 \\
368 \\
368 \\
368\end{array}$ & $\begin{array}{l}375 \\
283 \\
383 \\
375 \\
368\end{array}$ \\
\hline $\begin{array}{l}11 \\
12 \\
13 \\
14 \\
15\end{array}$ & $\begin{array}{l}314 \\
314 \\
314 \\
321 \\
327\end{array}$ & $\begin{array}{l}445 \\
455 \\
455 \\
455 \\
465\end{array}$ & $\begin{array}{l}595 \\
595 \\
595 \\
595 \\
595\end{array}$ & $\begin{array}{l}575 \\
575 \\
595 \\
835 \\
700\end{array}$ & $\begin{array}{r}810 \\
810 \\
910 \\
2,596 \\
2,850 \\
\end{array}$ & $\begin{array}{l}1,641 \\
1,947 \\
4,540 \\
3,740 \\
3,180\end{array}$ & $\begin{array}{l}4,4,80 \\
4,420 \\
4,290 \\
4,170 \\
4,170\end{array}$ & $\begin{array}{l}2,224 \\
1,998 \\
1,845 \\
1,692 \\
1,590\end{array}$ & $\begin{array}{l}665 \\
665 \\
645 \\
645 \\
630\end{array}$ & $\begin{array}{l}425 \\
1.25 \\
1,15 \\
1.15 \\
415\end{array}$ & $\begin{array}{l}368 \\
361 \\
368 \\
361 \\
368\end{array}$ & $\begin{array}{r}* 354 \\
347 \\
333 \\
321 \\
308\end{array}$ \\
\hline $\begin{array}{l}16 \\
17 \\
18 \\
19 \\
20\end{array}$ & $\begin{array}{l}333 \\
333 \\
340 \\
347 \\
347\end{array}$ & $\begin{array}{l}475 \\
475 \\
485 \\
485 \\
485\end{array}$ & $\begin{array}{l}595 \\
595 \\
595 \\
595 \\
595\end{array}$ & $\begin{array}{l}610 \\
560 \\
560 \\
575 \\
595\end{array}$ & $\begin{array}{r}1,896 \\
* 1,476 \\
1,400 \\
1,286 \\
1,286\end{array}$ & $\begin{array}{r}2,980 \\
* 2,596 \\
2,658 \\
2,658 \\
2,658\end{array}$ & $\begin{array}{l}4,230 \\
4,290 \\
4,600 \\
4,670 \\
4,51,0\end{array}$ & $\begin{array}{l}1,552 \\
1,5 U_{t} \\
1,476 \\
1,362 \\
1,362\end{array}$ & $\begin{array}{l}610 \\
595 \\
575 \\
560 \\
545\end{array}$ & $\begin{array}{l}415 \\
415 \\
415 \\
415 \\
415\end{array}$ & $\begin{array}{l}368 \\
368 \\
368 \\
368 \\
368\end{array}$ & $\begin{array}{r}301 \\
294 \\
* 288 \\
280 \\
280\end{array}$ \\
\hline $\begin{array}{l}21 \\
22 \\
23 \\
21 \\
25\end{array}$ & $\begin{array}{l}354 \\
361 \\
368 \\
375 \\
375\end{array}$ & $\begin{array}{l}4.95 \\
505 \\
505 \\
515 \\
515\end{array}$ & $\begin{array}{l}595 \\
595 \\
595 \\
595 \\
595\end{array}$ & $\begin{array}{r}630 \\
680 \\
665 \\
* 645 \\
630\end{array}$ & $\begin{array}{l}1,286 \\
1,248 \\
1,210 \\
1,210 \\
1,210\end{array}$ & $\begin{array}{l}2,472 \\
2,534 \\
2,658 \\
2,658 \\
2,596\end{array}$ & $\begin{array}{l}4,170 \\
3,920 \\
3,610 \\
3,490 \\
3,300\end{array}$ & $\begin{array}{l}1,324 \\
1,286 \\
1,2148 \\
1,150 \\
1,150\end{array}$ & $\begin{array}{l}530 \\
515 \\
505 \\
495 \\
505\end{array}$ & $\begin{array}{l}475 \\
435 \\
515 \\
545 \\
5145\end{array}$ & $\begin{array}{l}368 \\
361 \\
301 \\
361 \\
361 .\end{array}$ & $\begin{array}{r}267 \\
255 \\
232 \\
232 \\
r 232\end{array}$ \\
\hline $\begin{array}{l}26 \\
27 \\
28 \\
29 \\
30 \\
31 \\
\end{array}$ & $\begin{array}{l}383 \\
391 \\
391 \\
399 \\
407 \\
407\end{array}$ & $\begin{array}{l}515 \\
515 \\
545 \\
545 \\
560 \\
-\end{array}$ & $\begin{array}{l}595 \\
595 \\
630 \\
700 \\
\frac{605}{665} \\
645\end{array}$ & $\begin{array}{l}645 \\
665 \\
680 \\
700 \\
885 \\
940 \\
\end{array}$ & $\begin{array}{c}1,210 \\
1,210 \\
1,248 \\
1,286 \\
- \\
-\end{array}$ & $\begin{array}{l}2,870 \\
5,860 \\
5,160 \\
4,360 \\
4,360 \\
4,050\end{array}$ & $\begin{array}{c}3,2140 \\
3,300 \\
3,490 \\
3,430 \\
3,180 \\
-\end{array}$ & $\begin{array}{r}1,130 \\
1,090 \\
1,060 \\
1,030 \\
1,000 \\
970 \\
\end{array}$ & $\begin{array}{c}515 \\
515 \\
515 \\
515 \\
505 \\
-\end{array}$ & $\begin{array}{l}530 \\
495 \\
475 \\
465 \\
415 \\
435 \\
\end{array}$ & $\begin{array}{l}361 \\
368 \\
368 \\
368 \\
361 \\
361 \\
\end{array}$ & $\begin{array}{c}232 \\
243 \\
21: 3 \\
243 \\
243 \\
-\end{array}$ \\
\hline $\begin{array}{l}\text { Total } \\
\text { Wean } \\
\text { Ac-ft }\end{array}$ & $\begin{array}{r}10,578 \\
341 \\
20,980 \\
\end{array}$ & $\begin{array}{r}14,265 \\
476 \\
28,290 \\
\end{array}$ & $\begin{array}{r}18,525 \\
598 \\
36,740 \\
\end{array}$ & $\begin{array}{r}19,990 \\
645 \\
39,650 \\
\end{array}$ & $\begin{array}{r}34,664 \\
1,195 \\
68,750 \\
\end{array}$ & $\begin{array}{r}81,395 \\
2,625 \\
161,400 \\
\end{array}$ & $\begin{array}{r}122,050 \\
4,068 \\
242,100 \\
\end{array}$ & $\begin{array}{r}56, \overline{117} \\
1,811 \\
111,400 \\
\end{array}$ & $\begin{array}{r}19,115 \\
637 \\
37,910 \\
\end{array}$ & $\begin{array}{r}14,080 \\
454 \\
27,930\end{array}$ & $\begin{array}{r}11,573 \\
373 \\
22,950 \\
\end{array}$ & $\begin{array}{r}9,245 \\
308 \\
18,340 \\
\end{array}$ \\
\hline $\begin{array}{l}\text { Caler } \\
\text { Wate }\end{array}$ & or 195 & $\begin{array}{l}\text { Ma } \\
\mathrm{Ma}\end{array}$ & $5, \overline{860}$ & $\begin{array}{l}\text { Min } \\
\text { Mir }\end{array}$ & $\frac{-}{232}$ & $\begin{array}{l}\text { Mean } \\
\text { Mean }\end{array}$ & $\overline{1,}, \overline{125}$ & $\begin{array}{l}A c-f^{\prime} \\
A=-f t\end{array}$ & & & & \\
\hline
\end{tabular}

Peak discharge (base, 8,000 cfs). - No peak above the base.

* Discharge measurement miace on this day.

vote-Discharge corputed from nncriaily gage reading Oct. 1 to Jon. 23. 
HEIMAND RIVER BASIN

Arghandab River above Arghandab Reservoir, Afghanistan

Discharge, in cubic feet per second, water year October 1952 to Sept ember 1953

\begin{tabular}{|c|c|c|c|c|c|c|c|c|c|c|c|c|}
\hline Day & Oct. & Nov. & Dec. & Jan. & Feb. & Mar. & Apr. & May & June & July & Augs. & Sept. \\
\hline $\begin{array}{l}1 \\
2 \\
3 \\
4 \\
5\end{array}$ & $\begin{array}{r}\frac{253}{253} \\
264 \\
* 264 \\
275\end{array}$ & $\begin{array}{r}395 \\
395 \\
395 \\
410 \\
* 425\end{array}$ & $\begin{array}{l}\frac{495}{513} \\
495 \\
495 \\
495\end{array}$ & $\begin{array}{r}534 \\
534 \\
* 513 \\
513 \\
513\end{array}$ & $\begin{array}{l}\frac{478}{478} \\
534 \\
688 \\
596\end{array}$ & $\begin{array}{l}1,970 \\
1,890 \\
1,830 \\
1,860 \\
1,770\end{array}$ & $\begin{array}{l}1,480 \\
1,450 \\
1,420 \\
1,380 \\
1,350\end{array}$ & $\begin{array}{r}935 \\
* 857 \\
831 \\
805 \\
805\end{array}$ & $\begin{array}{r}* 805 \\
857 \\
1,220 \\
1,220 \\
1,060\end{array}$ & $\begin{array}{l}202 \\
202 \\
191 \\
179 \\
179\end{array}$ & $\begin{array}{l}\frac{71}{71} \\
78 \\
86 \\
86\end{array}$ & $\begin{array}{l}* 94 \\
a 95 \\
a 100 \\
a 100 \\
a 105\end{array}$ \\
\hline $\begin{array}{r}6 \\
7 \\
8 \\
9 \\
10\end{array}$ & $\begin{array}{l}286 \\
299 \\
299 \\
299 \\
324\end{array}$ & $\begin{array}{l}443 \\
443 \\
460 \\
460 \\
460\end{array}$ & $\begin{array}{l}495 \\
495 \\
495 \\
513 \\
513\end{array}$ & $\begin{array}{l}495 \\
495 \\
495 \\
513 \\
513\end{array}$ & $\begin{array}{r}555 \\
534 \\
555 \\
626 \\
4,130\end{array}$ & $\begin{array}{r}2,010 \\
2,330 \\
2,280 \\
* 2,130 \\
2,010\end{array}$ & $\begin{array}{r}1,350 \\
* 1,380 \\
1,380 \\
1,420 \\
1,520\end{array}$ & $\begin{array}{l}784 \\
763 \\
742 \\
721 \\
700\end{array}$ & $\begin{array}{r}909 \\
784 \\
a 670 \\
a 600 \\
a 540\end{array}$ & $\begin{array}{l}179 \\
179 \\
168 \\
191 \\
227\end{array}$ & $\begin{array}{r}93 \\
109 \\
145 \\
168 \\
156\end{array}$ & $\begin{array}{l}\text { a.105 } \\
a 105 \\
a 110 \\
\text { a.10 } \\
\text { a.115 }\end{array}$ \\
\hline $\begin{array}{l}11 \\
12 \\
13 \\
14 \\
15\end{array}$ & $\begin{array}{r}337 \\
350 \\
350 \\
* 350 \\
365\end{array}$ & $\begin{array}{l}478 \\
495 \\
495 \\
495 \\
495\end{array}$ & $\begin{array}{l}513 \\
513 \\
513 \\
513 \\
513\end{array}$ & $\begin{array}{l}534 \\
534 \\
622 \\
555 \\
534\end{array}$ & $\begin{array}{l}1,190 \\
4,010 \\
4,790 \\
3,860 \\
2,480\end{array}$ & $\begin{array}{l}1,970 \\
1,890 \\
1,770 \\
1,730 \\
1,730\end{array}$ & $\begin{array}{l}1,590 \\
1,810 \\
1,850 \\
1,850 \\
1,730\end{array}$ & $\begin{array}{l}700 \\
657 \\
657 \\
657 \\
636\end{array}$ & $\begin{array}{l}a 490 \\
a 450 \\
a 420 \\
a 390 \\
a 360\end{array}$ & $\begin{array}{l}\frac{252}{252} \\
227 \\
227 \\
215\end{array}$ & $\begin{array}{r}156 \\
162 \\
150 \\
140 \\
* 140\end{array}$ & $\begin{array}{l}a 120 \\
a 120 \\
a 125 \\
a 130 \\
a 130\end{array}$ \\
\hline $\begin{array}{l}16 \\
17 \\
18 \\
19 \\
20\end{array}$ & $\begin{array}{l}365 \\
365 \\
365 \\
365 \\
380\end{array}$ & $\begin{array}{l}495 \\
495 \\
478 \\
478 \\
478\end{array}$ & $\begin{array}{r}513 \\
* 534 \\
534 \\
534 \\
534\end{array}$ & $\begin{array}{l}534 \\
495 \\
495 \\
513 \\
513\end{array}$ & $\begin{array}{l}2,090 \\
1,930 \\
1,830 \\
1,660 \\
1,560\end{array}$ & $\begin{array}{l}1,700 \\
1,620 \\
1,520 \\
1,480 \\
1,420\end{array}$ & $\begin{array}{l}1,590 \\
1,450 \\
1,350 \\
1,290 \\
1,190\end{array}$ & $\begin{array}{l}615 \\
594 \\
576 \\
557 \\
576\end{array}$ & $\begin{array}{l}\text { a340 } \\
\text { a320 } \\
\text { a300 } \\
\text { a290 } \\
\text { a270 }\end{array}$ & $\begin{array}{l}202 \\
191 \\
179 \\
168 \\
156\end{array}$ & $\begin{array}{l}140 \\
132 \\
122 \\
114 \\
114\end{array}$ & $\begin{array}{r}8132 \\
136 \\
136 \\
136 \\
136\end{array}$ \\
\hline $\begin{array}{l}21 \\
22 \\
23 \\
24 \\
25\end{array}$ & $\begin{array}{r}380 \\
380 \\
380 \\
395 \\
* 380\end{array}$ & $\begin{array}{l}478 \\
478 \\
478 \\
478 \\
478\end{array}$ & $\begin{array}{l}513 \\
513 \\
513 \\
513 \\
513\end{array}$ & $\begin{array}{l}\frac{478}{478} \\
478 \\
478 \\
478\end{array}$ & $\begin{array}{l}1,480 \\
1,450 \\
1,420 \\
1,420 \\
1,480\end{array}$ & $\begin{array}{l}\frac{1,380}{1,380} \\
1,420 \\
2,050 \\
1,970\end{array}$ & $\begin{array}{r}1,190 \\
1,160 \\
1,130 \\
1,060 \\
987\end{array}$ & $\begin{array}{l}576 \\
557 \\
557 \\
538 \\
557\end{array}$ & $\begin{array}{l}a 260 \\
a 250 \\
\text { a240 } \\
\text { a230 } \\
\text { a225 }\end{array}$ & $\begin{array}{l}136 \\
136 \\
127 \\
118 \\
109\end{array}$ & $\begin{array}{r}104 \\
104 \\
104 \\
96 \\
104\end{array}$ & $\begin{array}{l}\frac{145}{145} \\
136 \\
136 \\
136\end{array}$ \\
\hline $\begin{array}{l}26 \\
27 \\
28 \\
29 \\
30 \\
31 \\
\end{array}$ & $\begin{array}{l}380 \\
380 \\
380 \\
380 \\
380 \\
380\end{array}$ & $\begin{array}{r}495 \\
* 513 \\
513 \\
513 \\
513 \\
-\end{array}$ & $\begin{array}{r}534 \\
534 \\
513 \\
513 \\
534 \\
534 \\
\end{array}$ & $\begin{array}{r}478 \\
478 \\
478 \\
478 \\
478 \\
* 478\end{array}$ & $\begin{array}{c}1,660 \\
1,810 \\
1,970 \\
- \\
- \\
-\end{array}$ & $\begin{array}{l}1,850 \\
1,770 \\
1,700 \\
1,620 \\
1,590 \\
1,520\end{array}$ & $\begin{array}{l}961 \\
961 \\
935 \\
935 \\
883 \\
-\end{array}$ & $\begin{array}{r}1,090 \\
1,290 \\
1,190 \\
1,060 \\
935 \\
857\end{array}$ & $\begin{array}{l}\text { a220 } \\
\text { a215 } \\
\text { a210 } \\
\text { a205 } \\
\text { *202 } \\
\text { - }\end{array}$ & $\begin{array}{r}100 \\
86 \\
78 \\
71 \\
71 \\
* 71\end{array}$ & $\begin{array}{l}104 \\
104 \\
104 \\
a 100 \\
\text { al00 } \\
\text { al00 }\end{array}$ & $\begin{array}{c}145 \\
145 \\
145 \\
145 \\
145 \\
-\end{array}$ \\
\hline $\begin{array}{l}\text { Total } \\
\text { Mean } \\
\text { Ac-ft }\end{array}$ & $\begin{array}{r}10,603 \\
342 \\
21,030\end{array}$ & $\begin{array}{r}14,105 \\
470 \\
27,980\end{array}$ & $\begin{array}{r}15,945 \\
514 \\
31,630\end{array}$ & $\begin{array}{r}15,705 \\
507 \\
31,150\end{array}$ & $\begin{array}{r}47,164 \\
1,684 \\
93,550\end{array}$ & $\begin{array}{r}55,110 \\
1,778 \\
109,300\end{array}$ & $\begin{array}{r}40,032 \\
1,334 \\
79,400\end{array}$ & $\begin{array}{r}23,375 \\
754 \\
46,360\end{array}$ & $\begin{array}{r}14,552 \\
485 \\
28,860\end{array}$ & $\begin{array}{r}5,069 \\
164 \\
10,050\end{array}$ & $\begin{array}{r}3,557 \\
115 \\
7,060\end{array}$ & $\begin{array}{r}3,763 \\
225 \\
7,460\end{array}$ \\
\hline
\end{tabular}

Celender year 1352: Max 5,860 Min 232 Mean 1,117 Ac-ft 811,000 Water year 1952-53: Max 4,790 Min 71 Mear 682 Ac-ft 493,800

Peak discharge (base, $8,000 \mathrm{cfs}$. - Feb. $10(1: 30$ a.m.) $11,600 \mathrm{cfs}(15.93 \mathrm{~m}) ;$ Feb. $12(7: 30 \mathrm{p} \cdot \mathrm{in}$ ) $12,500 \mathrm{cfs}$ $\left(16,00 \mathrm{~m}_{\bullet}\right)$; Feb. 14 (11 a.m. 5,800 cfs $(15.21 \mathrm{~m} \cdot)$.

No gage-height record; discharge estimated.

* Discharge measurement made on this day. 
HELMAND RIVER BASIN

Arghandab River above Arghandab Reservoir, Afghanistan

Discharge, in cubic feet per second, water year October 1953 to September 1954

\begin{tabular}{|c|c|c|c|c|c|c|c|c|c|c|c|c|}
\hline Day & Oct, & Nov. & Dec. & $\operatorname{Jan}_{0}$ & Feb. & Mar. & Apr. & May & June & July & Aug. & Sept. \\
\hline $\begin{array}{l}1 \\
2 \\
3 \\
4 \\
5\end{array}$ & $\begin{array}{r}145 \\
145 \\
* 152 \\
156 \\
162\end{array}$ & $\begin{array}{l}* 279 \\
308 \\
308 \\
323 \\
323\end{array}$ & $\begin{array}{r}\frac{380}{380} \\
* 388 \\
395 \\
402\end{array}$ & $\begin{array}{r}446 \\
* 428 \\
446 \\
628 \\
2,970 \\
\end{array}$ & $\begin{array}{l}657 \\
657 \\
657 \\
657 \\
636 \\
\end{array}$ & $\begin{array}{r}6,730 \\
a 4,600 \\
a 3,800 \\
a 3,500 \\
a 3,300\end{array}$ & $\begin{array}{l}9,050 \\
7,980 \\
7,250 \\
6,960 \\
6,680\end{array}$ & $\begin{array}{l}\frac{6,060}{6,000} \\
5,860 \\
5,670 \\
5,480\end{array}$ & $\begin{array}{l}\mathrm{al}, 700 \\
\mathrm{al}, 600 \\
\mathrm{al}, 500 \\
\mathrm{al}, 400 \\
\mathrm{al}, 300\end{array}$ & $\begin{array}{l}\frac{883}{870} \\
857 \\
883 \\
870\end{array}$ & $\begin{array}{l}464 \\
464 \\
464 \\
464 \\
464\end{array}$ & $\begin{array}{r}388 \\
* 373 \\
373 \\
373 \\
366\end{array}$ \\
\hline $\begin{array}{r}6 \\
7 \\
8 \\
9 \\
10\end{array}$ & $\begin{array}{l}174 \\
191 \\
191 \\
202 \\
208\end{array}$ & $\begin{array}{l}330 \\
330 \\
330 \\
337 \\
344\end{array}$ & $\begin{array}{l}402 \\
402 \\
418 \\
418 \\
418\end{array}$ & $\begin{array}{l}883 \\
831 \\
742 \\
628 \\
615\end{array}$ & $\begin{array}{r}657 \\
657 \\
657 \\
678 \\
4,690\end{array}$ & $\begin{array}{r}* 3,250 \\
3,000 \\
2,940 \\
2,940 \\
3,240\end{array}$ & $\begin{array}{l}6,540 \\
6,400 \\
6,470 \\
6,330 \\
6,470\end{array}$ & $\begin{array}{l}5,350 \\
5,350 \\
5,100 \\
5,100 \\
4,980\end{array}$ & $\begin{array}{r}a 1,500 \\
a 1,450 \\
* 1,420 \\
1,380 \\
1,350\end{array}$ & $\begin{array}{l}883 \\
870 \\
870 \\
870 \\
844\end{array}$ & $\begin{array}{l}464 \\
464 \\
464 \\
446 \\
446\end{array}$ & $\begin{array}{l}366 \\
358 \\
351 \\
330 \\
351\end{array}$ \\
\hline $\begin{array}{l}11 \\
12 \\
13 \\
14 \\
15\end{array}$ & $\begin{array}{l}215 \\
215 \\
227 \\
227 \\
227\end{array}$ & $\begin{array}{l}337 \\
351 \\
358 \\
358 \\
358\end{array}$ & $\begin{array}{l}418 \\
418 \\
437 \\
446 \\
455\end{array}$ & $\begin{array}{l}615 \\
594 \\
594 \\
615 \\
615\end{array}$ & $\begin{array}{l}4,980 \\
4,230 \\
6,400 \\
5,420 \\
4,230\end{array}$ & $\begin{array}{l}3,240 \\
3,180 \\
3,120 \\
3,120 \\
3,190\end{array}$ & $\begin{array}{l}6,270 \\
5,860 \\
6,060 \\
6,270 \\
6,270\end{array}$ & $\begin{array}{r}4,850 \\
4,480 \\
4,170 \\
a 3,800 \\
a 3,450\end{array}$ & $\begin{array}{l}1,320 \\
1,290 \\
1,250 \\
1,220 \\
x 1,160\end{array}$ & $\begin{array}{l}831 \\
818 \\
818 \\
805 \\
818\end{array}$ & $\begin{array}{l}446 \\
446 \\
446 \\
446 \\
446\end{array}$ & $\begin{array}{l}373 \\
366 \\
366 \\
366 \\
351\end{array}$ \\
\hline $\begin{array}{l}16 \\
17 \\
18 \\
19 \\
20\end{array}$ & $\begin{array}{r}234 \\
234 \\
234 \\
240 \\
a 245\end{array}$ & $\begin{array}{l}366 \\
380 \\
366 \\
380 \\
388\end{array}$ & $\begin{array}{l}455 \\
446 \\
437 \\
437 \\
428\end{array}$ & $\begin{array}{l}594 \\
615 \\
615 \\
636 \\
615\end{array}$ & $\begin{array}{l}3,360 \\
4,910 \\
5,040 \\
4,290 \\
3,920\end{array}$ & $\begin{array}{l}3,360 \\
3,860 \\
4,020 \\
4,540 \\
5,040\end{array}$ & $\begin{array}{l}6,470 \\
6,960 \\
6,610 \\
6,400 \\
6,270\end{array}$ & $\begin{array}{l}\text { a3,250 } \\
\text { a3,100 } \\
\text { a2,950 } \\
\text { a2,900 } \\
\text { a2,750 }\end{array}$ & $\begin{array}{l}1,130 \\
1,130 \\
1,100 \\
1,060 \\
1,040\end{array}$ & $\begin{array}{l}818 \\
794 \\
774 \\
763 \\
742\end{array}$ & $\begin{array}{l}428 \\
428 \\
428 \\
428 \\
428\end{array}$ & $\begin{array}{l}351 \\
351 \\
344 \\
337 \\
337\end{array}$ \\
\hline $\begin{array}{l}21 \\
22 \\
23 \\
24 \\
25\end{array}$ & $\begin{array}{l}\text { a250 } \\
\text { a250 } \\
\text { a260 } \\
\text { a260 } \\
\text { a260 }\end{array}$ & $\begin{array}{l}380 \\
380 \\
388 \\
388 \\
395 \\
\end{array}$ & $\begin{array}{l}428 \\
437 \\
828 \\
557 \\
520\end{array}$ & $\begin{array}{l}594 \\
594 \\
594 \\
615 \\
636\end{array}$ & $\begin{array}{l}3,550 \\
3,300 \\
3,300 \\
3,240 \\
3,000\end{array}$ & $\begin{array}{l}5,600 \\
5,800 \\
5,600 \\
5,480 \\
5,480\end{array}$ & $\begin{array}{l}6,400 \\
7,320 \\
8,280 \\
7,830 \\
7,100\end{array}$ & $\begin{array}{l}a 2,600 \\
a 2,500 \\
a 2,400 \\
a 2,300 \\
a 2,200\end{array}$ & $\begin{array}{l}1,040 \\
1,040 \\
1,060 \\
1,060 \\
1,040\end{array}$ & $\begin{array}{l}710 \\
689 \\
678 \\
678 \\
657\end{array}$ & $\begin{array}{l}428 \\
428 \\
428 \\
428 \\
409\end{array}$ & $\begin{array}{l}\frac{323}{323} \\
323 \\
330 \\
337\end{array}$ \\
\hline $\begin{array}{l}26 \\
27 \\
28 \\
29 \\
30 \\
31 \\
\end{array}$ & $\begin{array}{l}a 260 \\
a 260 \\
a 260 \\
a 265 \\
a 265 \\
a 270 \\
\end{array}$ & $\begin{array}{c}395 \\
388 \\
388 \\
380 \\
380 \\
-\end{array}$ & $\begin{array}{l}502 \\
464 \\
455 \\
446 \\
483 \\
474 \\
\end{array}$ & $\begin{array}{l}636 \\
657 \\
657 \\
657 \\
657 \\
657 \\
\end{array}$ & $\begin{array}{c}2,870 \\
2,630 \\
2,630 \\
- \\
- \\
-\end{array}$ & $\begin{array}{r}5,350 \\
5,290 \\
5,420 \\
6,810 \\
13,500 \\
11,100 \\
\end{array}$ & $\begin{array}{c}6,680 \\
6,400 \\
6,200 \\
6,130 \\
6,130 \\
-\end{array}$ & $\begin{array}{l}\mathrm{a} 2,150 \\
\mathrm{a} 2,050 \\
\mathrm{a} 1,950 \\
\mathrm{a} 1,900 \\
\mathrm{a} 1,850 \\
\mathrm{a} 1,800 \\
\end{array}$ & $\begin{array}{r}1,040 \\
987 \\
961 \\
935 \\
* 909 \\
-\end{array}$ & $\begin{array}{r}636 \\
615 \\
576 \\
* 520 \\
483 \\
483 \\
\end{array}$ & $\begin{array}{l}409 \\
409 \\
409 \\
409 \\
409 \\
395 \\
\end{array}$ & $\begin{array}{r}337 \\
351 \\
351 \\
351 \\
* 351 \\
-\end{array}$ \\
\hline $\begin{array}{l}\text { Total } \\
\text { Mean } \\
\text { AC-ft }\end{array}$ & $\begin{array}{r}6,878 \\
222 \\
13,640 \\
\end{array}$ & $\begin{array}{r}10,731 \\
358 \\
21,280 \\
\end{array}$ & $\begin{array}{r}13,974 \\
451 \\
27,720 \\
\end{array}$ & $\begin{array}{r}21,679 \\
699 \\
43,000 \\
\end{array}$ & $\begin{array}{r}81,903 \\
2,925 \\
162,500 \\
\end{array}$ & $\begin{array}{r}149,400 \\
4,819 \\
296,300 \\
\end{array}$ & $\begin{array}{r}202,040 \\
6,735 \\
400,700 \\
\end{array}$ & $\begin{array}{r}1 u_{4}, 350 \\
3,689 \\
226,800 \\
\end{array}$ & $\begin{array}{r}36,372 \\
1,212 \\
72, y_{4}\end{array}$ & $\begin{array}{r}23,406 \\
755 \\
46,430 \\
\end{array}$ & $\begin{array}{r}13,535 \\
437 \\
26,850 \\
\end{array}$ & $\begin{array}{r}10,548 \\
352 \\
20,920 \\
\end{array}$ \\
\hline
\end{tabular}

Calendar year 1953: Max 4,790 Min 71 Mean 657 Ac-ft 475,800

water year 1953-54: Max 13,500 lin 145 Mcan 1,876 Ac-ft 1,358,000

Peak discharge (base, 8,000 cfs).-Feb, 10 (8 a.m.) 9,960 cfs (15.77 m.); lar. 1 (7 a.m.) 11,600 cfs (15.93 m.); lar. 30 (about $12 \mathrm{~m}$ ) 14,100 efs $(16.1 \mathrm{in}$ ); Apr. 22 (4:30 p. fl.) 9,530 cfs (15.72 m.).

* Discharge measurement made on this day.

a No gace-height record; discharge estimated on basis of records of Archendab Reservoir and the station below dam. 
HEIMAND RIVER BASIN

Arghandab River above Arghandab Reservoir, Afghanistan

Discharge, in cubic feet per second, water year October 1954 to September 1955

\begin{tabular}{|c|c|c|c|c|c|c|c|c|c|c|c|c|}
\hline Day & Oct. & Nov. & Dec. & Jan. & Feb. & Mar. & Apr. & May & June & Julv & Aug. & Sept. \\
\hline $\begin{array}{l}1 \\
2 \\
3 \\
4 \\
5\end{array}$ & $\begin{array}{l}\frac{352}{352} \\
373 \\
373 \\
404\end{array}$ & $\begin{array}{l}\frac{540}{550} \\
560 \\
580 \\
580\end{array}$ & $\begin{array}{l}638 \\
638 \\
651 \\
\frac{664}{651}\end{array}$ & $\begin{array}{r}625 \\
* 754 \\
716 \\
690 \\
690\end{array}$ & $\begin{array}{r}* 612 \\
586 \\
599 \\
638 \\
625\end{array}$ & $\begin{array}{l}562 \\
550 \\
538 \\
527 \\
515\end{array}$ & $\begin{array}{l}1,560 \\
1,450 \\
1,380 \\
1,360 \\
1,320\end{array}$ & $\begin{array}{l}860 \\
846 \\
806 \\
793 \\
931\end{array}$ & $\begin{array}{r}599 \\
562 \\
550 \\
* 538 \\
550\end{array}$ & $\begin{array}{l}\frac{213}{206} \\
198 \\
198 \\
190\end{array}$ & $\begin{array}{l}\frac{120}{120} \\
120 \\
120 \\
120\end{array}$ & $\begin{array}{l}80 \\
80 \\
80 \\
80 \\
80\end{array}$ \\
\hline $\begin{array}{r}6 \\
7 \\
8 \\
9 \\
10\end{array}$ & $\begin{array}{l}404 \\
426 \\
436 \\
436 \\
457\end{array}$ & $\begin{array}{l}580 \\
580 \\
580 \\
600 \\
600\end{array}$ & $\begin{array}{l}651 \\
625 \\
625 \\
638 \\
638\end{array}$ & $\begin{array}{l}716 \\
716 \\
690 \\
664 \\
664\end{array}$ & $\begin{array}{l}612 \\
612 \\
612 \\
612 \\
599\end{array}$ & $\begin{array}{l}515 \\
503 \\
492 \\
515 \\
527\end{array}$ & $\begin{array}{l}1,260 \\
1,200 \\
1,150 \\
1,120 \\
1,090\end{array}$ & $\begin{array}{l}1,220 \\
1,590 \\
1,610 \\
1,560 \\
1,520\end{array}$ & $\begin{array}{l}562 \\
573 \\
573 \\
550 \\
527\end{array}$ & $\begin{array}{l}183 \\
177 \\
177 \\
177 \\
171\end{array}$ & $\begin{array}{l}115 \\
115 \\
115 \\
115 \\
115\end{array}$ & $\begin{array}{l}78 \\
78 \\
78 \\
78 \\
78\end{array}$ \\
\hline $\begin{array}{l}11 \\
12 \\
13 \\
14 \\
15\end{array}$ & $\begin{array}{l}457 \\
457 \\
468 \\
480 \\
492\end{array}$ & $\begin{array}{l}610 \\
620 \\
620 \\
620 \\
620\end{array}$ & $\begin{array}{l}638 \\
638 \\
638 \\
651 \\
651\end{array}$ & $\begin{array}{l}690 \\
703 \\
677 \\
651 \\
651\end{array}$ & $\begin{array}{l}599 \\
599 \\
586 \\
586 \\
573\end{array}$ & $\begin{array}{r}573 \\
677 \\
903 \\
3,680 \\
* 8,640 \\
\end{array}$ & $\begin{array}{l}1,080 \\
1,240 \\
1,240 \\
1,180 \\
1,120\end{array}$ & $\begin{array}{l}1,500 \\
1,430 \\
1,280 \\
1,150 \\
1,020\end{array}$ & $\begin{array}{l}503 \\
503 \\
480 \\
468 \\
457\end{array}$ & $\begin{array}{l}171 \\
165 \\
159 \\
154 \\
159\end{array}$ & $\begin{array}{l}110 \\
110 \\
110 \\
110 \\
110\end{array}$ & $\begin{array}{r}* 78 \\
74 \\
71 \\
64 \\
68\end{array}$ \\
\hline $\begin{array}{l}16 \\
17 \\
18 \\
19 \\
20\end{array}$ & $\begin{array}{l}503 \\
503 \\
503 \\
515 \\
527\end{array}$ & $\begin{array}{l}640 \\
640 \\
640 \\
640 \\
640\end{array}$ & $\begin{array}{l}651 \\
651 \\
651 \\
638 \\
638\end{array}$ & $\begin{array}{l}664 \\
651 \\
651 \\
651 \\
664\end{array}$ & $\begin{array}{l}573 \\
573 \\
573 \\
562 \\
562\end{array}$ & $\begin{array}{l}5,310 \\
3,490 \\
2,820 \\
2,440 \\
2,220\end{array}$ & $\begin{array}{r}1,090 \\
1,060 \\
1,030 \\
1,000 \\
945\end{array}$ & $\begin{array}{l}903 \\
793 \\
716 \\
664 \\
651 \\
\end{array}$ & $\begin{array}{l}436 \\
415 \\
394 \\
394 \\
373\end{array}$ & $\begin{array}{l}154 \\
148 \\
142 \\
142 \\
136\end{array}$ & $\begin{array}{l}110 \\
110 \\
110 \\
110 \\
110\end{array}$ & $\begin{array}{l}71 \\
71 \\
74 \\
78 \\
82\end{array}$ \\
\hline $\begin{array}{l}21 \\
22 \\
23 \\
24 \\
25\end{array}$ & $\begin{array}{l}527 \\
527 \\
527 \\
515 \\
527\end{array}$ & $\begin{array}{l}650 \\
660 \\
660 \\
660 \\
660\end{array}$ & $\begin{array}{l}638 \\
638 \\
638 \\
638 \\
638\end{array}$ & $\begin{array}{l}651 \\
651 \\
651 \\
638 \\
638\end{array}$ & $\begin{array}{l}562 \\
562 \\
562 \\
573 \\
573\end{array}$ & $\begin{array}{l}2,090 \\
1,960 \\
1,790 \\
1,720 \\
1,650\end{array}$ & $\begin{array}{l}889 \\
860 \\
860 \\
832 \\
846\end{array}$ & $\begin{array}{r}651 \\
651 \\
664 \\
974 \\
1,300\end{array}$ & $\begin{array}{l}353 \\
333 \\
324 \\
315 \\
296\end{array}$ & $\begin{array}{l}130 \\
130 \\
130 \\
124 \\
124\end{array}$ & $\begin{array}{l}100 \\
100 \\
100 \\
100 \\
100\end{array}$ & $\begin{array}{l}82 \\
87 \\
87 \\
92 \\
96\end{array}$ \\
\hline $\begin{array}{l}26 \\
27 \\
28 \\
29 \\
30 \\
31 \\
\end{array}$ & $\begin{array}{r}527 \\
538 \\
527 \\
527 \\
* 527 \\
530 \\
\end{array}$ & $\begin{array}{r}660 \\
660 \\
660 \\
650 \\
* 638 \\
- \\
\end{array}$ & $\begin{array}{l}625 \\
625 \\
625 \\
625 \\
625 \\
612 \\
\end{array}$ & $\begin{array}{r}612 \\
612 \\
638 \\
664 \\
664 \\
638 \\
\end{array}$ & $\begin{array}{r}573 \\
573 \\
* 573 \\
- \\
- \\
- \\
\end{array}$ & $\begin{array}{r}1,540 \\
1,470 \\
1,610 \\
1,920 \\
1,750 \\
* 1,610 \\
\end{array}$ & $\begin{array}{r}874 \\
860 \\
889 \\
889 \\
* 903 \\
- \\
\end{array}$ & $\begin{array}{r}1,480 \\
1,340 \\
1,180 \\
945 \\
846 \\
703 \\
\end{array}$ & $\begin{array}{r}278 \\
259 \\
* 252 \\
252 \\
236 \\
-\end{array}$ & $\begin{array}{r}124 \\
* 120 \\
120 \\
120 \\
120 \\
120\end{array}$ & $\begin{array}{l}95 \\
90 \\
90 \\
90 \\
90 \\
90 \\
\end{array}$ & $\begin{array}{r}106 \\
110 \\
115 \\
* 120 \\
120 \\
-\end{array}$ \\
\hline $\begin{array}{l}\text { Total } \\
\text { Mean } \\
\text { Ac-ft }\end{array}$ & $\begin{array}{r}14,717 \\
475 \\
29,190 \\
\end{array}$ & $\begin{array}{r}18,598 \\
620 \\
36,890 \\
\end{array}$ & $\begin{array}{r}19,791 \\
638 \\
39,250 \\
\end{array}$ & $\begin{array}{r}20,635 \\
666 \\
40,930 \\
\end{array}$ & $\begin{array}{r}16,444 \\
587 \\
32,620 \\
\end{array}$ & $\begin{array}{r}55,107 \\
1,778 \\
109,300 \\
\end{array}$ & $\begin{array}{r}32,577 \\
1,086 \\
64,620 \\
\end{array}$ & $\begin{array}{r}32,577 \\
1,050 \\
64,620 \\
\end{array}$ & $\begin{array}{r}12,905 \\
430 \\
25,600 \\
\end{array}$ & $\begin{array}{r}4,772 \\
154 \\
9,460 \\
\end{array}$ & $\begin{array}{r}3,320 \\
107 \\
6,590 \\
\end{array}$ & $\begin{array}{r}2,536 \\
85 \\
5,030 \\
\end{array}$ \\
\hline
\end{tabular}

Calendar year 1954: $\operatorname{Max} 13,500$ Min 323 Miean 1,935 Ac-ft 1,401,000

Water year 1954-55: Max 8,640 Nin 64 Mean 657 Ac-ft 464,100

Peak discharge (base, 8,000 cfs) .--Mar. $15\left(8\right.$ a.m.) 9,860 cfs $\left(1,115.76 \mathrm{~m}_{\bullet}\right)$.

* Discharge ineasurement made on this day.

Note. - lio gage-height record Oct. 31 to Nov. 29, July 28 to jept. 10; discharge estimated. 
HELMAND RIVER BASIN

Arghandab River above Arghandab Reservoir, ffghanistan

Discharge, in cubic feet per second, water year October 1955 to September 1956

\begin{tabular}{|c|c|c|c|c|c|c|c|c|c|c|c|c|}
\hline Day & Oct.1 & Nov. 1 & Dec. 1 & Jan. & Feb. & biar. & Apr. & May & June & July & Aug. & Sept. \\
\hline $\begin{array}{l}1 \\
2 \\
3 \\
4 \\
5\end{array}$ & $\begin{array}{l}\frac{136}{136} \\
112 \\
142 \\
148\end{array}$ & $\begin{array}{r}259 \\
259 \\
* 259 \\
259 \\
268\end{array}$ & $\begin{array}{l}\frac{415}{426} \\
426 \\
426 \\
436\end{array}$ & $\begin{array}{c}* 703 \\
917 \\
931 \\
832 \\
767\end{array}$ & $\begin{array}{l}677 \\
690 \\
690 \\
690 \\
677\end{array}$ & $\begin{array}{l}2,720 \\
2,500 \\
2,480 \\
\frac{2}{3,530} \\
6,120\end{array}$ & $\begin{array}{r}8,860 \\
10,100 \\
10,400 \\
9,800 \\
9,040\end{array}$ & $\begin{array}{l}\frac{5,300}{5,080} \\
4,930 \\
4,650 \\
4,340\end{array}$ & $\begin{array}{l}\frac{1,480}{1,440} \\
1,400 \\
1,300 \\
1,270\end{array}$ & $\begin{array}{l}970 \\
950 \\
920 \\
900 \\
880\end{array}$ & $\begin{array}{r}\frac{2,500}{2,300} \\
2,100 \\
* 1,660 \\
1,460\end{array}$ & $\begin{array}{l}592 \\
592 \\
580 \\
568 \\
568\end{array}$ \\
\hline $\begin{array}{r}6 \\
7 \\
8 \\
9 \\
10\end{array}$ & $\begin{array}{l}154 \\
159 \\
171 \\
177 \\
183\end{array}$ & $\begin{array}{l}278 \\
287 \\
296 \\
306 \\
306\end{array}$ & $\begin{array}{l}480 \\
538 \\
562 \\
538 \\
550\end{array}$ & $\begin{array}{l}716 \\
716 \\
716 \\
716 \\
703\end{array}$ & $\begin{array}{l}677 \\
677 \\
664 \\
651 \\
664\end{array}$ & $\begin{array}{l}7,180 \\
6,280 \\
5,380 \\
4,860 \\
4,510\end{array}$ & $\begin{array}{l}8,610 \\
8,610 \\
9,240 \\
9,140 \\
8,440\end{array}$ & $\begin{array}{l}4,170 \\
4,010 \\
3,810 \\
3,650 \\
3,470\end{array}$ & $\begin{array}{l}1,240 \\
1,220 \\
1,200 \\
1,190 \\
1,180\end{array}$ & $\begin{array}{l}850 \\
820 \\
800 \\
800 \\
820\end{array}$ & $\begin{array}{l}1,300 \\
1,220 \\
1,180 \\
1,140 \\
1,100\end{array}$ & $\begin{array}{l}542 \\
542 \\
530 \\
518 \\
492\end{array}$ \\
\hline $\begin{array}{l}17 \\
12 \\
13 \\
14 \\
15\end{array}$ & $\begin{array}{l}198 \\
206 \\
206 \\
213 \\
221\end{array}$ & $\begin{array}{l}315 \\
315 \\
324 \\
333 \\
333\end{array}$ & $\begin{array}{r}550 \\
550 \\
3,130 \\
2,420 \\
1,090\end{array}$ & $\begin{array}{l}703 \\
716 \\
716 \\
703 \\
703\end{array}$ & $\begin{array}{l}677 \\
677 \\
728 \\
754 \\
767\end{array}$ & $\begin{array}{l}4,400 \\
4,340 \\
4,270 \\
4,440 \\
4,440\end{array}$ & $\begin{array}{l}8,360 \\
8,440 \\
8,740 \\
9,040 \\
9,520\end{array}$ & $\begin{array}{l}3,380 \\
3,300 \\
3,330 \\
3,120 \\
2,950\end{array}$ & $\begin{array}{l}1,180 \\
1,180 \\
1,160 \\
1,140 \\
1,140\end{array}$ & $\begin{array}{r}850 \\
900 \\
1,000 \\
1,500 \\
3,000\end{array}$ & $\begin{array}{r}1,050 \\
995 \\
955 \\
902 \\
849\end{array}$ & $\begin{array}{l}480 \\
480 \\
492 \\
480 \\
480\end{array}$ \\
\hline $\begin{array}{l}16 \\
17 \\
18 \\
19 \\
20\end{array}$ & $\begin{array}{l}229 \\
229 \\
229 \\
229 \\
236\end{array}$ & $\begin{array}{l}342 \\
352 \\
352 \\
362 \\
362\end{array}$ & $\begin{array}{l}889 \\
780 \\
716 \\
690 \\
703\end{array}$ & $\begin{array}{l}716 \\
767 \\
767 \\
741 \\
716\end{array}$ & $\begin{array}{l}703 \\
690 \\
690 \\
703 \\
703\end{array}$ & $\begin{array}{r}4,100 \\
3,840 \\
3,780 \\
5,900 \\
11,000 \\
\end{array}$ & $\begin{array}{l}10,000 \\
10,300 \\
10,200 \\
10,300 \\
10,300\end{array}$ & $\begin{array}{l}2,850 \\
2,090 \\
2,430 \\
2,360 \\
2,240\end{array}$ & $\begin{array}{l}1,100 \\
1,080 \\
1,060 \\
1,040 \\
1,020\end{array}$ & $\begin{array}{r}3,700 \\
8,700 \\
10,100 \\
8,900 \\
4,000\end{array}$ & $\begin{array}{l}783 \\
743 \\
730 \\
718 \\
705\end{array}$ & $\begin{array}{l}480 \\
480 \\
480 \\
470 \\
470\end{array}$ \\
\hline $\begin{array}{l}21 \\
22 \\
23 \\
24 \\
25\end{array}$ & $\begin{array}{l}236 \\
236 \\
236 \\
244 \\
244\end{array}$ & $\begin{array}{l}373 \\
373 \\
384 \\
384 \\
394\end{array}$ & $\begin{array}{l}767 \\
728 \\
703 \\
677 \\
664\end{array}$ & $\begin{array}{l}716 \\
690 \\
703 \\
716 \\
716\end{array}$ & $\begin{array}{l}703 \\
690 \\
716 \\
728 \\
806\end{array}$ & $\begin{array}{r}9,330 \\
8,660 \\
* 7,700 \\
* 10,600 \\
8,900\end{array}$ & $\begin{array}{r}* 10,400 \\
9,900 \\
8,660 \\
8,060 \\
7,380\end{array}$ & $\begin{array}{l}2,150 \\
2,100 \\
2,010 \\
1,940 \\
1,860\end{array}$ & $\begin{array}{l}995 \\
982 \\
982 \\
982 \\
978\end{array}$ & $\begin{array}{l}3,400 \\
3,000 \\
3,500 \\
7,400 \\
7,400\end{array}$ & $\begin{array}{l}692 \\
680 \\
668 \\
655 \\
655\end{array}$ & $\begin{array}{l}\frac{460}{470} \\
480 \\
470 \\
470\end{array}$ \\
\hline $\begin{array}{l}26 \\
27 \\
28 \\
29 \\
30 \\
31 \\
\end{array}$ & $\begin{array}{l}244 \\
244 \\
252 \\
\frac{252}{252} \\
252 \\
252 \\
\end{array}$ & $\begin{array}{c}394 \\
404 \\
415 \\
415 \\
* 415 \\
-\end{array}$ & $\begin{array}{l}664 \\
690 \\
703 \\
690 \\
690 \\
703\end{array}$ & $\begin{array}{r}690 \\
703 \\
690 \\
677 \\
677 \\
* 664 \\
\end{array}$ & $\begin{array}{r}4,940 \\
5,750 \\
3,680 \\
* 2,950 \\
- \\
-\end{array}$ & $\begin{array}{r}7,940 \\
7,260 \\
6,740 \\
7,300 \\
8,610 \\
* 8,610 \\
\end{array}$ & $\begin{array}{l}7,060 \\
6,540 \\
6,120 \\
5,820 \\
5,600 \\
-\end{array}$ & $\begin{array}{r}1,840 \\
1,750 \\
1,710 \\
1,040 \\
1,580 \\
* 1,540 \\
\end{array}$ & $\begin{array}{r}973 \\
* 968 \\
1,000 \\
1,050 \\
1,000 \\
-\end{array}$ & $\begin{array}{r}3,000 \\
3,500 \\
15,500 \\
8,200 \\
4,400 \\
3,400\end{array}$ & $\begin{array}{r}642 \\
630 \\
618 \\
\times 618 \\
605 \\
592 \\
\end{array}$ & $\begin{array}{l}470 \\
470 \\
480 \\
480 \\
492 \\
-\end{array}$ \\
\hline $\begin{array}{l}\text { Total } \\
\text { Mean } \\
\text { Ac-ft }\end{array}$ & $\begin{array}{r}6,436 \\
208 \\
12,770 \\
\end{array}$ & $\begin{array}{r}10,118 \\
337 \\
20,070\end{array}$ & $\begin{array}{r}23,994 \\
774 \\
47,590\end{array}$ & $\begin{array}{r}22,607 \\
729 \\
44,840 \\
\end{array}$ & $\begin{array}{r}34,812 \\
1,200 \\
69,050\end{array}$ & $\begin{array}{r}187,720 \\
6,055 \\
372,300\end{array}$ & $\begin{array}{r}262,980 \\
8,766 \\
521,600\end{array}$ & $\begin{array}{r}92,200 \\
2,974 \\
182,900 \\
\end{array}$ & $\begin{array}{r}33,930 \\
1,131 \\
67,300\end{array}$ & $\begin{array}{r}114,060 \\
3,679 \\
220,200\end{array}$ & $\begin{array}{r}31,445 \\
1,014 \\
62,370\end{array}$ & $\begin{array}{r}15,058 \\
502 \\
29,870 \\
\end{array}$ \\
\hline
\end{tabular}
$\begin{array}{llrllllr}\text { Calendar year 1955: } & \text { Wax } 8,640 & \text { Hin } 64 & \text { Fean } 6,066 & \text { Ac-ft } & 439,200 \\ \text { Water year 1955-56: } & \text { Max } 15,500 & \text { Hin } 136 & \text { hear } & 2,282 & \text { Ac-ft } 1,657,000\end{array}$

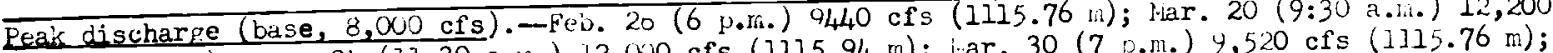

(1115.97 rn); Lar. 24 (11.30 a.i.) $12,000 \mathrm{cfs}(1315.94 \mathrm{~m})$; i.ar. 30 (7 p.m.) 4,520 cfs (1115.76 m); Apr. 3 (8 a.:. ) 10,900 cf

* Discharge measuremert mide or this day.

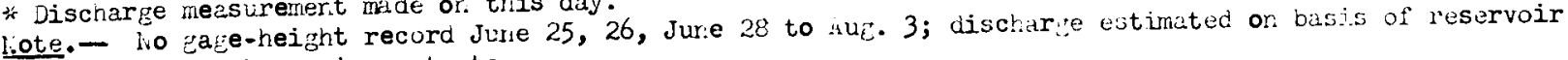
reltase and change in corterts. 
FEIMAND RIVER BASIN

Arohnrdah River above arghandab leservoin, if ghanistan

Discharge, in cibic feet per eecond, viter year cotober 1956 to Eepterber 1957

\begin{tabular}{|c|c|c|c|c|c|c|c|c|c|c|c|c|}
\hline Jay & Oct. & Nov. & Dec. & Ja:- & Teb. & lar. & Apr. & MaI & June & July & Aug. & Sept. \\
\hline$i$ & 492 & $* 1,010$ & 668 & 730 & 1,820 & $\because 2,2,1,0$ & 0,240 & 8,440 & $a 5,100$ & 1,940 & 1,160 & 662 \\
\hline 2 & $\overline{505}$ & 929 & 680 & 718 & 2,240 & 2,320 & 7,420 & 10,400 & 4,750 & 1,850 & $\overline{1,030}$ & 662 \\
\hline 3 & $* 18$ & $80 ?$ & 580 & 668 & 2,080 & 2,340 & 7,140 & 9,900 & 4,860 & $* 1,900$ & 986 & 696 \\
\hline 4 & 530 & 836 & 655 & 668 & 2,030 & 2,410 & 15,300 & 9,710 & 4,420 & 1,850 & 900 & 730 \\
\hline 5 & 555 & 823 & 668 & 680 & 2,030 & $?, 410$ & 22,000 & 8,700 & 4,310 & 1,760 & 900 & 730 \\
\hline 6 & 5.55 & 823 & $\approx 680$ & 680 & $* ?, 620$ & 2,560 & 21,100 & $* 8,78 n$ & 4,300 & 1,720 & 866 & 764 \\
\hline 7 & 555 & 823 & 682 & 705 & $\overline{2,500}$ & 2,660 & 20,000 & $\because, 440$ & 3,540 & 1,850 & 832 & 730 \\
\hline 8 & 568 & 810 & 692 & 718 & 2,320 & 2,850 & 20,400 & 8,270 & 3,970 & 1,850 & 866 & 730 \\
\hline 9 & 530 & 810 & 680 & 705 & 2,150 & 3,360 & 15,800 & 8,020 & 3,310 & 1,580 & 866 & 696 \\
\hline 10 & 592 & 783 & 680 & 668 & $2,19 n$ & $* 2,650$ & 14,500 & 100 & 3,310 & 1,500 & 798 & 662 \\
\hline 11 & 592 & 783 & 668 & 655 & 2,100 & $3,1: 70$ & 12,600 & 8,600 & 3,310 & 1,680 & $76 L:$ & 662 \\
\hline 12 & 592 & 770 & 680 & 668 & 2,050 & 3,230 & 11,000 & 8,78 & 3,260 & 1,680 & 764 & 662 \\
\hline 13 & 592 & 756 & 705 & 756 & 2,150 & 3,300 & 11,300 & 8,270 & 3,310 & 1,590 & 730 & 662 \\
\hline$u_{4}$ & 605 & 756 & 692 & 749 & 2,080 & 3,360 & 11,900 & 7,780 & 3,000 & 1,410 & 696 & 662 \\
\hline 15 & 618 & 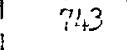 & 680 & 742 & 2,100 & 3,530 & 12,200 & 7,300 & 2,900 & 2,330 & 696 & 662 \\
\hline 16 & 630 & 730 & 600 & 692 & 2,100 & 3,650 & $1.1,000$ & $?, 100$ & 2,170 & 1,330 & 696 & 628 \\
\hline 17 & 630 & 730 & 692 & 655 & 2,150 & 12,200 & 10,800 & $\approx 7,000$ & 2,500 & 1,330 & 696 & $66 ?$ \\
\hline 18 & 642 & 718 & 668 & 668 & 2,240 & 21,400 & 9,800 & 27,000 & 2,600 & 1,200 & 696 & 560 \\
\hline 19 & 655 & 730 & 668 & 692 & 2,220 & 10,500 & 9,760 & 26,600 & $* 2,700$ & 1,160 & 696 & 560 \\
\hline 20 & 30 & 730 & 692 & 718 & 2,190 & 8,860 & 9,950 & $\therefore 6,500$ & 2,600 & 1,200 & 662 & 560 \\
\hline 21 & 630 & 718 & 680 & 810 & $\mathrm{a} 2,100$ & 8,480 & 9,650 & 26,400 & 2,500 & 1,200 & 628 & 560 \\
\hline 22 & 518 & 718 & 680 & 823 & $a 2,100$ & 7,980 & 9,760 & a6, 100 & 2,400 & 1,200 & 628 & 584 \\
\hline 23 & 530 & 718 & 655 & 862 & 2,190 & $?, 260$ & 9,090 & 26,000 & 2,400 & 1,200 & 628 & $58 / 4$ \\
\hline 24 & 630 & 730 & 730 & 902 & 22,100 & 6,700 & 9,000 & 35,700 & 2,200 & 1,120 & 662 & 628 \\
\hline 25 & 642 & 705 & 756 & 5,480 & a2,100 & 6,540 & $8,1.80$ & a 5,600 & 2,200 & 1,120 & 662 & 628 \\
\hline 26 & 630 & 705 & 680 & 4,070 & $\mathrm{a} 2,100$ & 6,320 & 9,040 & a 5,500 & 1,940 & 1,120 & 662 & 628 \\
\hline 27 & 630 & 692 & $* 655$ & 2,900 & 2,190 & 6,320 & 9,300 & $\mathrm{a} 5,100$ & 1,850 & 1,200 & 662 & 528 \\
\hline 28 & 642 & $\overline{71: 2}$ & $68 n$ & 2,410 & 2,190 & 7,100 & 8,400 & 25,200 & 1,940 & 1,200 & 662 & 560 \\
\hline 29 & 655 & 718 & 680 & 2,150 & - & 6,700 & 8,060 & 25,200 & 3,940 & 1,120 & 662 & 560 \\
\hline 30 & 680 & 692 & 642 & $\because 1,960$ & - & 6,540 & 8,020 & $a 5,300$ & 1,940 & 1,200 & 696 & 584 \\
\hline $3 I$ & 916 & - & 680 & 1,860 & - & $* 6,460$ & - & 25,300 & - & $* 1,200$ & $* 696$ & - \\
\hline $\begin{array}{l}\text { Tot } \equiv 1 \\
\text { Hosn }\end{array}$ & $\begin{array}{r}18,939 \\
611\end{array}$ & $\begin{array}{r}23,094 \\
770\end{array}$ & $\begin{array}{r}21,106 \\
681\end{array}$ & $\begin{array}{r}38,162 \\
1,231\end{array}$ & $\begin{array}{r}60,340 \\
2,155\end{array}$ & $\begin{array}{r}175,900 \\
5,706\end{array}$ & $\begin{array}{r}350,310 \\
11,680\end{array}$ & $\begin{array}{r}225,090 \\
7,261\end{array}$ & $\begin{array}{r}91,570 \\
3,05 ?\end{array}$ & $\begin{array}{r}44,690 \\
1,44: 2\end{array}$ & $\begin{array}{r}23,548 \\
760 \\
6,710\end{array}$ & $\begin{array}{r}19,296 \\
643 \\
38,250\end{array}$ \\
\hline & 37,560 & 45,210 & 41,860 & 75,690 & 119,700 & 350,900 & 694,370 & $44_{4} 6,500$ & 181,6 & $58,6,0$ & 6,710 & 230 \\
\hline
\end{tabular}

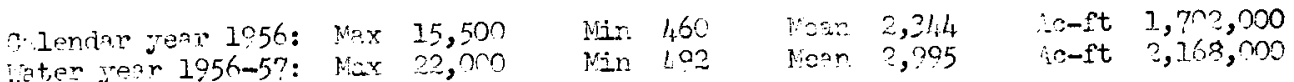

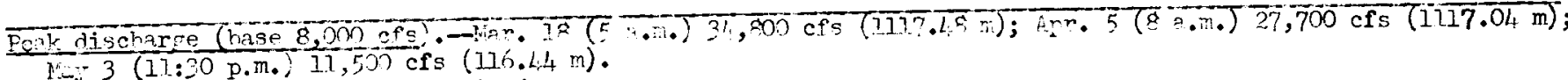

Disharce measuiement made or this day.

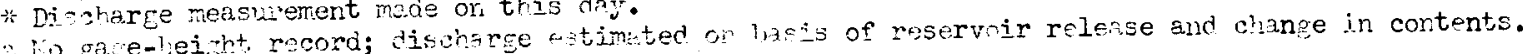


HETMAND RIVER BASIN

Arghandab River above Arghandab Reservoir, Afghanistan

Discharge, in cubic feet per second, water year October 1957 to September 1958

\begin{tabular}{|c|c|c|c|c|c|c|c|c|c|c|c|c|}
\hline Day & Qct. & Nov. & Dec. & Jan. & Feb. & Mar. & Apr. & May & June & July & Aug. & Sept. \\
\hline $\begin{array}{l}1 \\
2 \\
3 \\
4 \\
5\end{array}$ & $\begin{array}{r}594 \\
* 560 \\
565 \\
550 \\
560\end{array}$ & $\begin{array}{r}\frac{545}{635} \\
* 832 \\
832 \\
798\end{array}$ & $\begin{array}{r}1,100 \\
1,250 \\
1,460 \\
* 1,240 \\
1,330\end{array}$ & $\begin{array}{r}* 1,760 \\
1,680 \\
1,630 \\
1,680 \\
1,720\end{array}$ & $\begin{array}{l}3,860 \\
2,800 \\
2,400 \\
2,800 \\
2,650\end{array}$ & $\begin{array}{r}* 2,200 \\
2,200 \\
2,200 \\
2,160 \\
1,940 \\
\end{array}$ & $\begin{array}{l}4,190 \\
3,200 \\
3,260 \\
3,420 \\
3,420\end{array}$ & $\begin{array}{l}2,350 \\
2,700 \\
2,700 \\
2,650 \\
2,600\end{array}$ & $\begin{array}{r}1,160 \\
1,120 \\
* 986 \\
866 \\
832\end{array}$ & $\begin{array}{r}594 \\
560 \\
* 594 \\
662 \\
662\end{array}$ & $\begin{array}{r}* 375 \\
380 \\
380 \\
380 \\
380\end{array}$ & $\begin{array}{l}286 \\
294 \\
286 \\
286 \\
262 \\
\end{array}$ \\
\hline $\begin{array}{r}6 \\
7 \\
8 \\
9 \\
10\end{array}$ & $\begin{array}{l}615 \\
565 \\
595 \\
560 \\
600\end{array}$ & $\begin{array}{r}866 \\
1,030 \\
1,070 \\
1,030 \\
1,030\end{array}$ & $\begin{array}{l}4,000 \\
3,800 \\
3,100 \\
2,500 \\
5,000\end{array}$ & $\begin{array}{l}1,720 \\
1,850 \\
1,850 \\
1,760 \\
2,400\end{array}$ & $\begin{array}{l}2,350 \\
2,030 \\
2,160 \\
2,030 \\
1,720\end{array}$ & $\begin{array}{l}1,980 \\
2,110 \\
2,200 \\
2,600 \\
2,950\end{array}$ & $\begin{array}{l}3,260 \\
3,150 \\
2,600 \\
3,860 \\
4,020\end{array}$ & $\begin{array}{l}2,450 \\
2,450 \\
2,350 \\
2,250 \\
2,300\end{array}$ & $\begin{array}{r}848 \\
900 \\
900 \\
1,030 \\
1,030\end{array}$ & $\begin{array}{l}594 \\
594 \\
594 \\
594 \\
594\end{array}$ & $\begin{array}{r}380 \\
* 386 \\
386 \\
386 \\
386\end{array}$ & $\begin{array}{l}262 \\
262 \\
270 \\
270 \\
270\end{array}$ \\
\hline $\begin{array}{l}11 \\
12 \\
13 \\
14 \\
15\end{array}$ & $\begin{array}{l}650 \\
615 \\
600 \\
585 \\
735\end{array}$ & $\begin{array}{r}986 \\
1,120 \\
1,160 \\
1,070 \\
1,070\end{array}$ & $\begin{array}{l}3,700 \\
2,500 \\
2,000 \\
1,800 \\
1,600\end{array}$ & $\begin{array}{l}2,250 \\
2,200 \\
2,110 \\
1,940 \\
2,070\end{array}$ & $\begin{array}{l}2,030 \\
1,900 \\
2,030 \\
2,030 \\
1,760\end{array}$ & $\begin{array}{l}2,750 \\
2,600 \\
2,300 \\
2,850 \\
2,700\end{array}$ & $\begin{array}{l}3,530 \\
3,420 \\
3,100 \\
2,800 \\
2,400\end{array}$ & $\begin{array}{l}2,550 \\
2,350 \\
2,250 \\
2,110 \\
2,110\end{array}$ & $\begin{array}{l}986 \\
943 \\
943 \\
943 \\
900\end{array}$ & $\begin{array}{r}594 \\
696 \\
2,940 \\
4,180 \\
1,890\end{array}$ & $\begin{array}{l}362 \\
374 \\
374 \\
362 \\
362\end{array}$ & $\begin{array}{l}262 \\
262 \\
270 \\
262 \\
262\end{array}$ \\
\hline $\begin{array}{l}16 \\
17 \\
18 \\
19 \\
20\end{array}$ & $\begin{array}{l}590 \\
590 \\
680 \\
590 \\
750\end{array}$ & $\begin{array}{l}1,160 \\
1,070 \\
1,070 \\
9,000 \\
4,000\end{array}$ & $\begin{array}{l}1,500 \\
1,400 \\
1,330 \\
1,280 \\
1,160\end{array}$ & $\begin{array}{l}2,070 \\
2,070 \\
2,030 \\
2,160 \\
2,900 \\
\end{array}$ & $\begin{array}{l}1,590 \\
2,070 \\
2,200 \\
2,110 \\
2,070\end{array}$ & $\begin{array}{l}2,600 \\
2,900 \\
2,800 \\
2,200 \\
2,160\end{array}$ & $\begin{array}{l}2,600 \\
\frac{2,300}{2,400} \\
3,000 \\
3,200\end{array}$ & $\begin{array}{l}2,160 \\
1,980 \\
1,800 \\
1,680 \\
1,680\end{array}$ & $\begin{array}{l}943 \\
943 \\
986 \\
986 \\
900\end{array}$ & $\begin{array}{r}1,380 \\
1,020 \\
820 \\
730 \\
420\end{array}$ & $\begin{array}{l}342 \\
342 \\
350 \\
350 \\
342\end{array}$ & $\begin{array}{l}262 \\
270 \\
262 \\
270 \\
270\end{array}$ \\
\hline $\begin{array}{l}21 \\
22 \\
23 \\
24 \\
25\end{array}$ & $\begin{array}{l}635 \\
635 \\
735 \\
740 \\
791\end{array}$ & $\begin{array}{l}1,600 \\
1,400 \\
1,500 \\
1,700 \\
1,600\end{array}$ & $\begin{array}{l}1,240 \\
1,330 \\
1,160 \\
1,160 \\
1,240\end{array}$ & $\begin{array}{l}2,200 \\
1,850 \\
2,110 \\
2,350 \\
2,350\end{array}$ & $\begin{array}{l}1,980 \\
1,980 \\
2,030 \\
1,940 \\
2,030\end{array}$ & $\begin{array}{l}2,900 \\
2,250 \\
2,500 \\
2,900 \\
2,800\end{array}$ & $\begin{array}{l}3,860 \\
4,800 \\
4,860 \\
4,300 \\
4,080\end{array}$ & $\begin{array}{l}1,680 \\
1,760 \\
2,800 \\
2,400 \\
2,030\end{array}$ & $\begin{array}{l}832 \\
832 \\
832 \\
764 \\
696\end{array}$ & $\begin{array}{l}535 \\
305 \\
320 \\
220 \\
205 \\
\end{array}$ & $\begin{array}{l}326 \\
326 \\
326 \\
326 \\
318\end{array}$ & $\begin{array}{l}262 \\
262 \\
278 \\
278 \\
286\end{array}$ \\
\hline $\begin{array}{l}26 \\
27 \\
28 \\
29 \\
30 \\
31\end{array}$ & $\begin{array}{l}691 \\
856 \\
756 \\
821 \\
726 \\
780\end{array}$ & $\begin{array}{l}1,400 \\
1,300 \\
1,200 \\
1,150 \\
1,100\end{array}$ & $\begin{array}{l}1,370 \\
1,590 \\
1,590 \\
1,680 \\
1,850 \\
1,980\end{array}$ & $\begin{array}{l}2,110 \\
2,200 \\
1,900 \\
1,720 \\
1,940 \\
2,110\end{array}$ & $\begin{array}{c}2,400 \\
2,400 \\
2,250 \\
= \\
= \\
=\end{array}$ & $\begin{array}{l}2,950 \\
2,900 \\
3,480 \\
3,200 \\
3,050 \\
3,480\end{array}$ & $\begin{array}{c}3,530 \\
2,800 \\
3,800 \\
3,530 \\
3,530 \\
-\end{array}$ & $\begin{array}{l}1,720 \\
1,460 \\
1,280 \\
1,160 \\
1,070 \\
1,160\end{array}$ & $\begin{array}{l}560 \\
540 \\
560 \\
594 \\
594 \\
-\end{array}$ & $\begin{array}{l}230 \\
340 \\
360 \\
370 \\
375 \\
375\end{array}$ & $\begin{array}{r}294 \\
286 \\
278 \\
278 \\
278 \\
* 278\end{array}$ & $\begin{array}{l}286 \\
278 \\
286 \\
286 \\
286\end{array}$ \\
\hline $\begin{array}{l}\text { Total } \\
\text { Mean } \\
\text { Ac-ft } \\
\end{array}$ & $\begin{array}{r}20,315 \\
655 \\
40,290 \\
\end{array}$ & $\begin{array}{r}44,324 \\
1,477 \\
87,920 \\
\end{array}$ & $\begin{array}{r}59,240 \\
1,911 \\
117,500 \\
\end{array}$ & $\begin{array}{r}62,740 \\
2,024 \\
124,400 \\
\end{array}$ & $\begin{array}{r}61,600 \\
2,200 \\
122,200 \\
\end{array}$ & $\begin{array}{r}80,810 \\
2,607 \\
160,300 \\
\end{array}$ & $\begin{array}{r}102,220 \\
3,407 \\
202,800 \\
\end{array}$ & $\begin{array}{r}63,990 \\
2,064 \\
126,900 \\
\end{array}$ & $\begin{array}{r}25,949 \\
865 \\
51,470 \\
\end{array}$ & $\begin{array}{r}24,347 \\
785 \\
48,290\end{array}$ & $\begin{array}{r}10,693 \\
345 \\
21,210 \\
\end{array}$ & $\begin{array}{r}8,188 \\
273 \\
16,240 \\
\end{array}$ \\
\hline Wat & 8 & $\begin{array}{l}\operatorname{Max} \\
\operatorname{Max}\end{array}$ & $\begin{array}{r}22,000 \\
9,000\end{array}$ & $\begin{array}{l}\text { Min } 54 \\
\text { Min } 20\end{array}$ & $\begin{array}{l}\text { Wean } \\
\text { Nean }\end{array}$ & $\begin{array}{l}3,161 \\
1,546\end{array}$ & $\begin{array}{l}A c-f t \\
A c-f t\end{array}$ & $\begin{array}{l}88,000 \\
19,000\end{array}$ & & & & \\
\hline
\end{tabular}

Peak discharge (base 8,000 cfs). - Nov. 19 (10 p.m.) 9,100 cfs (1117.76 m); July 13 (5:30 p.1:. 8,060 cfs (1118.10 m).

* Discharge measurement made on this day.

outflow ard change in contents. 
EINWAND ZIVAP BASIN

Arghandab River atove Arghandigh Pfservoir, near Arghardab, Afgianistan

Discharge, in cubic feet per second, water yeer October 1958 to September 1959

\begin{tabular}{|c|c|c|c|c|c|c|c|c|c|c|c|c|}
\hline Day & Oct. & Nov. & Dec. & $J d r_{1}$ & Feb. & Mar. & Apr. & May & June & July & Aug. I & Sept. \\
\hline $\begin{array}{l}1 \\
2 \\
3 \\
4 \\
5\end{array}$ & $\begin{array}{l}\frac{318}{318} \\
326 \\
318 \\
326\end{array}$ & $\begin{array}{l}518 \\
518 \\
506 \\
518 \\
506\end{array}$ & $\begin{array}{r}\frac{590}{606} \\
622 \\
622 \\
* 622\end{array}$ & $\begin{array}{l}750 \\
766 \\
878 \\
814 \\
686\end{array}$ & $\begin{array}{l}862 \\
910 \\
988 \\
910 \\
798 \\
\end{array}$ & $\begin{array}{r}9,710 \\
11,900 \\
8,140 \\
6,160 \\
6,050\end{array}$ & $\begin{array}{l}4,660 \\
6,390 \\
7,060 \\
6,030 \\
5,400\end{array}$ & $\begin{array}{l}3,430 \\
3,420 \\
3,050 \\
3,030 \\
3,010\end{array}$ & $\begin{array}{l}1,300 \\
1,360 \\
\frac{1}{1,180} \\
1,340 \\
1,180\end{array}$ & $\begin{array}{r}816 \\
816 \\
884 \\
1,050 \\
1,120 \\
\end{array}$ & $\begin{array}{r}560 \\
* 540 \\
512 \\
468 \\
450\end{array}$ & $\begin{array}{r}\frac{290}{310} \\
* 320 \\
328 \\
320\end{array}$ \\
\hline $\begin{array}{r}6 \\
7 \\
8 \\
9 \\
10\end{array}$ & $\begin{array}{l}342 \\
350 \\
362 \\
374 \\
386\end{array}$ & $\begin{array}{r}* 506 \\
506 \\
542 \\
554 \\
578\end{array}$ & $\begin{array}{l}606 \\
622 \\
638 \\
638 \\
638\end{array}$ & $\begin{array}{l}\frac{566}{622} \\
686 \\
702 \\
702\end{array}$ & $\begin{array}{r}846 \\
910 \\
949 \\
1,010 \\
1,080\end{array}$ & $\begin{array}{l}5,250 \\
5,050 \\
5,200 \\
6,060 \\
8,230\end{array}$ & $\begin{array}{l}5,000 \\
4,750 \\
4,920 \\
5,300 \\
5,420\end{array}$ & $\begin{array}{l}2,840 \\
2,680 \\
2,590 \\
2,420 \\
?, 380\end{array}$ & $\begin{array}{l}1,130 \\
1,100 \\
1,090 \\
1,080 \\
1,030\end{array}$ & $\begin{array}{r}* 1,090 \\
1,020 \\
952 \\
884 \\
873\end{array}$ & $\begin{array}{l}468 \\
400 \\
370 \\
350 \\
340\end{array}$ & $\begin{array}{l}320 \\
336 \\
344 \\
344 \\
352\end{array}$ \\
\hline $\begin{array}{l}11 \\
12 \\
13 \\
14 \\
15\end{array}$ & $\begin{array}{l}410 \\
398 \\
398 \\
410 \\
422\end{array}$ & $\begin{array}{l}572 \\
622 \\
638 \\
622 \\
622\end{array}$ & $\begin{array}{r}686 \\
1,140 \\
949 \\
830 \\
718\end{array}$ & $\begin{array}{l}702 \\
702 \\
702 \\
718 \\
734\end{array}$ & $\begin{array}{r}1,010 \\
968 \\
1,030 \\
1,030 \\
1,010\end{array}$ & $\begin{array}{l}6,720 \\
6,300 \\
5,500 \\
5,470 \\
5,570\end{array}$ & $\begin{array}{l}5,610 \\
5,400 \\
5,070 \\
4,900 \\
4,920\end{array}$ & $\begin{array}{l}2,230 \\
2,320 \\
2,120 \\
2,240 \\
2,470\end{array}$ & $\begin{array}{r}1,050 \\
1,050 \\
1,000 \\
1,000 \\
977\end{array}$ & $\begin{array}{l}850 \\
816 \\
794 \\
732 \\
771\end{array}$ & $\begin{array}{l}330 \\
325 \\
320 \\
320 \\
315\end{array}$ & $\begin{array}{l}370 \\
370 \\
370 \\
361 \\
361\end{array}$ \\
\hline $\begin{array}{l}16 \\
17 \\
18 \\
19 \\
20\end{array}$ & $\begin{array}{l}434 \\
458 \\
446 \\
446 \\
482\end{array}$ & $\begin{array}{l}622 \\
606 \\
622 \\
638 \\
638\end{array}$ & $\begin{array}{l}702 \\
718 \\
734 \\
718 \\
750\end{array}$ & $\begin{array}{l}734 \\
702 \\
718 \\
766 \\
750\end{array}$ & $\begin{array}{l}1,050 \\
1,280 \\
1,120 \\
1,100 \\
1,180\end{array}$ & $\begin{array}{l}5,690 \\
5,370 \\
5,860 \\
6,170 \\
6,480\end{array}$ & $\begin{array}{l}4,720 \\
6,870 \\
4,760 \\
4,330 \\
4,360\end{array}$ & $\begin{array}{l}2,900 \\
3,160 \\
3,010 \\
3,470 \\
2,900\end{array}$ & $\begin{array}{r}977 \\
977 \\
977 \\
1,000 \\
977\end{array}$ & $\begin{array}{r}1,030 \\
1,000 \\
952 \\
918 \\
862\end{array}$ & $\begin{array}{l}310 \\
310 \\
310 \\
305 \\
300\end{array}$ & $\begin{array}{l}379 \\
379 \\
370 \\
379 \\
379\end{array}$ \\
\hline $\begin{array}{l}27 \\
22 \\
23 \\
24 \\
25\end{array}$ & $\begin{array}{l}470 \\
470 \\
470 \\
494 \\
494\end{array}$ & $\begin{array}{l}638 \\
606 \\
638 \\
638 \\
638\end{array}$ & $\begin{array}{r}1,030 \\
878 \\
798 \\
766 \\
766\end{array}$ & $\begin{array}{r}718 \\
734 \\
734 \\
1,280 \\
1,180\end{array}$ & $\begin{array}{l}1,180 \\
1,300 \\
1,340 \\
1,340 \\
1,360\end{array}$ & $\begin{array}{l}6,860 \\
6,860 \\
6,880 \\
6,680 \\
5,950\end{array}$ & $\begin{array}{l}4,410 \\
4,860 \\
4,820 \\
4,140 \\
4,080\end{array}$ & $\begin{array}{l}2,500 \\
2,380 \\
2,130 \\
1,760 \\
1,690\end{array}$ & $\begin{array}{l}952 \\
929 \\
907 \\
884 \\
862\end{array}$ & $\begin{array}{r}794 \\
738 \\
696 \\
1,080 \\
805\end{array}$ & $\begin{array}{l}300 \\
300 \\
300 \\
295 \\
295\end{array}$ & $\begin{array}{l}379 \\
388 \\
379 \\
388 \\
370\end{array}$ \\
\hline $\begin{array}{l}26 \\
27 \\
28 \\
29 \\
30 \\
31 \\
\end{array}$ & $\begin{array}{r}494 \\
494 \\
518 \\
530 \\
530 \\
* 530 \\
\end{array}$ & $\begin{array}{c}622 \\
606 \\
606 \\
578 \\
590 \\
- \\
\end{array}$ & $\begin{array}{r}790 \\
766 \\
750 \\
750 \\
750 \\
718 \\
\end{array}$ & $\begin{array}{r}1,010 \\
750 \\
686 \\
766 \\
814 \\
830 \\
\end{array}$ & $\begin{array}{c}1,400 \\
1,480 \\
1,620 \\
- \\
- \\
-\end{array}$ & $\begin{array}{l}6,860 \\
6,260 \\
6,4,20 \\
6,510 \\
6,410 \\
6,550\end{array}$ & $\begin{array}{c}3,740 \\
3,530 \\
3,520 \\
3,400 \\
3,280 \\
-\end{array}$ & $\begin{array}{l}1,420 \\
1,500 \\
1,500 \\
1,440 \\
1,470 \\
1,370 \\
1\end{array}$ & $\begin{array}{c}839 \\
839 \\
339 \\
839 \\
839 \\
-\end{array}$ & $\begin{array}{l}749 \\
687 \\
628 \\
589 \\
560 \\
540 \\
\end{array}$ & $\begin{array}{l}290 \\
290 \\
285 \\
285 \\
280 \\
280\end{array}$ & $\begin{array}{c}405 \\
4 y_{4} \\
450 \\
4114 \\
388 \\
-\end{array}$ \\
\hline $\begin{array}{l}\text { Total } \\
\text { Mean } \\
\text { Ac-ft }\end{array}$ & $\begin{array}{r}13,218 \\
426 \\
26,220 \\
\end{array}$ & $\begin{array}{r}17,620 \\
587 \\
34,950 \\
\end{array}$ & $\begin{array}{r}22, \overline{911} \\
739 \\
45,440\end{array}$ & $\begin{array}{r}23,902 \\
771 \\
1,7,410 \\
\end{array}$ & $\begin{array}{r}31,061 \\
1,109 \\
61,610 \\
\end{array}$ & $\begin{array}{r}204,620 \\
6,601 \\
4,05,900 \\
\end{array}$ & $\begin{array}{r}145,740 \\
4,859 \\
289,100\end{array}$ & $\begin{array}{r}74,880 \\
2,415 \\
148,500\end{array}$ & $\begin{array}{r}30,554 \\
1,018 \\
60,600\end{array}$ & $\begin{array}{r}26,156 \\
844 \\
51,880\end{array}$ & $\begin{array}{r}10,812 \\
349 \\
21,450\end{array}$ & $\begin{array}{r}10,957 \\
365 \\
21,730 \\
\end{array}$ \\
\hline $\begin{array}{l}\text { Calen } \\
\text { Water }\end{array}$ & $\begin{array}{l}\text { zrear } \\
\text { ar } 195\end{array}$ & $\begin{array}{l}\mathrm{Ma} \\
\mathrm{Ma}\end{array}$ & $\begin{array}{r}4,86 \\
17,90\end{array}$ & Mi & $\begin{array}{l}205 \\
280\end{array}$ & $\begin{array}{l}\text { Mzan } \\
\text { Mean }\end{array}$ & $\begin{array}{l}54 \\
78\end{array}$ & $\begin{array}{l}\text {-ft } \\
\text {-ft } \quad 1,2\end{array}$ & $\begin{array}{l}+\infty 0 \\
000\end{array}$ & & & \\
\hline
\end{tabular}

Fak discharae (base 8,000 cfs).-War. 2 mear dajily $11,900 \mathrm{cfs}$.

* Discharge measurument made on tris ing.

* Dich highwater rating was undefined and for sur. 7 to seni. 2

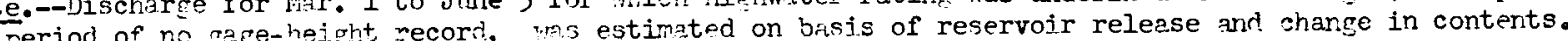


HEIMAND RIVER BASIN

Arghandab River above Arghandab Reservoir, Afghanistan

Discharge, in cubic feet per second, water year October 1959 to September 1960

\begin{tabular}{|c|c|c|c|c|c|c|c|c|c|c|c|c|}
\hline Dar & Oct. & Nov. & Dec. & Jan. & Feb. & Mar. & Apr. & May & June & July & $\mathrm{Aug}_{0}$ & Sept. \\
\hline $\begin{array}{l}1 \\
2 \\
3 \\
4 \\
5\end{array}$ & $\begin{array}{r}\frac{379}{379} \\
379 \\
388 \\
* 388\end{array}$ & $\begin{array}{l}\frac{459}{486} \\
550 \\
628 \\
794\end{array}$ & $\begin{array}{r}964 \\
1,540 \\
1,090 \\
1,060 \\
* 1,040\end{array}$ & $\begin{array}{r}794 \\
* 794 \\
771 \\
760 \\
782\end{array}$ & $\begin{array}{l}771 \\
771 \\
760 \\
\frac{749}{760}\end{array}$ & $\begin{array}{r}* 862 \\
850 \\
839 \\
929 \\
1,050\end{array}$ & $\begin{array}{r}2,230 \\
2,200 \\
* 2,260 \\
2,500 \\
2,640\end{array}$ & $\begin{array}{l}5,340 \\
5,400 \\
5,400 \\
5,280 \\
5,460 \\
\end{array}$ & $\begin{array}{l}1,930 \\
\frac{1,880}{1,790} \\
1,760 \\
1,680\end{array}$ & $\begin{array}{l}740 \\
730 \\
720 \\
730 \\
740\end{array}$ & $\begin{array}{l}a 480 \\
a 465 \\
a 450 \\
a 435 \\
a 420\end{array}$ & $\begin{array}{l}280 \\
284 \\
284 \\
272 \\
267 \\
\end{array}$ \\
\hline $\begin{array}{r}6 \\
7 \\
8 \\
9 \\
10\end{array}$ & $\begin{array}{l}388 \\
388 \\
396 \\
396 \\
414\end{array}$ & $\begin{array}{l}895 \\
907 \\
907 \\
918 \\
918\end{array}$ & $\begin{array}{r}1,000 \\
977 \\
952 \\
940 \\
929\end{array}$ & $\begin{array}{l}782 \\
771 \\
760 \\
760 \\
771\end{array}$ & $\begin{array}{l}782 \\
782 \\
794 \\
794 \\
805\end{array}$ & $\begin{array}{r}1,020 \\
964 \\
940 \\
952 \\
1,000\end{array}$ & $\begin{array}{l}2,860 \\
3,050 \\
3,280 \\
3,450 \\
3,530\end{array}$ & $\begin{array}{l}5,340 \\
4,880 \\
4,420 \\
4,180 \\
4,130\end{array}$ & $\begin{array}{l}1,660 \\
1,560 \\
1,580 \\
1,500 \\
1,400\end{array}$ & $\begin{array}{l}800 \\
850 \\
760 \\
730 \\
710\end{array}$ & $\begin{array}{l}a 405 \\
a 390 \\
a 375 \\
364 \\
364\end{array}$ & $\begin{array}{l}2844 \\
284 \\
280 \\
280 \\
276\end{array}$ \\
\hline $\begin{array}{l}11 \\
12 \\
13 \\
14 \\
15\end{array}$ & $\begin{array}{r}414 \\
423 \\
423 \\
423 \\
+441\end{array}$ & $\begin{array}{l}907 \\
907 \\
907 \\
907 \\
895\end{array}$ & $\begin{array}{l}918 \\
827 \\
884 \\
884 \\
929\end{array}$ & $\begin{array}{l}794 \\
850 \\
827 \\
794 \\
749\end{array}$ & $\begin{array}{l}827 \\
839 \\
850 \\
862 \\
918\end{array}$ & $\begin{array}{l}1,140 \\
1,200 \\
1,630 \\
1,630 \\
1,630\end{array}$ & $\begin{array}{l}3,650 \\
3,900 \\
4,000 \\
4,400 \\
4,600\end{array}$ & $\begin{array}{l}4,020 \\
3,900 \\
4,180 \\
4,240 \\
4,180\end{array}$ & $\begin{array}{l}1,380 \\
1,330 \\
1,210 \\
1,140 \\
1,120\end{array}$ & $\begin{array}{l}700 \\
700 \\
730 \\
740 \\
730\end{array}$ & $\begin{array}{l}354 \\
349 \\
344 \\
338 \\
344\end{array}$ & $\begin{array}{l}288 \\
288 \\
288 \\
288 \\
288\end{array}$ \\
\hline $\begin{array}{l}16 \\
17 \\
18 \\
19 \\
20\end{array}$ & $\begin{array}{l}447 \\
432 \\
432 \\
423 \\
423\end{array}$ & $\begin{array}{l}884 \\
873 \\
873 \\
862 \\
862\end{array}$ & $\begin{array}{r}1,030 \\
952 \\
907 \\
907 \\
929\end{array}$ & $\begin{array}{l}737 \\
749 \\
760 \\
760 \\
760\end{array}$ & $\begin{array}{l}\frac{964}{907} \\
895 \\
873 \\
873\end{array}$ & $\begin{array}{l}1,510 \\
1,460 \\
1,660 \\
1,600 \\
1,600\end{array}$ & $\begin{array}{l}6,660 \\
9,900 \\
7,470 \\
6,950 \\
6,890\end{array}$ & $\begin{array}{l}4,130 \\
4,240 \\
4,300 \\
4,300 \\
4,180\end{array}$ & $\begin{array}{r}1,070 \\
1,040 \\
1,000 \\
987 \\
958\end{array}$ & $\begin{array}{l}700 \\
632 \\
632 \\
572 \\
572\end{array}$ & $\begin{array}{l}318 \\
302 \\
292 \\
308 \\
292\end{array}$ & $\begin{array}{l}302 \\
297 \\
292 \\
297 \\
297\end{array}$ \\
\hline $\begin{array}{l}21 \\
22 \\
23 \\
24 \\
25\end{array}$ & $\begin{array}{l}423 \\
423 \\
423 \\
423 \\
423\end{array}$ & $\begin{array}{l}862 \\
862 \\
850 \\
850 \\
862\end{array}$ & $\begin{array}{l}929 \\
918 \\
918 \\
895 \\
834\end{array}$ & $\begin{array}{l}726 \\
706 \\
716 \\
726 \\
726\end{array}$ & $\begin{array}{l}884 \\
940 \\
918 \\
895 \\
895\end{array}$ & $\begin{array}{l}1,630 \\
1,570 \\
1,540 \\
1,540 \\
1,600\end{array}$ & $\begin{array}{l}7,120 \\
6,660 \\
6,540 \\
6,260 \\
4,880\end{array}$ & $\begin{array}{r}3,750 \\
2,990 \\
3,140 \\
* 2,860 \\
2,670\end{array}$ & $\begin{array}{l}958 \\
944 \\
987 \\
987 \\
944\end{array}$ & $\begin{array}{l}615 \\
547 \\
547 \\
556 \\
556\end{array}$ & $\begin{array}{r}* 284 \\
284 \\
280 \\
\frac{267}{267}\end{array}$ & $\begin{array}{l}313 \\
318 \\
323 \\
313 \\
302\end{array}$ \\
\hline $\begin{array}{l}26 \\
27 \\
28 \\
29 \\
30 \\
31\end{array}$ & $\begin{array}{r}423 \\
432 \\
432 \\
432 \\
441 \\
\times 450 \\
\end{array}$ & $\begin{array}{r}895 \\
1,040 \\
1,050 \\
988 \\
952 \\
-\end{array}$ & $\begin{array}{l}873 \\
862 \\
862 \\
862 \\
839 \\
816 \\
\end{array}$ & $\begin{array}{r}737 \\
726 \\
749 \\
782 \\
* 782 \\
749\end{array}$ & $\begin{array}{c}895 \\
884 \\
884 \\
884 \\
- \\
-\end{array}$ & $\begin{array}{l}1,800 \\
1,830 \\
1,660 \\
2,230 \\
2,180 \\
2,230 \\
\end{array}$ & $\begin{array}{c}4,590 \\
4,480 \\
4,480 \\
* 4,540 \\
4,880 \\
-\end{array}$ & $\begin{array}{l}2,520 \\
2,340 \\
2,220 \\
2,150 \\
2,010 \\
1,960 \\
\end{array}$ & $\begin{array}{r}900 \\
850 \\
810 \\
760 \\
* 730 \\
-\end{array}$ & $\begin{array}{r}538 \\
556 \\
2540 \\
a 525 \\
* a 510 \\
2495\end{array}$ & $\begin{array}{l}276 \\
272 \\
276 \\
276 \\
272 \\
272\end{array}$ & $\begin{array}{c}308 \\
313 \\
308 \\
308 \\
318 \\
-\end{array}$ \\
\hline $\begin{array}{l}\text { Total } \\
\text { Mean } \\
\text { Ac-ft }\end{array}$ & $\begin{array}{r}12,895 \\
416 \\
25,580 \\
\end{array}$ & $\begin{array}{r}25,550 \\
852 \\
50,680 \\
\end{array}$ & $\begin{array}{r}29,317 \\
946 \\
58,150 \\
\end{array}$ & $\begin{array}{r}23,650 \\
763 \\
46,910 \\
\end{array}$ & $\begin{array}{r}21,655 \\
850 \\
48,900 \\
\end{array}$ & $\begin{array}{r}44,276 \\
1,428 \\
87,820 \\
\end{array}$ & $\begin{array}{r}140,850 \\
4,695 \\
279,400 \\
\end{array}$ & $\begin{array}{r}120,110 \\
3,874 \\
238,200\end{array}$ & $\begin{array}{r}36,845 \\
1,228 \\
73,080 \\
\end{array}$ & $\begin{array}{r}20,203 \\
652 \\
40,070\end{array}$ & $\begin{array}{r}10,415 \\
336 \\
20,660\end{array}$ & $\begin{array}{r}8,840 \\
295 \\
17,540 \\
\end{array}$ \\
\hline $\begin{array}{l}\text { Caler } \\
\text { viater }\end{array}$ & & $\begin{array}{l}\text { lia } \\
\text { lia }\end{array}$ & $\begin{array}{r}11,900 \\
9,900\end{array}$ & $\begin{array}{l}\text { lin } \\
\text { lin }\end{array}$ & $\begin{array}{l}2 \varepsilon 0 \\
267\end{array}$ & $\begin{array}{l}\text { liear: } \\
\text { Mean }\end{array}$ & $\begin{array}{l}1,716 \\
1,360\end{array}$ & $\begin{array}{l}A C-f t \quad I, \\
A C-f t\end{array}$ & $\begin{array}{l}, 000 \\
, 000\end{array}$ & & & \\
\hline
\end{tabular}

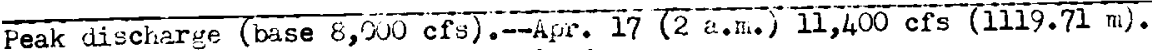

* Discharge measurenerit made on this day.

a l.o gage-hej ght $^{2}$ record; aischarge estimated. 
Arghandab Reservoir near Kandahar, ifghari star

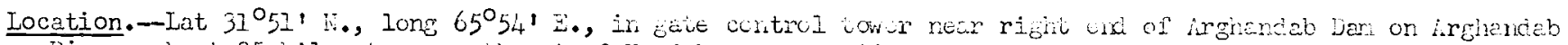
iiver, about $35 \mathrm{kilmeters} \mathrm{northeast} \mathrm{of} \mathrm{landaher,} \mathrm{wbout} 90$ kilometere urstreas from Deri Ii ver, and about 185 kiloneters urstroan fron Helmand River.

Drainare area.-6,790 sq ni, apripoximately (from irver of India mans) wi wich about 640 sa ni is rrobably non-contributing.

Pccords available.-February 1952 to sertente r 1960.

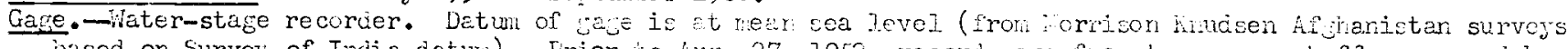
Dased on Survey of India datuil). Frior to lug. 27, 1952, recorde ere fron temorary staff gages and by levels at same daturn.

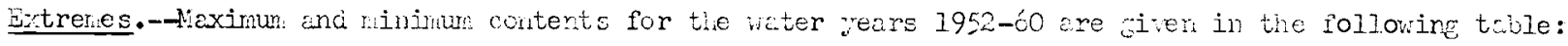

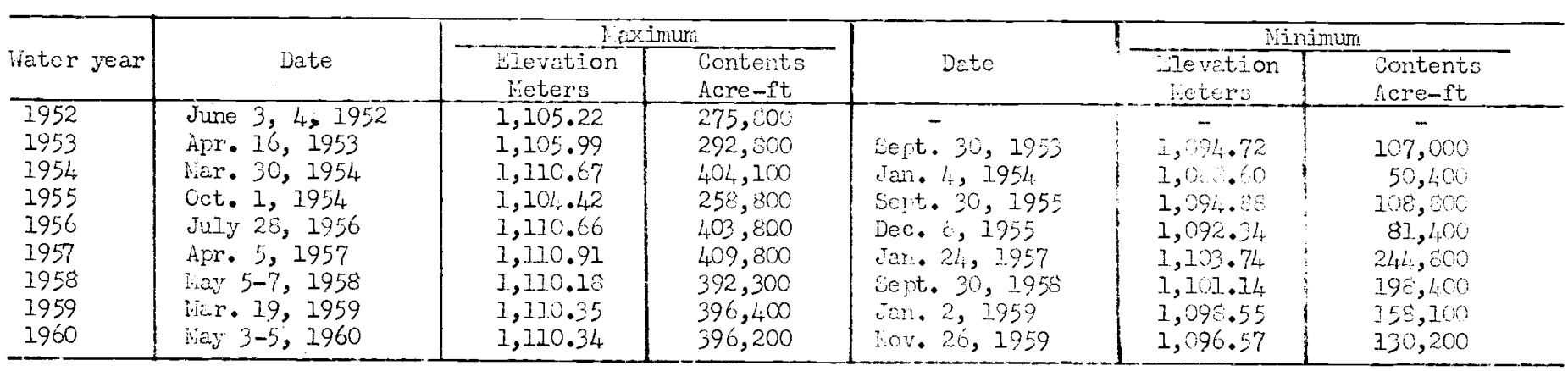

Remank.--Reservoir is formed by earth-fill dan; storage vegar: Feb. 24, 1952; dem conpletea in 1952. Capacity 388,000 acre-ft between elevation 1,06́6.0 neters, center line of irrigation outlet, and 1l10.0 jieters, crest of ungated spilliwa, above mean sea level. lecervoir is for irrigation of up to 150,000 acres in the Arghandab River Valley and for a future power plant for which proposed minimuri opereiting 7 evel. vould be 1088.0 neters. 
FELENIND RIVER BASIH

Arghandab Reservoir near Kandahex, Afghanistan

Contents in huncireds of acre-feet on last dia oi nonth, of Grghandab leservoir ne ir indahar, hfgharistan

\begin{tabular}{|c|c|c|c|c|c|c|c|c|c|c|c|c|}
\hline Year & Oct. & Nov. & Dec. & JEn. & Feb. & MaI'. & $A \mathrm{pr}$ & Hay & June & JuIy & AUE. & Sept. \\
\hline $\begin{array}{l}1952 \\
1953 \\
1954_{4} \\
1955 \\
1956 \\
1957 \\
1958 \\
1959 \\
1960\end{array}$ & $\begin{array}{r}1,795 \\
774 \\
2,277 \\
914 \\
2,791 \\
2,944 \\
1,084 \\
1,430\end{array}$ & $\begin{array}{r}1,737 \\
578 \\
2,100 \\
823 \\
2,743 \\
3,222 \\
1,616 \\
1,306\end{array}$ & $\begin{array}{r}1,722 \\
513 \\
1,936 \\
1,065 \\
2,620 \\
3,261 \\
1,587 \\
1,306\end{array}$ & $\begin{array}{r}1,528 \\
64,2 \\
1,813 \\
1,266 \\
2,816 \\
3,075 \\
1,690 \\
1,340\end{array}$ & $\begin{array}{r}1,03 \\
2,157 \\
2,189 \\
1,752 \\
1,803 \\
3,158 \\
3,316 \\
2,049 \\
1,597\end{array}$ & $\begin{array}{l}1,137 \\
2,802 \\
4,012 \\
2,500 \\
4,000 \\
3,964 \\
3,909 \\
3,938 \\
2,079\end{array}$ & $\begin{array}{l}2,4,02 \\
2,349 \\
3,947 \\
2,354 \\
3,966 \\
3,978 \\
3,909 \\
3,912 \\
3,952\end{array}$ & $\begin{array}{l}2,748 \\
2,611 \\
3,368 \\
2,314 \\
3,904 \\
3,959 \\
3,846 \\
3,301 \\
3,921\end{array}$ & $\begin{array}{l}2,519 \\
2,346 \\
3,698 \\
2,064 \\
3,800 \\
3,603 \\
3,360 \\
3,158 \\
3,885\end{array}$ & $\begin{array}{l}2,226 \\
1,915 \\
3,4,4 \\
1,693 \\
3,91,2 \\
3,4,98 \\
2,94.8 \\
2,857 \\
3,626\end{array}$ & $\begin{array}{l}1,994 \\
1,447 \\
3,010 \\
1,356 \\
3,556 \\
3,374 \\
2,290 \\
2,318 \\
3,104\end{array}$ & $\begin{array}{l}1,327 \\
1,070 \\
2,588 \\
1,088 \\
3,045 \\
3,128 \\
1,984 \\
1,822 \\
2,723\end{array}$ \\
\hline
\end{tabular}



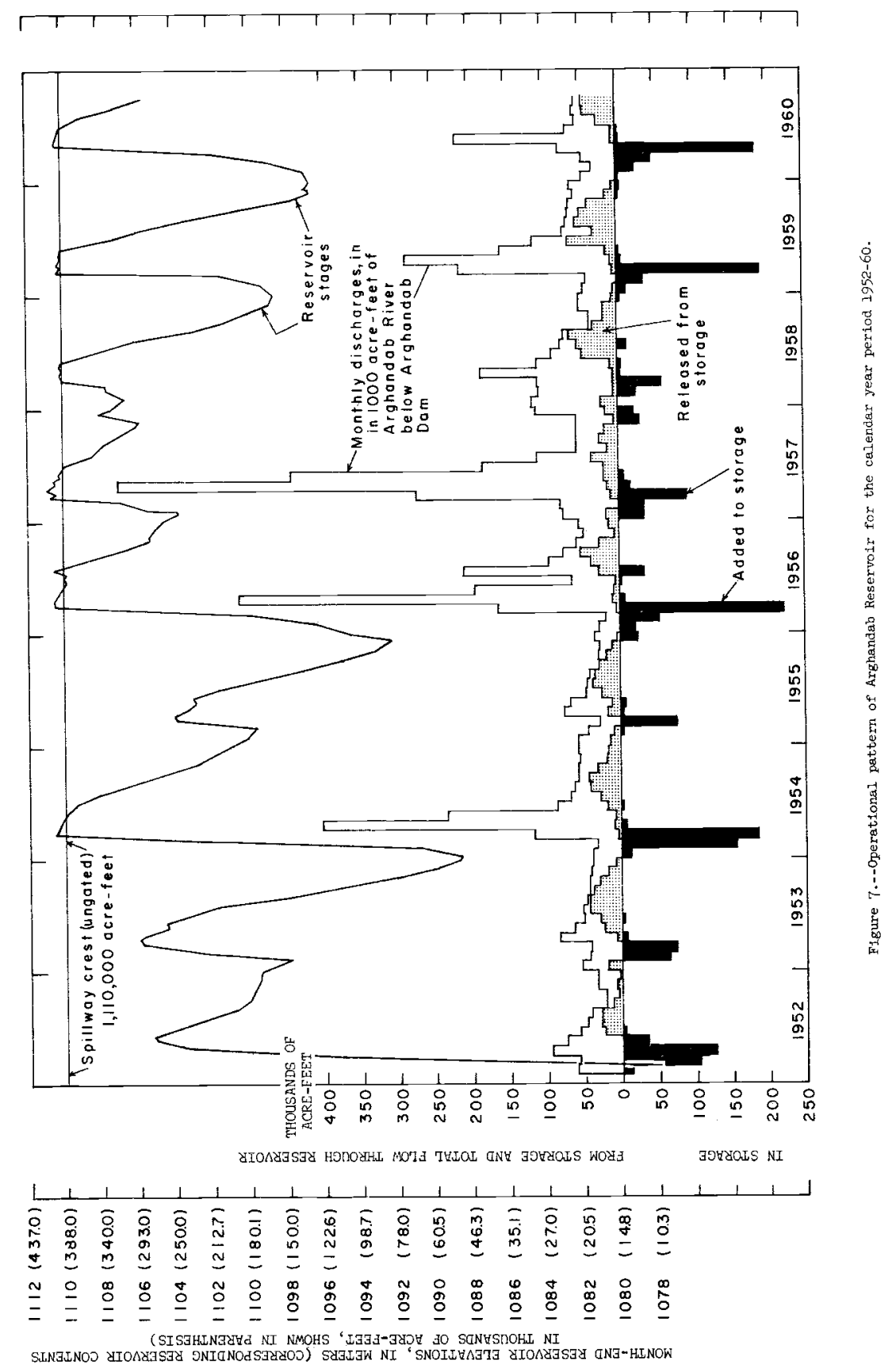


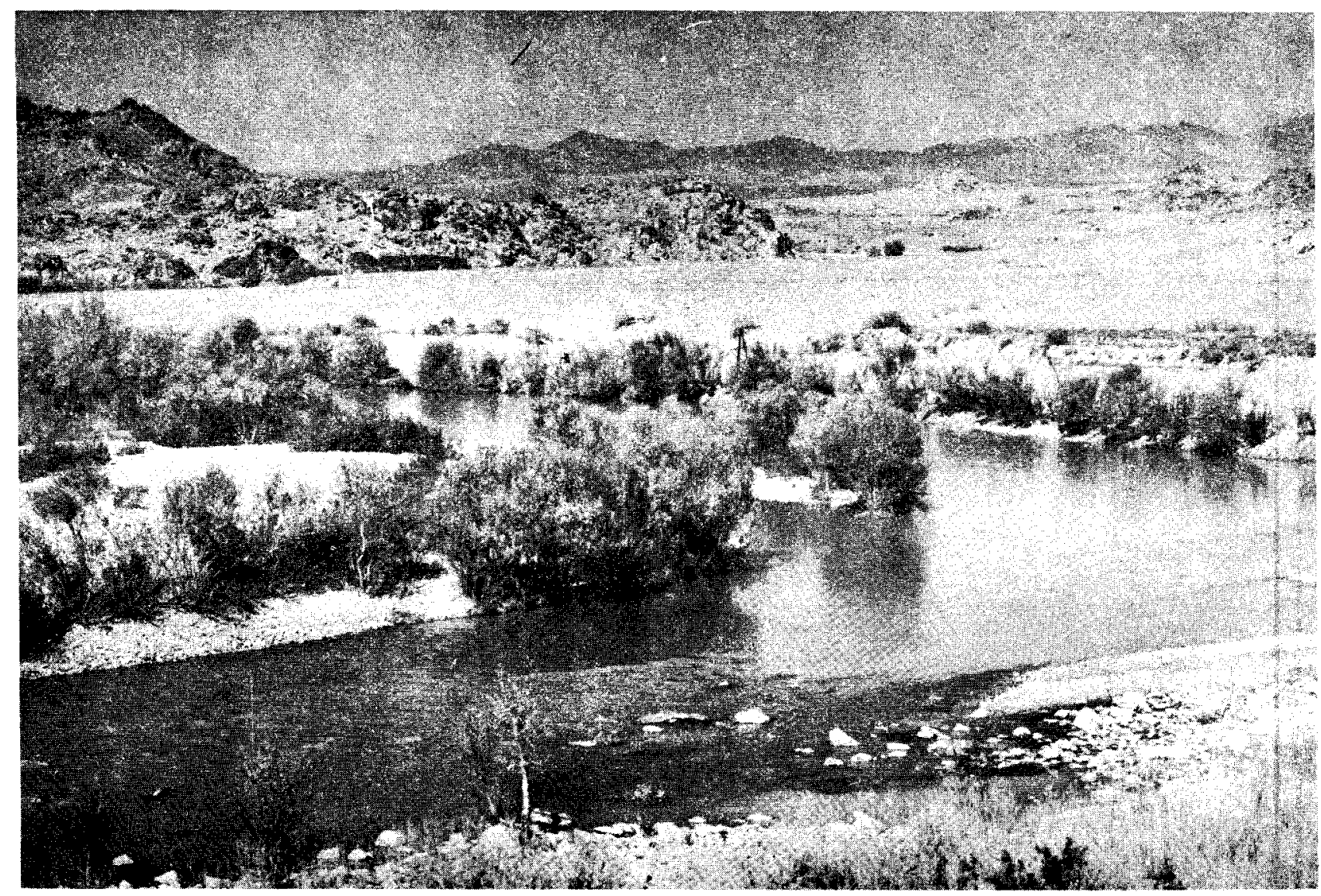

Figure 8.--Arghandab River below Arghandab Dam showing cableway and control. 
HBIXAND RIVER BASTI

Arghandab River belon Arghandab Dan, Afghanistan

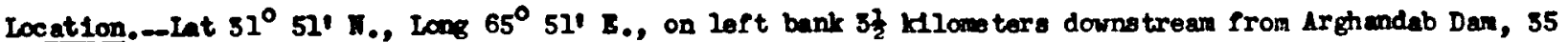
Hlow ters northeast of Kandahar, 90 klloweters upotrean from Dorl Rud, and 180 k1loweters upstream Trom Belmand River.

Drathage area.--6,870 oq at (Trom Survey of India maps) of which 640 sq int is probably non-contributing. Records avallable.--October 1947 to September 1960.

Gap.-Hater-tage recorder. Datw of gage is at mean sea level (fron Morrison-Kmudsen Afghanistan surveys

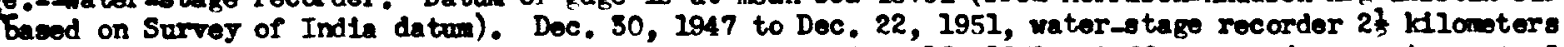
up trean at datun 10 meters higher. Dec. 23, 1951 to $\alpha t$. 16, 1952, staff gage and concrete control 3 Ulanoters upotrean at datun 10 metors higher.

Average discharge.--15 jears, $1446 \mathrm{cfa}$ (adjueted for storage) 1,047,nM acre-ft per joar.

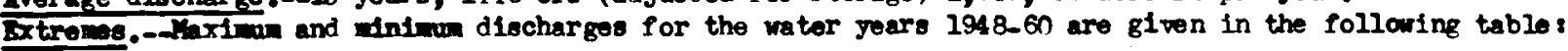

\begin{tabular}{|c|c|c|c|c|c|}
\hline \multirow[b]{2}{*}{ Water soar } & \multirow[b]{2}{*}{ Date } & \multicolumn{2}{|c|}{ landn } & \multicolumn{2}{|c|}{ Findina vaits } \\
\hline & & $\begin{array}{l}\text { Gage Holght } \\
\text { (netors) }\end{array}$ & $\begin{array}{c}\text { Dischargo } \\
\text { (cro) }\end{array}$ & Dato & $\begin{array}{c}\text { Dischar } \\
\text { (crs) }\end{array}$ \\
\hline $\begin{array}{l}1948 \\
1919 \\
1950 \\
1951 \\
1952 \\
1953 \\
1954 \\
1955 \\
1956 \\
1957 \\
1958 \\
1959\end{array}$ & 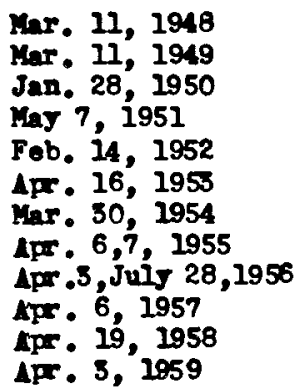 & $\begin{array}{c}1042.71 \\
21043.98 \\
1042.87 \\
1042.92 \\
- \\
1055.58 \\
1057.35 \\
1055.92 \\
1057.52 \\
1058.54 \\
1056.44 \\
1057.02\end{array}$ & $\begin{array}{r}12,700 \\
28,000 \\
14,700 \\
15,300 \\
b 5,100 \\
1,570 \\
15,800 \\
1,600 \\
10,100 \\
22,500 \\
5,550 \\
7,520\end{array}$ & $\begin{array}{l}\text { Sept. 7-8, } 1948 \\
\text { Sept. 5-9, } 1949 \\
\text { Oct. 1, 2, } 1949 \\
\text { Sept. 30, } 1951 \\
\text { Aug. 11, } 1952 \\
\text { Oct. 4-6, } 1952 \\
\text { Feb. 28, 1954 } \\
\text { Feb. 6, 1955 } \\
\text { Feb. 21-23, } 1956 \\
\text { Mar. 11, 1957 } \\
\text { Sept. 19.20, } 1958 \\
\text { Oct. 23, 1958 }\end{array}$ & $\begin{array}{r}44 \\
156 \\
243 \\
285 \\
0 \\
280 \\
406 \\
84 \\
242 \\
152 \\
600 \\
585\end{array}$ \\
\hline 1960 & $\operatorname{MaJ} 4,1960$ & 1056.75 & 5,050 & Jan. 30 to Fob. 2 & 422 \\
\hline
\end{tabular}

- Iron eloodmark

b From records of Morrison-Kmudsen Afghanistan

Remarke.-Recards good except those above 15,000 cfs and those for periods of no gage-height record, which are fatr. Flo regulated since February 24, 1952 by Arghandab Dam. No diverelons between dam and gage. Regulated recervolr capeity 18 used to irrigate up to 150,000 acres in the Arghandab Vallay. 
HEIMAND RIVER BASIN

Arghandab River Below Arghandab Dam, Afghanistan

Discharge, in cubic feet per second, water year October 1947 to September 1948

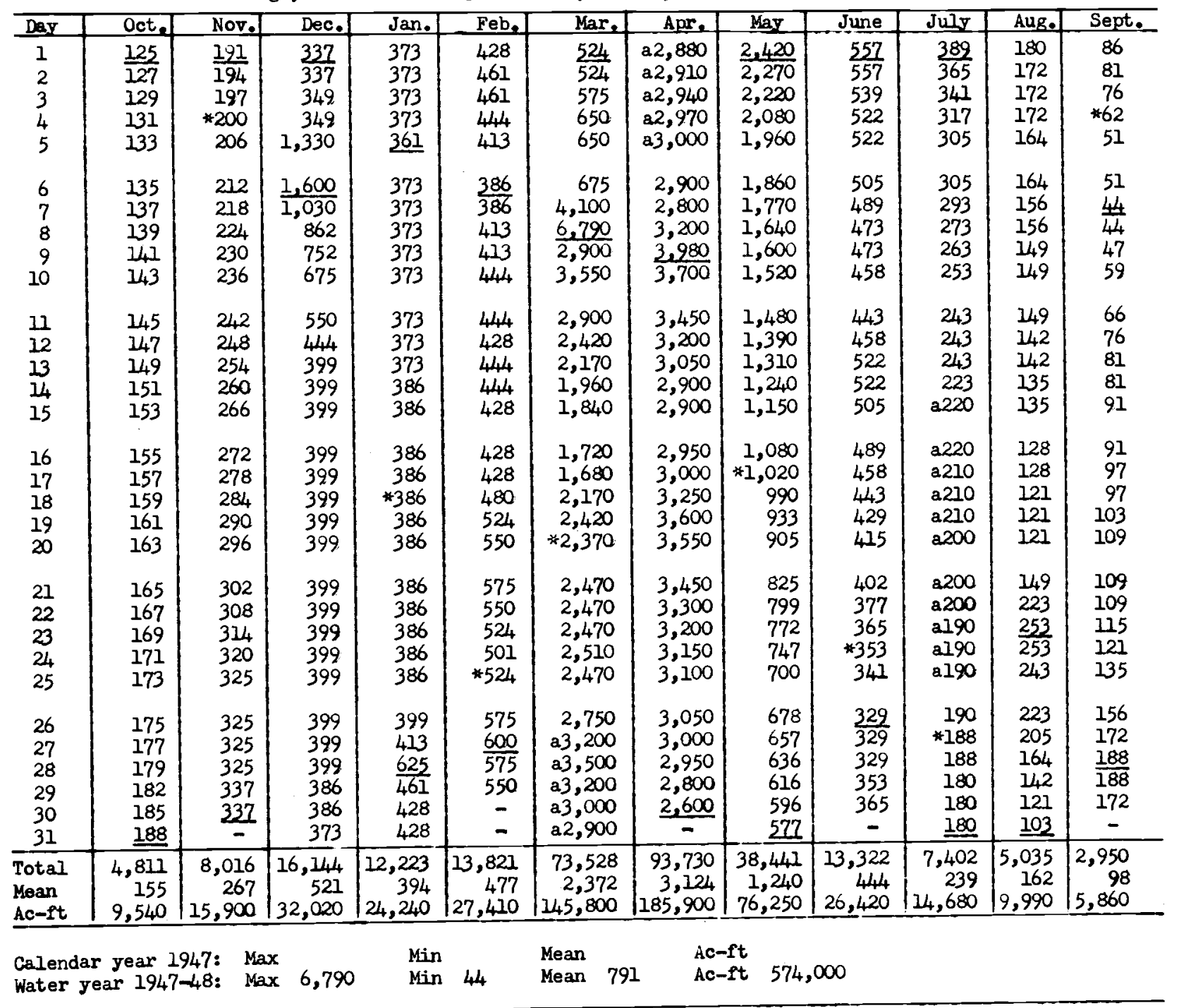

* Discharge measurement made on this day.

a No gage-height record; discharge estimated on basis of weather records.

a No - water-stage recorder installed Dec. 30; occasional staff gage readings only prior to Dec. 30. 
HEIMAND RIVER BASIN

Arghandab River below Arghandab Dam, Afghanistan

Discharge, in cubic feet per second, water year October 1948 to September 1949

\begin{tabular}{|c|c|c|c|c|c|c|c|c|c|c|c|c|}
\hline Day & Cots. & Nov. & Dec. & Jan. & Feb. & Mar. & Apr. & May & June & July & Aug. & Sept. \\
\hline $\begin{array}{l}1 \\
2 \\
3 \\
4 \\
5\end{array}$ & $\begin{array}{r}* 164 \\
156 \\
149 \\
2150 \\
a 150\end{array}$ & 370 & 480 & 550 & $\begin{array}{r}a 580 \\
a 1,000 \\
a 900 \\
a 700 \\
a 650\end{array}$ & $\begin{array}{l}640 \\
670 \\
670 \\
640 \\
620 \\
\end{array}$ & $\begin{array}{r}* 4,340 \\
4,520 \\
4,820 \\
4,940 \\
4,940\end{array}$ & $\begin{array}{l}3,000 \\
2,800 \\
2,600 \\
2,500 \\
2,350\end{array}$ & 550 & $\begin{array}{l}\frac{130}{4} \\
2420 \\
2410 \\
2400 \\
* 389\end{array}$ & $\begin{array}{l}196 \\
196 \\
196 \\
196 \\
317\end{array}$ & $\begin{array}{l}180 \\
172 \\
164 \\
156 \\
156\end{array}$ \\
\hline $\begin{array}{r}6 \\
7 \\
8 \\
9 \\
10\end{array}$ & $\begin{array}{l}a 150 \\
a 160 \\
\text { al60 } \\
\text { al60 } \\
\text { al70 }\end{array}$ & & & & $\begin{array}{l}\text { a600 } \\
\text { a } 580 \\
\text { a580 } \\
\text { a } 580 \\
\text { a } 580\end{array}$ & $\begin{array}{r}670 \\
670 \\
825 \\
2,000 \\
a 2,000\end{array}$ & $\begin{array}{l}4,880 \\
5,420 \\
5,180 \\
5,120 \\
5,620\end{array}$ & $\begin{array}{l}2,200 \\
2,100 \\
2,000 \\
1,900 \\
1,800\end{array}$ & & $\begin{array}{l}377 \\
365 \\
353 \\
341 \\
329\end{array}$ & $\begin{array}{r}1,680 \\
1,390 \\
933 \\
723 \\
577\end{array}$ & $\begin{array}{r}156 \\
156 \\
156 \\
156 \\
* 164\end{array}$ \\
\hline $\begin{array}{l}11 \\
12 \\
13 \\
14 \\
15\end{array}$ & $\begin{array}{l}2170 \\
a 170 \\
a 180 \\
a 180 \\
a 180\end{array}$ & & & & $\begin{array}{l}a 580 \\
a 580 \\
a 580 \\
* 577 \\
a 580\end{array}$ & $\begin{array}{r}a 15,000 \\
a 12,000 \\
a 8,000 \\
a 7,000 \\
a 6,500\end{array}$ & $\begin{array}{r}6,440 \\
6,440 \\
5,420 \\
* 5,180 \\
4,940\end{array}$ & $\begin{array}{l}1,800 \\
1,700 \\
1,600 \\
1,500 \\
1,400\end{array}$ & & $\begin{array}{l}317 \\
293 \\
293 \\
283 \\
273\end{array}$ & $\begin{array}{r}473 \\
389 \\
329 \\
* 283 \\
253\end{array}$ & $\begin{array}{l}\text { al70 } \\
\text { al70 } \\
\text { al70 } \\
\text { al75 } \\
\text { al75 }\end{array}$ \\
\hline $\begin{array}{l}16 \\
17 \\
18 \\
19 \\
20\end{array}$ & $\begin{array}{l}2190 \\
a 190 \\
a 200 \\
a 200 \\
a 210\end{array}$ & & & & $\begin{array}{l}\text { a } 580 \\
\text { a } 590 \\
\text { a.590 } \\
\text { a } 590 \\
\text { a } 600\end{array}$ & $\begin{array}{l}a 6,000 \\
a 5,800 \\
a 5,600 \\
a 5,400 \\
a 5,200\end{array}$ & $\begin{array}{l}4,760 \\
4,700 \\
4,700 \\
4,520 \\
4,400\end{array}$ & $\begin{array}{l}1,300 \\
1,200 \\
1,200 \\
1,100 \\
1,100\end{array}$ & & $\begin{array}{l}273 \\
263 \\
263 \\
253 \\
243\end{array}$ & $\begin{array}{l}233 \\
205 \\
188 \\
172 \\
164\end{array}$ & $\begin{array}{l}\text { al75 } \\
\text { al } \\
\text { al } \\
\text { al } 80 \\
\text { al } 85\end{array}$ \\
\hline $\begin{array}{l}21 \\
22 \\
23 \\
24 \\
25\end{array}$ & $\begin{array}{l}\text { a220 } \\
\text { a230 } \\
\text { a240 } \\
\text { a250 } \\
\text { a260 }\end{array}$ & & & & $\begin{array}{l}a 600 \\
a 600 \\
a 600 \\
8610 \\
a 620\end{array}$ & $\begin{array}{l}a 6,000 \\
85,500 \\
a 5,000 \\
a 4,800 \\
a 4,600\end{array}$ & $\begin{array}{l}4,220 \\
3,980 \\
3,650 \\
3,600 \\
3,550\end{array}$ & $\begin{array}{r}1,000 \\
* 990 \\
950 \\
910 \\
880\end{array}$ & & $\begin{array}{l}233 \\
223 \\
214 \\
214 \\
205\end{array}$ & $\begin{array}{l}\frac{156}{156} \\
156 \\
156 \\
164\end{array}$ & $\begin{array}{l}a 185 \\
a 188 \\
a 196 \\
a 196 \\
a 205\end{array}$ \\
\hline $\begin{array}{l}26 \\
27 \\
28 \\
29 \\
30 \\
31\end{array}$ & $\begin{array}{l}* 263 \\
2270 \\
a 270 \\
a 280 \\
a 290 \\
a 290\end{array}$ & $\begin{array}{r}370 \\
* 458 \\
-\end{array}$ & $1_{4 \infty 0}$ & $\underset{550}{1}$ & $\begin{array}{c}a 620 \\
a 630 \\
* 636 \\
- \\
= \\
-\end{array}$ & $\begin{array}{l}24,500 \\
24,400 \\
24,300 \\
24,300 \\
24,300 \\
24,300\end{array}$ & $\begin{array}{r}3,450 \\
\text { a3,350 } \\
\text { a3,300 } \\
\mathrm{a} 3,200 \\
\mathrm{a} 3,100 \\
-\end{array}$ & $\begin{array}{l}860 \\
830 \\
810 \\
790 \\
774 \\
760 \\
\end{array}$ & $\left.\right|_{550}$ & $\begin{array}{l}205 \\
205 \\
205 \\
196 \\
196 \\
196\end{array}$ & $\begin{array}{l}180 \\
205 \\
214 \\
214 \\
205 \\
188\end{array}$ & $\begin{array}{l}\mathrm{a} 205 \\
\mathrm{a} 21 \mathrm{u}_{4} \\
\mathrm{a} 214_{4} \\
\mathrm{a} 223 \\
233 \\
-\end{array}$ \\
\hline $\begin{array}{l}\text { Total } \\
\text { Mean } \\
\text { Ac-ft } \\
\end{array}$ & $\begin{array}{r}6,302 \\
203 \\
12,500 \\
\end{array}$ & $\begin{array}{r}11,188 \\
373 \\
22,190\end{array}$ & $\begin{array}{r}14,880 \\
480 \\
29,510 \\
\end{array}$ & $\begin{array}{r}17,050 \\
550 \\
33,820 \\
\end{array}$ & $\begin{array}{r}17,513 \\
625 \\
34,740 \\
\end{array}$ & $\begin{array}{r}147,905 \\
4,770 \\
293,400\end{array}$ & $\begin{array}{r}136,680 \\
4,556 \\
271,100 \\
\end{array}$ & $\begin{array}{r}46,704 \\
1,507 \\
92,640 \\
\end{array}$ & $\begin{array}{r}16,500 \\
550 \\
32,730 \\
\end{array}$ & $\begin{array}{r}8,860 \\
286 \\
17,570 \\
\end{array}$ & $\begin{array}{r}11,087 \\
358 \\
21,990\end{array}$ & $\begin{array}{r}5,435 \\
181 \\
10,780 \\
\end{array}$ \\
\hline $\begin{array}{l}\text { Calen } \\
\text { Water }\end{array}$ & $\begin{array}{l}\text { year } \\
\text { ar } 19\end{array}$ & $\begin{array}{l}\mathrm{Ma} \\
\mathrm{Maz}\end{array}$ & $\begin{array}{r}6,790 \\
15,000\end{array}$ & Min & $\begin{array}{r}44 \\
149\end{array}$ & $\begin{array}{lr}\text { Mean } & 800 \\
\text { Mean } & 1,200\end{array}$ & $\begin{array}{l}A c-f t \\
A c-f t\end{array}$ & $\begin{array}{l}580,800 \\
873,000\end{array}$ & & & & \\
\hline
\end{tabular}

* Discharge measurement made on this day.

No gage-height record; discharge estimated on basis of discharge measurements and weather records. 
HETWAND RIVER BASIN

Arghandab River below Arghandab Dam, Afghanistan

Discharge, in cubic feet per second, water year October 1949 to September 1950

\begin{tabular}{|c|c|c|c|c|c|c|c|c|c|c|c|c|}
\hline Day & Oct. & Nove & Dec. & Jan. & Feb. & Mar. & Apr 0 & May & June & July & Aug. & Sept. \\
\hline $\begin{array}{l}1 \\
2 \\
3 \\
4 \\
5\end{array}$ & $\begin{array}{l}\frac{243}{243} \\
253 \\
253 \\
263\end{array}$ & $\begin{array}{l}429 \\
429 \\
429 \\
429 \\
429\end{array}$ & $\begin{array}{l}\frac{522}{505} \\
505 \\
505 \\
505\end{array}$ & $\begin{array}{l}522 \\
539 \\
522 \\
505 \\
505\end{array}$ & $\begin{array}{l}2,220 \\
2,120 \\
2,000 \\
1,908 \\
1,770\end{array}$ & $\begin{array}{l}1,311 \\
1,311 \\
1,311 \\
1,311 \\
1,348\end{array}$ & $\begin{array}{r}8,500 \\
10,200 \\
7,780 \\
6,860 \\
6,230\end{array}$ & $\begin{array}{l}5,300 \\
5,240 \\
5,180 \\
5,000 \\
5,300\end{array}$ & $\begin{array}{l}\frac{2,120}{1,955} \\
1,861 \\
1,815 \\
1,726\end{array}$ & $\begin{array}{l}700 \\
678 \\
657 \\
636 \\
616\end{array}$ & $\begin{array}{l}377 \\
415 \\
429 \\
443 \\
429\end{array}$ & $\begin{array}{l}317 \\
317 \\
317 \\
317 \\
317\end{array}$ \\
\hline $\begin{array}{r}6 \\
7 \\
8 \\
9 \\
10\end{array}$ & $\begin{array}{l}263 \\
273 \\
273 \\
273 \\
283\end{array}$ & $\begin{array}{l}429 \\
429 \\
429 \\
429 \\
415\end{array}$ & $\begin{array}{l}522 \\
522 \\
505 \\
505 \\
505\end{array}$ & $\begin{array}{l}505 \\
505 \\
522 \\
522 \\
522\end{array}$ & $\begin{array}{l}1,641 \\
1,558 \\
1,437 \\
1,348 \\
1,240\end{array}$ & $\begin{array}{l}1,348 \\
1,437 \\
1,477 \\
1,512 \\
1,860\end{array}$ & $\begin{array}{l}5,740 \\
5,420 \\
5,300 \\
5,180 \\
5,180\end{array}$ & $\begin{array}{l}5,620 \\
5,740 \\
5,420 \\
5,420 \\
6,020\end{array}$ & $\begin{array}{l}1,680 \\
1,726 \\
1,683 \\
1,641 \\
1,599\end{array}$ & $\begin{array}{l}616 \\
596 \\
577 \\
557 \\
539\end{array}$ & $\begin{array}{l}473 \\
473 \\
489 \\
522 \\
522\end{array}$ & $\begin{array}{l}317 \\
317 \\
317 \\
317 \\
317\end{array}$ \\
\hline $\begin{array}{l}\frac{11}{12} \\
13 \\
14 \\
15\end{array}$ & $\begin{array}{l}283 \\
273 \\
283 \\
293 \\
305\end{array}$ & $\begin{array}{l}\frac{402}{402} \\
415 \\
415 \\
415\end{array}$ & $\begin{array}{l}505 \\
505 \\
505 \\
505 \\
505\end{array}$ & $\begin{array}{l}522 \\
522 \\
522 \\
522 \\
539\end{array}$ & $\begin{array}{r}1,149 \\
1,084 \\
1,020 \\
961 \\
990\end{array}$ & $\begin{array}{l}2,120 \\
1,908 \\
2,080 \\
2,170 \\
2,040\end{array}$ & $\begin{array}{l}5,240 \\
5,360 \\
5,480 \\
5,420 \\
5,480\end{array}$ & $\begin{array}{l}6,860 \\
7,000 \\
6,160 \\
5,680 \\
5,300\end{array}$ & $\begin{array}{l}1,558 \\
1,477 \\
1,386 \\
1,311 \\
1,216\end{array}$ & $\begin{array}{l}522 \\
522 \\
522 \\
522 \\
678\end{array}$ & $\begin{array}{l}505 \\
473 \\
443 \\
429 \\
429\end{array}$ & $\begin{array}{l}317 \\
317 \\
317 \\
317 \\
317\end{array}$ \\
\hline $\begin{array}{l}16 \\
17 \\
18 \\
19 \\
20\end{array}$ & $\begin{array}{l}305 \\
305 \\
329 \\
341 \\
341\end{array}$ & $\begin{array}{l}429 \\
429 \\
429 \\
429 \\
443\end{array}$ & $\begin{array}{l}505 \\
489 \\
489 \\
489 \\
489\end{array}$ & $\begin{array}{l}539 \\
539 \\
539 \\
539 \\
539\end{array}$ & $\begin{array}{l}961 \\
961 \\
990 \\
990 \\
961\end{array}$ & $\begin{array}{l}1,955 \\
1,861 \\
1,815 \\
1,955 \\
2,320\end{array}$ & $\begin{array}{l}5,480 \\
5,240 \\
5,000 \\
5,000 \\
4,820\end{array}$ & $\begin{array}{l}5,000 \\
4,820 \\
4,640 \\
4,520 \\
4,460\end{array}$ & $\begin{array}{r}1,149 \\
1,084 \\
990 \\
905 \\
905\end{array}$ & $\begin{array}{l}825 \\
851 \\
772 \\
723 \\
678\end{array}$ & $\begin{array}{l}415 \\
415 \\
402 \\
389 \\
377\end{array}$ & $\begin{array}{l}317 \\
317 \\
317 \\
317 \\
317\end{array}$ \\
\hline $\begin{array}{l}21 \\
22 \\
23 \\
24 \\
25\end{array}$ & $\begin{array}{l}353 \\
365 \\
365 \\
377 \\
389\end{array}$ & $\begin{array}{l}443 \\
443 \\
443 \\
458 \\
458\end{array}$ & $\begin{array}{l}489 \\
489 \\
489 \\
489 \\
489\end{array}$ & $\begin{array}{l}522 \\
489 \\
522 \\
539 \\
539\end{array}$ & $\begin{array}{l}961 \\
\frac{933}{933} \\
933 \\
961\end{array}$ & $\begin{array}{l}2,600 \\
2,900 \\
3,250 \\
3,450 \\
3,400\end{array}$ & $\begin{array}{l}4,640 \\
4,520 \\
4,400 \\
4,280 \\
4,220 \\
\end{array}$ & $\begin{array}{l}4,220 \\
3,980 \\
3,800 \\
3,600 \\
3,400\end{array}$ & $\begin{array}{l}878 \\
851 \\
825 \\
799 \\
799\end{array}$ & $\begin{array}{l}616 \\
557 \\
522 \\
489 \\
473\end{array}$ & $\begin{array}{l}377 \\
365 \\
365 \\
353 \\
353\end{array}$ & $\begin{array}{l}317 \\
317 \\
317 \\
317 \\
317\end{array}$ \\
\hline $\begin{array}{l}26 \\
27 \\
28 \\
29 \\
30 \\
31\end{array}$ & $\begin{array}{l}389 \\
402 \\
402 \\
402 \\
415 \\
415\end{array}$ & $\begin{array}{l}473 \\
489 \\
505 \\
505 \\
522 \\
-\end{array}$ & $\begin{array}{l}489 \\
489 \\
489 \\
489 \\
505 \\
505\end{array}$ & $\begin{array}{r}557 \\
3,150 \\
* 11,800 \\
9,600 \\
6,230 \\
3,600\end{array}$ & $\begin{array}{c}1,020 \\
1,084 \\
1,240 \\
- \\
-\end{array}$ & $\begin{array}{l}3,200 \\
3,000 \\
3,000 \\
3,050 \\
3,100 \\
3,100\end{array}$ & $\begin{array}{c}4,280 \\
4,400 \\
4,700 \\
5,120 \\
5,300 \\
=\end{array}$ & $\begin{array}{l}3,200 \\
3,100 \\
2,800 \\
2,650 \\
2,470 \\
2,220 \\
\end{array}$ & $\begin{array}{l}772 \\
772 \\
772 \\
747 \\
723 \\
-\end{array}$ & $\begin{array}{l}443 \\
415 \\
402 \\
389 \\
389 \\
377\end{array}$ & $\begin{array}{l}341 \\
341 \\
341 \\
329 \\
329 \\
317\end{array}$ & $\begin{array}{l}317 \\
317 \\
317 \\
317 \\
317 \\
-\end{array}$ \\
\hline $\begin{array}{l}\text { Total } \\
\text { Mean } \\
\text { Ac-ft }\end{array}$ & $\begin{array}{r}9,953 \\
321 \\
19,740\end{array}$ & $\begin{array}{r}13,223 \\
441 \\
26,230\end{array}$ & $\begin{array}{r}15,498 \\
500 \\
30,740\end{array}$ & $\begin{array}{r}48,039 \\
1,550 \\
95,280\end{array}$ & $\begin{array}{r}35,374 \\
1,263 \\
70,160\end{array}$ & $\begin{array}{r}68,500 \\
2,210 \\
135,900\end{array}$ & $\begin{array}{r}164,770 \\
5,492 \\
326,800\end{array}$ & $\begin{array}{r}144,120 \\
4,649 \\
285,900\end{array}$ & $\begin{array}{r}37,725 \\
1,258 \\
74,830\end{array}$ & $\begin{array}{r}17,859 \\
576 \\
35,420\end{array}$ & $\begin{array}{r}12,660 \\
408 \\
25,110\end{array}$ & $\begin{array}{r}9,510 \\
317 \\
18,860\end{array}$ \\
\hline
\end{tabular}

$\begin{array}{lllllll}\text { Calendar year 1949: } & \operatorname{Max} 15,000 & \text { Min } 156 & \text { Mean 1,223 } & \text { Ac-ft } 885,500 \\ \text { Ac-ft 1, 145,000 }\end{array}$

* Discharge measurement made on this day. 
HEIMAND RIVER BASIN

Arghandab River below Arghandab Dam, Afghanistan

Discharge, in cubic feet per second, water year October 1950 to September 1951

\begin{tabular}{|c|c|c|c|c|c|c|c|c|c|c|c|c|}
\hline Day & Oct. & Nov, & Dec. & Jan. & Feb. & Mar. & Apr. & May & June & $\mathrm{Ju}] \mathrm{y}$ & Aug. & Sept. \\
\hline $\begin{array}{l}1 \\
2 \\
3 \\
4 \\
5\end{array}$ & $\begin{array}{l}\frac{329}{353} \\
365 \\
377 \\
377\end{array}$ & $\begin{array}{l}458 \\
473 \\
473 \\
458 \\
443\end{array}$ & $\begin{array}{l}657 \\
657 \\
657 \\
657 \\
657\end{array}$ & $\begin{array}{l}596 \\
596 \\
596 \\
596 \\
596\end{array}$ & $\begin{array}{l}596 \\
596 \\
577 \\
557 \\
577\end{array}$ & $\begin{array}{r}\frac{933}{933} \\
990 \\
1,020 \\
1,051\end{array}$ & $\begin{array}{l}3,980 \\
4,700 \\
4,820 \\
4,220 \\
3,920\end{array}$ & $\begin{array}{l}5,120 \\
5,240 \\
4,880 \\
4,640 \\
5,000\end{array}$ & $\begin{array}{r}\frac{2,700}{2,470} \\
2,170 \\
2,000 \\
1,770\end{array}$ & $\begin{array}{l}\frac{723}{700} \\
700 \\
700 \\
678\end{array}$ & $\begin{array}{l}\frac{317}{317} \\
329 \\
329 \\
341\end{array}$ & $\begin{array}{l}317 \\
317 \\
317 \\
329 \\
317\end{array}$ \\
\hline $\begin{array}{r}6 \\
7 \\
8 \\
9 \\
10\end{array}$ & $\begin{array}{l}389 \\
389 \\
389 \\
389 \\
389\end{array}$ & $\begin{array}{l}473 \\
489 \\
522 \\
522 \\
522\end{array}$ & $\begin{array}{l}657 \\
678 \\
678 \\
678 \\
678\end{array}$ & $\begin{array}{l}596 \\
616 \\
636 \\
616 \\
700\end{array}$ & $\begin{array}{r}596 \\
1,410 \\
1,560 \\
1,275 \\
1,116\end{array}$ & $\begin{array}{l}1,182 \\
1,348 \\
1,437 \\
2,000 \\
1,815\end{array}$ & $\begin{array}{l}3,860 \\
4,280 \\
4,160 \\
3,860 \\
3,750\end{array}$ & $\begin{array}{r}7,040 \\
11,100 \\
9,650 \\
6,720 \\
6,090\end{array}$ & $\begin{array}{l}1,683 \\
1,599 \\
1,599 \\
1,599 \\
1,558\end{array}$ & $\begin{array}{l}657 \\
636 \\
636 \\
636 \\
636\end{array}$ & $\begin{array}{l}353 \\
353 \\
353 \\
353 \\
341\end{array}$ & $\begin{array}{l}329 \\
329 \\
317 \\
317 \\
329\end{array}$ \\
\hline $\begin{array}{l}\frac{11}{12} \\
13 \\
14 \\
15\end{array}$ & $\begin{array}{l}377 \\
389 \\
389 \\
389 \\
389\end{array}$ & $\begin{array}{l}539 \\
539 \\
539 \\
539 \\
557\end{array}$ & $\begin{array}{l}678 \\
678 \\
678 \\
657 \\
657\end{array}$ & $\begin{array}{l}\frac{878}{772} \\
723 \\
678 \\
678\end{array}$ & $\begin{array}{l}961 \\
878 \\
825 \\
799 \\
772\end{array}$ & $\begin{array}{l}1,599 \\
1,517 \\
1,517 \\
1,517 \\
1,558\end{array}$ & $\begin{array}{l}3,800 \\
4,100 \\
4,460 \\
4,640 \\
4,520\end{array}$ & $\begin{array}{l}5,550 \\
5,480 \\
6,020 \\
5,680 \\
5,120\end{array}$ & $\begin{array}{l}1,477 \\
1,437 \\
1,348 \\
1,317 \\
1,275\end{array}$ & $\begin{array}{l}616 \\
616 \\
616 \\
596 \\
577\end{array}$ & $\begin{array}{l}341 \\
329 \\
329 \\
317 \\
329\end{array}$ & $\begin{array}{l}\frac{341}{341} \\
341 \\
341 \\
329\end{array}$ \\
\hline $\begin{array}{l}16 \\
17 \\
18 \\
19 \\
20\end{array}$ & $\begin{array}{l}389 \\
389 \\
389 \\
389 \\
389\end{array}$ & $\begin{array}{l}557 \\
557 \\
557 \\
577 \\
577\end{array}$ & $\begin{array}{l}657 \\
657 \\
657 \\
657 \\
657\end{array}$ & $\begin{array}{l}657 \\
636 \\
616 \\
616 \\
616\end{array}$ & $\begin{array}{l}772 \\
747 \\
723 \\
772 \\
851\end{array}$ & $\begin{array}{l}2,120 \\
2,220 \\
2,040 \\
1,955 \\
1,815\end{array}$ & $\begin{array}{l}4,460 \\
4,580 \\
4,760 \\
4,640 \\
4,520\end{array}$ & $\begin{array}{l}4,700 \\
4,400 \\
4,280 \\
4,160 \\
4,220\end{array}$ & $\begin{array}{l}1,240 \\
1,084 \\
1,084 \\
1,084 \\
1,051\end{array}$ & $\begin{array}{l}577 \\
577 \\
557 \\
539 \\
539\end{array}$ & $\begin{array}{l}353 \\
365 \\
365 \\
377 \\
365\end{array}$ & $\begin{array}{l}341 \\
329 \\
329 \\
317 \\
305\end{array}$ \\
\hline $\begin{array}{l}21 \\
22 \\
23 \\
24 \\
25\end{array}$ & $\begin{array}{l}389 \\
389 \\
389 \\
389 \\
377\end{array}$ & $\begin{array}{l}577 \\
577 \\
577 \\
577 \\
577\end{array}$ & $\begin{array}{l}636 \\
636 \\
616 \\
616 \\
616\end{array}$ & $\begin{array}{l}596 \\
577 \\
577 \\
596 \\
596\end{array}$ & $\begin{array}{l}878 \\
851 \\
851 \\
851 \\
878\end{array}$ & $\begin{array}{r}1,683 \\
1,726 \\
1,727 \\
2,080 \\
* 3,650\end{array}$ & $\begin{array}{l}4,280 \\
4,280 \\
4,580 \\
4,460 \\
4,280\end{array}$ & $\begin{array}{r}4,160 \\
4,040 \\
a 3,920 \\
a 3,750 \\
a 3,550\end{array}$ & $\begin{array}{r}1,020 \\
1,020 \\
990 \\
961 \\
905\end{array}$ & $\begin{array}{l}a 539 \\
a 505 \\
a 473 \\
a 458 \\
a 429\end{array}$ & $\begin{array}{l}365 \\
365 \\
353 \\
341 \\
341\end{array}$ & $\begin{array}{l}305 \\
293 \\
293 \\
293 \\
293\end{array}$ \\
\hline $\begin{array}{l}26 \\
27 \\
28 \\
29 \\
30 \\
31\end{array}$ & $\begin{array}{l}377 \\
377 \\
389 \\
402 \\
429 \\
458\end{array}$ & $\begin{array}{l}577 \\
596 \\
596 \\
616 \\
657 \\
-\end{array}$ & $\begin{array}{l}616 \\
616 \\
616 \\
616 \\
616 \\
616\end{array}$ & $\begin{array}{l}596 \\
596 \\
596 \\
596 \\
596 \\
616\end{array}$ & $\begin{array}{l}878 \\
905 \\
905 \\
- \\
- \\
-\end{array}$ & $\begin{array}{l}6,510 \\
6,650 \\
\frac{5,300}{4,640} \\
4,220 \\
3,980\end{array}$ & $\begin{array}{l}4,280 \\
4,460 \\
4,640 \\
4,880 \\
5,000 \\
-\end{array}$ & $\begin{array}{r}a 3,400 \\
a 3,250 \\
a 3,050 \\
a 2,850 \\
a 2,700 \\
2,550 \\
\end{array}$ & $\begin{array}{l}878 \\
851 \\
799 \\
772 \\
747 \\
-\end{array}$ & $\begin{array}{l}a 415 \\
a 402 \\
a 377 \\
a 365 \\
a 341 \\
a 329\end{array}$ & $\begin{array}{l}329 \\
329 \\
317 \\
317 \\
317 \\
317\end{array}$ & $\begin{array}{l}293 \\
305 \\
293 \\
293 \\
283 \\
-\end{array}$ \\
\hline $\begin{array}{l}\text { Total } \\
\text { Mean } \\
\text { Ac-ft }\end{array}$ & $\begin{array}{r}11,989 \\
387 \\
23,780\end{array}$ & $\begin{array}{r}16,298 \\
543 \\
32,330\end{array}$ & $\begin{array}{r}20,103 \\
648 \\
39,870 \\
\end{array}$ & $\begin{array}{r}19,552 \\
631 \\
38,870 \\
\end{array}$ & $\begin{array}{r}23,957 \\
856 \\
47,520 \\
\end{array}$ & $\begin{array}{r}72,733 \\
2,353 \\
y_{4}, 300 \\
\end{array}$ & $\begin{array}{r}131,170 \\
4,372 \\
260,200\end{array}$ & $\begin{array}{r}152,310 \\
4,913 \\
302,100 \\
\end{array}$ & $\begin{array}{r}40,482 \\
1,346 \\
80,290\end{array}$ & 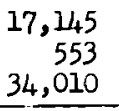 & $\begin{array}{r}10,547 \\
340 \\
20,920\end{array}$ & $\begin{array}{r}9,476 \\
316 \\
18,800\end{array}$ \\
\hline $\begin{array}{l}\text { Caler } \\
\text { Wate? }\end{array}$ & 195 & $\begin{array}{l}\text { Max } \\
\operatorname{Max}\end{array}$ & $\begin{array}{l}11,800 \\
11,100\end{array}$ & $\begin{array}{l}\text { Mirn } \\
\text { Min }\end{array}$ & $\begin{array}{l}317 \\
283\end{array}$ & $\begin{array}{l}1,608 \\
1,4,40\end{array}$ & $\begin{array}{l}A c-f t \\
A c-f t\end{array}$ & $\begin{array}{l}1,164,0 \\
1,043,0\end{array}$ & & & & \\
\hline
\end{tabular}

* Discharge measurement made on this day.

a No gage-height record; discharge interpolated. 
HELMAND RIVER BASIN

Arghandab River below Arghandab Darn, Afgharistan

Discharge, in cubic feet per second, water year October 1951 to September 1952

\begin{tabular}{|c|c|c|c|c|c|c|c|c|c|c|c|c|}
\hline Day & Oct. & Nov. & Dec. & Jan. & Feb. & Mar. & Apr. & May & June & July & Auge & Sept. \\
\hline $\begin{array}{l}1 \\
2 \\
3 \\
4 \\
5\end{array}$ & $\begin{array}{l}\frac{223}{317} \\
314 \\
365 \\
365\end{array}$ & $\begin{array}{l}539 \\
539 \\
522 \\
505 \\
505\end{array}$ & $\begin{array}{l}577 \\
577 \\
577 \\
577 \\
577\end{array}$ & $\begin{array}{l}650 \\
650 \\
650 \\
650 \\
650\end{array}$ & $\begin{array}{l}800 \\
770 \\
750 \\
770 \\
750\end{array}$ & $\begin{array}{l}700 \\
700 \\
700 \\
680 \\
680\end{array}$ & $\begin{array}{r}1,515 \\
\frac{830}{570} \\
1,570 \\
1,570 \\
1,585\end{array}$ & $\begin{array}{l}1,725 \\
1,725 \\
1,725 \\
1,725 \\
1,740\end{array}$ & $\begin{array}{r}690 \\
765 \\
930 \\
960 \\
1,050\end{array}$ & $\begin{array}{r}855 \\
795 \\
795 \\
810 \\
1,065 \\
\end{array}$ & $\begin{array}{l}750 \\
750 \\
750 \\
750 \\
750\end{array}$ & $\begin{array}{r}\frac{189}{189} \\
304 \\
* 322 \\
\frac{292}{392}\end{array}$ \\
\hline $\begin{array}{r}6 \\
7 \\
8 \\
9 \\
10\end{array}$ & $\begin{array}{l}377 \\
389 \\
402 \\
420 \\
415\end{array}$ & $\begin{array}{l}522 \\
522 \\
522 \\
522 \\
522\end{array}$ & $\begin{array}{l}577 \\
577 \\
577 \\
577 \\
596\end{array}$ & $\begin{array}{l}640 \\
630 \\
620 \\
600 \\
610\end{array}$ & $\begin{array}{l}720 \\
720 \\
720 \\
720 \\
750\end{array}$ & $\begin{array}{l}710 \\
765 \\
815 \\
830 \\
830\end{array}$ & $\begin{array}{l}1,585 \\
1,600 \\
1,600 \\
1,615 \\
1,630\end{array}$ & $\begin{array}{l}1,740 \\
1,740 \\
1,740 \\
1,740 \\
1,740\end{array}$ & $\begin{array}{r}1,050 \\
975 \\
915 \\
930 \\
930\end{array}$ & $\begin{array}{l}765 \\
810 \\
810 \\
810 \\
780\end{array}$ & $\begin{array}{l}735 \\
735 \\
720 \\
720 \\
12\end{array}$ & $\begin{array}{l}379 \\
379 \\
366 \\
366 \\
366\end{array}$ \\
\hline $\begin{array}{l}11 \\
12 \\
13 \\
14 \\
15\end{array}$ & $\begin{array}{l}415 \\
429 \\
415 \\
429 \\
429\end{array}$ & $\begin{array}{l}522 \\
522 \\
522 \\
522 \\
522\end{array}$ & $\begin{array}{l}596 \\
577 \\
596 \\
596 \\
596\end{array}$ & $\begin{array}{l}610 \\
630 \\
670 \\
800 \\
700\end{array}$ & $\begin{array}{r}750 \\
780 \\
1,000 \\
5,100 \\
2,500\end{array}$ & $\begin{array}{l}830 \\
845 \\
870 \\
900 \\
915\end{array}$ & $\begin{array}{l}1,630 \\
1,645 \\
1,645 \\
1,660 \\
1,675\end{array}$ & $\begin{array}{l}1,740 \\
1,400 \\
1,240 \\
1,240 \\
1,240\end{array}$ & $\begin{array}{l}1,030 \\
1,180 \\
1,225 \\
\frac{1,225}{1,225}\end{array}$ & $\begin{array}{l}750 \\
750 \\
750 \\
735 \\
735\end{array}$ & $\begin{array}{r}0 \\
379 \\
509 \\
645 \\
1,065\end{array}$ & $\begin{array}{l}366 \\
366 \\
366 \\
366 \\
366\end{array}$ \\
\hline $\begin{array}{l}16 \\
17 \\
18 \\
19 \\
20\end{array}$ & $\begin{array}{l}443 \\
443 \\
443 \\
443 \\
458\end{array}$ & $\begin{array}{l}522 \\
522 \\
522 \\
539 \\
539\end{array}$ & $\begin{array}{l}616 \\
616 \\
616 \\
616 \\
616\end{array}$ & $\begin{array}{l}650 \\
640 \\
640 \\
640 \\
640\end{array}$ & $\begin{array}{l}1,700 \\
1,500 \\
1,400 \\
1,300 \\
1,280\end{array}$ & $\begin{array}{l}930 \\
945 \\
945 \\
960 \\
975\end{array}$ & $\begin{array}{l}1,675 \\
1,675 \\
1,675 \\
1,690 \\
1,690\end{array}$ & $\begin{array}{r}1,015 \\
795 \\
795 \\
795 \\
795\end{array}$ & $\begin{array}{l}1,225 \\
1,150 \\
1,030 \\
1,030 \\
1,030\end{array}$ & $\begin{array}{l}735 \\
750 \\
750 \\
750 \\
750\end{array}$ & $\begin{array}{r}1,215 \\
1,050 \\
810 \\
870 \\
870\end{array}$ & $\begin{array}{l}366 \\
366 \\
366 \\
366 \\
366\end{array}$ \\
\hline $\begin{array}{l}21 \\
22 \\
23 \\
24 \\
25\end{array}$ & $\begin{array}{l}473 \\
489 \\
489 \\
489 \\
505\end{array}$ & $\begin{array}{l}539 \\
539 \\
539 \\
539 \\
557\end{array}$ & $\begin{array}{r}636 \\
* 636 \\
630 \\
630 \\
640\end{array}$ & $\begin{array}{l}630 \\
620 \\
600 \\
580 \\
560 \\
\end{array}$ & $\begin{array}{r}1,250 \\
1,230 \\
1,200 \\
800 \\
2\end{array}$ & $\begin{array}{l}975 \\
975 \\
975 \\
990 \\
990\end{array}$ & $\begin{array}{l}1,690 \\
1,690 \\
1,710 \\
1,710 \\
1,710\end{array}$ & $\begin{array}{l}795 \\
795 \\
795 \\
795 \\
795\end{array}$ & $\begin{array}{r}1,030 \\
1,030 \\
1,030 \\
1,030 \\
990\end{array}$ & $\begin{array}{l}750 \\
750 \\
750 \\
750 \\
750\end{array}$ & $\begin{array}{l}870 \\
870 \\
870 \\
615 \\
825\end{array}$ & $\begin{array}{l}366 \\
366 \\
366 \\
366 \\
366\end{array}$ \\
\hline $\begin{array}{l}26 \\
27 \\
28 \\
29 \\
30 \\
31\end{array}$ & $\begin{array}{l}522 \\
522 \\
522 \\
522 \\
522 \\
532 \\
\end{array}$ & $\begin{array}{c}557 \\
577 \\
577 \\
577 \\
577 \\
-\end{array}$ & $\begin{array}{l}650 \\
650 \\
650 \\
650 \\
660 \\
660\end{array}$ & $\begin{array}{l}570 \\
600 \\
650 \\
730 \\
\frac{880}{840}\end{array}$ & $\begin{array}{r}2 \\
160 \\
700 \\
700 \\
- \\
-\end{array}$ & $\begin{array}{r}990 \\
1,310 \\
1,480 \\
1,495 \\
1,510 \\
1,510\end{array}$ & $\begin{array}{c}1,710 \\
1,710 \\
1,725 \\
1,725 \\
1,725 \\
-\end{array}$ & $\begin{array}{l}795 \\
750 \\
690 \\
690 \\
690 \\
690\end{array}$ & $\begin{array}{l}855 \\
815 \\
780 \\
780 \\
840 \\
-\end{array}$ & $\begin{array}{l}750 \\
750 \\
750 \\
750 \\
750 \\
750\end{array}$ & $\begin{array}{l}810 \\
457 \\
280 \\
244 \\
220 \\
200\end{array}$ & $\begin{array}{l}366 \\
366 \\
366 \\
316 \\
292 \\
-\end{array}$ \\
\hline $\begin{array}{l}\text { Total } \\
\text { Mean } \\
\text { Ac-ft }\end{array}$ & $\begin{array}{r}13,580 \\
438 \\
26,940 \\
\end{array}$ & $\begin{array}{r}16,053 \\
535 \\
31,840 \\
\end{array}$ & $\begin{array}{r}18,922 \\
610 \\
37,530 \\
\end{array}$ & $\begin{array}{r}20,230 \\
653 \\
40,130\end{array}$ & $\begin{array}{r}30,824 \\
1,063 \\
61,140\end{array}$ & $\begin{array}{r}29,725 \\
959 \\
58,960 \\
\end{array}$ & $\begin{array}{r}48,865 \\
1,629 \\
96,920\end{array}$ & $\begin{array}{r}37,120 \\
1,197 \\
73,630\end{array}$ & $\begin{array}{r}29,725 \\
991 \\
58,960\end{array}$ & $\begin{array}{r}23,985 \\
774 \\
47,570 \\
\end{array}$ & $\begin{array}{r}20,346 \\
656 \\
40,360 \\
\end{array}$ & $\begin{array}{r}10,505 \\
350 \\
20,840\end{array}$ \\
\hline
\end{tabular}

Calendar year 1951: $\operatorname{Max} 11,100$ Min 283 Mean 1,441 Ac-ft 1,043,000

water year 1951-52: Max 5,100 Min 0 Mean 819 Ac-ft 594,800

* Discharge measurement made on this day.

Note. - Arghandab reservoir closed and storage begun Feb. 24; regulated flow thereafter. 
HEIMAND RIVER BASIN

Arghandas fiver below Arghandab Dam, Afgharistan

Discharge, in cubic feet per second, water year October 1952 to jeptember 1953

\begin{tabular}{|c|c|c|c|c|c|c|c|c|c|c|c|c|}
\hline Day & Oct. & Nov. & Dec. & Jan. & Feb. & Mar. & Apr. & May & June & July & Aug. & Sept. \\
\hline 1 & a292 & $* 492$ & 554 & 554 & $\approx 1,010$ & 447 & 1,170 & 1,250 & 384 & $\because 828$ & 809 & 772 \\
\hline 2 & $* 292$ & $\overline{492}$ & 554 & 554 & 1,010 & $\sqrt{447}$ & 1,380 & 3,250 & 847 & $\overline{828}$ & $* 809$ & $\frac{1}{772}$ \\
\hline 3 & a292 & 492 & 554 & 554 & 1,010 & 447 & 1,350 & $* 1,250$ & 847 & 828 & 809 & 755 \\
\hline 4 & 2280 & 492 & 554 & 554 & 990 & 42,7 & 1,360 & 1,250 & 847 & 828 & 809 & 755 \\
\hline 5 & $a \overline{280}$ & 492 & 554 & 554 & 1,000 & 447 & 1,360 & 1,250 & 847 & 828 & 790 & 720 \\
\hline 6 & $a 280$ & 538 & 554 & 554 & 1,000 & 447 & $* 1,510$ & 1,220 & 847 & 828 & 790 & 702 \\
\hline 7 & a292 & 562 & 554 & 554 & 1,000 & 447 & 1,510 & 1,220 & 847 & 828 & 790 & 702 \\
\hline 8 & a292 & 562 & 554 & 554 & 1,000 & 447 & 1,510 & 1,220 & 847 & 828 & 790 & 702 \\
\hline 9 & a292 & $\star 562$ & 554 & 554 & 1,080 & 447 & 1,300 & 1,220 & 847 & 828 & 790 & 702 \\
\hline 10 & 2292 & 639 & 554 & 554 & 1,150 & 454 & 1,510 & 1,220 & $2 x_{4} 7$ & 828 & 790 & 702 \\
\hline 11 & a292 & 554 & 554 & 796 & 1,030 & $* 447$ & 1,510 & 1,220 & 847 & 828 & 790 & $* 702$ \\
\hline 12 & 2292 & 554 & 554 & $* 1,010$ & 1,080 & 447 & 1,460 & 1,220 & 847 & 818 & 790 & 702 \\
\hline 13 & 2292 & 554 & 554 & 1,030 & 1,080 & 447 & 1,510 & 1,170 & 847 & 818 & 790 & 702 \\
\hline$u_{4}$ & a292 & 554 & 554 & 1,030 & 1,150 & 447 & 1,510 & 1,150 & 847 & 818 & 790 & 702 \\
\hline 15 & 2350 & 554 & 554 & 1,030 & 852 & 447 & 1,510 & 979 & $84 ?$ & 809 & 790 & 702 \\
\hline 16 & 382 & 554 & 554 & 1,030 & $* 432$ & 461 & 1,570 & 847 & 847 & 809 & 790 & 702 \\
\hline 17 & 382 & 554 & 554 & 1,030 & 432 & 461 & 3,510 & 847 & 847 & 809 & 781 & 702 \\
\hline 28 & 382 & 554 & $* 554$ & 1,030 & 432 & 461 & $* 1,510$ & 84.7 & 847 & 809 & 781 & 702 \\
\hline 19 & 382 & 554 & 554 & 1,030 & 432 & 461 & 1,400 & 847 & $82 \varepsilon$ & 809 & 781 & 702 \\
\hline 20 & 382 & 554 & 554 & 1,030 & 432 & 618 & 1,460 & 847 & 847 & 809 & 781 & 702 \\
\hline 21 & 382 & 554 & 554 & 1,030 & 425 & 737 & 1,300 & 847 & 847 & 809 & 772 & 702 \\
\hline 22 & 382 & 554 & 554 & 1,030 & $\frac{12}{432}$ & 847 & 1,360 & 847 & 828 & 809 & $\frac{17}{772}$ & 702 \\
\hline 23 & 382 & 554 & 554 & 1,030 & 432 & 1,030 & 1,350 & 847 & 828 & 809 & 772 & 702 \\
\hline 24 & 382 & 554 & 554 & 1,030 & 432 & 1,030 & 1,350 & 847 & 828 & 809 & 772 & 702 \\
\hline 25 & 382 & 554 & 554 & 1,030 & 432 & $* 1,060$ & 1,350 & 847 & 828 & 809 & 772 & 702 \\
\hline 26 & 389 & 554 & 554 & 1,030 & 432 & 1,030 & 1,350 & 847 & 828 & 809 & 772 & 685 \\
\hline 27 & 389 & 554 & 554 & 1,020 & 432 & 1,030 & 1,350 & 847 & 828 & 809 & 772 & 685 \\
\hline 28 & 389 & 554 & 554 & 1,020 & 432 & 1,060 & 1,320 & 847 & 828 & 809 & 772 & 685 \\
\hline 29 & 389 & 554 & 554 & 1,020 & - & 1,060 & 1,270 & 847 & 828 & 809 & 772 & 685 \\
\hline 30 & 418 & 554 & 554 & 1,020 & - & 1,030 & 1,250 & 847 & 828 & 809 & 772 & 685 \\
\hline 31 & 492 & - & 554 & 1,020 & - & 1,220 & - & 847 & - & 809 & 772 & - \\
\hline Total & 10,688 & 16,403 & 17,174 & 26,866 & 21,051 & 20,308 & 42,160 & 31,641 & 25,220 & 25,315 & 24,332 & 21,239 \\
\hline Mean & 345 & 547 & 554 & 867 & 752 & 655 & 1,405 & 1,021 & 841 & 817 & 785 & 708 \\
\hline$A c-f t$ & 21,200 & 32,530 & 34,060 & 53,290 & 41,750 & 40,280 & 83,620 & 62,760 & 50,020 & 50,210 & 48,260 & 42,130 \\
\hline Ca] & חב & $\operatorname{Max}$ & 100 & Nin & & 808 & $A c-f t$ & 586,300 & & & & \\
\hline & & Max & 570 & Min & & 774 & $A c-f t$ & 560,110 & & & & \\
\hline
\end{tabular}

* Discharge measurement made or this day.

a No gage-height record; discharge computed from gage readings. 
HETMAND RIVER BASIN

Arghandab River below Arghandab Dam, Afghanistan

Discharge, in cubic feet per second, water year October 1953 to September 1954

\begin{tabular}{|c|c|c|c|c|c|c|c|c|c|c|c|c|}
\hline Day & Oct. & Nov. & Dec. & Jan. & Feb。 & Mar. & Apr. & May & June & July & Aug. & Sept. \\
\hline $\begin{array}{l}1 \\
2 \\
3 \\
4 \\
5\end{array}$ & $\begin{array}{l}\frac{711}{702} \\
702 \\
702 \\
702\end{array}$ & $\begin{array}{l}\frac{676}{676} \\
676 \\
676 \\
676\end{array}$ & $\begin{array}{r}\frac{652}{652} \\
652 \\
* 652 \\
652\end{array}$ & $\begin{array}{r}602 \\
* 602 \\
602 \\
618 \\
694 \\
\end{array}$ & $\begin{array}{l}554 \\
546 \\
530 \\
523 \\
523\end{array}$ & $\begin{array}{l}* 476 \\
702 \\
737 \\
755 \\
755\end{array}$ & $\begin{array}{r}9,300 \\
8,400 \\
7,700 \\
7,300 \\
* 7,000\end{array}$ & $\begin{array}{l}\frac{6,000}{5,900} \\
5,800 \\
5,600 \\
5,500\end{array}$ & $\begin{array}{r}1,870 \\
* 1,870 \\
1,840 \\
1,830 \\
1,830\end{array}$ & $\begin{array}{r}* 1,150 \\
1,160 \\
d 1,160 \\
d 1,160 \\
d 1,160\end{array}$ & $\begin{array}{l}951 \\
951 \\
951 \\
951 \\
951\end{array}$ & $\begin{array}{l}1,000 \\
\frac{1}{1,000} \\
1,000 \\
1,000 \\
1,000\end{array}$ \\
\hline $\begin{array}{r}6 \\
7 \\
8 \\
9 \\
10\end{array}$ & $\begin{array}{l}702 \\
702 \\
702 \\
694 \\
694\end{array}$ & $\begin{array}{l}676 \\
676 \\
676 \\
668 \\
668\end{array}$ & $\begin{array}{l}652 \\
652 \\
652 \\
652 \\
652\end{array}$ & $\begin{array}{l}660 \\
652 \\
652 \\
644 \\
635\end{array}$ & $\begin{array}{l}523 \\
523 \\
523 \\
523 \\
586\end{array}$ & $\begin{array}{l}764 \\
764 \\
772 \\
781 \\
781\end{array}$ & $\begin{array}{l}6,800 \\
6,700 \\
6,600 \\
6,500 \\
6,500\end{array}$ & $\begin{array}{l}5,400 \\
5,300 \\
5,200 \\
5,150 \\
5,080\end{array}$ & $\begin{array}{l}1,820 \\
1,820 \\
1,800 \\
1,800 \\
1,790\end{array}$ & $\begin{array}{r}\mathrm{d} l, 160 \\
\mathrm{dl}, 160 \\
\mathrm{dl}, 160 \\
\mathrm{dl}, 160 \\
1,160\end{array}$ & $\begin{array}{r}951 \\
951 \\
* 1,280 \\
988 \\
988\end{array}$ & $\begin{array}{r}1,000 \\
1,000 \\
1,000 \\
988 \\
988\end{array}$ \\
\hline $\begin{array}{l}11 \\
12 \\
13 \\
14 \\
15\end{array}$ & $\begin{array}{l}624 \\
694 \\
694 \\
694 \\
694\end{array}$ & $\begin{array}{l}668 \\
668 \\
668 \\
668 \\
668\end{array}$ & $\begin{array}{l}644 \\
644 \\
644 \\
644 \\
644\end{array}$ & $\begin{array}{l}626 \\
626 \\
626 \\
626 \\
626\end{array}$ & $\begin{array}{l}586 \\
602 \\
626 \\
635 \\
635\end{array}$ & $\begin{array}{l}790 \\
790 \\
790 \\
790 \\
809\end{array}$ & $\begin{array}{l}6,500 \\
6,300 \\
6,200 \\
6,300 \\
6,300\end{array}$ & $\begin{array}{r}5,020 \\
4,700 \\
4,360 \\
* 3,980 \\
3,620\end{array}$ & $\begin{array}{l}1,790 \\
1,790 \\
1,710 \\
1,120 \\
1,120\end{array}$ & $\begin{array}{l}1,160 \\
1,160 \\
1,150 \\
1,150 \\
1,150\end{array}$ & $\begin{array}{l}988 \\
988 \\
988 \\
988 \\
988\end{array}$ & $\begin{array}{l}988 \\
976 \\
976 \\
976 \\
964\end{array}$ \\
\hline $\begin{array}{l}16 \\
17 \\
18 \\
19 \\
20\end{array}$ & $\begin{array}{l}694 \\
694 \\
694 \\
694 \\
685\end{array}$ & $\begin{array}{l}668 \\
668 \\
668 \\
668 \\
668\end{array}$ & $\begin{array}{l}644 \\
644 \\
635 \\
635 \\
635\end{array}$ & $\begin{array}{l}626 \\
626 \\
626 \\
626 \\
618\end{array}$ & $\begin{array}{l}626 \\
652 \\
6444 \\
\frac{644}{644}\end{array}$ & $\begin{array}{l}1,220 \\
1,030 \\
1,300 \\
1,400 \\
1,460\end{array}$ & $\begin{array}{l}6,300 \\
6,800 \\
6,600 \\
6,400 \\
6,300\end{array}$ & $\begin{array}{l}3,400 \\
3,250 \\
3,110 \\
3,030 \\
2,820\end{array}$ & $\begin{array}{l}1,120 \\
* 1,120 \\
* 1,400 \\
* 1,320 \\
* 1,150\end{array}$ & $\begin{array}{l}1,150 \\
1,150 \\
1,150 \\
1,140 \\
1,140\end{array}$ & $\begin{array}{l}988 \\
988 \\
988 \\
988 \\
988\end{array}$ & $\begin{array}{l}964 \\
964 \\
964 \\
951 \\
927\end{array}$ \\
\hline $\begin{array}{l}21 \\
22 \\
23 \\
24 \\
25\end{array}$ & $\begin{array}{l}685 \\
685 \\
685 \\
685 \\
685\end{array}$ & $\begin{array}{l}668 \\
668 \\
668 \\
668 \\
660 \\
\end{array}$ & $\begin{array}{l}635 \\
635 \\
635 \\
626 \\
626\end{array}$ & $\begin{array}{l}618 \\
618 \\
618 \\
610 \\
610\end{array}$ & $\begin{array}{l}644 \\
635 \\
635 \\
570 \\
432\end{array}$ & $\begin{array}{l}1,430 \\
1,570 \\
1,540 \\
1,570 \\
1,520\end{array}$ & $\begin{array}{l}6,300 \\
6,900 \\
7,500 \\
7,600 \\
7,000\end{array}$ & $\begin{array}{l}2,710 \\
2,640 \\
2,540 \\
2,450 \\
2,320\end{array}$ & $\begin{array}{r}1,120 \\
1,120 \\
1,120 \\
* 1,000 \\
* 805\end{array}$ & $\begin{array}{l}1,140 \\
1,140 \\
1,120 \\
1,120 \\
1,110\end{array}$ & $\begin{array}{l}988 \\
988 \\
988 \\
988 \\
988\end{array}$ & $\begin{array}{l}927 \\
927 \\
914 \\
902 \\
902\end{array}$ \\
\hline $\begin{array}{l}26 \\
27 \\
28 \\
29 \\
30 \\
31\end{array}$ & $\begin{array}{r}685 \\
685 \\
750 \\
685 \\
676 \\
* 676\end{array}$ & $\begin{array}{l}660 \\
660 \\
660 \\
660 \\
660 \\
-\end{array}$ & $\begin{array}{l}626 \\
626 \\
626 \\
626 \\
626 \\
618 \\
\end{array}$ & $\begin{array}{l}610 \\
610 \\
602 \\
562 \\
546 \\
* 546\end{array}$ & $\begin{array}{l}410 \\
410 \\
403 \\
- \\
- \\
-\end{array}$ & $\begin{array}{r}1,590 \\
2,220 \\
3,320 \\
6,700 \\
9,610 \\
10,600 \\
\end{array}$ & $\begin{array}{c}6,600 \\
6,400 \\
6,300 \\
6,300 \\
6,200 \\
-\end{array}$ & $\begin{array}{l}2,2100 \\
2,140 \\
2,070 \\
2,020 \\
1,960 \\
1,900\end{array}$ & $\begin{array}{c}1,150 \\
1,150 \\
1,150 \\
1,150 \\
1,150 \\
=\end{array}$ & $\begin{array}{r}1,050 \\
* 951 \\
951 \\
951 \\
951 \\
951\end{array}$ & $\begin{array}{r}988 \\
988 \\
988 \\
988 \\
1,000 \\
* 1,000\end{array}$ & $\begin{array}{r}902 \\
780 \\
843 \\
* 939 \\
939 \\
-\end{array}$ \\
\hline $\begin{array}{l}\text { Total } \\
\text { Mean } \\
\text { Ac-ft }\end{array}$ & $\begin{array}{r}21,526 \\
694 \\
42,700\end{array}$ & $\begin{array}{r}20,056 \\
669 \\
39,780\end{array}$ & $\begin{array}{r}19,838 \\
640 \\
39,350\end{array}$ & $\begin{array}{r}19,163 \\
619 \\
38,010\end{array}$ & $\begin{array}{r}15,787 \\
564 \\
31,310\end{array}$ & $\begin{array}{r}59,336 \\
1,914 \\
117,700 \\
\end{array}$ & $\begin{array}{r}203,900 \\
6,797 \\
404,400\end{array}$ & $\begin{array}{r}117,210 \\
3,781 \\
232,500\end{array}$ & $\begin{array}{r}42,825 \\
1,428 \\
84,940\end{array}$ & $\begin{array}{r}34,525 \\
1,124 \\
68,480\end{array}$ & $\begin{array}{r}30,685 \\
990 \\
60,860\end{array}$ & $\begin{array}{r}28,601 \\
953 \\
56,730\end{array}$ \\
\hline
\end{tabular}

Calendar year 1953: Max 1,570 Min 425 Mean 820 Ac-ft 594,200

Water year 1953-54: Max 10,600 Min 403 Mean 1,681 Ac-ft 1,217,000

* Discharge measurement made on this day.

d Doubtful gage-height record; discharge estimated on basis of recession curve or interpolated.

Note.- No gage-height record Apr. 2 to Way 8; discharge estimated by reservoir operation. 
HEJMAND RIVER BASIN

Arghandab River below Arghandab Dam, Afghanistan

Discharge, in cubic feet per second, water year October 1954 to September 1955

\begin{tabular}{|c|c|c|c|c|c|c|c|c|c|c|c|c|}
\hline Dar & Oct. & Nov. & Dec. & Jan. & Feb. & Mar & Apr. & May & June & July & AUg. & Sept. \\
\hline $\begin{array}{l}1 \\
2 \\
3 \\
4 \\
5\end{array}$ & $\begin{array}{l}939 \\
939 \\
939 \\
939 \\
939\end{array}$ & $\begin{array}{l}927 \\
927 \\
927 \\
927 \\
927\end{array}$ & $\begin{array}{l}* 902 \\
902 \\
902 \\
902 \\
902\end{array}$ & $\begin{array}{r}* 902 \\
902 \\
902 \\
902 \\
902\end{array}$ & $\begin{array}{l}\frac{902}{902} \\
902 \\
902 \\
882\end{array}$ & $\begin{array}{l}480 \\
418 \\
410 \\
410 \\
410\end{array}$ & $\begin{array}{r}\frac{854}{930} \\
1,050 \\
1,120 \\
1,400\end{array}$ & $\begin{array}{l}1,4,10 \\
1,400 \\
1,300 \\
1,300 \\
1,300\end{array}$ & $\begin{array}{r}866 \\
* 866 \\
866 \\
854 \\
805\end{array}$ & $\begin{array}{r}744 \\
744 \\
* 744 \\
744 \\
744\end{array}$ & $\begin{array}{l}\frac{665}{665} \\
665 \\
665 \\
654\end{array}$ & $\begin{array}{l}\frac{621}{528} \\
\frac{434}{442} \\
442\end{array}$ \\
\hline $\begin{array}{r}6 \\
7 \\
8 \\
9 \\
10\end{array}$ & $\begin{array}{l}939 \\
939 \\
939 \\
939 \\
939\end{array}$ & $\begin{array}{l}927 \\
91_{4} \\
9 u_{4} \\
91 u_{4} \\
9 u_{4}\end{array}$ & $\begin{array}{l}902 \\
902 \\
902 \\
902 \\
902\end{array}$ & $\begin{array}{l}902 \\
902 \\
902 \\
902 \\
902\end{array}$ & $\begin{array}{l}1 \frac{84}{83} \\
902 \\
902 \\
902\end{array}$ & $\begin{array}{l}\frac{401}{401} \\
401 \\
401 \\
401\end{array}$ & $\begin{array}{l}1,600 \\
1,600 \\
1,420 \\
1,300 \\
1,300\end{array}$ & $\begin{array}{l}1,300 \\
1,300 \\
1,280 \\
1,210 \\
1,210\end{array}$ & $\begin{array}{l}792 \\
756 \\
710 \\
688 \\
688\end{array}$ & $\begin{array}{l}710 \\
688 \\
688 \\
688 \\
676\end{array}$ & $\begin{array}{l}654 \\
654 \\
654 \\
654 \\
654\end{array}$ & $\begin{array}{r}442 \\
442 \\
442 \\
442 \\
* 442\end{array}$ \\
\hline $\begin{array}{l}11 \\
12 \\
13 \\
14 \\
15\end{array}$ & $\begin{array}{l}939 \\
939 \\
939 \\
939 \\
939\end{array}$ & $\begin{array}{l}9 u_{4} \\
9 u_{4} \\
91 u_{4} \\
91 u_{4} \\
9 u_{4}\end{array}$ & $\begin{array}{l}902 \\
902 \\
902 \\
902 \\
902\end{array}$ & $\begin{array}{l}902 \\
902 \\
902 \\
902 \\
902\end{array}$ & $\begin{array}{l}902 \\
902 \\
902 \\
902 \\
902\end{array}$ & $\begin{array}{l}401 \\
401 \\
418 \\
410 \\
410\end{array}$ & $\begin{array}{l}1,300 \\
1,180 \\
1,090 \\
1,080 \\
1,080\end{array}$ & $\begin{array}{r}1,210 \\
1,210 \\
1,210 \\
* 1,210 \\
* 1,120\end{array}$ & $\begin{array}{l}688 \\
688 \\
688 \\
688 \\
676 \\
\end{array}$ & $\begin{array}{l}676 \\
676 \\
676 \\
676 \\
676\end{array}$ & $\begin{array}{l}654 \\
654 \\
654 \\
654 \\
621 \\
\end{array}$ & $\begin{array}{r}442 \\
442 \\
* 452 \\
461 \\
470\end{array}$ \\
\hline $\begin{array}{l}16 \\
17 \\
18 \\
19 \\
20\end{array}$ & $\begin{array}{l}939 \\
939 \\
927 \\
927 \\
927\end{array}$ & $\begin{array}{l}910 \\
910 \\
910 \\
910 \\
910\end{array}$ & $\begin{array}{l}902 \\
902 \\
902 \\
902 \\
902\end{array}$ & $\begin{array}{l}902 \\
902 \\
902 \\
902 \\
902\end{array}$ & $\begin{array}{l}902 \\
902 \\
902 \\
902 \\
902\end{array}$ & $\begin{array}{l}426 \\
434 \\
442 \\
461 \\
470\end{array}$ & $\begin{array}{l}1,080 \\
1,080 \\
1,080 \\
1,080 \\
1,280\end{array}$ & $\begin{array}{r}1,050 \\
* 1,010 \\
976 \\
939 \\
902\end{array}$ & $\begin{array}{l}676 \\
676 \\
733 \\
780 \\
792\end{array}$ & $\begin{array}{l}676 \\
665 \\
665 \\
665 \\
665\end{array}$ & $\begin{array}{l}621 \\
621 \\
621 \\
621 \\
621\end{array}$ & $\begin{array}{r}500 \\
* 500 \\
500 \\
500 \\
500\end{array}$ \\
\hline $\begin{array}{l}21 \\
22 \\
23 \\
24 \\
25\end{array}$ & $\begin{array}{l}927 \\
927 \\
927 \\
927 \\
927\end{array}$ & $\begin{array}{l}905 \\
905 \\
905 \\
905 \\
905\end{array}$ & $\begin{array}{l}902 \\
902 \\
902 \\
902 \\
902\end{array}$ & $\begin{array}{l}902 \\
902 \\
902 \\
902 \\
902\end{array}$ & $\begin{array}{l}902 \\
631 \\
519 \\
519 \\
519\end{array}$ & $\begin{array}{l}490 \\
500 \\
500 \\
500 \\
500\end{array}$ & $\begin{array}{l}1,450 \\
1,450 \\
1,450 \\
1,450 \\
1,450\end{array}$ & $\begin{array}{l}\frac{866}{866} \\
866 \\
890 \\
866\end{array}$ & $\begin{array}{l}805 \\
805 \\
805 \\
805 \\
805\end{array}$ & $\begin{array}{l}665 \\
665 \\
665 \\
665 \\
665\end{array}$ & $\begin{array}{l}621 \\
621 \\
621 \\
621 \\
621\end{array}$ & $\begin{array}{l}500 \\
500 \\
500 \\
500 \\
500\end{array}$ \\
\hline $\begin{array}{l}26 \\
27 \\
28 \\
29 \\
30 \\
31 \\
\end{array}$ & $\begin{array}{r}927 \\
927 \\
927 \\
927 \\
* 927 \\
927 \\
\end{array}$ & $\begin{array}{r}\frac{902}{902} \\
902 \\
+902 \\
902 \\
- \\
\end{array}$ & $\begin{array}{l}902 \\
902 \\
902 \\
902 \\
902 \\
902 \\
\end{array}$ & $\begin{array}{r}902 \\
902 \\
902 \\
902 \\
902 \\
\times 902\end{array}$ & $\begin{array}{r}519 \\
519 \\
* 519 \\
- \\
- \\
-\end{array}$ & $\begin{array}{r}500 \\
500 \\
500 \\
528 \\
722 \\
* 756 \\
\end{array}$ & $\begin{array}{c}1,450 \\
1,450 \\
1,440 \\
1,440 \\
\times 1,440 \\
-\end{array}$ & $\begin{array}{l}866 \\
866 \\
866 \\
866 \\
866 \\
866\end{array}$ & $\begin{array}{l}805 \\
805 \\
780 \\
756 \\
744 \\
-\end{array}$ & $\begin{array}{r}665 \\
665 \\
* 665 \\
665 \\
665 \\
565 \\
\end{array}$ & $\begin{array}{l}621 \\
621 \\
621 \\
621 \\
621 \\
621\end{array}$ & $\begin{array}{c}500 \\
500 \\
* 500 \\
490 \\
490 \\
-\end{array}$ \\
\hline $\begin{array}{l}\text { Total } \\
\text { Mean } \\
\text { Ac-ft }\end{array}$ & $\begin{array}{r}28,941 \\
934 \\
57,400 \\
\end{array}$ & $\begin{array}{r}27,373 \\
912 \\
54,290 \\
\end{array}$ & $\begin{array}{r}27,962 \\
902 \\
55,460 \\
\end{array}$ & $\begin{array}{r}27,962 \\
902 \\
55,460 \\
\end{array}$ & $\begin{array}{r}21,130 \\
754 \\
41,910 \\
\end{array}$ & $\begin{array}{r}14,402 \\
464 \\
28,560 \\
\end{array}$ & {$\left[\begin{array}{r}38,374 \\
1,279 \\
76,110\end{array}\right.$} & $\begin{array}{r}33,397 \\
1,077 \\
66,240 \\
\end{array}$ & $\begin{array}{r}22,891 \\
763 \\
45,400 \\
\end{array}$ & $\begin{array}{r}21,201 \\
684 \\
42,050 \\
\end{array}$ & $\begin{array}{r}19,757 \\
637 \\
39,190\end{array}$ & $\begin{array}{r}14,424 \\
480 \\
28,610 \\
\end{array}$ \\
\hline $\begin{array}{l}\text { Calen } \\
\text { Water }\end{array}$ & $(17)$ & $\begin{array}{l}\operatorname{Max} \\
\operatorname{Max}\end{array}$ & $\begin{array}{r}10,600 \\
1,600\end{array}$ & $\begin{array}{l}\text { Min } \\
\text { liin }\end{array}$ & $\begin{array}{r}403 \\
84\end{array}$ & an & & $\begin{array}{r}1,26 \\
59\end{array}$ & & & & \\
\hline
\end{tabular}

* Discharge measurement nade on this day.

Note. - No gage-height record Nov. 9-28, Nov. 30, Jarl. 2-30; discharge estimated on basis of reservoir release and discharge measurements. 
HELMANE RIVER BASIN

Arghandab River below Arghandab Dam, Afghanistan

Discharge, in cubic feet per second, water year October 1955 to September 1956

\begin{tabular}{|c|c|c|c|c|c|c|c|c|c|c|c|c|}
\hline Dav & Oct. & Nov. & Dec. & $\operatorname{Jan}$. & Feb. & $\operatorname{Mar}_{2}$ & Apr. & May & June & July & Aug. & Sept. \\
\hline $\begin{array}{l}1 \\
2 \\
3 \\
4 \\
5\end{array}$ & $\begin{array}{l}\frac{480}{480} \\
480 \\
480 \\
480\end{array}$ & $\begin{array}{l}\frac{42}{* 142} \\
442 \\
442 \\
442\end{array}$ & $\begin{array}{l}\frac{480}{480} \\
480 \\
480 \\
480\end{array}$ & $\begin{array}{l}\frac{500}{500} \\
500 \\
500 \\
500\end{array}$ & $\begin{array}{l}288 \\
288 \\
288 \\
282 \\
270\end{array}$ & $\begin{array}{l}\frac{654}{665} \\
665 \\
676 \\
676\end{array}$ & $\begin{array}{r}8,630 \\
9,320 \\
10,100 \\
9,950 \\
9,230\end{array}$ & $\begin{array}{r}5,620 \\
5,320 \\
* 5,120 \\
4,930 \\
4,660\end{array}$ & $\begin{array}{l}1,510 \\
\frac{1,450}{1,400} \\
1,320 \\
1,270\end{array}$ & $\begin{array}{l}853 \\
862 \\
834 \\
815 \\
786\end{array}$ & $\begin{array}{l}3,320 \\
a 3,000 \\
a 2,600 \\
a 2,000 \\
a 1,850\end{array}$ & $\begin{array}{l}\frac{1,260}{1,260} \\
1,260 \\
1,260 \\
1,260\end{array}$ \\
\hline $\begin{array}{r}6 \\
7 \\
8 \\
9 \\
10\end{array}$ & $\begin{array}{l}480 \\
480 \\
480 \\
480 \\
480\end{array}$ & $\begin{array}{l}442 \\
442 \\
442 \\
452 \\
452\end{array}$ & $\begin{array}{l}480 \\
480 \\
480 \\
480 \\
480\end{array}$ & $\begin{array}{l}500 \\
500 \\
500 \\
500 \\
500\end{array}$ & $\begin{array}{l}270 \\
270 \\
270 \\
270 \\
270\end{array}$ & $\begin{array}{l}688 \\
699 \\
710 \\
710 \\
722\end{array}$ & $\begin{array}{l}8,710 \\
8,630 \\
8,970 \\
9,050 \\
8,790\end{array}$ & $\begin{array}{l}4,360 \\
4,240 \\
4,060 \\
3,880 \\
3,720\end{array}$ & $\begin{array}{l}1,200 \\
1,160 \\
1,150 \\
1,120 \\
1,100\end{array}$ & $\begin{array}{l}739 \\
712 \\
720 \\
712 \\
696 \\
\end{array}$ & $\begin{array}{l}a 1,850 \\
a 1,700 \\
a 1,300 \\
a 1,300 \\
a 1,300\end{array}$ & $\begin{array}{l}1,240 \\
1,240 \\
1,240 \\
1,240 \\
1,240\end{array}$ \\
\hline $\begin{array}{l}11 \\
12 \\
13 \\
14 \\
15\end{array}$ & $\begin{array}{l}480 \\
480 \\
480 \\
480 \\
480\end{array}$ & $\begin{array}{l}452 \\
452 \\
461 \\
461 \\
461\end{array}$ & $\begin{array}{r}* 480 \\
480 \\
570 \\
519 \\
510\end{array}$ & $\begin{array}{l}490 \\
500 \\
500 \\
500 \\
490\end{array}$ & $\begin{array}{l}265 \\
265 \\
259 \\
259 \\
253\end{array}$ & $\begin{array}{l}722 \\
733 \\
733 \\
744 \\
744\end{array}$ & $\begin{array}{l}8,300 \\
8,380 \\
8,460 \\
8,710 \\
8,970\end{array}$ & $\begin{array}{l}3,500 \\
3,380 \\
3,320 \\
3,260 \\
3,150\end{array}$ & $\begin{array}{l}1,080 \\
1,070 \\
1,060 \\
1,060 \\
1,040\end{array}$ & $\begin{array}{r}696 \\
720 \\
739 \\
931 \\
1,570\end{array}$ & $\begin{array}{l}a 1,300 \\
a 1,300 \\
a 1,000 \\
a 1,150 \\
a 1,300\end{array}$ & $\begin{array}{l}1,240 \\
1,240 \\
1,200 \\
1,230 \\
1,240\end{array}$ \\
\hline $\begin{array}{l}16 \\
17 \\
18 \\
19 \\
20\end{array}$ & $\begin{array}{l}480 \\
480 \\
480 \\
\frac{397}{461}\end{array}$ & $\begin{array}{l}461 \\
470 \\
470 \\
470 \\
470\end{array}$ & $\begin{array}{l}510 \\
500 \\
500 \\
500 \\
500\end{array}$ & $\begin{array}{l}442 \\
401 \\
401 \\
401 \\
392\end{array}$ & $\begin{array}{l}253 \\
253 \\
253 \\
253 \\
253\end{array}$ & $\begin{array}{l}744 \\
744 \\
756 \\
805 \\
768\end{array}$ & $\begin{array}{l}9,230 \\
9,670 \\
9,760 \\
9,860 \\
9,950\end{array}$ & $\begin{array}{l}3,000 \\
2,820 \\
2,660 \\
2,540 \\
2,480\end{array}$ & $\begin{array}{r}1,010 \\
994 \\
962 \\
920 \\
900\end{array}$ & $\begin{array}{l}2,580 \\
6,220 \\
9,100 \\
9,520 \\
4,980\end{array}$ & $\begin{array}{l}a l, 300 \\
a l, 300 \\
a l, 300 \\
a l, 300 \\
a l, 300\end{array}$ & $\begin{array}{l}1,240 \\
1,240 \\
1,240 \\
1,240 \\
1,240\end{array}$ \\
\hline $\begin{array}{l}21 \\
22 \\
23 \\
24 \\
25\end{array}$ & $\begin{array}{l}461 \\
461 \\
461 \\
461 \\
461\end{array}$ & $\begin{array}{l}470 \\
470 \\
470 \\
470 \\
470\end{array}$ & $\begin{array}{l}500 \\
500 \\
500 \\
490 \\
490\end{array}$ & $\begin{array}{l}392 \\
392 \\
392 \\
392 \\
392\end{array}$ & $\begin{array}{l}\frac{242}{242} \\
242 \\
248 \\
352\end{array}$ & $\begin{array}{r}768 \\
768 \\
1,700 \\
7,020 \\
8,970 \\
\end{array}$ & $\begin{array}{r}10,000 \\
9,760 \\
8,880 \\
8,220 \\
7,740\end{array}$ & $\begin{array}{l}2,360 \\
2,260 \\
2,160 \\
2,060 \\
1,990\end{array}$ & $\begin{array}{l}882 \\
872 \\
853 \\
834 \\
872\end{array}$ & $\begin{array}{l}3,320 \\
3,440 \\
3,320 \\
4,740 \\
7,140\end{array}$ & $\begin{array}{l}a 1,280 \\
a 1,280 \\
a 1,280 \\
a 1,280 \\
a 1,280\end{array}$ & $\begin{array}{l}1,240 \\
1,240 \\
1,240 \\
1,240 \\
1,230\end{array}$ \\
\hline $\begin{array}{l}26 \\
27 \\
28 \\
29 \\
30 \\
31 \\
\end{array}$ & $\begin{array}{l}461 \\
452 \\
452 \\
442 \\
442 \\
442 \\
\end{array}$ & $\begin{array}{c}480 \\
480 \\
480 \\
* 480 \\
480 \\
-\end{array}$ & $\begin{array}{r}490 \\
490 \\
* 490 \\
490 \\
490 \\
500\end{array}$ & $\begin{array}{r}392 \\
392 \\
* 392 \\
392 \\
338 \\
288 \\
\end{array}$ & $\begin{array}{r}799 \\
654 \\
* 632 \\
632 \\
- \\
-\end{array}$ & $\begin{array}{r}8,790 \\
* 8,050 \\
7,510 \\
7,440 \\
8,300 \\
8,380\end{array}$ & $\begin{array}{c}7,360 \\
6,930 \\
6,440 \\
6,090 \\
5,800 \\
-\end{array}$ & $\begin{array}{r}1,920 \\
1,810 \\
1,760 \\
1,710 \\
* 1,660 \\
1,560 \\
\end{array}$ & $\begin{array}{r}872 \\
796 \\
* 815 \\
834 \\
853 \\
- \\
\end{array}$ & $\begin{array}{r}5,420 \\
* 3,080 \\
10,100 \\
9,740 \\
5,830 \\
4,280\end{array}$ & $\begin{array}{r}a 1,270 \\
a 1,270 \\
* 1,260 \\
1,260 \\
1,260 \\
1,260\end{array}$ & $\begin{array}{c}1,230 \\
1,230 \\
1,230 \\
1,230 \\
1,230 \\
- \\
\end{array}$ \\
\hline $\begin{array}{l}\text { Total } \\
\text { Mean } \\
\text { Ac-ft }\end{array}$ & $\begin{array}{r}14,494 \\
468 \\
28,750\end{array}$ & $\begin{array}{r}13,818 \\
461 \\
27,410\end{array}$ & $\begin{array}{r}15,299 \\
494 \\
30,350 \\
\end{array}$ & $\begin{array}{r}13,671 \\
442 \\
27,120 \\
\end{array}$ & $\begin{array}{r}9,375 \\
323 \\
18,600 \\
\end{array}$ & $\begin{array}{r}81,754 \\
2,637 \\
162,200 \\
\end{array}$ & $\begin{array}{r}259,630 \\
8,654 \\
515,000 \\
\end{array}$ & $\begin{array}{r}97,270 \\
3,138 \\
192,900 \\
\end{array}$ & $\begin{array}{r}31,259 \\
1,042 \\
62,000\end{array}$ & $\begin{array}{r}105,195 \\
3,393 \\
208,700\end{array}$ & $\begin{array}{r}46,750 \\
1,508 \\
92,730 \\
\end{array}$ & $\begin{array}{r}37,190 \\
1,240 \\
73,770 \\
\end{array}$ \\
\hline wate & $1 /$ & $\begin{array}{l}\text { Kax } \\
\operatorname{Max}\end{array}$ & $\begin{array}{r}1,600 \\
10,100\end{array}$ & $\begin{array}{l}\text { Min } \\
\text { Mini }\end{array}$ & & $\begin{array}{r}705 \\
1,983\end{array}$ & $\begin{array}{l}A c-f t \\
A c-f t\end{array}$ & $\begin{array}{r}510, \\
1,440 \text {, }\end{array}$ & & & & \\
\hline
\end{tabular}

* Discharge measurement made on this day.

a Gage-height record doubtful; discharge computed from Arghandab Reservoir gate release. 
HEIMAND RIVER BASIN

Arghandab River below Arghandab Dam, Afghanistan

Discharge, in cubic feet per second, water year October 1956 to September 1957

\begin{tabular}{|c|c|c|c|c|c|c|c|c|c|c|c|c|}
\hline Day & Oct. & Nov. & Dec. & Jan. & $\mathrm{Feb}$. & Mar. & Apr. & May & June & July & Aug. & Sept. \\
\hline $\begin{array}{l}1 \\
2 \\
3 \\
4 \\
5\end{array}$ & $\begin{array}{l}* 1,230 \\
1,230 \\
1,230 \\
1,230 \\
1,230\end{array}$ & $\begin{array}{l}* 806 \\
806 \\
806 \\
806 \\
806\end{array}$ & $\begin{array}{l}800 \\
800 \\
800 \\
800 \\
800\end{array}$ & $\begin{array}{l}1,210 \\
1,210 \\
1,200 \\
1,200 \\
1,200\end{array}$ & $\begin{array}{l}1,230 \\
1,230 \\
1,230 \\
1,230 \\
1,230\end{array}$ & $\begin{array}{r}1,790 \\
* 1,790 \\
1,790 \\
1,790 \\
1,790\end{array}$ & $\begin{array}{r}6,760 \\
7,080 \\
7,360 \\
12,500 \\
18,900\end{array}$ & $\begin{array}{r}8,080 \\
9,570 \\
11,200 \\
10,400 \\
* 9,930\end{array}$ & $\begin{array}{l}4,950 \\
4,890 \\
2,470 \\
4,900 \\
4,300\end{array}$ & $\begin{array}{r}1,900 \\
1,900 \\
* 1,900 \\
1,900 \\
1,900\end{array}$ & $\begin{array}{r}* 1,280 \\
1,280 \\
1,020 \\
802 \\
802\end{array}$ & $\begin{array}{l}932 \\
932 \\
932 \\
932 \\
932\end{array}$ \\
\hline $\begin{array}{r}6 \\
7 \\
8 \\
9 \\
10\end{array}$ & $\begin{array}{r}1,120 \\
910 \\
910 \\
900 \\
900\end{array}$ & $\begin{array}{l}806 \\
806 \\
806 \\
806 \\
806\end{array}$ & $\begin{array}{r}800 \\
* 796 \\
796 \\
796 \\
796\end{array}$ & $\begin{array}{l}1,200 \\
1,200 \\
1,200 \\
1,200 \\
1,200\end{array}$ & $\begin{array}{l}1,230 \\
1,230 \\
1,230 \\
1,230 \\
1,230\end{array}$ & $\begin{array}{r}1,790 \\
1,790 \\
1,790 \\
1,050 \\
210\end{array}$ & $\begin{array}{l}\frac{22,100}{20,900} \\
19,500 \\
10,000 \\
14,100\end{array}$ & $\begin{array}{l}9,030 \\
8,320 \\
8,000 \\
7,880 \\
7,920\end{array}$ & $\begin{array}{l}4,100 \\
3,900 \\
3,800 \\
3,600 \\
3,500\end{array}$ & $\begin{array}{l}1,900 \\
1,900 \\
1,900 \\
1,900 \\
1,880\end{array}$ & $\begin{array}{l}\frac{793}{793} \\
793 \\
793 \\
793\end{array}$ & $\begin{array}{l}932 \\
932 \\
932 \\
932 \\
932\end{array}$ \\
\hline $\begin{array}{l}11 \\
12 \\
13 \\
14 \\
15\end{array}$ & $\begin{array}{l}900 \\
900 \\
900 \\
900 \\
900\end{array}$ & $\begin{array}{l}806 \\
806 \\
806 \\
806 \\
806\end{array}$ & $\begin{array}{l}796 \\
796 \\
796 \\
796 \\
796\end{array}$ & $\begin{array}{l}1,200 \\
1,210 \\
1,220 \\
1,210 \\
1,210\end{array}$ & $\begin{array}{l}1,240 \\
1,240 \\
1,240 \\
1,240 \\
1,240\end{array}$ & $\begin{array}{l}* 132 \\
\frac{1320}{520} \\
1,800 \\
1,800 \\
1,800\end{array}$ & $\begin{array}{l}12,000 \\
11,100 \\
11,100 \\
11,300 \\
12,000\end{array}$ & $\begin{array}{l}8,240 \\
8,670 \\
8,320 \\
7,600 \\
7,040\end{array}$ & $\begin{array}{l}3,300 \\
3,200 \\
3,100 \\
2,900 \\
2,800\end{array}$ & $\begin{array}{l}1,880 \\
1,880 \\
1,880 \\
1,870 \\
1,870\end{array}$ & $\begin{array}{l}793 \\
793 \\
793 \\
793 \\
793\end{array}$ & $\begin{array}{l}932 \\
932 \\
921 \\
910 \\
910\end{array}$ \\
\hline $\begin{array}{l}16 \\
17 \\
18 \\
19 \\
20\end{array}$ & $\begin{array}{l}900 \\
853 \\
815 \\
815 \\
815\end{array}$ & $\begin{array}{l}805 \\
805 \\
805 \\
805 \\
805\end{array}$ & $\begin{array}{l}796 \\
796 \\
796 \\
796 \\
796\end{array}$ & $\begin{array}{l}1,210 \\
1,210 \\
1,210 \\
1,200 \\
1,200\end{array}$ & $\begin{array}{l}1,240 \\
1,260 \\
1,260 \\
1,260 \\
1,540\end{array}$ & $\begin{array}{r}1,800 \\
2,380 \\
5,680 \\
11,500 \\
11,200\end{array}$ & $\begin{array}{r}11,700 \\
10,500 \\
9,840 \\
9,660 \\
9,660\end{array}$ & $\begin{array}{r}6,840 \\
6,700 \\
* 6,660 \\
6,520 \\
5,610\end{array}$ & $\begin{array}{l}2,700 \\
2,600 \\
2,500 \\
2,500 \\
2,400\end{array}$ & $\begin{array}{l}1,870 \\
1,860 \\
1,860 \\
1,850 \\
1,850\end{array}$ & $\begin{array}{l}793 \\
793 \\
793 \\
793 \\
793\end{array}$ & $\begin{array}{l}910 \\
910 \\
910 \\
910 \\
910\end{array}$ \\
\hline $\begin{array}{l}21 \\
22 \\
23 \\
24 \\
25\end{array}$ & $\begin{array}{l}815 \\
815 \\
815 \\
815 \\
815\end{array}$ & $\begin{array}{l}805 \\
805 \\
805 \\
805 \\
805\end{array}$ & $\begin{array}{l}796 \\
796 \\
796 \\
796 \\
796\end{array}$ & $\begin{array}{l}1,200 \\
1,200 \\
1,200 \\
1,210 \\
1,220\end{array}$ & $\begin{array}{l}1,790 \\
\frac{1}{1,790} \\
1,790 \\
1,790 \\
1,790\end{array}$ & $\begin{array}{l}9,030 \\
8,850 \\
8,000 \\
7,560 \\
7,280\end{array}$ & $\begin{array}{l}3,480 \\
9,120 \\
8,940 \\
8,650 \\
8,490\end{array}$ & $\begin{array}{r}* 6,310 \\
6,520 \\
5,960 \\
5,720 \\
5,610\end{array}$ & $\begin{array}{l}2,300 \\
2,200 \\
2,200 \\
2,000 \\
1,900\end{array}$ & $\begin{array}{l}1,840 \\
1,840 \\
1,830 \\
1,820 \\
1,640\end{array}$ & $\begin{array}{l}793 \\
862 \\
932 \\
932 \\
932\end{array}$ & $\begin{array}{l}910 \\
910 \\
910 \\
910 \\
910\end{array}$ \\
\hline $\begin{array}{l}26 \\
27 \\
28 \\
29 \\
30 \\
31 \\
\end{array}$ & $\begin{array}{l}815 \\
815 \\
815 \\
815 \\
806 \\
806 \\
\end{array}$ & $\begin{array}{c}805 \\
805 \\
805 \\
805 \\
805 \\
- \\
\end{array}$ & $\begin{array}{r}1,020 \\
1,210 \\
* 1,210 \\
1,210 \\
1,210 \\
1,210\end{array}$ & $\begin{array}{l}1,220 \\
1,220 \\
1,220 \\
1,220 \\
1,220 \\
* 1,230 \\
\end{array}$ & $\begin{array}{c}1,790 \\
1,790 \\
1,790 \\
= \\
= \\
=\end{array}$ & $\begin{array}{r}7,160 \\
7,080 \\
7,040 \\
7,040 \\
* 7,040 \\
6,880\end{array}$ & $\begin{array}{c}8,160 \\
9,210 \\
8,940 \\
8,090 \\
7,760 \\
-\end{array}$ & $\begin{array}{r}5,400 \\
5,250 \\
* 4,920 \\
4,500 \\
4,920 \\
4,920\end{array}$ & $\begin{array}{c}1,900 \\
1,900 \\
1,900 \\
1,900 \\
1,900 \\
\end{array}$ & $\begin{array}{l}1,300 \\
1,300 \\
1,300 \\
1,300 \\
1,280 \\
1,280 \\
\end{array}$ & $\begin{array}{r}932 \\
932 \\
932 \\
* 932 \\
932 \\
932 \\
\end{array}$ & $\begin{array}{l}910 \\
910 \\
910 \\
910 \\
910 \\
-\end{array}$ \\
\hline $\begin{array}{l}\text { Total } \\
\text { Mean } \\
\text { Ac-ft }\end{array}$ & $\begin{array}{r}28,535 \\
920 \\
56,600 \\
\end{array}$ & $\begin{array}{r}24,165 \\
806 \\
47,930\end{array}$ & $\begin{array}{r}26,994 \\
871 \\
53,540 \\
\end{array}$ & $\begin{array}{r}37,460 \\
1,208 \\
74,300 \\
\end{array}$ & $\begin{array}{r}39,380 \\
1,410 \\
78,110 \\
\end{array}$ & $\begin{array}{r}137,152 \\
4,424 \\
272,000 \\
\end{array}$ & $\begin{array}{r}341,090 \\
11,370 \\
676,500 \\
\end{array}$ & $\begin{array}{r}222,560 \\
7,179 \\
441,400 \\
\end{array}$ & $\begin{array}{r}91,510 \\
3,050 \\
181,500 \\
\end{array}$ & $\begin{array}{r}54, \overline{380} \\
1,734 \\
107,900\end{array}$ & $\begin{array}{r}27, \frac{122}{875} \\
53,800 \\
\end{array}$ & $\begin{array}{r}27,575 \\
919 \\
54,690 \\
\end{array}$ \\
\hline ate & 1 & $7:$ & & & $\begin{array}{l}242 \\
132\end{array}$ & $\begin{array}{l}\text { liean } \\
\text { Mean }\end{array}$ & & $\begin{array}{ll}\text {-ft } & 1, \\
\text {-ft } & 2,\end{array}$ & $\begin{array}{l}000 \\
000\end{array}$ & & & \\
\hline
\end{tabular}

* Discharge measurement made on tris day.

Note. - No gage-height record Nov, 16 to Dec. 6, June 3 to July I, July 12-31; discharge conputed on basis of reservoir release. 
HEIMAND RIVER BASIN

Arghandab River below Arghandab Dam, Afghanistan

Discharge, in cubic feet per second, water year October 1957 to September 1958

\begin{tabular}{|c|c|c|c|c|c|c|c|c|c|c|c|c|}
\hline Day & oct. & Nov. & Dec. & Jan. & Feb. & Mar. & Apr. & May & June & JuIy & Aug. & Sept. \\
\hline $\begin{array}{l}1 \\
2 \\
3 \\
4 \\
5\end{array}$ & $\begin{array}{r}910 \\
910 \\
* 910 \\
910 \\
910\end{array}$ & $\begin{array}{r}900 \\
890 \\
890 \\
* 852 \\
866\end{array}$ & $\begin{array}{r}1,080 \\
1,080 \\
* 1,070 \\
1,070 \\
1,270\end{array}$ & $\begin{array}{l}1,880 \\
1,880 \\
1,880 \\
1,880 \\
1,880\end{array}$ & $\begin{array}{r}* 1,860 \\
1,860 \\
1,880 \\
1,880 \\
1,880\end{array}$ & $\begin{array}{r}1,900 \\
* 1,910 \\
1,910 \\
1,910 \\
1,910\end{array}$ & $\begin{array}{r}2,900 \\
3,260 \\
* 3,240 \\
3,220 \\
3,060\end{array}$ & $\begin{array}{r}\frac{2,700}{2,620} \\
* 1,920 \\
1,690 \\
1,970\end{array}$ & $\begin{array}{r}1,490 \\
* 1,490 \\
1,490 \\
1,490 \\
1,490\end{array}$ & $\begin{array}{r}1,470 \\
1,470 \\
1,470 \\
1,470 \\
* 1,470\end{array}$ & $\begin{array}{r}1,150 \\
1,150 \\
1,150 \\
1,170 \\
* 1,170\end{array}$ & $\begin{array}{r}1,180 \\
1,170 \\
1,060 \\
620 \\
620\end{array}$ \\
\hline $\begin{array}{r}6 \\
7 \\
8 \\
9 \\
10\end{array}$ & $\begin{array}{l}910 \\
910 \\
910 \\
910 \\
910\end{array}$ & $\begin{array}{l}910 \\
910 \\
910 \\
910 \\
910\end{array}$ & $\begin{array}{l}\frac{2,000}{1,970} \\
1,940 \\
1,910 \\
2,000\end{array}$ & $\begin{array}{l}1,880 \\
1,880 \\
1,880 \\
1,880 \\
1,880\end{array}$ & $\begin{array}{l}1,880 \\
1,880 \\
1,880 \\
1,880 \\
1,880\end{array}$ & $\begin{array}{l}1,880 \\
1,900 \\
1,900 \\
1,900 \\
1,900\end{array}$ & $\begin{array}{l}2,940 \\
2,880 \\
3,020 \\
3,060 \\
3,060\end{array}$ & $\begin{array}{l}1,970 \\
1,940 \\
1,850 \\
1,740 \\
1,680\end{array}$ & $\begin{array}{l}1,470 \\
1,480 \\
1,490 \\
1,490 \\
1,490\end{array}$ & $\begin{array}{l}1,470 \\
1,470 \\
1,470 \\
1,330 \\
1,250\end{array}$ & $\begin{array}{l}1,180 \\
1,190 \\
1,190 \\
1,190 \\
1,190\end{array}$ & $\begin{array}{l}620 \\
620 \\
620 \\
620 \\
620\end{array}$ \\
\hline $\begin{array}{l}11 \\
12 \\
13 \\
14 \\
15\end{array}$ & $\begin{array}{l}910 \\
910 \\
900 \\
900 \\
900\end{array}$ & $\begin{array}{l}921 \\
921 \\
921 \\
921 \\
921\end{array}$ & $\begin{array}{l}1,920 \\
1,910 \\
1,910 \\
1,910 \\
1,910\end{array}$ & $\begin{array}{l}1,880 \\
1,880 \\
1,880 \\
1,880 \\
1,880\end{array}$ & $\begin{array}{l}1,880 \\
1,880 \\
1,880 \\
1,880 \\
1,880\end{array}$ & $\begin{array}{l}1,910 \\
1,910 \\
1,910 \\
1,910 \\
1,910\end{array}$ & $\begin{array}{l}2,960 \\
2,880 \\
2,880 \\
2,800 \\
2,820\end{array}$ & $\begin{array}{l}1,680 \\
1,740 \\
1,640 \\
1,520 \\
1,530\end{array}$ & $\begin{array}{l}\frac{1,460}{1,480} \\
1,490 \\
1,490 \\
1,490\end{array}$ & $\begin{array}{l}1,240 \\
1,240 \\
1,300 \\
1,400 \\
1,500 \\
\end{array}$ & $\begin{array}{l}1,190 \\
1,180 \\
1,180 \\
1,170 \\
1,160\end{array}$ & $\begin{array}{l}620 \\
620 \\
620 \\
610 \\
610\end{array}$ \\
\hline $\begin{array}{l}16 \\
17 \\
18 \\
19 \\
20\end{array}$ & $\begin{array}{l}900 \\
900 \\
900 \\
900 \\
890 \\
\end{array}$ & $\begin{array}{r}921 \\
921 \\
921 \\
1,150 \\
964\end{array}$ & $\begin{array}{l}1,910 \\
1,910 \\
1,910 \\
1,910 \\
1,910\end{array}$ & $\begin{array}{l}1,880 \\
1,880 \\
1,860 \\
1,860 \\
1,880\end{array}$ & $\begin{array}{l}1,880 \\
1,880 \\
1,840 \\
\frac{1}{1,900} \\
1,900\end{array}$ & $\begin{array}{l}1,800 \\
1,070 \\
1,670 \\
1,570 \\
1,500 \\
1\end{array}$ & $\begin{array}{l}2,820 \\
3,060 \\
3,400 \\
3,380 \\
3,280\end{array}$ & $\begin{array}{l}1,660 \\
2,240 \\
2,090 \\
1,720 \\
1,580\end{array}$ & $\begin{array}{l}1,490 \\
1,480 \\
1,480 \\
1,480 \\
1,480\end{array}$ & $\begin{array}{l}1,300 \\
1,200 \\
1,200 \\
1,200 \\
1,200\end{array}$ & $\begin{array}{l}1,160 \\
1,160 \\
1,150 \\
1,150 \\
1,150\end{array}$ & $\begin{array}{l}610 \\
610 \\
610 \\
600 \\
600\end{array}$ \\
\hline $\begin{array}{l}21 \\
22 \\
23 \\
24 \\
25\end{array}$ & $\begin{array}{l}890 \\
890 \\
890 \\
900 \\
910\end{array}$ & $\begin{array}{l}953 \\
953 \\
953 \\
953 \\
953\end{array}$ & $\begin{array}{l}1,910 \\
1,910 \\
1,910 \\
1,900 \\
1,900\end{array}$ & $\begin{array}{l}1,880 \\
1,880 \\
1,880 \\
1,880 \\
1,880\end{array}$ & $\begin{array}{l}1,900 \\
1,900 \\
1,900 \\
1,900 \\
1,900\end{array}$ & $\begin{array}{l}1,500 \\
1,500 \\
1,500 \\
1,500 \\
1,520\end{array}$ & $\begin{array}{l}3,320 \\
3,380 \\
3,320 \\
3,300 \\
3,280\end{array}$ & $\begin{array}{l}1,540 \\
1,530 \\
1,520 \\
1,540 \\
1,580\end{array}$ & $\begin{array}{l}1,480 \\
1,480 \\
1,480 \\
1,480 \\
1,480\end{array}$ & $\begin{array}{l}1,200 \\
1,200 \\
1,200 \\
1,200 \\
1,200\end{array}$ & $\begin{array}{l}1,150 \\
1,150 \\
1,150 \\
1,150 \\
1,150\end{array}$ & $\begin{array}{l}600 \\
600 \\
600 \\
600 \\
590 \\
\end{array}$ \\
\hline $\begin{array}{l}26 \\
27 \\
28 \\
29 \\
30 \\
31 \\
\end{array}$ & $\begin{array}{l}910 \\
910 \\
910 \\
910 \\
910 \\
900\end{array}$ & $\begin{array}{r}953 \\
953 \\
1,080 \\
1,080 \\
1,080 \\
-\end{array}$ & $\begin{array}{r}1,900 \\
1,900 \\
1,900 \\
1,900 \\
1,900 \\
* 1,900\end{array}$ & $\begin{array}{l}1,880 \\
1,880 \\
1,860 \\
1,860 \\
1,860 \\
1,860\end{array}$ & $\begin{array}{l}1,900 \\
1,900 \\
1,900 \\
- \\
= \\
-\end{array}$ & $\begin{array}{l}1,520 \\
1,520 \\
1,520 \\
1,520 \\
1,540 \\
2,260 \\
\end{array}$ & $\begin{array}{l}3,220 \\
3,220 \\
3,080 \\
2,880 \\
\frac{2,780}{-}\end{array}$ & $\begin{array}{l}1,600 \\
1,570 \\
1,530 \\
1,500 \\
1,490 \\
1,490\end{array}$ & $\begin{array}{c}1,480 \\
1,470 \\
1,470 \\
1,470 \\
1,470 \\
-\end{array}$ & $\begin{array}{l}1,200 \\
1,150 \\
\frac{1}{1,150} \\
1,150 \\
1,150 \\
1,150\end{array}$ & $\begin{array}{l}1,150 \\
1,150 \\
1,150 \\
1,170 \\
1,190 \\
1,180\end{array}$ & $\begin{array}{r}590 \\
590 \\
590 \\
* 590 \\
590 \\
-\end{array}$ \\
\hline $\begin{array}{l}\text { Total } \\
\text { Mean } \\
\text { Ac-ft } \\
\end{array}$ & $\begin{array}{r}28,040 \\
905 \\
55,620 \\
\end{array}$ & $\begin{array}{r}28,341 \\
945 \\
56,210 \\
\end{array}$ & $\begin{array}{r}55,430 \\
1,788 \\
109,900 \\
\end{array}$ & $\begin{array}{r}58,160 \\
1,876 \\
115,400 \\
\end{array}$ & $\begin{array}{r}52,760 \\
1,884 \\
104,600 \\
\end{array}$ & $\begin{array}{r}54, \overline{180} \\
1,748 \\
107,500 \\
\end{array}$ & $\begin{array}{r}92,730 \\
3,091 \\
183,900 \\
\end{array}$ & $\begin{array}{r}54,370 \\
1,754 \\
107,800 \\
\end{array}$ & $\begin{array}{r}44,450 \\
1,482 \\
88,170\end{array}$ & $\begin{array}{r}40,070 \\
1,293 \\
79,480\end{array}$ & $\begin{array}{r}36,120 \\
1,166 \\
71,640\end{array}$ & $\begin{array}{r}19,800 \\
660 \\
39,270 \\
\end{array}$ \\
\hline
\end{tabular}

$\begin{array}{llllllll}\text { Calendar year 1957: } & \text { Max } 22,100 & \text { Min } 132 & \text { Mean 2,986 } & \text { Ac-ft 2,162,000 } \\ \text { water year 1957-58: } & \text { Max } 3,400 & \text { Min 590 } & \text { Mean 1,546 } & \text { Ac-ft 1,119,000 }\end{array}$

* Discharge measurement made or this day.

Note.- No gage-height record July Il to Aug. 3, Sept. 4-30 and doubtful gage-height record June 19 to July 4; discharge computed or basis of reservoir release. 
HELMAND RIVER BASIN

Arghandab River below Arghandab Dam, Afghanistan

Discharge, in cubic feet per second, water year October 1958 to September 1959

\begin{tabular}{|c|c|c|c|c|c|c|c|c|c|c|c|c|}
\hline Dar & oct. & Nov. & Dec. & Jan. & Feb. & Mar. & Apr. & May & June & JuIV & Aug. & Sept. \\
\hline $\begin{array}{l}1 \\
2 \\
3 \\
4 \\
5\end{array}$ & $\begin{array}{r}583 \\
583 \\
583 \\
* 583 \\
583\end{array}$ & $\begin{array}{l}\frac{852}{852} \\
852 \\
852 \\
852\end{array}$ & $\begin{array}{l}\frac{812}{812} \\
812 \\
812 \\
812\end{array}$ & $\begin{array}{l}812 \\
812 \\
822 \\
812 \\
812\end{array}$ & $\begin{array}{l}734 \\
734 \\
734 \\
734 \\
734\end{array}$ & $\begin{array}{r}974 \\
822 \\
812 \\
* 822 \\
822\end{array}$ & $\begin{array}{l}5,100 \\
5,610 \\
7,080 \\
5,960 \\
5,890\end{array}$ & $\begin{array}{r}3,380 \\
3,300 \\
3,140 \\
* 2,900 \\
2,920\end{array}$ & $\begin{array}{r}1,980 \\
1,980 \\
1,970 \\
* 1,970 \\
1,970\end{array}$ & $\begin{array}{r}1,500 \\
1,500 \\
1,500 \\
1,500 \\
* 1,500\end{array}$ & $\begin{array}{l}* 1,010 \\
1,010 \\
1,010 \\
1,010 \\
1,010\end{array}$ & $\begin{array}{l}1,150 \\
1,150 \\
1,150 \\
1,150 \\
1,150\end{array}$ \\
\hline $\begin{array}{r}6 \\
7 \\
8 \\
9 \\
10\end{array}$ & $\begin{array}{l}583 \\
583 \\
583 \\
583 \\
583\end{array}$ & $\begin{array}{l}852 \\
852 \\
852 \\
852 \\
852\end{array}$ & $\begin{array}{l}812 \\
812 \\
812 \\
812 \\
812\end{array}$ & $\begin{array}{r}774 \\
715 \\
* 715 \\
715 \\
715\end{array}$ & $\begin{array}{l}734 \\
734 \\
734 \\
734 \\
734\end{array}$ & $\begin{array}{l}832 \\
632 \\
832 \\
842 \\
862\end{array}$ & $\begin{array}{l}5,280 \\
4,920 \\
4,920 \\
4,980 \\
5,160\end{array}$ & $\begin{array}{l}2,860 \\
2,740 \\
2,620 \\
2,520 \\
2,430\end{array}$ & $\begin{array}{l}1,970 \\
1,960 \\
1,960 \\
1,960 \\
1,940\end{array}$ & $\begin{array}{l}1,500 \\
1,500 \\
1,500 \\
1,490 \\
1,050\end{array}$ & $\begin{array}{l}1,010 \\
1,010 \\
1,090 \\
\frac{1,170}{1,170}\end{array}$ & $\begin{array}{l}1,140 \\
1,140 \\
1,140 \\
1,140 \\
1,140\end{array}$ \\
\hline $\begin{array}{l}11 \\
12 \\
13 \\
14 \\
15\end{array}$ & $\begin{array}{l}583 \\
583 \\
583 \\
583 \\
583\end{array}$ & $\begin{array}{l}852 \\
852 \\
852 \\
852 \\
852\end{array}$ & $\begin{array}{l}812 \\
871 \\
822 \\
822 \\
822\end{array}$ & $\begin{array}{l}715 \\
715 \\
715 \\
715 \\
715\end{array}$ & $\begin{array}{l}734 \\
734 \\
734 \\
734 \\
734\end{array}$ & $\begin{array}{l}862 \\
871 \\
890 \\
890 \\
890\end{array}$ & $\begin{array}{r}5,280 \\
5,340 \\
5,400 \\
5,040 \\
* 4,500\end{array}$ & $\begin{array}{l}2,320 \\
2,280 \\
2,220 \\
2,200 \\
2,260\end{array}$ & $\begin{array}{l}1,940 \\
1,940 \\
1,940 \\
1,940 \\
1,940\end{array}$ & $\begin{array}{r}1,050 \\
1,040 \\
1,040 \\
1,0216 \\
1,020\end{array}$ & $\begin{array}{l}1,170 \\
1,170 \\
1,170 \\
1,160 \\
1,160\end{array}$ & $\begin{array}{r}1,140 \\
1,140 \\
1,125 \\
1,125 \\
* 1,110\end{array}$ \\
\hline $\begin{array}{l}16 \\
17 \\
18 \\
19 \\
20\end{array}$ & $\begin{array}{l}583 \\
583 \\
583 \\
583 \\
583\end{array}$ & $\begin{array}{l}852 \\
852 \\
852 \\
852 \\
852\end{array}$ & $\begin{array}{l}822 \\
822 \\
822 \\
822 \\
822\end{array}$ & $\begin{array}{l}715 \\
715 \\
715 \\
724 \\
724\end{array}$ & $\begin{array}{l}734 \\
744 \\
832 \\
754 \\
754\end{array}$ & $\begin{array}{l}1,090 \\
3,530 \\
5,440 \\
6,000 \\
6,510\end{array}$ & $\begin{array}{l}5,070 \\
4,800 \\
4,700 \\
4,500 \\
4,320\end{array}$ & $\begin{array}{l}2,430 \\
2,740 \\
3,280 \\
3,440 \\
3,350\end{array}$ & $\begin{array}{l}1,940 \\
1,940 \\
1,940 \\
1,920 \\
1,920\end{array}$ & $\begin{array}{l}1,020 \\
1,020 \\
1,020 \\
1,020 \\
1,020\end{array}$ & $\begin{array}{l}1,160 \\
1,160 \\
1,160 \\
1,160 \\
1,160\end{array}$ & $\begin{array}{l}1,110 \\
1,110 \\
1,110 \\
1,100 \\
1,100\end{array}$ \\
\hline $\begin{array}{l}21 \\
22 \\
23 \\
24 \\
25\end{array}$ & $\begin{array}{l}583 \\
583 \\
616 \\
715 \\
706\end{array}$ & $\begin{array}{l}852 \\
852 \\
832 \\
832 \\
832\end{array}$ & $\begin{array}{l}822 \\
822 \\
822 \\
822 \\
822\end{array}$ & $\begin{array}{l}724 \\
724 \\
724 \\
724 \\
724\end{array}$ & $\begin{array}{l}754 \\
754 \\
754 \\
754 \\
754\end{array}$ & $\begin{array}{l}6,760 \\
6,760 \\
6,800 \\
6,760 \\
6,730\end{array}$ & $\begin{array}{l}4,300 \\
4,600 \\
4,750 \\
4,300 \\
4,250\end{array}$ & $\begin{array}{l}2,820 \\
2,520 \\
2,290 \\
2,120 \\
2,020\end{array}$ & $\begin{array}{l}1,920 \\
1,920 \\
1,920 \\
1,910 \\
1,910\end{array}$ & $\begin{array}{l}1,020 \\
1,020 \\
1,020 \\
1,020 \\
1,020\end{array}$ & $\begin{array}{l}1,160 \\
1,160 \\
1,160 \\
1,150 \\
1,150\end{array}$ & $\begin{array}{l}1,110 \\
1,110 \\
1,040 \\
1,100 \\
1,100\end{array}$ \\
\hline $\begin{array}{l}26 \\
27 \\
28 \\
29 \\
30 \\
31 \\
\end{array}$ & $\begin{array}{r}802 \\
852 \\
852 \\
852 \\
852 \\
* 852\end{array}$ & $\begin{array}{r}832 \\
832 \\
822 \\
822 \\
* 812 \\
- \\
\end{array}$ & $\begin{array}{l}822 \\
822 \\
822 \\
812 \\
812 \\
812 \\
\end{array}$ & $\begin{array}{r}724 \\
715 \\
* 734 \\
734 \\
734 \\
734 \\
\end{array}$ & $\begin{array}{c}754 \\
764 \\
764 \\
- \\
- \\
- \\
\end{array}$ & $\begin{array}{r}6,840 \\
* 6,700 \\
6,280 \\
6,060 \\
5,780 \\
5,500 \\
\end{array}$ & $\begin{array}{c}3,950 \\
3,710 \\
3,500 \\
3,440 \\
3,440 \\
-\end{array}$ & $\begin{array}{l}1,980 \\
1,980 \\
1,980 \\
1,980 \\
1,980 \\
1,980\end{array}$ & $\begin{array}{l}1,910 \\
1,660 \\
1,520 \\
1,520 \\
1,500 \\
\frac{-}{1}\end{array}$ & $\begin{array}{l}1,020 \\
1,020 \\
1,020 \\
1,020 \\
1,020 \\
1,020\end{array}$ & $\begin{array}{l}1,150 \\
1,150 \\
1,150 \\
1,150 \\
1,150 \\
1,150\end{array}$ & $\begin{array}{c}1,110 \\
1,110 \\
1,100 \\
1,100 \\
1,100 \\
-\end{array}$ \\
\hline $\begin{array}{l}\text { Total } \\
\text { Mean } \\
\text { de-ft }\end{array}$ & $\begin{array}{r}19,925 \\
643 \\
39,520 \\
\end{array}$ & $\begin{array}{r}25,360 \\
845 \\
50,300 \\
\end{array}$ & $\begin{array}{r}25,391 \\
819 \\
50,360 \\
\end{array}$ & $\begin{array}{r}22,867 \\
738 \\
45,360 \\
\end{array}$ & $\begin{array}{r}20,880 \\
746 \\
41,420\end{array}$ & $\begin{array}{r}106,395 \\
3,432 \\
211,030 \\
\end{array}$ & $\begin{array}{r}144,090 \\
4,803 \\
285,800 \\
\end{array}$ & $\begin{array}{r}78,980 \\
2,548 \\
156,650 \\
\end{array}$ & $\begin{array}{r}56,690 \\
1,890 \\
112,440\end{array}$ & $\begin{array}{c}35,926 \\
1,159 \\
71,260\end{array}$ & $\begin{array}{r}34,810 \\
1,125 \\
69,050\end{array}$ & $\begin{array}{r}33,600 \\
1,120 \\
66,640 \\
\end{array}$ \\
\hline
\end{tabular}

\# Discharge measurement made on this day. 
HEIMAND RIVER BASIN

Arghandab Piver below Àrghandab Dam, Afghanistan

Discharge, in cubic feet per second, water year October 1959 to september 1960

\begin{tabular}{|c|c|c|c|c|c|c|c|c|c|c|c|c|}
\hline Day & Oct. & Nov. & Dec & $J_{\operatorname{Jan}}$ & $\overline{\mathrm{Feb}}$. & Miar. & Apr $f$ & May & June & July & Aug. & Sept. \\
\hline $\begin{array}{l}1 \\
2 \\
3 \\
4 \\
5\end{array}$ & $\begin{array}{l}\frac{1,1,0}{1,140} \\
1,140 \\
1,090 \\
1,030\end{array}$ & $\begin{array}{l}\frac{1}{1,000} \\
1,000 \\
1,000 \\
1,000 \\
1,000\end{array}$ & $\begin{array}{r}990 \\
1,030 \\
1,010 \\
1,010 \\
1,010\end{array}$ & $\begin{array}{r}773 \\
* 773 \\
773 \\
773 \\
773\end{array}$ & $\begin{array}{l}\frac{422}{422} \\
452 \\
530 \\
530\end{array}$ & $\begin{array}{r}791 \\
* 791 \\
782 \\
782 \\
782\end{array}$ & $\begin{array}{l}692 \\
692 \\
638 \\
560 \\
560\end{array}$ & $\begin{array}{l}4,360 \\
4,660 \\
4,880 \\
5,000 \\
4,750\end{array}$ & $\begin{array}{l}\frac{1,910}{1,840} \\
1,770 \\
1,670 \\
1,630\end{array}$ & $\begin{array}{l}701 \\
683 \\
683 \\
764 \\
737\end{array}$ & $\begin{array}{l}\frac{971}{971} \\
971 \\
971 \\
971\end{array}$ & $\begin{array}{l}\frac{952}{952} \\
952 \\
952 \\
952\end{array}$ \\
\hline $\begin{array}{r}6 \\
7 \\
8 \\
9 \\
10\end{array}$ & $\begin{array}{l}1,030 \\
1,030 \\
1,030 \\
1,030 \\
1,020\end{array}$ & $\begin{array}{r}1,000 \\
990 \\
990 \\
990 \\
990\end{array}$ & $\begin{array}{r}* 1,010 \\
1,010 \\
1,010 \\
1,010 \\
1,010\end{array}$ & $\begin{array}{l}773 \\
773 \\
773 \\
773 \\
773\end{array}$ & $\begin{array}{l}530 \\
530 \\
530 \\
538 \\
538\end{array}$ & $\begin{array}{l}782 \\
782 \\
782 \\
782 \\
791\end{array}$ & $\begin{array}{r}* 560 \\
545 \\
545 \\
545 \\
552\end{array}$ & $\begin{array}{l}4,410 \\
4,220 \\
3,900 \\
3,720 \\
3,610\end{array}$ & $\begin{array}{l}1,580 \\
1,530 \\
1,450 \\
1,380 \\
1,320\end{array}$ & $\begin{array}{l}728 \\
737 \\
857 \\
857 \\
857\end{array}$ & $\begin{array}{l}971 \\
962 \\
962 \\
962 \\
962\end{array}$ & $\begin{array}{l}952 \\
942 \\
942 \\
942 \\
942\end{array}$ \\
\hline $\begin{array}{l}17 \\
12 \\
13 \\
14 \\
15\end{array}$ & $\begin{array}{l}1,020 \\
1,020 \\
1,020 \\
1,020 \\
1,020\end{array}$ & $\begin{array}{l}990 \\
990 \\
990 \\
990 \\
990\end{array}$ & $\begin{array}{l}1,010 \\
1,010 \\
1,010 \\
1,010 \\
1,010\end{array}$ & $\begin{array}{l}773 \\
773 \\
773 \\
764 \\
773\end{array}$ & $\begin{array}{l}538 \\
538 \\
538 \\
545 \\
545\end{array}$ & $\begin{array}{l}791 \\
800 \\
791 \\
791 \\
800\end{array}$ & $\begin{array}{l}612 \\
764 \\
848 \\
722 \\
560\end{array}$ & $\begin{array}{l}3,540 \\
3,610 \\
3,680 \\
3,770 \\
3,820\end{array}$ & $\begin{array}{l}1,230 \\
1,130 \\
1,040 \\
1,020 \\
1,030\end{array}$ & $\begin{array}{l}929 \\
\frac{980}{980} \\
971 \\
971\end{array}$ & $\begin{array}{l}962 \\
962 \\
962 \\
962 \\
962\end{array}$ & $\begin{array}{l}942 \\
942 \\
942 \\
\frac{233}{933}\end{array}$ \\
\hline $\begin{array}{l}16 \\
17 \\
18 \\
19 \\
20\end{array}$ & $\begin{array}{l}1,020 \\
1,020 \\
1,010 \\
1,010 \\
1,010\end{array}$ & $\begin{array}{l}990 \\
990 \\
990 \\
990 \\
990\end{array}$ & $\begin{array}{l}1,010 \\
1,010 \\
1,010 \\
1,010 \\
1,010\end{array}$ & $\begin{array}{l}773 \\
773 \\
773 \\
773 \\
773\end{array}$ & $\begin{array}{l}545 \\
545 \\
545 \\
545 \\
552\end{array}$ & $\begin{array}{l}800 \\
800 \\
800 \\
800 \\
800\end{array}$ & $\begin{array}{l}870 \\
692 \\
590 \\
582 \\
582\end{array}$ & $\begin{array}{l}3,820 \\
3,790 \\
3,900 \\
3,790 \\
3,610\end{array}$ & $\begin{array}{l}980 \\
962 \\
933 \\
895 \\
886\end{array}$ & $\begin{array}{l}971 \\
971 \\
971 \\
971 \\
971\end{array}$ & $\begin{array}{l}962 \\
962 \\
962 \\
\frac{952}{952}\end{array}$ & $\begin{array}{l}933 \\
933 \\
933 \\
933 \\
933\end{array}$ \\
\hline $\begin{array}{l}21 \\
22 \\
23 \\
24 \\
25\end{array}$ & $\begin{array}{l}1,010 \\
1,010 \\
1,010 \\
1,010 \\
1,010\end{array}$ & $\begin{array}{l}990 \\
990 \\
990 \\
990 \\
990\end{array}$ & $\begin{array}{l}1,010 \\
1,010 \\
1,010 \\
1,010 \\
1,010\end{array}$ & $\begin{array}{l}773 \\
773 \\
773 \\
773 \\
773\end{array}$ & $\begin{array}{l}605 \\
701 \\
710 \\
710 \\
701\end{array}$ & $\begin{array}{l}\frac{810}{810} \\
810 \\
810 \\
810\end{array}$ & $\begin{array}{r}620 \\
582 \\
575 \\
1,080 \\
* 3,340\end{array}$ & $\begin{array}{r}3,450 \\
3,170 \\
2,960 \\
2,790 \\
* 2,730\end{array}$ & $\begin{array}{l}886 \\
866 \\
886 \\
911_{4} \\
914\end{array}$ & $\begin{array}{l}971 \\
971 \\
971 \\
971 \\
962\end{array}$ & $\begin{array}{r}952 \\
* 952 \\
952 \\
952 \\
952\end{array}$ & $\begin{array}{l}933 \\
933 \\
933 \\
933 \\
933\end{array}$ \\
\hline $\begin{array}{l}26 \\
27 \\
28 \\
29 \\
30 \\
31\end{array}$ & $\begin{array}{l}1,010 \\
1,010 \\
1,010 \\
1,010 \\
\frac{1,000}{1,000}\end{array}$ & $\begin{array}{l}990 \\
990 \\
990 \\
990 \\
990 \\
-\end{array}$ & $\begin{array}{r}1,010 \\
1,010 \\
1,010 \\
1,010 \\
2,010 \\
\quad 887\end{array}$ & $\begin{array}{l}773 \\
773 \\
773 \\
539 \\
422 \\
422\end{array}$ & $\begin{array}{l}728 \\
8000 \\
800 \\
800 \\
- \\
-\end{array}$ & $\begin{array}{l}782 \\
692 \\
692 \\
671 \\
683 \\
692\end{array}$ & $\begin{array}{c}4,170 \\
4,240 \\
4,220 \\
4,170 \\
4,200 \\
-\end{array}$ & $\begin{array}{l}2,580 \\
2,370 \\
2,260 \\
2,180 \\
3,100 \\
1,990\end{array}$ & $\begin{array}{r}876 \\
942 \\
* 924 \\
819 \\
746 \\
-\end{array}$ & $\begin{array}{r}962 \\
962 \\
962 \\
962 \\
* 962 \\
971\end{array}$ & $\begin{array}{l}952 \\
952 \\
952 \\
952 \\
952 \\
952\end{array}$ & $\begin{array}{l}933 \\
933 \\
933 \\
933 \\
933 \\
-\end{array}$ \\
\hline $\begin{array}{l}\text { Total } \\
\text { Mean } \\
\text { Ac-ft }\end{array}$ & $\begin{array}{r}31,940 \\
1,030 \\
63,350\end{array}$ & $\begin{array}{r}29,760 \\
992 \\
59,030\end{array}$ & $\begin{array}{r}31,187 \\
1,006 \\
61,860\end{array}$ & $\begin{array}{r}23,018 \\
743 \\
45,660\end{array}$ & $\begin{array}{r}16,468 \\
568 \\
32,660\end{array}$ & $\begin{array}{r}24,082 \\
777 \\
47,770\end{array}$ & $\begin{array}{r}39,908 \\
1,330 \\
79,160\end{array}$ & $\begin{array}{r}109,420 \\
3,530 \\
217,030\end{array}$ & $\begin{array}{r}34,959 \\
1,165 \\
69,340\end{array}$ & $\begin{array}{r}27,912 \\
900 \\
55,360\end{array}$ & $\begin{array}{r}29,746 \\
960 \\
59,000\end{array}$ & $\begin{array}{r}28,167 \\
939 \\
55,870\end{array}$ \\
\hline & & $\begin{array}{l}\mathrm{Ma} \\
\mathrm{Ma}\end{array}$ & $\begin{array}{l}7,080 \\
5,000\end{array}$ & $\begin{array}{l}\text { Min } \\
\text { Min }\end{array}$ & $\begin{array}{l}715 \\
422\end{array}$ & $\begin{array}{l}\text { liean } \\
\text { Mean }\end{array}$ & & $\begin{array}{l}\mathrm{Ac}-\mathrm{ft} \\
\mathrm{Ac}-\mathrm{ft}\end{array}$ & 846,100 & & & \\
\hline
\end{tabular}

* Discharge measurement made on this day. 
HELMAND RIVER BASIN

Ghazni River near Ghazni, Afghanistan

Location.--Lat $33^{\circ} 45^{\prime} \mathrm{N}$, long $68^{\circ} 24^{\prime} \mathrm{E} ., 500$ meters downstream from Seraj dam and about 24 kilometers upstream from Ghazni.

Drainage area.-- $-495 \mathrm{sq} \mathrm{mi}$, approximately.

Records available..-January to May 1948, March 1949 to April 1952.

Gage.-water-stage recorder and staff gage read once daily. Jan. Il to May 10, 1948 at site 14 kilometers

downstream at different datum. Altitude of gage is about 2,300 meters from topographic map.

Extremes.-Maximum and minimum discharges for the water years 1948-52 are contained in the following table:

\begin{tabular}{|c|c|c|c|c|c|c|}
\hline \multirow[b]{2}{*}{ Water Year } & \multirow[b]{2}{*}{ Date } & \multicolumn{2}{|c|}{ Maximum } & \multirow[b]{2}{*}{ Date. } & \multicolumn{2}{|c|}{ Minimum } \\
\hline & & $\begin{array}{l}\text { Gage Height } \\
\text { (meters) }\end{array}$ & $\begin{array}{c}\text { Discharge } \\
\text { (cfs) }\end{array}$ & & $\begin{array}{c}\text { Gage Height } \\
\text { (meters) }\end{array}$ & $\begin{array}{c}\text { Discharge } \\
\text { (cfs) }\end{array}$ \\
\hline $\begin{array}{l}1948 \\
1949 \\
1950 \\
1951 \\
1952\end{array}$ & $\begin{array}{l}\text { Apr. } 2,3 \\
\text { Apr. } 10 \\
\text { Apr. } 28 \\
\text { May } 6 \\
\text { Apr. } 7\end{array}$ & $\begin{array}{r}* 0.30 \\
10.46 \\
10.52 \\
10.75 \\
10.61\end{array}$ & $\begin{array}{l}122 \\
200 \\
242 \\
454 \\
317\end{array}$ & $\begin{array}{l}\text { May } 10 \\
\text { Many Lays } \\
\text { July } 9 \\
\text { July } 10\end{array}$ & $\begin{array}{l}*-0.11 \\
10.05 \\
10.04 \\
10.12\end{array}$ & $\begin{array}{l}2 \\
1.4 \\
12 \\
29\end{array}$ \\
\hline
\end{tabular}

* At different datum.

Remarks.--Records are fair except those for 1948 which are poor. Flow regulated by Seraj dam. Many diversions for irrigation upstream. hecords collected by Morrison-Knudsen Afghanistan. 
HELAAND RIVER BASIN

Gheznt River near Ghazni, Afghanistan

Discharge in cubic feet per second, water year October 1947 to September 1948

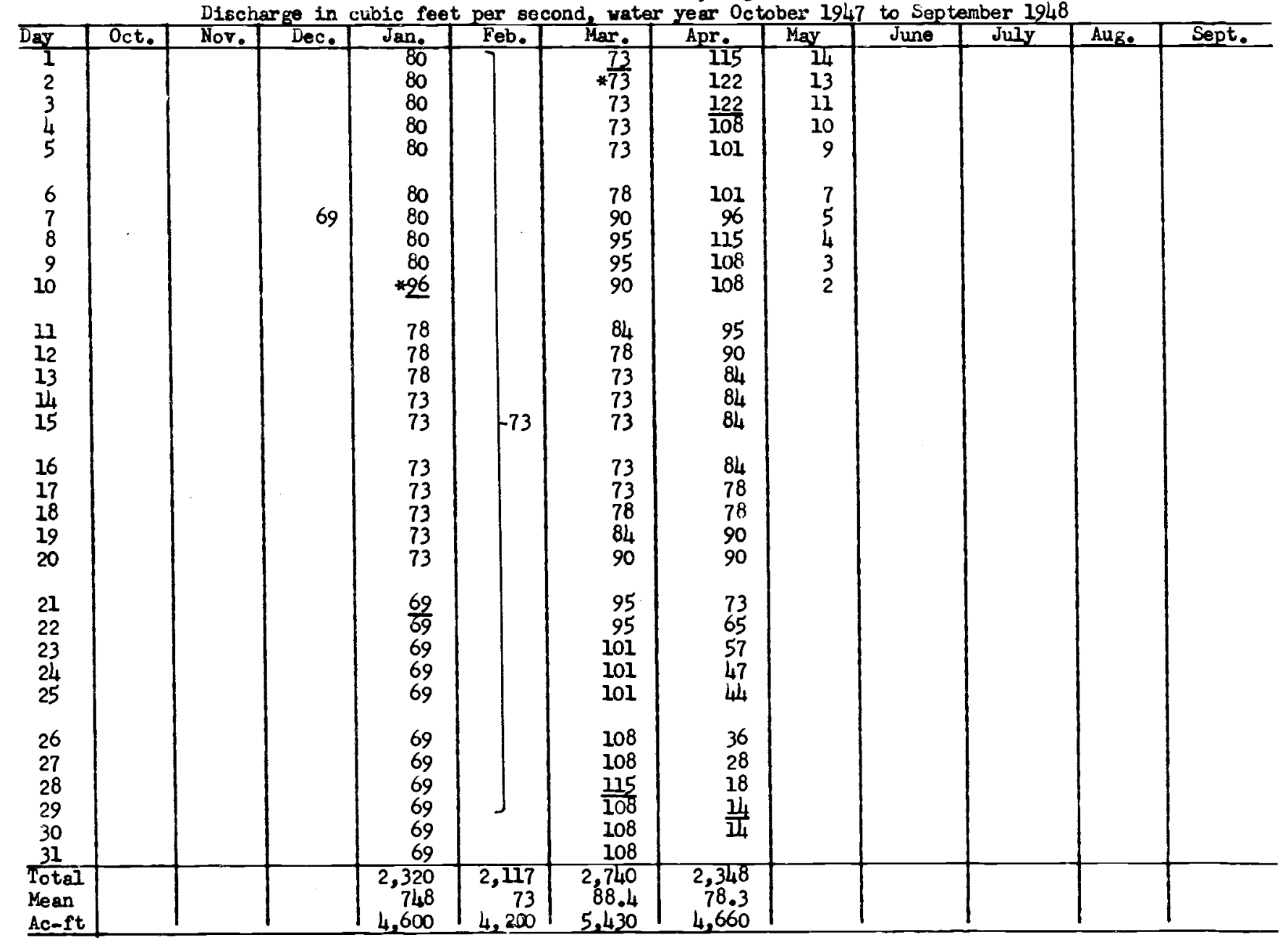

* Discharge measurement made on this day.

Note.-No gage-heights Jan. 1-9, Jan. 26 to Mar. 1, May 2-8; discharge est1mated. 
HELMAND RIVER BASIN

Ghazni River near Ghazni, Afghanistan

Discharge, in cubic feet per second water year October 1948 to September 1949

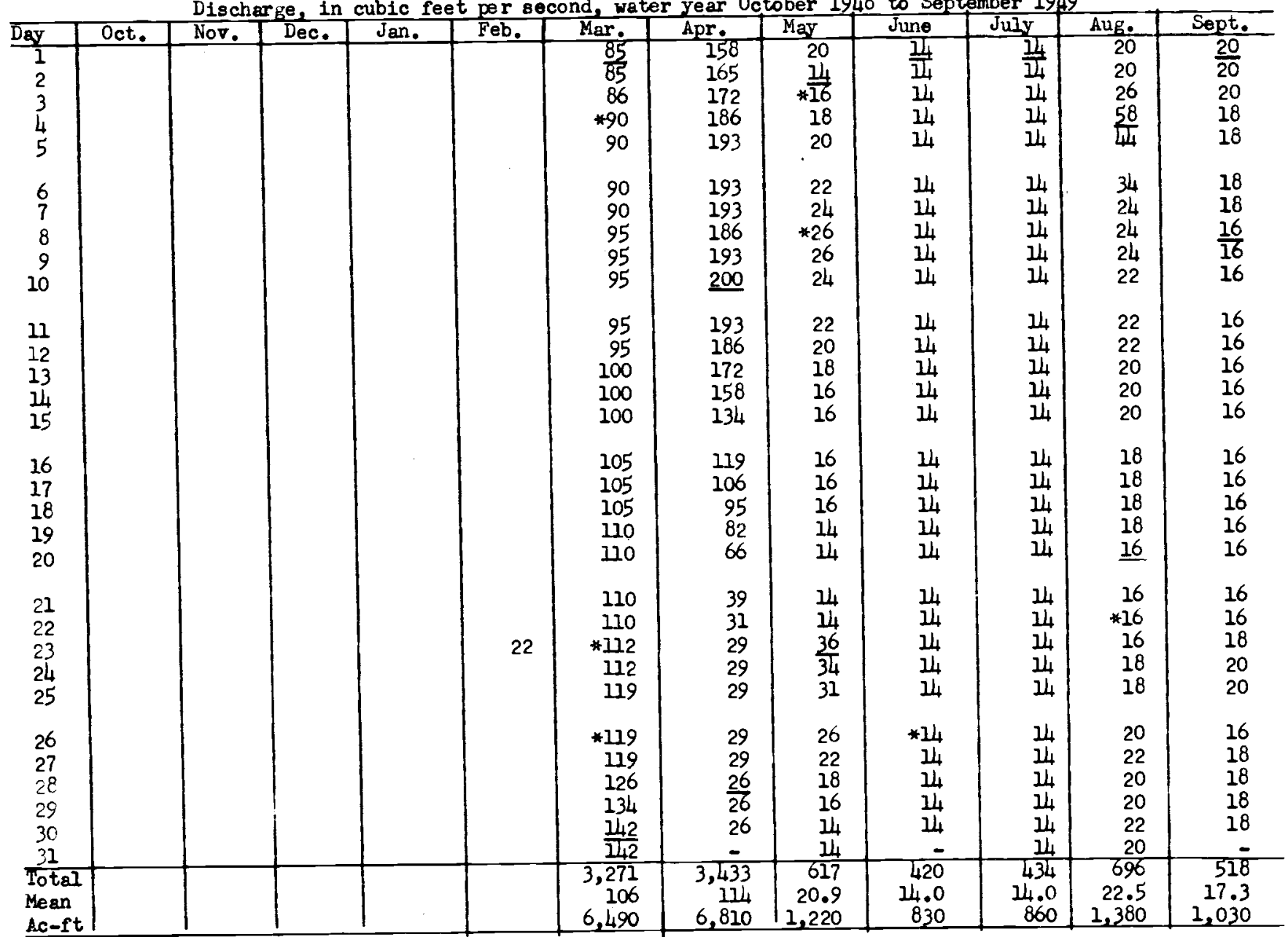

* Discharge measurement made on this day.

Note.-No gage-height record Mar. 1-22, May 4-7; discharge estimated on basis of discharge measurements of Mar. 4,23 and May 3, 8 . 
HELMAND RIVER BASIN

Ghasnt River near Ghazni, Afghanistan

Discharge, in cubic feet per second, water year October 1949 to September 1950

\begin{tabular}{|c|c|c|c|c|c|c|c|c|c|c|c|c|}
\hline Dgy & Oct. & Nov. & Dec. & Jan. & Feb. & Mar. & Apr. & Mgy & June & July & Aug. & Sept. \\
\hline $\begin{array}{l}1 . \\
2 \\
3 \\
4 \\
5\end{array}$ & $\begin{array}{l}\frac{20}{29} \\
29 \\
29 \\
31\end{array}$ & $\begin{array}{l}54 \\
58 \\
62 \\
62 \\
62\end{array}$ & $\begin{array}{l}74 \\
74 \\
74 \\
78 \\
78\end{array}$ & $\begin{array}{l}82 \\
82 \\
82 \\
82 \\
82\end{array}$ & $\begin{array}{l}74 \\
70 \\
70 \\
70 \\
70\end{array}$ & $\begin{array}{l}\frac{82}{82} \\
82 \\
82 \\
82\end{array}$ & $\begin{array}{r}150 \\
* 158 \\
142 \\
126 \\
126\end{array}$ & $\begin{array}{l}186 \\
172 \\
119 \\
106 \\
172\end{array}$ & $\begin{array}{l}50 \\
54 \\
47 \\
39 \\
34\end{array}$ & $\begin{array}{l}16 \\
16 \\
16 \\
16 \\
16\end{array}$ & $\begin{array}{l}22 \\
22 \\
23 \\
24 \\
25\end{array}$ & $\begin{array}{l}18 \\
\frac{18}{18} \\
18 \\
18 \\
18\end{array}$ \\
\hline $\begin{array}{r}6 \\
7 \\
8 \\
9 \\
10\end{array}$ & $\begin{array}{l}31 \\
31 \\
31 \\
31 \\
34\end{array}$ & $\begin{array}{l}66 \\
66 \\
66 \\
66 \\
66\end{array}$ & $\begin{array}{l}78 \\
78 \\
78 \\
78 \\
78\end{array}$ & $\begin{array}{l}\frac{142}{95} \\
90 \\
90 \\
90\end{array}$ & $\begin{array}{l}70 \\
70 \\
70 \\
70 \\
70\end{array}$ & $\begin{array}{l}82 \\
86 \\
90 \\
90 \\
90\end{array}$ & $\begin{array}{l}158 \\
165 \\
179 \\
193 \\
193\end{array}$ & $\begin{array}{r}200 \\
* 186 \\
134 \\
150 \\
214\end{array}$ & $\begin{array}{l}29 \\
29 \\
29 \\
24 \\
26\end{array}$ & $\begin{array}{r}16 \\
14 \\
14 \\
* 12 \\
\frac{11}{16}\end{array}$ & $\begin{array}{l}26 \\
35 \\
41 \\
39 \\
41\end{array}$ & $\begin{array}{l}18 \\
18 \\
18 \\
18 \\
18\end{array}$ \\
\hline $\begin{array}{l}11 \\
12 \\
13 \\
14 \\
15\end{array}$ & $\begin{array}{r}34 \\
* 34 \\
34 \\
36 \\
36\end{array}$ & $\begin{array}{l}66 \\
66 \\
66 \\
66 \\
66\end{array}$ & $\begin{array}{l}78 \\
78 \\
78 \\
78 \\
78\end{array}$ & $\begin{array}{r}90 \\
90 \\
112 \\
90 \\
95\end{array}$ & $\begin{array}{l}70 \\
70 \\
70 \\
70 \\
70\end{array}$ & $\begin{array}{r}95 \\
100 \\
100 \\
95 \\
95\end{array}$ & $\begin{array}{r}200 \\
* 200 \\
200 \\
207 \\
228\end{array}$ & $\begin{array}{r}200 \\
235 \\
193 \\
207 \\
86\end{array}$ & $\begin{array}{l}24 \\
22 \\
24 \\
22 \\
22\end{array}$ & $\begin{array}{l}16 \\
16 \\
16 \\
16 \\
24 \\
\end{array}$ & $\begin{array}{l}36 \\
36 \\
29 \\
29 \\
20\end{array}$ & $\begin{array}{l}18 \\
18 \\
18 \\
18 \\
18\end{array}$ \\
\hline $\begin{array}{l}16 \\
17 \\
18 \\
19 \\
20\end{array}$ & $\begin{array}{l}36 \\
36 \\
39 \\
39 \\
39\end{array}$ & $\begin{array}{r}* 70 \\
70 \\
70 \\
70 \\
70\end{array}$ & $\begin{array}{l}\frac{82}{82} \\
82 \\
82 \\
82\end{array}$ & $\begin{array}{l}90 \\
90 \\
82 \\
70 \\
74\end{array}$ & $\begin{array}{l}75 \\
75 \\
75 \\
75 \\
75\end{array}$ & $\begin{array}{l}95 \\
95 \\
95 \\
90 \\
82\end{array}$ & $\begin{array}{l}235 \\
228 \\
228 \\
214 \\
214\end{array}$ & $\begin{array}{l}74 \\
62 \\
54 \\
44 \\
44\end{array}$ & $\begin{array}{r}20 \\
* 22 \\
20 \\
22 \\
20\end{array}$ & $\begin{array}{l}16 \\
16 \\
16 \\
16 \\
17\end{array}$ & $\begin{array}{r}* 22 \\
22 \\
20 \\
20 \\
20\end{array}$ & $\begin{array}{r}18 \\
* 18 \\
16 \\
16 \\
16\end{array}$ \\
\hline $\begin{array}{l}21 \\
22 \\
23 \\
24 \\
25\end{array}$ & $\begin{array}{l}39 \\
39 \\
39 \\
39 \\
41\end{array}$ & $\begin{array}{l}70 \\
70 \\
70 \\
70 \\
74\end{array}$ & $\begin{array}{l}82 \\
82 \\
82 \\
82 \\
82\end{array}$ & $\begin{array}{l}74 \\
78 \\
78 \\
82 \\
82\end{array}$ & $\begin{array}{r}75 \\
80 \\
* 82 \\
82 \\
86 \\
\end{array}$ & $\begin{array}{l}90 \\
100 \\
100 \\
106 \\
106\end{array}$ & $\begin{array}{l}207 \\
193 \\
193 \\
193 \\
200\end{array}$ & $\begin{array}{l}39 \\
44 \\
47 \\
47 \\
44\end{array}$ & $\begin{array}{l}18 \\
16 \\
16 \\
14 \\
12\end{array}$ & $\begin{array}{l}16 \\
16 \\
20 \\
22 \\
22\end{array}$ & $\begin{array}{l}20 \\
20 \\
20 \\
20 \\
20\end{array}$ & $\begin{array}{l}16 \\
16 \\
16 \\
16 \\
16\end{array}$ \\
\hline $\begin{array}{l}26 \\
27 \\
28 \\
29 \\
30 \\
31 \\
\end{array}$ & $\begin{array}{l}41 \\
44 \\
47 \\
47 \\
50 \\
50\end{array}$ & $\begin{array}{l}74 \\
74 \\
74 \\
74 \\
74\end{array}$ & $\begin{array}{r}82 \\
82 \\
* 82 \\
82 \\
82 \\
82 \\
\end{array}$ & $\begin{array}{l}82 \\
82 \\
82 \\
74 \\
70 \\
74\end{array}$ & $\begin{array}{l}82 \\
82 \\
82 \\
-\end{array}$ & $\begin{array}{r}134 \\
150 \\
119 \\
106 \\
106 \\
* 119 \\
\end{array}$ & $\begin{array}{l}165 \\
165 \\
242 \\
172 \\
179\end{array}$ & $\begin{array}{l}44 \\
47 \\
41 \\
39 \\
47 \\
54 \\
\end{array}$ & $\begin{array}{l}14 \\
14 \\
16 \\
12 \\
12\end{array}$ & $\begin{array}{l}22 \\
22 \\
22 \\
22 \\
24 \\
24\end{array}$ & $\begin{array}{l}20 \\
20 \\
20 \\
18 \\
\frac{18}{18} \\
18\end{array}$ & $\begin{array}{l}16 \\
16 \\
16 \\
16 \\
16\end{array}$ \\
\hline $\begin{array}{l}\text { Total } \\
\text { Mean } \\
\text { Ac-ft }\end{array}$ & $\begin{array}{r}1,135 \\
36,6 \\
2,250 \\
\end{array}$ & $\begin{array}{r}2,032 \\
67.7 \\
4,030 \\
\end{array}$ & $\begin{array}{r}2,470 \\
79.7 \\
4,900\end{array}$ & $\begin{array}{r}2,658 \\
85.7 \\
5,270 \\
\end{array}$ & $\begin{array}{r}2,080 \\
74.3 \\
4,130 \\
\end{array}$ & $\begin{array}{r}3,026 \\
97.6 \\
6,000 \\
\end{array}$ & $\begin{array}{r}5,653 \\
188 \\
11,200\end{array}$ & $\begin{array}{r}3,331 \\
107 \\
6,610\end{array}$ & $\begin{array}{r}723 \\
24.1 \\
1,430 \\
\end{array}$ & $\begin{array}{r}553 \\
17.8 \\
1,100 \\
\end{array}$ & $\begin{array}{r}766 \\
24.7 \\
1,520 \\
\end{array}$ & $\begin{array}{r}514 \\
17.1 \\
1,020 \\
\end{array}$ \\
\hline
\end{tabular}

Calendar year 1949: Max Min Mean Ac-ft

Water year 1949-50: Max 242 Min 12 Mean 68.3 Ac-ft 49,460

* Discharse measurement made on this day.

Note.--No gage-heicht record Feb. 5-22; discharge estimated. 
HELMAND RIVER BASIN

Ghazni hiver near thazni, Afghanistan

ischarge in cubic feet per second, water year October 1950 to September 1951

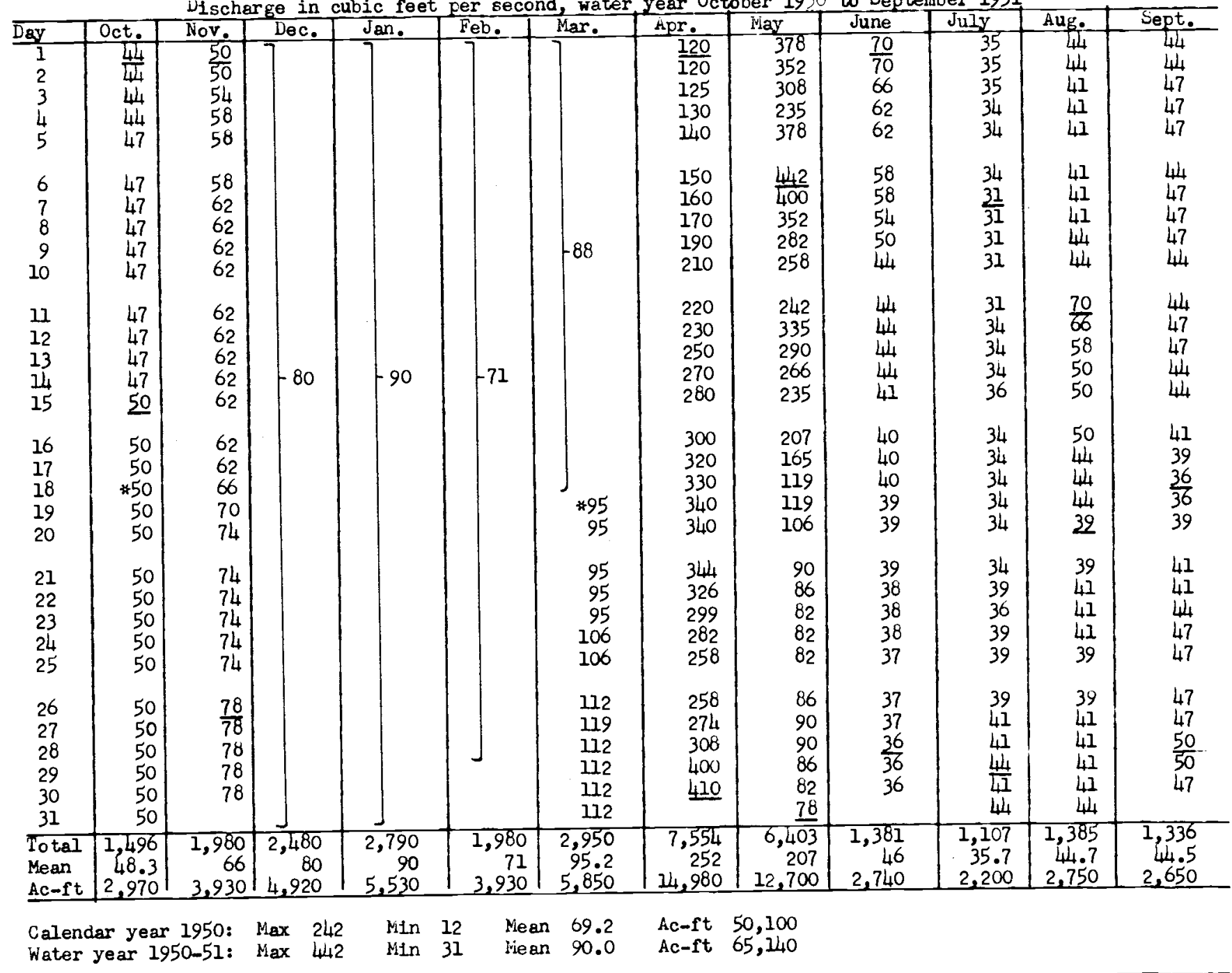

* Vischarge measurement made on this day.

Note.--No gage-helght record Dec. 1 to Mar. 18, April 1-20, June 16 to July 4; discharge estimated. 
HEIMAND RIVER BASIN

Ghazni River near Ghazni, Afghanistan

Discharge, in cubic feet per second, water year October 1951 to September 1952

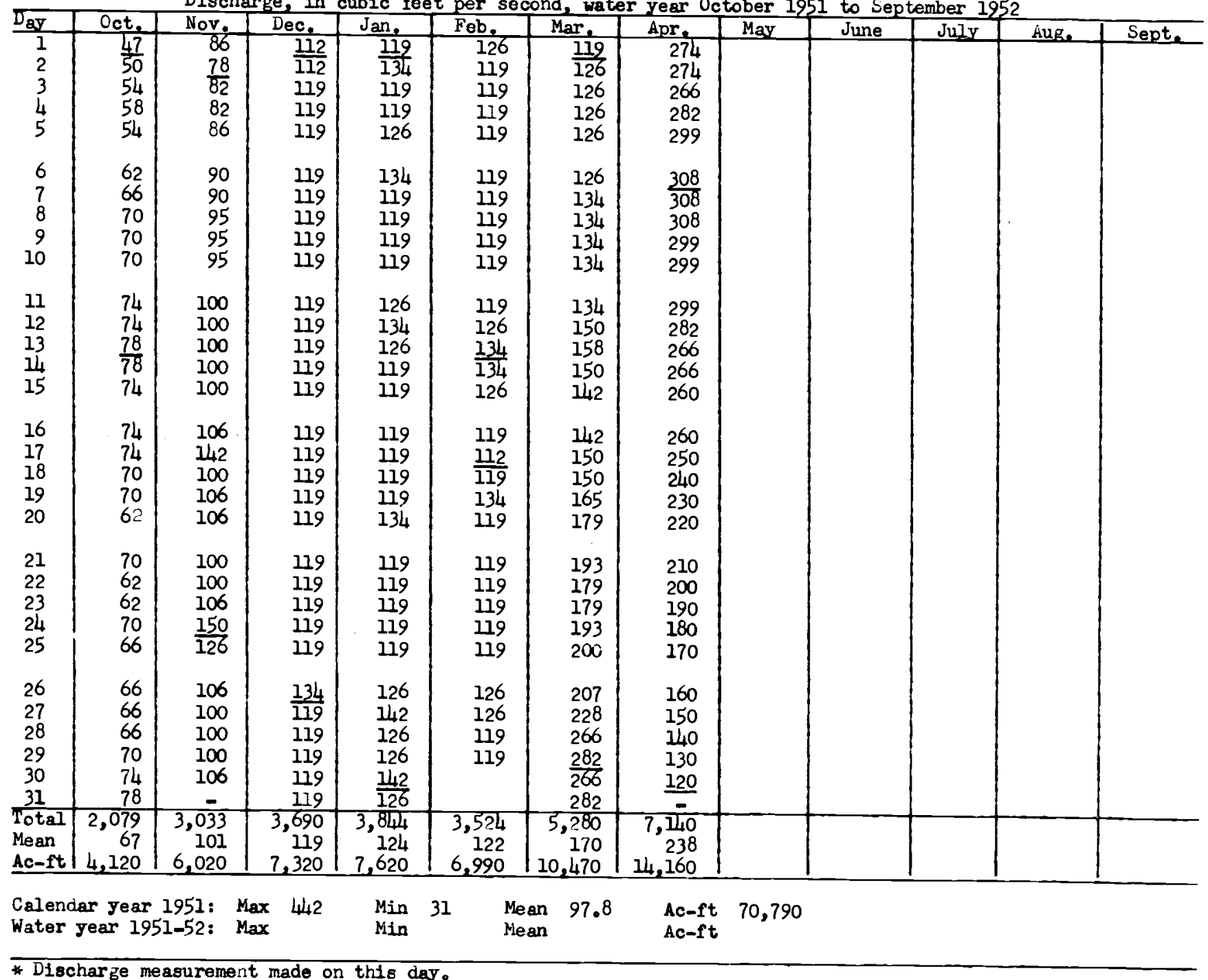

Note.--No gage-heights Apr. 15-30; discharge estimated. 
HEIMAND RIVER BASTN

Paltu River near Gardez, Afghanistan

Location.--Lat $33^{\circ} 08^{\prime N}$, long $69^{\circ} 05^{\prime} \mathrm{E}$, on right bank of Paltu River, one kilometer downstream from the Paltu dan-site, bout 19 kilometers east of Serafsar, 51 kilometers south of Gerdez, and 72 kilometors southesest of Ghasni.

Drelinge erea.-130 oq in, approximately

Eocords arailable.-May 1949 to March 1952.

Gage.-.Water-stage recorder. Altitude, of gage, about 2,100 neters.

Frtremes.-1949-50: Kaxima discharge during year, $540 \mathrm{cfs}$ Apr. 3 (gage-height 2.19 meters); minimum, 1 cfs Sept. 13-30. 1950-51: Maxjmum discharge during jear, 780 cfs Aug. 11 (gage-height, 2.24 meter8) minime, 2 cfs 0ct. 1-18.

Remarks.--Records are fair except those for periods of no gage-height record, which are poor. Becords collected by Morrison-Knudsen Afghanistan, Inc. 
HEIMAND RIVER BASIN

Paltu River near Gardez, Afghanistan

Discharge, in cubic feet per second, woter year October 1948 to September 1949

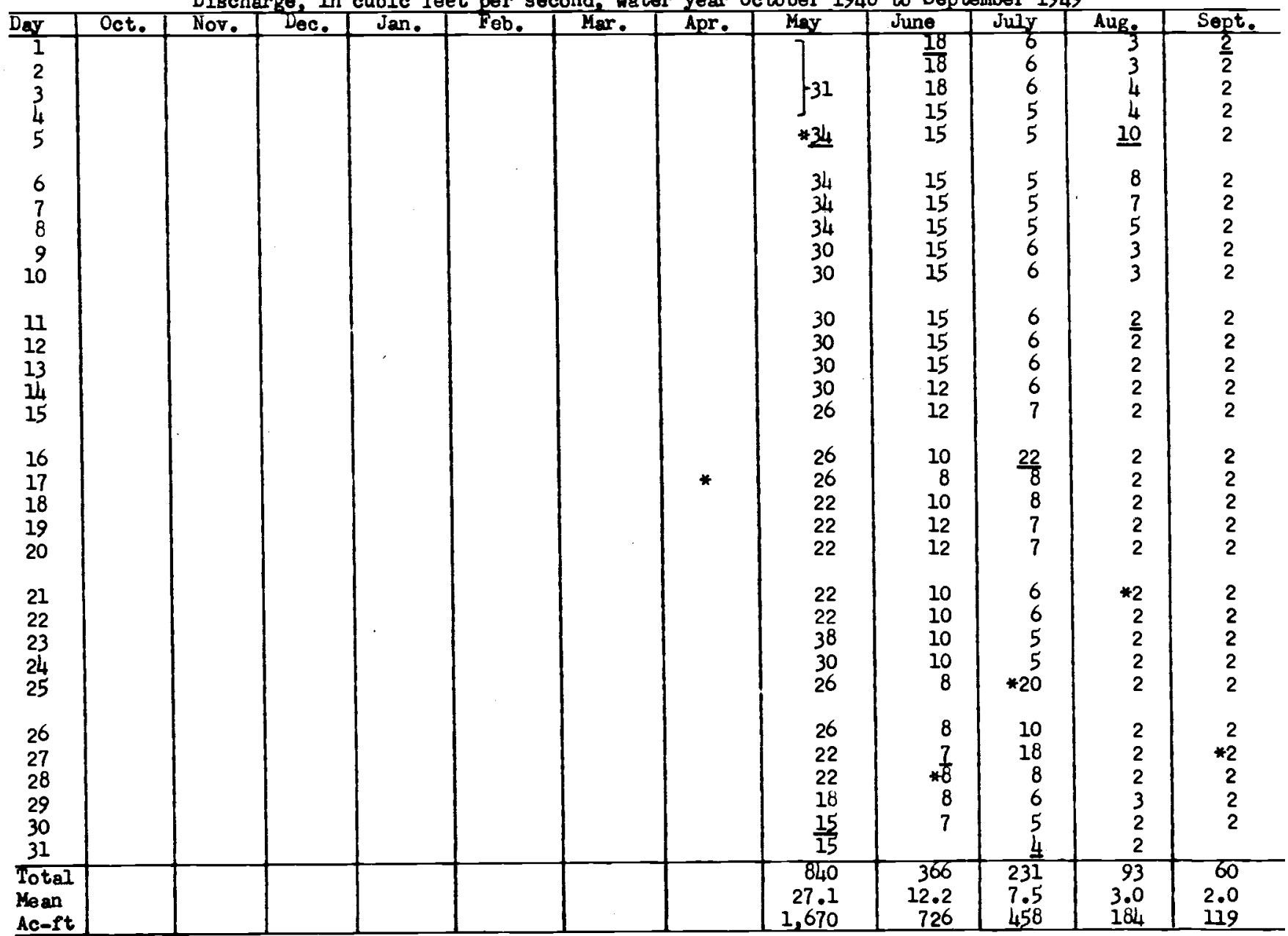

* Discharge measurement made on this day.

Note.--No gage-helght record May 1-4; discharge estimated. 
HELMAND RIVER BASIN

Paltu River near liardez, Afghanistan

Discharge, in cubic feet per second, vater year October 1949 to September 1950

\begin{tabular}{|c|c|c|c|c|c|c|c|c|c|c|c|c|}
\hline Day & Oct. & Hov. & Dec. & Jan. & Feb. & Mar. & Apr. & $\mathrm{Mgy}$ & Jun $\theta$ & July & Aug. & Sept. \\
\hline $\begin{array}{l}1 . \\
2 \\
3 \\
4 \\
5\end{array}$ & $\begin{array}{l}\frac{2}{2} \\
2 \\
2 \\
2\end{array}$ & $\begin{array}{l}\frac{3}{3} \\
3 \\
3 \\
3\end{array}$ & $\begin{array}{l}\frac{3}{3} \\
3 \\
3 \\
3\end{array}$ & $\begin{array}{l}\frac{2}{2} \\
2 \\
2 \\
2\end{array}$ & $-\underline{2}$ & 26 & $\begin{array}{l}245 \\
292 \\
344 \\
374 \\
344\end{array}$ & $\begin{array}{l}176 \\
176 \\
176 \\
176 \\
199\end{array}$ & $\begin{array}{l}\frac{43}{39} \\
39 \\
35 \\
32\end{array}$ & $\begin{array}{l}9 \\
9 \\
9 \\
9 \\
9\end{array}$ & $\begin{array}{r}15 \\
13 \\
17 \\
6 \\
5\end{array}$ & $\begin{array}{l}\frac{3}{3} \\
2 \\
2 \\
2\end{array}$ \\
\hline $\begin{array}{r}6 \\
7 \\
8 \\
9 \\
10\end{array}$ & $\begin{array}{l}2 \\
2 \\
2 \\
2 \\
2\end{array}$ & $\begin{array}{l}4 \\
4 \\
3 \\
3 \\
4\end{array}$ & $\begin{array}{l}3 \\
4 \\
4 \\
4 \\
4\end{array}$ & $\begin{array}{l}2 \\
2 \\
2 \\
2 \\
2\end{array}$ & & -31 & $\begin{array}{l}* 344 \\
344 \\
318 \\
292 \\
268\end{array}$ & $\begin{array}{l}199 \\
176 \\
153 \\
268 \\
222\end{array}$ & $\begin{array}{l}32 \\
29 \\
26 \\
26 \\
23\end{array}$ & $\begin{array}{r}9 \\
7 \\
7 \\
7 \\
* 7\end{array}$ & $\begin{array}{l}5 \\
6 \\
6 \\
5 \\
5\end{array}$ & $\begin{array}{l}2 \\
2 \\
2 \\
2 \\
2\end{array}$ \\
\hline $\begin{array}{l}11 \\
12 \\
13 \\
14 \\
15\end{array}$ & $\begin{array}{r}2 \\
2 \\
* 2 \\
2 \\
2\end{array}$ & $\begin{array}{l}4 \\
4 \\
3 \\
3 \\
4\end{array}$ & $\begin{array}{l}4 \\
4 \\
4 \\
3 \\
3\end{array}$ & $\begin{array}{l}2 \\
2 \\
2 \\
2 \\
3\end{array}$ & -4 & $\begin{array}{l}J \\
* 34 \\
42 \\
42 \\
42\end{array}$ & $\begin{array}{r}268 \\
* 268 \\
245 \\
222 \\
245\end{array}$ & $\begin{array}{r}* 175 \\
175 \\
152 \\
152 \\
132\end{array}$ & $\begin{array}{r}23 \\
* 20 \\
20 \\
13 \\
13\end{array}$ & $\begin{array}{r}11 \\
5 \\
15 \\
9 \\
9\end{array}$ & $\begin{array}{l}4 \\
4 \\
3 \\
3 \\
4\end{array}$ & $\begin{array}{l}2 \\
2 \\
1 \\
\frac{1}{1} \\
1\end{array}$ \\
\hline $\begin{array}{l}16 \\
17 \\
18 \\
19 \\
20\end{array}$ & $\begin{array}{l}\frac{3}{3} \\
3 \\
3 \\
3\end{array}$ & $\begin{array}{r}4 \\
3 \\
* 3 \\
3 \\
3\end{array}$ & $\begin{array}{l}4 \\
4 \\
3 \\
3 \\
3\end{array}$ & $\begin{array}{l}3 \\
2 \\
3 \\
3 \\
2\end{array}$ & & $\begin{array}{l}42 \\
42 \\
42 \\
46 \\
51\end{array}$ & $\begin{array}{l}222 \\
199 \\
199 \\
199 \\
199\end{array}$ & $\begin{array}{r}115 \\
115 \\
101 \\
90 \\
90\end{array}$ & $\begin{array}{l}13 \\
13 \\
13 \\
11 \\
11\end{array}$ & $\begin{array}{r}13 \\
7 \\
5 \\
4 \\
4\end{array}$ & $\begin{array}{r}3 \\
* 3 \\
\frac{2}{2} \\
2\end{array}$ & $\begin{array}{r}1 \\
1 \\
* 1 \\
1 \\
1\end{array}$ \\
\hline $\begin{array}{l}21 \\
22 \\
23 \\
24 \\
25\end{array}$ & $\begin{array}{l}3 \\
3 \\
3 \\
3 \\
3\end{array}$ & $\begin{array}{l}3 \\
3 \\
3 \\
3 \\
4\end{array}$ & $\begin{array}{l}3 \\
3 \\
3 \\
3 \\
3\end{array}$ & $\begin{array}{l}2 \\
2 \\
2 \\
3 \\
3\end{array}$ & -8 & $\begin{array}{l}56 \\
51 \\
51 \\
51 \\
46\end{array}$ & $\begin{array}{l}176 \\
176 \\
153 \\
131 \\
\frac{136}{176}\end{array}$ & $\begin{array}{l}80 \\
72 \\
72 \\
66 \\
66\end{array}$ & $\begin{array}{r}11 \\
11 \\
11 \\
11 \\
9\end{array}$ & $\begin{array}{l}4 \\
4 \\
4 \\
4 \\
3\end{array}$ & $\begin{array}{l}2 \\
2 \\
2 \\
2 \\
2\end{array}$ & $\begin{array}{l}1 \\
1 \\
1 \\
1 \\
1\end{array}$ \\
\hline $\begin{array}{l}26 \\
27 \\
28 \\
29 \\
30 \\
31 \\
\end{array}$ & $\begin{array}{l}3 \\
3 \\
3 \\
3 \\
3 \\
3\end{array}$ & $\begin{array}{l}\frac{5}{4} \\
4 \\
4 \\
3\end{array}$ & $\begin{array}{r}4 \\
* 3 \\
3 \\
3 \\
3 \\
3 \\
\end{array}$ & $\begin{array}{l}3 \\
3 \\
3 \\
3 \\
2 \\
2 \\
\end{array}$ & - & $\begin{array}{r}46 \\
* 46 \\
51 \\
51 \\
51 \\
61 \\
\end{array}$ & $\begin{array}{l}176 \\
222 \\
199 \\
222 \\
199\end{array}$ & $\begin{array}{l}61 \\
56 \\
51 \\
51 \\
47 \\
47\end{array}$ & $\begin{array}{l}9 \\
9 \\
7 \\
7 \\
7\end{array}$ & $\begin{array}{l}3 \\
3 \\
3 \\
4 \\
4 \\
5\end{array}$ & $\begin{array}{r}2 \\
2 \\
2 \\
2 \\
5 \\
4 \\
\end{array}$ & $\begin{array}{l}1 \\
1 \\
1 \\
1 \\
1\end{array}$ \\
\hline $\begin{array}{l}\text { Total } \\
\text { Mean } \\
\text { Ac-ft }\end{array}$ & $\begin{array}{r}78 \\
2.5 \\
155\end{array}$ & $\begin{array}{l}103 \\
3.4 \\
204\end{array}$ & $\begin{array}{l}103 \\
3.4 \\
204\end{array}$ & $\begin{array}{r}72 \\
2.3 \\
143 \\
\end{array}$ & $\begin{array}{r}124 \\
4.43 \\
246\end{array}$ & $\begin{array}{r}1,260 \\
40.6 \\
2,500\end{array}$ & $\begin{array}{r}7,236 \\
241 \\
14,350 \\
\end{array}$ & $\begin{array}{r}3,887 \\
125 \\
7,710 \\
\end{array}$ & $\begin{array}{r}566 \\
19 \\
1,120 \\
\end{array}$ & $\begin{array}{l}205 \\
6.6 \\
407\end{array}$ & $\begin{array}{l}140 \\
4.5 \\
278\end{array}$ & $\begin{array}{r}44 \\
1.5 \\
87 \\
\end{array}$ \\
\hline
\end{tabular}

$\begin{array}{lllll}\text { Calendar year 1949: Max } & \text { Min } & \text { Mean } & \text { Ac-ft } \\ \text { Water year 1949-50: } & \text { Miax } 374 & \text { Min } 1 & \text { Mean 37.9 Ac-ft 27,410 }\end{array}$

* Discharge measurement made on this day.

Note.-No gage-height record Feb. 1 to Mar. 1l; discharte estimated. 
HEIMAND RIVER BASIN

Paltu River near Gardez, Afghanistan

Discharge, in cubic feet per second, water year October 1950 to September 1951

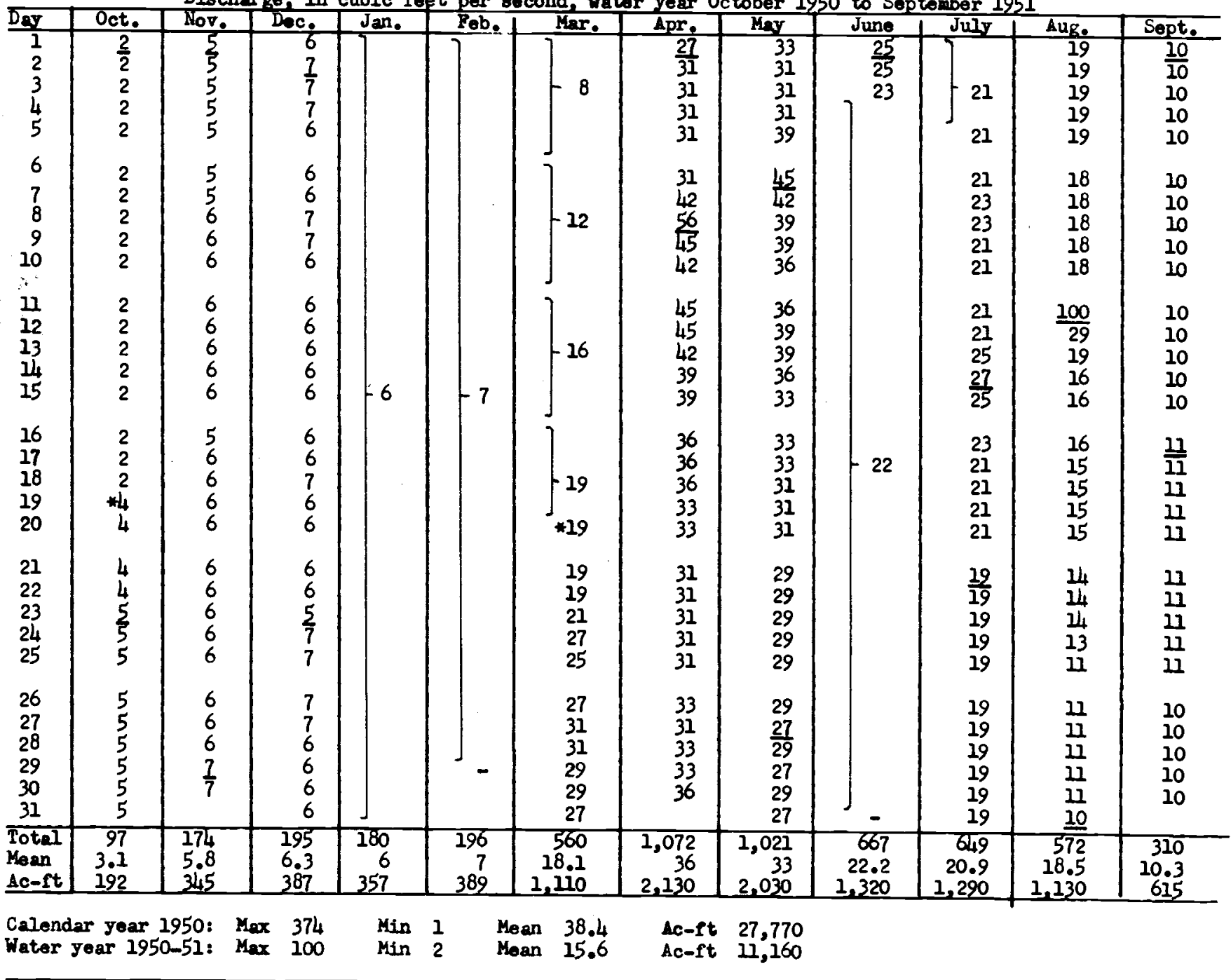

* Discharge meacurement made on this day.

Note.-No gage-height record Jan. 1 to Mar. 19, June 4 to July 4 ; discharge estimated on basis of records for Seraj River near Ghazni. 
HEIMAND RIVER BASIN

Paltu River near Gardez, Afghanistan

Discharge, in cubic feet per second, water year October 1951 to September 1952

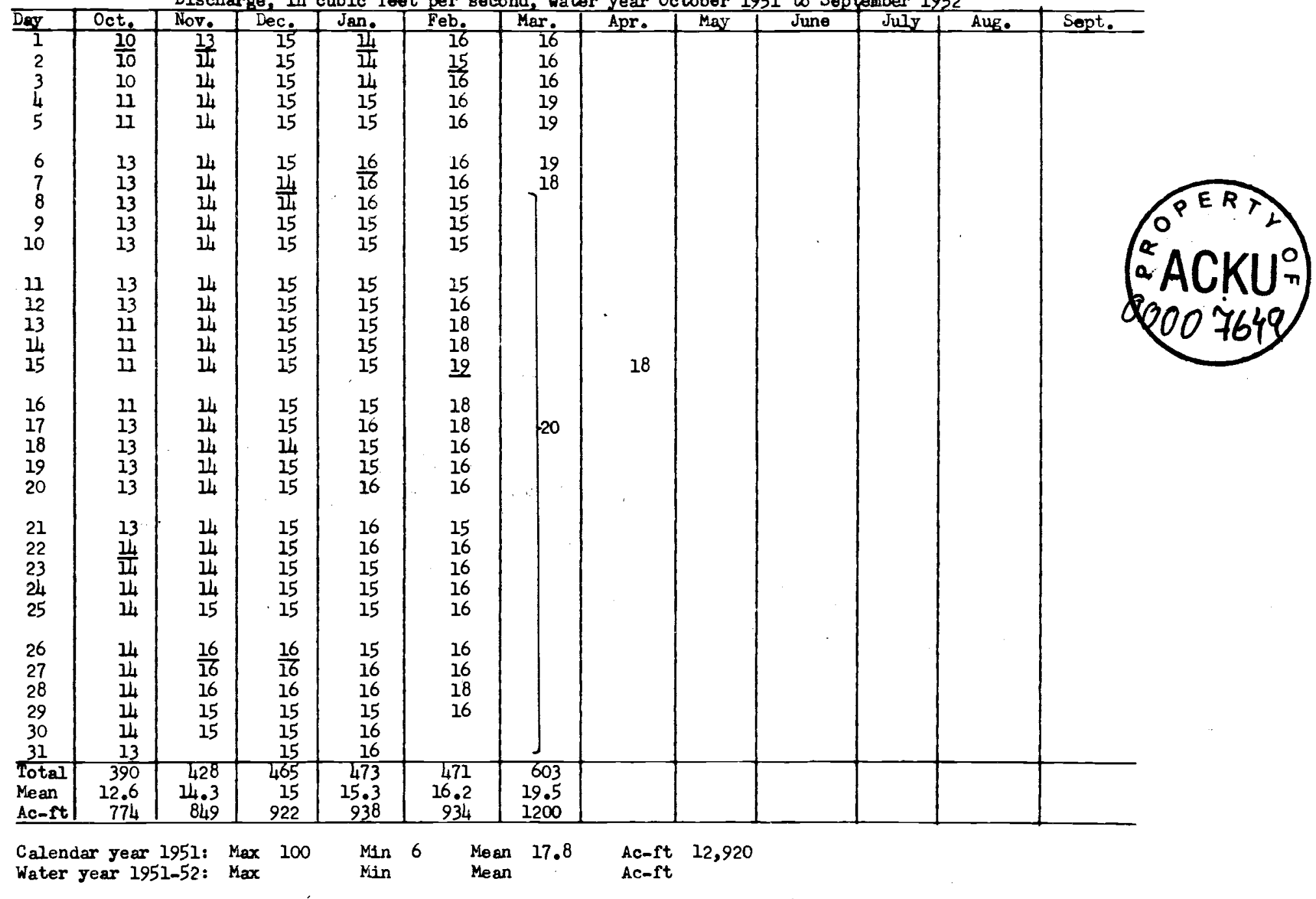

Note.-No gage-helght racord Mar. 8 to Apr. 14; discharge for Mar. 8-31 estimated. 


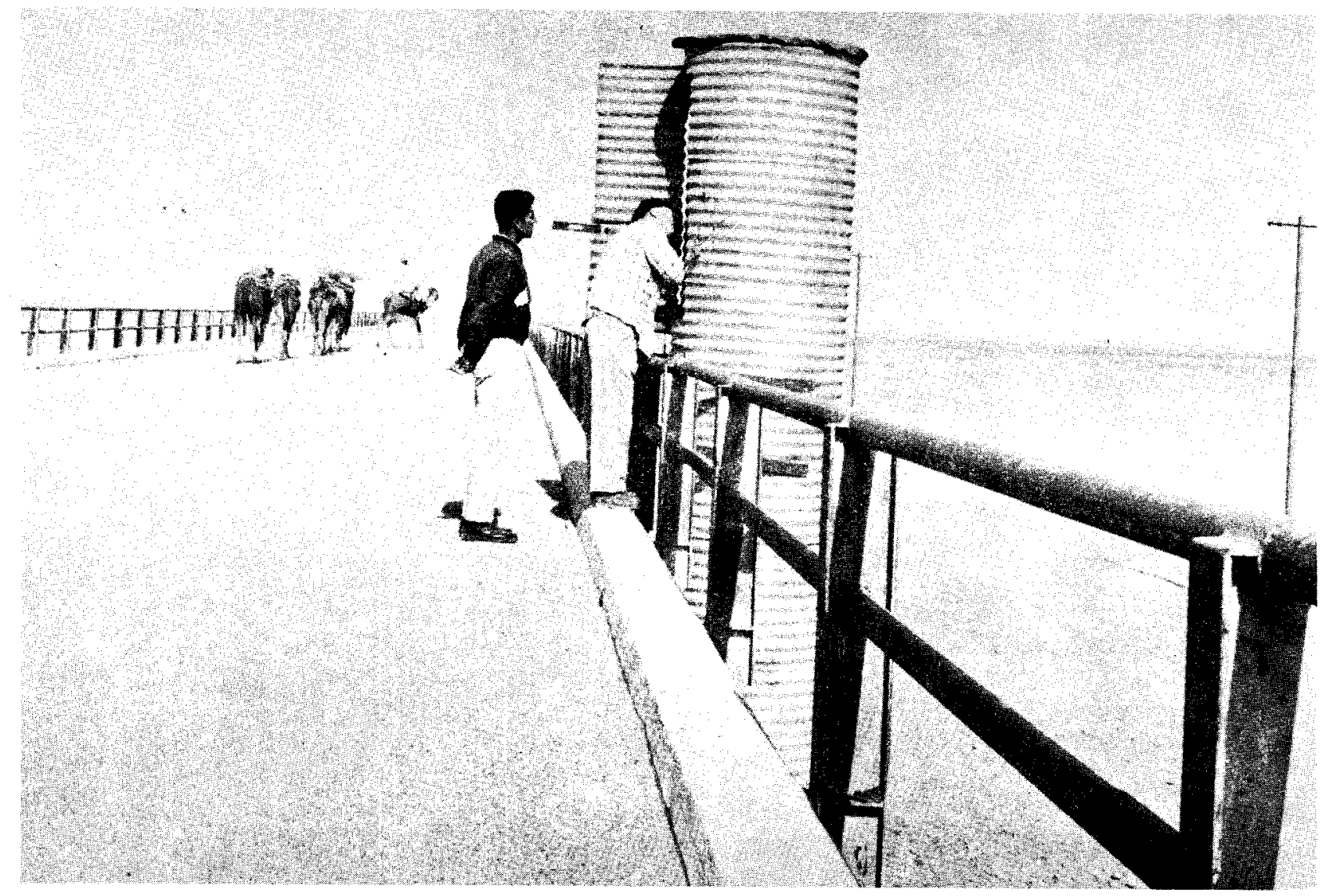

Figure 9.--Arghastan River near Kandahar showing water-stage recorder well and shelter. 
HEIMAND RIVER BASIN

Arghastan River near Kandahar, Afghanistan

Location.-Lat $31^{\circ} 261 \mathrm{~N}$. , long $65^{\circ} 54^{\prime}$ E., on upstream side of bridge on Kandahar to Chaman (Pakistan) road, about 22 kilometers upstream from Dori River, and 28 kilometers southeast of Kandahar.

Drainage area. $-12,820 \mathrm{sq} \mathrm{mi}$, approximately, of which about 6,200 sq mi (above outlet of Lake Ab-i-Istada)

is probably noncontributing.

Records available.-October 1952 to September 1959; extremes only for water jear 1954 and 1959.

Gage.-Water-stage recorder. Datum of gage is at mean sea level (în MKA surveys based on Survey of India datum).

Average discharge.-5 years (1952-53, 1954-58) 250 cfs (181,000 acre-ft per year).

Extremes.- Maximum discharge for the water years 1953-59 are given in the following table:

\begin{tabular}{c|c|c|c}
\hline Water Year & Date & $\begin{array}{c}\text { Gage Height } \\
\text { (meters) }\end{array}$ & $\begin{array}{c}\text { Discharge } \\
\text { (cfs) }\end{array}$ \\
\hline 1953 & Feb. 14, 1953 & 1020.0 & 11,100 \\
1954 & Feb.13,1954 & 1021.09 & 100 \\
1955 & Mar. 16, 1955 & 1019.98 & 2,870 \\
1956 & July 20,1956 & 1020.80 & 21,900 \\
1957 & Apr. 8, 1957 & 1020.01 & 5,180 \\
1958 & Dec. 14, 1957 & 1020.40 & 5,980 \\
1959 & About Mar. 2, 1959 & 1019.98 & 4,320 \\
\hline
\end{tabular}

Remarks.-Records are fair. Extensive diversions for irrigation above the station. Discharge measurements listed below were made in wąter year 1959.

\begin{tabular}{c|c|c}
\hline Date & $\begin{array}{c}\text { Gage Height } \\
\text { (meters) }\end{array}$ & $\begin{array}{c}\text { Discharge } \\
\text { (cfs) }\end{array}$ \\
\hline Dec. 25, 1958 & 1019.00 & 78.2 \\
Jan. 27, 1959 & 1019.155 & 192 \\
Feb. 19, 1959 & 1019.60 & 879 \\
Mar. 25, 1959 & 1018.79 & 175 \\
Apr. 4, 1959 & 1019.74 & 2,230 \\
\hline
\end{tabular}


HFTMAND RIVER BASIN

Arghastan River near Kandahar, Afghanistan

Discharge, in cubic feet per second, water year October 1952 to September 1953

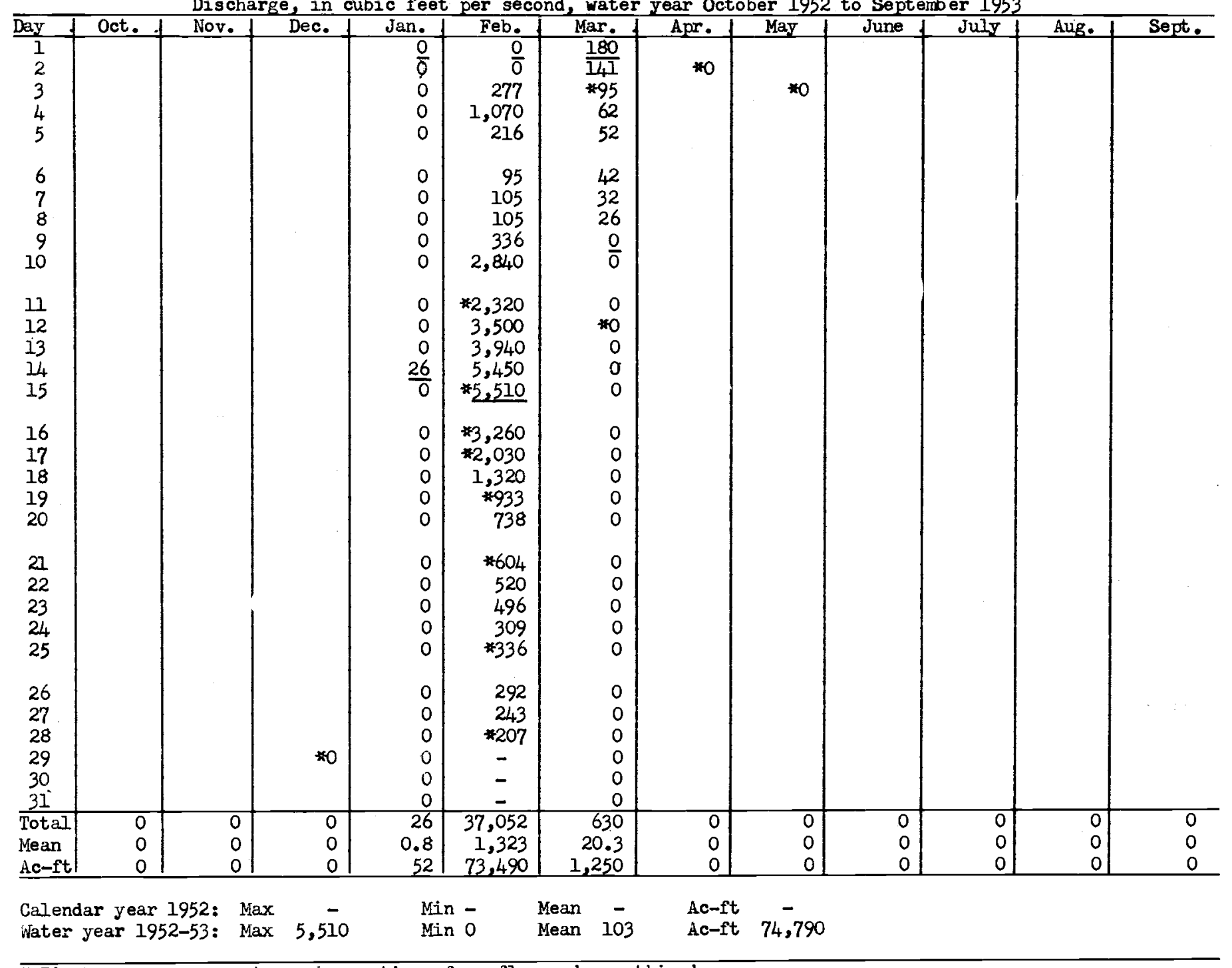

* Discharge measurement or observation of no flow made on this day. 
HGTMAND RIVER BASIN

Argnastan River near Kandahar, Afghanistan

Discharge, in cubic feet per second, water year October 1954 to September 1955

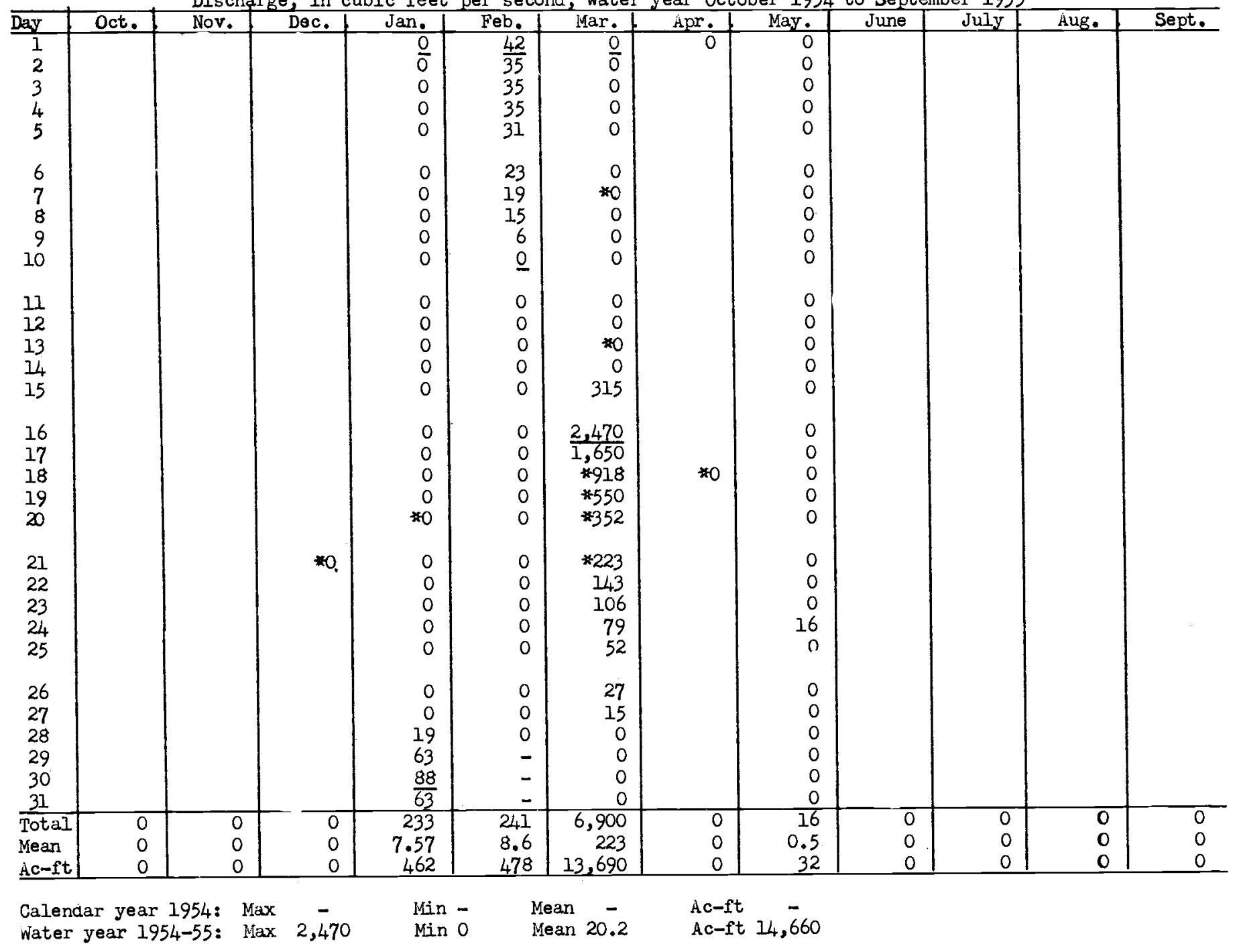

* Discharge measurement or observation of no flow made on this day. 
HELWIAND RIVER BASIN

Arghastan kiver near Kandahar, af ghanistan

Discharge, in cuoic feet per second, water year October 1955 to September 1956

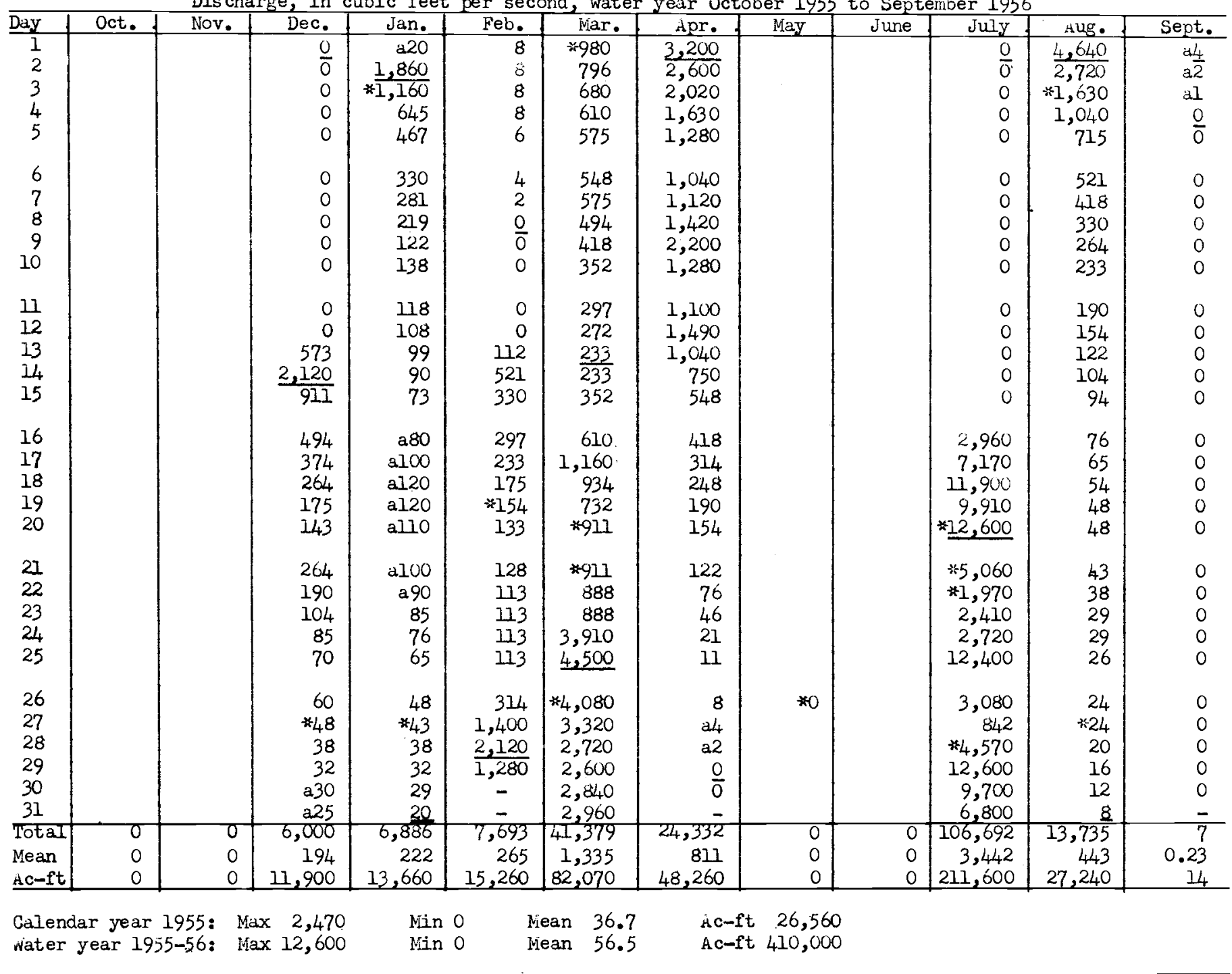

* Discharge messurenent or observation of no flow made on this day.

a No gage-height record; discharge estinated. 
HETWAND RTVER BASIN

Arghastan Kiver near Kandahar, Afghanistan

Discharge, in cubic feet er second, water year October 1956 to September 1957

\begin{tabular}{|c|c|c|c|c|c|c|c|c|c|c|c|c|}
\hline Ddy & Oct. & Nov. & Dec. & tan. & Feb. & Mar. & Apr. & May. & June & July & Aug. & Sept. \\
\hline 1 & & & & 0 & 1,000 & 1,020 & 1,500 & 400 & 110 & & & \\
\hline 2 & & & & $\overline{0}$ & $\overline{1,500}$ & $\overline{1,240}$ & 1,000 & 370 & $\overline{105}$ & & & \\
\hline 3 & & & & 0 & 2,000 & 1,370 & 900 & 1,290 & 100 & & & \\
\hline 4 & & & & 0 & 2,030 & 1,370 & 800 & 3,480 & 100 & & & \\
\hline 5 & & & & 0 & 2,000 & 1,520 & 700 & $\overline{1,930}$ & 90 & & & \\
\hline 6 & & & & 0 & 2,160 & 1,370 & 600 & 767 & 80 & & & \\
\hline 7 & & & & 0 & 2,920 & $* 1,370$ & 2,000 & 500 & 70 & & & \\
\hline 8 & & & & 315 & 2,440 & 1,320 & 4,000 & 400 & 60 & & & \\
\hline 9 & & & & 325 & 2,360 & 1,300 & $* 3,480$ & 370 & 50 & & & \\
\hline 10 & & & & $* 287$ & 2,060 & 2,030 & 2,560 & 350 & 30 & & & \\
\hline 11 & & & & 260 & 2,240 & 2,160 & 2,680 & 636 & 10 & & & \\
\hline 12 & & & $* 0$ & 278 & 2,100 & 1,860 & 1,320 & 500 & 10 & & & \\
\hline 13 & & & & $* 1,560$ & 2,000 & 1,470 & 942 & 400 & 10 & & & \\
\hline 14 & & & & $* 598$ & 1,900 & 1,340 & 1,170 & 350 & $\underline{0}$ & & & \\
\hline 15 & & & & 415 & 1,820 & 1,170 & 2,130 & 330 & 0 & & & \\
\hline 16 & & & & 367 & 1,790 & 1,020 & 1,470 & 300 & 0 & & & \\
\hline 17 & & & & 335 & 1,720 & 1,140 & 796 & 280 & 0 & & & \\
\hline 18 & & & & 325 & 1,790 & 2,160 & 669 & 260 & 0 & & & \\
\hline 19 & & & & 335 & 1,930 & $2,4,40$ & 553 & $* 203$ & 0 & & & \\
\hline 20 & $* 0$ & & & 345 & 1,860 & 1,930 & 500 & $* 200$ & 0 & & & \\
\hline 21 & & & & 361 & 1,690 & 1,860 & 480 & 180 & 0 & & & \\
\hline 22 & & & & 367 & 1,440 & 1,790 & 470 & 170 & 0 & & & \\
\hline 23 & & & & 403 & 1,400 & 1,790 & 460 & 160 & 0 & & & \\
\hline 24 & & & & 464 & 1,220 & 1,860 & 450 & 150 & 0 & & & \\
\hline 25 & & & & 680 & 1,100 & 1,520 & 450 & 140 & 0 & & & \\
\hline 26 & & & & 1,470 & 1,020 & 1,370 & 450 & 135 & 0 & & & \\
\hline 27 & & & & 2,000 & 1,020 & 1,300 & 553 & 130 & 0 & & & \\
\hline 28 & & & & 1,720 & 1,000 & 2,200 & 500 & 125 & 0 & & & \\
\hline 29 & & & & $* 1,520$ & - & $* 2,680$ & 450 & 120 & 0 & & & \\
\hline 30 & & & & 1,320 & - & 3,000 & 430 & 115 & 0 & & & \\
\hline 31 & & & & 1,140 & - & 2,000 & - & 110 & - & & & \\
\hline Total & 0 & 0 & 0 & 17,190 & 49,570 & $51, \overline{970}$ & 34,463 & 14,851 & 825 & 0 & 0 & 0 \\
\hline Mean & 0 & 0 & 0 & 555 & 1,770 & 1,676 & 7,149 & 479 & 27.5 & 0 & 0 & 0 \\
\hline$A c-f t$ & 0 & 0 & 0 & 34,100 & 98,320 & 103,100 & 68,360 & 29,460 & 1,636 & 0 & 0 & 0 \\
\hline
\end{tabular}

$\begin{array}{lllll}\text { Calendar year 1956: } & \text { Max 12,600 } & \text { Min 0 } & \text { Mean 548 } & \text { Ac-ft 398,100 } \\ \text { Water year 1956-57: } & \text { Max 4,000 } & \text { Min 0 } & \text { Mean 463 } & \text { Ac-ft 335,000 }\end{array}$

* Discharge measurement or observation of no flow made on this day.

Note.-No gage-height record Mar. 30 to Apr. 8; Apr. 20-26; Apr. 28 to May 2; May 7-10, 12-18, May 21 to June 13; discharge estinated on basis of discharge measurements and records for Arghandab River above Arghandab Reservoir. 
HETMAND RIVER BASIN

Arghastan River near Kandahar, Afghanistan

Discharge, in cubic feet per second, water year October 1957 to September 1958

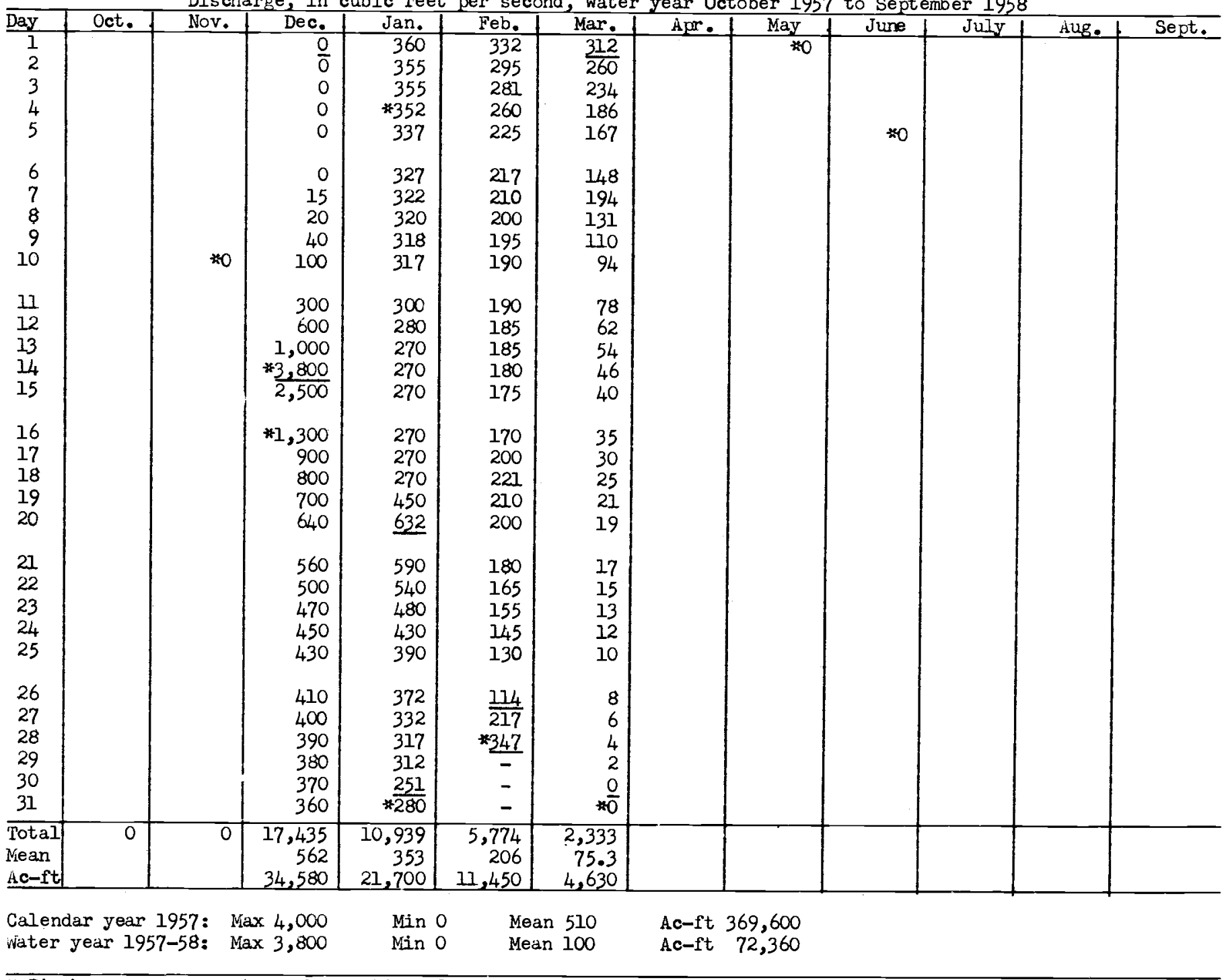

* Discharge measurement or observation of no flow made on this day.

Note.-No gage-height record Dec. 7 to Jan. 3; Jan. 8, 9, 11-25; Feb. 8-25; Mar. 8-29; discharge estimated

by available record, rainfall and records for station Arghandab River above Arghandab Reservoir. 


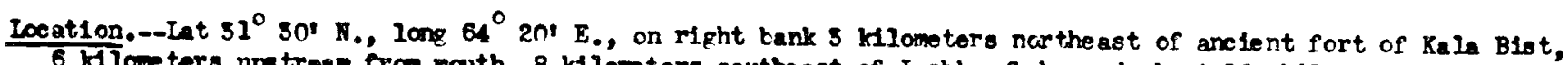
6 kliometers ups troam from mouth, 8 kilometers southeast of Lashkar $G$ ah, and about 185 kllome ter a downs tream Trom Arghandab Dam.

Draina area. $-31,600 \mathrm{sq} \mathrm{m}$, approximetely, freludes that of $4 b-1$-Iatads (about 6,800 sq mi is probably nonocards available.--October 1947 to Septenter 1960.

Gage.- blater stige recardor. Altitade of gage is 720 me tars from survey of India map.

Iverage discharpo.--13 pors, 1069 cfs (774,570 acre-pt per year).

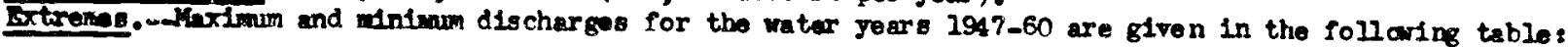

\begin{tabular}{|c|c|c|c|c|c|c|}
\hline Wher jeax & Dato & $\begin{array}{l}\text { Gexilu } \\
\text { Gage Hejght } \\
\text { (Haters) }\end{array}$ & $\begin{array}{c}\text { Dischargo } \\
\text { (cre) }\end{array}$ & Date & $\begin{array}{c}\text { Cugning Helght } \\
\text { (Meters) }\end{array}$ & $\begin{array}{c}\text { Discharge } \\
\text { (cre) }\end{array}$ \\
\hline $\begin{array}{l}1948 \\
1949 \\
1950 \\
1951 \\
1952 \\
1953 \\
1954 \\
1955 \\
1956 \\
1957 \\
1958 \\
1959 \\
1960\end{array}$ & $\begin{array}{l}\text { (a) } \\
\text { (a) } \\
\text { Jan. } 50,1950 \\
\text { Mar. 28, 1951 } \\
\text { Fob. 16, 1952 } \\
\text { Fob. 16, 1953 } \\
\text { Fob. 14, 1954 } \\
\text { Jan. 31, } 1955 \\
\text { July 27, } 1956 \\
\text { Apr. 6, 1957 } \\
\text { Dec. 12, } 1957 \\
\text { Yar.2; Ipr.5, } 1959 \\
\text { Apr. 18, } 1960\end{array}$ & $\begin{array}{c}= \\
b 3.80 \\
1.54 \\
.97 \\
2.11 \\
3.30 \\
.63 \\
3.15 \\
2.90 \\
3.015 \\
1.64 \\
1.85\end{array}$ & $\begin{array}{r}- \\
- \\
60,000 \\
9,660 \\
2,010 \\
18,700 \\
44,400 \\
991 \\
55,000 \\
32,700 \\
34,900 \\
9,040 \\
12,600\end{array}$ & $\begin{array}{l}\text { Sept. 8-19, } 1957 \\
\text { oct. 1, 1957 } \\
\text { Aug. 17, } 1959 \\
\text { Mar.10_12; Aug.10_29, } \\
1960\end{array}$ & \begin{tabular}{|l} 
many daye \\
do \\
do \\
do \\
do \\
do \\
do \\
do \\
do \\
0.18 \\
.34 \\
.37 \\
-
\end{tabular} & $\begin{array}{r}18 \\
41 \\
2 \\
3\end{array}$ \\
\hline
\end{tabular}

- Not determined.

From ploodmark.

Remark--Records good except those for 1947-48, 1949-51 and those for per1ods of no gage-heights which are fair. Flow is regulated by Irghandab Roservoir. Extensive divarsions for irrifation upotrean. 
HELMIDD RIVER BASIN

Arghandab River neer Kala Bist, Arghanistan

Discharge, in cubic feet per secud, water year October 1947 to Senterber 1948

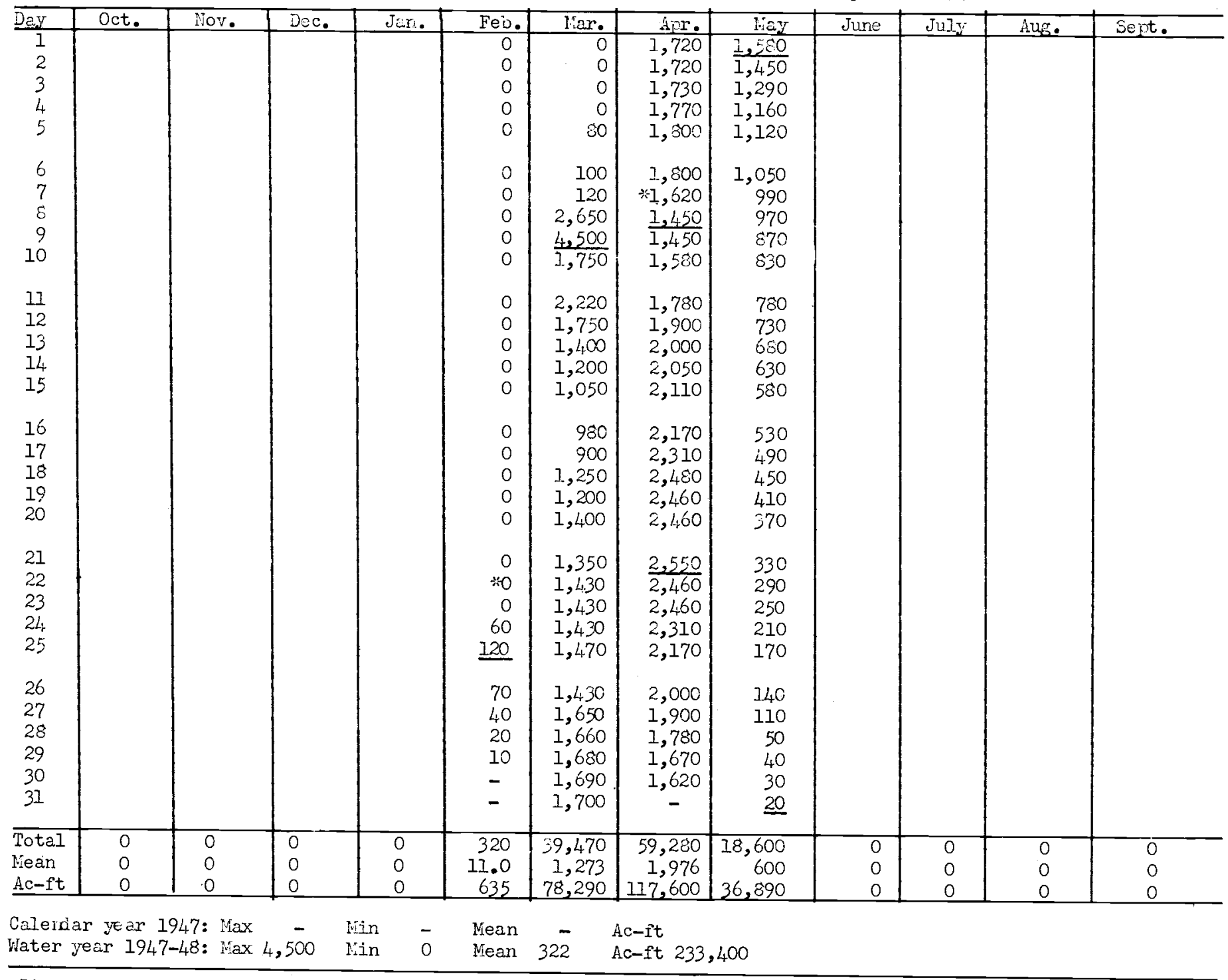

*Discharge measurement or observation of no flow made on this day.

Note.-No gage-height record liar. 5-17, Nar. 19 to Apr. 6, May 5-31; discharge estinated on basis of records for Arghandao River at darisite. 
HETRATD RIVLR BASTN

Arshandai River nesr Kala Bist, Arghanistan

Discharge, in cubic feet par second, water ycar october 1948 to septeiber 1949

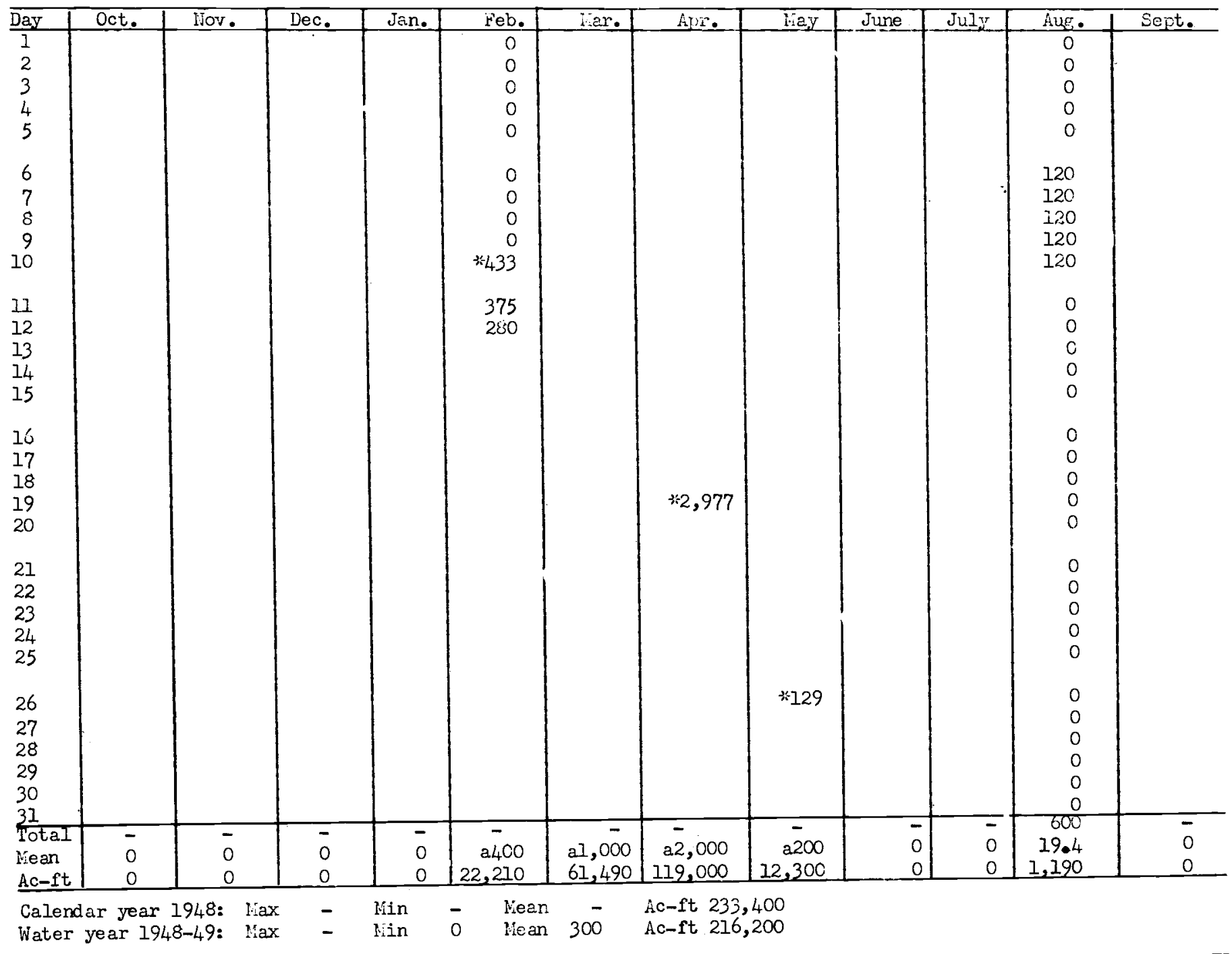

Fischarge measurement made on this day.

a Estimated.

Note.-No gage-height recond Feb. 13 to Apr. 18, Apr. 20 to May 25, Way 27-30; discharge e stimated. 
HETMAND RIVAR BASIN

Arghandab River near Kala Bist, Afghanistan

Discharge, in cubic feet per second, water year October 1949 to September 1950

\begin{tabular}{|c|c|c|c|c|c|c|c|c|c|c|c|c|}
\hline Dar & Oct. & Nov, & Dec. & Jan. & Feb? & Mare $_{2}$ & Apr. & May & June & Julr & Aug. & Sept. \\
\hline $\begin{array}{l}1 \\
2 \\
3 \\
4 \\
5\end{array}$ & & & & $\begin{array}{l}0 \\
0 \\
0 \\
0 \\
0\end{array}$ & $\begin{array}{r}* 3,300 \\
3,200 \\
3,100 \\
3,100 \\
3,100\end{array}$ & $\begin{array}{r}* 3,800 \\
3,800 \\
4,000 \\
4,000 \\
4,100\end{array}$ & $\begin{array}{r}7,390 \\
11,200 \\
18,700 \\
15,100 \\
12,400\end{array}$ & $\begin{array}{l}3,770 \\
3,860 \\
3,860 \\
3,860 \\
3,860\end{array}$ & $\begin{array}{r}1,150 \\
1,020 \\
954 \\
920 \\
859\end{array}$ & $\begin{array}{l}100 \\
90 \\
80 \\
60 \\
50\end{array}$ & & \\
\hline $\begin{array}{r}6 \\
7 \\
8 \\
9 \\
10\end{array}$ & & & & $\begin{array}{l}0 \\
0 \\
0 \\
0 \\
0\end{array}$ & $\begin{array}{l}3,100 \\
3,100 \\
3,000 \\
3,000 \\
2,600 \\
\end{array}$ & $\begin{array}{l}4,200 \\
4,200 \\
4,400 \\
4,500 \\
4,600\end{array}$ & $\begin{array}{r}11,000 \\
9,240 \\
8,420 \\
7,630 \\
7,030\end{array}$ & $\begin{array}{l}3,860 \\
3,860 \\
4,140 \\
4,620 \\
4,930\end{array}$ & $\begin{array}{l}828 \\
768 \\
706 \\
676 \\
646\end{array}$ & $\begin{array}{r}30 \\
20 \\
10 \\
0 \\
0\end{array}$ & & \\
\hline $\begin{array}{l}12 \\
12 \\
13 \\
14 \\
15\end{array}$ & & & & $\begin{array}{l}0 \\
0 \\
0 \\
0 \\
0\end{array}$ & $\begin{array}{l}2,600 \\
2,600 \\
2,600 \\
2,600 \\
2,600\end{array}$ & $\begin{array}{l}4,900 \\
5,000 \\
5,500 \\
6,000 \\
9,200\end{array}$ & $\begin{array}{l}6,440 \\
6,110 \\
6,220 \\
5,670 \\
5,560\end{array}$ & $\begin{array}{l}5,030 \\
5,140 \\
5,140 \\
5,450 \\
5,780 \\
\end{array}$ & $\begin{array}{l}615 \\
587 \\
559 \\
530 \\
500\end{array}$ & $\begin{array}{l}0 \\
0 \\
0 \\
0 \\
0\end{array}$ & & \\
\hline $\begin{array}{l}16 \\
17 \\
18 \\
19 \\
20\end{array}$ & & & & $\begin{array}{l}0 \\
0 \\
0 \\
0 \\
0\end{array}$ & $\begin{array}{l}2,600 \\
2,600 \\
2,600 \\
2,600 \\
2,600\end{array}$ & $\begin{array}{l}6,600 \\
5,700 \\
5,100 \\
4,600 \\
4,500\end{array}$ & $\begin{array}{l}5,450 \\
5,450 \\
5,450 \\
5,350 \\
5,140\end{array}$ & $\begin{array}{l}5,560 \\
5,250 \\
4,510 \\
4,050 \\
3,770\end{array}$ & $\begin{array}{l}460 \\
430 \\
400 \\
380 \\
350\end{array}$ & $\begin{array}{l}0 \\
0 \\
0 \\
0 \\
0\end{array}$ & & \\
\hline $\begin{array}{l}21 \\
22 \\
23 \\
24 \\
25\end{array}$ & & & & $\begin{array}{l}0 \\
0 \\
0 \\
0 \\
0\end{array}$ & $\begin{array}{l}2,600 \\
2,600 \\
2,600 \\
2,900 \\
3,000\end{array}$ & $\begin{array}{l}4,600 \\
4,800 \\
5,600 \\
6,000 \\
2,700 \\
\end{array}$ & $\begin{array}{l}5,030 \\
4,510 \\
4,320 \\
4,140 \\
4,050\end{array}$ & $\begin{array}{l}3,500 \\
2,940 \\
2,640 \\
2,280 \\
1,960\end{array}$ & $\begin{array}{l}330 \\
300 \\
280 \\
260 \\
2140\end{array}$ & $\begin{array}{l}0 \\
0 \\
0 \\
0 \\
0\end{array}$ & & \\
\hline $\begin{array}{l}26 \\
27 \\
28 \\
29 \\
30 \\
31\end{array}$ & & & & $\begin{array}{r}0 \\
0 \\
0 \\
300 \\
* 19,000 \\
21,000 \\
\end{array}$ & $\begin{array}{c}3,300 \\
3,500 \\
3,800 \\
- \\
- \\
-\end{array}$ & $\begin{array}{l}9,100 \\
6,800 \\
4,720 \\
5,030 \\
5,890 \\
7,150\end{array}$ & $\begin{array}{c}4,050 \\
3,860 \\
3,860 \\
3,770 \\
3,770 \\
=\end{array}$ & $\begin{array}{r}1,820 \\
1,590 \\
1,480 \\
1,370 \\
* 1,260 \\
1,220 \\
\end{array}$ & $\begin{array}{l}220 \\
200 \\
180 \\
160 \\
130 \\
-\end{array}$ & $\begin{array}{l}0 \\
0 \\
0 \\
0 \\
0 \\
0\end{array}$ & & \\
\hline $\begin{array}{l}\text { Total } \\
\text { Moan } \\
\text { Ac-ft }\end{array}$ & $\begin{array}{l}0 \\
0 \\
0\end{array}$ & $\begin{array}{l}0 \\
0 \\
0\end{array}$ & $\begin{array}{l}0 \\
0 \\
0\end{array}$ & $\begin{array}{r}40,300 \\
1,300 \\
79,930\end{array}$ & $\begin{array}{r}77,800 \\
2,779 \\
154,300\end{array}$ & $\begin{array}{r}168,290 \\
5,429 \\
333,800\end{array}$ & $\begin{array}{r}206,310 \\
6,877 \\
409,200\end{array}$ & $\begin{array}{r}112,350 \\
3,624 \\
222,800\end{array}$ & $\begin{array}{r}15,638 \\
521 \\
31,020\end{array}$ & $\begin{array}{r}450 \\
14.5 \\
893\end{array}$ & $\begin{array}{l}0 \\
0 \\
0\end{array}$ & $\begin{array}{l}0 \\
0 \\
0\end{array}$ \\
\hline
\end{tabular}

Calendar year 1849: Max - Min 0 Mean - Ac-ft 216,200

Water year 1949-50: Max 21,000 Min 0 Mean 1,702 Ac-rt 1,232,000

* Discharge measurament made on this day.

Note. - Doubtful or no gage-height record Jan. 29 to Mar. 27; Jurie $u_{4}$ to July 8; discharge estimated on basis of records for station below Arghandab damsite. 
HMTYAND RIVER BASIN

Arghandab River near Kala Blst, Lfghaniotan

Discharge, in cuble feet per second, water year October 1950 to September 1951

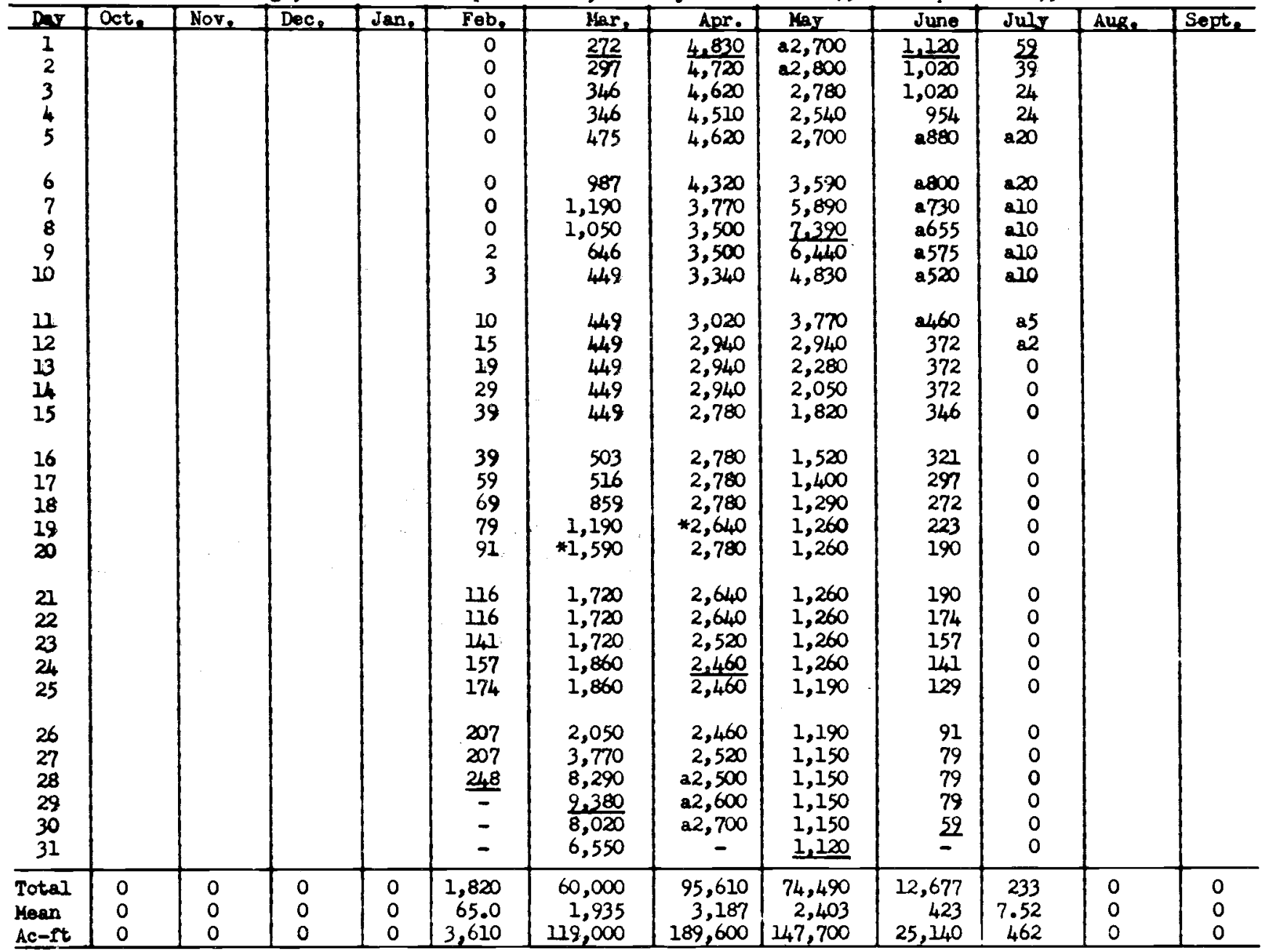

Calendar year 1950: Max 21,000 Min 0 Mean 1,702 Ac-ft 1,232,000

Water year 1950-51: Max 9,380 Min 0 Mean 671 Ac-ft 485,500

* Discharge measurement made on this day.

No gage-height record; discharge interpolated.

Note. - Discharge for period Feb. 8 to Mar. 19 computed from graph based on information fumished by watchman. 
HELMAND RIVER BASIN

Arghandab River near Kala Bist, Afghanistan

Discharge, in cubic feet per second, water year October 1951 to September 1952

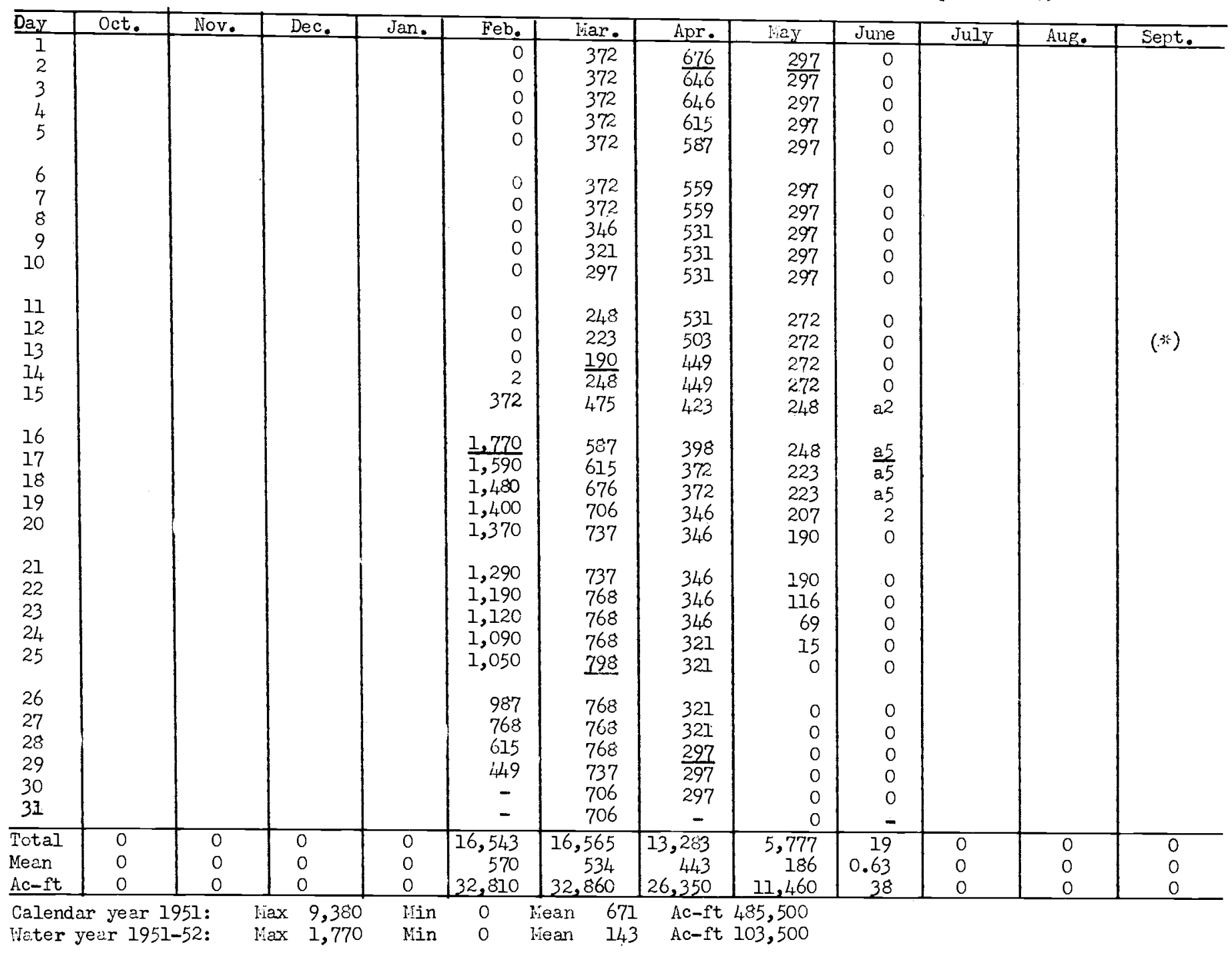

* Observation of no flow made on this day.

a No gage-height record; discharge estimated on basis of recorded range in stage. 
HIIIMAND RIVER BASIN

Arghandab River near Kala Bist, Afghanistan

Discharge, in cubic feet per second, water year October 1952 to September 1953

\begin{tabular}{|c|c|c|c|c|c|c|c|c|c|c|c|c|}
\hline Day & Oct. & Nov. & Dec. & Jan. & Feb. & liar. & Apr. & May & June & Julv & Aug. & Sept. \\
\hline 1 & & & & 0 & 503 & 200 & 100 & 69 & 0 & & & \\
\hline 2 & & & & 0 & 503 & $\overline{170}$ & 100 & 69 & 2 & & & \\
\hline 3 & & & & 0 & 503 & 130 & 100 & 64 & 0 & & & \\
\hline 4 & & & & 0 & 503 & 100 & 110 & 59 & 0 & & & \\
\hline 5 & & & & 0 & 503 & 80 & 130 & 54 & 0 & & & \\
\hline 6 & & & & 0 & 859 & 60 & 140 & 59 & 0 & & & \\
\hline 7 & & & & 0 & 646 & 50 & $U_{1} O$ & 54 & 197 & & & \\
\hline 8 & & & & 0 & $\because 559$ & 40 & 140 & 54 & 141 & & & \\
\hline 9 & & & & 0 & 630 & 30 & 140 & 49 & 98 & & & \\
\hline 10 & & & & 0 & 1,050 & 20 & 140 & 44 & 69 & & & \\
\hline 11 & & & & 0 & 8,370 & 10 & 0 & 39 & 49 & & & \\
\hline 12 & & & & 0 & 8,200 & 5 & *14 & 39 & 34 & & & \\
\hline 13 & & & & 0 & 6,010 & 0 & 157 & 34 & 24 & & & \\
\hline 14 & & & & 0 & 12,400 & 0 & 182 & 39 & 19 & & & \\
\hline 15 & & & & 70 & 13,000 & 0 & 174 & 29 & 15 & & & \\
\hline 16 & & & & 372 & 12,600 & 0 & 190 & $* 29$ & $* 12$ & & & \\
\hline 17 & & & & 423 & 8,000 & 0 & $\frac{12}{174}$ & 26 & 10 & & & \\
\hline 18 & & & & 423 & 5,000 & 0 & 166 & 24 & 8 & & & \\
\hline 19 & & & & $=475$ & 3,000 & 0 & 157 & 22 & 3 & & & \\
\hline 20 & & & & 475 & $* 1,890$ & 0 & 141 & 17 & 24 & & & \\
\hline 21 & & & & 475 & 1,000 & 0 & 129 & 10 & 39 & & & \\
\hline 22 & & & & 503 & 700 & 0 & $\begin{array}{l}129 \\
135\end{array}$ & 10 & 34 & & & \\
\hline 23 & & & & 503 & 550 & 0 & 129 & 10 & 24 & & & \\
\hline 24 & & & & 503 & 450 & 0 & 110 & 8 & 19 & & & \\
\hline 25 & & & & 503 & 350 & 0 & 170 & 8 & 8 & & & \\
\hline 26 & & & & 531 & 300 & 30 & 116 & 5 & 5 & & & \\
\hline 27 & & & & 531 & 250 & 60 & $1 \alpha_{4}$ & 4 & 2 & & & $(*)$ \\
\hline 28 & & & & 531 & 230 & 100 & 98 & 3 & 1 & & & \\
\hline 29 & & & & 503 & - & 100 & 91 & 2 & 0 & & & \\
\hline 30 & & & & 503 & - & 100 & 79 & $\underline{0}$ & 0 & & & \\
\hline 31 & & & & 503 & $\therefore-$ & 100 & & $\frac{\pi}{1}$ & - & & & \\
\hline TotaI & 0 & 0 & 0 & 7,827 & $\overline{88,559}$ & 1,385 & 3,963 & 924 & 835 & 0 & 0 & 0 \\
\hline Miean & 0 & 0 & 0 & 252 & 3,163 & 44.7 & 132 & 29.8 & 27.8 & 0 & 0 & 0 \\
\hline$A c-f t$ & 0 & 0 & 0 & 15,520 & 175,700 & 2,740 & 7,860 & 1,830 & 1,660 & 0 & 0 & 0 \\
\hline Calend & $r$ year & 52: If & $I, 770$ & Wijn & Riean & 143 & -ft 10 & & & & & \\
\hline Water & rear 1 & 53: hia & 13,000 & Min & Hean & 284 & ft 205 & & & & & \\
\hline
\end{tabular}

xischarge neasurement or observation of no flow made on this day.

Hote. -ivo gage-height record Feb. 17-19, Feb. 21 to Apr. 1l; discharge estimated on basis of records for station below Arghandab Dam and weather records. 
HETMAND RIVER BASIN

Arghandab River noar Kala Bist, Afghanistan

Discharge, in cubic feet per second, water year October 1953 to September 1954

\begin{tabular}{|c|c|c|c|c|c|c|c|c|c|c|c|c|}
\hline Dry & oet. & $\mathrm{Nov}_{r}$ & Dec. & $\operatorname{Jan}$ & Febof & kar. & $\Delta p r$, & $\mathrm{Mg}$ & June & JuLY & Aug. & Sept. \\
\hline $\begin{array}{l}1 \\
2 \\
3 \\
4 \\
5\end{array}$ & & $\begin{array}{l}0 \\
0 \\
0 \\
5 \\
5\end{array}$ & $\begin{array}{l}49 \\
54 \\
49 \\
79 \\
49\end{array}$ & $\begin{array}{l}260 \\
215 \\
198 \\
215 \\
813\end{array}$ & $\begin{array}{l}859 \\
920 \\
768 \\
691 \\
615\end{array}$ & $\begin{array}{r}3,000 \\
3,500 \\
6,000 \\
10,000 \\
8,000\end{array}$ & $\begin{array}{r}19,000 \\
13,000 \\
* 10,500 \\
8,830 \\
7,750\end{array}$ & $\begin{array}{r}5,240 \\
5,080 \\
* 5,030 \\
5,030 \\
4,930\end{array}$ & $\begin{array}{l}1,350 \\
1,300 \\
1,300 \\
1,300 \\
1,200\end{array}$ & $\begin{array}{r}* 248 \\
207 \\
207 \\
207 \\
190\end{array}$ & $\begin{array}{r}116 \\
* 92 \\
98 \\
98 \\
98\end{array}$ & $\begin{array}{l}* 98 \\
104 \\
104 \\
104 \\
110\end{array}$ \\
\hline $\begin{array}{r}6 \\
7 \\
8 \\
9 \\
10\end{array}$ & & $\begin{array}{r}8 \\
10 \\
+10 \\
12 \\
15\end{array}$ & $\begin{array}{l}49 \\
49 \\
49 \\
54 \\
69\end{array}$ & $\begin{array}{l}4,830 \\
2,030 \\
4,620 \\
3,860 \\
2,640\end{array}$ & $\begin{array}{l}615 \\
503 \\
462 \\
449 \\
475\end{array}$ & $\begin{array}{l}5,000 \\
4,000 \\
3,300 \\
2,900 \\
2,600\end{array}$ & $\begin{array}{r}* 7,450 \\
6,910 \\
6,220 \\
5,890 \\
5,670\end{array}$ & $\begin{array}{l}4,930 \\
4,830 \\
4,830 \\
4,800 \\
4,800\end{array}$ & $\begin{array}{l}1,200 \\
1,200 \\
1,150 \\
1,150 \\
1,150\end{array}$ & $\begin{array}{l}190 \\
190 \\
190 \\
190 \\
190\end{array}$ & $\begin{array}{l}98 \\
91 \\
85 \\
\frac{74}{91}\end{array}$ & $\begin{array}{r}98 \\
104 \\
98 \\
122 \\
129\end{array}$ \\
\hline $\begin{array}{l}11 \\
12 \\
13 \\
14 \\
15\end{array}$ & & $\begin{array}{l}17 \\
19 \\
19 \\
22 \\
22\end{array}$ & $\begin{array}{l}69 \\
64 \\
74 \\
74 \\
69\end{array}$ & $\begin{array}{r}1,630 \\
1,250 \\
954 \\
828 \\
737\end{array}$ & $\begin{array}{r}1,920 \\
12,100 \\
28,600 \\
* 42,100 \\
30,000\end{array}$ & $\begin{array}{l}2,400 \\
2,200 \\
2,100 \\
2,000 \\
1,900\end{array}$ & $\begin{array}{l}5,450 \\
5,350 \\
5,240 \\
5,240 \\
5,190\end{array}$ & $\begin{array}{l}4,700 \\
4,600 \\
4,400 \\
4,000 \\
3,600\end{array}$ & $\begin{array}{r}1,100 \\
1,100 \\
1,100 \\
* 1,040 \\
1,000\end{array}$ & $\begin{array}{l}190 \\
190 \\
190 \\
190 \\
190\end{array}$ & $\begin{array}{l}\frac{182}{182} \\
157 \\
149 \\
147\end{array}$ & $\begin{array}{l}129 \\
116 \\
129 \\
135 \\
* 157\end{array}$ \\
\hline $\begin{array}{l}16 \\
17 \\
18 \\
19 \\
20\end{array}$ & & $\begin{array}{l}22 \\
24 \\
24 \\
24 \\
24\end{array}$ & $\begin{array}{l}69 \\
74 \\
85 \\
85 \\
79\end{array}$ & $\begin{array}{l}676 \\
676 \\
706 \\
706 \\
676\end{array}$ & $\begin{array}{r}20,000 \\
12,000 \\
8,000 \\
6,000 \\
5,000\end{array}$ & $\begin{array}{r}1,800 \\
* 1,800 \\
1,800 \\
1,700 \\
1,700\end{array}$ & $\begin{array}{l}5,140 \\
5,140 \\
5,140 \\
5,190 \\
5,240\end{array}$ & $\begin{array}{l}3,400 \\
3,200 \\
3,000 \\
2,800 \\
2,600\end{array}$ & $\begin{array}{r}980 \\
+640 \\
610 \\
600 \\
600\end{array}$ & $\begin{array}{l}248 \\
297 \\
272 \\
248 \\
207\end{array}$ & $\begin{array}{l}129 \\
129 \\
135 \\
129 \\
129\end{array}$ & $\begin{array}{l}141 \\
135 \\
141 \\
157 \\
157\end{array}$ \\
\hline $\begin{array}{l}21 \\
22 \\
23 \\
24 \\
25\end{array}$ & & $\begin{array}{l}24 \\
24 \\
29 \\
26 \\
24\end{array}$ & $\begin{array}{r}79 \\
85 \\
104 \\
122 \\
149\end{array}$ & $\begin{array}{r}661 \\
587 \\
531 \\
475 \\
* 436\end{array}$ & $\begin{array}{l}4,500 \\
4,000 \\
3,600 \\
3,400 \\
3,200\end{array}$ & $\begin{array}{l}1,700 \\
1,700 \\
1,700 \\
1,700 \\
1,800\end{array}$ & $\begin{array}{l}5,240 \\
5,240 \\
5,450 \\
5,670 \\
6,380\end{array}$ & $\begin{array}{r}2,400 \\
2,200 \\
* 2,110 \\
2,000 \\
1,900\end{array}$ & $\begin{array}{l}640 \\
600 \\
500 \\
500 \\
500\end{array}$ & $\begin{array}{l}174 \\
157 \\
141 \\
157 \\
157\end{array}$ & $\begin{array}{r}122 \\
116 \\
110 \\
104 \\
98\end{array}$ & $\begin{array}{l}\frac{174}{174} \\
157 \\
166 \\
157\end{array}$ \\
\hline $\begin{array}{l}26 \\
27 \\
28 \\
29 \\
30 \\
31\end{array}$ & & $\begin{array}{l}26 \\
24 \\
26 \\
44 \\
44 \\
-\end{array}$ & $\begin{array}{r}* 340 \\
531 \\
545 \\
462 \\
* 346 \\
309 \\
\end{array}$ & $\begin{array}{r}423 \\
398 \\
372 \\
449 \\
960 \\
* 963\end{array}$ & $\begin{array}{c}3,000 \\
3,000 \\
3,000 \\
= \\
=\end{array}$ & $\begin{array}{r}2,000 \\
2,400 \\
2,900 \\
4,180 \\
7,300 \\
17,600\end{array}$ & $\begin{array}{c}6,850 \\
6,550 \\
6,110 \\
5,560 \\
5,240 \\
-\end{array}$ & $\begin{array}{l}1,850 \\
1,800 \\
1,700 \\
1,600 \\
1,500 \\
1,400\end{array}$ & $\begin{array}{c}500 \\
300 \\
250 \\
250 \\
250 \\
- \\
\end{array}$ & $\begin{array}{l}111 \\
129 \\
129 \\
116 \\
116 \\
116\end{array}$ & $\begin{array}{l}98 \\
98 \\
91 \\
91 \\
91 \\
91\end{array}$ & $\begin{array}{c}* 174 \\
150 \\
140 \\
140 \\
140 \\
-\end{array}$ \\
\hline $\begin{array}{l}\text { Total } \\
\text { Yean } \\
\text { Ae-rt }\end{array}$ & $\begin{array}{l}0 \\
0 \\
0\end{array}$ & $\begin{array}{r}573 \\
19.1 \\
1,140 \\
\end{array}$ & $\begin{array}{r}4,3644 \\
141 \\
8,660 \\
\end{array}$ & $\begin{array}{r}39,675 \\
1,280 \\
78,690\end{array}$ & $\begin{array}{r}199,777 \\
7,235 \\
396,300\end{array}$ & $\begin{array}{r}112,760 \\
3,636 \\
223,700\end{array}$ & $\begin{array}{r}201,830 \\
6,728 \\
400,300 \\
\end{array}$ & $\begin{array}{r}106,260 \\
3,428 \\
210,800\end{array}$ & $\begin{array}{r}25,360 \\
845 \\
50,300 \\
\end{array}$ & $\begin{array}{r}5,764 \\
186 \\
11,430 \\
\end{array}$ & $\begin{array}{r}3,521 \\
114 \\
6,990\end{array}$ & $\begin{array}{r}4,040 \\
135 \\
8,010 \\
\end{array}$ \\
\hline & & & $2 x$ & & $\begin{array}{ll}\text { Mat } & 0 \\
\text { Matr } & 0\end{array}$ & $\begin{array}{l}\text { Mean } \\
\text { Mean }\end{array}$ & $\begin{array}{l}97 \\
29\end{array}$ & $\begin{array}{l}-f t \\
-f t \quad 1,\end{array}$ & $\begin{array}{l}5,100 \\
5,000\end{array}$ & & & \\
\hline
\end{tabular}

* Dischargo reasurement made on this day.

Note - No gage-netght caccrt Ezb. 15 to Mar. 27, Kay 9 to June 30, Sept. 27-30; discharge estimated on basis of peco-ris Jr atation below Arghandab Dam and weather records. 
HELIRND RIVLET BASTN

Arghandab River near Kala Bist, Af shanistan

Discharge, in cubic feet per second, water vear October 1954 to Septenber 1955

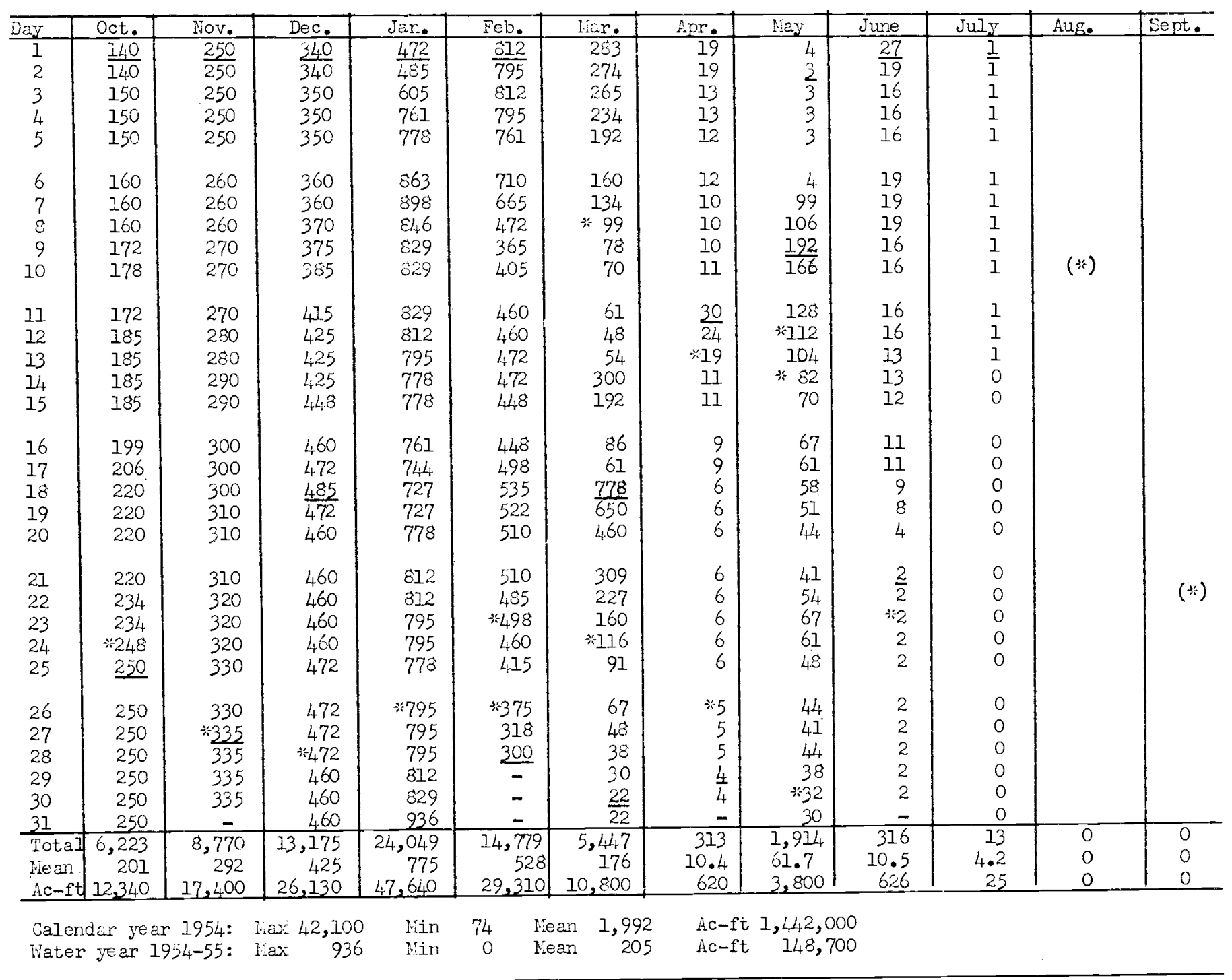

Fischarge neasurement or observation of no flow nade on this day.

Note.-No gase-heicht record oct. 1-8, Oct. 25 to Nov. 26, Nov. 28 to Dec. 8, June 24 to July 5 ; discharge

estiriated on basis of records for station below Arghandab Dan or interpolated. 
HETHADD RIVIR BASIN

Arghandab River near Kala Bist, Afghanistan

Discharge, in cubic feet per second, water year Octoider 1955 to Septeniver 1956

\begin{tabular}{|c|c|c|c|c|c|c|c|c|c|c|c|c|}
\hline Day & oct. & Nov. & Doc. & Jan. & $\mathrm{Feb}$. & riar. & Apr. & Vay & June & July & AUE. & Sept. \\
\hline $1 \cdot$ & & & $\underline{0}$ & 405 & 740 & 1,910 & 10,800 & 5,530 & $=, 000$ & 51 & a?,000 & 506 \\
\hline 2 & & & $\overline{0}$ & $39 i_{i}$ & 125 & $1,64,0$ & 11,$4 ; 00$ & 5,760 & 9140 & 45 & 25,000 & $\longdiv { 1 9 5 }$ \\
\hline 3 & & & 0 & 568 & 107 & 1,420 & 11,100 & $i_{i}, 820$ & 887 & 41 & $a_{4}, 000$ & 484 \\
\hline 4 & & & 0 & 1,120 & 92 & 1,170 & $\because 17,700$ & 4,570 & $\mathrm{BO}_{2}$ & 36 & 23,500 & 473 \\
\hline 5 & & & 0 & $1,1,80$ & 80 & 1,000 & $\overline{11,700}$ & 4,250 & 730 & 30 & 3,000 & 450 \\
\hline 6 & & & 0 & $1,34,0$ & 57 & 930 & 10,700 & $\because 4,020$ & 673 & 23 & 22,500 & 439 \\
\hline 7 & & & 0 & 1,080 & 57 & 901 & 9,790 & 3,750 & 619 & 20 & 32,200 & 439 \\
\hline 8 & & & 0 & *e: 14 & 51 & 858 & $10,1,00$ & 3,520 & $59 \%$ & 17 & $32 ; 000$ & 428 \\
\hline 9 & & & 1 & 730 & 51 & 802 & 11,400 & 3,360 & 556 & 15 & a], 900 & $* \longdiv { 2 0 }$ \\
\hline 10 & & & 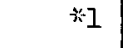 & 6itit & 54 & 730 & 11,500 & $\because 3,220$ & 530 & 14 & al,, 600 & 439 \\
\hline 17 & & & 3 & 594. & 40 & 673 & 10,800 & 3,180 & 506 & 10 & all, 500 & 439 \\
\hline 12 & & & 7 & 568 & 5 & 619 & 9,860 & 3,120 & 173 & $\delta$ & a.t,, 400 & 450 \\
\hline 13 & & & 84 & 515 & 51 & 531 & 9,790 & 3,020 & $: 50$ & 6 & 21,300 & 4.50 \\
\hline 14 & & & 2,100 & 404 & 48 & 495 & 10,000 & $2,0 ह 0$ & 45 & +5 & al, 100 & 462 \\
\hline 15 & & & 3,080 & 462 & 48 & 516 & 9,660 & 2,700 & 386 & 6 & al,000 & 4,62 \\
\hline 16 & & & 3,360 & 450 & 216 & 506 & 9,590 & 2,640 & 360 & 1,750 & 930 & 450 \\
\hline 17 & & & 2,580 & 428 & 301 & 404 & $* 9,140$ & 2,520 & $* 338$ & $4,4,0$ & 816 & 450 \\
\hline 18 & & & 1,910 & 405 & 484 & $* \overline{632}$ & 9,320 & 2,400 & 301 & 24,600 & 730 & 450 \\
\hline 19 & & & 1,300 & 386 & 310 & 901 & 9,380 & 2,280 & 262 & 20,900 & 687 & 462 \\
\hline 20 & (*) & & 1,080 & 367 & 270 & 1,500 & 9,590 & 2,230 & 224 & 19,700 & 632 & 14062 \\
\hline 21 & & & $* 716$ & 357 & 230 & 1,750 & 9,450 & 2,070 & 198 & 19,400 & 594 & 473 \\
\hline 22 & & & 632 & $* 338$ & 166 & 1,750 & $* 9,320$ & 1,910 & 179 & $* 13,100$ & 606 & 473 \\
\hline 23 & & & 619 & 318 & *130 & 1,720 & 9,020 & 1,500 & 156 & 8,090 & 568 & 473 \\
\hline 24 & & & $* 606$ & 293 & 111 & 1,860 & 8,320 & 1,660 & 135 & 7,420 & 556 & 462 \\
\hline 25 & & & 594 & 277 & 92 & 7,460 & 7,480 & 1,520 & 120 & 20,000 & 530 & 462 \\
\hline 26 & & & 543 & 262 & 92 & 16,700 & 7,300 & 1,390 & 99 & 27,500 & 506 & 473 \\
\hline 27 & & & 495 & 238 & 96 & $\overline{15,900}$ & 7,030 & 다 280 & 88 & $=30,000$ & 495 & 473 \\
\hline 28 & & & 506 & 224 & 493 & 11,500 & 6,730 & 1,230 & 76 & $2 \overline{25,000}$ & $\overline{495}$ & 473 \\
\hline 29 & & & 450 & 204 & 1,750 & 9,720 & 6,380 & 1,170 & 70 & a27,000 & 495 & 484 \\
\hline 30 & & & 428 & 179 & - & 9,020 & $* 5,900$ & 1,120 & $\underline{60}$ & 220,000 & 506 & 495 \\
\hline 31 & & & -416 & 162 & - & 9,360 & - & $1,04,0$ & - & 210,000 & 506 & - \\
\hline Total & 0 & 0 & 21,509 & $16, \overline{113}$ & 5,819 & 105,460 & 284,550 & 85,450 & 12,325 & 279,227 & 40,652 & 13,559 \\
\hline Mean & 0 & 0 & 694 & 520 & 201 & 3,402 & $9,4,55$ & 2,756 & 411 & 9,007 & 1,569 & 462 \\
\hline $\mathrm{Ac}-\mathrm{ft}$ & 0 & 0 & 42,660 & 31,970 & 11,540 & 209,200 & 564,400 & 169,500 & 24,450 & 553,800 & 96,500 & 27,490 \\
\hline
\end{tabular}

Calendar year 1955: $\operatorname{lax} 3,360$ Nin 0 Nean 187 Ac-ft 135,500 Water vear 1955-56: $\mathrm{iax} 16,700$ lin o liean 2,385 Ac-ft 1,732,000

* Discharge heasurenert or observation of no flow hade on this day.

a Wo gase-height record; discharge estimated on basis of peak stage and records for station below Arghandab Dam and Arghastan River near Kandahar. 
HELMAND RIVER BASIN

Arghandab River near Kala Bist, Afghanistan

Discharge, in cubic feet per second, water year Cctober 1956 to Septenber 1957

\begin{tabular}{|c|c|c|c|c|c|c|c|c|c|c|c|c|}
\hline Day & Oct. & Nov. & Dec. & Jan. & Feb. & Mar. & Apr. & May & June & JuIy & Aug. & Sept. \\
\hline 1 & 495 & 376 & 376 & 758 & 3,020 & 2,610 & 7,420 & 8,000 & 4,500 & 1,120 & 482 & 22 \\
\hline 2 & $4 \longdiv { 4 9 5 }$ & 396 & 376 & 830 & 2,880 & 2,670 & 7,140 & 8,000 & 4,500 & 1,100 & 410 & 22 \\
\hline 3 & 495 & 416 & 376 & 858 & 2,850 & 2,700 & 3,030 & 8,400 & 4,400 & 1,080 & 374 & 21 \\
\hline 4 & 495 & 428 & 386 & 916 & 2,950 & 2,820 & 7,030 & 9,000 & 4,200 & 1,060 & 320 & 20 \\
\hline 5 & 495 & $\overline{416}$ & 386 & 984 & 3,080 & $* 2,920$ & 10,800 & 2,400 & 4,000 & 1,040 & 280 & 20 \\
\hline 6 & 495 & 405 & 386 & 1,000 & 3,150 & 2,920 & 17.200 & 9,000 & 3,800 & 7,030 & 250 & 20 \\
\hline 7 & 495 & 405 & 386 & 1,000 & 3,150 & 2,850 & 19,000 & 8,600 & 3,600 & 995 & $\because 213$ & 19 \\
\hline 8 & 495 & 405 & 386 & 1,080 & 3,150 & 2,880 & 18,000 & 8,200 & 3,500 & 995 & 164 & 18 \\
\hline 9 & 495 & 396 & 386 & 1,390 & 3,220 & 2,880 & 17,000 & 8,000 & $* 3,260$ & 995 & 135 & $\overline{18}$ \\
\hline 10 & 462 & 396 & 386 & 2,100 & 3,220 & 2,880 & 15,000 & 7,800 & 2,950 & 995 & 110 & 18 \\
\hline II & 450 & $\approx 386$ & 386 & 2,020 & 3,220 & 2,280 & 13,000 & 7,800 & 2,870 & 995 & 87 & 18 \\
\hline 12 & 428 & 386 & 396 & 1,990 & 3,160 & 2,430 & 12,000 & 7,800 & 2,790 & 1,030 & 70 & 18 \\
\hline 13 & $\div 428$ & 386 & 396 & 3,150 & 3,150 & 2,170 & 11,000 & 7,800 & 2,710 & 1,040 & 61 & 18 \\
\hline 14 & 416 & 386 & 396 & 2,860 & 3,080 & $\frac{2,1}{2,460}$ & 31,000 & 7,600 & 2,630 & 1,060 & $\div 52$ & 15 \\
\hline 15 & 405 & 376 & $* 386$ & $x 5,800$ & 2,950 & 2,760 & 11,000 & 7,200 & 2,230 & 1,060 & 44 & 18 \\
\hline 16 & 405 & 376 & 386 & 3,360 & 2,880 & 2,640 & 11,000 & 7,000 & 2,090 & 1,030 & 37 & 18 \\
\hline 17 & 396 & 376 & 336 & $* 2,760$ & 2,790 & 2,700 & 11,000 & 6,600 & 2,000 & $* 1,030$ & 32 & 18 \\
\hline 18 & 396 & 376 & 386 & 2,520 & 2,700 & $* 3,360$ & 10,000 & 6,600 & 1,940 & 1,000 & 30 & 18 \\
\hline 19 & 396 & 367 & 386 & 2,310 & 2,500 & $* 10,000$ & 9,600 & 6,400 & 1,790 & 1,000 & 28 & 18 \\
\hline 20 & 396 & 367 & 396 & 2,200 & 2,550 & 18,200 & 9,400 & 6,200 & 1,670 & 1,000 & 26 & 22 \\
\hline 21 & 405 & 367 & 4,05 & 2,140 & $\underline{2,520}$ & 13,200 & 9,200 & 6,200 & 1,610 & 1,000 & 26 & 25 \\
\hline 22 & 396 & 376 & 405 & 2,120 & 2,580 & 10,400 & 9,000 & 6,000 & 1,400 & 960 & 26 & $\approx 27$ \\
\hline 23 & 386 & 386 & 405 & 2,100 & 2,640 & 10,300 & 8,000 & 5,800 & $1,4,20$ & 930 & 25 & 26 \\
\hline 24 & 386 & 386 & 416 & 2,120 & 2,670 & 9,450 & 8,600 & 5,600 & 1,420 & 900 & 24 & 27 \\
\hline 25 & 376 & 386 & 416 & 2,260 & $* 2,580$ & 8,200 & 8,400 & 5,400 & 1,320 & 855 & 24 & 28 \\
\hline 26 & 376 & 396 & 428 & 2,490 & 2,580 & 7,750 & 6,000 & 5,200 & 1,260 & 785 & 23 & 28 \\
\hline 27 & 376 & 396 & 428 & 2,760 & 2,530 & 7,420 & $\times 7,600$ & 5,000 & 1,190 & 750 & 23 & 28 \\
\hline 28 & 367 & 386 & 428 & 3,320 & 2,580 & 7,240 & 8,000 & 4,800 & 1,190 & $* 700$ & 23 & 29 \\
\hline 29 & 367 & 376 & 543 & 3,390 & - & 7,640 & 8,200 & 4,700 & 1,190 & $\div 625$ & 22 & 31 \\
\hline 30 & 367 & 376 & 619 & 3,320 & - & 8,040 & 8,000 & 4,500 & 1,140 & 600 & 22 & 40 \\
\hline 31 & 3607 & - & 658 & 3,160 & - & 7,860 & - & $\overline{4,500}$ & - & 562 & 22 & - \\
\hline Total & 13,202 & 11,652 & 12,881 & 76,086 & 80,480 & 174,630 & 317,620 & 213,100 & 74,650 & 29,322 & $3,4,55$ & 671 \\
\hline Fean & 426 & 388 & 416 & 2,454 & 2,874 & 5,633 & 10,590 & 6,874 & 2,488 & 946 & 112 & 22.4 \\
\hline$A c-i t$ & 26,190 & 23,110 & 25,550 & 150,900 & 159,600 & 346,400 & 630,000 & 422,700 & $1, \varepsilon, 100$ & 58,160 & 6,873 & 1,331 \\
\hline & dar year & 1956: liax & 16,700 & Iin & Nean & 2,429 & \multirow{2}{*}{\multicolumn{6}{|c|}{$\begin{array}{l}\therefore c-\text { ft } 1,764,000 \\
\text { Ac-ft } 1,999,000\end{array}$}} \\
\hline Water & 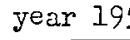 & 57: liax & 19,000 & Win & [viean & 2,761 & & & & & & \\
\hline
\end{tabular}

* Discharge measurement made on this day.

Note.-No gaze-height record hpr. 7 to June 8, July 18-24; dischrge estimated on basis of recards for

station below Arghandab Dam. 
HET FAMD RIVRR BASIN

Arghandab River near Kala Bist, Afghanistan

Discharge, in cubic feet per second, water year Cctoiver 1957 to September 1958

\begin{tabular}{|c|c|c|c|c|c|c|c|c|c|c|c|c|}
\hline Day & Oct. & Mov. & Dec. & Jan. & Feb. & lar. & Apr. & May & June & July & Aug. & Sept. \\
\hline 1 & 50 & 300 & 1,300 & 2,300 & 2,000 & 1,350 & 134 & 1,640 & 690 & 392 & 294 & 343 \\
\hline 2 & 67 & 280 & $\overline{1,300}$ & 2,200 & 2,000 & 1,860 & $1, \longdiv { 4 5 0 }$ & $\overline{1,470}$ & 690 & 392 & 287 & 329 \\
\hline 3 & 100 & 260 & 1,300 & 2,200 & 2,000 & 1,840 & 2,000 & 1,550 & 676 & 392 & 274 & 322 \\
\hline 4 & 110 & 250 & 1,300 & 2,200 & 2,150 & 1,760 & 2,400 & 1,380 & 632 & 392 & 267 & 329 \\
\hline 5 & 120 & 213 & 1,300 & 2,200 & 2,150 & 1,710 & 2,400 & 1,060 & 632 & 392 & $\overline{274}$ & 322 \\
\hline 6 & 130 & 240 & 1,300 & 2,200 & 2,090 & 1,670 & 2,400 & 866 & 632 & 392 & 267 & 294 \\
\hline 7 & 150 & 220 & 1,790 & 2,200 & 2,000 & 1,600 & $2,4,00$ & 883 & 690 & 382 & 267 & 287 \\
\hline 8 & 178 & 220 & 2,630 & 2,200 & 2,000 & 1,490 & 2,300 & 883 & 662 & 382 & 267 & 280 \\
\hline 9 & 185 & $* 220$ & 15,000 & 2,200 & 2,000 & $1,4,30$ & 2,200 & 821 & 647 & 392 & 280 & 280 \\
\hline 10 & 178 & 240 & 9,760 & 2,200 & 1,970 & 1,360 & 2,300 & 763 & 632 & 392 & 294 & 267 \\
\hline .11 & 164 & 230 & $10,4,00$ & 2,200 & 1,970 & 1,320 & $2,1,00$ & 734 & 618 & 392 & 294 & 254 \\
\hline 12 & 164 & 240 & 27,800 & 2,200 & 1,910 & 1,320 & 2,400 & 705 & 618 & 350 & 287 & 248 \\
\hline 13 & $16_{i t}^{i}$ & 250 & 27,200 & 2,200 & 1,860 & 1,280 & 2,300 & 705 & 589 & 350 & 287 & 234 \\
\hline 14 & 178 & 260 & 27,700 & 2,200 & 1,880 & 1,260 & 2,300 & 734 & 574 & 336 & 280 & 215 \\
\hline 15 & 206 & 260 & 28,100 & 2,200 & 1,880 & 1,260 & 2,200 & 705 & 550 & 315 & 280 & 203 \\
\hline 16 & 206 & 250 & 15,000 & $\because 2,150$ & 1,880 & 1,300 & 2,100 & 705 & 550 & 308 & 287 & 185 \\
\hline 17 & 213 & 250 & 7,000 & 2,090 & 1,880 & 1,280 & $* 2,000$ & 705 & 539 & 543 & 287 & 155 \\
\hline 18 & 220 & 250 & 5,000 & 2,060 & 1,970 & $* 1,220$ & 2,020 & 705 & 520 & 508 & $* 294$ & $* 144$ \\
\hline 19 & 220 & 290 & 3,500 & 2,090 & 2,000 & 1,100 & 2,500 & 1,110 & 500 & $\approx 476$ & 294 & 133 \\
\hline 20 & 220 & $* 785$ & 3,000 & 2,060 & 1,970 & 1,020 & 2,670 & 1,160 & 450 & 455 & 287 & 128 \\
\hline 21 & 220 & 1,610 & 2,700 & 2,060 & 1,880 & 966 & 2,500 & 982 & 420 & 402 & 287 & 122 \\
\hline 22 & 220 & 1,320 & 2,500 & 3,060 & 2,230 & 866 & 2,500 & $\because 850$ & 380 & 360 & 287 & 116 \\
\hline 23 & 220 & 1,280 & 2,400 & 2,830 & 2,060 & $\$ 21$ & 2,570 & 850 & 350 & 343 & 294 & 111 \\
\hline 24 & $2 ! 3$ & 1,550 & 2,400 & 2,630 & 2,060 & 792 & $2,74,0$ & 866 & $* 329$ & 322 & 280 & 100 \\
\hline 25 & 260 & 1,760 & 2,300 & 2,470 & 2,030 & 720 & 2,440 & 836 & 336 & 315 & 280 & 89 \\
\hline 26 & 370 & 1,730 & 2,300 & 2,310 & 1,910 & 676 & 2,120 & 806 & 322 & 315 & 294 & 84 \\
\hline 27 & 347 & 3,460 & 2,300 & 2,150 & 1,850 & $\overline{720}$ & 2,050 & 792 & $\overline{322}$ & 315 & 322 & 73 \\
\hline 28 & $\therefore 365$ & $1,4,00$ & 2,300 & 2,090 & $\overline{1,940}$ & 763 & 1,980 & 778 & 329 & 315 & $3 E 2$ & 64 \\
\hline 29 & 329 & 1,400 & 2,300 & 2,030 & & 792 & 1,950 & 734 & 371 & 315 & 392 & 53 \\
\hline 30 & 290 & 1,300 & 2,300 & 1,970 & & 734 & 1,880 & 705 & 392 & 301 & 413 & 48 \\
\hline 31 & 290 & & 2,300 & 1,970 & & 690 & & 690 & & 301 & 382 & \\
\hline Total & 6,287 & 20,318 & 217,780 & 69,120 & 55,540 & 37,470 & 66,204 & 28,173 & 15,642 & 11,537 & 9,261 & 5,812 \\
\hline Hean & 203 & 677 & 7,025 & 2,230 & 1,984 & 1,209 & 2,207 & 909 & 521 & 372 & 299 & 194 \\
\hline$A c-i t$ & 12,470 & 40,300 & 432,000 & 137,100 & 110,200 & 74,320 & 131,300 & 55,880 & 31,030 & 22,880 & 18,370 & 11,530 \\
\hline
\end{tabular}

Calendar year 1957: lar 28,100 lin 13 liean 3,327 Ac-ft 2,409,000 Vater year 1957-58: liax 28,100 Win 48 hean 1,488 Ac-ft 1,077,000

*Discharge measurement made on this day.

lote.-Doubtful gase-height record Nov. 26 to Dec. 6 and no gage-height record Dec. 16 to Jan. 15, Apr. 3-16,

June 18-23; discharge estinuted on the basis oi reccrds nor station below Arghand ab Dam. 


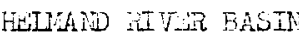

Arginandab River neur Kala Bist, Afcharictan

Dischare, in cuvic feet per second, waier year October 1958 to september 1959

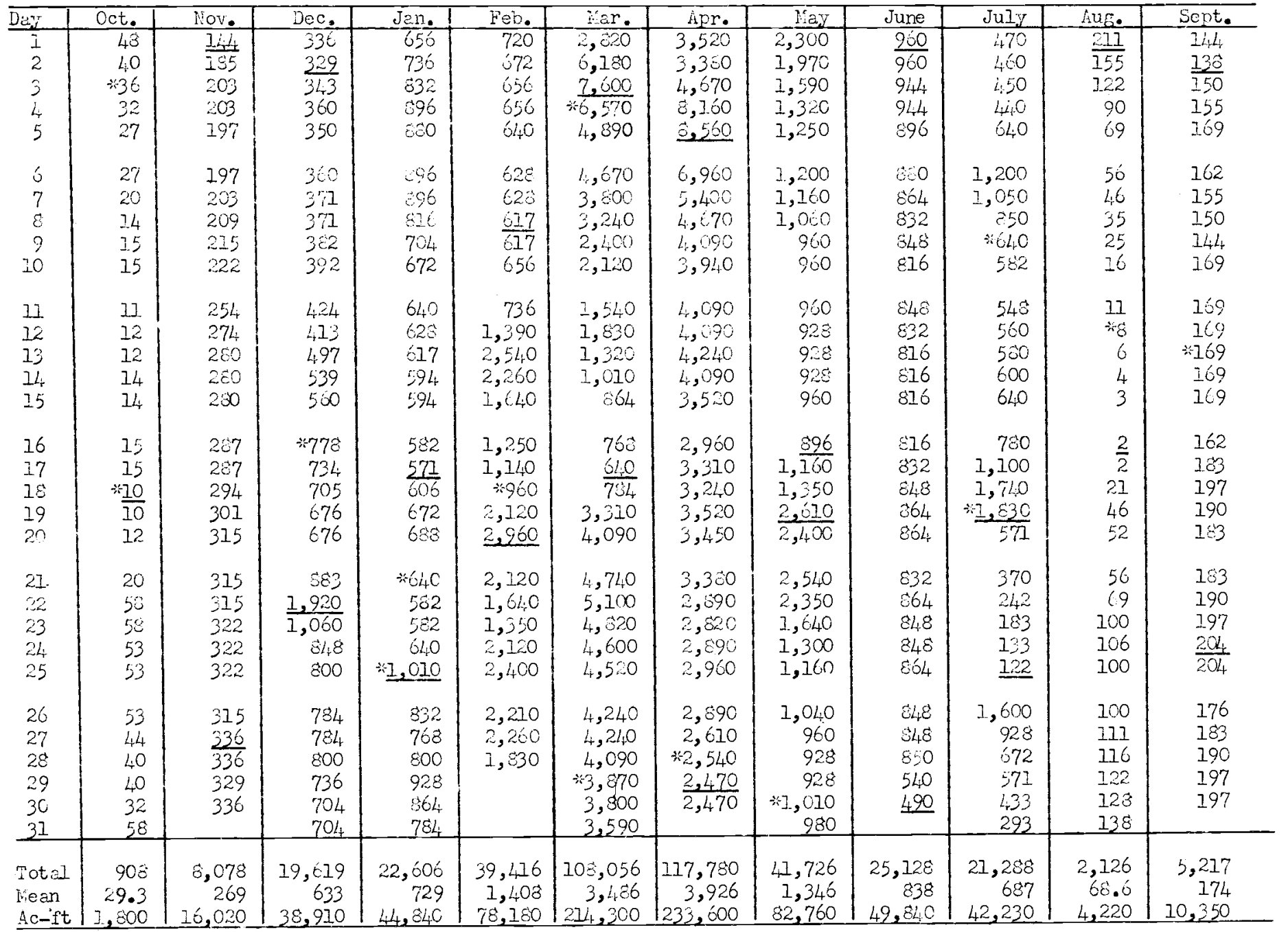

Calender yeer 1958: $\operatorname{kex} 3,060$ kin 10 kean 897 Ac-it 649,300 Vaton Jear 1958-59: lax 3,500 hin 2 lican 1,129 Ac-ft 617,200

\#ischaxge reasurerent made on this day

note - No rage-neight record June 28 to July 8 , July 12-18, July 30 to Aug. 11; discharge estinated on basis of recorded range in stage, recession curres, and comparison with records for station below Arginandeb Dan. 
HEIMHID IITVR BASTV

Arghandab River near Kala Bist, Afgharistan

Discharge, in cubic feet per second, water year October 1959 to September 1960

\begin{tabular}{|c|c|c|c|c|c|c|c|c|c|c|c|c|}
\hline Day & Oct. & Nov. & Dec. & Jan. & Feb. & lar. & AEr. & $16 y$ & June & JuIy & hugr. & Sent. \\
\hline $\begin{array}{l}1 \\
2 \\
3 \\
4 \\
5\end{array}$ & $\begin{array}{l}\frac{20 L_{1}}{211} \\
218 \\
225 \\
225\end{array}$ & $\begin{array}{l}* \frac{215}{260} \\
276 \\
330 \\
400\end{array}$ & $\begin{array}{l}\frac{502}{560} \\
640 \\
640 \\
604\end{array}$ & $\begin{array}{l}628 \\
606 \\
525 \\
456 \\
422\end{array}$ & $\begin{array}{l}310 \\
310 \\
320 \\
310 \\
310\end{array}$ & $\begin{array}{l}86 \\
60 \\
52 \\
46 \\
38\end{array}$ & $\begin{array}{l}434 \\
300 \\
301 \\
240 \\
203\end{array}$ & $\begin{array}{l}2,960 \\
2,820 \\
3,030 \\
3,240 \\
3,310\end{array}$ & $\begin{array}{r}1,110 \\
1,000 \\
949 \\
916 \\
566\end{array}$ & $\begin{array}{r}9.0 \\
* 9.5 \\
8.5 \\
7.5 \\
6.5\end{array}$ & $\begin{array}{l}4.0 \\
4.0 \\
4.0 \\
4.0 \\
4.0\end{array}$ & $\begin{array}{l}\frac{30}{58} \\
58 \\
43 \\
53\end{array}$ \\
\hline $\begin{array}{r}6 \\
7 \\
8 \\
9 \\
10\end{array}$ & $\begin{array}{l}225 \\
225 \\
234 \\
225 \\
218\end{array}$ & $\begin{array}{l}433 \\
433 \\
410 \\
390 \\
390\end{array}$ & $\begin{array}{l}656 \\
628 \\
605 \\
582 \\
571\end{array}$ & $\begin{array}{l}390 \\
390 \\
390 \\
370 \\
370\end{array}$ & $\begin{array}{l}320 \\
310 \\
302 \\
310 \\
310\end{array}$ & $\begin{array}{r}33 \\
28 \\
18 \\
7 \\
3\end{array}$ & $\begin{array}{l}173 \\
173 \\
173 \\
173 \\
167\end{array}$ & $\begin{array}{l}3,170 \\
2,750 \\
2,540 \\
2,470 \\
2,400\end{array}$ & $\begin{array}{l}821 \\
792 \\
778 \\
734 \\
705\end{array}$ & $\begin{array}{l}5.5 \\
5.0 \\
5.0 \\
5.0 \\
5.0\end{array}$ & $\begin{array}{l}4.0 \\
4.0 \\
4.0 \\
3.5 \\
3.0 \\
\end{array}$ & $\begin{array}{l}58 \\
63 \\
50 \\
44 \\
44\end{array}$ \\
\hline $\begin{array}{l}11 \\
12 \\
13 \\
14 \\
15\end{array}$ & $\begin{array}{l}204 \\
204 \\
204 \\
211 \\
211\end{array}$ & $\begin{array}{l}433 \\
433 \\
422 \\
400 \\
400\end{array}$ & $\begin{array}{l}560 \\
560 \\
548 \\
548 \\
560\end{array}$ & $\begin{array}{l}360 \\
350 \\
350 \\
330 \\
350\end{array}$ & $\begin{array}{l}302 \\
310 \\
302 \\
310 \\
293\end{array}$ & $\begin{array}{r}3 \\
3 \\
4 \\
1,940 \\
2,610 \\
\end{array}$ & $\begin{array}{l}\frac{161}{167} \\
167 \\
167 \\
173\end{array}$ & $\begin{array}{l}2,470 \\
2,360 \\
2,360 \\
2,470 \\
2,890\end{array}$ & $\begin{array}{l}662 \\
300 \\
480 \\
424 \\
371\end{array}$ & $\begin{array}{l}5.0 \\
5.0 \\
5.0 \\
5.0 \\
5.0\end{array}$ & $\begin{array}{l}3.0 \\
3.0 \\
3.0 \\
3.0 \\
3.0\end{array}$ & $\begin{array}{r}53 \\
* 53 \\
4.8 \\
4.4 \\
44\end{array}$ \\
\hline $\begin{array}{l}16 \\
17 \\
18 \\
19 \\
20\end{array}$ & $\begin{array}{l}218 \\
225 \\
225 \\
218 \\
218\end{array}$ & $\begin{array}{l}400 \\
390 \\
380 \\
370 \\
370\end{array}$ & $\begin{array}{l}640 \\
688 \\
720 \\
816 \\
816\end{array}$ & $\begin{array}{r}340 \\
340 \\
330 \\
* 330 \\
330\end{array}$ & $\begin{array}{l}276 \\
242 \\
218 \\
183 \\
176\end{array}$ & $\begin{array}{r}1,940 \\
1,320 \\
1,040 \\
900 \\
883\end{array}$ & $\begin{array}{r}155 \\
2,920 \\
10,800 \\
4,520 \\
1,500\end{array}$ & $\begin{array}{l}2,750 \\
2,820 \\
3,170 \\
3,520 \\
2,890\end{array}$ & $\begin{array}{l}329 \\
294 \\
260 \\
241 \\
228\end{array}$ & $\begin{array}{l}4.5 \\
4.5 \\
4.0 \\
4.0 \\
4.0\end{array}$ & $\begin{array}{l}3.0 \\
3.0 \\
3.0 \\
3.0 \\
3.0\end{array}$ & $\begin{array}{l}44 \\
48 \\
\frac{68}{53} \\
1.4\end{array}$ \\
\hline $\begin{array}{l}21 \\
22 \\
23 \\
24 \\
25\end{array}$ & $\begin{array}{l}218 \\
218 \\
218 \\
218 \\
218\end{array}$ & $\begin{array}{l}380 \\
390 \\
390 \\
390 \\
390\end{array}$ & $\begin{array}{r}* 800 \\
784 \\
784 \\
768 \\
800\end{array}$ & $\begin{array}{l}320 \\
330 \\
320 \\
310 \\
302\end{array}$ & $\begin{array}{l}* 176 \\
183 \\
176 \\
169 \\
155\end{array}$ & $\begin{array}{l}350 \\
773 \\
705 \\
705 \\
574\end{array}$ & $\begin{array}{l}1,550 \\
1,550 \\
1,500 \\
1,500 \\
1,440\end{array}$ & $\begin{array}{r}2,750 \\
2,680 \\
2,360 \\
2,210 \\
* 1,830\end{array}$ & $\begin{array}{l}209 \\
185 \\
16.1 \\
133 \\
106\end{array}$ & $\begin{array}{l}4.0 \\
4.0 \\
4.0 \\
4.0 \\
4.0\end{array}$ & $\begin{array}{l}3.0 \\
3.0 \\
3.0 \\
3.0 \\
3.0\end{array}$ & $\begin{array}{l}44 \\
44 \\
44 \\
48 \\
40\end{array}$ \\
\hline $\begin{array}{l}26 \\
27 \\
28 \\
29 \\
30 \\
31 \\
\end{array}$ & $\begin{array}{l}211 \\
218 \\
218 \\
268 \\
250 \\
242\end{array}$ & $\begin{array}{l}390 \\
400 \\
433 \\
444 \\
468 \\
\end{array}$ & $\begin{array}{l}752 \\
736 \\
720 \\
704 \\
680 \\
656\end{array}$ & $\begin{array}{l}302 \\
293 \\
293 \\
276 \\
276 \\
293\end{array}$ & $\begin{array}{l}133 \\
116 \\
111 \\
25\end{array}$ & $\begin{array}{r}329 \\
329 \\
329 \\
329 \\
336 \\
336 \\
\end{array}$ & $\begin{array}{l}1,590 \\
2,680 \\
2,750 \\
2,820 \\
3,030\end{array}$ & $\begin{array}{l}1,680 \\
1,570 \\
1,410 \\
1,270 \\
1,230 \\
1,160 \\
\end{array}$ & $\begin{array}{l}89 \\
68 \\
48 \\
30 \\
18 \\
\end{array}$ & $\begin{array}{r}4.0 \\
4.0 \\
4.0 \\
4.0 \\
4.0 \\
4.0\end{array}$ & $\begin{array}{r}3.0 \\
3.0 \\
3.0 \\
3.0 \\
27 \\
30 \\
\end{array}$ & $\begin{array}{l}44 \\
53 \\
53 \\
48 \\
48\end{array}$ \\
\hline $\begin{array}{l}\text { Total } \\
\text { Nean } \\
\text { Ac-ft }\end{array}$ & $\begin{array}{r}6,845 \\
221 \\
13,580 \\
\end{array}$ & $\begin{array}{r}11,618 \\
387 \\
23,040 \\
\end{array}$ & $\begin{array}{r}20,716 \\
668 \\
41,090 \\
\end{array}$ & $\begin{array}{r}11,272 \\
364 \\
22,360\end{array}$ & $\begin{array}{r}7,038 \\
243 \\
13,960 \\
\end{array}$ & $\begin{array}{r}16,620 \\
536 \\
32,970 \\
\end{array}$ & $\begin{array}{r}43,545 \\
1,452 \\
86,370 \\
\end{array}$ & $\begin{array}{c}76,540 \\
2,469 \\
151,820\end{array}$ & $\begin{array}{r}13,563 \\
452 \\
26,900\end{array}$ & $\begin{array}{c}156.5 \\
5.05 \\
310\end{array}$ & $\begin{array}{c}152.5 \\
4.92 \\
300\end{array}$ & $\begin{array}{r}1,479 \\
49.3 \\
2,930 \\
\end{array}$ \\
\hline $\begin{array}{l}\text { Calen } \\
\text { Water }\end{array}$ & $\begin{array}{l}\text { dar year } \\
\text { year } 19\end{array}$ & $\begin{array}{l}1959: \mathrm{Ma} \\
7-60: \mathrm{Ma}\end{array}$ & $\begin{array}{r}8,560 \\
10,800\end{array}$ & $\begin{array}{l}\text { Min } \\
\text { Lin }\end{array}$ & $\begin{array}{l}\text { liean } \\
\text { liean }\end{array}$ & $\begin{array}{r}1,158 \\
573\end{array}$ & $\begin{array}{ll}-\mathrm{ft} & 838 \\
-\mathrm{ft} & 4 \mathrm{~T} 5\end{array}$ & $\begin{array}{l}000 \\
600\end{array}$ & & & & \\
\hline
\end{tabular}

*Discharge measurement made on this diar. Note.-Doubtful Eage-height Apr. 19-23; discharge estimated on basis of partial recorder record and record
for station below Arghendar Dam. 
HELMAND RIVER BASIN

Helmand River at Darweshan, Afghanistan

Location.-Lat $31^{\circ} \mathrm{Ol} \cdot \mathrm{N}$, , long $64^{\circ} 05^{\prime} \mathrm{E}$, at highway bridge in Darweshan, about 55 kilometers downstream from Arghandab River, about 65 kilometers downstrean from Lashkar Gah and about 185 kilometers downstream from Kajakai Dam.

Drainage area.-56,920 sq $\mathrm{mi}$ approximately, of which about 7,800 sq mi (above Lake Ab-i-Istada on the Arghastan River and probebly parts of the Registan desert) are probably non-contributing. Records available.-October 1956 to September 1960

Gage.-Water-stage recorder. Datum of gage is mean sea level (from Morrison-Knudsen Afghanistan surveys based on Survey of India datum•)

Extreme 8.--Maximum and minimum discharges for water years 1957-1960 are given in the following table:

\begin{tabular}{|c|c|c|c|c|c|c|}
\hline \multirow[b]{2}{*}{ Water Year } & \multirow[b]{2}{*}{ Dato } & \multicolumn{2}{|c|}{ Maximum } & \multirow[b]{2}{*}{ Date } & \multicolumn{2}{|c|}{ Minimum } \\
\hline & & $\begin{array}{r}\text { Elevation } \\
\text { (meters) }\end{array}$ & $\begin{array}{c}\text { Discharge } \\
\text { (cfs) }\end{array}$ & & $\begin{array}{r}\text { Elevation } \\
\text { (meters) }\end{array}$ & $\begin{array}{c}\text { Discharge } \\
\text { (cfs) }\end{array}$ \\
\hline $\begin{array}{l}1957 \\
1958 \\
1959 \\
1960 \\
\end{array}$ & $\begin{array}{l}\text { Mar. 18, } 1957 \\
\text { Dec. 13, } 1957 \\
\text { Kar. 3, } 1959 \\
\text { May 19, } 1960\end{array}$ & $\begin{array}{l}709.80 \\
707.44 \\
707.49 \\
706.34\end{array}$ & $\begin{array}{r}133,000 \\
46,600 \\
47,800 \\
24,000 \\
\end{array}$ & $\begin{array}{l}\text { Aug. } 21,1957 \\
\text { Nov. } 16,1957 \\
\text { Nov. 18, } 1958 \\
\text { Mar. } 9,1960\end{array}$ & $\begin{array}{l}703.96 \\
704.07 \\
704.425 \\
704.36\end{array}$ & $\begin{array}{r}614 \\
837 \\
2040 \\
1580 \\
\end{array}$ \\
\hline
\end{tabular}

Remarks.-Records good. 
HEIMAND RIVER BASIN

Helmand River at Darweshan, Afghanistan

Discharge, in cubic feet per second, water year October 1956 to September 1957

\begin{tabular}{|c|c|c|c|c|c|c|c|c|c|c|c|c|}
\hline Day & Oct. & Nov. & Dec. & $\operatorname{Jan}$. & Feb. & Mar. & Apr. & May & June & July & Aug. & Sept. \\
\hline $\begin{array}{l}1 \\
2 \\
3 \\
4 \\
5\end{array}$ & $\begin{array}{l}7,400 \\
7,400 \\
7,400 \\
7,400 \\
7,400\end{array}$ & $\begin{array}{l}4,540 \\
4,800 \\
4,800 \\
4,800 \\
4,840\end{array}$ & $\begin{array}{l}5,380 \\
5,380 \\
5,340 \\
5,340 \\
5,300\end{array}$ & $\begin{array}{l}7,600 \\
7,700 \\
7,700 \\
7,700 \\
7,700\end{array}$ & $\begin{array}{l}11,600 \\
11,700 \\
11,900 \\
12,300 \\
12,400\end{array}$ & $\begin{array}{r}4,630 \\
9,500 \\
9,930 \\
10,200 \\
* 10,600\end{array}$ & $\begin{array}{l}\frac{20,500}{20,500} \\
23,500 \\
43,400 \\
67,400\end{array}$ & $\begin{array}{l}48,500 \\
50,900 \\
54,700 \\
69,000 \\
80,600 \\
\end{array}$ & $\begin{array}{l}33,400 \\
33,600 \\
33,200 \\
33,200 \\
33,600\end{array}$ & $\begin{array}{l}\frac{11,100}{10,800} \\
10,700 \\
10,500 \\
10,400\end{array}$ & $\begin{array}{l}6,510 \\
6,420 \\
6,420 \\
6,330 \\
6,330\end{array}$ & $\begin{array}{l}6,900 \\
6,800 \\
6,800 \\
6,800 \\
6,700\end{array}$ \\
\hline $\begin{array}{r}6 \\
7 \\
8 \\
9 \\
10\end{array}$ & $\begin{array}{l}7,400 \\
7,400 \\
7,400 \\
7,400 \\
7,300\end{array}$ & $\begin{array}{r}4,840 \\
4,890 \\
* 4,840 \\
4,890 \\
4,890\end{array}$ & $\begin{array}{l}\frac{5,250}{5,250} \\
5,250 \\
5,250 \\
5,250\end{array}$ & $\begin{array}{l}7,700 \\
7,800 \\
8,000 \\
8,400 \\
9,600\end{array}$ & $\begin{array}{l}12,400 \\
12,500 \\
13,100 \\
\frac{13,400}{12,800}\end{array}$ & $\begin{array}{l}10,600 \\
10,500 \\
10,500 \\
10,700 \\
11,000\end{array}$ & $\begin{array}{l}71,100 \\
72,000 \\
\frac{69,900}{67,800} \\
64,000\end{array}$ & $\begin{array}{l}80,600 \\
72,600 \\
69,600 \\
63,800 \\
58,600\end{array}$ & $\begin{array}{l}31,600 \\
29,300 \\
29,100 \\
28,500 \\
26,200\end{array}$ & $\begin{array}{l}9,820 \\
9,600 \\
9,300 \\
9,300 \\
9,100\end{array}$ & $\begin{array}{l}6,240 \\
6,240 \\
6,240 \\
6,240 \\
6,150\end{array}$ & $\begin{array}{l}6,600 \\
6,600 \\
\frac{7,100}{6,510} \\
\frac{6,560}{6,50}\end{array}$ \\
\hline $\begin{array}{l}11 \\
12 \\
13 \\
14 \\
15\end{array}$ & $\begin{array}{r}* 7,200 \\
7,200 \\
4,600 \\
2,300 \\
* 2,100\end{array}$ & $\begin{array}{l}4,890 \\
4,890 \\
4,890 \\
4,940 \\
4,980\end{array}$ & $\begin{array}{l}5,250 \\
5,250 \\
5,250 \\
5,250 \\
5,250\end{array}$ & $\begin{array}{r}9,400 \\
8,500 \\
11,600 \\
* 18,500 \\
15,900\end{array}$ & $\begin{array}{l}12,000 \\
11,700 \\
11,400 \\
11,100 \\
11,000\end{array}$ & $\begin{array}{l}11,000 \\
10,800 \\
10,500 \\
10,200 \\
10,600\end{array}$ & $\begin{array}{l}60,100 \\
59,100 \\
57,800 \\
58,600 \\
63,500\end{array}$ & $\begin{array}{l}57,800 \\
58,100 \\
59,400 \\
57,300 \\
54,200\end{array}$ & $\begin{array}{r}* 25,100 \\
24,200 \\
23,100 \\
21,900 \\
20,600\end{array}$ & $\begin{array}{l}8,700 \\
8,600 \\
8,500 \\
8,400 \\
8,200\end{array}$ & $\begin{array}{l}6,060 \\
6,330 \\
6,600 \\
6,420 \\
2,590\end{array}$ & $\begin{array}{l}6,600 \\
6,600 \\
6,650 \\
6,600 \\
6,560\end{array}$ \\
\hline $\begin{array}{l}16 \\
17 \\
18 \\
19 \\
20\end{array}$ & $\begin{array}{l}1,800 \\
1,700 \\
1,700 \\
1,700 \\
1,200\end{array}$ & $\begin{array}{l}5,020 \\
5,020 \\
5,070 \\
5,160 \\
5,160\end{array}$ & $\begin{array}{l}5,250 \\
5,250 \\
5,250 \\
5,300 \\
5,340\end{array}$ & $\begin{array}{r}10,600 \\
9,600 \\
9,300 \\
9,710 \\
9,800\end{array}$ & $\begin{array}{l}10,900 \\
10,900 \\
10,800 \\
10,700 \\
10,700\end{array}$ & $\begin{array}{l}10,600 \\
10,800 \\
66,600 \\
\frac{53,800}{42,500}\end{array}$ & $\begin{array}{l}68,100 \\
69,600 \\
66,000 \\
64,900 \\
60,100\end{array}$ & $\begin{array}{l}50,600 \\
47,500 \\
46,300 \\
45,200 \\
44,100\end{array}$ & $\begin{array}{l}19,900 \\
19,100 \\
18,400 \\
17,600 \\
16,900\end{array}$ & $\begin{array}{l}8,000 \\
7,700 \\
7,600 \\
7,400 \\
7,300\end{array}$ & $\begin{array}{r}1,220 \\
950 \\
975 \\
879 \\
816\end{array}$ & $\begin{array}{l}6,560 \\
6,650 \\
6,700 \\
6,800 \\
6,700\end{array}$ \\
\hline $\begin{array}{l}21 \\
22 \\
23 \\
24 \\
25\end{array}$ & $\begin{array}{r}1,200 \\
1,100 \\
* 1,000 \\
1,000 \\
1,000\end{array}$ & $\begin{array}{l}5,070 \\
5,160 \\
5,250 \\
5,250 \\
5,300\end{array}$ & $\begin{array}{r}5,340 \\
* 5,300 \\
5,660 \\
6,060 \\
5,970\end{array}$ & $\begin{array}{r}9,930 \\
9,930 \\
10,000 \\
10,700 \\
13,700\end{array}$ & $\begin{array}{l}10,800 \\
10,700 \\
10,700 \\
10,600 \\
10,400\end{array}$ & $\begin{array}{r}36,000 \\
30,400 \\
* 28,100 \\
23,000 \\
22,600\end{array}$ & $\begin{array}{l}59,400 \\
58,100 \\
57,000 \\
56,000 \\
55,700\end{array}$ & $\begin{array}{l}43,400 \\
43,000 \\
42,300 \\
42,100 \\
41,200\end{array}$ & $\begin{array}{l}16,200 \\
15,700 \\
15,100 \\
14,300 \\
13,800\end{array}$ & $\begin{array}{l}7,200 \\
7,200 \\
7,100 \\
6,900 \\
7,000\end{array}$ & $\begin{array}{l}5,974 \\
6,240 \\
6,240 \\
6,330\end{array}$ & $\begin{array}{l}6,700 \\
6,700 \\
6,700 \\
6,700 \\
6,700\end{array}$ \\
\hline $\begin{array}{l}26 \\
27 \\
28 \\
29 \\
30 \\
31 \\
\end{array}$ & $\begin{array}{r}1,000 \\
900 \\
900 \\
1, \frac{858}{270} \\
4,120 \\
\end{array}$ & $\begin{array}{c}5,340 \\
5,340 \\
5,340 \\
5,340 \\
5,380 \\
- \\
\end{array}$ & $\begin{array}{l}5,970 \\
5,970 \\
5,880 \\
6,060 \\
7,000 \\
7,400\end{array}$ & $\begin{array}{l}\frac{22,100}{17,600} \\
16,000 \\
13,800 \\
12,600 \\
12,000 \\
\end{array}$ & $\begin{array}{c}9,300 \\
5,160 \\
4,380 \\
- \\
- \\
-\end{array}$ & $\begin{array}{l}21,000 \\
20,000 \\
21,000 \\
23,300 \\
23,700 \\
22,600\end{array}$ & $\begin{array}{c}53,300 \\
51,800 \\
51,600 \\
50,400 \\
47,000 \\
-\end{array}$ & $\begin{array}{l}39,200 \\
36,200 \\
34,400 \\
33,600 \\
32,400 \\
32,400\end{array}$ & $\begin{array}{l}13,500 \\
12,800 \\
11,400 \\
10,900 \\
10,700 \\
-\end{array}$ & $\begin{array}{r}7,000 \\
* 7,100 \\
7,000 \\
6,900 \\
6,700 \\
6,600 \\
\end{array}$ & $\begin{array}{l}6,420 \\
6,510 \\
6,510 \\
6,510 \\
6,560 \\
6,700\end{array}$ & $\begin{array}{c}6,800 \\
6,700 \\
6,750 \\
6,750 \\
6,750 \\
-\end{array}$ \\
\hline $\begin{array}{l}\text { Total } \\
\text { Mean } \\
\text { Ac-ft }\end{array}$ & $\begin{array}{r}119,748 \\
3,863 \\
237,500 \\
\end{array}$ & $\begin{array}{r}150,920 \\
5,031 \\
299,300\end{array}$ & $\begin{array}{r}172,240 \\
5,556 \\
34,600\end{array}$ & $\begin{array}{r}341,190 \\
17,010 \\
676,700\end{array}$ & $\begin{array}{r}307,340 \\
10,980 \\
609,600\end{array}$ & $\begin{array}{r}607,260 \\
9,590 \\
2,20 \% \\
\end{array}$ & $\begin{array}{r}1,688,200 \\
56,270 \\
3,31,8,000\end{array}$ & $\begin{array}{r}1,589,600 \\
51,280 \\
3.153,000\end{array}$ & $\begin{array}{r}652,900 \\
21,760 \\
1295,000\end{array}$ & $\begin{array}{r}257,720 \\
8,314 \\
51,200 \\
\end{array}$ & $\begin{array}{r}160,724 \\
5,185 \\
318,800\end{array}$ & $\begin{array}{r}201,040 \\
6,701 \\
398,800\end{array}$ \\
\hline $\begin{array}{l}\text { Cale I } \\
\text { Water }\end{array}$ & ear & $\begin{array}{ll}1956: & M z \\
-57: & M e\end{array}$ & $x \quad 80,600$ & Min $\quad \overline{7}$ & $\begin{array}{l}\text { Mean } \\
\text { Hean } 17\end{array}$ & $\overline{7,070 \mathrm{Ac}-\mathrm{A}}$ & & & & & & \\
\hline
\end{tabular}

* Discharge measurement made on this day.

Note.-No gage-heights Uct. 1-27; discharge estinated on basis of reco. is at Chahar Burjak. 
HELMAND RTVER BASIN

Helmand River at Darweshan, Afghanistan

Discharge, in cubic feet per secono, water year October 1957 to September 1958

\begin{tabular}{|c|c|c|c|c|c|c|c|c|c|c|c|c|}
\hline Day & Oct. & Nov. & Dec. & Jan. & Feb. & Mar. & Apr. & May & June & July & Aug. & Sept. \\
\hline $\begin{array}{l}1 \\
2 \\
3 \\
4 \\
5\end{array}$ & $\begin{array}{l}6,800 \\
6,700 \\
6,750 \\
6,950 \\
7,000\end{array}$ & $\begin{array}{l}6,600 \\
6,700 \\
6,700 \\
6,700 \\
6,600\end{array}$ & $\begin{array}{l}5,880 \\
5,700 \\
5,610 \\
5,610 \\
5,610\end{array}$ & $\begin{array}{r}10,200 \\
10,200 \\
10,000 \\
9,930 \\
9,820\end{array}$ & $\begin{array}{r}9,500 \\
10,000 \\
11,000 \\
10,300 \\
9,930\end{array}$ & $\begin{array}{l}\frac{8,200}{8,100} \\
8,200 \\
8,100 \\
8,000\end{array}$ & $\begin{array}{l}\frac{7,400}{7,800} \\
8,500 \\
8,700 \\
8,700\end{array}$ & $\begin{array}{l}\frac{23,000}{21,800} \\
20,800 \\
19,800 \\
18,800\end{array}$ & $\begin{array}{l}9,500 \\
9,400 \\
9,500 \\
8,800 \\
8,300\end{array}$ & $\begin{array}{l}6,700 \\
6,700 \\
6,700 \\
6,700 \\
6,800\end{array}$ & $\begin{array}{l}6,100 \\
6,060 \\
6,020 \\
5,920 \\
5,920\end{array}$ & $\begin{array}{l}\frac{6,460}{6,380} \\
6,240 \\
6,060 \\
5,880\end{array}$ \\
\hline $\begin{array}{r}6 \\
7 \\
8 \\
9 \\
10\end{array}$ & $\begin{array}{r}7,000 \\
6,850 \\
* 6,800 \\
6,900 \\
6,850\end{array}$ & $\begin{array}{r}6,700 \\
6,330 \\
2,280 \\
1,320 \\
* 1,100\end{array}$ & $\begin{array}{r}5,790 \\
8,000 \\
10,400 \\
18,000 \\
17,600\end{array}$ & $\begin{array}{l}9,710 \\
9,710 \\
9,710 \\
9,710 \\
9,820\end{array}$ & $\begin{array}{l}9,710 \\
9,500 \\
9,400 \\
9,300 \\
9,200\end{array}$ & $\begin{array}{l}8,000 \\
7,900 \\
7,800 \\
7,700 \\
7,700\end{array}$ & $\begin{array}{l}8,600 \\
8,500 \\
8,400 \\
8,400 \\
8,200\end{array}$ & $\begin{array}{l}17,600 \\
17,000 \\
16,400 \\
15,700 \\
15,000\end{array}$ & $\begin{array}{l}8,400 \\
8,400 \\
8,150 \\
7,950 \\
7,750\end{array}$ & $\begin{array}{l}\frac{6,950}{6,750} \\
6,600 \\
5,840 \\
3,010 \\
\end{array}$ & $\begin{array}{l}5,970 \\
5,970 \\
5,970 \\
5,970 \\
5,970\end{array}$ & $\begin{array}{l}5,790 \\
5,520 \\
5,520 \\
5,790 \\
5,700\end{array}$ \\
\hline $\begin{array}{l}11 \\
12 \\
13 \\
14 \\
15\end{array}$ & $\begin{array}{l}7,100 \\
7,500 \\
7,000 \\
6,420 \\
\frac{6,600}{6}\end{array}$ & $\begin{array}{r}1,000 \\
938 \\
900 \\
879 \\
858\end{array}$ & $\begin{array}{r}21,000 \\
* 34,100 \\
34,200 \\
19,100 \\
14,000\end{array}$ & $\begin{array}{r}10,200 \\
10,200 \\
10,000 \\
9,820 \\
9,820\end{array}$ & $\begin{array}{l}9,000 \\
8,900 \\
8,800 \\
8,800 \\
8,900\end{array}$ & $\begin{array}{l}7,700 \\
7,700 \\
7,700 \\
7,700 \\
7,700\end{array}$ & $\begin{array}{r}8,300 \\
* 8,400 \\
8,400 \\
8,400 \\
8,300\end{array}$ & $\begin{array}{l}14,400 \\
14,200 \\
13,600 \\
13,300 \\
12,600\end{array}$ & $\begin{array}{l}7,750 \\
7,500 \\
7,250 \\
7,200 \\
7,050\end{array}$ & $\begin{array}{l}3,980 \\
6,280 \\
6,380 \\
6,420 \\
6,240\end{array}$ & $\begin{array}{r}6,060 \\
6,060 \\
6,020 \\
5,920 \\
* 5,880\end{array}$ & $\begin{array}{l}5,700 \\
5,700 \\
5,920 \\
6,150 \\
5,920\end{array}$ \\
\hline $\begin{array}{l}16 \\
17 \\
18 \\
19 \\
20\end{array}$ & $\begin{array}{l}6,600 \\
6,700 \\
6,900 \\
6,700 \\
6,560\end{array}$ & $\begin{array}{l}\frac{837}{1,480} \\
3,630 \\
4,120 \\
7,700\end{array}$ & $\begin{array}{l}12,400 \\
11,800 \\
11,400 \\
11,000 \\
10,800\end{array}$ & $\begin{array}{r}9,820 \\
9,820 \\
9,710 \\
* 9,710 \\
9,820\end{array}$ & $\begin{array}{l}8,600 \\
8,600 \\
8,500 \\
8,400 \\
8,400\end{array}$ & $\begin{array}{r}7,600 \\
7,600 \\
7,500 \\
* 7,300 \\
7,200\end{array}$ & $\begin{array}{r}8,400 \\
10,200 \\
12,300 \\
14,000 \\
15,600\end{array}$ & $\begin{array}{l}12,800 \\
12,900 \\
12,600 \\
12,600 \\
12,300\end{array}$ & $\begin{array}{l}7,050 \\
7,000 \\
7,000 \\
7,100 \\
7,050\end{array}$ & $\begin{array}{l}6,280 \\
6,420 \\
6,560 \\
6,510 \\
6,600\end{array}$ & $\begin{array}{l}5,880 \\
5,880 \\
5,880 \\
5,880 \\
5,880\end{array}$ & $\begin{array}{l}6,420 \\
6,100 \\
5,520 \\
5,520 \\
5,480\end{array}$ \\
\hline $\begin{array}{l}21 \\
22 \\
23 \\
24 \\
25\end{array}$ & $\begin{array}{l}6,700 \\
6,700 \\
6,650 \\
6,700 \\
6,700\end{array}$ & $\begin{array}{r}10,200 \\
7,700 \\
6,700 \\
6,700 \\
7,700\end{array}$ & $\begin{array}{l}10,700 \\
10,500 \\
10,700 \\
10,600 \\
10,500\end{array}$ & $\begin{array}{r}9,820 \\
10,200 \\
10,400 \\
\frac{10,200}{10,000}\end{array}$ & $\begin{array}{r}8,400 \\
8,500 \\
8,400 \\
* 8,200 \\
8,000 \\
\end{array}$ & $\begin{array}{l}7,100 \\
7,100 \\
7,000 \\
6,950 \\
6,850\end{array}$ & $\begin{array}{l}16,600 \\
18,500 \\
19,700 \\
20,500 \\
21,100\end{array}$ & $\begin{array}{r}11,900 \\
11,400 \\
10,900 \\
* 11,400 \\
11,200\end{array}$ & $\begin{array}{l}7,000 \\
7,000 \\
6,800 \\
6,600 \\
6,600\end{array}$ & $\begin{array}{l}6,800 \\
6,510 \\
6,240 \\
6,150 \\
6,150\end{array}$ & $\begin{array}{l}5,840 \\
5,790 \\
5,790 \\
5,790 \\
5,880\end{array}$ & $\begin{array}{l}5,480 \\
5,480 \\
5,520 \\
5,520 \\
5,380\end{array}$ \\
\hline $\begin{array}{l}26 \\
27 \\
28 \\
29 \\
30 \\
31\end{array}$ & $\begin{array}{l}6,420 \\
6,700 \\
6,850 \\
6,800 \\
6,700 \\
6,600\end{array}$ & $\begin{array}{c}7,900 \\
6,900 \\
6,330 \\
6,060 \\
5,970 \\
-\end{array}$ & $\begin{array}{l}10,400 \\
10,300 \\
10,200 \\
10,200 \\
10,300 \\
10,200\end{array}$ & $\begin{array}{l}9,930 \\
9,820 \\
9,820 \\
9,710 \\
9,500 \\
2,400\end{array}$ & $\begin{array}{c}8,000 \\
8,000 \\
8,000 \\
- \\
= \\
-\end{array}$ & $\begin{array}{l}6,800 \\
6,900 \\
7,000 \\
7,300 \\
7,600 \\
7,300\end{array}$ & $\begin{array}{c}21,900 \\
23,100 \\
24,000 \\
\frac{24,400}{23,700} \\
-\end{array}$ & $\begin{array}{r}11,000 \\
10,800 \\
10,600 \\
10,300 \\
10,000 \\
2,710\end{array}$ & $\begin{array}{c}6,600 \\
6,510 \\
6,600 \\
6,600 \\
6,650 \\
-\end{array}$ & $\begin{array}{l}6,150 \\
6,100 \\
6,060 \\
6,150 \\
6,150 \\
6,150\end{array}$ & $\begin{array}{l}5,880 \\
5,920 \\
6,150 \\
6,200 \\
6,240 \\
6,460 \\
\end{array}$ & $\begin{array}{c}5,340 \\
5,480 \\
5,520 \\
5,520 \\
5,560 \\
-\end{array}$ \\
\hline Total & $\begin{array}{r}210,200 \\
6,781 \\
6\end{array}$ & $\begin{array}{r}145,532 \\
4,851 \\
288,700\end{array}$ & $\begin{array}{r}382,600 \\
12,340 \\
758,900\end{array}$ & $\begin{array}{r}306,530 \\
9,888 \\
608,000\end{array}$ & $\begin{array}{r}250,240 \\
8,937 \\
496,300 \\
\end{array}$ & $\begin{array}{r}233,300 \\
7,526 \\
462,700\end{array}$ & $\begin{array}{r}399,000 \\
13,270 \\
791,400 \\
\end{array}$ & $\begin{array}{r}436,410 \\
14,080 \\
865,600 \\
\end{array}$ & $\begin{array}{r}225,060 \\
7,502 \\
446,400\end{array}$ & $\begin{array}{r}193,030 \\
6,227 \\
382,900 \\
\end{array}$ & $\begin{array}{r}185,150 \\
5,973 \\
367,200 \\
\end{array}$ & $\begin{array}{r}172,520 \\
5,751 \\
342,200 \\
\end{array}$ \\
\hline
\end{tabular}

Calendar year 1957: Viax 80,600 Min 774 Mean 17,930 Ac-ft 12,980,000

* Dir

Note.-Gage read once daily January $7-18$, March 3-17. 
HELMAND RIVER BASIN

Helmand River at Darweshan, Afghanistan

Discharge, in cublc feet per second, water year October 1958 to Septemer 1959

\begin{tabular}{|c|c|c|c|c|c|c|c|c|c|c|c|c|}
\hline Day & Oct. & Nov. & Dec. & Jan. & Fob. & Mar. & Apr. & May & June & July & Aug. & Sept. \\
\hline $\begin{array}{l}1 \\
2 \\
3 \\
4 \\
5\end{array}$ & $\begin{array}{l}5,520 \\
5,520 \\
5,520 \\
5,560 \\
5,560\end{array}$ & $\begin{array}{l}5,160 \\
5,160 \\
5,160 \\
5,160 \\
5,160\end{array}$ & $\begin{array}{l}\frac{3,040}{3,120} \\
3,150 \\
3,150 \\
3,150\end{array}$ & $\begin{array}{l}\frac{3,920}{4,000} \\
4,080 \\
4,240 \\
4,400\end{array}$ & $\begin{array}{l}4,580 \\
4,490 \\
4,490 \\
4,400 \\
4,440\end{array}$ & $\begin{array}{r}5,120 \\
18,600 \\
* 27,170 \\
15,500 \\
12,100\end{array}$ & $\begin{array}{l}11,500 \\
11,200 \\
12,100 \\
15,000 \\
16,300\end{array}$ & $\begin{array}{l}19,000 \\
18,500 \\
17,800 \\
16,900 \\
16,300\end{array}$ & $\begin{array}{l}\frac{9,900}{9,580} \\
9,360 \\
9,140 \\
8,920\end{array}$ & $\begin{array}{l}6,600 \\
6,500 \\
6,500 \\
6,400 \\
6,300\end{array}$ & $\begin{array}{l}5,570 \\
5,390 \\
5,340 \\
5,300 \\
5,210\end{array}$ & $\begin{array}{l}5,080 \\
5,030 \\
4,980 \\
4,980 \\
4,980\end{array}$ \\
\hline $\begin{array}{r}6 \\
7 \\
8 \\
9 \\
10\end{array}$ & $\begin{array}{l}5,520 \\
5,480 \\
5,480 \\
5,480 \\
5,430\end{array}$ & $\begin{array}{l}5,160 \\
5,120 \\
5,120 \\
5,120 \\
5,120\end{array}$ & $\begin{array}{l}3,190 \\
3,230 \\
3,190 \\
3,190 \\
3,230\end{array}$ & $\begin{array}{l}4,580 \\
4,580 \\
4,670 \\
4,440 \\
4,400\end{array}$ & $\begin{array}{l}4,080 \\
4,000 \\
3,880 \\
3,920 \\
4,080\end{array}$ & $\begin{array}{r}10,900 \\
9,960 \\
9,300 \\
8,420 \\
9,190\end{array}$ & $\begin{array}{l}14,500 \\
13,300 \\
12,400 \\
11,700 \\
11,500\end{array}$ & $\begin{array}{l}16,000 \\
15,600 \\
15,300 \\
u_{4}, 900 \\
u_{4}, 300\end{array}$ & $\begin{array}{r}8,920 \\
8,700 \\
8,360 \\
8,150 \\
* 8,050\end{array}$ & $\begin{array}{l}6,400 \\
6,900 \\
6,700 \\
6,400 \\
6,300\end{array}$ & $\begin{array}{l}5,160 \\
5,120 \\
5,030 \\
5,080 \\
5,260\end{array}$ & $\begin{array}{l}4,980 \\
4,980 \\
4,900 \\
4,360 \\
4,280\end{array}$ \\
\hline $\begin{array}{l}11 \\
12 \\
13 \\
14 \\
15\end{array}$ & $\begin{array}{l}5,430 \\
5,430 \\
5,430 \\
5,480 \\
5,520\end{array}$ & $\begin{array}{l}5,120 \\
5,120 \\
4,630 \\
3,080 \\
2,840\end{array}$ & $\begin{array}{r}3,270 \\
3,550 \\
5,250 \\
4,540 \\
* 4,200\end{array}$ & $\begin{array}{l}4,320 \\
4,320 \\
4,320 \\
4,240 \\
4,320\end{array}$ & $\begin{array}{l}4,160 \\
4,400 \\
5,210 \\
5,120 \\
4,670\end{array}$ & $\begin{array}{r}11,600 \\
9,960 \\
8,970 \\
8,000 \\
7,300\end{array}$ & $\begin{array}{l}11,400 \\
11,400 \\
11,500 \\
11,600 \\
11,000\end{array}$ & $\begin{array}{l}14,100 \\
13,700 \\
13,300 \\
13,000 \\
12,800\end{array}$ & $\begin{array}{l}7,700 \\
7,400 \\
7,200 \\
7,000 \\
6,900\end{array}$ & $\begin{array}{r}6,200 \\
* 6,200 \\
6,200 \\
6,110 \\
6,020\end{array}$ & $\begin{array}{r}5,750 \\
5,570 \\
5,030 \\
5,030 \\
* 4,940\end{array}$ & $\begin{array}{l}4,280 \\
4,280 \\
4,280 \\
4,280 \\
4,320\end{array}$ \\
\hline $\begin{array}{l}16 \\
17 \\
18 \\
19 \\
20\end{array}$ & $\begin{array}{l}5,480 \\
5,520 \\
5,430 \\
5,340 \\
4,980\end{array}$ & $\begin{array}{l}2,730 \\
2,590 \\
2,620 \\
2,800 \\
2,800\end{array}$ & $\begin{array}{l}4,000 \\
3,870 \\
3,790 \\
3,790 \\
3,870\end{array}$ & $\begin{array}{l}4,320 \\
4,320 \\
4,580 \\
4,580 \\
4,580\end{array}$ & $\begin{array}{l}4,400 \\
4,160 \\
5,040 \\
\frac{8,200}{5,660}\end{array}$ & $\begin{array}{l}6,800 \\
6,400 \\
6,020 \\
7,800 \\
8,750\end{array}$ & $\begin{array}{l}\frac{10,600}{11,700} \\
14,600 \\
17,400 \\
19,700\end{array}$ & $\begin{array}{l}12,800 \\
12,850 \\
14,000 \\
14,150 \\
14,950\end{array}$ & $\begin{array}{l}7,000 \\
6,900 \\
7,000 \\
7,000 \\
7,000\end{array}$ & $\begin{array}{l}5,930 \\
5,840 \\
5,750 \\
6,300 \\
6,400\end{array}$ & $\begin{array}{l}5,030 \\
5,030 \\
4,940 \\
4,940 \\
4,940\end{array}$ & $\begin{array}{l}4,670 \\
4,670 \\
4,670 \\
4,620 \\
4,120\end{array}$ \\
\hline $\begin{array}{l}21 \\
22 \\
23 \\
24 \\
25\end{array}$ & $\begin{array}{r}3,710 \\
* 4,800 \\
5,430 \\
5,480 \\
5,520\end{array}$ & $\begin{array}{l}2,870 \\
2,800 \\
2,840 \\
2,800 \\
2,840\end{array}$ & $\begin{array}{l}4,420 \\
4,670 \\
4,400 \\
3,920 \\
3,760\end{array}$ & $\begin{array}{r}4,490 \\
4,400 \\
4,400 \\
4,490 \\
* 5,030 \\
\end{array}$ & $\begin{array}{l}5,120 \\
4,760 \\
4,580 \\
4,940 \\
5,750\end{array}$ & $\begin{array}{l}9,300 \\
9,740 \\
9,740 \\
9,740 \\
9,630\end{array}$ & $\begin{array}{l}* 21,100 \\
22,800 \\
23,800 \\
\frac{24}{24,700}\end{array}$ & $\begin{array}{l}14,950 \\
14,250 \\
13,500 \\
12,850 \\
12,550\end{array}$ & $\begin{array}{l}6,900 \\
6,800 \\
6,800 \\
6,800 \\
7,000\end{array}$ & $\begin{array}{l}5,880 \\
5,800 \\
5,840 \\
5,750 \\
5,750\end{array}$ & $\begin{array}{l}5,030 \\
4,940 \\
4,940 \\
4,940 \\
4,940\end{array}$ & $\begin{array}{l}4,120 \\
4,160 \\
4,240 \\
4,120 \\
3,880\end{array}$ \\
\hline $\begin{array}{l}26 \\
27 \\
28 \\
29 \\
30 \\
31\end{array}$ & $\begin{array}{l}5,480 \\
5,430 \\
5,070 \\
5,430 \\
5,160 \\
5,160\end{array}$ & $\begin{array}{c}2,800 \\
2,870 \\
3,080 \\
3,120 \\
3,120 \\
-\end{array}$ & $\begin{array}{l}3,680 \\
3,680 \\
4,160 \\
4,160 \\
4,080 \\
4,000\end{array}$ & $\begin{array}{l}4,850 \\
4,850 \\
4,760 \\
4,850 \\
4,720 \\
4,620\end{array}$ & $\begin{array}{c}5,390 \\
5,120 \\
5,120 \\
- \\
= \\
-\end{array}$ & $\begin{array}{r}9,410 \\
9,300 \\
9,960 \\
11,700 \\
12,400 \\
12,000\end{array}$ & $\begin{array}{c}24,000 \\
22,800 \\
21,300 \\
20,300 \\
19,600 \\
-\end{array}$ & $\begin{array}{l}12,050 \\
11,550 \\
11,150 \\
10,850 \\
10,550 \\
10,250\end{array}$ & $\begin{array}{l}7,200 \\
6,900 \\
6,900 \\
6,800 \\
6,700 \\
-\end{array}$ & $\begin{array}{l}6,400 \\
7,200 \\
6,250 \\
5,840 \\
5,660 \\
5,570\end{array}$ & $\begin{array}{l}4,900 \\
4,900 \\
4,900 \\
4,940 \\
4,980 \\
5,030\end{array}$ & $\begin{array}{r}3,960 \\
3,960 \\
3,920 \\
4,000 \\
* 3,520 \\
-\end{array}$ \\
\hline $\begin{array}{l}\text { Total } \\
\text { Mean } \\
\text { Ac-ft } \\
\end{array}$ & $\begin{array}{r}165,780 \\
5,348 \\
328,800 \\
\end{array}$ & $\begin{array}{r}114,910 \\
3,830 \\
227,900 \\
\end{array}$ & $\begin{array}{r}116,700 \\
3,765 \\
231,500 \\
\end{array}$ & $\begin{array}{r}138,670 \\
4,473 \\
275,000 \\
\end{array}$ & $\begin{array}{r}134,160 \\
4,791 \\
266,100\end{array}$ & $\begin{array}{r}320,780 \\
10,350 \\
636,300\end{array}$ & $\begin{array}{r}485,500 \\
16,180 \\
963,000\end{array}$ & $\begin{array}{r}434,750 \\
14,020 \\
862,300 \\
\end{array}$ & $\begin{array}{r}228,980 \\
7,633 \\
454,200 \\
\end{array}$ & $\begin{array}{r}191,890 \\
6,190 \\
380,600 \\
\end{array}$ & $\begin{array}{r}158,100 \\
5,100 \\
313,600\end{array}$ & $\begin{array}{r}132,620 \\
4,421 \\
263,000\end{array}$ \\
\hline $\begin{array}{l}\text { Calend } \\
\text { Water }\end{array}$ & year 17 & $\begin{array}{l}\text { 1958: } \operatorname{Max} \\
\text { 8-59: } \operatorname{Max}\end{array}$ & $\begin{array}{l}34,200 \\
27,170\end{array}$ & $\begin{array}{ll}\text { Min } & 2,590 \\
\text { Min } & 2,59\end{array}$ & $\begin{array}{ll}0 & \text { Mean } 7 \\
0 & \text { Mean } 7\end{array}$ & $\begin{array}{ll}667 & \mathrm{Ac}- \\
186 \mathrm{Ac}-\end{array}$ & $\begin{array}{l}\text { ft } 5,550 \text {, } \\
\text { ft } 5,202 \text {, }\end{array}$ & 500 & & & & \\
\hline
\end{tabular}

*Discharge measurement made on this day. 
Helmand River at Darweshan, Afghanistan

Discharge, in cubic feet per second, water year October 1959 to September 1960

\begin{tabular}{|c|c|c|c|c|c|c|c|c|c|c|c|c|}
\hline Eay & Oct. & Nov. & Dec. & Jan. & Feb. & Mar. & Apr. & May & June & July & Aug. & Sept. \\
\hline $\begin{array}{l}1 \\
2 \\
3 \\
4 \\
5\end{array}$ & $\begin{array}{l}3,520 \\
3,600 \\
3,360 \\
3,280 \\
3,240\end{array}$ & $\begin{array}{l}3,160 \\
3,160 \\
3,200 \\
3,240 \\
3,400\end{array}$ & $\begin{array}{l}\frac{3,120}{3,440} \\
\frac{8,4,80}{5,120} \\
4,240\end{array}$ & $\begin{array}{l}6,200 \\
6,800 \\
6,800 \\
6,600 \\
6,500\end{array}$ & $\begin{array}{r}4,000 \\
4,000 \\
3,960 \\
* 3,870 \\
3,520\end{array}$ & $\begin{array}{l}2,250 \\
2,250 \\
2,190 \\
2,140 \\
1,940\end{array}$ & $\begin{array}{l}3,800 \\
3,840 \\
3,600 \\
3,560 \\
3,680\end{array}$ & $\begin{array}{l}6,950 \\
6,650 \\
6,650 \\
7,100 \\
7,150\end{array}$ & $\begin{array}{l}14,200 \\
13,600 \\
13,100 \\
12,600 \\
12,400\end{array}$ & $\begin{array}{l}\frac{4,200}{4,040} \\
3,800 \\
3,560 \\
3,400\end{array}$ & $\begin{array}{l}2,060 \\
2,060 \\
2,040 \\
2,040 \\
2,040\end{array}$ & $\begin{array}{l}2,120 \\
2,120 \\
2,090 \\
2,020 \\
2,020\end{array}$ \\
\hline $\begin{array}{r}6 \\
7 \\
8 \\
9 \\
10\end{array}$ & $\begin{array}{l}3,200 \\
3,200 \\
3,200 \\
3,160 \\
3,120\end{array}$ & $\begin{array}{l}\frac{3,480}{3,440} \\
3,440 \\
3,440 \\
3,320\end{array}$ & $\begin{array}{l}4,080 \\
3,840 \\
3,760 \\
3,680 \\
3,640\end{array}$ & $\begin{array}{l}6,400 \\
6,440 \\
6,300 \\
6,200 \\
6,160\end{array}$ & $\begin{array}{l}3,560 \\
3,680 \\
3,560 \\
3,560 \\
3,520\end{array}$ & $\begin{array}{l}2,090 \\
2,060 \\
2,020 \\
\frac{1,820}{2,040}\end{array}$ & $\begin{array}{l}3,520 \\
3,280 \\
3,120 \\
3,040 \\
3,000\end{array}$ & $\begin{array}{l}7,050 \\
6,650 \\
6,250 \\
6,260 \\
7,800\end{array}$ & $\begin{array}{l}12,000 \\
11,600 \\
11,400 \\
11,200 \\
10,900\end{array}$ & $\begin{array}{r}3,360 \\
* 3,280 \\
3,120 \\
3,040 \\
2,920\end{array}$ & $\begin{array}{l}2,040 \\
2,040 \\
2,020 \\
1,990 \\
1,960\end{array}$ & $\begin{array}{l}2,020 \\
2,340 \\
2,400 \\
2,370 \\
2,370\end{array}$ \\
\hline $\begin{array}{l}11 \\
12 \\
13 \\
14 \\
15\end{array}$ & $\begin{array}{l}3,120 \\
3,120 \\
3,120 \\
3,120 \\
3,120\end{array}$ & $\begin{array}{r}2,960 \\
* 2,880 \\
2,840 \\
2,800 \\
2,770\end{array}$ & $\begin{array}{l}3,840 \\
5,840 \\
5,980 \\
5,980 \\
6,200\end{array}$ & $\begin{array}{l}4,800 \\
4,440 \\
4,440 \\
4,360 \\
4,280\end{array}$ & $\begin{array}{l}3,360 \\
3,200 \\
3,120 \\
3,040 \\
2,960\end{array}$ & $\begin{array}{r}2,250 \\
2,370 \\
* 2,930 \\
* 6,110 \\
7,500 \\
\end{array}$ & $\begin{array}{l}2,960 \\
2,880 \\
2,880 \\
3,040 \\
3,600\end{array}$ & $\begin{array}{r}10,100 \\
12,400 \\
14,400 \\
16,300 \\
* 18,200\end{array}$ & $\begin{array}{r}10,600 \\
10,300 \\
9,900 \\
9,360 \\
8,800\end{array}$ & $\begin{array}{l}2,770 \\
2,680 \\
2,620 \\
2,560 \\
2,500\end{array}$ & $\begin{array}{l}1,960 \\
1,940 \\
1,940 \\
1,940 \\
1,900\end{array}$ & $\begin{array}{r}* 2,370 \\
2,250 \\
2,250 \\
2,250 \\
2,250\end{array}$ \\
\hline $\begin{array}{l}16 \\
17 \\
18 \\
19 \\
20\end{array}$ & $\begin{array}{l}3,120 \\
3,040 \\
3,000 \\
3,040 \\
3,040\end{array}$ & $\begin{array}{l}2,770 \\
2,770 \\
2,770 \\
2,740 \\
2,740\end{array}$ & $\begin{array}{l}6,400 \\
6,500 \\
6,450 \\
6,500 \\
6,450\end{array}$ & $\begin{array}{l}4,280 \\
4,230 \\
4,280 \\
4,280 \\
4,120\end{array}$ & $\begin{array}{l}2,800 \\
2,620 \\
2,560 \\
2,460 \\
2,500\end{array}$ & $\begin{array}{l}6,300 \\
5,120 \\
4,490 \\
4,160 \\
3,960\end{array}$ & $\begin{array}{r}4,400 \\
12,200 \\
20,200 \\
17,400 \\
12,500\end{array}$ & $\begin{array}{l}19,700 \\
21,000 \\
22,300 \\
23,500 \\
23,700\end{array}$ & $\begin{array}{l}8,200 \\
8,000 \\
7,600 \\
7,000 \\
6,600\end{array}$ & $\begin{array}{l}2,400 \\
2,250 \\
2,220 \\
2,190 \\
2,190\end{array}$ & $\begin{array}{l}1,900 \\
1,920 \\
1,920 \\
1,940 \\
1,990\end{array}$ & $\begin{array}{l}2,310 \\
2,310 \\
2,250 \\
2,310 \\
2,280\end{array}$ \\
\hline $\begin{array}{l}21 \\
22 \\
23 \\
24 \\
25\end{array}$ & $\begin{array}{l}\frac{2,960}{2,960} \\
3,000 \\
3,040 \\
3,040\end{array}$ & $\begin{array}{l}2,740 \\
2,740 \\
2,740 \\
2,740 \\
2,740\end{array}$ & $\begin{array}{r}6,400 \\
6,350 \\
6,350 \\
* 6,250 \\
6,200\end{array}$ & $\begin{array}{l}4,160 \\
4,160 \\
4,160 \\
4,160 \\
4,080\end{array}$ & $\begin{array}{l}2,500 \\
2,500 \\
2,460 \\
2,430 \\
2,430\end{array}$ & $\begin{array}{l}4,320 \\
3,480 \\
3,280 \\
3,200 \\
3,040\end{array}$ & $\begin{array}{r}* 9,900 \\
10,400 \\
9,680 \\
9,240 \\
7,200\end{array}$ & $\begin{array}{l}23,300 \\
23,000 \\
22,300 \\
21,300 \\
20, \infty 00\end{array}$ & $\begin{array}{l}6,250 \\
5,980 \\
5,700 \\
5,480 \\
5,260\end{array}$ & $\begin{array}{l}2,190 \\
2,250 \\
2,310 \\
2,310 \\
2,280\end{array}$ & $\begin{array}{l}2,020 \\
1,960 \\
1,940 \\
2,140 \\
2,160\end{array}$ & $\begin{array}{l}2,280 \\
2,280 \\
2,310 \\
2,310 \\
2,310\end{array}$ \\
\hline $\begin{array}{l}26 \\
27 \\
28 \\
29 \\
30 \\
31 \\
\end{array}$ & $\begin{array}{l}3,040 \\
3,000 \\
3,040 \\
3,080 \\
3,120 \\
3,160 \\
\end{array}$ & $\begin{array}{c}2,770 \\
2,960 \\
3,040 \\
3,080 \\
3,160 \\
- \\
\end{array}$ & $\begin{array}{r}6,200 \\
6,200 \\
6,110 \\
6,110 \\
6,110 \\
6,110 \\
\end{array}$ & $\begin{array}{l}4,000 \\
4,000 \\
4,000 \\
4,000 \\
4,000 \\
4,000\end{array}$ & $\begin{array}{c}2,370 \\
2,370 \\
2,370 \\
2,310 \\
- \\
- \\
\end{array}$ & $\begin{array}{l}3,080 \\
3,320 \\
4,800 \\
4,800 \\
4,160 \\
3,920 \\
\end{array}$ & $\begin{array}{c}6,700 \\
7,900 \\
7,650 \\
7,650 \\
7,450 \\
-\end{array}$ & $\begin{array}{l}18,900 \\
17,900 \\
17,000 \\
16,000 \\
15,500 \\
14,700\end{array}$ & $\begin{array}{l}5,080 \\
4,800 \\
4,620 \\
4,400 \\
4,360 \\
\end{array}$ & $\begin{array}{l}2,340 \\
2,340 \\
2,220 \\
2,120 \\
2,060 \\
2,090 \\
\end{array}$ & $\begin{array}{l}2,160 \\
2,160 \\
2,140 \\
2,140 \\
2,160 \\
2,220 \\
\end{array}$ & $\begin{array}{l}2,370 \\
2,400 \\
2,370 \\
2,400 \\
2,500 \\
-\end{array}$ \\
\hline $\begin{array}{l}\text { Total } \\
\text { Hean }\end{array}$ & $\begin{array}{r}97,160 \\
3,134\end{array}$ & $\begin{array}{r}90,030 \\
3,001 \\
178,600\end{array}$ & $\begin{array}{r}171,930 \\
5,546 \\
341,000\end{array}$ & $\begin{array}{r}152,640 \\
4,924 \\
302,800\end{array}$ & $\begin{array}{r}87,590 \\
3,020 \\
173,700 \\
\end{array}$ & $\begin{array}{r}107,390 \\
3,464 \\
213,000\end{array}$ & $\begin{array}{r}200,270 \\
6,676 \\
397,200 \\
\end{array}$ & $\begin{array}{r}459,910 \\
14,840 \\
912,200\end{array}$ & $\begin{array}{r}261,290 \\
8,710 \\
518,300 \\
\end{array}$ & $\begin{array}{r}83,610 \\
2,697 \\
165,800 \\
\end{array}$ & $\begin{array}{r}62,840 \\
2,027 \\
124,600 \\
\end{array}$ & $\begin{array}{r}68,230 \\
2,274 \\
135,300 \\
\end{array}$ \\
\hline
\end{tabular}

Ac-ft $192,700 \quad 178,600 \quad 341,0001$

Calendar year 1959: $\operatorname{Max} 27,170$ Min 2,740 Mean 7,081 Ac-ft 5,126,000

Water year 1959-60: Max 23,700 Min 1,8

*Discharge measurement made on this day. 
METXAND RIVER BASTN

Helnand River at Chahar Burjak, Afghanistan

Iecetzog.- Lat $30^{\circ} 15^{\prime} \mathrm{H}$, , long $62^{\circ}$ 00 B., on right bank 1 k kilomoters downstrean from Chahar Burjak, about

00 dilomoters from Bud-i-Seletan and Iran boundary, and about 320 kdlometers downstream from Arghandab River.

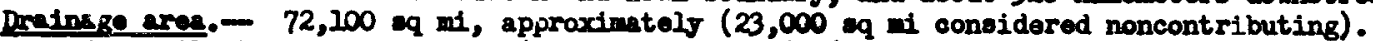

Pecorde available.- October 1946 to September 1960 (monthly discharge only Dctober 1946 to September 1948,

Dotobai 1951 to April 1952, July to Septamber 1952, Fobruary, Karch 1954).

fare.- Water-tage recorder. Altitude of gage 500 meters, approximately.

Arese discharge.- 14 years 7,157 of (adjurted for storege) 5,181,000 acre-ft per year.

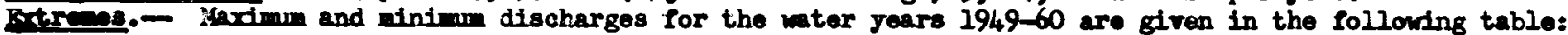

\begin{tabular}{|c|c|c|c|c|c|c|}
\hline & & \multicolumn{2}{|c|}{$\frac{\text { Yaxdmim }}{2}$} & \multicolumn{3}{|c|}{ Giniman } \\
\hline $\begin{array}{l}\text { Whter } \\
\text { Tear }\end{array}$ & Date & $\begin{array}{l}\text { Cage Height } \\
\text { (meters) }\end{array}$ & $\begin{array}{c}\text { Discharge } \\
\text { (ofe) }\end{array}$ & Dete & $\begin{array}{l}\text { Gege Helght } \\
\text { (moters) }\end{array}$ & $\begin{array}{c}\text { Discharge } \\
\text { (cfs) }\end{array}$ \\
\hline $\begin{array}{l}1949 \\
1950 \\
1951 \\
1952 \\
1953 \\
1954 \\
1955 \\
1956 \\
1957 \\
1958 \\
1959 \\
1960\end{array}$ & 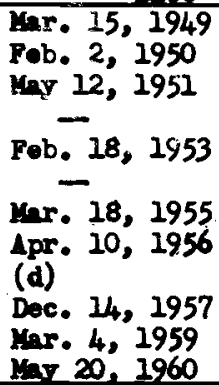 & $\begin{array}{c}12.23 \\
11.30 \\
- \\
- \\
- \\
10.02 \\
11.25 \\
21.98 \\
10.20 \\
9.31 \\
9.55\end{array}$ & $\begin{array}{c}80,000 \\
57,300 \\
\mathrm{~b} 56,000 \\
(\mathrm{c}) \\
\mathrm{b} 10,000 \\
(\mathrm{c}) \\
30,400 \\
57,200 \\
117,000 \\
31,900 \\
32,300 \\
25,400\end{array}$ & $\begin{array}{l}\text { Oct. } 1,1948 \\
\text { Oct. } 1,2,7-9,1949 \\
\text { Oct. } 1,1950 \\
\text { Oct. } 1-8,1952 \\
\text { Dec. } 11,1953 \\
\text { Dec. } 13,1954 \\
\text { Oct. } 5,1955 \\
\text { Aug. } 24,25,1957 \\
\text { Nov. } 20,1957 \\
\text { Nov. } 20,1958 \\
\text { Lug. } 7-9,1960\end{array}$ & $\begin{array}{l}= \\
= \\
= \\
z \\
\overline{7.565} \\
\bar{z} \\
7.10 \\
7.535 \\
7.23\end{array}$ & $\begin{array}{r}\mathrm{b} 510 \\
760 \\
\mathrm{~b} 860 \\
(\mathrm{c}) \\
\mathrm{b} 1,000 \\
\mathrm{~b} 740 \\
\mathrm{~b} 700 \\
2,420 \\
\mathrm{~b} 1,000 \\
812 \\
2,820 \\
1,660\end{array}$ \\
\hline
\end{tabular}

* From rating curve extended above 30,000 cfs on besis of velocity-area studies.

Proa rloodertes.

b Kan daily discharge.

c lot deterrined.

d Yay have oceurred on Kar. 21 or Apr. 7 or May 7, 2957.

A discharg of $91,500 \mathrm{cfs}$ in 1903 and a discharge in excess of $600,000 \mathrm{cfs}$ in 1885 were computed by slopearea nothod in the lower Helmand River by a Britioh river commiseion in 1903-5.

Renrks.- Records good except those for water years 1950, 1952, which are fair, and those for periods of no gage-holght record, wioh are poor. Discharge regulated by Kajakai Reservoir on Helmand River and Arghandab Beserrotr on Arghandab River, also by extensive irrigation throughout the river basin. 
Helmand River at Chahar Burjak, Afghanistan

Monthly discharge, water year October 1946 to September 1947

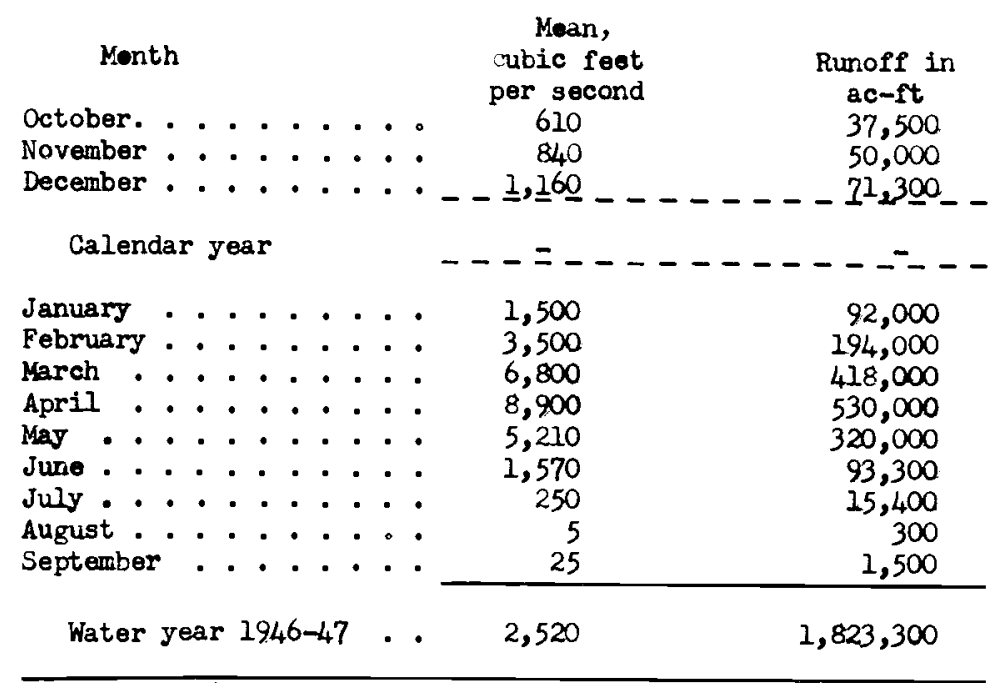

Note. - Discharge estimated on basis of streamflow data collected at upstream sites.

Monthly discharge, water year October 1947 to September 1948

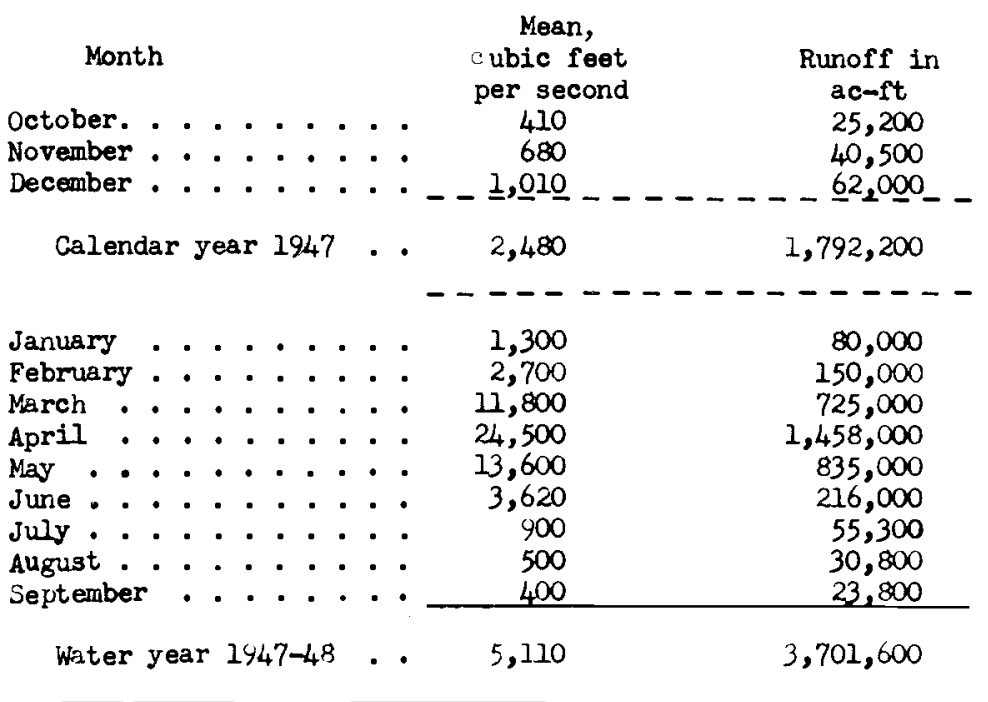

Note. - Discharge estimated on basis of streanflow data collected at upstrean sites. 
HEIMAND RIVER BASIN

Helmand River at Chahar Burjak, Afghanistan

Discharge, in cubic feet per second, water year October 1948 to September 1949

\begin{tabular}{|c|c|c|c|c|c|c|c|c|c|c|c|c|}
\hline Dar & Oct.a & Nor, & Dec. & Jan. & Feb. & Mar. $_{0}$ & Apr. & May & June & July & Aug. & Sept. \\
\hline $\begin{array}{l}1 \\
2 \\
3 \\
4 \\
5\end{array}$ & $\begin{array}{l}\frac{510}{520} \\
520 \\
530 \\
540\end{array}$ & $\begin{array}{l}\frac{780}{790} \\
800 \\
810 \\
820\end{array}$ & $\begin{array}{l}\frac{1,100}{1,100} \\
1,100 \\
1,100 \\
1,100\end{array}$ & $\begin{array}{l}1,500 \\
1,600 \\
1,500 \\
1,400 \\
1,550\end{array}$ & $\begin{array}{r}2,180 \\
2,130 \\
2,180 \\
6,400 \\
10,300 \\
\end{array}$ & $\begin{array}{l}4,910 \\
4,450 \\
3,960 \\
3,760 \\
3,690\end{array}$ & $\begin{array}{l}\frac{15,000}{18,000} \\
24,000 \\
22,000 \\
27,000\end{array}$ & $\begin{array}{l}\frac{28,860}{27,920} \\
27,020 \\
26,120 \\
25,220\end{array}$ & $\begin{array}{l}\frac{8,390}{8,040} \\
7,780 \\
7,530 \\
7,280\end{array}$ & $\begin{array}{l}\frac{2,960}{2,850} \\
2,740 \\
2,630 \\
2,530\end{array}$ & $\begin{array}{l}1,060 \\
1,040 \\
1,040 \\
1,040 \\
1,040\end{array}$ & $\begin{array}{l}760 \\
760 \\
744 \\
728 \\
712\end{array}$ \\
\hline $\begin{array}{r}6 \\
7 \\
8 \\
9 \\
10\end{array}$ & $\begin{array}{l}540 \\
550 \\
550 \\
560 \\
560\end{array}$ & $\begin{array}{l}830 \\
840 \\
850 \\
860 \\
870\end{array}$ & $\begin{array}{l}1,100 \\
1,100 \\
1,100 \\
1,100 \\
1,100\end{array}$ & $\begin{array}{r}1,700 \\
* 1,800 \\
1,700 \\
1,600 \\
1,600\end{array}$ & $\begin{array}{l}7,700 \\
6,440 \\
5,650 \\
5,320 \\
4,760\end{array}$ & $\begin{array}{l}3,560 \\
3,440 \\
3,560 \\
3,690 \\
3,820\end{array}$ & $\begin{array}{l}31,000 \\
30,000 \\
32,000 \\
32,000 \\
32,000\end{array}$ & $\begin{array}{l}24,330 \\
23,480 \\
22,630 \\
21,780 \\
20,940\end{array}$ & $\begin{array}{l}7,040 \\
6,800 \\
6,560 \\
6,340 \\
6,110\end{array}$ & $\begin{array}{l}2,420 \\
2,320 \\
2,220 \\
2,120 \\
2,080\end{array}$ & $\begin{array}{r}1,040 \\
994 \\
928 \\
928 \\
906\end{array}$ & $\begin{array}{l}712 \\
696 \\
712 \\
696 \\
696\end{array}$ \\
\hline $\begin{array}{l}11 \\
12 \\
13 \\
14 \\
15\end{array}$ & $\begin{array}{l}570 \\
580 \\
580 \\
590 \\
600\end{array}$ & $\begin{array}{l}880 \\
890 \\
900 \\
920 \\
930\end{array}$ & $\begin{array}{l}1,100 \\
1,100 \\
1,150 \\
1,150 \\
1,150\end{array}$ & $\begin{array}{r}1,550 \\
1,550 \\
1,500 \\
* 1,440 \\
1,250 \\
\end{array}$ & $\begin{array}{l}4,170 \\
3,690 \\
3,500 \\
3,440 \\
3,320\end{array}$ & $\begin{array}{r}3,960 \\
6,710 \\
28,670 \\
42,530 \\
80,000 \\
\end{array}$ & $\begin{array}{l}31,000 \\
32,000 \\
32,000 \\
45,000 \\
74,600\end{array}$ & $\begin{array}{l}20,140 \\
19,390 \\
18,590 \\
17,750 \\
17,000\end{array}$ & $\begin{array}{l}5,880 \\
5,660 \\
5,450 \\
5,230 \\
5,020\end{array}$ & $\begin{array}{l}2,040 \\
1,990 \\
1,940 \\
1,900 \\
1,860\end{array}$ & $\begin{array}{r}884 \\
928 \\
1,350 \\
1,320 \\
1,230\end{array}$ & $\begin{array}{l}696 \\
712 \\
712 \\
712 \\
728\end{array}$ \\
\hline $\begin{array}{l}16 \\
17 \\
18 \\
19 \\
20\end{array}$ & $\begin{array}{l}600 \\
610 \\
620 \\
630 \\
640\end{array}$ & $\begin{array}{r}250 \\
970 \\
990 \\
1,000 \\
1,000\end{array}$ & $\begin{array}{l}1,200 \\
1,200 \\
1,200 \\
1,200 \\
1,200\end{array}$ & $\begin{array}{l}1,290 \\
1,320 \\
1,290 \\
1,400 \\
1,470\end{array}$ & $\begin{array}{l}3,260 \\
3,260 \\
3,320 \\
3,200 \\
3,140\end{array}$ & $\begin{array}{l}70,000 \\
50,000 \\
30,000 \\
20,000 \\
\omega_{4}, 000\end{array}$ & $\begin{array}{l}70,600 \\
55,000 \\
46,000 \\
44,000 \\
41,000\end{array}$ & $\begin{array}{l}16,260 \\
15,560 \\
14,820 \\
14,220 \\
13,580\end{array}$ & $\begin{array}{l}4,820 \\
4,680 \\
4,550 \\
4,420 \\
4,290\end{array}$ & $\begin{array}{l}1,810 \\
1,760 \\
1,720 \\
1,680 \\
1,650\end{array}$ & $\begin{array}{r}1,090 \\
994 \\
906 \\
840 \\
808\end{array}$ & $\begin{array}{l}728 \\
696 \\
696 \\
712 \\
680 \\
\end{array}$ \\
\hline $\begin{array}{l}21 \\
22 \\
23 \\
24 \\
25\end{array}$ & $\begin{array}{l}650 \\
660 \\
670 \\
680 \\
700\end{array}$ & $\begin{array}{r}1,000 \\
1,000 \\
1,000 \\
1,000 \\
* 1,000\end{array}$ & $\begin{array}{l}1,200 \\
1,200 \\
1,200 \\
1,200 \\
1,200\end{array}$ & $\begin{array}{r}1,470 \\
1,550 \\
1,620 \\
* 1,620 \\
1,700\end{array}$ & $\begin{array}{r}3,080 \\
3,080 \\
3,020 \\
* 3,020 \\
3,080\end{array}$ & $\begin{array}{l}12,000 \\
11,500 \\
11,000 \\
11,500 \\
11,500\end{array}$ & $\begin{array}{l}38,000 \\
36,000 \\
35,000 \\
34,000 \\
34,000\end{array}$ & $\begin{array}{l}12,980 \\
12,380 \\
11,780 \\
11,190 \\
10,640\end{array}$ & $\begin{array}{l}4,160 \\
4,030 \\
3,900 \\
3,780 \\
3,660\end{array}$ & $\begin{array}{r}1,570 \\
1,540 \\
1,500 \\
* 1,460 \\
1,320\end{array}$ & $\begin{array}{l}808 \\
792 \\
792 \\
760 \\
760\end{array}$ & $\begin{array}{r}728 \\
744 \\
728 \\
+728 \\
744\end{array}$ \\
\hline $\begin{array}{l}26 \\
27 \\
28 \\
29 \\
30 \\
31\end{array}$ & $\begin{array}{l}710 \\
720 \\
730 \\
740 \\
760 \\
770 \\
\end{array}$ & $\begin{array}{c}1,050 \\
1,050 \\
1,050 \\
1,050 \\
1,050 \\
=\end{array}$ & $\begin{array}{l}1,200 \\
1,200 \\
1,200 \\
1,250 \\
1,300 \\
1,400 \\
1\end{array}$ & $\begin{array}{l}1,790 \\
1,910 \\
1,960 \\
2,220 \\
\frac{2,270}{2,180}\end{array}$ & $\begin{array}{c}3,440 \\
5,060 \\
5,480 \\
= \\
= \\
=\end{array}$ & $\begin{array}{l}11,500 \\
12,000 \\
13,000 \\
16,000 \\
15,000 \\
15,000\end{array}$ & $\begin{array}{r}34,000 \\
36,000 \\
40,000 \\
\times 30,810 \\
29,810 \\
-\end{array}$ & $\begin{array}{r}10,100 \\
10,640 \\
10,100 \\
9,610 \\
9,130 \\
8,760\end{array}$ & $\begin{array}{c}3,540 \\
3,420 \\
3,300 \\
3,180 \\
\frac{3,070}{-}\end{array}$ & $\begin{array}{l}1,290 \\
1,230 \\
1,180 \\
1,150 \\
1,120 \\
1,040 \\
1\end{array}$ & $\begin{array}{l}766 \\
776 \\
776 \\
760 \\
760 \\
744 \\
\end{array}$ & $\begin{array}{c}712 \\
728 \\
776 \\
776 \\
760 \\
-\end{array}$ \\
\hline $\begin{array}{l}\text { Total } \\
\text { Mean } \\
\text { Ac-ft }\end{array}$ & $\begin{array}{r}19,190 \\
619 \\
38,060\end{array}$ & $\begin{array}{r}27,930 \\
931 \\
55,400\end{array}$ & $\begin{array}{r}36,200 \\
1,168 \\
71,800\end{array}$ & $\begin{array}{r}50,300 \\
1,623 \\
99,770\end{array}$ & $\begin{array}{r}116,620 \\
4,165 \\
231,300\end{array}$ & $\begin{array}{r}526,010 \\
16,970 \\
1,043,000\end{array}$ & $\begin{array}{r}1,081,820 \\
36,060 \\
2,146,000\end{array}$ & $\begin{array}{r}532,870 \\
17,190 \\
1,057,000 \\
\end{array}$ & $\begin{array}{r}157,910 \\
5,264 \\
313,200\end{array}$ & $\begin{array}{r}57,620 \\
1,859 \\
114,300\end{array}$ & $\begin{array}{r}28,854 \\
731 \\
57,230 \\
\end{array}$ & $\begin{array}{r}21,712 \\
724 \\
43,060\end{array}$ \\
\hline
\end{tabular}

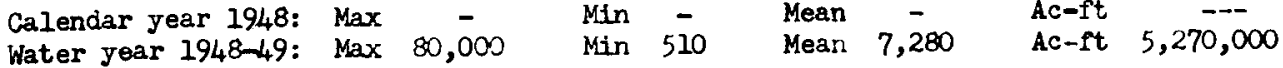

* Discharge measurement made on this day.

Note. - lo gage-height record Oct. 1 to Jan. 13, Mar. 15 to Aur. $\mathcal{U}_{4}$, Apr. 16-28, Apr. 29 to July 23; discharge estimated on basis of estimated gage heights during recession, discharge measurements, flocdmark stages Mar. 15 and Apr. 15, and records for stations upstream. 
HELMAND RIVER BASIN

Helmand River at Chahar Burjak, Afghanistan

Discharge, in cubic feet per second, water year October 1949 to September 1950

\begin{tabular}{|c|c|c|c|c|c|c|c|c|c|c|c|c|}
\hline Day & Oct. & Nove & $\mathrm{Dec}_{2}$ & Jan. & Feb. & Mar. & Apr. & May & June & July & Aug. & Sept. \\
\hline $\begin{array}{l}1 \\
2 \\
3 \\
4 \\
5\end{array}$ & $\begin{array}{l}\frac{760}{760} \\
776 \\
792 \\
808\end{array}$ & $\begin{array}{r}\frac{950}{972} \\
994 \\
994 \\
1,020\end{array}$ & $\begin{array}{l}1,180 \\
1,230 \\
1,320 \\
1,320 \\
1,320\end{array}$ & $\begin{array}{l}1,320 \\
1,290 \\
1,320 \\
1,350 \\
1,420\end{array}$ & $\begin{array}{r}21,100 \\
55,000 \\
28,290 \\
6,640 \\
5,520\end{array}$ & $\begin{array}{l}4,700 \\
5,000 \\
5,400 \\
5,800 \\
6,400\end{array}$ & $\begin{array}{l}\frac{9,800}{10,000} \\
11,000 \\
14,000 \\
28,000\end{array}$ & $\begin{array}{l}\frac{18,060}{19,820} \\
21,610 \\
22,800 \\
23,990\end{array}$ & $\begin{array}{l}\frac{20,000}{19,500} \\
18,500 \\
18,000 \\
17,000\end{array}$ & $\begin{array}{l}4,600 \\
\frac{4}{4,400} \\
4,200 \\
4,000 \\
3,800\end{array}$ & $\begin{array}{l}1,580 \\
1,580 \\
1,510 \\
1,470 \\
1,440\end{array}$ & $\begin{array}{l}\frac{880}{880} \\
860 \\
840 \\
820\end{array}$ \\
\hline $\begin{array}{r}6 \\
7 \\
8 \\
9 \\
10\end{array}$ & $\begin{array}{l}776 \\
760 \\
760 \\
760 \\
776\end{array}$ & $\begin{array}{l}1,020 \\
1,020 \\
1,040 \\
1,040 \\
1,040\end{array}$ & $\begin{array}{l}1,290 \\
1,320 \\
1,290 \\
1,260 \\
1,230\end{array}$ & $\begin{array}{l}1,460 \\
1,420 \\
1,420 \\
1,350 \\
1,290\end{array}$ & $\begin{array}{l}5,000 \\
5,160 \\
5,020 \\
5,020 \\
6,340\end{array}$ & $\begin{array}{l}6,800 \\
7,000 \\
7,000 \\
6,500 \\
6,300\end{array}$ & $\begin{array}{l}\frac{40,000}{28,000} \\
22,000 \\
19,000 \\
17,000\end{array}$ & $\begin{array}{l}24,330 \\
25,040 \\
27,020 \\
28,480 \\
31,000\end{array}$ & $\begin{array}{l}15,000 \\
15,500 \\
14,500 \\
14,000 \\
13,500\end{array}$ & $\begin{array}{l}3,600 \\
3,400 \\
3,200 \\
3,100 \\
3,000\end{array}$ & $\begin{array}{l}1,400 \\
1,360 \\
1,360 \\
1,320 \\
1,290\end{array}$ & $\begin{array}{l}800 \\
800 \\
800 \\
800 \\
800\end{array}$ \\
\hline $\begin{array}{l}11 \\
12 \\
13 \\
14 \\
15\end{array}$ & $\begin{array}{l}840 \\
840 \\
840 \\
884 \\
884\end{array}$ & $\begin{array}{l}1,060 \\
1,060 \\
1,060 \\
1,120 \\
1,150\end{array}$ & $\begin{array}{l}1,260 \\
1,260 \\
1,290 \\
1,390 \\
1,420\end{array}$ & $\begin{array}{l}1,260 \\
1,260 \\
1,290 \\
1,390 \\
1,500\end{array}$ & $\begin{array}{l}6,600 \\
7,000 \\
6,800 \\
6,000 \\
5,300\end{array}$ & $\begin{array}{l}6,200 \\
6,400 \\
6,900 \\
7,700 \\
9,500\end{array}$ & $\begin{array}{l}16,000 \\
16,000 \\
16,000 \\
18,000 \\
20,000\end{array}$ & $\begin{array}{l}35,000 \\
33,000 \\
31,000 \\
34,000 \\
39,000\end{array}$ & $\begin{array}{l}13,000 \\
12,000 \\
11,500 \\
11,000 \\
10,500\end{array}$ & $\begin{array}{r}2,900 \\
* 2,760 \\
2,760 \\
2,710 \\
2,600\end{array}$ & $\begin{array}{l}1,220 \\
1,190 \\
1,130 \\
1,100 \\
1,080\end{array}$ & $\begin{array}{l}800 \\
800 \\
800 \\
\frac{780}{780}\end{array}$ \\
\hline $\begin{array}{l}16 \\
17 \\
18 \\
19 \\
20\end{array}$ & $\begin{array}{l}884 \\
884 \\
862 \\
884 \\
840\end{array}$ & $\begin{array}{l}1,150 \\
1,180 \\
1,090 \\
1,090 \\
1,090\end{array}$ & $\begin{array}{l}\frac{1,500}{1,500} \\
1,460 \\
1,420 \\
1,420\end{array}$ & $\begin{array}{l}1,540 \\
1,570 \\
1,610 \\
1,610 \\
1,570\end{array}$ & $\begin{array}{l}5,000 \\
4,800 \\
4,700 \\
4,600 \\
4,500\end{array}$ & $\begin{array}{r}11,500 \\
10,500 \\
9,500 \\
9,000 \\
6,600\end{array}$ & $\begin{array}{l}23,000 \\
26,000 \\
30,000 \\
27,000 \\
24,000\end{array}$ & $\begin{array}{l}\frac{41,480}{39,000} \\
37,000 \\
36,000 \\
35,000\end{array}$ & $\begin{array}{l}9,800 \\
9,400 \\
9,000 \\
8,500 \\
8,000\end{array}$ & $\begin{array}{l}2,500 \\
2,410 \\
2,320 \\
2,320 \\
2,270\end{array}$ & $\begin{array}{r}1,050 \\
989 \\
960 \\
960 \\
989\end{array}$ & $\begin{array}{l}780 \\
800 \\
800 \\
800 \\
800\end{array}$ \\
\hline $\begin{array}{l}21 \\
22 \\
23 \\
24 \\
25\end{array}$ & $\begin{array}{l}862 \\
840 \\
840 \\
862 \\
884\end{array}$ & $\begin{array}{l}1,090 \\
1,090 \\
1,120 \\
1,120 \\
1,120\end{array}$ & $\begin{array}{l}1,460 \\
1,420 \\
1,390 \\
1,390 \\
1,350\end{array}$ & $\begin{array}{l}1,540 \\
1,570 \\
1,570 \\
1,610 \\
1,540\end{array}$ & $\begin{array}{l}4,400 \\
4,300 \\
4,300 \\
4,300 \\
4,300\end{array}$ & $\begin{array}{r}8,4,00 \\
8,400 \\
8,600 \\
11,000 \\
13,000\end{array}$ & $\begin{array}{r}* 20,300 \\
19,820 \\
18,700 \\
17,450 \\
17,000\end{array}$ & $\begin{array}{l}36,000 \\
35,000 \\
34,000 \\
32,000 \\
30,000\end{array}$ & $\begin{array}{l}7,600 \\
7,200 \\
6,800 \\
6,500 \\
6,200\end{array}$ & $\begin{array}{l}2,220 \\
2,130 \\
2,040 \\
2,040 \\
1,960\end{array}$ & $\begin{array}{l}989 \\
989 \\
989 \\
960 \\
960\end{array}$ & $\begin{array}{l}800 \\
800 \\
800 \\
800 \\
800\end{array}$ \\
\hline $\begin{array}{l}26 \\
27 \\
28 \\
29 \\
30 \\
31 \\
\end{array}$ & $\begin{array}{l}884 \\
906 \\
906 \\
294 \\
950 \\
928\end{array}$ & $\begin{array}{c}1,150 \\
1,180 \\
1,230 \\
1,200 \\
1,200 \\
-\end{array}$ & $\begin{array}{l}1,390 \\
1,460 \\
1,420 \\
1,390 \\
1,390 \\
1,350 \\
\end{array}$ & $\begin{array}{l}1,570 \\
1,610 \\
1,500 \\
1,540 \\
1,570 \\
1,610\end{array}$ & $\begin{array}{c}4,300 \\
4,400 \\
4,500 \\
- \\
=\end{array}$ & $\begin{array}{r}17,000 \\
14,000 \\
12,000 \\
11,000 \\
10,000 \\
9,800\end{array}$ & $\begin{array}{c}16,120 \\
15,560 \\
15,140 \\
15,420 \\
16,260 \\
-\end{array}$ & $\begin{array}{l}28,000 \\
26,000 \\
25,000 \\
23,000 \\
22,000 \\
21,000\end{array}$ & $\begin{array}{c}5,800 \\
5,500 \\
5,200 \\
5,000 \\
4,800 \\
-\end{array}$ & $\begin{array}{l}1,910 \\
1,910 \\
1,870 \\
1,830 \\
1,750 \\
1,700 \\
\end{array}$ & $\begin{array}{l}960 \\
960 \\
940 \\
940 \\
920 \\
900 \\
\end{array}$ & $\begin{array}{c}800 \\
800 \\
820 \\
820 \\
840 \\
-\end{array}$ \\
\hline $\begin{array}{l}\text { Total } \\
\text { Mean } \\
\text { sic-ft }\end{array}$ & $\begin{array}{r}26,226 \\
846 \\
52,020\end{array}$ & $\begin{array}{r}32,640 \\
1,088 \\
64,740\end{array}$ & $\begin{array}{r}42,090 \\
1,358 \\
83,480\end{array}$ & $\begin{array}{r}45,470 \\
1,457 \\
90,1 \times 0\end{array}$ & $\begin{array}{r}233,490 \\
8,339 \\
463,100\end{array}$ & $\begin{array}{r}269,900 \\
8,706 \\
535,300\end{array}$ & $\begin{array}{r}586,570 \\
19,550 \\
1,163,000\end{array}$ & $\begin{array}{r}914,630 \\
29,500 \\
1,814,000\end{array}$ & $\begin{array}{r}329,800 \\
10,990 \\
654,100\end{array}$ & $\begin{array}{r}84,210 \\
2,716 \\
167,000\end{array}$ & $\begin{array}{r}35,485 \\
1,145 \\
70,300\end{array}$ & $\begin{array}{r}24,300 \\
810 \\
1+8,200\end{array}$ \\
\hline
\end{tabular}

Calendar year 1949: Max 80,000 Mir. 680 Mear 7,328 Ac-ft 5,305,000 Valendar year 1949: Max 80,000 Mir 760 liean 7,191 Ac-ft 5,206,000

\footnotetext{
* Discharge measurement made on this day.

Dote- - Vo lote- No tage-height record dar: 20 to Apr.
} 
HETMAND RIVER BASIN

Helmand River at Chahar Burjak, Afghanistan

Discharge, in cubic feet per second, water year October 1950 to September 1951

\begin{tabular}{|c|c|c|c|c|c|c|c|c|c|c|c|c|}
\hline Day & Oct. & Nov. & Dec. & Jan. & Feb. & Mar. & Apr. & May & June & July & Auge & Sept. \\
\hline $\begin{array}{l}1 \\
2 \\
3 \\
4 \\
5\end{array}$ & $\begin{array}{l}\frac{880}{900} \\
900 \\
920 \\
920\end{array}$ & $\begin{array}{l}1,300 \\
1,300 \\
1,300 \\
1,300 \\
* 1,320\end{array}$ & $\begin{array}{l}1,470 \\
1,500 \\
1,550 \\
1,580 \\
1,580\end{array}$ & $\begin{array}{l}1,420 \\
1,420 \\
1,420 \\
1,440 \\
1,440\end{array}$ & $\begin{array}{l}1,690 \\
\frac{1}{1,750} \\
1,750 \\
1,750 \\
1,750\end{array}$ & $\begin{array}{l}3,000 \\
3,100 \\
3,300 \\
3,500 \\
3,700\end{array}$ & $\begin{array}{l}25,000 \\
21,000 \\
19,000 \\
18,000 \\
18,000\end{array}$ & $\begin{array}{l}29,100 \\
30,400 \\
31,500 \\
32,400 \\
32,900\end{array}$ & $\begin{array}{l}\frac{24,000}{24,000} \\
23,000 \\
22,000 \\
21,000\end{array}$ & $\begin{array}{l}\frac{6,000}{5,600} \\
5,400 \\
5,200 \\
5,000\end{array}$ & $\begin{array}{l}1,900 \\
1,800 \\
1,700 \\
1,700 \\
1,600\end{array}$ & $\begin{array}{l}1,100 \\
1,100 \\
1,100 \\
1,100 \\
1,100\end{array}$ \\
\hline $\begin{array}{r}6 \\
7 \\
8 \\
9 \\
10\end{array}$ & $\begin{array}{r}920 \\
940 \\
960 \\
960 \\
1,000\end{array}$ & $\begin{array}{l}1,340 \\
1,370 \\
1,370 \\
1,370 \\
1,340\end{array}$ & $\begin{array}{l}1,580 \\
1,580 \\
1,550 \\
1,500 \\
1,470\end{array}$ & $\begin{array}{l}1,470 \\
1,470 \\
1,500 \\
1,500 \\
1,520\end{array}$ & $\begin{array}{l}1,880 \\
3,070 \\
2,700 \\
2,410 \\
2,550\end{array}$ & $\begin{array}{l}4,000 \\
4,500 \\
5,000 \\
5,500 \\
6,000\end{array}$ & $\begin{array}{l}18,000 \\
17,000 \\
17,000 \\
16,000 \\
15,000\end{array}$ & $\begin{array}{l}32,500 \\
31,500 \\
31,500 \\
33,500 \\
40,000\end{array}$ & $\begin{array}{l}20,000 \\
19,000 \\
18,000 \\
17,000 \\
16,000\end{array}$ & $\begin{array}{l}4,800 \\
4,600 \\
4,400 \\
4,300 \\
4,200\end{array}$ & $\begin{array}{l}1,600 \\
1,600 \\
1,500 \\
1,500 \\
1,500\end{array}$ & $\begin{array}{l}1,100 \\
1,100 \\
1,100 \\
1,100 \\
1,100\end{array}$ \\
\hline $\begin{array}{l}11 \\
12 \\
13 \\
14 \\
15\end{array}$ & $\begin{array}{l}1,050 \\
1,050 \\
1,100 \\
1,100 \\
1,100\end{array}$ & $\begin{array}{l}1,370 \\
1,370 \\
1,370 \\
1,390 \\
1,420\end{array}$ & $\begin{array}{l}1,470 \\
1,470 \\
1,470 \\
1,500 \\
1,520\end{array}$ & $\begin{array}{l}1,520 \\
1,520 \\
1,550 \\
1,600 \\
1,650\end{array}$ & $\begin{array}{l}\frac{4,670}{3,500} \\
3,000 \\
2,700 \\
2,500\end{array}$ & $\begin{array}{r}7,000 \\
9,000 \\
10,000 \\
11,000 \\
10,000\end{array}$ & $\begin{array}{l}15,000 \\
15,000 \\
17,000 \\
20,000 \\
24,000\end{array}$ & $\begin{array}{l}50,000 \\
56,000 \\
50,000 \\
45,000 \\
40,000\end{array}$ & $\begin{array}{l}15,000 \\
14,000 \\
13,000 \\
13,000 \\
12,000\end{array}$ & $\begin{array}{l}4,000 \\
3,800 \\
3,600 \\
3,500 \\
3,300\end{array}$ & $\begin{array}{l}1,400 \\
1,400 \\
1,300 \\
1,300 \\
1,300\end{array}$ & $\begin{array}{l}1,100 \\
1,100 \\
1,100 \\
1,100 \\
1,100\end{array}$ \\
\hline $\begin{array}{l}16 \\
17 \\
18 \\
19 \\
20\end{array}$ & $\begin{array}{l}1,150 \\
1,150 \\
1,150 \\
1,150 \\
1,150\end{array}$ & $\begin{array}{l}1,390 \\
1,370 \\
1,370 \\
1,370 \\
1,370\end{array}$ & $\begin{array}{l}1,520 \\
1,550 \\
1,520 \\
1,500 \\
1,470\end{array}$ & $\begin{array}{l}1,650 \\
1,700 \\
1,700 \\
1,700 \\
1,650\end{array}$ & $\begin{array}{l}2,400 \\
2,400 \\
2,500 \\
2,400 \\
2,400\end{array}$ & $\begin{array}{r}8,600 \\
8,000 \\
8,400 \\
12,000 \\
14,000\end{array}$ & $\begin{array}{l}26,000 \\
27,000 \\
27,000 \\
27,000 \\
27,000\end{array}$ & $\begin{array}{l}36,000 \\
37,000 \\
32,200 \\
30,400 \\
29,600\end{array}$ & $\begin{array}{l}11,000 \\
11,000 \\
10,500 \\
10,000 \\
10,000\end{array}$ & $\begin{array}{l}3,200 \\
3,100 \\
2,900 \\
2,800 \\
2,700\end{array}$ & $\begin{array}{l}1,250 \\
1,200 \\
1,200 \\
1,200 \\
1,150\end{array}$ & $\begin{array}{l}1,100 \\
1,100 \\
1,100 \\
1,100 \\
1,100\end{array}$ \\
\hline $\begin{array}{l}21 \\
22 \\
23 \\
24 \\
25\end{array}$ & $\begin{array}{l}1,180 \\
1,200 \\
1,220 \\
1,240 \\
1,250\end{array}$ & $\begin{array}{l}1,370 \\
1,370 \\
1,390 \\
1,390 \\
1,420\end{array}$ & $\begin{array}{l}1,470 \\
1,440 \\
1,440 \\
1,440 \\
1,440\end{array}$ & $\begin{array}{r}1,640 \\
1,640 \\
1,660 \\
1,660 \\
* 1,660\end{array}$ & $\begin{array}{l}2,400 \\
2,500 \\
2,700 \\
3,000 \\
2,700\end{array}$ & $\begin{array}{l}13,700 \\
13,000 \\
12,200 \\
11,400 \\
11,000\end{array}$ & $\begin{array}{l}28,000 \\
\frac{29,000}{28,000} \\
27,000 \\
27,000\end{array}$ & $\begin{array}{l}29,200 \\
29,100 \\
29,100 \\
28,800 \\
28,400\end{array}$ & $\begin{array}{l}9,600 \\
9,000 \\
8,600 \\
8,000 \\
7,500\end{array}$ & $\begin{array}{l}2,600 \\
2,500 \\
2,400 \\
2,400 \\
2,300\end{array}$ & $\begin{array}{l}1,150 \\
1,150 \\
1,150 \\
1,150 \\
1,150\end{array}$ & $\begin{array}{l}1,100 \\
1,100 \\
1,100 \\
1,100 \\
1,100\end{array}$ \\
\hline $\begin{array}{l}26 \\
27 \\
28 \\
29 \\
30 \\
31\end{array}$ & $\begin{array}{l}1,260 \\
1,260 \\
1,270 \\
1,300 \\
1,300 \\
1,300\end{array}$ & $\begin{array}{l}1,440 \\
1,440 \\
1,470 \\
1,500 \\
1,500 \\
\end{array}$ & $\begin{array}{l}1,440 \\
1,470 \\
1,440 \\
1,440 \\
1,440 \\
1,420 \\
\end{array}$ & $\begin{array}{l}1,660 \\
1,640 \\
1,640 \\
1,660 \\
1,690 \\
1,660\end{array}$ & $\begin{array}{c}2,600 \\
2,700 \\
2,800 \\
= \\
- \\
-\end{array}$ & $\begin{array}{l}11,500 \\
12,700 \\
14,000 \\
18,000 \\
27,000 \\
29,000 \\
\end{array}$ & $\begin{array}{c}29,000 \\
28,200 \\
27,600 \\
27,200 \\
28,100 \\
-\end{array}$ & $\begin{array}{l}27,800 \\
27,200 \\
26,600 \\
25,800 \\
25,200 \\
24,500\end{array}$ & $\begin{array}{l}7,200 \\
7,000 \\
6,800 \\
6,600 \\
6,400 \\
-\end{array}$ & $\begin{array}{l}2,200 \\
2,200 \\
2,100 \\
2,100 \\
2,000 \\
1,900 \\
\end{array}$ & $\begin{array}{l}\frac{1,100}{1,100} \\
1,100 \\
1,100 \\
1,100 \\
1,100\end{array}$ & $\begin{array}{c}1,100 \\
1,100 \\
1,100 \\
1,100 \\
1,100 \\
-\end{array}$ \\
\hline $\begin{array}{l}\text { Total } \\
\text { Mean } \\
\text { Ac-ft }\end{array}$ & $\begin{array}{r}34,230 \\
1,104 \\
67,890\end{array}$ & $\begin{array}{r}41,390 \\
1,380 \\
82,100\end{array}$ & $\begin{array}{r}46,230 \\
1,491 \\
91,700\end{array}$ & $\begin{array}{r}49,050 \\
1,582 \\
97,290\end{array}$ & $\begin{array}{r}71,170 \\
2,542 \\
141,200 \\
\end{array}$ & $\begin{array}{r}313,100 \\
10,100 \\
621,000\end{array}$ & $\begin{array}{r}688,100 \\
22,940 \\
1,365,000\end{array}$ & $\begin{array}{r}1,033,200 \\
33,330 \\
2,049,000\end{array}$ & $\begin{array}{r}400,200 \\
13,340 \\
793,800\end{array}$ & $\begin{array}{r}107,100 \\
3,455 \\
212,400\end{array}$ & $\begin{array}{r}41,450 \\
1,337 \\
82,210\end{array}$ & $\begin{array}{r}33,000 \\
1,100 \\
65,450\end{array}$ \\
\hline$t \in I$ & & & & & 880 & Mean & 831 & $-f t$ & 00 & & & \\
\hline
\end{tabular}

* Discharge measurement mad on this day.

Note. - No gage-height record Oct. 1-31, Jan. 5-24, Feb. 12 to Apr. 26, May 10-17, May 31 to Sept. 30 ; discharge estimated on basis of upstream records at Kajakai Dam and Arghandab River near Kala Bist

and discharge measurements. 
Helmand River at Chahar Burjak, Afgharistan

Discharge, in cubic feet per second, water year October 1951 to september 1952

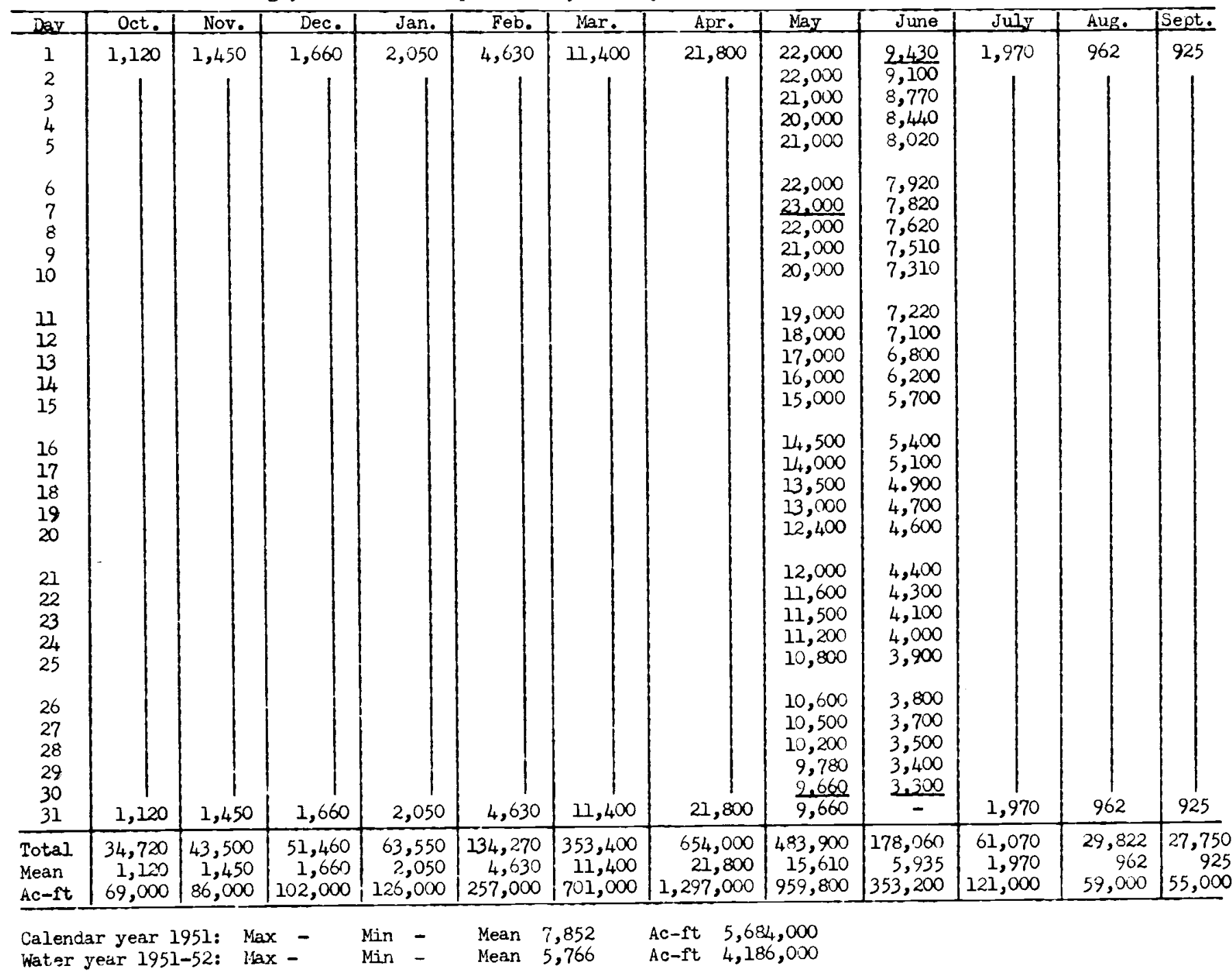

( June 12 to Sept. 30 ; discharge for May 1-19, June 12-30 estimated or basis of upstream records; montrily discharge for remainder of year computed on basis of upstream $r \in c o r d s$ and discharge ratios for other years. 
HEIMAND RIVER BASIN

Helmand River at Chahar Burjak, Afghanistan

Discharge, in cubic feet per second, water jear October 1952 to September 1953

\begin{tabular}{|c|c|c|c|c|c|c|c|c|c|c|c|c|}
\hline Dar & Oct, & Nov. & Dec. & Jan. & Feb, & Mar. & Apr. & May & Jurie & July & Aug. & Septe. \\
\hline $\begin{array}{l}1 \\
2 \\
3 \\
4 \\
5\end{array}$ & $\begin{array}{l}1,000 \\
1,000 \\
1,000 \\
1,000 \\
1,000\end{array}$ & $\begin{array}{l}1,300 \\
1,350 \\
1,350 \\
1,350 \\
1,350\end{array}$ & $\begin{array}{l}1,450 \\
1,450 \\
1,450 \\
1,450 \\
1,450\end{array}$ & $\begin{array}{l}1,500 \\
1,600 \\
1,650 \\
1,650 \\
1,700\end{array}$ & $\begin{array}{l}2,670 \\
2,620 \\
2,250 \\
2,170 \\
2,120 \\
\end{array}$ & $\begin{array}{l}3,030 \\
3,900 \\
3,960 \\
3,840 \\
3,590\end{array}$ & $\begin{array}{l}\frac{2,970}{3,900} \\
4,020 \\
4,140 \\
4,080\end{array}$ & $\begin{array}{l}5,260 \\
5,040 \\
5,040 \\
5,110 \\
5,180\end{array}$ & $\begin{array}{l}5,560 \\
5,980 \\
6,060 \\
5,890 \\
6,140\end{array}$ & $\begin{array}{l}\frac{5,180}{5,180} \\
5,080 \\
5,080 \\
5,080\end{array}$ & $\begin{array}{l}4,680 \\
4,740 \\
4,680 \\
4,740 \\
4,610\end{array}$ & $\begin{array}{l}4,480 \\
4,540 \\
4,540 \\
4,510 \\
4,410\end{array}$ \\
\hline $\begin{array}{r}6 \\
7 \\
8 \\
9 \\
10\end{array}$ & $\begin{array}{l}1,000 \\
1,000 \\
1,000 \\
1,050 \\
1,050\end{array}$ & $\begin{array}{l}1,350 \\
1,350 \\
1,350 \\
1,350 \\
1,350\end{array}$ & $\begin{array}{l}1,450 \\
1,450 \\
1,450 \\
1,500 \\
1,500\end{array}$ & $\begin{array}{l}1,700 \\
1,700 \\
1,700 \\
1,700 \\
1,650\end{array}$ & $\begin{array}{l}2,120 \\
2,120 \\
2,120 \\
2,170 \\
2,300\end{array}$ & $\begin{array}{l}3,530 \\
3,530 \\
3,530 \\
3,530 \\
3,420\end{array}$ & $\begin{array}{l}4,080 \\
4,210 \\
4,210 \\
4,410 \\
4,210\end{array}$ & $\begin{array}{l}5,110 \\
5,180 \\
5,180 \\
5,260 \\
5,110\end{array}$ & $\begin{array}{l}5,810 \\
5,730 \\
5,640 \\
5,640 \\
5,730\end{array}$ & $\begin{array}{l}4,140 \\
2,970 \\
2,620 \\
2,870 \\
4,480\end{array}$ & $\begin{array}{l}4,580 \\
4,610 \\
4,610 \\
4,540 \\
4,580\end{array}$ & $\begin{array}{l}4,340 \\
4,410 \\
4,410 \\
4,480 \\
4,540\end{array}$ \\
\hline $\begin{array}{l}11 \\
12 \\
13 \\
14 \\
15\end{array}$ & $\begin{array}{l}1,050 \\
1,050 \\
1,100 \\
1,100 \\
1,100\end{array}$ & $\begin{array}{l}1,350 \\
1,350 \\
1,350 \\
1,350 \\
1,350\end{array}$ & $\begin{array}{l}1,500 \\
1,500 \\
1,500 \\
1,500 \\
1,500\end{array}$ & $\begin{array}{l}1,650 \\
1,600 \\
1,600 \\
1,600 \\
1,600\end{array}$ & $\begin{array}{l}2,340 \\
2,470 \\
4,530 \\
9,900 \\
7,210\end{array}$ & $\begin{array}{l}3,530 \\
3,770 \\
3,840 \\
3,770 \\
3,710\end{array}$ & $\begin{array}{l}4,270 \\
4,210 \\
4,410 \\
4,740 \\
5,320\end{array}$ & $\begin{array}{l}5,110 \\
5,180 \\
5,260 \\
5,110 \\
5,180\end{array}$ & $\begin{array}{r}* 5,730 \\
5,730 \\
5,640 \\
5,640 \\
5,640\end{array}$ & $\begin{array}{l}4,740 \\
4,810 \\
4,840 \\
4,740 \\
4,880\end{array}$ & $\begin{array}{r}4,680 \\
* 4,710 \\
4,610 \\
4,610 \\
4,680\end{array}$ & $\begin{array}{l}\frac{4,610}{4,440} \\
4,340 \\
4,340 \\
4,340\end{array}$ \\
\hline $\begin{array}{l}16 \\
17 \\
18 \\
19 \\
20\end{array}$ & $\begin{array}{l}1,100 \\
1,100 \\
1,100 \\
1,100 \\
1,100\end{array}$ & $\begin{array}{l}1,350 \\
1,350 \\
1,350 \\
1,350 \\
1,400\end{array}$ & $\begin{array}{l}1,500 \\
1,500 \\
1,500 \\
1,550 \\
1,550\end{array}$ & $\begin{array}{l}1,700 \\
1,900 \\
2,200 \\
2,400 \\
2,450\end{array}$ & $\begin{array}{r}6,800 \\
7,600 \\
10,000 \\
8,000 \\
6,500\end{array}$ & $\begin{array}{l}3,710 \\
3,710 \\
3,710 \\
3,710 \\
3,770\end{array}$ & $\begin{array}{l}\frac{7,410}{5,980} \\
5,560 \\
5,410 \\
5,340\end{array}$ & $\begin{array}{l}5,260 \\
5,260 \\
5,260 \\
5,180 \\
5,180\end{array}$ & $\begin{array}{l}5,560 \\
5,520 \\
5,410 \\
5,340 \\
5,340\end{array}$ & $\begin{array}{l}4,860 \\
4,840 \\
4,920 \\
5,000 \\
4,880\end{array}$ & $\begin{array}{l}4,610 \\
4,540 \\
4,610 \\
4,480 \\
4,610\end{array}$ & $\begin{array}{l}4,340 \\
4,410 \\
4,020 \\
3,250 \\
2,940\end{array}$ \\
\hline $\begin{array}{l}21 \\
22 \\
23 \\
24 \\
25\end{array}$ & $\begin{array}{l}1,100 \\
1,100 \\
1,100 \\
1,100 \\
1,100\end{array}$ & $\begin{array}{l}1,400 \\
1,400 \\
1,400 \\
1,400 \\
1,400\end{array}$ & $\begin{array}{l}1,550 \\
1,600 \\
1,600 \\
1,600 \\
1,600\end{array}$ & $\begin{array}{r}* 2,470 \\
2,470 \\
2,470 \\
2,470 \\
2,470\end{array}$ & $\begin{array}{l}5,500 \\
4,800 \\
4,300 \\
3,900 \\
3,700\end{array}$ & $\begin{array}{l}3,770 \\
3,770 \\
3,770 \\
3,710 \\
3,710\end{array}$ & $\begin{array}{l}5,260 \\
5,260 \\
5,260 \\
5,340 \\
5,340\end{array}$ & $\begin{array}{l}5,180 \\
5,260 \\
5,260 \\
5,340 \\
5,410\end{array}$ & $\begin{array}{l}5,180 \\
5,180 \\
5,180 \\
5,340 \\
5,480\end{array}$ & $\begin{array}{l}4,810 \\
4,810 \\
4,740 \\
4,680 \\
4,740\end{array}$ & $\begin{array}{l}4,740 \\
4,680 \\
4,640 \\
4,640 \\
4,680\end{array}$ & $\begin{array}{l}2,820 \\
2,820 \\
2,870 \\
2,820 \\
2,770\end{array}$ \\
\hline $\begin{array}{l}26 \\
27 \\
28 \\
29 \\
30 \\
31\end{array}$ & $\begin{array}{l}1,150 \\
1,200 \\
1,200 \\
1,200 \\
1,250 \\
1,300\end{array}$ & $\begin{array}{c}1,400 \\
1,400 \\
1,400 \\
1,450 \\
1,450 \\
=\end{array}$ & $\begin{array}{l}1,600 \\
1,600 \\
1,600 \\
1,600 \\
1,600 \\
1,600\end{array}$ & $\begin{array}{l}2,520 \\
2,520 \\
2,470 \\
2,470 \\
2,520 \\
2,570 \\
\end{array}$ & $\begin{array}{c}3,500 \\
3,300 \\
* 3,140 \\
- \\
- \\
-\end{array}$ & $\begin{array}{l}3,710 \\
3,770 \\
3,530 \\
2,670 \\
2,300 \\
2,080 \\
\end{array}$ & $\begin{array}{c}5,180 \\
5,180 \\
5,260 \\
5,180 \\
5,110 \\
-\end{array}$ & $\begin{array}{l}5,560 \\
5,480 \\
5,480 \\
5,410 \\
5,480 \\
5,560\end{array}$ & $\begin{array}{c}5,410 \\
5,180 \\
5,040 \\
5,000 \\
5,040 \\
\end{array}$ & $\begin{array}{l}4,740 \\
4,740 \\
4,840 \\
4,680 \\
4,610 \\
4,610\end{array}$ & $\begin{array}{l}4,680 \\
4,610 \\
4,810 \\
4,740 \\
4,740 \\
4,610\end{array}$ & $\begin{array}{c}\frac{2,720}{2,770} \\
2,820 \\
2,820 \\
2,720 \\
-\end{array}$ \\
\hline $\begin{array}{l}\text { Total } \\
\text { Mean } \\
\text { Ac-ft }\end{array}$ & $\begin{array}{r}33,800 \\
1,090 \\
67,040\end{array}$ & $\begin{array}{r}41,100 \\
1,370 \\
81,520\end{array}$ & $\begin{array}{r}47,250 \\
1,524 \\
93,720\end{array}$ & $\begin{array}{r}62,770 \\
2,025 \\
124,500\end{array}$ & $\begin{array}{r}119,550 \\
4,270 \\
237,100\end{array}$ & $\begin{array}{r}109,880 \\
3,545 \\
217,900\end{array}$ & $\begin{array}{r}144,250 \\
4,808 \\
286,100\end{array}$ & $\begin{array}{r}162,870 \\
5,254 \\
323,000\end{array}$ & $\begin{array}{r}165,760 \\
5,525 \\
328,800\end{array}$ & $\begin{array}{r}143,210 \\
4,620 \\
284,100\end{array}$ & $\begin{array}{r}144,080 \\
4,648 \\
285,800\end{array}$ & $\begin{array}{r}113,640 \\
3,788 \\
225,400\end{array}$ \\
\hline
\end{tabular}

Calendar jear 1952: Max - Min - $\quad$ Mean 5,759 Ac-ft 4, 171,000

Water year 1952-53: $\operatorname{Max} 10,000$ Min 1,000 Mean 3,529 Ac-ft 2,555,000

* Discharge measurement made on this day.

Note. - No gage-hejght record 0ct. I to Jan, 20, Feb. 16-27, Apr. 15; discharge estimated on basis of records for below Kajakal Reservoir, Arghandab River near Kala Bist and discharge measurements. 
HEIMAND RIVER BASIN

Helmand River at Chahar Burjak, Afghanistan

Discharge, in cubic feet per second, water year October 1953 to September 1954

\begin{tabular}{|c|c|c|c|c|c|c|c|c|c|c|c|c|}
\hline Day & oct. & Nov. & Dec. & $\operatorname{Jan}$. & Feb. & Mar. & Apr. & May & June & Jury & Aug. & Sept. \\
\hline $\begin{array}{l}1 \\
2 \\
3 \\
4 \\
5\end{array}$ & $\begin{array}{l}2,670 \\
2,670 \\
2,380 \\
2,080 \\
1,840\end{array}$ & $\begin{array}{l}2,740 \\
2,700 \\
2,670 \\
2,640 \\
2,620\end{array}$ & $\begin{array}{l}\frac{2,800}{2,800} \\
2,800 \\
2,800 \\
2,800\end{array}$ & $\begin{array}{l}773 \\
756 \\
756 \\
823 \\
815\end{array}$ & 18,910 & 12,200 & $\begin{array}{l}25,000 \\
33,000 \\
32,000 \\
27,000 \\
23,000\end{array}$ & $\begin{array}{l}\frac{32,000}{32,000} \\
32,000 \\
32,000 \\
32,000\end{array}$ & $\begin{array}{l}\frac{15,000}{15,000} \\
15,000 \\
14,500 \\
14,000\end{array}$ & $\begin{array}{l}\frac{7,000}{5,000} \\
2,500 \\
1,500 \\
1,200\end{array}$ & $\begin{array}{l}3,100 \\
3,040 \\
3,100 \\
3,100 \\
3,180\end{array}$ & $\begin{array}{l}\frac{3,400}{3,400} \\
3,400 \\
3,250 \\
3,250\end{array}$ \\
\hline $\begin{array}{r}6 \\
7 \\
8 \\
9 \\
10\end{array}$ & $\begin{array}{l}1,690 \\
2,200 \\
2,520 \\
2,570 \\
2,620\end{array}$ & $\begin{array}{l}2,600 \\
2,570 \\
2,600 \\
2,600 \\
2,600\end{array}$ & $\begin{array}{r}2,800 \\
2,800 \\
1,600 \\
1,110 \\
* 839\end{array}$ & $\begin{array}{r}764 \\
881 \\
1,290 \\
1,340 \\
1,400\end{array}$ & & & $\begin{array}{l}20,000 \\
18,000 \\
17,000 \\
16,000 \\
15,000\end{array}$ & $\begin{array}{l}32,000 \\
32,000 \\
32,000 \\
32,000 \\
32,000\end{array}$ & $\begin{array}{l}13,500 \\
13,000 \\
13,000 \\
12,500 \\
12,500\end{array}$ & $\begin{array}{r}1,100 \\
* 1,080 \\
2,000 \\
3,550 \\
3,480\end{array}$ & $\begin{array}{l}3,180 \\
3,180 \\
3,180 \\
3,040 \\
2,980\end{array}$ & $\begin{array}{l}3,100 \\
3,040 \\
2,980 \\
2,980 \\
2,980\end{array}$ \\
\hline $\begin{array}{l}11 \\
12 \\
13 \\
14 \\
15\end{array}$ & $\begin{array}{r}2,620 \\
2,570 \\
2,520 \\
* 2,520 \\
2,540\end{array}$ & $\begin{array}{l}2,600 \\
2,600 \\
2,620 \\
2,620 \\
2,540\end{array}$ & $\begin{array}{l}740 \\
1,380 \\
2,040 \\
2,140 \\
2,190\end{array}$ & $\begin{array}{l}1,470 \\
3,670 \\
4,680 \\
4,680 \\
4,680\end{array}$ & & & $\begin{array}{l}15,000 \\
14,000 \\
14,000 \\
13,000 \\
13,000\end{array}$ & $\begin{array}{l}32,000 \\
31,000 \\
30,000 \\
29,000 \\
29,000\end{array}$ & $\begin{array}{l}12,000 \\
12,000 \\
11,500 \\
11,000 \\
11,000\end{array}$ & $\begin{array}{l}3,100 \\
2,920 \\
2,800 \\
2,800 \\
2,980\end{array}$ & $\begin{array}{l}\frac{2,920}{2,980} \\
2,980 \\
2,980 \\
3,040\end{array}$ & $\begin{array}{l}2,860 \\
2,740 \\
2,770 \\
2,770 \\
2,770\end{array}$ \\
\hline $\begin{array}{l}16 \\
17 \\
18 \\
19 \\
20\end{array}$ & $\begin{array}{l}2,540 \\
2,540 \\
2,600 \\
2,600 \\
2,600\end{array}$ & $\begin{array}{r}\mathrm{dl}, 250 \\
\mathrm{~d} 820 \\
\mathrm{~d} \overline{8200} \\
\mathrm{dl}, 200 \\
\mathrm{~d} 2,000\end{array}$ & $\begin{array}{l}2,230 \\
2,300 \\
2,300 \\
2,300 \\
2,300\end{array}$ & $\begin{array}{l}4,680 \\
4,680 \\
4,680 \\
4,610 \\
4,680\end{array}$ & & & $\begin{array}{l}13,000 \\
13,000 \\
13,000 \\
13,000 \\
13,000\end{array}$ & $\begin{array}{l}27,000 \\
25,000 \\
23,000 \\
22,000 \\
21,000\end{array}$ & $\begin{array}{r}10,500 \\
10,500 \\
9,500 \\
9,000 \\
8,500\end{array}$ & $\begin{array}{l}2,980 \\
2,980 \\
2,980 \\
3,040 \\
3,040\end{array}$ & $\begin{array}{l}3,040 \\
3,100 \\
3,100 \\
3,040 \\
3,040\end{array}$ & $\begin{array}{l}2,770 \\
2,770 \\
2,830 \\
2,890 \\
2,830\end{array}$ \\
\hline $\begin{array}{l}21 \\
22 \\
23 \\
24 \\
25\end{array}$ & $\begin{array}{l}2,570 \\
2,570 \\
2,600 \\
2,600 \\
2,600\end{array}$ & $\begin{array}{l}2,210 \\
2,520 \\
2,570 \\
2,570 \\
2,600\end{array}$ & $\begin{array}{l}2,300 \\
2,340 \\
2,340 \\
2,340 \\
2,340\end{array}$ & $\begin{array}{l}4,680 \\
4,740 \\
4,960 \\
5,340 \\
5,680\end{array}$ & & & $\begin{array}{l}\frac{12,500}{13,300} \\
16,100 \\
19,700 \\
24,000\end{array}$ & $\begin{array}{l}20,500 \\
20,000 \\
19,500 \\
19,000 \\
18,500\end{array}$ & $\begin{array}{l}8,500 \\
8,500 \\
8,200 \\
8,100 \\
7,900\end{array}$ & $\begin{array}{l}2,800 \\
2,680 \\
2,620 \\
2,620 \\
2,620\end{array}$ & $\begin{array}{l}2,980 \\
3,040 \\
3,320 \\
3,320 \\
3,320\end{array}$ & $\begin{array}{l}2,830 \\
2,800 \\
2,770 \\
2,740 \\
2,680\end{array}$ \\
\hline $\begin{array}{l}26 \\
27 \\
28 \\
29 \\
30 \\
31\end{array}$ & $\begin{array}{l}2,600 \\
2,670 \\
2,700 \\
2,700 \\
2,670 \\
2,670\end{array}$ & $\begin{array}{c}2,600 \\
2,800 \\
2,800 \\
2,800 \\
2,800 \\
-\end{array}$ & $\begin{array}{r}2,340 \\
2,340 \\
1,730 \\
1,210 \\
966 \\
839\end{array}$ & $\begin{array}{l}\frac{5,890}{5,770} \\
5,680 \\
5,640 \\
5,170 \\
5,000\end{array}$ & $\begin{array}{c}18,910 \\
- \\
- \\
-\end{array}$ & $\left.\right|_{12,200}$ & $\begin{array}{c}27,400 \\
31,200 \\
33,300 \\
34,100 \\
34,000 \\
-\end{array}$ & $\begin{array}{l}18,000 \\
17,000 \\
16,500 \\
16,000 \\
16,000 \\
15,500 \\
\end{array}$ & $\begin{array}{l}7,800 \\
7,700 \\
7,700 \\
7,700 \\
7,600 \\
-\end{array}$ & $\begin{array}{l}2,740 \\
2,800 \\
2,740 \\
2,620 \\
2,680 \\
3,100\end{array}$ & $\begin{array}{l}3,320 \\
3,320 \\
3,320 \\
3,180 \\
3,360 \\
3,400 \\
\end{array}$ & $\begin{array}{c}\frac{2,590}{2,710} \\
2,770 \\
2,770 \\
2,620 \\
-\end{array}$ \\
\hline $\begin{array}{l}\text { Total } \\
\text { Mean } \\
\text { Ac-It }\end{array}$ & $\begin{array}{r}77,840 \\
2,510 \\
154,400 \\
\end{array}$ & $\begin{array}{r}71,680 \\
2,389 \\
142,200 \\
\end{array}$ & $\begin{array}{r}64,144 \\
2,069 \\
127,200\end{array}$ & $\begin{array}{r}110,658 \\
3,569 \\
219,500 \\
\end{array}$ & $\begin{array}{r}529,480 \\
18,910 \\
1,050,000 \\
\end{array}$ & $\begin{array}{r}378,200 \\
12,200 \\
750,000\end{array}$ & $\begin{array}{r}605,600 \\
20,190 \\
1,201,000\end{array}$ & $\begin{array}{r}786,500 \\
25,370 \\
1,560,000 \\
\end{array}$ & $\begin{array}{r}322,700 \\
10,760 \\
640,000\end{array}$ & $\begin{array}{r}88,050 \\
2,840 \\
174,000\end{array}$ & $\begin{array}{r}97,180 \\
3,135 \\
192,800 \\
\end{array}$ & $\begin{array}{r}87,050 \\
2,902 \\
172,700 \\
\end{array}$ \\
\hline & $r 19$ & $\begin{array}{l}\text { : Max } \\
\text { Max }\end{array}$ & $\begin{array}{c}10,000 \\
-\end{array}$ & $\begin{array}{l}\text { Min } \\
\text { Min }\end{array}$ & 740 & $\begin{array}{l}3,780 \\
8,819\end{array}$ & $\begin{array}{l}A c-f t \\
A c-f t\end{array}$ & $\begin{array}{l}2,736,000 \\
6,394,000\end{array}$ & & & & \\
\hline
\end{tabular}

* Discharge measurement made on this day.

Doubtful gage height record; discharge estimated from study of discharge records for Helmand River below Kajakat Reservolr.

Note.- No gage-height record Nov, 25 to Dec. 9, Feb. 1 to Apr. 20, Apr. 30 to July 6; discharge estimated from study of discharge records for Helmaind River below Kajakai Dam, Arghandab River near Kala Bist, and temporary station on Helmand R. at Lashkar Gah and discharge measurements. 
HEIMAND RIVER BASTN

Helmand River at Chahar Burjak, Afghanistan

Discharge, in cubic feet per second, water year October 1954 to September 1955

\begin{tabular}{|c|c|c|c|c|c|c|c|c|c|c|c|c|}
\hline Dar & Oct. & Nov. & Dec. & $\operatorname{Jan}_{2}$ & Feb. & Mar. & Apr. & May & June & July & Aug. & Sept. \\
\hline $\begin{array}{l}1 \\
2 \\
3 \\
4 \\
5\end{array}$ & $\begin{array}{l}2,540 \\
2,510 \\
2,460 \\
2,460 \\
2,460\end{array}$ & $\begin{array}{l}2,650 \\
2,680 \\
2,680 \\
2,680 \\
2,720\end{array}$ & $\begin{array}{l}2,830 \\
2,800 \\
2,830 \\
2,830 \\
2,830\end{array}$ & $\begin{array}{l}4,350 \\
5,280 \\
5,520 \\
5,620 \\
5,620\end{array}$ & $\begin{array}{l}6,780 \\
6,780 \\
6,820 \\
6,660 \\
6,190\end{array}$ & $\begin{array}{l}4,620 \\
1,420 \\
4,280 \\
4,220 \\
4,180\end{array}$ & $\begin{array}{l}3,890 \\
4,090 \\
3,950 \\
3,890 \\
3,820\end{array}$ & $\begin{array}{l}3,760 \\
3,760 \\
3,760 \\
3,820 \\
3,760\end{array}$ & $\begin{array}{l}a 7,000 \\
a 7,000 \\
a 6,800 \\
a 6,800 \\
a 6,600\end{array}$ & $\begin{array}{l}2,100 \\
2,100 \\
2,000 \\
2,000 \\
2,000\end{array}$ & $\begin{array}{l}2,480 \\
2,460 \\
2,560 \\
2,600 \\
2,620\end{array}$ & $\begin{array}{l}2,700 \\
2,650 \\
2,680 \\
2,680 \\
2,650\end{array}$ \\
\hline $\begin{array}{r}6 \\
7 \\
8 \\
9 \\
10\end{array}$ & $\begin{array}{l}2,460 \\
2,460 \\
2,460 \\
2,480 \\
2,460\end{array}$ & $\begin{array}{l}2,720 \\
2,750 \\
2,750 \\
2,750 \\
2,750\end{array}$ & $\begin{array}{r}2,860 \\
a 2,700 \\
a 2,500 \\
a 1,500 \\
a 1,100\end{array}$ & $\begin{array}{l}5,960 \\
6,140 \\
6,290 \\
6,360 \\
6,360\end{array}$ & $\begin{array}{l}6,740 \\
6,870 \\
6,820 \\
6,740 \\
6,620\end{array}$ & $\begin{array}{l}4,180 \\
4,150 \\
4,150 \\
4,120 \\
4,090\end{array}$ & $\begin{array}{l}3,820 \\
3,820 \\
3,760 \\
3,760 \\
3,760\end{array}$ & $\begin{array}{l}3,820 \\
3,890 \\
4,020 \\
4,420 \\
4,720\end{array}$ & $\begin{array}{r}a 6,600 \\
* 6,500 \\
6,400 \\
6,200 \\
6,000\end{array}$ & $\begin{array}{l}1,900 \\
1,900 \\
1,900 \\
1,900 \\
1,800\end{array}$ & $\begin{array}{l}2,650 \\
2,650 \\
2,600 \\
2,560 \\
2,510\end{array}$ & $\begin{array}{l}2,700 \\
2,750 \\
2,650 \\
2,700 \\
2,720\end{array}$ \\
\hline $\begin{array}{l}11 \\
12 \\
13 \\
14 \\
15\end{array}$ & $\begin{array}{r}2,460 \\
* 2,480 \\
2,460 \\
2,510 \\
2,510\end{array}$ & $\begin{array}{l}2,800 \\
2,860 \\
2,880 \\
2,880 \\
2,880\end{array}$ & $\begin{array}{l}2900 \\
a 800 \\
a 700 \\
4, \frac{220}{5,020}\end{array}$ & $\begin{array}{r}6,360 \\
6,360 \\
* 6,360 \\
6,360 \\
6,360\end{array}$ & $\begin{array}{l}6,360 \\
6,060 \\
6,140 \\
6,100 \\
6,020\end{array}$ & $\begin{array}{l}4,060 \\
4,060 \\
3,980 \\
3,980 \\
3,950\end{array}$ & $\begin{array}{l}3,690 \\
3,690 \\
3,690 \\
3,690 \\
3,690\end{array}$ & $\begin{array}{l}4,720 \\
5,020 \\
4,950 \\
4,800 \\
4,650\end{array}$ & $\begin{array}{l}5,800 \\
5,700 \\
5,600 \\
5,500 \\
5,400\end{array}$ & $\begin{array}{r}1,800 \\
1,800 \\
* 1,790 \\
1,930 \\
1,630\end{array}$ & $\begin{array}{l}2,510 \\
2,600 \\
2,600 \\
2,560 \\
2,560\end{array}$ & $\begin{array}{l}\frac{2,780}{2,780} \\
2,750 \\
2,720 \\
2,680\end{array}$ \\
\hline $\begin{array}{l}16 \\
17 \\
18 \\
19 \\
20\end{array}$ & $\begin{array}{l}2,510 \\
2,540 \\
2,540 \\
2,540 \\
2,560\end{array}$ & $\begin{array}{l}2,860 \\
2,860 \\
2,880 \\
2,910 \\
2,910\end{array}$ & $\begin{array}{l}5,180 \\
5,280 \\
5,280 \\
5,280 \\
5,440\end{array}$ & $\begin{array}{l}6,360 \\
6,320 \\
6,530 \\
6,740 \\
6,780 \\
\end{array}$ & $\begin{array}{r}6,020 \\
* 5,900 \\
5,700 \\
5,700 \\
5,580\end{array}$ & $\begin{array}{r}3,950 \\
16,100 \\
28,200 \\
\frac{17,600}{210,000}\end{array}$ & $\begin{array}{l}3,630 \\
3,630 \\
3,630 \\
3,570 \\
3,570\end{array}$ & $\begin{array}{l}4,580 \\
4,500 \\
4,420 \\
4,420 \\
4,500\end{array}$ & $\begin{array}{l}5,300 \\
5,200 \\
5,100 \\
5,000 \\
4,900\end{array}$ & $\begin{array}{l}1,620 \\
1,580 \\
1,560 \\
1,950 \\
2,280\end{array}$ & $\begin{array}{l}2,600 \\
2,580 \\
2,540 \\
2,280 \\
2,580\end{array}$ & $\begin{array}{l}2,620 \\
2,700 \\
2,780 \\
2,680 \\
2,620\end{array}$ \\
\hline $\begin{array}{l}21 \\
22 \\
23 \\
24 \\
25\end{array}$ & $\begin{array}{l}2,560 \\
2,580 \\
2,580 \\
2,580 \\
2,600\end{array}$ & $\begin{array}{r}* 2,910 \\
2,860 \\
2,860 \\
2,860 \\
2,860\end{array}$ & $\begin{array}{l}5,520 \\
5,550 \\
5,550 \\
5,060 \\
4,380\end{array}$ & $\begin{array}{l}6,780 \\
6,700 \\
6,700 \\
6,660 \\
6,480\end{array}$ & $\begin{array}{l}5,580 \\
5,320 \\
4,960 \\
4,920 \\
4,800\end{array}$ & $\begin{array}{l}a 7,000 \\
a 6,000 \\
a 5,400 \\
a 5,600 \\
a 5,200\end{array}$ & $\begin{array}{l}3,820 \\
4,150 \\
4,020 \\
4,020 \\
3,820\end{array}$ & $\begin{array}{l}4,500 \\
4,580 \\
4,500 \\
4,500 \\
4,500\end{array}$ & $\begin{array}{l}4,000 \\
3,000 \\
2,100 \\
\frac{2,000}{2,100}\end{array}$ & $\begin{array}{l}2,330 \\
\frac{2,560}{2,460} \\
2,330 \\
2,280\end{array}$ & $\begin{array}{r}2,650 \\
2,650 \\
2,680 \\
* 2,720 \\
2,720\end{array}$ & $\begin{array}{r}2,560 \\
2,510 \\
2,510 \\
2,560 \\
* 2,600\end{array}$ \\
\hline $\begin{array}{l}26 \\
27 \\
28 \\
29 \\
30 \\
31\end{array}$ & $\begin{array}{l}2,580 \\
2,650 \\
2,650 \\
2,650 \\
2,650 \\
2,650\end{array}$ & $\begin{array}{c}2,860 \\
2,860 \\
2,860 \\
2,860 \\
2,860 \\
-\end{array}$ & $\begin{array}{l}4,250 \\
4,090 \\
3,820 \\
3,690 \\
3,690 \\
3,690\end{array}$ & $\begin{array}{l}6,620 \\
6,660 \\
6,660 \\
6,660 \\
6,700 \\
6,740\end{array}$ & $\begin{array}{c}4,720 \\
4,650 \\
4,720 \\
= \\
=\end{array}$ & $\begin{array}{r}a_{4}, 800 \\
a_{4}, 500 \\
a 4,300 \\
* 4,150 \\
3,950 \\
3,820 \\
\end{array}$ & $\begin{array}{c}3,760 \\
3,760 \\
3,820 \\
3,820 \\
3,820 \\
-\end{array}$ & $\begin{array}{r}4,650 \\
\text { a5,000 } \\
\text { a5,500 } \\
\text { a6,000 } \\
\text { a6,700 } \\
\text { az,000 }\end{array}$ & $\begin{array}{c}2,200 \\
2,200 \\
2,200 \\
2,200 \\
2,200 \\
-\end{array}$ & $\begin{array}{l}2,220 \\
2,240 \\
2,440 \\
2,280 \\
2,460 \\
2,540\end{array}$ & $\begin{array}{l}2,700 \\
2,680 \\
2,650 \\
2,680 \\
2,700 \\
2,720\end{array}$ & $\begin{array}{c}2,620 \\
2,650 \\
2,650 \\
2,650 \\
2,600 \\
-\end{array}$ \\
\hline $\begin{array}{l}\text { Total } \\
\text { Mean } \\
\text { Ac-ft }\end{array}$ & $\begin{array}{r}78,590 \\
2,535 \\
155,900\end{array}$ & $\begin{array}{r}84,500 \\
2,817 \\
167,600\end{array}$ & $\begin{array}{r}112,170 \\
3,618 \\
222,500\end{array}$ & $\begin{array}{r}195,400 \\
6,303 \\
387,600\end{array}$ & $\begin{array}{r}166,290 \\
5,939 \\
329,800\end{array}$ & $\begin{array}{r}193,010 \\
6,226 \\
382,800\end{array}$ & $\begin{array}{r}113,850 \\
3,795 \\
225,800\end{array}$ & $\begin{array}{r}143,720 \\
4,636 \\
285,100\end{array}$ & $\begin{array}{r}143,600 \\
1,787 \\
284,800\end{array}$ & $\begin{array}{r}63,680 \\
2,054 \\
126,300\end{array}$ & $\begin{array}{r}80,650 \\
2,602 \\
160,000\end{array}$ & $\begin{array}{r}79,900 \\
2,663 \\
158,500\end{array}$ \\
\hline
\end{tabular}

Calendar year 1954: Max 34,100 Min 700 Mean 8,988 Ac-ft 6,507,000

Water year 1954-55: $\operatorname{Max} 28,200$ Min 700 Mean 3,987 Ac-ft 2,887,000

* Discharge measurement made on this daj.

a No gage-height record; discharge estimated. 
HETMAND RIVER BASIN

Helmand River at Chahar Burjak, Afghanistan

Discharge, in cubic feet yer second, water year October 1955 to Septeuber 1956

\begin{tabular}{|c|c|c|c|c|c|c|c|c|c|c|c|c|}
\hline Day & Oct, & Novo & Dec. & Jan. & $\mathrm{Feb}_{2}$ & Ma & Apr. & May & June & July & Aug. & Sept. \\
\hline $\begin{array}{l}1 \\
2 \\
3 \\
4 \\
5\end{array}$ & $\begin{array}{l}2,600 \\
2,600 \\
2,580 \\
2,540 \\
2,460 \\
\end{array}$ & $\begin{array}{l}2,620 \\
2,620 \\
2,620 \\
2,680 \\
2,600\end{array}$ & $\begin{array}{l}\frac{2,750}{2,780} \\
2,800 \\
2,780 \\
2,800\end{array}$ & $\begin{array}{r}4,280 \\
4,220 \\
4,150 \\
4,220 \\
* 4,620\end{array}$ & $\begin{array}{l}3,890 \\
3,820 \\
3,760 \\
3,690 \\
3,630\end{array}$ & $\begin{array}{l}5,360 \\
5,550 \\
5,980 \\
5,700 \\
5,320\end{array}$ & $\begin{array}{r}\frac{18,000}{26,000} \\
22,000 \\
24,000 \\
* 26,800\end{array}$ & $\begin{array}{l}\frac{41,000}{40,000} \\
38,000 \\
35,000 \\
33,000\end{array}$ & $\begin{array}{l}\frac{12,000}{11,000} \\
11,000 \\
10,000 \\
10,000\end{array}$ & $\begin{array}{l}5,800 \\
5,700 \\
5,600 \\
5,500 \\
5,400\end{array}$ & $\begin{array}{l}\frac{36,000}{30,000} \\
18,000 \\
14,000 \\
13,000\end{array}$ & $\begin{array}{l}6,500 \\
6,400 \\
6,400 \\
6,400 \\
6,300\end{array}$ \\
\hline $\begin{array}{r}6 \\
7 \\
8 \\
9 \\
10\end{array}$ & $\begin{array}{l}2,480 \\
2,540 \\
2,540 \\
2,580 \\
2,650\end{array}$ & $\begin{array}{l}2,560 \\
2,540 \\
2,540 \\
2,540 \\
2,540\end{array}$ & $\begin{array}{l}2,800 \\
2,800 \\
2,800 \\
2,910 \\
2,980\end{array}$ & $\begin{array}{l}6,360 \\
6,620 \\
5,940 \\
5,020 \\
4,680\end{array}$ & $\begin{array}{l}3,530 \\
3,570 \\
3,520 \\
3,520 \\
3,460\end{array}$ & $\begin{array}{l}5,060 \\
8,220 \\
9,450 \\
8,640 \\
7,640\end{array}$ & $\begin{array}{l}25,500 \\
24,600 \\
27,400 \\
31,700 \\
34,700\end{array}$ & $\begin{array}{l}32,000 \\
31,000 \\
30,000 \\
29,000 \\
28,000\end{array}$ & $\begin{array}{l}8,000 \\
7,000 \\
7,200 \\
7,400 \\
7,600\end{array}$ & $\begin{array}{l}5,300 \\
5,200 \\
5,200 \\
5,100 \\
5,000\end{array}$ & $\begin{array}{l}12,000 \\
11,000 \\
11,000 \\
11,000 \\
10,000\end{array}$ & $\begin{array}{l}6,300 \\
6,300 \\
4,000 \\
2,500 \\
2,000 \\
\end{array}$ \\
\hline $\begin{array}{l}11 \\
12 \\
13 \\
14 \\
15\end{array}$ & $\begin{array}{l}\frac{2,700}{2,620} \\
2,580 \\
2,650 \\
2,600\end{array}$ & $\begin{array}{r}2,540 \\
* 2,560 \\
2,560 \\
2,560 \\
2,560\end{array}$ & $\begin{array}{l}3,460 \\
3,400 \\
3,520 \\
3,490 \\
3,460\end{array}$ & $\begin{array}{l}4,460 \\
4,280 \\
4,220 \\
4,220 \\
4,180\end{array}$ & $\begin{array}{l}3,400 \\
3,340 \\
3,280 \\
3,280 \\
3,170\end{array}$ & $\begin{array}{l}6,700 \\
6,020 \\
5,550 \\
5,220 \\
4,950\end{array}$ & $\begin{array}{l}37,700 \\
40,700 \\
45,000 \\
48,000 \\
49,000\end{array}$ & $\begin{array}{l}27,000 \\
26,000 \\
24,000 \\
23,000 \\
22,000\end{array}$ & $\begin{array}{l}7,800 \\
7,800 \\
7,800 \\
7,800 \\
7,600\end{array}$ & $\begin{array}{l}5,900 \\
4,900 \\
4,900 \\
4,800 \\
4,800\end{array}$ & $\begin{array}{l}9,800 \\
9,400 \\
9,000 \\
8,800 \\
8,600\end{array}$ & $\begin{array}{l}2,000 \\
2,000 \\
2,000 \\
4,500 \\
5,400\end{array}$ \\
\hline $\begin{array}{l}16 \\
17 \\
18 \\
19 \\
20\end{array}$ & $\begin{array}{l}2,600 \\
2,650 \\
2,600 \\
2,600 \\
2,620\end{array}$ & $\begin{array}{l}2,560 \\
2,560 \\
2,580 \\
2,580 \\
2,580\end{array}$ & $\begin{array}{r}12,200 \\
12,100 \\
9,020 \\
7,120 \\
6,020\end{array}$ & $\begin{array}{l}4,150 \\
4,120 \\
4,180 \\
4,150 \\
4,120\end{array}$ & $\begin{array}{l}3,110 \\
3,110 \\
3,060 \\
3,110 \\
3,260\end{array}$ & $\begin{array}{l}4,840 \\
5,060 \\
6,020 \\
5,180 \\
5,600\end{array}$ & $\begin{array}{l}49,000 \\
47,000 \\
48,000 \\
50,000 \\
50,000\end{array}$ & $\begin{array}{l}21,000 \\
21,000 \\
20,000 \\
19,000 \\
19,000\end{array}$ & $\begin{array}{l}7,600 \\
7,400 \\
7,200 \\
7,200 \\
7,000\end{array}$ & $\begin{array}{r}4,800 \\
4,700 \\
4,700 \\
4,700 \\
10,000\end{array}$ & $\begin{array}{l}8,400 \\
8,000 \\
7,800 \\
7,800 \\
7,600\end{array}$ & $\begin{array}{l}6,100 \\
6,200 \\
6,300 \\
6,300 \\
6,300\end{array}$ \\
\hline $\begin{array}{l}21 \\
22 \\
23 \\
24 \\
25\end{array}$ & $\begin{array}{l}2,650 \\
2,650 \\
2,620 \\
2,650 \\
2,680\end{array}$ & $\begin{array}{l}2,600 \\
2,600 \\
2,600 \\
2,650 \\
2,750 \\
\end{array}$ & $\begin{array}{l}5,550 \\
5,250 \\
4,980 \\
4,720 \\
4,650\end{array}$ & $\begin{array}{l}4,420 \\
4,350 \\
4,250 \\
4,180 \\
4,150\end{array}$ & $\begin{array}{l}3,370 \\
3,260 \\
3,110 \\
2,960 \\
2,960\end{array}$ & $\begin{array}{r}7,000 \\
13,000 \\
14,000 \\
14,000 \\
12,000\end{array}$ & $\begin{array}{l}50,000 \\
51,000 \\
53,000 \\
55,000 \\
55,000\end{array}$ & $\begin{array}{l}18,000 \\
17,000 \\
16,000 \\
16,000 \\
15,000\end{array}$ & $\begin{array}{l}6,800 \\
6,800 \\
6,600 \\
6,600 \\
6,400\end{array}$ & $\begin{array}{l}20,000 \\
30,000 \\
\frac{34,000}{34,000} \\
31,000\end{array}$ & $\begin{array}{l}7,400 \\
7,300 \\
7,200 \\
7,100 \\
6,800\end{array}$ & $\begin{array}{r}6,300 \\
* 6,300 \\
* 6,300 \\
6,300 \\
6,200\end{array}$ \\
\hline $\begin{array}{l}26 \\
27 \\
28 \\
29 \\
30 \\
31\end{array}$ & $\begin{array}{l}2,620 \\
2,600 \\
2,620 \\
2,680 \\
2,620 \\
2,620\end{array}$ & $\begin{array}{c}2,720 \\
2,750 \\
2,750 \\
2,750 \\
2,750 \\
-\end{array}$ & $\begin{array}{l}4,580 \\
4,580 \\
4,620 \\
4,540 \\
4,420 \\
4,420\end{array}$ & $\begin{array}{l}4,090 \\
4,060 \\
4,020 \\
3,980 \\
3,950 \\
3,920 \\
\end{array}$ & $\begin{array}{c}2,980 \\
2,980 \\
3,110 \\
3,060 \\
= \\
=\end{array}$ & $\begin{array}{l}11,000 \\
12,000 \\
18,000 \\
27,500 \\
\frac{25,000}{20,000}\end{array}$ & $\begin{array}{c}52,000 \\
49,000 \\
46,000 \\
44,000 \\
42,000 \\
-\end{array}$ & $\begin{array}{l}14,000 \\
14,000 \\
13,000 \\
13,000 \\
\frac{12,000}{12,000}\end{array}$ & $\begin{array}{l}6,300 \\
6,200 \\
6,200 \\
6,100 \\
6,000 \\
-\end{array}$ & $\begin{array}{l}29,000 \\
28,000 \\
26,000 \\
32,000 \\
38,000 \\
40,000\end{array}$ & $\begin{array}{l}6,800 \\
6,700 \\
6,600 \\
6,600 \\
6,600 \\
6,600\end{array}$ & $\begin{array}{c}6,200 \\
6,200 \\
6,200 \\
6,200 \\
6,200 \\
=\end{array}$ \\
\hline $\begin{array}{l}\text { Total } \\
\text { Mean } \\
\text { Ac-ft }\end{array}$ & $\begin{array}{r}80,800 \\
2,606 \\
160,300 \\
\end{array}$ & $\begin{array}{r}78,320 \\
2,611 \\
155,300 \\
\end{array}$ & $\begin{array}{r}144,300 \\
4,655 \\
286,200 \\
\end{array}$ & $\begin{array}{r}137,560 \\
4,437 \\
272,800\end{array}$ & $\begin{array}{r}96,350 \\
3,322 \\
191,100 \\
\end{array}$ & $\begin{array}{r}295,560 \\
9,534 \\
586,200\end{array}$ & $\begin{array}{r}1,216,100 \\
40,540 \\
2,412,000\end{array}$ & $\begin{array}{r}719,000 \\
23,190 \\
1,426,000\end{array}$ & $\begin{array}{r}230,400 \\
7,680 \\
457,000\end{array}$ & $\begin{array}{r}4,49,100 \\
14,490 \\
890,800\end{array}$ & $\begin{array}{r}326,900 \\
10,550 \\
648,400\end{array}$ & $\begin{array}{r}162,600 \\
5,420 \\
322,500 \\
\end{array}$ \\
\hline vate & & $\begin{array}{l}\text { Max } \\
\operatorname{Max}\end{array}$ & $\begin{array}{l}28,200 \\
55,000\end{array}$ & $\begin{array}{l}\text { Min } \\
\text { Min }\end{array}$ & & an 4,0 & $A C-$ & $7,80^{\circ}, 0$ & & & & \\
\hline
\end{tabular}

* Discharge measurement made on this day.

Note. - lo gage-height, record Mar. 20 to Apr. L, hr. 13 to Sent. 30; discharge estimated on basis of records for

temporary gage at Lashkar Gah, Arghandab River at Kala Bist and weather records. 
HETMAND RIVER BASTN

Helmand River at Chahar Burjak, Afghanistan

Discharge, in cubic feet per second, water year October 1956 to september 1957

\begin{tabular}{|c|c|c|c|c|c|c|c|c|c|c|c|c|}
\hline Day & oct. & Nove & $\mathrm{Dec}_{2}$ & Jan. & Feb. & Mar. & Apr. & May & June & Julv & Aug, & Sept. \\
\hline \begin{tabular}{l|}
1 \\
2 \\
3 \\
4 \\
5
\end{tabular} & $\begin{array}{l}\frac{6,200}{6,200} \\
6,800 \\
6,200 \\
6,200\end{array}$ & $\begin{array}{l}\frac{1,480}{1,480} \\
3,010 \\
3,660 \\
3,760\end{array}$ & $\begin{array}{l}3,760 \\
3,760 \\
3,760 \\
3,790 \\
3,820\end{array}$ & $\begin{array}{r}4,760 \\
5,740 \\
* 5,940 \\
6,100 \\
6,100\end{array}$ & $\begin{array}{l}10,000 \\
10,000 \\
10,000 \\
10,000 \\
10,000\end{array}$ & $\begin{array}{r}5,000 \\
* 4,500 \\
4,150 \\
6,500 \\
8,120\end{array}$ & $\begin{array}{l}22,000 \\
22,000 \\
20,000 \\
\frac{19,000}{25,000}\end{array}$ & $\begin{array}{l}47,000 \\
46,000 \\
47,000 \\
50,000 \\
60,000\end{array}$ & $\begin{array}{l}\frac{32,000}{32,000} \\
32,000 \\
32,000 \\
32,000\end{array}$ & $\begin{array}{l}10,500 \\
10,000 \\
10,500 \\
10,500 \\
10,000\end{array}$ & $\begin{array}{l}\frac{6,100}{6,000} \\
5,700 \\
5,700 \\
5,700\end{array}$ & $\begin{array}{l}6,100 \\
6,100 \\
6,200 \\
6,200 \\
6,000\end{array}$ \\
\hline $\begin{array}{r}6 \\
7 \\
8 \\
9 \\
10\end{array}$ & $\begin{array}{l}6,100 \\
6,100 \\
6,100 \\
6,100 \\
6,100\end{array}$ & $\begin{array}{l}3,760 \\
3,790 \\
3,860 \\
3,860 \\
3,860\end{array}$ & $\begin{array}{l}3,820 \\
3,820 \\
3,790 \\
3,790 \\
3,790\end{array}$ & $\begin{array}{l}6,190 \\
6,320 \\
6,360 \\
6,400 \\
6,660\end{array}$ & $\begin{array}{l}10,000 \\
10,000 \\
10,000 \\
10,500 \\
11,000\end{array}$ & $\begin{array}{l}8,310 \\
8,550 \\
8,450 \\
8,400 \\
8,310\end{array}$ & $\begin{array}{l}45,000 \\
\frac{80,000}{78,000} \\
76,000 \\
70,000\end{array}$ & $\begin{array}{l}70,000 \\
80,000 \\
\frac{85,000}{80,000} \\
75,000\end{array}$ & $\begin{array}{l}32,000 \\
32,000 \\
31,000 \\
30,000 \\
28,000\end{array}$ & $\begin{array}{r}10,000 \\
9,500 \\
9,500 \\
9,500 \\
9,000\end{array}$ & $\begin{array}{l}5,600 \\
5,600 \\
5,500 \\
5,500 \\
5,400\end{array}$ & $\begin{array}{l}6,000 \\
6,000 \\
6,200 \\
6,400 \\
7,000 \\
\end{array}$ \\
\hline $\begin{array}{l}11 \\
12 \\
13 \\
14 \\
15\end{array}$ & $\begin{array}{l}6,000 \\
6,000 \\
6,000 \\
6,000 \\
6,000\end{array}$ & $\begin{array}{l}3,820 \\
3,860 \\
3,820 \\
3,790 \\
3,760\end{array}$ & $\begin{array}{l}3,820 \\
3,820 \\
3,860 \\
3,860 \\
3,860\end{array}$ & $\begin{array}{l}6,780 \\
7,550 \\
8,070 \\
7,550 \\
9,730\end{array}$ & $\begin{array}{l}11,500 \\
12,000 \\
\frac{12,000}{11,500} \\
11,500\end{array}$ & $\begin{array}{l}8,400 \\
8,500 \\
8,600 \\
8,400 \\
8,120\end{array}$ & $\begin{array}{l}66,000 \\
62,000 \\
62,000 \\
60,000 \\
60,000\end{array}$ & $\begin{array}{l}68,000 \\
62,000 \\
56,000 \\
56,000 \\
58,000\end{array}$ & $\begin{array}{l}27,000 \\
25,000 \\
23,000 \\
22,000 \\
21,000\end{array}$ & $\begin{array}{l}8,800 \\
8,600 \\
8,500 \\
8,200 \\
8,000\end{array}$ & $\begin{array}{l}5,400 \\
5,400 \\
5,400 \\
5,600 \\
5,700\end{array}$ & $\begin{array}{l}6,200 \\
6,000 \\
6,000 \\
6,000 \\
6,000\end{array}$ \\
\hline $\begin{array}{l}16 \\
17 \\
18 \\
19 \\
20\end{array}$ & $\begin{array}{l}2,300 \\
2,200 \\
2,100 \\
2,000 \\
1,900\end{array}$ & $\begin{array}{l}3,760 \\
3,760 \\
3,760 \\
3,760 \\
3,760\end{array}$ & $\begin{array}{l}3,860 \\
3,860 \\
3,860 \\
3,860 \\
3,860\end{array}$ & $\begin{array}{r}12,000 \\
15,000 \\
13,000 \\
11,000 \\
9,000\end{array}$ & $\begin{array}{r}11,000 \\
10,000 \\
9,500 \\
9,200 \\
9,000\end{array}$ & $\begin{array}{r}7,880 \\
8,400 \\
8,310 \\
8,430 \\
10,000\end{array}$ & $\begin{array}{l}62,000 \\
71,000 \\
74,000 \\
74,000 \\
70,000\end{array}$ & $\begin{array}{l}58,000 \\
54,000 \\
50,000 \\
46,000 \\
45,000\end{array}$ & $\begin{array}{l}20,000 \\
19,000 \\
18,000 \\
17,000 \\
17,000\end{array}$ & $\begin{array}{l}8,000 \\
7,800 \\
7,400 \\
7,300 \\
7,300\end{array}$ & $\begin{array}{l}5,800 \\
6,000 \\
2,500 \\
1,300 \\
1,200\end{array}$ & $\begin{array}{r}6,000 \\
* 5,910 \\
5,910 \\
5,990 \\
6,070\end{array}$ \\
\hline $\begin{array}{l}21 \\
22 \\
23 \\
24 \\
25\end{array}$ & $\begin{array}{r}1,800 \\
1,800 \\
1,700 \\
* 1,700 \\
1,600\end{array}$ & $\begin{array}{l}3,760 \\
3,760 \\
3,760 \\
3,690 \\
3,690\end{array}$ & $\begin{array}{l}3,860 \\
3,860 \\
3,860 \\
3,950 \\
4,020\end{array}$ & $\begin{array}{l}8,600 \\
8,600 \\
8,500 \\
8,600 \\
9,000\end{array}$ & $\begin{array}{l}9,000 \\
9,000 \\
9,000 \\
9,000 \\
9,000\end{array}$ & $\begin{array}{l}\frac{80,000}{70,000} \\
50,000 \\
35,000 \\
30,000\end{array}$ & $\begin{array}{l}66,000 \\
64,000 \\
62,000 \\
60,000 \\
53,000\end{array}$ & $\begin{array}{l}44,000 \\
44,000 \\
44,000 \\
43,000 \\
43,000\end{array}$ & $\begin{array}{l}16,000 \\
16,000 \\
15,000 \\
1 i_{4}, 000 \\
14,000\end{array}$ & $\begin{array}{l}7,200 \\
7,200 \\
7,200 \\
7,200 \\
7,000\end{array}$ & $\begin{array}{l}1,150 \\
1,100 \\
1,050 \\
\frac{1}{1,000} \\
1,000\end{array}$ & $\begin{array}{l}6,070 \\
5,990 \\
5,950 \\
5,990 \\
6,030\end{array}$ \\
\hline $\begin{array}{l}26 \\
27 \\
28 \\
29 \\
30 \\
31\end{array}$ & $\begin{array}{l}1,510 \\
1,480 \\
1,480 \\
1,480 \\
1,450 \\
1,460\end{array}$ & $\begin{array}{c}3,720 \\
3,760 \\
3,760 \\
3,760 \\
3,760 \\
=\end{array}$ & $\begin{array}{l}4,220 \\
4,320 \\
4,320 \\
4,320 \\
4,320 \\
4,380 \\
\end{array}$ & $\begin{array}{r}9,000 \\
9,500 \\
14,000 \\
18,000 \\
15,000 \\
12,000\end{array}$ & $\begin{array}{c}9,000 \\
9,000 \\
7,000 \\
- \\
- \\
-\end{array}$ & $\begin{array}{l}25,000 \\
22,000 \\
2.1,000 \\
20,000 \\
20,000 \\
22,000\end{array}$ & $\begin{array}{c}55,000 \\
54,000 \\
52,000 \\
52,000 \\
50,000 \\
-\end{array}$ & $\begin{array}{l}1+2,000 \\
1+2,000 \\
38,000 \\
36,000 \\
35,000 \\
33,000 \\
\end{array}$ & $\begin{array}{c}13,000 \\
13,000 \\
12,000 \\
\frac{12,000}{12,000} \\
-\end{array}$ & $\begin{array}{l}6,500 \\
6,600 \\
6,500 \\
6,500 \\
6,500 \\
6,400 \\
\end{array}$ & $\begin{array}{l}5,700 \\
,, 000 \\
, 000 \\
6,000 \\
0,100 \\
6,100\end{array}$ & $\begin{array}{c}5,990 \\
5,870 \\
6,030 \\
5,990 \\
6,030 \\
-\end{array}$ \\
\hline $\begin{array}{l}\text { Total } \\
\text { Nean } \\
\text { Ac-ft }\end{array}$ & $\begin{array}{r}19,460 \\
3,854 \\
36,900 \\
\end{array}$ & $\begin{array}{r}107,790 \\
3,593 \\
213,0 \mathrm{~m}\end{array}$ & $\begin{array}{r}121,910 \\
3,933 \\
21,1, \text {, }\end{array}$ & $\begin{array}{r}281,050 \\
9,066 \\
557,500 \\
\end{array}$ & $\begin{array}{r}27^{8}, 700 \\
9,954 \\
552,8 \times 0 \\
\end{array}$ & $\begin{array}{r}550,330 \\
17,750 \\
1,092,000 \\
\end{array}$ & $\begin{array}{r}1,691,000 \\
56,370 \\
3,354,000\end{array}$ & $\begin{array}{r}1,637,000 \\
52,810 \\
3,21,7,000\end{array}$ & $\begin{array}{r}65,000 \\
21,970 \\
1,307,000\end{array}$ & $\begin{array}{r}254,220 \\
8, ? 10 \\
504,200\end{array}$ & $\begin{array}{r}L_{i}=, 300 \\
4,500 \\
28,200 \\
\end{array}$ & $\begin{array}{r}182,220 \\
6,074 \\
361,400 \\
\end{array}$ \\
\hline
\end{tabular}

Calentar yezr 1956: Max 55,000 Min 1,450 liean 10,9ln Ac-ft 7,899,000

Aater year 1956-57: Max 85,000 Min 1,000 liean 16,510 Ac-ft 1],950,000

* Dischirae messigenent made on this day.

Hote gare-hei ht record Oct. 1-24, Jur. 16 to Mr. 1, Mar. 20 to Sent. 16; discherge astimated on basis

of records for stations upstream and discharge measurements. 
HEIMAND RIVER BASIN

Helmand River at Chahar Burjak, Afghanistan

Discharge, in cubic feet per second, water year October 1957 to Septenber 1958

\begin{tabular}{|c|c|c|c|c|c|c|c|c|c|c|c|c|}
\hline$\overline{D_{3 y}}$ & oct, & Novo & Dec. & Jan. & $\mathrm{Feb}_{\mathrm{Q}}$ & Mar, & Apr. & May & June & JuTy & Alig. & Sept. \\
\hline $\begin{array}{l}1 \\
2 \\
3 \\
4 \\
5\end{array}$ & $\begin{array}{l}6,070 \\
5,990 \\
5,990 \\
6,070 \\
5,910\end{array}$ & $\begin{array}{l}5,910 \\
5,830 \\
5,830 \\
5,830 \\
5,910\end{array}$ & $\begin{array}{l}5,600 \\
5,380 \\
5,300 \\
5,220 \\
5,080\end{array}$ & $\begin{array}{l}9,200 \\
9,000 \\
9,000 \\
9,000 \\
8,900\end{array}$ & $\begin{array}{l}8,600 \\
8,600 \\
8,600 \\
8,800 \\
2,600 \\
\end{array}$ & $\begin{array}{l}6,890 \\
6,860 \\
6,980 \\
6,880 \\
6,890\end{array}$ & $\begin{array}{l}\frac{5,830}{5,950} \\
5,910 \\
5,870 \\
5,990\end{array}$ & $\begin{array}{l}\frac{19,900}{19,500} \\
18,900 \\
18,100 \\
17,300\end{array}$ & $\begin{array}{l}\frac{8,350}{8,120} \\
7,980 \\
7,940 \\
8,000\end{array}$ & $\begin{array}{l}5,870 \\
5,870 \\
5,910 \\
5,950 \\
5,950\end{array}$ & $\begin{array}{l}\frac{5,480}{5,450} \\
5,450 \\
5,380 \\
5,260\end{array}$ & $\begin{array}{l}5,450 \\
5,520 \\
5,640 \\
5,640 \\
5,480\end{array}$ \\
\hline $\begin{array}{r}6 \\
7 \\
8 \\
9 \\
10\end{array}$ & $\begin{array}{l}6,150 \\
6,230 \\
6,230 \\
6,150 \\
6,070\end{array}$ & $\begin{array}{l}5,910 \\
5,910 \\
5,910 \\
5,910 \\
4,620\end{array}$ & $\begin{array}{l}5,000 \\
5,000 \\
4,960 \\
5,910 \\
9,700\end{array}$ & $\begin{array}{l}8,800 \\
8,600 \\
8,600 \\
\frac{8,400}{8,500}\end{array}$ & $\begin{array}{l}9,400 \\
9,200 \\
8,900 \\
8,700 \\
8,500\end{array}$ & $\begin{array}{l}6,940 \\
6.980 \\
6,860 \\
6,860 \\
6,720\end{array}$ & $\begin{array}{l}6,720 \\
6,980 \\
7,060 \\
7,020 \\
7,060\end{array}$ & $\begin{array}{l}16,600 \\
15,700 \\
15,000 \\
14,400 \\
14,000\end{array}$ & $\begin{array}{l}8,000 \\
7,700 \\
7,400 \\
7,200 \\
7,200\end{array}$ & $\begin{array}{l}5,870 \\
5,790 \\
5,830 \\
5,870 \\
5,790\end{array}$ & $\begin{array}{l}5,180 \\
5,150 \\
5,180 \\
5,300 \\
5,300\end{array}$ & $\begin{array}{l}5,260 \\
5,080 \\
5,080 \\
4,920 \\
4,960\end{array}$ \\
\hline $\begin{array}{l}11 \\
12 \\
13 \\
14 \\
15\end{array}$ & $\begin{array}{l}6,230 \\
6,230 \\
6,110 \\
6,550 \\
6,720 \\
\end{array}$ & $\begin{array}{r}2,100 \\
\frac{854}{868} \\
929 \\
* 1,000\end{array}$ & $\begin{array}{l}16,800 \\
15,000 \\
20,000 \\
\frac{27,500}{23,200}\end{array}$ & $\begin{array}{l}8,500 \\
8,700 \\
8,800 \\
8,800 \\
8,900\end{array}$ & $\begin{array}{l}8,200 \\
8,000 \\
7,900 \\
7,700 \\
7,670\end{array}$ & $\begin{array}{l}6,470 \\
6,310 \\
6,390 \\
6,430 \\
6,430\end{array}$ & $\begin{array}{r}6,880 \\
6,890 \\
6,860 \\
6,640 \\
* 6,720\end{array}$ & $\begin{array}{l}13,400 \\
12,800 \\
12,200 \\
11,800 \\
11,600\end{array}$ & $\begin{array}{l}7,000 \\
6,700 \\
6,600 \\
6,500 \\
6,400\end{array}$ & $\begin{array}{l}5,680 \\
4,250 \\
3,220 \\
5,150 \\
5,750\end{array}$ & $\begin{array}{r}5,220 \\
5,180 \\
5,300 \\
* 5,380 \\
5,260\end{array}$ & $\begin{array}{l}5,150 \\
5,080 \\
4,850 \\
4,850 \\
4,820\end{array}$ \\
\hline $\begin{array}{l}16 \\
17 \\
18 \\
19 \\
20\end{array}$ & $\begin{array}{l}5,990 \\
5,910 \\
6,070 \\
6,070 \\
6,150\end{array}$ & $\begin{array}{l}956 \\
910 \\
896 \\
882 \\
910\end{array}$ & $\begin{array}{l}15,300 \\
12,900 \\
11,500 \\
10,700 \\
10,400\end{array}$ & $\begin{array}{l}8,300 \\
8,700 \\
8,600 \\
8,600 \\
8,600\end{array}$ & $\begin{array}{l}7,580 \\
7,670 \\
7,490 \\
7,320 \\
7,320\end{array}$ & $\begin{array}{l}6,390 \\
6,390 \\
6,230 \\
6,190 \\
6,190\end{array}$ & $\begin{array}{r}6,720 \\
6,790 \\
6,790 \\
8,030 \\
10,600\end{array}$ & $\begin{array}{l}11,400 \\
10,800 \\
10,800 \\
10,800 \\
10,500\end{array}$ & $\begin{array}{r}6,300 \\
6,200 \\
6,100 \\
* 6,070 \\
6,030\end{array}$ & $\begin{array}{l}5,680 \\
5,600 \\
5,600 \\
5,640 \\
5,830\end{array}$ & $\begin{array}{l}5,180 \\
5,150 \\
5,150 \\
5,180 \\
5,220\end{array}$ & $\begin{array}{l}5,300 \\
5,300 \\
5,420 \\
5,600 \\
4,960\end{array}$ \\
\hline $\begin{array}{l}21 \\
22 \\
23 \\
24 \\
25\end{array}$ & $\begin{array}{l}6,070 \\
5,910 \\
5,910 \\
6,070 \\
6,070\end{array}$ & $\begin{array}{l}2,700 \\
3,500 \\
8,300 \\
7,490 \\
6,310\end{array}$ & $\begin{array}{r}10,100 \\
9,700 \\
9,500 \\
9,300 \\
9,400\end{array}$ & $\begin{array}{l}8,700 \\
8,800 \\
8,800 \\
8,900 \\
9,000\end{array}$ & $\begin{array}{l}7,320 \\
7,180 \\
7,280 \\
7,230 \\
7,180\end{array}$ & $\begin{array}{l}6,110 \\
5,990 \\
5,830 \\
5,790 \\
5,720\end{array}$ & $\begin{array}{l}12,700 \\
14,000 \\
14,900 \\
16,100 \\
16,900\end{array}$ & $\begin{array}{r}10,400 \\
10,300 \\
9,950 \\
9,550 \\
9,100\end{array}$ & $\begin{array}{l}6,150 \\
6,070 \\
6,030 \\
6,070 \\
5,990\end{array}$ & $\begin{array}{l}5,830 \\
5,830 \\
5,990 \\
5,950 \\
5,640\end{array}$ & $\begin{array}{l}5,150 \\
5,120 \\
5,180 \\
5,040 \\
5,080\end{array}$ & $\begin{array}{l}4,740 \\
4,700 \\
4,700 \\
4,700 \\
4,740\end{array}$ \\
\hline $\begin{array}{l}26 \\
27 \\
28 \\
29 \\
30 \\
31 \\
\end{array}$ & $\begin{array}{l}6,070 \\
6,070 \\
6,070 \\
5,000 \\
6,030 \\
6,030\end{array}$ & $\begin{array}{c}5,910 \\
6,550 \\
7,060 \\
6,550 \\
5,910 \\
-\end{array}$ & $\begin{array}{l}9,300 \\
9,200 \\
9,100 \\
9,100 \\
9,000 \\
9,200\end{array}$ & $\begin{array}{l}8,900 \\
8,800 \\
8,800 \\
8,800 \\
8,800 \\
8,700\end{array}$ & $\begin{array}{r}7,140 \\
6,980 \\
6,890 \\
- \\
- \\
-\end{array}$ & $\begin{array}{l}5,600 \\
5,560 \\
5,600 \\
5,520 \\
5,520 \\
5,600\end{array}$ & $\begin{array}{l}17,500 \\
18,100 \\
18,600 \\
19,500 \\
\frac{19,800}{-}\end{array}$ & $\begin{array}{l}9,200 \\
9,250 \\
9,100 \\
8,950 \\
8,700 \\
8,500 \\
\end{array}$ & $\begin{array}{c}5,910 \\
5,910 \\
5,870 \\
5,790 \\
5,830 \\
-\end{array}$ & $\begin{array}{l}5,520 \\
5,450 \\
5,420 \\
5,450 \\
5,450 \\
5,450\end{array}$ & $\begin{array}{l}5,080 \\
5,080 \\
5,120 \\
5,080 \\
5,260 \\
5,420\end{array}$ & $\begin{array}{c}4,740 \\
4,740 \\
4,550 \\
4,660 \\
4,740 \\
=\end{array}$ \\
\hline $\begin{array}{l}\text { Total } \\
\text { Mean } \\
\text { Ac-ft }\end{array}$ & $\begin{array}{r}188,190 \\
6,071 \\
373,300\end{array}$ & $\begin{array}{r}128,157 \\
4,272 \\
254,200\end{array}$ & $\begin{array}{r}323,350 \\
10,430 \\
641,400\end{array}$ & $\begin{array}{r}272,000 \\
8,774 \\
539,500\end{array}$ & $\begin{array}{r}222,950 \\
7,962 \\
442,200\end{array}$ & $\begin{array}{r}195,120 \\
6,294 \\
387,000\end{array}$ & $\begin{array}{r}305,410 \\
10,180 \\
605,800\end{array}$ & $\begin{array}{r}388,500 \\
12,530 \\
770,600\end{array}$ & $\begin{array}{r}201,410 \\
6,714 \\
399,500\end{array}$ & $\begin{array}{r}173,080 \\
5,583 \\
343,300\end{array}$ & $\begin{array}{r}161,960 \\
5,225 \\
321,300\end{array}$ & $\begin{array}{r}151,370 \\
5,046 \\
300,300\end{array}$ \\
\hline
\end{tabular}

$\begin{array}{llllllll}\text { Calendar year 1957: } & \text { Max } 85,000 & \text { Min } 854 & \text { Mean 17,300 } & \text { Ac-ft } & 12,530,000 \\ \text { Water year 1957-58: } & \text { Max 25,500 } & \text { Min 854 } & \text { Mean 7,429 } & \text { Ac-ft } 5,378,000\end{array}$

* Discharge measurement made on this day.

Note. - No gage-height record Jan. $u_{4}$ to Feb. $\mu_{4}$, June 4-18; discharge estimated on basis of records for stations upstream. 
HETMAND RIVER BASIN

Helmand River at Chahar Burjak, Afghanistan

Discharge, in cuble feet per second, water year October 1958 to September 1959

\begin{tabular}{|c|c|c|c|c|c|c|c|c|c|c|c|c|}
\hline Dar & $\operatorname{act}_{6}$ & Nove & Dec. & Jan. & Feb. & Mar. & Apr. & May & June & July & Aug & Sept. \\
\hline $\begin{array}{l}1 \\
2 \\
3 \\
4 \\
5\end{array}$ & $\begin{array}{l}4,930 \\
4,930 \\
5,000 \\
5,000 \\
5,000\end{array}$ & $\begin{array}{l}\frac{5,000}{5,000} \\
4,900 \\
4,800 \\
4,800\end{array}$ & $\begin{array}{l}\frac{2,990}{3,050} \\
3,050 \\
3,020 \\
3,050\end{array}$ & $\begin{array}{l}4,180 \\
4,120 \\
4,120 \\
4,090 \\
4,120\end{array}$ & $\begin{array}{l}4,600 \\
4,530 \\
4,460 \\
4,400 \\
4,330\end{array}$ & $\begin{array}{r}5,280 \\
5,120 \\
5,160 \\
16,600 \\
19,500 \\
\end{array}$ & $\begin{array}{l}13,000 \\
12,500 \\
12,200 \\
12,300 \\
13,100\end{array}$ & $\begin{array}{l}\frac{21,200}{20,400} \\
19,800 \\
19,400 \\
18,300\end{array}$ & $\begin{array}{l}\frac{11}{10,100} \\
10,600 \\
10,300 \\
10,000\end{array}$ & $\begin{array}{l}7,060 \\
7,010 \\
6,620 \\
6,460 \\
6,300\end{array}$ & $\begin{array}{l}5,200 \\
5,160 \\
5,160 \\
5,040 \\
5,000\end{array}$ & $\begin{array}{l}4,360 \\
4,460 \\
4,460 \\
4,460 \\
4,400\end{array}$ \\
\hline $\begin{array}{r}6 \\
7 \\
8 \\
9 \\
10\end{array}$ & $\begin{array}{l}5,000 \\
5,000 \\
5,000 \\
5,000 \\
5,000\end{array}$ & $\begin{array}{l}4,800 \\
4,700 \\
4,700 \\
4,700 \\
4,600\end{array}$ & $\begin{array}{l}3,100 \\
3,080 \\
3,080 \\
3,050 \\
3,050\end{array}$ & $\begin{array}{l}4,210 \\
4,330 \\
4,430 \\
4,460 \\
4,400\end{array}$ & $\begin{array}{l}4,330 \\
4,400 \\
4,330 \\
4,020 \\
3,960\end{array}$ & $\begin{array}{r}13,800 \\
13,300 \\
11,900 \\
10,800 \\
9,920\end{array}$ & $\begin{array}{l}16,000 \\
17,200 \\
15,700 \\
14,4,00 \\
13,400\end{array}$ & $\begin{array}{l}17,700 \\
17,100 \\
16,500 \\
16,200 \\
15,600\end{array}$ & $\begin{array}{l}9,760 \\
9,650 \\
9,600 \\
9,430 \\
9,100\end{array}$ & $\begin{array}{l}6,130 \\
5,910 \\
5,910 \\
6,130 \\
6,400\end{array}$ & $\begin{array}{r}4,900 \\
4,870 \\
* 4,800 \\
4,660 \\
4,600\end{array}$ & $\begin{array}{l}4,400 \\
4,430 \\
4,400 \\
4,400 \\
4,330\end{array}$ \\
\hline $\begin{array}{l}11 \\
12 \\
13 \\
14 \\
15\end{array}$ & $\begin{array}{l}5,000 \\
5,000 \\
5,000 \\
5,000 \\
5,000\end{array}$ & $\begin{array}{r}4,600 \\
4,600 \\
* 4,600 \\
* 4,660 \\
4,660\end{array}$ & $\begin{array}{l}3,050 \\
3,050 \\
3,080 \\
3,240 \\
3,610\end{array}$ & $\begin{array}{l}4,320 \\
4,240 \\
4,210 \\
4,150 \\
4,150\end{array}$ & $\begin{array}{l}\frac{3,930}{4,020} \\
4,210 \\
1,4,30 \\
4,760\end{array}$ & $\begin{array}{r}8,990 \\
9,920 \\
11,900 \\
10,400 \\
9,380\end{array}$ & $\begin{array}{l}12,800 \\
12,400 \\
12,400 \\
12,100 \\
12,000\end{array}$ & $\begin{array}{l}15,200 \\
14,900 \\
14,500 \\
11,900 \\
14,000\end{array}$ & $\begin{array}{l}8,820 \\
8,720 \\
8,440 \\
7,940 \\
7,890\end{array}$ & $\begin{array}{l}6,180 \\
5,960 \\
5,960 \\
5,800 \\
5,720\end{array}$ & $\begin{array}{l}4,560 \\
4,600 \\
4,930 \\
5,150 \\
4,730\end{array}$ & $\begin{array}{l}4,150 \\
3,870 \\
3,870 \\
3,870 \\
3,870\end{array}$ \\
\hline $\begin{array}{l}16 \\
17 \\
18 \\
19 \\
20\end{array}$ & $\begin{array}{l}5,000 \\
5,000 \\
5,000 \\
5,000 \\
5,000\end{array}$ & $\begin{array}{r}4,630 \\
3,260 \\
3,020 \\
* 2,940 \\
2,820 \\
\end{array}$ & $\begin{array}{l}4,400 \\
3,900 \\
3,720 \\
3,750 \\
3,870\end{array}$ & $\begin{array}{l}4,150 \\
4,150 \\
4,150 \\
4,210 \\
4,240\end{array}$ & $\begin{array}{l}5,240 \\
5,000 \\
4,660 \\
4,460 \\
4,180\end{array}$ & $\begin{array}{l}8,440 \\
7,840 \\
7,230 \\
6,900 \\
6,520\end{array}$ & $\begin{array}{l}11,800 \\
11,300 \\
10,800 \\
12,200 \\
15,100\end{array}$ & $\begin{array}{r}13,600 \\
13,300 \\
12,900 \\
* 12,700 \\
13,500\end{array}$ & $\begin{array}{l}7,560 \\
7,280 \\
7,450 \\
7,500 \\
7,500\end{array}$ & $\begin{array}{l}5,600 \\
5,560 \\
5,460 \\
5,260 \\
5,160 \\
\end{array}$ & $\begin{array}{l}4,500 \\
4,430 \\
4,460 \\
4,460 \\
4,400\end{array}$ & $\begin{array}{l}3,810 \\
3,810 \\
3,930 \\
4,090 \\
4,020\end{array}$ \\
\hline $\begin{array}{l}21 \\
22 \\
23 \\
24 \\
25\end{array}$ & $\begin{array}{l}4,900 \\
4,600 \\
4,500 \\
4,700 \\
5,000\end{array}$ & $\begin{array}{l}2,840 \\
2,920 \\
2,940 \\
2,920 \\
2,890\end{array}$ & $\begin{array}{l}3,720 \\
3,750 \\
3,840 \\
4,210 \\
4,730\end{array}$ & $\begin{array}{l}4,360 \\
4,360 \\
4,360 \\
4,330 \\
4,300\end{array}$ & $\begin{array}{r}\frac{8,450}{6,620} \\
5,230 \\
5,040 \\
* 4,760\end{array}$ & $\begin{array}{r}3,110 \\
9,480 \\
9,920 \\
10,200 \\
10,200\end{array}$ & $\begin{array}{l}18,200 \\
20,200 \\
21,700 \\
23,000 \\
23,600\end{array}$ & $\begin{array}{l}u_{4}, 300 \\
u_{4}, 300 \\
u_{4}, 100 \\
u_{4}, 400 \\
13,700\end{array}$ & $\begin{array}{l}7,500 \\
7,400 \\
7,400 \\
7,230 \\
7,180\end{array}$ & $\begin{array}{l}5,240 \\
6,080 \\
5,800 \\
5,320 \\
5,400\end{array}$ & $\begin{array}{l}\frac{4,300}{4,400} \\
4,500 \\
4,400 \\
4,360\end{array}$ & $\begin{array}{l}3,900 \\
3,780 \\
3,580 \\
3,580 \\
3,610\end{array}$ \\
\hline $\begin{array}{l}26 \\
27 \\
28 \\
29 \\
30 \\
31\end{array}$ & $\begin{array}{l}5,000 \\
5,000 \\
5,000 \\
5,000 \\
5,000 \\
5,000\end{array}$ & $\begin{array}{l}2,890 \\
2,860 \\
2,860 \\
2,840 \\
2,860 \\
-\end{array}$ & $\begin{array}{l}4,270 \\
4,020 \\
3,870 \\
3,840 \\
3,870 \\
4,210\end{array}$ & $\begin{array}{l}4,300 \\
4,4,00 \\
4,630 \\
4,600 \\
4,530 \\
4,530\end{array}$ & $\begin{array}{c}* 4,700 \\
5,360 \\
5,520 \\
- \\
=\end{array}$ & $\begin{array}{r}10,200 \\
10,100 \\
10,000 \\
9,980 \\
10,600 \\
12,800\end{array}$ & $\begin{array}{c}24,400 \\
24,600 \\
24,000 \\
23,000 \\
22,000 \\
-\end{array}$ & $\begin{array}{l}13,000 \\
12,700 \\
12,200 \\
12,000 \\
11,700 \\
11,200 \\
\end{array}$ & $\begin{array}{c}7,230 \\
7,450 \\
7,670 \\
7,450 \\
7,060 \\
-\end{array}$ & $\begin{array}{l}5,320 \\
5,200 \\
5,200 \\
7,460 \\
6,350 \\
5,520\end{array}$ & $\begin{array}{l}4,360 \\
4,400 \\
4,360 \\
4,400 \\
4,360 \\
4,330\end{array}$ & $\begin{array}{l}3,660 \\
3,440 \\
3,050 \\
2,940 \\
\frac{2,920}{-}\end{array}$ \\
\hline $\begin{array}{l}\text { Iotial } \\
\text { Mean } \\
\text { Ac-fi }\end{array}$ & $\begin{array}{r}153,560 \\
4,954 \\
304,600\end{array}$ & $\begin{array}{r}116,610 \\
3,887 \\
231,300\end{array}$ & $\begin{array}{r}110,520 \\
3,565 \\
219,200\end{array}$ & $\begin{array}{r}133,140 \\
4,295 \\
264,100\end{array}$ & $\begin{array}{r}133,980 \\
4,785 \\
265,700\end{array}$ & $\begin{array}{r}310,390 \\
10,010 \\
615,600\end{array}$ & $\begin{array}{r}487,400 \\
16,250 \\
966,700\end{array}$ & $\begin{array}{r}464,600 \\
14,990 \\
921,500\end{array}$ & $\begin{array}{r}253,010 \\
8,434 \\
501,800\end{array}$ & $\begin{array}{r}182,520 \\
5,920 \\
364,000\end{array}$ & $\begin{array}{r}143,790 \\
4,638 \\
285,200\end{array}$ & $\begin{array}{r}117,850 \\
3,928 \\
233,800\end{array}$ \\
\hline
\end{tabular}

$\begin{array}{llllllll}\text { Calendar year 1956: Mex 19,900 } & \text { Min 2,820 } & \text { Mean 6,719 } & \text { Ac-ft } t_{i}, 865,000 \\ \text { Weter year 1958-59: Mex 24,600 } & \text { Min 2,820 } & \text { Mean 7,146 } & \text { Ac-ft 5, } 174,000\end{array}$

* Discrerge measurement made on this day.

Note.- No gaze-height record Cct. 3 to Nov. 13; discharge estimated or besis nf rocusded lange in stage and reccrds

for station at Darweshan. 
HETMAND RIVER BASIN!

Helmand River at Chahar Burjak, Hfghanistan

Discharge, in cubic feet per second, water year October 1959 to September 1960

\begin{tabular}{|c|c|c|c|c|c|c|c|c|c|c|c|c|}
\hline Dav & Oct. & Nov. & Dec, & Jan. & Feb. & Mar. & Apr. & Mar & June & July & Aug. & Sept. \\
\hline $\begin{array}{l}1 \\
2 \\
3 \\
4 \\
5\end{array}$ & $\begin{array}{l}3,330 \\
3,490 \\
3,180 \\
3,290 \\
3,240\end{array}$ & $\begin{array}{l}2,730 \\
2,790 \\
2,890 \\
2,890 \\
2,840\end{array}$ & $\begin{array}{l}\frac{2,730}{2,760} \\
2,790 \\
2,730 \\
5,600\end{array}$ & $\begin{array}{l}5,560 \\
5,480 \\
5,520 \\
5,960 \\
6,300 \\
\end{array}$ & $\begin{array}{l}3,4,40 \\
3,440 \\
3,550 \\
3,490 \\
3,460\end{array}$ & $\begin{array}{l}2,200 \\
2,200 \\
2,200 \\
2,200 \\
2,200\end{array}$ & $\begin{array}{l}4,200 \\
3,700 \\
3,4,00 \\
3,100 \\
3,000\end{array}$ & $\begin{array}{l}7,010 \\
6,790 \\
6,570 \\
6,080 \\
6,020\end{array}$ & $\begin{array}{l}17,500 \\
17,600 \\
\frac{17,600}{17,400} \\
17,000\end{array}$ & $\begin{array}{l}4,600 \\
4,400 \\
4,200 \\
4,000 \\
3,800\end{array}$ & $\begin{array}{l}\frac{2,080}{2,080} \\
2,060 \\
2,060 \\
2,060\end{array}$ & $\begin{array}{l}1,900 \\
1,900 \\
1,900 \\
1,900 \\
1,850\end{array}$ \\
\hline $\begin{array}{r}6 \\
7 \\
8 \\
9 \\
10\end{array}$ & $\begin{array}{l}3,100 \\
3,080 \\
3,050 \\
2,990 \\
2,940\end{array}$ & $\begin{array}{l}2,860 \\
2,890 \\
2,390 \\
\frac{3,020}{2,990}\end{array}$ & $\begin{array}{l}5,910 \\
4,210 \\
3,810 \\
3,550 \\
3,410\end{array}$ & $\begin{array}{r}* 6,080 \\
5,910 \\
5,760 \\
5,680 \\
5,600\end{array}$ & $\begin{array}{l}3,440 \\
3,260 \\
3,200 \\
3,150 \\
3,150\end{array}$ & $\begin{array}{l}2,100 \\
2,000 \\
1,900 \\
1,900 \\
1,900\end{array}$ & $\begin{array}{r}* 2,920 \\
2,890 \\
2,630 \\
\frac{2}{2}, 820 \\
2,940\end{array}$ & $\begin{array}{r}5,960 \\
6,300 \\
* 6,240 \\
5,960 \\
5,910\end{array}$ & $\begin{array}{l}16,800 \\
16,400 \\
15,900 \\
15,600 \\
13,400\end{array}$ & $\begin{array}{l}3,600 \\
3,500 \\
3,400 \\
3,300 \\
3,200\end{array}$ & $\begin{array}{l}1,920 \\
1,660 \\
1,660 \\
1,660 \\
1,680\end{array}$ & $\begin{array}{l}\frac{1,800}{1,800} \\
1,800 \\
1,850 \\
1,900\end{array}$ \\
\hline $\begin{array}{l}11 \\
12 \\
13 \\
14 \\
15\end{array}$ & $\begin{array}{l}2,990 \\
2,890 \\
2,860 \\
2,840 \\
2,860\end{array}$ & $\begin{array}{l}2,960 \\
2,940 \\
2,840 \\
2,660 \\
2,660\end{array}$ & $\begin{array}{l}3,350 \\
3,260 \\
3,180 \\
3,720 \\
4,660\end{array}$ & $\begin{array}{l}5,600 \\
5,560 \\
4,570 \\
4,020 \\
3,840\end{array}$ & $\begin{array}{l}3,150 \\
3,150 \\
3,100 \\
2,840 \\
2,840\end{array}$ & $\begin{array}{l}\frac{1,800}{1,800} \\
1,900 \\
2,000 \\
2,300\end{array}$ & $\begin{array}{l}3,020 \\
3,080 \\
3,080 \\
3,120 \\
3,150\end{array}$ & $\begin{array}{r}5,910 \\
6,460 \\
9,870 \\
12,400 \\
13,700\end{array}$ & $\begin{array}{r}11,200 \\
11,200 \\
10,600 \\
10,100 \\
9,870\end{array}$ & $\begin{array}{l}3,000 \\
2,900 \\
2,800 \\
2,700 \\
2,600\end{array}$ & $\begin{array}{l}1,700 \\
1,700 \\
1,700 \\
1,700 \\
1,700\end{array}$ & $\begin{array}{l}1,950 \\
2,000 \\
2,000 \\
2,000 \\
2,000\end{array}$ \\
\hline $\begin{array}{l}16 \\
17 \\
18 \\
19 \\
20\end{array}$ & $\begin{array}{r}2,860 \\
2,840 \\
2,860 \\
* 2,840 \\
2,700\end{array}$ & $\begin{array}{l}2,680 \\
2,700 \\
2,760 \\
2,760 \\
2,760\end{array}$ & $\begin{array}{l}5,480 \\
5,600 \\
5,960 \\
6,180 \\
6,130\end{array}$ & $\begin{array}{l}3,780 \\
3,720 \\
3,660 \\
3,610 \\
3,610\end{array}$ & $\begin{array}{l}2,840 \\
2,840 \\
2,700 \\
2,500 \\
2,500\end{array}$ & $\begin{array}{l}2,600 \\
5,000 \\
6,000 \\
5,400 \\
4,600\end{array}$ & $\begin{array}{r}3,120 \\
2,990 \\
3,020 \\
10,500 \\
17,100 \\
\end{array}$ & $\begin{array}{l}16,400 \\
18,300 \\
20,000 \\
21,100 \\
22,200\end{array}$ & $\begin{array}{l}9,700 \\
9,480 \\
9,540 \\
9,480 \\
9,600\end{array}$ & $\begin{array}{r}2,500 \\
* 2,400 \\
2,260 \\
2,200 \\
2,160\end{array}$ & $\begin{array}{l}1,700 \\
1,700 \\
1,700 \\
1,700 \\
1,700\end{array}$ & $\begin{array}{r}2,000 \\
2,000 \\
* 2,000 \\
2,020 \\
2,040\end{array}$ \\
\hline $\begin{array}{l}21 \\
22 \\
23 \\
24 \\
25\end{array}$ & $\begin{array}{l}2,680 \\
2,660 \\
2,680 \\
2,700 \\
2,730\end{array}$ & $\begin{array}{l}2,790 \\
2,820 \\
2,840 \\
2,840 \\
2,840\end{array}$ & $\begin{array}{l}6,020 \\
6,020 \\
5,910 \\
5,800 \\
5,800\end{array}$ & $\begin{array}{l}3,580 \\
3,550 \\
3,490 \\
3,520 \\
3,460\end{array}$ & $\begin{array}{l}2,400 \\
2,300 \\
2,300 \\
2,300 \\
2,300\end{array}$ & $\begin{array}{l}4,000 \\
3,800 \\
3,700 \\
3,600 \\
3,200\end{array}$ & $\begin{array}{r}15,600 \\
11,200 \\
9,820 \\
9,160 \\
8,720\end{array}$ & $\begin{array}{l}23,300 \\
\frac{23}{23}, 400 \\
23,400 \\
22,7000\end{array}$ & $\begin{array}{l}9,430 \\
8,880 \\
8,820 \\
8,820 \\
7,000\end{array}$ & $\begin{array}{l}2,1,0 \\
2,080 \\
2,060 \\
\frac{2,040}{2,060}\end{array}$ & $\begin{array}{l}1,700 \\
1,700 \\
1,750 \\
1,750 \\
1,750\end{array}$ & $\begin{array}{l}2,000 \\
2,020 \\
1,940 \\
1,920 \\
1,940\end{array}$ \\
\hline $\begin{array}{l}26 \\
27 \\
28 \\
29 \\
30 \\
31 \\
\end{array}$ & $\begin{array}{l}2,700 \\
2,730 \\
2,730 \\
2,730 \\
2,730 \\
2,700 \\
\end{array}$ & $\begin{array}{c}2,840 \\
2,820 \\
2,820 \\
2,790 \\
2,680 \\
-\end{array}$ & $\begin{array}{l}5,800 \\
5,720 \\
5,680 \\
5,640 \\
5,640 \\
5,640 \\
\end{array}$ & $\begin{array}{l}3,470 \\
3,460 \\
3,440 \\
3,460 \\
3,440 \\
3,440\end{array}$ & $\begin{array}{c}2,300 \\
2,300 \\
2,300 \\
2,300 \\
- \\
-\end{array}$ & $\begin{array}{l}3,100 \\
3,000 \\
2,900 \\
3,000 \\
3,200 \\
4,400\end{array}$ & $\begin{array}{c}8,230 \\
7,840 \\
7,560 \\
7,340 \\
7,230 \\
-\end{array}$ & $\begin{array}{l}21,800 \\
20,700 \\
19,600 \\
18,800 \\
17,900 \\
17,600\end{array}$ & $\begin{array}{l}6,500 \\
6,000 \\
5,500 \\
5,200 \\
\frac{4,900}{-}\end{array}$ & $\begin{array}{l}2,060 \\
2,060 \\
2,080 \\
2,080 \\
2,080 \\
2,080\end{array}$ & $\begin{array}{l}1,800 \\
1,850 \\
1,900 \\
1,900 \\
1,900 \\
1,900\end{array}$ & $\begin{array}{l}1,960 \\
1,960 \\
1,980 \\
2,040 \\
2,080 \\
-\end{array}$ \\
\hline $\begin{array}{l}\text { Total } \\
\text { Mean } \\
\text { Ac-ft }\end{array}$ & $\begin{array}{r}90,000 \\
2,903 \\
178,500 \\
\end{array}$ & $\begin{array}{r}84,690 \\
2,823 \\
168,000 \\
\end{array}$ & $\begin{array}{r}148,690 \\
4,796 \\
294,900\end{array}$ & $\begin{array}{r}138,450 \\
4,466 \\
274,600\end{array}$ & $\begin{array}{r}82,940 \\
2,860 \\
164,500\end{array}$ & $\begin{array}{r}92,100 \\
2,971 \\
182,700 \\
\end{array}$ & $\begin{array}{r}176,470 \\
5,882 \\
350,000 \\
\end{array}$ & $\begin{array}{r}41_{1}, 380 \\
u_{4}, 240 \\
875,500\end{array}$ & $\begin{array}{r}337,020 \\
11,230 \\
668,500\end{array}$ & $\begin{array}{r}86,340 \\
2,785 \\
171,300\end{array}$ & $\begin{array}{r}55,820 \\
1,801 \\
110,700\end{array}$ & $\begin{array}{r}58,450 \\
1,945 \\
115,900\end{array}$ \\
\hline
\end{tabular}

Galendar year 1959: Max 24,600 Min 2,660 Moan 6,989 Ac-ft 5,060,000

Tater year 1959-60: Max 23,400 Min 1,660 Mean 4,897 Ac-ft 3,555,000

* Discharge measurement made on this day.

Note.-- No gage-height record Feb. 8-14, Feb. 14 to Apr. 5, June 25 to July 17, Aug. 11 to Sept. 17; discharge

estimated on basis of recorded range ir. stage and records for station at Darweshan. 


\section{HETMAND RIVER BASTN}

Helmand River at Shals Charkh, Afghanistan

Ination. - Iat $31^{\circ} 021 \mathrm{M} .$, long $61^{\circ} 52^{\prime}$ E., on left bank at bridge aite at village of Shela Charkh about 8 kilometers south of $\mathrm{Kala} \mathrm{Kang}$, about 24 kilometers southwest from Chakansur, about $28 \mathrm{kllometers}$ downstream from Seistan Diversion, and about 105 kilometers downstroam from Chahar Burjak.

Bncords arajlable.m August 1955 to September 1957, September 1960. Occasional discharge meagurements fren Feb. 27, 1953 to August 23, 1955 and May 20, 1959 to Sept. 17, 1960.

fare.- Staff gage or roference point.

Butreng.

1956: Kaximm daily discharge $12,000 \mathrm{cfs}$ May 8-1l, July 23, 24, 30; minimum daily, 214 cfs July 12

1957: vaximm daily discharge, 36,000 cfs kay 13, from rating curve extended above 3,200 cfs; minimum dails $260 \mathrm{crs}$ Aug. 24, 25.

8enarica. - Records fair except those for periods of no gage height and those above 6,000 crs whjch are poor. Extensive diversions for irrigation upstream. Discharge measurements listed below were made outside period of record.

\begin{tabular}{|c|c|c|c|c|c|}
\hline Date & $\begin{array}{c}\text { Gage Height } \\
\text { (motere) }\end{array}$ & $\begin{array}{c}\text { Diecharge } \\
\text { (crs) }\end{array}$ & Date & $\begin{array}{c}\text { Gage Height } \\
\text { (moters) }\end{array}$ & $\begin{array}{c}\text { Discharge } \\
\text { (crs) }\end{array}$ \\
\hline $\begin{array}{l}\text { Feb. } 27,1953 \\
\text { Jwe } 9,1953 \\
\text { Aug. } 13,1953 \\
\text { Oct. } 14,1953 \\
\text { Jul. } 8,1954 \\
\text { Sept. } 3,1954 \\
\text { Oct. 11, 1954 } \\
\text { Nov. 22, } 1954 \\
\text { Jan. 14, } 1955 \\
\text { Peb. 18, } 1955 \\
\text { Yar. } 30,1955 \\
\text { June } 7,1955\end{array}$ & $\begin{array}{l}= \\
= \\
= \\
0.82 \\
.465 \\
.42 \\
1.50 \\
1.045 \\
.66 \\
1.225\end{array}$ & $\begin{array}{r}217 \\
1240 \\
1080 \\
456 \\
732 \\
1880 \\
900 \\
888 \\
3380 \\
2230 \\
1960 \\
2610\end{array}$ & $\begin{array}{l}\text { July } 13,1955 \\
\text { Aug. } 23,1955 \\
\text { Sept. } 26,1955 \\
\text { May } 20,1959 \\
\text { Aug. } 7,1959 \\
\text { Oct. } 22,1959 \\
\text { Jan. } 7,1960 \\
\text { Apr. } 9,1960 \\
\text { Nay 15, } 1960 \\
\text { July 19, } 1960 \\
\text { Sept. 17, } 1960\end{array}$ & $\begin{array}{l}1.35 \\
1.66 \\
1.64 \\
1.01 \\
- \\
.96 \\
.55 \\
1.46 \\
.25 \\
.16\end{array}$ & $\begin{array}{r}577 \\
1190 \\
1070 \\
6010 \\
2290 \\
1330 \\
3340 \\
1840 \\
4560 \\
1210 \\
900\end{array}$ \\
\hline
\end{tabular}


HEIMANE RIVER BASIN

Helinand River ai Shela Charkh, Af ghanistan

Discharge, in cubic feet per second, water year October 1955 to September 1956

\begin{tabular}{|c|c|c|c|c|c|c|c|c|c|c|c|c|}
\hline DaY & det. & Nore & Doc. & Jan. & Fob. & Mar. & Apr. & $\mathrm{MaY}$ & June & July & Aug. & Sept. \\
\hline $\begin{array}{l}1 \\
2 \\
3 \\
4 \\
5\end{array}$ & $\begin{array}{l}1,100 \\
1,310 \\
1,220 \\
1,190 \\
1,240\end{array}$ & $\begin{array}{r}1,120 \\
1,100 \\
1,120 \\
1,100 \\
850\end{array}$ & $\begin{array}{r}760 \\
850 \\
850 \\
1,320 \\
1,320\end{array}$ & $\begin{array}{r}2,240 \\
1,540 \\
1,610 \\
* 1,540 \\
1,500\end{array}$ & $\begin{array}{l}1,680 \\
1,680 \\
1,600 \\
1,580 \\
1,790\end{array}$ & $\begin{array}{l}1,080 \\
1,100 \\
2,620 \\
2,430 \\
2,380\end{array}$ & $\begin{array}{l}2,730 \\
2,730 \\
2,730 \\
2,730 \\
2,730\end{array}$ & $\begin{array}{l}850 \\
850 \\
425 \\
425 \\
425\end{array}$ & $\begin{array}{l}3,260 \\
3,050 \\
2,950 \\
2,620 \\
1,990\end{array}$ & $\begin{array}{l}464 \\
432 \\
425 \\
412 \\
335\end{array}$ & $\begin{array}{l}7,860 \\
5,700 \\
4,500 \\
3,940 \\
3,000\end{array}$ & $\begin{array}{r}2,570 \\
1,750 \\
1,310 \\
810 \\
770\end{array}$ \\
\hline $\begin{array}{r}6 \\
7 \\
8 \\
9 \\
10\end{array}$ & $\begin{array}{l}1,250 \\
1,240 \\
1,160 \\
1,060 \\
1,090\end{array}$ & $\begin{array}{l}850 \\
850 \\
850 \\
850 \\
800\end{array}$ & $\begin{array}{l}1,340 \\
1,280 \\
1,280 \\
1,200 \\
1,520\end{array}$ & $\begin{array}{l}1,520 \\
2,600 \\
2,620 \\
2,580 \\
2,030\end{array}$ & $\begin{array}{l}1,910 \\
1,890 \\
1,330 \\
1,870 \\
2,050 \\
\end{array}$ & $\begin{array}{l}2,340 \\
2,290 \\
2,290 \\
3,360 \\
4,220\end{array}$ & $\begin{array}{r}586 \\
850 \\
406 \\
850 \\
I, 580\end{array}$ & $\begin{array}{r}425 \\
\frac{243}{12,000} \\
\frac{12,000}{12,000} \\
12,000\end{array}$ & $\begin{array}{l}1,680 \\
1,640 \\
1,580 \\
1,500 \\
1,500\end{array}$ & $\begin{array}{l}260 \\
230 \\
226 \\
226 \\
226\end{array}$ & $\begin{array}{l}2,900 \\
2,620 \\
2,620 \\
3,960 \\
3,060\end{array}$ & $\begin{array}{r}780 \\
2,520 \\
2,340 \\
2,840 \\
2,840\end{array}$ \\
\hline $\begin{array}{l}11 \\
12 \\
13 \\
14 \\
15\end{array}$ & $\begin{array}{l}1,200 \\
1,280 \\
1,230 \\
1,220 \\
1,240\end{array}$ & $\begin{array}{r}790 \\
780 \\
* 750 \\
750 \\
710\end{array}$ & $\begin{array}{l}1,330 \\
1,330 \\
2,110 \\
2,070 \\
1,390\end{array}$ & $\begin{array}{l}1,830 \\
1,660 \\
1,460 \\
1,360 \\
1,320\end{array}$ & $\begin{array}{l}2,010 \\
1,950 \\
1,970 \\
1,850 \\
1,750\end{array}$ & $\begin{array}{l}3,800 \\
2,900 \\
2,810 \\
2,870 \\
2,600\end{array}$ & $\begin{array}{l}2,620 \\
2,620 \\
2,620 \\
2,620 \\
2,620\end{array}$ & $\begin{array}{r}12,000 \\
3,000 \\
2,900 \\
2,620 \\
2,620\end{array}$ & $\begin{array}{l}1,420 \\
1,250 \\
1,250 \\
1,380 \\
1,250\end{array}$ & $\begin{array}{l}226 \\
214 \\
310 \\
340 \\
335\end{array}$ & $\begin{array}{l}3,060 \\
3,060 \\
3,060 \\
2,950 \\
2,340\end{array}$ & $\begin{array}{l}2,840 \\
2,840 \\
2,950 \\
3,060 \\
2,950\end{array}$ \\
\hline $\begin{array}{l}16 \\
17 \\
18 \\
19 \\
20\end{array}$ & $\begin{array}{l}1,340 \\
1,300 \\
1,320 \\
1,280 \\
1,220\end{array}$ & $\begin{array}{l}690 \\
690 \\
690 \\
680 \\
660\end{array}$ & $\begin{array}{l}1,810 \\
2,900 \\
7,480 \\
4,010 \\
3,550\end{array}$ & $\begin{array}{l}1,300 \\
1,250 \\
1,240 \\
1,250 \\
1,280\end{array}$ & $\begin{array}{l}1,680 \\
1,660 \\
1,750 \\
1,750 \\
1,610\end{array}$ & $\begin{array}{l}2,620 \\
2,480 \\
2,400 \\
2,530 \\
2,870\end{array}$ & $\begin{array}{l}2,620 \\
1,580 \\
1,580 \\
1,580 \\
1,580\end{array}$ & $\begin{array}{l}3,060 \\
3,060 \\
3,060 \\
2,950 \\
2,950\end{array}$ & $\begin{array}{r}1,250 \\
1,130 \\
1,080 \\
975 \\
875\end{array}$ & $\begin{array}{r}325 \\
7,200 \\
12,000 \\
12,000 \\
2,950\end{array}$ & $\begin{array}{l}2,620 \\
2,570 \\
2,460 \\
2,380 \\
2,430\end{array}$ & $\begin{array}{l}2,730 \\
2,730 \\
2,800 \\
2,900 \\
2,900\end{array}$ \\
\hline $\begin{array}{l}21 \\
22 \\
23 \\
24 \\
25\end{array}$ & $\begin{array}{l}1,300 \\
1,250 \\
1,240 \\
1,300 \\
1,200\end{array}$ & $\begin{array}{l}634 \\
634 \\
618 \\
634 \\
670\end{array}$ & $\begin{array}{l}2,810 \\
2,700 \\
2,400 \\
2,400 \\
2,550\end{array}$ & $\begin{array}{l}1,360 \\
1,380 \\
1,420 \\
1,400 \\
1,400\end{array}$ & $\begin{array}{l}1,640 \\
1,700 \\
1,580 \\
1,460 \\
1,320\end{array}$ & $\begin{array}{l}2,650 \\
2,620 \\
3,360 \\
2,730 \\
7,530\end{array}$ & $\begin{array}{l}1,580 \\
1,580 \\
1,580 \\
2,520 \\
2,620\end{array}$ & $\begin{array}{l}2,950 \\
2,840 \\
2,840 \\
2,840 \\
4,500\end{array}$ & $\begin{array}{l}860 \\
830 \\
790 \\
750 \\
634\end{array}$ & $\begin{array}{r}2,900 \\
2,950 \\
12,000 \\
12,200 \\
425\end{array}$ & $\begin{array}{l}2,460 \\
2,380 \\
2,380 \\
2,340 \\
2,340\end{array}$ & $\begin{array}{r}3,000 \\
3,100 \\
3,100 \\
+3,120 \\
2,900\end{array}$ \\
\hline $\begin{array}{l}26 \\
27 \\
28 \\
29 \\
30 \\
31\end{array}$ & $\begin{array}{l}1,200 \\
1,360 \\
1,2,260 \\
1,220 \\
1,220 \\
1,190\end{array}$ & $\begin{array}{l}690 \\
690 \\
690 \\
690 \\
670 \\
-\end{array}$ & $\begin{array}{l}2,530 \\
2,340 \\
2,580 \\
2,380 \\
2,570 \\
2,620\end{array}$ & $\begin{array}{l}1,370 \\
1,680 \\
1,660 \\
1,640 \\
1,680 \\
1,660\end{array}$ & $\begin{array}{c}1,090 \\
1,040 \\
1,220 \\
1,150 \\
= \\
=\end{array}$ & $\begin{array}{r}5,840 \\
5,440 \\
6,200 \\
11,000 \\
2,900 \\
3,900\end{array}$ & $\begin{array}{c}2,620 \\
2,620 \\
1,580 \\
1,580 \\
1,180 \\
=\end{array}$ & $\begin{array}{l}4,500 \\
4,500 \\
4,500 \\
3,940 \\
3,940 \\
3,060\end{array}$ & $\begin{array}{l}570 \\
562 \\
554 \\
514 \\
498 \\
-\end{array}$ & $\begin{array}{r}425 \\
850 \\
850 \\
1,320 \\
12, \infty 00 \\
10,500\end{array}$ & $\begin{array}{l}2,430 \\
2,430 \\
2,460 \\
2,530 \\
2,620 \\
2,840\end{array}$ & $\begin{array}{l}2,840 \\
2,340 \\
2,840 \\
2,950 \\
2,950 \\
=\end{array}$ \\
\hline $\begin{array}{l}\text { Total } \\
\text { Mean } \\
\text { Ac-et }\end{array}$ & $\begin{array}{r}38,280 \\
1,235 \\
75,930\end{array}$ & $\begin{array}{r}23,280 \\
778 \\
46,280\end{array}$ & $\begin{array}{r}68,220 \\
2,201 \\
135,300 \\
\end{array}$ & $\begin{array}{r}50,330 \\
1,625 \\
99,930\end{array}$ & $\begin{array}{r}47,940 \\
1,671 \\
95,090\end{array}$ & $\begin{array}{r}106,160 \\
3,425 \\
213,500\end{array}$ & $\begin{array}{r}59,522 \\
1,984 \\
18,100 \\
\end{array}$ & $\begin{array}{r}118,273 \\
3,815 \\
234,600\end{array}$ & $\begin{array}{r}40,002 \\
1,353 \\
79,340\end{array}$ & $\begin{array}{r}95,556 \\
3,082 \\
189,500 \\
\end{array}$ & $\begin{array}{r}93,260 \\
3,008 \\
185,000\end{array}$ & $\begin{array}{r}77,470 \\
2,582 \\
153,600\end{array}$ \\
\hline & & & & & & 214 & & 2,256 & & & $3, \infty 00$ & \\
\hline
\end{tabular}

* Discharge measurement made on this day.

Hote.-No gage-height recard Sept. 17-23; discharge estimated on basis of records for otation at Chahar Burjak. 
HETMAND RIVER BASTI

Holmand River at Sbola Charih, Mghaniotan

Discharge, in cubic feet per second, water year October 1956 to September 1957

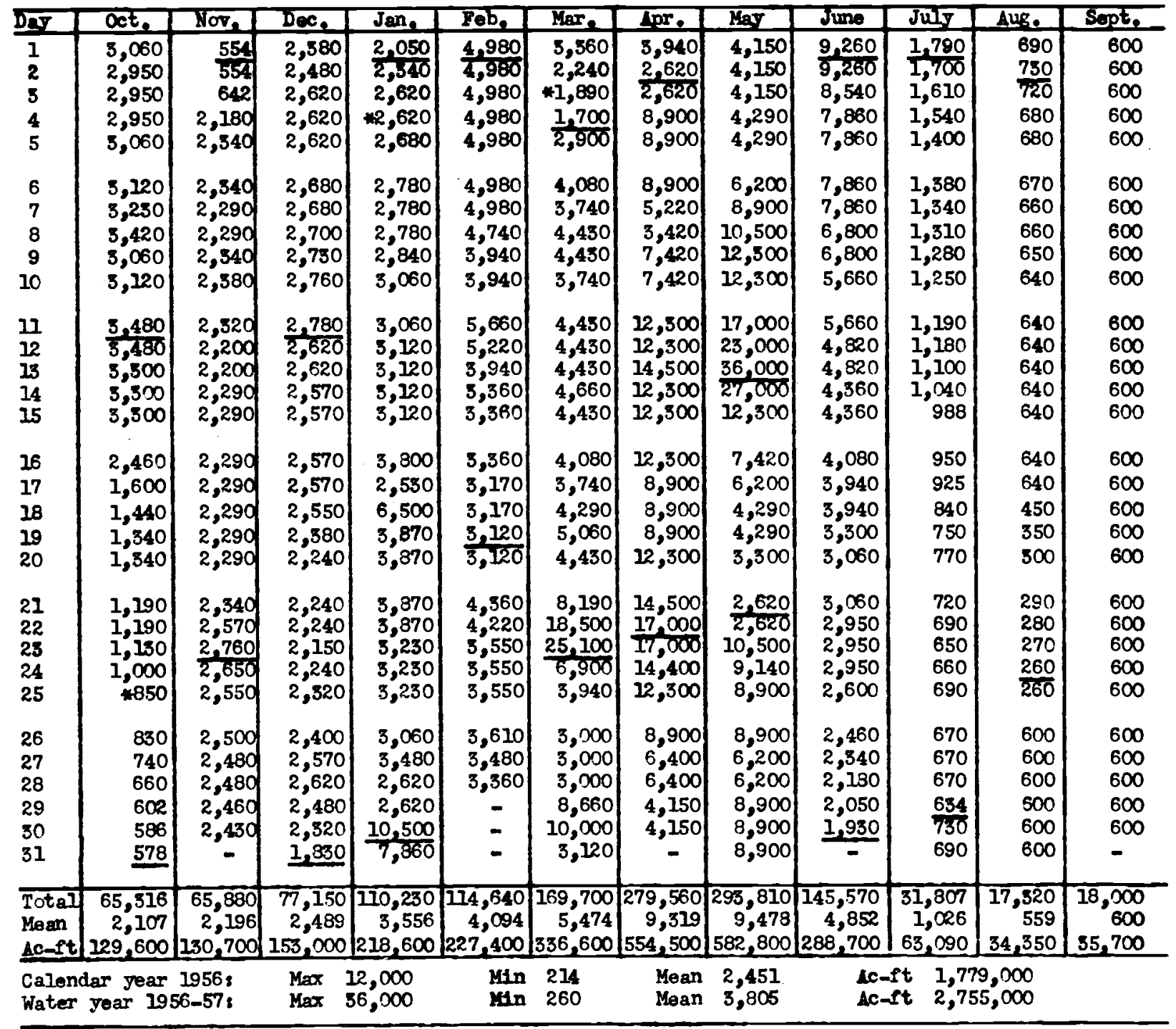

* Discharge measurement made on this day.

Note.-No gage-helght record Aug. 2 to Sept. 30; discharge estimated on basis of records for station at Chahar Burjak. 
Lecation.- Lat. $32^{\circ} 10^{\prime} \mathrm{N}$, long $63^{\circ} 25^{\prime} \mathrm{E}$, , on right bank about 300 meters downstream from highway bridge at DIlaram, about 180 kilometers upstream from Chakhansur.

Drainage area.- 2,080 oq mi, approximately.

Recorde aveilable.- October 1952 to September 1960.

Gage.- Water-stage recorder. Prior to Aug. 1, 1958, water-stage recorder on left bank about 3 kilometers downstream at same datum.

Average discharge.- 8 years, 712 cfs $(515,500$ acre-ft per year).

Extremes.- Hadimm and minimum discharges for the water years $1952-60$ are contained in the following table:

\begin{tabular}{|c|c|c|c|c|c|c|}
\hline \multirow[b]{2}{*}{ Whter year } & \multirow[b]{2}{*}{ Date } & \multicolumn{2}{|c|}{ Kaximm } & \multirow[b]{2}{*}{ Dete } & \multicolumn{2}{|c|}{ Minjinum } \\
\hline & & $\begin{array}{l}\text { Gege Height } \\
\text { (meters) }\end{array}$ & $\begin{array}{c}\text { Discharge } \\
\text { (crs) }\end{array}$ & & $\begin{array}{l}\text { Gage Height } \\
\text { (meters) }\end{array}$ & $\begin{array}{c}\text { Discharge } \\
\text { (cfa) }\end{array}$ \\
\hline $\begin{array}{l}1953 \\
1954 \\
1955 \\
1956 \\
1957 \\
1958 \\
1959 \\
1960\end{array}$ & $\begin{array}{l}\text { Feb. 14, } 1953 \\
\text { Feb. 13, } 1954 \\
\text { Mar. 15, } 1955 \\
\text { Mar. 4, } 1956 \\
\text { Kar. 17, } 1957 \\
\text { Dec. 6, } 1957 \\
\text { Yar. 2, } 1959 \\
\text { Apr. 16, } 1960 \\
\end{array}$ & $\begin{array}{c}2.52 \\
1.83 \\
2.97 \\
2.56 \\
- \\
1.90 \\
b 6.444 \\
7.515 \\
\end{array}$ & $\begin{array}{r}26,700 \\
9,380 \\
49,000 \\
35,100 \\
245,000 \\
15,100 \\
8,100 \\
21,600 \\
\end{array}$ & $\begin{array}{l}\text { Kany days } \\
\text { Kany days } \\
\text { Kany days } \\
\text { Many day: } \\
\text { Many day: } \\
\text { Sept. 6-11, } 1958 \\
\text { Sept. 23, } 1959 \\
\text { Oct. 1-31, } 1959\end{array}$ & $\begin{array}{c}\text { b5. } 11_{4} \\
5.245 \\
5.26\end{array}$ & $\begin{array}{c}0 \\
0 \\
22 \\
24 \\
210 \\
5 \\
4.4 \\
25\end{array}$ \\
\hline
\end{tabular}

a Mean daily discharge.

b Present site.

Bemarke.- Records fair except those for periods of doubtful or no gage-height record, which are poor. Many all diversions for irrigation upstream. 
HELMAND RIVER BASIN

Kash River near Dilaram, Afghanistan

Discharge, in cubic feet per second, water year October 1952 to September 1953

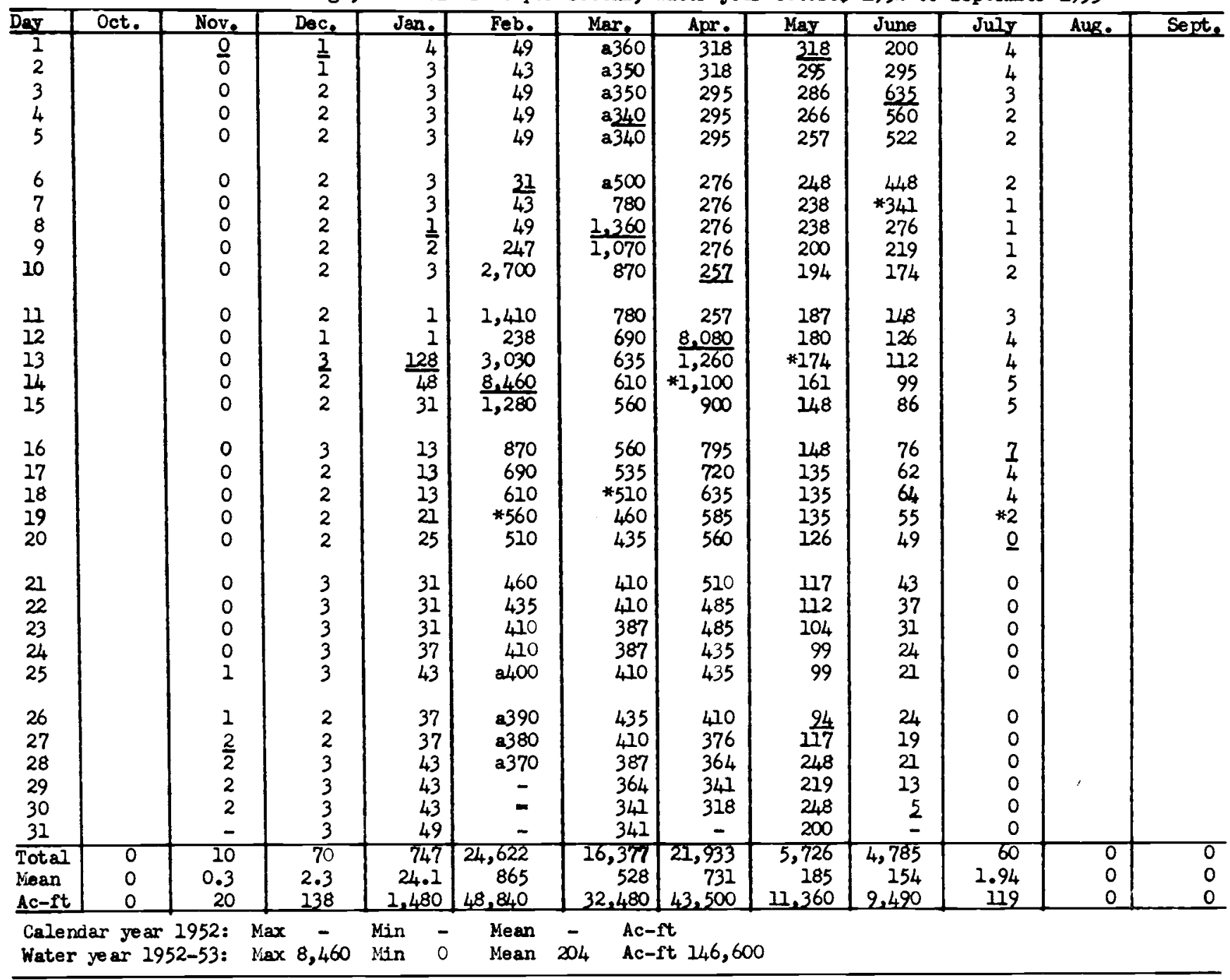

* Discharge measurement made on this day.

a No gage-hei ght record; discharge inter polated. 
HETHAND RIVWR BASIN

Khash River near Dilarail, Lfghanistan

Discharge, in cubic feet per second, water year October 1953 to jepteniber 1954

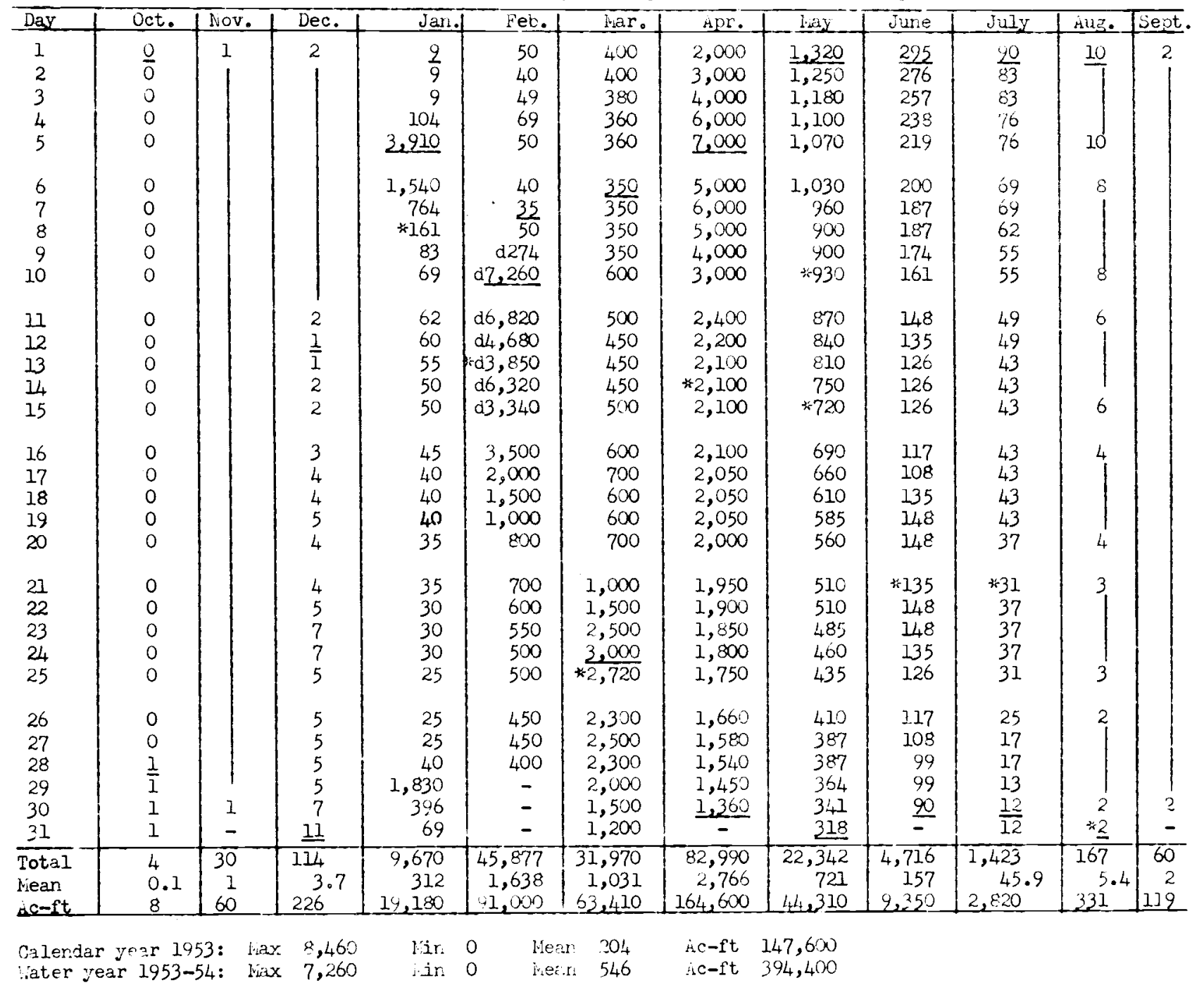

* Discharge measurement mide on tris day.

d Jischarge computed from doubtful cege hej ght record.

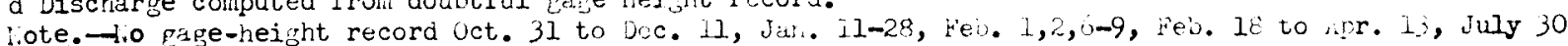
to jept. 30 , dischare estinated or bisis of recession curves, and con prison with recorjs fur liusa

ala River, Helnand liver and irghandab River. 
HETMAND RIVER BASIN

Khash River near Dilaram, Afghanistan

Discharge, in cubic feet per second, water year October 1954 to September 1955

\begin{tabular}{|c|c|c|c|c|c|c|c|c|c|c|c|c|}
\hline Day & Oct. & Nov. & Dec. & Jan. & Feb. & Mar. & Apr. & May & June & July & Aug. & Sept. \\
\hline $\begin{array}{l}1 \\
2 \\
3 \\
4 \\
5\end{array}$ & 2 & 2 & 4 & $\begin{array}{r}\frac{7}{8} \\
9 \\
10 \\
20\end{array}$ & $\begin{array}{l}65 \\
65 \\
65 \\
65 \\
59\end{array}$ & $\begin{array}{l}39 \\
39 \\
39 \\
39 \\
33\end{array}$ & $\begin{array}{l}\frac{908}{825} \\
782 \\
739 \\
718\end{array}$ & $\begin{array}{l}480 \\
499 \\
480 \\
480 \\
480\end{array}$ & $\begin{array}{l}159 \\
149 \\
140 \\
131 \\
122\end{array}$ & $\begin{array}{l}\frac{21}{21} \\
21 \\
18 \\
18\end{array}$ & $\begin{array}{l}5 \\
5 \\
5 \\
5 \\
5\end{array}$ & $\begin{array}{l}5 \\
5 \\
5 \\
5 \\
5\end{array}$ \\
\hline $\begin{array}{r}6 \\
7 \\
8 \\
9 \\
10\end{array}$ & & & & $\begin{array}{r}* 33 \\
39 \\
33 \\
36 \\
33\end{array}$ & $\begin{array}{l}56 \\
52 \\
49 \\
49 \\
44\end{array}$ & $\begin{array}{l}33 \\
30 \\
\frac{28}{49} \\
36\end{array}$ & $\begin{array}{l}696 \\
674 \\
653 \\
653 \\
632\end{array}$ & $\begin{array}{r}462 \\
632 \\
573 \\
499 \\
* 480\end{array}$ & $\begin{array}{r}112 \\
103 \\
94 \\
84 \\
84\end{array}$ & $\begin{array}{l}18 \\
16 \\
16 \\
14 \\
12\end{array}$ & $\begin{array}{l}5 \\
5 \\
5 \\
5 \\
5\end{array}$ & $\begin{array}{l}5 \\
5 \\
5 \\
5 \\
5\end{array}$ \\
\hline $\begin{array}{l}11 \\
12 \\
13 \\
14 \\
15\end{array}$ & & & & $\begin{array}{l}36 \\
39 \\
39 \\
36 \\
36\end{array}$ & $\begin{array}{l}39 \\
39 \\
33 \\
33 \\
28\end{array}$ & $\begin{array}{r}59 \\
84 \\
316 \\
25,900 \\
* 36,200 \\
\end{array}$ & $\begin{array}{l}610 \\
610 \\
592 \\
592 \\
573\end{array}$ & $\begin{array}{l}450 \\
425 \\
410 \\
395 \\
380\end{array}$ & $\begin{array}{r}* 89 \\
84 \\
75 \\
70 \\
70\end{array}$ & $\begin{array}{r}12 \\
12 \\
12 \\
9 \\
* 8\end{array}$ & $\begin{array}{l}5 \\
5 \\
5 \\
5 \\
5\end{array}$ & $\begin{array}{l}5 \\
5 \\
5 \\
5 \\
5\end{array}$ \\
\hline $\begin{array}{l}16 \\
17 \\
18 \\
19 \\
20\end{array}$ & * & * & & $\begin{array}{l}30 \\
28 \\
26 \\
26 \\
23\end{array}$ & $\begin{array}{r}28 \\
28 \\
28 \\
23 \\
+28\end{array}$ & $\begin{array}{l}26,500 \\
12,400 \\
* 6,220 \\
* 4,210 \\
* 3,250\end{array}$ & $\begin{array}{l}554 \\
554 \\
536 \\
518 \\
518\end{array}$ & $\begin{array}{l}365 \\
365 \\
350 \\
335 \\
320\end{array}$ & $\begin{array}{l}65 \\
65 \\
59 \\
54 \\
54\end{array}$ & $\begin{array}{l}7 \\
7 \\
7 \\
6 \\
6\end{array}$ & $\begin{array}{l}5 \\
5 \\
5 \\
5 \\
5\end{array}$ & $\begin{array}{l}5 \\
5 \\
5 \\
5 \\
5\end{array}$ \\
\hline $\begin{array}{l}21 \\
22 \\
23 \\
24 \\
25\end{array}$ & & & & $\begin{array}{r}23 \\
23 \\
26 \\
23 \\
* 23\end{array}$ & $\begin{array}{l}28 \\
28 \\
33 \\
30 \\
33\end{array}$ & $\begin{array}{r}2,890 \\
2,530 \\
* 2,060 \\
1,630 \\
1,360\end{array}$ & $\begin{array}{l}499 \\
499 \\
480 \\
462 \\
480\end{array}$ & $\begin{array}{l}305 \\
275 \\
264 \\
254 \\
243\end{array}$ & $\begin{array}{l}54 \\
49 \\
49 \\
44 \\
44\end{array}$ & $\begin{array}{l}6 \\
5 \\
5 \\
5 \\
6\end{array}$ & $\begin{array}{r}5 \\
5 \\
5 \\
* 7 \\
\frac{7}{5}\end{array}$ & $\begin{array}{l}5 \\
5 \\
5 \\
4 \\
4\end{array}$ \\
\hline $\begin{array}{l}26 \\
27 \\
28 \\
29 \\
30 \\
31 \\
\end{array}$ & $\begin{array}{l}1 \\
2\end{array}$ & $\begin{array}{l}1 \\
2 \\
-\end{array}$ & 4 & $\begin{array}{l}23 \\
28 \\
28 \\
30 \\
\frac{75}{70}\end{array}$ & $\begin{array}{r}36 \\
33 \\
39 \\
- \\
- \\
-\end{array}$ & $\begin{array}{r}1,210 \\
1,040 \\
962 \\
990 \\
1,140 \\
1,020\end{array}$ & $\begin{array}{l}518 \\
499 \\
480 \\
480 \\
462 \\
\frac{-}{-}\end{array}$ & $\begin{array}{l}232 \\
222 \\
211 \\
189 \\
179 \\
168 \\
\end{array}$ & $\begin{array}{l}44 \\
39 \\
33 \\
28 \\
23 \\
-\end{array}$ & $\begin{array}{l}6 \\
6 \\
6 \\
5 \\
5 \\
5\end{array}$ & $\begin{array}{l}5 \\
5 \\
5 \\
5 \\
5 \\
5\end{array}$ & $\begin{array}{l}4 \\
4 \\
5 \\
5 \\
5 \\
-\end{array}$ \\
\hline $\begin{array}{l}\text { Total } \\
\text { Mean } \\
\text { ic-ft } \\
\end{array}$ & $\begin{array}{r}62 \\
2 \\
123 \\
\end{array}$ & $\begin{array}{r}60 \\
2 \\
119 \\
\end{array}$ & $\begin{array}{r}124 \\
4 \\
246 \\
\end{array}$ & $\begin{array}{r}919 \\
29.6 \\
1,820 \\
\end{array}$ & $\begin{array}{r}1,136 \\
40.6 \\
2,250 \\
\end{array}$ & $\begin{array}{r}132,336 \\
4,269 \\
262,500 \\
\end{array}$ & $\begin{array}{r}17,796 \\
593 \\
35,300 \\
\end{array}$ & $\begin{array}{r}11,402 \\
368 \\
22,620 \\
\end{array}$ & $\begin{array}{r}2,270 \\
76.0 \\
4,500 \\
\end{array}$ & $\begin{array}{r}321 \\
10.4 \\
637 \\
\end{array}$ & $\begin{array}{l}157 \\
5.1 \\
311 \\
\end{array}$ & $\begin{array}{l}146 \\
4.9 \\
290 \\
\end{array}$ \\
\hline $\begin{array}{l}\text { Calend } \\
\text { Water }\end{array}$ & $\begin{array}{l}\text { ear } \\
195\end{array}$ & $\begin{array}{l}\text { 4: } \\
5:\end{array}$ & 7, & $\begin{array}{l}\text { Mi } \\
\text { Mi }\end{array}$ & $\begin{array}{l}2 \\
2\end{array}$ & $\begin{array}{ll}\text { Mean } & 546 \\
\text { Mean } & 457\end{array}$ & $\begin{array}{l}A C-f t \\
A C-f t\end{array}$ & $\begin{array}{l}395,600 \\
330,720\end{array}$ & & & & \\
\hline
\end{tabular}

* Discharge measurement me de on this day.

Hote lio

ote.-Alo gase read twice daily, Mar. 20 to llay 10 , afier recorder measurements and know 
HEIWAND RIVIR BASIN

Khash River near Dilaram, ifghanistan

Discharge, in cubic feet per second, water year October 1955 to september 1956

\begin{tabular}{|c|c|c|c|c|c|c|c|c|c|c|c|c|}
\hline Day & Oct. & Nov. & Dec. & Jan. & Feb. & liar. & Apr. & May & June & July & Aug. & Sept \\
\hline $\begin{array}{l}1 \\
2 \\
3 \\
4 \\
5\end{array}$ & 4 & 4 & 4 & $\begin{array}{l}\frac{211}{4 \sqrt{4}} \\
450 \\
500 \\
908\end{array}$ & $\begin{array}{l}545 \\
527 \\
508 \\
499 \\
400\end{array}$ & $\begin{array}{r}2,810 \\
3,030 \\
9,600 \\
23,200 \\
23,200\end{array}$ & $\begin{array}{l}1,940 \\
2,600 \\
2,530 \\
2,470 \\
2,500\end{array}$ & $\begin{array}{l}1,080 \\
1,040 \\
1,140 \\
1,210 \\
1,140\end{array}$ & $\begin{array}{l}\frac{264}{264} \\
254 \\
232 \\
232\end{array}$ & $\begin{array}{r}84 \\
103 \\
131 \\
144\end{array}$ & $\begin{array}{l}140 \\
144 \\
136 \\
122 \\
103\end{array}$ & $\begin{array}{l}36 \\
42 \\
44 \\
44 \\
46 \\
\end{array}$ \\
\hline $\begin{array}{r}6 \\
7 \\
8 \\
9 \\
10\end{array}$ & & & & $\begin{array}{l}739 \\
610 \\
564 \\
518 \\
471\end{array}$ & $\begin{array}{l}462 \\
434 \\
434 \\
425 \\
418\end{array}$ & $\begin{array}{r}19,500 \\
21,900 \\
12,800 \\
9,760 \\
7,880\end{array}$ & $\begin{array}{l}2,740 \\
3,100 \\
3,320 \\
3,940 \\
5,160\end{array}$ & $\begin{array}{l}1,160 \\
1,120 \\
1,080 \\
1,040 \\
1,000\end{array}$ & $\begin{array}{l}254 \\
211 \\
227 \\
254 \\
211\end{array}$ & $\begin{array}{l}131 \\
149 \\
131 \\
140 \\
140\end{array}$ & $\begin{array}{r}131 \\
* 131 \\
108 \\
94 \\
84\end{array}$ & $\begin{array}{l}44 \\
42 \\
42 \\
46 \\
36\end{array}$ \\
\hline $\begin{array}{l}11 \\
12 \\
13 \\
14 \\
15\end{array}$ & & & $\begin{array}{r}1 \\
4 \\
400 \\
* 718 \\
\end{array}$ & $\begin{array}{l}434 \\
410 \\
388 \\
358 \\
372\end{array}$ & $\begin{array}{l}410 \\
402 \\
395 \\
395 \\
402\end{array}$ & $\begin{array}{r}7,230 \\
8,870 \\
10,100 \\
15,100 \\
18,700\end{array}$ & $\begin{array}{l}\frac{6,710}{5,980} \\
5,160 \\
4,410 \\
4,030\end{array}$ & $\begin{array}{l}948 \\
908 \\
574 \\
554 \\
573\end{array}$ & $\begin{array}{l}189 \\
164 \\
131 \\
122 \\
122\end{array}$ & $\begin{array}{l}131 \\
140 \\
154 \\
159 \\
168\end{array}$ & $\begin{array}{l}75 \\
72 \\
70 \\
75 \\
62\end{array}$ & $\begin{array}{l}23 \\
20 \\
18 \\
18 \\
17\end{array}$ \\
\hline $\begin{array}{l}16 \\
17 \\
18 \\
19 \\
20\end{array}$ & & * & $\begin{array}{r}500 \\
400 \\
300 \\
200 \\
* 211\end{array}$ & $\begin{array}{r}518 \\
2,470 \\
* 2,890 \\
2,400 \\
1,940\end{array}$ & $\begin{array}{l}402 \\
395 \\
395 \\
388 \\
380 \\
\end{array}$ & $\begin{array}{r}21,300 \\
20,700 \\
16,400 \\
7,360 \\
3,660\end{array}$ & $\begin{array}{l}3,170 \\
2,810 \\
2,470 \\
2,100 \\
1,830\end{array}$ & $\begin{array}{r}601 \\
642 \\
696 \\
* 718 \\
642\end{array}$ & $\begin{array}{r}242 \\
116 \\
+189 \\
179 \\
194\end{array}$ & $\begin{array}{l}159 \\
174 \\
159 \\
159 \\
179\end{array}$ & $\begin{array}{l}54 \\
46 \\
44 \\
42 \\
39\end{array}$ & $\begin{array}{l}16 \\
16 \\
15 \\
15 \\
13\end{array}$ \\
\hline $\begin{array}{l}21 \\
22 \\
23 \\
24 \\
25\end{array}$ & & & $\begin{array}{l}243 \\
238 \\
211 \\
270 \\
211\end{array}$ & $\begin{array}{r}* 1,470 \\
1,250 \\
1,080 \\
976 \\
880\end{array}$ & $\begin{array}{l}380 \\
380 \\
380 \\
380 \\
402\end{array}$ & $\begin{array}{l}2,600 \\
3,170 \\
3,660 \\
4,300 \\
6,710\end{array}$ & $\begin{array}{r}1,540 \\
1,340 \\
1,080 \\
935 \\
804\end{array}$ & $\begin{array}{l}621 \\
564 \\
518 \\
450 \\
444\end{array}$ & $\begin{array}{l}184 \\
122 \\
131 \\
144 \\
112\end{array}$ & $\begin{array}{l}179 \\
144 \\
136 \\
168 \\
200 \\
\end{array}$ & $\begin{array}{l}39 \\
39 \\
36 \\
\frac{33}{44}\end{array}$ & $\begin{array}{r}13 \\
13 \\
12 \\
12 \\
* 10 \\
\end{array}$ \\
\hline $\begin{array}{l}26 \\
27 \\
28 \\
29 \\
30 \\
31\end{array}$ & 4 & $\begin{array}{l}1 \\
4 \\
-\end{array}$ & $\begin{array}{l}200 \\
206 \\
200 \\
194 \\
189 \\
227\end{array}$ & $\begin{array}{l}782 \\
728 \\
674 \\
632 \\
592 \\
573\end{array}$ & $\begin{array}{r}4,910 \\
10,100 \\
* 4,210 \\
3,030 \\
- \\
-\end{array}$ & $\begin{array}{l}7,880 \\
7,620 \\
6,220 \\
4,950 \\
3,100 \\
1,940 \\
\end{array}$ & $\begin{array}{r}718 \\
642 \\
573 \\
610 \\
1,100 \\
-\end{array}$ & $\begin{array}{l}425 \\
410 \\
388 \\
393 \\
372 \\
222 \\
\end{array}$ & $\begin{array}{r}144 \\
140 \\
103 \\
98 \\
89 \\
-\end{array}$ & $\begin{array}{l}136 \\
103 \\
108 \\
159 \\
179 \\
144\end{array}$ & $\begin{array}{l}59 \\
46 \\
36 \\
36 \\
42 \\
36\end{array}$ & $\begin{array}{l}10 \\
10 \\
10 \\
10 \\
10 \\
-\end{array}$ \\
\hline $\begin{array}{l}\text { Total } \\
\text { Mean } \\
\text { de-ft }\end{array}$ & $\begin{array}{r}124 \\
4 \\
246 \\
\end{array}$ & $\begin{array}{r}120 \\
4 \\
238 \\
\end{array}$ & $\begin{array}{r}5,170 \\
166 \\
10,250 \\
\end{array}$ & $\begin{array}{r}27,232 \\
878 \\
54,010 \\
\end{array}$ & $\begin{array}{r}32,868 \\
1,130 \\
65,190\end{array}$ & $\begin{array}{r}315,250 \\
10,170 \\
625,300 \\
\end{array}$ & $\begin{array}{r}78,412 \\
2,610 \\
155,500\end{array}$ & $\begin{array}{r}22,773 \\
734 \\
45,170 \\
\end{array}$ & $\begin{array}{r}5,318 \\
171 \\
10,550\end{array}$ & $\begin{array}{r}4,466 \\
144 \\
, 560 \\
\end{array}$ & $\begin{array}{r}2,218 \\
71.5 \\
4,400 \\
\end{array}$ & $\begin{array}{r}703 \\
23.4 \\
+390 \\
\end{array}$ \\
\hline
\end{tabular}

Calendar year 1955: liax 36,200 lijin 4 liean 472 fc-ft 340,960 water year 1955-56: liax 23,200 lin 4 liean 1,352 Ac-ft 981,100

* Discharge measurement mide on this day.

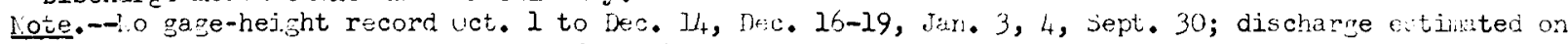
basis of discilarge inessuremerts and weather. 
HELM:ND RIVIR BASIN

Witash River nean Dilaram, Afghianistan

Discharge, in cubic feet per second, water year October 1956 to Sentember 1957

\begin{tabular}{|c|c|c|c|c|c|c|c|c|c|c|c|c|}
\hline Day & Oct. & Nov. & Dec. & Jali. 1 & $\mathrm{Feb}_{4}$ & Nar. & Apr. & May & June & Julv & Aug. & Sept. \\
\hline $\begin{array}{l}1 \\
2 \\
3 \\
4 \\
5\end{array}$ & 10 & 30 & 30 & $\begin{array}{c}30 \\
1\end{array}$ & $\begin{array}{l}1,900 \\
1,900 \\
1,900 \\
1,800 \\
1,800\end{array}$ & $\begin{array}{l}1,540 \\
1,540 \\
1,51,0 \\
1,5300 \\
1,6,30\end{array}$ & $\begin{array}{r}6,800 \\
6,600 \\
6,800 \\
7,000 \\
20,000\end{array}$ & $\begin{array}{r}\frac{13,000}{7,000} \\
3,500 \\
2,000 \\
1,000\end{array}$ & $\begin{array}{l}305 \\
298 \\
282 \\
259 \\
243\end{array}$ & $\begin{array}{l}372 \\
305 \\
298 \\
254 \\
275\end{array}$ & $\begin{array}{l}140 \\
159 \\
160 \\
150 \\
150\end{array}$ & $\begin{array}{l}108 \\
98 \\
80 \\
89 \\
89\end{array}$ \\
\hline $\begin{array}{r}6 \\
7 \\
8 \\
9 \\
10\end{array}$ & & $\frac{1}{30}$ & & $\begin{array}{l}30 \\
35 \\
100 \\
250 \\
200\end{array}$ & $\begin{array}{l}1,800 \\
1,730 \\
1,630 \\
1,2,20 \\
1,4,50\end{array}$ & $\begin{array}{l}1,680 \\
1,630 \\
1,630 \\
1,830 \\
1,520 \\
1.920\end{array}$ & $\begin{array}{r}\frac{40,000}{30,050} \\
15,000 \\
8,000 \\
7,000\end{array}$ & $\begin{array}{l}800 \\
720 \\
700 \\
700 \\
800\end{array}$ & $\begin{array}{l}236 \\
216 \\
189 \\
174 \\
168\end{array}$ & $\begin{array}{l}290 \\
282 \\
264 \\
264 \\
254\end{array}$ & $\begin{array}{l}140 \\
140 \\
140 \\
131 \\
168\end{array}$ & $\begin{array}{l}84 \\
84 \\
80 \\
84 \\
5 ?\end{array}$ \\
\hline $\begin{array}{l}11 \\
12 \\
13 \\
1 i \\
15\end{array}$ & $\frac{1}{2 n}$ & 40 & & $\begin{array}{r}190 \\
180 \\
190 \\
2,000 \\
1,500\end{array}$ & $\begin{array}{l}1,430 \\
1,430 \\
1,400 \\
1,400 \\
1,400\end{array}$ & $\begin{array}{l}2,470 \\
4,500 \\
3,500 \\
3,200 \\
3,000\end{array}$ & $\begin{array}{l}6,500 \\
6,500 \\
6,600 \\
6,800 \\
7,000\end{array}$ & $\begin{array}{r}1,000 \\
800 \\
720 \\
680 \\
653\end{array}$ & $\begin{array}{r}164 \\
140 \\
122 \\
103 \\
84\end{array}$ & $\begin{array}{l}254 \\
254 \\
248 \\
254 \\
254\end{array}$ & $\begin{array}{l}265 \\
140 \\
126 \\
126 \\
136\end{array}$ & $\begin{array}{r}45 \\
44 \\
+54 \\
52 \\
42\end{array}$ \\
\hline $\begin{array}{l}16 \\
17 \\
18 \\
19 \\
20\end{array}$ & 20 & * & * & $\begin{array}{r}1,000 \\
500 \\
400 \\
350 \\
340\end{array}$ & $\begin{array}{l}1,400 \\
1,470 \\
1,1,00 \\
1,360 \\
\frac{1,360}{1,36}\end{array}$ & $\begin{array}{r}3,100 \\
45,000 \\
40,000 \\
25,000 \\
15,000\end{array}$ & $\begin{array}{r}10,000 \\
10,000 \\
9,000 \\
8,000 \\
7,000\end{array}$ & $\begin{array}{l}653 \\
632 \\
653 \\
642 \\
621\end{array}$ & $\begin{array}{l}72 \\
65 \\
56 \\
49 \\
39 \\
\end{array}$ & $\begin{array}{l}243 \\
222 \\
189 \\
184 \\
298\end{array}$ & $\begin{array}{l}\frac{68}{126} \\
177 \\
126 \\
140\end{array}$ & $\begin{array}{l}54 \\
54 \\
62 \\
62 \\
54\end{array}$ \\
\hline $\begin{array}{l}21 \\
22 \\
23 \\
24 \\
25\end{array}$ & $*$ & 13 & & $\begin{array}{r}330 \\
330 \\
350 \\
760 \\
2,0^{\prime} 0\end{array}$ & $\begin{array}{l}1,360 \\
1,360 \\
1,360 \\
1,360 \\
1,360\end{array}$ & $\begin{array}{r}12,000 \\
10,000 \\
8,500 \\
7,500 \\
7,000\end{array}$ & $\begin{array}{l}6,700 \\
6,500 \\
5,200 \\
6,200 \\
6,200\end{array}$ & $\begin{array}{l}592 \\
564 \\
527 \\
480 \\
453\end{array}$ & $\begin{array}{r}03 \\
108 \\
* 388 \\
499 \\
554\end{array}$ & $\begin{array}{r}320 \\
335 \\
335 \\
* 164 \\
164\end{array}$ & $\begin{array}{l}131 \\
174 \\
12: 9 \\
159 \\
184 \\
\end{array}$ & $\begin{array}{l}59 \\
59 \\
49 \\
47 \\
44\end{array}$ \\
\hline $\begin{array}{l}26 \\
27 \\
28 \\
29 \\
30 \\
3 \pm \\
\end{array}$ & $\mid \begin{array}{r}1 \\
1 \\
1 \\
20\end{array}$ & $\begin{array}{r}30 \\
1 \\
30 \\
-\end{array}$ & 30 & $\begin{array}{r}* 7,880 \\
46,810 \\
4,620 \\
2,810 \\
2,060 \\
2,000\end{array}$ & $\begin{array}{c}1,360 \\
1,360 \\
1,420 \\
- \\
= \\
=\end{array}$ & $\begin{array}{l}0,500 \\
4,500 \\
6,000 \\
7,600 \\
7,200 \\
7,000\end{array}$ & $\begin{array}{c}6,200 \\
5,200 \\
6,200 \\
5,500 \\
6,000 \\
-\end{array}$ & $\begin{array}{l}425 \\
410 \\
388 \\
372 \\
358 \\
328 \\
\end{array}$ & $\begin{array}{c}518 \\
462 \\
410 \\
350 \\
390 \\
-\end{array}$ & $\begin{array}{l}164 \\
159 \\
149 \\
149 \\
131 \\
139\end{array}$ & $\begin{array}{r}154 \\
144 \\
108 \\
108 \\
29 \\
112\end{array}$ & $\begin{array}{l}39 \\
39 \\
44 \\
36 \\
44 \\
-\end{array}$ \\
\hline $\begin{array}{l}\text { Total } \\
\text { Mean } \\
\text { Ac-fu }\end{array}$ & $\begin{array}{r}130 \\
150 \\
422\end{array}$ & $\begin{array}{r}1,050 \\
35.0 \\
2,083 \\
\end{array}$ & $\begin{array}{r}930 \\
30.0 \\
1,845 \\
\end{array}$ & $\begin{array}{r}37,1,35 \\
1,2013 \\
74,250\end{array}$ & $\begin{array}{r}42,4,00 \\
1,515 \\
84,140\end{array}$ & $\begin{array}{r}246,640 \\
7,956 \\
1.89,200 \\
\end{array}$ & $\begin{array}{r}979,500 \\
9,317 \\
554,400\end{array}$ & $\begin{array}{r}4,2,171 \\
1,360 \\
83,640 \\
\end{array}$ & $\begin{array}{r}7,031 \\
234 \\
12,950 \\
\end{array}$ & $\begin{array}{r}7,487 \\
24: 2 \\
u_{1}, 850 \\
\end{array}$ & $\begin{array}{r}4,221 \\
136 \\
8,372 \\
\end{array}$ & $\begin{array}{r}1,830 \\
67.0 \\
3,630 \\
\end{array}$ \\
\hline $\begin{array}{l}\text { raler } \\
\text { Vater }\end{array}$ & $i_{\alpha} \mathbf{r} I$ & $\begin{array}{r}56: \\
-57:\end{array}$ & $\begin{array}{l}35 \\
45\end{array}$ & $\begin{array}{l}10 \\
0\end{array}$ & $\begin{array}{l}10 \\
10\end{array}$ & $\begin{array}{l}\text { Mean } 1 \\
\text { Mean } 1\end{array}$ & $\begin{array}{l}43 \\
39\end{array}$ & $t$ & $\begin{array}{l}200 \\
500\end{array}$ & & & \\
\hline
\end{tabular}

* Discrarge measurement mide on this day.

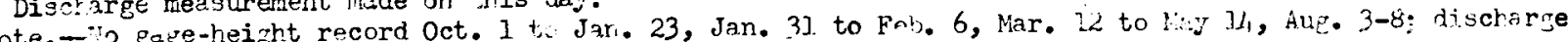

estimater on basis of dischare neas'derents and wenter informition. 
HTIYND RIVER BASIN

Thesh Rivor near Dilaram, Afghanioten

DAscharge, in cubic feot per second, water year October 1957 to September 1958

\begin{tabular}{|c|c|c|c|c|c|c|c|c|c|c|c|c|}
\hline Dar & Dot. & Nov. & Dec. & Jan. & $\mathrm{Feb}$ & Mar. & $\Delta \mathrm{pr}$ & Nas & June & July & Aug. & Sopt. \\
\hline $\begin{array}{l}1 \\
2 \\
3 \\
4 \\
5\end{array}$ & $\begin{array}{l}52 \\
75 \\
39 \\
33 \\
26\end{array}$ & $\begin{array}{l}39 \\
36 \\
36 \\
36 \\
142\end{array}$ & $\begin{array}{l}434 \\
425 \\
444 \\
434 \\
444\end{array}$ & $\begin{array}{l}275 \\
282 \\
264 \\
264 \\
264\end{array}$ & $\begin{array}{r}564 \\
1,410 \\
\times 1,230 \\
1,060 \\
948\end{array}$ & $\begin{array}{l}328 \\
328 \\
328 \\
328 \\
320\end{array}$ & $\begin{array}{l}592 \\
582 \\
545 \\
518 \\
490\end{array}$ & $\begin{array}{l}\frac{290}{290} \\
280 \\
270 \\
270\end{array}$ & $\begin{array}{r}122 \\
122 \\
112 \\
98 \\
84\end{array}$ & $\begin{array}{l}206 \\
200 \\
194 \\
194 \\
194\end{array}$ & $\begin{array}{l}\frac{25}{25} \\
25 \\
24 \\
22\end{array}$ & $\begin{array}{r}6 \\
6 \\
6 \\
-6 \\
6\end{array}$ \\
\hline $\begin{array}{r}6 \\
7 \\
8 \\
9 \\
10\end{array}$ & $\begin{array}{l}33 \\
30 \\
22 \\
21 \\
17\end{array}$ & $\begin{array}{l}46 \\
42 \\
48 \\
54 \\
52\end{array}$ & $\begin{array}{r}5,440 \\
935 \\
637 \\
462 \\
3,330\end{array}$ & $\begin{array}{l}259 \\
259 \\
254 \\
\frac{254}{635} \\
\end{array}$ & $\begin{array}{l}838 \\
782 \\
707 \\
664 \\
592\end{array}$ & $\begin{array}{l}320 \\
\frac{322}{328} \\
365 \\
564\end{array}$ & $\begin{array}{l}480 \\
480 \\
480 \\
462 \\
444\end{array}$ & $\begin{array}{l}270 \\
270 \\
270 \\
260 \\
260\end{array}$ & $\begin{array}{l}84 \\
75 \\
72 \\
72 \\
72\end{array}$ & $\begin{array}{l}194 \\
189 \\
189 \\
179 \\
179\end{array}$ & $\begin{array}{l}22 \\
20 \\
18 \\
18 \\
18\end{array}$ & $\begin{array}{l}5 \\
5 \\
5 \\
5 \\
5\end{array}$ \\
\hline $\begin{array}{l}11 \\
12 \\
13 \\
14 \\
15\end{array}$ & $\begin{array}{l}21 \\
39 \\
28 \\
49 \\
46\end{array}$ & $\begin{array}{l}49 \\
46 \\
44 \\
46 \\
46\end{array}$ & $\begin{array}{r}2,740 \\
1,100 \\
771 \\
674 \\
582\end{array}$ & $\begin{array}{l}632 \\
554 \\
573 \\
527 \\
490\end{array}$ & $\begin{array}{l}592 \\
545 \\
518 \\
490 \\
480\end{array}$ & $\begin{array}{l}621 \\
642 \\
621 \\
601 \\
582\end{array}$ & $\begin{array}{l}425 \\
410 \\
402 \\
402 \\
372\end{array}$ & $\begin{array}{l}260 \\
250 \\
250 \\
250 \\
240\end{array}$ & $\begin{array}{l}\frac{68}{84} \\
131 \\
122 \\
103\end{array}$ & $\begin{array}{r}189 \\
174 \\
211 \\
211 \\
* 213\end{array}$ & $\begin{array}{r}* 16 \\
16 \\
16 \\
15 \\
15\end{array}$ & $\begin{array}{l}5 \\
6 \\
6 \\
6 \\
6\end{array}$ \\
\hline $\begin{array}{l}16 \\
17 \\
18 \\
19 \\
20\end{array}$ & $\begin{array}{l}44 \\
39 \\
42 \\
44 \\
54\end{array}$ & $\begin{array}{r}42 \\
39 \\
39 \\
26 \\
+261 \\
\end{array}$ & $\begin{array}{l}518 \\
462 \\
434 \\
418 \\
395\end{array}$ & $\begin{array}{l}462 \\
434 \\
410 \\
410 \\
410\end{array}$ & $\begin{array}{l}462 \\
434 \\
418 \\
410 \\
395\end{array}$ & $\begin{array}{l}564 \\
564 \\
582 \\
621 \\
621\end{array}$ & $\begin{array}{l}372 \\
388 \\
372 \\
365 \\
350\end{array}$ & $\begin{array}{l}240 \\
240 \\
240 \\
230 \\
230\end{array}$ & $\begin{array}{r}89 \\
80 \\
140 \\
184 \\
179\end{array}$ & $\begin{array}{r}350 \\
200 \\
100 \\
80 \\
70\end{array}$ & $\begin{array}{l}14 \\
14 \\
12 \\
10 \\
10\end{array}$ & $\begin{array}{l}6 \\
6 \\
6 \\
6 \\
6\end{array}$ \\
\hline $\begin{array}{l}21 \\
22 \\
23 \\
24 \\
25\end{array}$ & $\begin{array}{r}54 \\
52 \\
52 \\
46 \\
-46\end{array}$ & $\begin{array}{l}395 \\
243 \\
320 \\
380 \\
365\end{array}$ & $\begin{array}{r}372 \\
358 \\
350 \\
335 \\
* 320\end{array}$ & $\begin{array}{l}434 \\
434 \\
410 \\
410 \\
402\end{array}$ & $\begin{array}{l}380 \\
402 \\
350 \\
342 \\
342\end{array}$ & $\begin{array}{r}621 \\
601 \\
621 \\
564 \\
+527\end{array}$ & $\begin{array}{l}365 \\
350 \\
328 \\
320 \\
312\end{array}$ & $\begin{array}{l}230 \\
220 \\
220 \\
210 \\
210\end{array}$ & $\begin{array}{l}179 \\
184 \\
179 \\
168 \\
184\end{array}$ & $\begin{array}{l}60 \\
50 \\
40 \\
35 \\
33\end{array}$ & $\begin{array}{r}10 \\
8 \\
8 \\
8 \\
8\end{array}$ & $\begin{array}{l}6 \\
6 \\
6 \\
6 \\
6\end{array}$ \\
\hline $\begin{array}{l}26 \\
27 \\
28 \\
29 \\
30 \\
31 \\
\end{array}$ & $\begin{array}{l}44 \\
39 \\
39 \\
39 \\
36 \\
36 \\
\end{array}$ & $\begin{array}{c}328 \\
328 \\
471 \\
462 \\
444 \\
- \\
\end{array}$ & $\begin{array}{l}320 \\
312 \\
305 \\
305 \\
298 \\
290 \\
\end{array}$ & $\begin{array}{l}418 \\
418 \\
410 \\
410 \\
410 \\
402\end{array}$ & $\begin{array}{l}350 \\
342 \\
335 \\
= \\
= \\
=\end{array}$ & $\begin{array}{l}518 \\
545 \\
728 \\
990 \\
750 \\
632 \\
\end{array}$ & $\begin{array}{c}305 \\
312 \\
305 \\
290 \\
290 \\
-\end{array}$ & $\begin{array}{r}* 194 \\
184 \\
168 \\
122 \\
149 \\
131 \\
\end{array}$ & $\begin{array}{c}184 \\
179 \\
189 \\
200 \\
194 \\
- \\
\end{array}$ & $\begin{array}{l}32 \\
32 \\
32 \\
31 \\
31 \\
30 \\
\end{array}$ & $\begin{array}{l}6 \\
6 \\
6 \\
6 \\
6 \\
6\end{array}$ & $\begin{array}{l}6 \\
6 \\
6 \\
6 \\
6 \\
-\end{array}$ \\
\hline $\begin{array}{l}\text { Totel } \\
\text { Yoan } \\
\text { le-ft } \\
\end{array}$ & $\begin{array}{r}1,237 \\
39.9 \\
2,454 \\
\end{array}$ & $\begin{array}{r}5,509 \\
184 \\
10,930\end{array}$ & $\begin{array}{r}24,644 \\
795 \\
48,880 \\
\end{array}$ & $\begin{array}{r}12,480 \\
403 \\
24,750 \\
\end{array}$ & $\begin{array}{r}16,382 \\
585 \\
32,490 \\
\end{array}$ & $\begin{array}{r}16,637 \\
537 \\
33,000\end{array}$ & $\begin{array}{r}12,108 \\
404 \\
24,020 \\
\end{array}$ & $\begin{array}{r}7,198 \\
232 \\
14,200 \\
\end{array}$ & $\begin{array}{r}3,933 \\
131 \\
7,801 \\
\end{array}$ & $\begin{array}{r}4,152 \\
134 \\
8,235 \\
\end{array}$ & $\begin{array}{r}433 \\
14.0 \\
859 \\
\end{array}$ & $\begin{array}{l}174 \\
5.8 \\
345 \\
\end{array}$ \\
\hline
\end{tabular}

* Discharge neasurement made on this day.

Note. -No gage-helght record Apr. 30 to May 25, July 16-30; discharge estimated on basis of discharge measurements, weather and records for Farah River at Farah. 
HETMAND RIVER BASIN

Khash River at Dilaram, Afghanistan

Discharge, in cubic feet per second, water year October 1958 to September 1959

\begin{tabular}{|c|c|c|c|c|c|c|c|c|c|c|c|c|}
\hline Dey & Oct. & Nov. & Dec. & Jan. & Feb. & Kax. & Apr. & May & June & July & Aug. & Sept. \\
\hline $\begin{array}{l}1 \\
2 \\
3 \\
4 \\
5\end{array}$ & $\begin{array}{l}8.5 \\
8.5 \\
8.5 \\
8.5 \\
8.5\end{array}$ & $\begin{array}{l}\frac{16}{16} \\
17 \\
17 \\
17\end{array}$ & $\begin{array}{l}\frac{31}{31} \\
31 \\
38 \\
38\end{array}$ & $\begin{array}{l}\frac{47}{47} \\
53 \\
61 \\
69\end{array}$ & $\begin{array}{l}142 \\
135 \\
130 \\
130 \\
135\end{array}$ & $\begin{array}{r}2,290 \\
4,580 \\
4,580 \\
3,200 \\
* 2,650\end{array}$ & $\begin{array}{l}2,160 \\
2,220 \\
2,870 \\
2,870 \\
2,870\end{array}$ & $\begin{array}{r}1,000 \\
930 \\
895 \\
860 \\
825\end{array}$ & $\begin{array}{l}302 \\
295 \\
288 \\
\frac{325}{325}\end{array}$ & $\begin{array}{l}\frac{75}{75} \\
71 \\
67 \\
67\end{array}$ & $\begin{array}{l}\frac{25}{23} \\
21 \\
19 \\
19\end{array}$ & $\begin{array}{l}7.4 \\
7.4 \\
7.4 \\
7.4 \\
7.4\end{array}$ \\
\hline $\begin{array}{r}6 \\
7 \\
8 \\
9 \\
10\end{array}$ & $\begin{array}{l}7.4 \\
7.4 \\
7.4 \\
7.4 \\
7.4\end{array}$ & $\begin{array}{l}18 \\
18 \\
18 \\
21 \\
21\end{array}$ & $\begin{array}{l}38 \\
38 \\
38 \\
38 \\
38\end{array}$ & $\begin{array}{l}65 \\
61 \\
53 \\
53 \\
50\end{array}$ & $\begin{array}{l}130 \\
126 \\
122 \\
\frac{118}{151}\end{array}$ & $\begin{array}{l}2,430 \\
2,240 \\
1,890 \\
2,930 \\
4,680\end{array}$ & $\begin{array}{l}2,840 \\
2,520 \\
2,490 \\
2,540 \\
2,490\end{array}$ & $\begin{array}{l}790 \\
764 \\
720 \\
699 \\
660\end{array}$ & $\begin{array}{l}310 \\
265 \\
250 \\
228 \\
212\end{array}$ & $\begin{array}{l}67 \\
63 \\
59 \\
59 \\
56\end{array}$ & $\begin{array}{l}17 \\
17 \\
16 \\
15 \\
14\end{array}$ & $\begin{array}{l}7.4 \\
7.4 \\
7.4 \\
7.4 \\
7.4\end{array}$ \\
\hline $\begin{array}{l}11 \\
12 \\
13 \\
14 \\
15\end{array}$ & $\begin{array}{l}7.4 \\
7.4 \\
7.4 \\
7.4 \\
7.4\end{array}$ & $\begin{array}{l}21 \\
21 \\
22 \\
22 \\
22\end{array}$ & $\begin{array}{l}36 \\
50 \\
50 \\
\frac{80}{72}\end{array}$ & $\begin{array}{l}50 \\
50 \\
47 \\
47 \\
61\end{array}$ & $\begin{array}{l}600 \\
464 \\
374 \\
318 \\
272\end{array}$ & $\begin{array}{l}4,300 \\
3,260 \\
2,500 \\
2,070 \\
1,760\end{array}$ & $\begin{array}{l}2,380 \\
2,220 \\
2,100 \\
2,060 \\
2,000\end{array}$ & $\begin{array}{l}647 \\
621 \\
608 \\
608 \\
634\end{array}$ & $\begin{array}{l}198 \\
190 \\
170 \\
160 \\
155\end{array}$ & $\begin{array}{l}56 \\
52 \\
52 \\
48 \\
44\end{array}$ & $\begin{array}{l}11 \\
11 \\
11 \\
10 \\
10\end{array}$ & $\begin{array}{l}7.4 \\
7.4 \\
7.4 \\
7.4 \\
7.4\end{array}$ \\
\hline $\begin{array}{l}16 \\
17 \\
18 \\
19 \\
20\end{array}$ & $\begin{array}{r}7.4 \\
7.4 \\
* 7.4 \\
7.4 \\
8.5\end{array}$ & $\begin{array}{l}24 \\
24 \\
24 \\
24 \\
25\end{array}$ & $\begin{array}{l}65 \\
57 \\
57 \\
57 \\
57\end{array}$ & $\begin{array}{r}57 \\
72 \\
365 \\
84 \\
* 84\end{array}$ & $\begin{array}{r}245 \\
257 \\
1,030 \\
660 \\
515\end{array}$ & $\begin{array}{l}1,640 \\
1,520 \\
1,390 \\
1,330 \\
1,300\end{array}$ & $\begin{array}{l}1,950 \\
1,840 \\
1,740 \\
1,630 \\
1,540\end{array}$ & $\begin{array}{r}673 \\
1,070 \\
1,070 \\
790 \\
660\end{array}$ & $\begin{array}{l}145 \\
140 \\
135 \\
135 \\
130\end{array}$ & $\begin{array}{l}44 \\
36 \\
36 \\
36 \\
34\end{array}$ & $\begin{array}{l}10 \\
8.6 \\
8.6 \\
8.6 \\
8.6\end{array}$ & $\begin{array}{l}7.4 \\
5 \\
5 \\
5 \\
5\end{array}$ \\
\hline $\begin{array}{l}21 \\
22 \\
23 \\
24 \\
25\end{array}$ & $\begin{array}{l}8.5 \\
9.6 \\
9.6 \\
12 \\
12\end{array}$ & $\begin{array}{r}25 \\
25 \\
* 28 \\
28 \\
28\end{array}$ & $\begin{array}{l}65 \\
65 \\
65 \\
65 \\
61\end{array}$ & $\begin{array}{r}96 \\
92 \\
92 \\
503 \\
464\end{array}$ & $\begin{array}{l}481 \\
464 \\
481 \\
498 \\
464\end{array}$ & $\begin{array}{r}1,250 \\
1,280 \\
1,220 \\
1,100 \\
925\end{array}$ & $\begin{array}{l}1,580 \\
1,900 \\
1,740 \\
1,490 \\
1,360\end{array}$ & $\begin{array}{r}582 \\
530 \\
510 \\
468 \\
* 448\end{array}$ & $\begin{array}{l}125 \\
125 \\
125 \\
120 \\
115\end{array}$ & $\begin{array}{r}* 36 \\
36 \\
34 \\
32 \\
32\end{array}$ & $\begin{array}{l}8.6 \\
8.6 \\
8.6 \\
8.6 \\
8.6\end{array}$ & $\begin{array}{l}5 \\
5 \\
* 4.8 \\
5 \\
5\end{array}$ \\
\hline $\begin{array}{l}26 \\
27 \\
28 \\
29 \\
30 \\
31 \\
\end{array}$ & $\begin{array}{l}13 \\
13 \\
14 \\
14 \\
15 \\
15\end{array}$ & $\begin{array}{l}28 \\
28 \\
28 \\
31 \\
\frac{31}{-}\end{array}$ & $\begin{array}{l}57 \\
53 \\
53 \\
53 \\
50 \\
47\end{array}$ & $\begin{array}{l}332 \\
272 \\
277 \\
194 \\
174 \\
154\end{array}$ & $\begin{array}{c}464 \\
515 \\
583 \\
- \\
-\end{array}$ & $\begin{array}{r}760 \\
583 \\
2,280 \\
3,360 \\
* 2,870 \\
2,430\end{array}$ & $\begin{array}{c}1,230 \\
1,120 \\
1,040 \\
1,040 \\
1,040 \\
-\end{array}$ & $\begin{array}{l}428 \\
418 \\
386 \\
356 \\
336 \\
318 \\
\end{array}$ & $\begin{array}{r}110 \\
105 \\
95 \\
90 \\
80 \\
\end{array}$ & $\begin{array}{l}32 \\
30 \\
30 \\
28 \\
28 \\
25 \\
\end{array}$ & $\begin{array}{l}8.6 \\
8.6 \\
8.6 \\
8.6 \\
8.6 \\
8.6 \\
\end{array}$ & $\begin{array}{l}5 \\
5 \\
5 \\
5 \\
5 \\
-\end{array}$ \\
\hline $\begin{array}{l}\text { Total } \\
\text { Koan } \\
\text { Ac-ft }\end{array}$ & $\begin{array}{r}290.3 \\
9.4 \\
576\end{array}$ & $\begin{array}{r}688 \\
22.9 \\
1,360 \\
\end{array}$ & $\begin{array}{r}1,547 \\
49.9 \\
3,070 \\
\end{array}$ & $\begin{array}{r}4,126 \\
133 \\
8,170 \\
\end{array}$ & $\begin{array}{r}10,004 \\
357 \\
19,840 \\
\end{array}$ & $\begin{array}{r}70,618 \\
2,278 \\
140,070 \\
\end{array}$ & $\begin{array}{r}58,870 \\
1,960 \\
116,770 \\
\end{array}$ & $\begin{array}{r}20,304 \\
655 \\
40,270 \\
\end{array}$ & $\begin{array}{r}5,448 \\
182 \\
10,810 \\
\end{array}$ & $\begin{array}{r}1,440 \\
46.4 \\
2,860 \\
\end{array}$ & $\begin{array}{c}378 \\
12.2 \\
750\end{array}$ & $\begin{array}{c}188.2 \\
6.27 \\
373\end{array}$ \\
\hline Wat & 1 & & 4, & & $\begin{array}{l}5 \\
4.8\end{array}$ & $\begin{array}{l}\text { Mean } \\
\text { Mean }\end{array}$ & The & $\begin{array}{l}150 \\
344\end{array}$ & & & & \\
\hline
\end{tabular}

* Discharge measurement made on this day.

Note. - Gage-heights estimated June 18 to July 20 when float was disengaged. No rainfall in period. 
HELMAND RIVER BASIN

Kash River at Dilaram, Afghaniatan

Discharge, in cubic feet per second, water year October 1959 to September 1960

\begin{tabular}{|c|c|c|c|c|c|c|c|c|c|c|c|c|}
\hline Day & Oct. & Nov, & Dec. & Jan. & Feb. & Mar. & Apr. & May & June & July & Aug. & Sept. \\
\hline $\begin{array}{l}1 \\
2 \\
3 \\
4 \\
5\end{array}$ & $\begin{array}{l}5.0 \\
5.0 \\
5.0 \\
5.0 \\
5.0\end{array}$ & $\begin{array}{l}\frac{8.6}{8.6} \\
8.6 \\
8.6 \\
8.6\end{array}$ & $\begin{array}{r}578 \\
2,970 \\
* 825 \\
384 \\
* 295\end{array}$ & $\begin{array}{l}\frac{185}{176} \\
170 \\
164 \\
164\end{array}$ & $\begin{array}{l}136 \\
130 \\
126 \\
126 \\
123\end{array}$ & $\begin{array}{l}188 \\
185 \\
188 \\
188 \\
212\end{array}$ & $\begin{array}{l}1,470 \\
1,470 \\
1,490 \\
1,450 \\
1,450\end{array}$ & $\begin{array}{l}\frac{2,940}{2,620} \\
2,330 \\
2,080 \\
1,900\end{array}$ & $\begin{array}{l}520 \\
510 \\
500 \\
490 \\
480\end{array}$ & $\begin{array}{l}\frac{144}{139} \\
134 \\
129 \\
129\end{array}$ & $\begin{array}{l}47 \\
47 \\
44 \\
42 \\
39\end{array}$ & $\begin{array}{l}2 \\
9 \\
9 \\
9 \\
8\end{array}$ \\
\hline $\begin{array}{r}6 \\
7 \\
8 \\
9 \\
10\end{array}$ & $\begin{array}{l}5.0 \\
5.0 \\
5.0 \\
5.0 \\
5.0\end{array}$ & $\begin{array}{c}8.6 \\
8.6 \\
8.6 \\
12 \\
16\end{array}$ & $\begin{array}{l}238 \\
198 \\
179 \\
160 \\
157\end{array}$ & $\begin{array}{l}164 \\
160 \\
157 \\
157 \\
157\end{array}$ & $\begin{array}{l}123 \\
120 \\
120 \\
120 \\
120\end{array}$ & $\begin{array}{l}242 \\
250 \\
238 \\
224 \\
216\end{array}$ & $\begin{array}{l}1,360 \\
1,300 \\
1,340 \\
1,380 \\
1,320\end{array}$ & $\begin{array}{l}1,760 \\
1,690 \\
1,580 \\
1,580 \\
1,540\end{array}$ & $\begin{array}{l}470 \\
450 \\
440 \\
420 \\
400\end{array}$ & $\begin{array}{r}124 \\
114 \\
108 \\
98 \\
98\end{array}$ & $\begin{array}{l}36 \\
36 \\
33 \\
30 \\
28\end{array}$ & $\begin{array}{l}8 \\
8 \\
8 \\
8 \\
8\end{array}$ \\
\hline $\begin{array}{l}11 \\
12 \\
13 \\
14 \\
15\end{array}$ & $\begin{array}{l}5.0 \\
5.0 \\
5.0 \\
5.0 \\
5.0\end{array}$ & $\begin{array}{l}19 \\
21 \\
23 \\
25 \\
28\end{array}$ & $\begin{array}{l}151 \\
145 \\
136 \\
133 \\
164\end{array}$ & $\begin{array}{l}157 \\
157 \\
157 \\
154 \\
151\end{array}$ & $\begin{array}{l}223 \\
123 \\
126 \\
130 \\
142\end{array}$ & $\begin{array}{r}212 \\
604 \\
2,270 \\
1,320 \\
* 1,400\end{array}$ & $\begin{array}{l}1,200 \\
1,140 \\
1,340 \\
1,850 \\
2,430\end{array}$ & $\begin{array}{l}1,450 \\
1,360 \\
1,300 \\
1,300 \\
1,140\end{array}$ & $\begin{array}{l}390 \\
380 \\
360 \\
350 \\
330\end{array}$ & $\begin{array}{l}88 \\
88 \\
85 \\
82 \\
79\end{array}$ & $\begin{array}{l}28 \\
26 \\
24 \\
23 \\
23\end{array}$ & $\begin{array}{l}8 \\
8 \\
8 \\
8 \\
8\end{array}$ \\
\hline $\begin{array}{l}16 \\
17 \\
18 \\
19 \\
20\end{array}$ & $\begin{array}{l}5.0 \\
5.0 \\
5.0 \\
5.0 \\
5.0\end{array}$ & $\begin{array}{l}28 \\
28 \\
28 \\
28 \\
30\end{array}$ & $\begin{array}{l}268 \\
220 \\
194 \\
188 \\
191\end{array}$ & $\begin{array}{r}145 \\
142 \\
139 \\
139 \\
\times 136\end{array}$ & $\begin{array}{l}176 \\
203 \\
212 \\
203 \\
198\end{array}$ & $\begin{array}{r}1,320 \\
1,160 \\
1,070 \\
1,000 \\
930\end{array}$ & $\begin{array}{r}* 7,250 \\
8,300 \\
8,000 \\
6,530 \\
5,850\end{array}$ & $\begin{array}{r}1,070 \\
965 \\
860 \\
840 \\
800\end{array}$ & $\begin{array}{l}310 \\
290 \\
280 \\
260 \\
250\end{array}$ & $\begin{array}{l}76 \\
74 \\
71 \\
71 \\
71\end{array}$ & $\begin{array}{l}21 \\
21 \\
19 \\
19 \\
19\end{array}$ & $\begin{array}{l}8 \\
8 \\
8 \\
8 \\
8\end{array}$ \\
\hline $\begin{array}{l}21 \\
22 \\
23 \\
24 \\
25\end{array}$ & $\begin{array}{l}5.0 \\
5.0 \\
5.0 \\
5.0 \\
5.0\end{array}$ & $\begin{array}{r}32 \\
32 \\
32 \\
* 30 \\
28\end{array}$ & $\begin{array}{l}203 \\
212 \\
216 \\
212 \\
208\end{array}$ & $\begin{array}{l}133 \\
133 \\
130 \\
128 \\
128\end{array}$ & $\begin{array}{l}188 \\
188 \\
188 \\
185 \\
185\end{array}$ & $\begin{array}{l}860 \\
790 \\
736 \\
693 \\
682\end{array}$ & $\begin{array}{l}5,100 \\
4,500 \\
4,400 \\
4,020 \\
3,660\end{array}$ & $\begin{array}{l}780 \\
760 \\
730 \\
710 \\
690\end{array}$ & $\begin{array}{l}230 \\
220 \\
210 \\
200 \\
180\end{array}$ & $\begin{array}{r}71 \\
70 \\
68 \\
67 \\
* 65\end{array}$ & $\begin{array}{l}16 \\
14 \\
12 \\
12 \\
11\end{array}$ & $\begin{array}{l}7 \\
7 \\
7 \\
7 \\
7\end{array}$ \\
\hline $\begin{array}{l}26 \\
27 \\
28 \\
29 \\
30 \\
31 \\
\end{array}$ & $\begin{array}{l}5.0 \\
5.0 \\
5.0 \\
5.0 \\
5.0 \\
5.0 \\
\end{array}$ & $\begin{array}{r}30 \\
2.300 \\
639 \\
359 \\
308 \\
-\end{array}$ & $\begin{array}{l}198 \\
203 \\
194 \\
194 \\
191 \\
188 \\
\end{array}$ & $\begin{array}{l}126 \\
123 \\
126 \\
130 \\
142 \\
139\end{array}$ & $\begin{array}{r}182 \\
185 \\
* 188 \\
188 \\
- \\
- \\
\end{array}$ & $\begin{array}{r}747 \\
2,220 \\
1,850 \\
1,690 \\
1,600 \\
1,520 \\
\end{array}$ & $\begin{array}{l}3,470 \\
3,090 \\
2,900 \\
3,020 \\
3,020\end{array}$ & $\begin{array}{r}660 \\
640 \\
610 \\
580 \\
560 \\
540 \\
\end{array}$ & $\begin{array}{r}175 \\
165 \\
160 \\
+159 \\
154 \\
-\end{array}$ & $\begin{array}{l}65 \\
59 \\
59 \\
53 \\
53 \\
50 \\
\end{array}$ & $\begin{array}{l}11 \\
11 \\
11 \\
10 \\
10\end{array}$ & $\begin{array}{r}\frac{6}{6} \\
6 \\
6 \\
6 \\
-\end{array}$ \\
\hline $\begin{array}{l}\text { Total } \\
\text { Mean } \\
\text { Ac-ft }\end{array}$ & $\begin{array}{r}155 \\
5 \\
310 \\
\end{array}$ & $\begin{array}{c}4, \frac{144.8}{138} \\
8,220 \\
\end{array}$ & $\begin{array}{r}9,993 \\
322 \\
19,820 \\
\end{array}$ & $\begin{array}{r}4,569 \\
147 \\
9,060 \\
\end{array}$ & $\begin{array}{r}4,557 \\
157 \\
9,040 \\
\end{array}$ & $\begin{array}{r}26,805 \\
865 \\
53,170\end{array}$ & $\begin{array}{r}95,100 \\
3,170 \\
188,600 \\
\end{array}$ & $\begin{array}{r}38,365 \\
1,238 \\
76,100\end{array}$ & $\begin{array}{r}9,733 \\
324 \\
19,310 \\
\end{array}$ & $\begin{array}{r}2,682 \\
86.5 \\
5,320 \\
\end{array}$ & $\begin{array}{r}732 \\
23.6 \\
1,450 \\
\end{array}$ & $\begin{array}{c}229 \\
76.3 \\
455 \\
\end{array}$ \\
\hline
\end{tabular}

Calendar Year 1959: Max 4,680 Min 4.8 Mean 509 Ac-ft 368,300

Water year 1959-60: iviax 8,300 in 5 Mean 538 Ac-ft 390,900

* Discharge measurement made on this day.

Note. - No gage-height record liay 19 to June 29, JuIy 22-24, Sept. 21-30; discharge estimated on basis of recorded

range in stage ard records for Farah River at Farah. 
Farah River near icrab, Afghanistar.

Lecstion.- Lat $32^{\circ} 20^{\prime} \mathrm{N} .$, Iong $62^{\circ} 00 \mathrm{k}$., on downstream side of highway bridge on farah to herat rcad, 8 kilometers southwest of Farah and about 130 kilometers upstream from Hamun-i-3abari in the ChakarsurSeistan basin.

Drainage area.- 10,400 sq $\mathrm{mi}$, approximately, from Survey of India base maps.

Recoras available.- April 1953 to September 1960.

Gage.- Water-stage recorder. Altitude of gage is about 760 meters. Prior to July $\mathcal{U}_{4}, 1958$, staff gafes about 300 meters upstream at datum 0.55 meters higher.

Average discharge. -8 years $(1952-60) 1,705$ cfe $(1,235,490$ acre-ft per year).

Bxtremes.- Kaximum and minimum discharges for the water years 1953 to 1960 are contained in the following table:

\begin{tabular}{|c|c|c|c|c|c|}
\hline Water Year & Dete & \multicolumn{2}{|c|}{ Naximum } & Date & Minimum Dajly \\
\hline $\begin{array}{l}1953 \\
1954 \\
1955 \\
1956 \\
1957 \\
1958 \\
1959 \\
1960\end{array}$ & $\begin{array}{l}\text { Apr. } 11,1953 \\
\text { Feb. } 10,1954 \\
\text { Mar. 14, } 1955 \\
\text { Mar. } 5,1956 \\
\text { Mar. 17, } 1957 \\
\text { Mar. 29, } 1958 \\
\text { Mar. 29, } 1959 \\
\text { Apr. } 17,1960\end{array}$ & $\begin{array}{l}5.75 \\
5.35 \\
5.85 \\
- \\
- \\
2.22 \\
3.10 \\
3.51\end{array}$ & $\begin{array}{r}74,300 \\
65,000 \\
76,800 \\
\text { b78,000 } \\
\text { ac40,000 } \\
6,010 \\
26,000 \\
38,4,00\end{array}$ & $\begin{array}{l}\text { Many days } \\
\text { Many days } \\
\text { Many days } \\
\text { jept. } 23-30,1956 \\
\text { Many days } \\
\text { Sept. } 3,4,7,1958 \\
\text { Many days } \\
\text { Sept. } 16-30,1960\end{array}$ & $\begin{array}{l}2 \\
2 \\
2 \\
1 \\
2 \\
1.5 \\
2 \\
1\end{array}$ \\
\hline
\end{tabular}

a Mear dajly discharge.

b slope-area measuremert.

c Estimated.

Renarics. - kecords good except those for periods of no gage-heights or fiows belcw ic cfs wnich are $:$ cr. liany diversions for irrigation upstrean. 
FARAH RIVEIR BASTN

Farah River near Farah, Afghanistan

Discharge, in cubic feet per second, water year October 1952 to September 1953

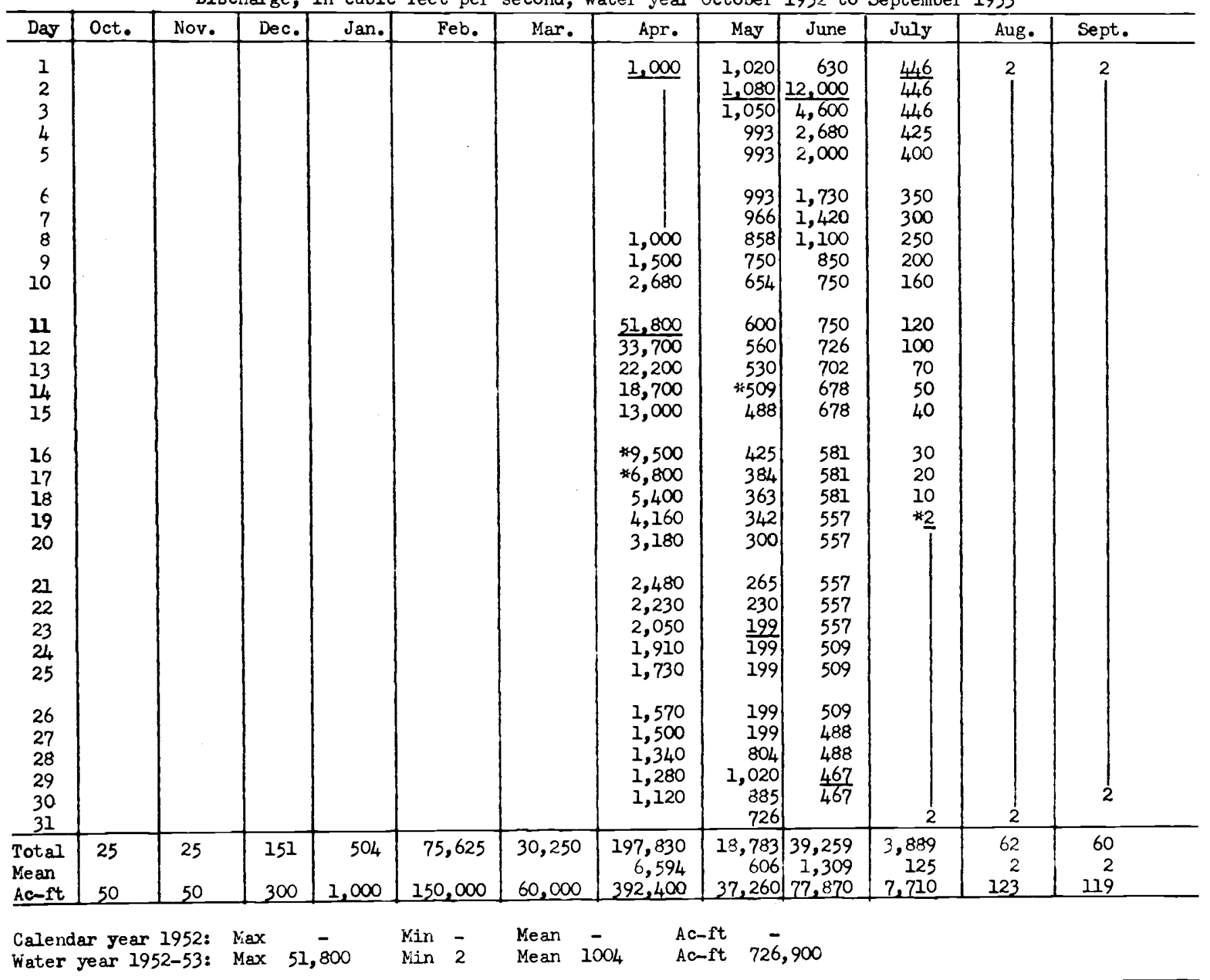

* Discharge measurement made on this day.

Note.- lio gage-heights Oct. 1 to $\mathrm{fpr}$. 9, July 5-18, July 20 to Sept. 30; discharge estimated on basis of records for Khash River near Dilaram and observed flows. 
FAKAH RIVER EASIN

Farah River near Farah, Af chanistan

Discharge, in cubic feet per second, water year October 1953 to September 1954

\begin{tabular}{|c|c|c|c|c|c|c|c|c|c|c|c|c|}
\hline Day & Oct. & Nov. & Dec. & Jan. & $\mathrm{Feb}$ & Mar. & Apr. & May & June & July & Aug. & Sept. \\
\hline $\begin{array}{l}1 \\
2 \\
3 \\
4 \\
5\end{array}$ & 2 & 2 & 2 & $\begin{array}{r}9 \\
9 \\
15 \\
60 \\
1,000\end{array}$ & 9 & $\begin{array}{l}2,200 \\
1,900 \\
1,600 \\
1,400 \\
1,300\end{array}$ & $\begin{array}{r}5,000 \\
7,000 \\
10,000 \\
15,000 \\
19,000\end{array}$ & $\begin{array}{l}6,100 \\
6,000 \\
5,800 \\
5,600 \\
5,500\end{array}$ & $\begin{array}{l}\frac{8,580}{8,580} \\
8,580 \\
4,550 \\
4,550\end{array}$ & $\begin{array}{c}\frac{450}{400} \\
1 \\
400 \\
350\end{array}$ & $\frac{25}{1}$ & 2 \\
\hline $\begin{array}{r}6 \\
7 \\
8 \\
9 \\
10\end{array}$ & & & $\begin{array}{l}1 \\
2 \\
4 \\
1\end{array}$ & $\begin{array}{r}600 \\
300 \\
150 \\
60 \\
30\end{array}$ & $\begin{array}{r}10 \\
15 \\
20 \\
135 \\
\times 57,300 \\
\end{array}$ & $\begin{array}{l}1,200 \\
1,100 \\
1,000 \\
1,000 \\
1,200\end{array}$ & $\begin{array}{l}20,000 \\
\frac{21,000}{20,000} \\
18,000 \\
16,000\end{array}$ & $\begin{array}{l}5,400 \\
5,300 \\
5,200 \\
5,100 \\
5,000\end{array}$ & $\begin{array}{l}4,550 \\
3,030 \\
3,030 \\
2,770 \\
2,770\end{array}$ & $\left.\right|_{300} ^{1}$ & 10 & \\
\hline $\begin{array}{l}11 \\
12 \\
13 \\
14 \\
15\end{array}$ & & & $\begin{array}{r}1 \\
4 \\
10 \\
10 \\
8\end{array}$ & $\begin{array}{r}20 \\
15 \\
10 \\
6 \\
5\end{array}$ & $\begin{array}{l}50,000 \\
45,000 \\
40,000 \\
37,000 \\
25,000\end{array}$ & $\begin{array}{l}1,500 \\
1,800 \\
1,900 \\
2,100 \\
2,800\end{array}$ & $\begin{array}{r}14,000 \\
12,000 \\
10,000 \\
9,000 \\
8,4,00\end{array}$ & $\begin{array}{r}4,900 \\
4,800 \\
4,700 \\
\times 4,520 \\
4,410\end{array}$ & $\begin{array}{l}2,770 \\
2,530 \\
2,530 \\
2,530 \\
2,300\end{array}$ & $\begin{array}{c}250 \\
1 \\
250 \\
200 \\
1\end{array}$ & 1 & \\
\hline $\begin{array}{l}16 \\
17 \\
18 \\
19 \\
20\end{array}$ & • & & $\begin{array}{r}115 \\
16 \\
2 \\
25 \\
45\end{array}$ & & $\begin{array}{l}18,000 \\
13,000 \\
11,500 \\
11,000 \\
10,000\end{array}$ & $\begin{array}{r}4,000 \\
5,200 \\
6,600 \\
8,400 \\
10,000\end{array}$ & $\begin{array}{l}7,900 \\
7,600 \\
7,400 \\
7,200 \\
7,100\end{array}$ & $\begin{array}{l}4,410 \\
4,410 \\
4,090 \\
4,090 \\
4,090\end{array}$ & $\begin{array}{r}2,300 \\
2,210 \\
1,890 \\
1,200 \\
916\end{array}$ & $\begin{array}{r}200 \\
150 \\
150 \\
* 117\end{array}$ & 3 & \\
\hline $\begin{array}{l}21 \\
22 \\
23 \\
24 \\
25\end{array}$ & & & $\begin{array}{r}37 \\
29 \\
16 \\
9 \\
1\end{array}$ & $\begin{array}{l}1 \\
5 \\
6 \\
8\end{array}$ & $\begin{array}{l}8,600 \\
7,000 \\
6,000 \\
5,000 \\
4,200\end{array}$ & $\begin{array}{l}12,000 \\
14,000 \\
15,000 \\
15,000 \\
13,000\end{array}$ & $\begin{array}{l}7,000 \\
7,000 \\
6,900 \\
6,800 \\
6,700\end{array}$ & $\begin{array}{l}3,510 \\
2,930 \\
2,930 \\
2,930 \\
2,720\end{array}$ & $\begin{array}{r}442 \\
* 562 \\
550 \\
550\end{array}$ & $\begin{array}{l}115 \\
115 \\
100 \\
1 \\
100\end{array}$ & & \\
\hline $\begin{array}{l}26 \\
27 \\
28 \\
29 \\
30 \\
31 \\
\end{array}$ & 2 & $\begin{array}{r}1 \\
2 \\
- \\
\end{array}$ & 9 & $\begin{array}{r}10 \\
30 \\
100 \\
600 \\
200 \\
80 \\
\end{array}$ & $\begin{array}{c}3,500 \\
3,000 \\
2,600 \\
= \\
- \\
=\end{array}$ & $\begin{array}{r}\times 10,400 \\
7,500 \\
5,400 \\
4,600 \\
4,200 \\
4,000 \\
\end{array}$ & $\begin{array}{l}6,000 \\
6,500 \\
6,400 \\
6,300 \\
6,200\end{array}$ & $\begin{array}{r}2,720 \\
2,720 \\
2,260 \\
2,260 \\
2,720 \\
8,490 \\
\end{array}$ & $\begin{array}{l}500 \\
500 \\
1.50 \\
+50\end{array}$ & $\begin{array}{l}75 \\
1 \\
75 \\
50 \\
1 \\
50\end{array}$ & $\begin{array}{r}1 \\
3 \\
3 \\
\end{array}$ & $\begin{array}{l}1 \\
2\end{array}$ \\
\hline $\begin{array}{l}\text { Total } \\
\text { Mean } \\
\text { Ac-ft }\end{array}$ & $\begin{array}{r}62 \\
2 \\
123 \\
\end{array}$ & $\begin{array}{r}60 \\
2 \\
119 \\
\end{array}$ & $\begin{array}{r}419 \\
13.5 \\
931 \\
\end{array}$ & $\begin{array}{r}3,363 \\
108 \\
6,670 \\
\end{array}$ & $\begin{array}{r}35 \%, 925 \\
12,780 \\
709,900\end{array}$ & $\begin{array}{r}163,300 \\
5,268 \\
323,900\end{array}$ & $\begin{array}{r}308,000 \\
1,027 \\
610,900\end{array}$ & $\begin{array}{r}135,610 \\
, 375 \\
269,000\end{array}$ & $\begin{array}{r}77,220 \\
2,574 \\
253,200 \\
\end{array}$ & $\begin{array}{r}6,422 \\
307 \\
17,740 \\
\end{array}$ & $\begin{array}{l}248 \\
2.0 \\
492 \\
\end{array}$ & $\begin{array}{r}60 \\
2 \\
119 \\
\end{array}$ \\
\hline $\begin{array}{l}\text { Calend } \\
\text { Water }\end{array}$ & r 195 & $\begin{array}{l}\mathrm{Ma} \\
\mathrm{Ma}\end{array}$ & $\begin{array}{l}51,80 \\
57,30\end{array}$ & & $\begin{array}{ll}\text { in } & 2 \\
\text { in } & 2\end{array}$ & $\begin{array}{ll}\operatorname{ear} & 1,002 \\
\text { ean } & 2,88 i\end{array}$ & $\begin{array}{l}\mathrm{Ac}-1 \\
\mathrm{~A}-\mathrm{C}-1\end{array}$ & $\begin{array}{r}727,5 \\
+2,028,0\end{array}$ & & & & \\
\hline
\end{tabular}

* Discharge neasurement made or. this day.

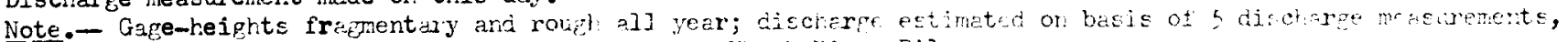
ore slope-aree measurement and recoras for hhast hiver DiJ aram. 
FARAH KIVER BASIN

Farah River near Farain, Afghanistan

Discharge, in cubic feet per second, water year October 1954 to September 1955

\begin{tabular}{|c|c|c|c|c|c|c|c|c|c|c|c|c|}
\hline Day & Oct. & Nov. & Dec. & Jan. & Feb. & Mar. & Apr. & May & June & July & Aug. & Sept. \\
\hline $\begin{array}{l}1 \\
2 \\
3 \\
4 \\
5\end{array}$ & $\frac{2}{1}$ & $\frac{2}{1}$ & $\frac{4}{1}$ & $\begin{array}{r}5 \\
5 \\
5 \\
51 \\
43\end{array}$ & $\begin{array}{l}235 \\
207 \\
214 \\
155 \\
145\end{array}$ & $\begin{array}{l}155 \\
140 \\
135 \\
140 \\
130\end{array}$ & $\begin{array}{l}\frac{2,800}{2,320} \\
2,150 \\
1,850 \\
1,700\end{array}$ & $\begin{array}{r}1,000 \\
1,000 \\
1,030 \\
986 \\
1,020\end{array}$ & $\begin{array}{l}\frac{785}{785} \\
685 \\
568 \\
516\end{array}$ & $\begin{array}{l}\frac{19}{16} \\
16 \\
12 \\
10\end{array}$ & 3 & $\begin{array}{l}6 \\
6 \\
6 \\
6 \\
5\end{array}$ \\
\hline $\begin{array}{r}6 \\
7 \\
8 \\
9 \\
10\end{array}$ & & $\begin{array}{l}1 \\
2 \\
2\end{array}$ & & $\begin{array}{r}75 \\
140 \\
207 \\
276 \\
318 \\
\end{array}$ & $\begin{array}{l}145 \\
140 \\
135 \\
135 \\
115\end{array}$ & $\begin{array}{r}107 \\
35 \\
10 \\
12 \\
12\end{array}$ & $\begin{array}{l}1,580 \\
1,560 \\
1,520 \\
1,460 \\
1,240\end{array}$ & $\begin{array}{l}1,040 \\
1,400 \\
1,300 \\
1,300 \\
1,170\end{array}$ & $\begin{array}{r}474 \\
484 \\
452 \\
* 432 \\
350\end{array}$ & $\begin{array}{r}10 \\
9 \\
9 \\
10 \\
9\end{array}$ & & $\begin{array}{l}\frac{3}{3} \\
1\end{array}$ \\
\hline $\begin{array}{l}11 \\
12 \\
13 \\
14 \\
15\end{array}$ & & $\begin{array}{l}2 \\
3 \\
4 \\
5 \\
5\end{array}$ & $\frac{1}{4}$ & $\begin{array}{l}200 \\
140 \\
140 \\
115 \\
115\end{array}$ & $\begin{array}{l}\frac{57}{172} \\
292 \\
334 \\
228\end{array}$ & $\begin{array}{r}2,250 \\
7,080 \\
* 5,050 \\
* 49,700 \\
55,100 \\
\end{array}$ & $\begin{array}{r}986 \\
1,060 \\
1,760 \\
1,760 \\
1,620\end{array}$ & $\begin{array}{r}1,120 \\
1,040 \\
1,030 \\
874 \\
848\end{array}$ & $\begin{array}{l}310 \\
284 \\
276 \\
260 \\
221\end{array}$ & $\begin{array}{r}9 \\
9 \\
8 \\
* 6 \\
5\end{array}$ & 3 & \\
\hline $\begin{array}{l}16 \\
17 \\
18 \\
19 \\
20\end{array}$ & $\begin{array}{r}1 \\
* 2 \\
2 \\
5\end{array}$ & $\begin{array}{r}* 4 \\
3 \\
3 \\
3 \\
3\end{array}$ & 2 & $\begin{array}{r}111 \\
99 \\
99 \\
91 \\
75\end{array}$ & $\begin{array}{r}221 \\
221 \\
214 \\
193 \\
* 200\end{array}$ & $\begin{array}{r}34,500 \\
11,300 \\
* 9,800 \\
6,860 \\
8,180\end{array}$ & $\begin{array}{l}1,440 \\
1,340 \\
1,200 \\
1,110 \\
1,000\end{array}$ & $\begin{array}{l}810 \\
810 \\
810 \\
810 \\
810\end{array}$ & $\begin{array}{r}155 \\
125 \\
115 \\
111 \\
99\end{array}$ & $\begin{array}{l}5 \\
5 \\
5 \\
4 \\
4\end{array}$ & 3 & \\
\hline $\begin{array}{l}27 \\
22 \\
23 \\
24 \\
25\end{array}$ & $\begin{array}{l}5 \\
4 \\
2 \\
1\end{array}$ & $\begin{array}{l}2 \\
2 \\
3 \\
2 \\
2\end{array}$ & & $\begin{array}{r}95 \\
111 \\
75 \\
72 \\
69\end{array}$ & $\begin{array}{l}186 \\
172 \\
193 \\
207 \\
155\end{array}$ & $\begin{array}{r}8,400 \\
* 5,700 \\
5,100 \\
4,000 \\
3,770\end{array}$ & $\begin{array}{l}944 \\
944 \\
972 \\
930 \\
930\end{array}$ & $\begin{array}{l}785 \\
785 \\
785 \\
760 \\
760\end{array}$ & $\begin{array}{l}83 \\
75 \\
69 \\
63 \\
63\end{array}$ & $\begin{array}{l}\frac{3}{3} \\
3 \\
3 \\
1\end{array}$ & * & \\
\hline $\begin{array}{l}26 \\
27 \\
28 \\
29 \\
30 \\
31 \\
\end{array}$ & $\begin{array}{r}1 \\
2 \\
\end{array}$ & $\begin{array}{l}2 \\
3 \\
4 \\
3 \\
3 \\
\end{array}$ & $\begin{array}{r}1 \\
5 \\
\end{array}$ & $\begin{array}{r}* 69 \\
69 \\
135 \\
130 \\
165 \\
200 \\
\end{array}$ & $\begin{array}{c}160 \\
165 \\
165 \\
- \\
- \\
- \\
\end{array}$ & $\begin{array}{r}3,030 \\
2,300 \\
2,130 \\
2,580 \\
3,280 \\
3,000 \\
\end{array}$ & $\begin{array}{r}916 \\
9144 \\
944 \\
972 \\
972 \\
- \\
\end{array}$ & $\begin{array}{l}785 \\
760 \\
760 \\
785 \\
785 \\
772 \\
\end{array}$ & $\begin{array}{r}51 \\
35 \\
29 \\
25 \\
25 \\
- \\
\end{array}$ & $\begin{array}{r}1 \\
3 \\
\end{array}$ & 3 & $\begin{array}{l}1 \\
3 \\
2 \\
\end{array}$ \\
\hline $\begin{array}{l}\text { Total } \\
\text { Mean } \\
\text { Ac-ft } \\
\end{array}$ & $\begin{array}{c}70 \\
2.3 \\
139 \\
\end{array}$ & $\begin{array}{r}81 \\
2.7 \\
161 \\
\end{array}$ & $\begin{array}{r}140 \\
4.5 \\
278 \\
\end{array}$ & $\begin{array}{r}3,500 \\
113 \\
6,942 \\
\end{array}$ & $\begin{array}{r}5,161 \\
184 \\
20,240\end{array}$ & $\begin{array}{r}233,986 \\
7,548 \\
464,100 \\
\end{array}$ & $\begin{array}{r}40,924 \\
1,364 \\
81.170\end{array}$ & $\begin{array}{r}28,730 \\
927 \\
56,980\end{array}$ & $\begin{array}{r}8,005 \\
267 \\
15,880 \\
\end{array}$ & $\begin{array}{r}213 \\
6.9 \\
422 \\
\end{array}$ & $\begin{array}{r}93 \\
3 \\
184 \\
\end{array}$ & $\begin{array}{c}110 \\
3.7 \\
218\end{array}$ \\
\hline $\begin{array}{l}\text { Caler } \\
\text { Wate }\end{array}$ & $\operatorname{ar} 1$ & $\begin{array}{l}54: \\
55:\end{array}$ & $\begin{array}{l}57, \\
55,\end{array}$ & & $\begin{array}{l}2 \\
2\end{array}$ & $\begin{array}{l}\text { Mean } 2 \\
\text { Mean }\end{array}$ & $\begin{array}{l}\mathrm{AC}- \\
\mathrm{AC}-\end{array}$ & $\begin{array}{r}2,08 \\
63\end{array}$ & 100 & & & \\
\hline
\end{tabular}

* Discharge measurement made on this day. 
FARAH RIVER BASIN

Farah River near Farah, Afghanistan

Discharge, in cubic feet per second, water year October 1955 to Septeniber 1956

\begin{tabular}{|c|c|c|c|c|c|c|c|c|c|c|c|c|}
\hline Das & Oct. & Nov. & Dec. & Jan. & $\mathrm{F} \in \mathrm{b}$. & Mar. & Apr. & May & June & July & Aug. & Sept. \\
\hline $\begin{array}{l}1 \\
2 \\
3 \\
4 \\
5\end{array}$ & $\begin{array}{r}69 \\
125 \\
6 \\
7 \\
6\end{array}$ & $\frac{2}{1}$ & $\frac{5}{1}$ & $\begin{array}{r}\frac{145}{160} \\
1,330 \\
1,460 \\
1,140\end{array}$ & $\begin{array}{l}698 \\
622 \\
600 \\
568 \\
526\end{array}$ & $\begin{array}{r}4,920 \\
4,030 \\
3,960 \\
8,310 \\
\times 35,000 \\
\end{array}$ & $\begin{array}{r}10,400 \\
14,000 \\
12,500 \\
9,900 \\
10,600\end{array}$ & $\begin{array}{l}9,700 \\
9,400 \\
9,200 \\
8,700 \\
8,200\end{array}$ & $\begin{array}{l}\frac{334}{334} \\
350 \\
350 \\
334\end{array}$ & $\begin{array}{l}318 \\
284 \\
214 \\
200 \\
179\end{array}$ & $\begin{array}{l}\frac{179}{165} \\
155 \\
155 \\
155\end{array}$ & $\begin{array}{l}18 \\
\frac{20}{19} \\
18 \\
12\end{array}$ \\
\hline $\begin{array}{r}6 \\
7 \\
8 \\
9 \\
10\end{array}$ & $\begin{array}{l}3 \\
2 \\
\underline{1}\end{array}$ & & $\begin{array}{r}5 \\
6 \\
5 \\
7 \\
45\end{array}$ & $\begin{array}{l}810 \\
822 \\
622 \\
558 \\
536\end{array}$ & $\begin{array}{l}494 \\
463 \\
392 \\
342 \\
326\end{array}$ & $\begin{array}{r}18,700 \\
15,500 \\
12,900 \\
9,900 \\
8,040\end{array}$ & $\begin{array}{l}14,300 \\
21,700 \\
25,000 \\
30,000 \\
37,000\end{array}$ & $\begin{array}{l}7,600 \\
6,800 \\
6,000 \\
5,400 \\
4,800\end{array}$ & $\begin{array}{l}334 \\
334 \\
334 \\
334 \\
358\end{array}$ & $\begin{array}{r}160 \\
135 \\
6,370 \\
5,360 \\
2,210\end{array}$ & $\begin{array}{r}155 \\
145 \\
165 \\
165 \\
165\end{array}$ & $\begin{array}{l}5 \\
4 \\
4 \\
4 \\
4\end{array}$ \\
\hline $\begin{array}{l}11 \\
12 \\
13 \\
14 \\
15\end{array}$ & & * & $\begin{array}{l}2,390 \\
2,010 \\
1,460 \\
2,770 \\
5,700 \\
\end{array}$ & $\begin{array}{l}442 \\
400 \\
367 \\
292 \\
494\end{array}$ & $\begin{array}{l}268 \\
260 \\
252 \\
235 \\
235\end{array}$ & $\begin{array}{l}7,380 \\
6,740 \\
6,330 \\
6,210 \\
6,820\end{array}$ & $\begin{array}{l}\frac{50,000}{42,000} \\
36,000 \\
30,000 \\
26,000\end{array}$ & $\begin{array}{l}4,000 \\
3,300 \\
2,500 \\
1,900 \\
1,500\end{array}$ & $\begin{array}{l}350 \\
350 \\
350 \\
350 \\
350\end{array}$ & $\begin{array}{r}474 \\
547 \\
326 \\
172 \\
2,320\end{array}$ & $\begin{array}{l}135 \\
135 \\
130 \\
125 \\
115\end{array}$ & $\begin{array}{l}3 \\
3 \\
3 \\
3 \\
3\end{array}$ \\
\hline $\begin{array}{l}16 \\
17 \\
18 \\
19 \\
20\end{array}$ & & & $\begin{array}{r}1,890 \\
860 \\
610 \\
400 \\
1,330\end{array}$ & $\begin{array}{r}568 \\
17,300 \\
8,040 \\
5,550 \\
3,830\end{array}$ & $\begin{array}{l}235 \\
235 \\
221 \\
160 \\
135\end{array}$ & $\begin{array}{r}6,530 \\
5,360 \\
4,890 \\
21,800 \\
16,800\end{array}$ & $\begin{array}{l}23,000 \\
19,000 \\
17,000 \\
15,000 \\
13,000\end{array}$ & $\begin{array}{r}1,000 \\
800 \\
540 \\
420 \\
326\end{array}$ & $\begin{array}{r}350 \\
452 \\
410 \\
* 400 \\
392\end{array}$ & $\begin{array}{l}2,240 \\
2,170 \\
2,260 \\
\frac{2,420}{2,320}\end{array}$ & $\begin{array}{l}99 \\
99 \\
91 \\
91 \\
87\end{array}$ & $\begin{array}{l}3 \\
2 \\
2 \\
2 \\
2\end{array}$ \\
\hline $\begin{array}{l}27 \\
22 \\
23 \\
24 \\
25\end{array}$ & & $\begin{array}{l}1 \\
2 \\
3\end{array}$ & $\begin{array}{r}1,000 \\
* 860 \\
635 \\
474 \\
376\end{array}$ & $\begin{array}{l}2,260 \\
2,130 \\
1,780 \\
1,450 \\
1,360\end{array}$ & $\begin{array}{l}135 \\
135 \\
135 \\
135 \\
179\end{array}$ & $\begin{array}{l}14,400 \\
14,900 \\
12,500 \\
12,800 \\
13,700\end{array}$ & $\begin{array}{r}11,000 \\
10,000 \\
9,000 \\
8,400 \\
7,600\end{array}$ & $\begin{array}{r}\frac{310}{635} \\
2,280 \\
848 \\
660\end{array}$ & $\begin{array}{l}367 \\
367 \\
367 \\
410 \\
589\end{array}$ & $\begin{array}{r}1,110 \\
284 \\
235 \\
235 \\
484\end{array}$ & $\begin{array}{l}69 \\
69 \\
66 \\
63 \\
51\end{array}$ & $\begin{array}{r}2 \\
2 \\
\frac{1}{1} \\
* 1\end{array}$ \\
\hline $\begin{array}{l}26 \\
27 \\
23 \\
29 \\
30 \\
31 \\
\end{array}$ & $\begin{array}{l}1 \\
2 \\
\end{array}$ & $\begin{array}{l}4 \\
5 \\
6 \\
8 \\
6 \\
- \\
\end{array}$ & $\begin{array}{l}252 \\
244 \\
172 \\
150 \\
140 \\
135 \\
\end{array}$ & $\begin{array}{r}1,270 \\
1,160 \\
930 \\
902 \\
810 \\
722 \\
\end{array}$ & $\begin{array}{r}193 \\
5,650 \\
5,740 \\
5,180 \\
- \\
=\end{array}$ & $\begin{array}{r}10,400 \\
8,720 \\
7,590 \\
7,680 \\
8,260 \\
9,400 \\
\end{array}$ & $\begin{array}{r}6,800 \\
6,200 \\
5,600 \\
7,000 \\
10,000\end{array}$ & $\begin{array}{l}547 \\
334 \\
334 \\
334 \\
334 \\
334\end{array}$ & $\begin{array}{l}589 \\
589 \\
589 \\
547 \\
494 \\
- \\
\end{array}$ & $\begin{array}{l}292 \\
600 \\
342 \\
228 \\
214 \\
193\end{array}$ & $\begin{array}{l}37 \\
31 \\
24 \\
22 \\
22 \\
20\end{array}$ & $\begin{array}{l}1 \\
1 \\
1 \\
1 \\
1\end{array}$ \\
\hline $\begin{array}{l}\text { Total } \\
\text { Mean } \\
\text { Ac-ft }\end{array}$ & $\begin{array}{r}266 \\
8.6 \\
528 \\
\end{array}$ & $\begin{array}{c}80 \\
2.7 \\
159\end{array}$ & $\begin{array}{r}23,950 \\
773 \\
47,500 \\
\end{array}$ & $\begin{array}{r}59,640 \\
1,924 \\
118,300 \\
\end{array}$ & $\begin{array}{r}24,750 \\
853 \\
49,090 \\
\end{array}$ & $\begin{array}{r}330,370 \\
10,660 \\
655,300 \\
\end{array}$ & $\begin{array}{r}538,000 \\
17,930 \\
1,067,000 \\
\end{array}$ & $\begin{array}{r}99,040 \\
3,195 \\
196,400 \\
\end{array}$ & $\begin{array}{r}12,060 \\
4,020 \\
23,920 \\
\end{array}$ & $\begin{array}{r}39,896 \\
1,287 \\
79,130\end{array}$ & $\begin{array}{r}3,185 \\
103 \\
6,317\end{array}$ & $\begin{array}{l}146 \\
4.9 \\
290 \\
\end{array}$ \\
\hline
\end{tabular}

Calendar year 1955: $\operatorname{Max} 55,100$ Min 2 liean 945 Ac-ft 684,200

* Discharge measurement made on this day.

Note. - No gage-helght record Apr. 8 to May 19, Seft. 22-30; discharge estiniated on basis of records for Khash River at Dilaram. 
FAHAH RIVER BASIN

Farat, River near Farah, Afghanistar

Discharge, in cubic feet per second, water year Uctober 1956 to September 1957

\begin{tabular}{|c|c|c|c|c|c|c|c|c|c|c|c|c|}
\hline Day & Oct. & Nov. & Dec. & $\mathrm{Jar}_{.}$ & Feb。 & Mar. & Apr. & May & June & July & Aug. & Sept. \\
\hline $\begin{array}{l}1 \\
2 \\
3 \\
4 \\
5\end{array}$ & 1 & 3 & $\begin{array}{l}13 \\
13 \\
16 \\
16 \\
18\end{array}$ & $\begin{array}{l}\frac{252}{252} \\
252 \\
252 \\
252\end{array}$ & $\begin{array}{l}2,210 \\
2,510 \\
2,560 \\
3,120 \\
5,550 \\
\end{array}$ & $\begin{array}{l}2,560 \\
2,600 \\
2,600 \\
2,560 \\
2,560\end{array}$ & $\begin{array}{r}6,800 \\
6,900 \\
7,000 \\
7,400 \\
12,000\end{array}$ & $\begin{array}{l}\frac{7,200}{5,000} \\
2,500 \\
1,300 \\
1,000\end{array}$ & $\begin{array}{l}480 \\
460 \\
430 \\
400 \\
380\end{array}$ & $\begin{array}{l}1,540 \\
1,430 \\
1,360 \\
1,300 \\
1,270\end{array}$ & $\begin{array}{l}\frac{568}{516} \\
494 \\
474 \\
463\end{array}$ & $\begin{array}{l}\frac{221}{221} \\
221 \\
221 \\
214\end{array}$ \\
\hline $\begin{array}{r}6 \\
7 \\
8 \\
9 \\
10\end{array}$ & & & $\begin{array}{l}19 \\
20 \\
29 \\
31 \\
51\end{array}$ & $\begin{array}{l}252 \\
252 \\
252 \\
252 \\
252\end{array}$ & $\begin{array}{l}4,150 \\
2,530 \\
2,560 \\
2,420 \\
2,320\end{array}$ & $\begin{array}{l}2,580 \\
2,620 \\
2,670 \\
2,640 \\
2,580\end{array}$ & $\begin{array}{r}\frac{30,000}{28,000} \\
17,000 \\
10,000 \\
9,000\end{array}$ & $\begin{array}{l}950 \\
920 \\
900 \\
900 \\
900\end{array}$ & $\begin{array}{l}350 \\
330 \\
300 \\
270 \\
250\end{array}$ & $\begin{array}{l}1,1,0 \\
1,070 \\
1,060 \\
1,060 \\
1,060\end{array}$ & $\begin{array}{l}463 \\
463 \\
432 \\
421 \\
410\end{array}$ & $\begin{array}{l}207 \\
207 \\
207 \\
207 \\
207\end{array}$ \\
\hline $\begin{array}{l}11 \\
12 \\
13 \\
14 \\
15\end{array}$ & $\begin{array}{l}1 \\
1\end{array}$ & & $\begin{array}{l}57 \\
57 \\
63 \\
69 \\
83\end{array}$ & $\begin{array}{l}252 \\
384 \\
318 \\
301 \\
284\end{array}$ & $\begin{array}{l}2,260 \\
2,150 \\
1,660 \\
1,600 \\
\frac{1,830}{3,8}\end{array}$ & $\begin{array}{l}2,530 \\
2,490 \\
2,440 \\
2,440 \\
3,000\end{array}$ & $\begin{array}{l}8,700 \\
8,400 \\
8,200 \\
7,900 \\
7,700\end{array}$ & $\begin{array}{l}900 \\
900 \\
900 \\
900 \\
900\end{array}$ & $\begin{array}{l}230 \\
220 \\
200 \\
200 \\
190\end{array}$ & $\begin{array}{l}1,040 \\
1,030 \\
1,030 \\
1,030 \\
1,000\end{array}$ & $\begin{array}{l}400 \\
400 \\
392 \\
384 \\
384\end{array}$ & $\begin{array}{r}207 \\
207 \\
207 \\
* 207 \\
207\end{array}$ \\
\hline $\begin{array}{l}16 \\
17 \\
18 \\
19 \\
20\end{array}$ & 2 & $*$ & $\begin{array}{l}83 \\
83 \\
91 \\
91 \\
91\end{array}$ & $\begin{array}{r}284 \\
276 \\
252 \\
252 \\
4,150\end{array}$ & $\begin{array}{l}2,240 \\
2,130 \\
2,130 \\
2,150 \\
2,170\end{array}$ & $\begin{array}{r}7,000 \\
40,000 \\
35,000 \\
27,000 \\
20,000\end{array}$ & $\begin{array}{l}7,400 \\
7,200 \\
7,100 \\
7,000 \\
6,800\end{array}$ & $\begin{array}{l}880 \\
860 \\
820 \\
770 \\
720\end{array}$ & $\begin{array}{l}180 \\
170 \\
150 \\
130 \\
120 \\
\end{array}$ & $\begin{array}{l}986 \\
944 \\
944 \\
916 \\
916\end{array}$ & $\begin{array}{l}384 \\
358 \\
334 \\
326 \\
292\end{array}$ & $\begin{array}{l}207 \\
207 \\
207 \\
207 \\
207\end{array}$ \\
\hline $\begin{array}{l}21 \\
22 \\
23 \\
24 \\
25\end{array}$ & & & $\begin{array}{r}91 \\
95 \\
160 \\
252 \\
252\end{array}$ & $\begin{array}{l}8,490 \\
8,720 \\
7,860 \\
6,330 \\
5,740\end{array}$ & $\begin{array}{l}1,780 \\
1,760 \\
1,720 \\
1,700 \\
1,670\end{array}$ & $\begin{array}{r}13,000 \\
11,000 \\
9,000 \\
7,600 \\
7,100\end{array}$ & $\begin{array}{l}6,600 \\
6,500 \\
6,400 \\
6,300 \\
6,200 \\
\end{array}$ & $\begin{array}{l}680 \\
640 \\
600 \\
580 \\
560\end{array}$ & $\begin{array}{l}1,000 \\
1,600 \\
1,780 \\
\frac{1}{1,780} \\
1,780\end{array}$ & $\begin{array}{r}772 \\
748 \\
735 \\
* 685 \\
635\end{array}$ & $\begin{array}{l}276 \\
268 \\
268 \\
260 \\
252\end{array}$ & $\begin{array}{l}207 \\
207 \\
207 \\
207 \\
207\end{array}$ \\
\hline $\begin{array}{l}26 \\
27 \\
28 \\
29 \\
30 \\
31 \\
\end{array}$ & $\begin{array}{l}1 \\
2 \\
\end{array}$ & $\begin{array}{l}1 \\
3\end{array}$ & $\begin{array}{l}235 \\
244 \\
252 \\
252 \\
252 \\
252 \\
\end{array}$ & $\begin{array}{l}4,360 \\
3,250 \\
2,880 \\
2,670 \\
2,490 \\
2,320 \\
\end{array}$ & $\begin{array}{l}2,090 \\
2,260 \\
2,370\end{array}$ & $\begin{array}{l}6,900 \\
6,800 \\
6,800 \\
6,800 \\
6,800 \\
6,800\end{array}$ & $\begin{array}{l}6,200 \\
6,200 \\
6,400 \\
7,000 \\
8,000\end{array}$ & $\begin{array}{l}540 \\
530 \\
520 \\
510 \\
510 \\
500 \\
\end{array}$ & $\begin{array}{l}1,780 \\
1,780 \\
1,780 \\
1,740 \\
1,680\end{array}$ & $\begin{array}{l}622 \\
589 \\
589 \\
589 \\
589 \\
589\end{array}$ & $\begin{array}{l}252 \\
252 \\
244 \\
235 \\
235 \\
221\end{array}$ & $\begin{array}{l}214 \\
221 \\
207 \\
\frac{179}{179}\end{array}$ \\
\hline $\begin{array}{l}\text { Total } \\
\text { Mean } \\
\text { Ac-ft }\end{array}$ & $\begin{array}{c}47 \\
1.5 \\
93\end{array}$ & $\begin{array}{r}90 \\
3 \\
179 \\
\end{array}$ & $\begin{array}{r}3,331 \\
108 \\
6,607\end{array}$ & $\begin{array}{r}64,883 \\
2,093 \\
128,700 \\
\end{array}$ & $\begin{array}{r}67,600 \\
2,414 \\
134,100 \\
\end{array}$ & $\begin{array}{r}256,470 \\
8,273 \\
508,700 \\
\end{array}$ & $\begin{array}{r}276,300 \\
9,210 \\
548,000\end{array}$ & $\begin{array}{r}36,290 \\
1,271 \\
71,980\end{array}$ & $\begin{array}{r}22, \overline{140} \\
738 \\
43,910 \\
\end{array}$ & $\begin{array}{r}29,268 \\
744 \\
58,050\end{array}$ & $\begin{array}{r}11, \frac{121}{359} \\
22,060\end{array}$ & $\begin{array}{r}6,238 \\
208 \\
12,370 \\
\end{array}$ \\
\hline
\end{tabular}

Calendar year 1956: Nax 50,000 Kin 1 kean 3,043 Ac-f't 2,203,000

water year 1956-57: Max 40,000 Win 1 Mean 2,120 Ac-ft 1,535,000

* Discharge measurement or field estimate made on this day.

Note.- No gage-height record Oct. 1 to Nov. 30, Mar. 15 to June 22; discharge estimated on basis of records for Khash Fiver at "ilaram and discharge measureinents. 
FARAH RIVER BASIN

Farah River near Farah, Afghanistan

Discharge, in cuble feet per second, water year October 1957 to September 1958

\begin{tabular}{|c|c|c|c|c|c|c|c|c|c|c|c|c|}
\hline Dar & oct. & $\mathrm{Nor}_{2}$ & Dec. & Jan. & Febe & Mar, & Apr, & Mar & June & July & Aug. & Sept. \\
\hline $\begin{array}{l}1 \\
2 \\
3 \\
4 \\
5\end{array}$ & $\begin{array}{r}\frac{150}{110} \\
80 \\
70 \\
60\end{array}$ & $\begin{array}{l}\frac{62}{63} \\
66 \\
68 \\
70\end{array}$ & $\begin{array}{l}350 \\
360 \\
360 \\
360 \\
360\end{array}$ & $\begin{array}{l}221 \\
221 \\
207 \\
193 \\
179\end{array}$ & $\begin{array}{r}568 \\
3,770 \\
3,620 \\
2,770 \\
2,090\end{array}$ & $\begin{array}{l}494 \\
442 \\
421 \\
410 \\
400\end{array}$ & $\begin{array}{l}2,320 \\
1,970 \\
1,640 \\
1,780 \\
1,740\end{array}$ & $\begin{array}{l}\frac{3,190}{3,190} \\
2,300 \\
2,210 \\
1,950\end{array}$ & $\begin{array}{l}\frac{760}{760} \\
748 \\
672 \\
648\end{array}$ & $\begin{array}{l}33 \\
33 \\
33 \\
\frac{37}{20}\end{array}$ & $\begin{array}{l}\frac{4.2}{4.2} \\
3.8 \\
3.2 \\
3.0\end{array}$ & $\begin{array}{r}1.6 \\
1.6 \\
1.5 \\
* 1.5 \\
1.6\end{array}$ \\
\hline $\begin{array}{r}6 \\
7 \\
8 \\
9 \\
10\end{array}$ & $\begin{array}{l}52 \\
46 \\
40 \\
36 \\
35\end{array}$ & $\begin{array}{l}72 \\
74 \\
76 \\
78 \\
80\end{array}$ & $\begin{array}{l}1,500 \\
1,800 \\
2,000 \\
1,900 \\
1,700\end{array}$ & $\begin{array}{r}\frac{63}{99} \\
103 \\
107 \\
95\end{array}$ & $\begin{array}{l}1,660 \\
1,420 \\
1,300 \\
1,160 \\
1,040\end{array}$ & $\begin{array}{l}400 \\
358 \\
318 \\
301 \\
\frac{848}{48}\end{array}$ & $\begin{array}{l}1,670 \\
1,760 \\
2,210 \\
2,210 \\
1,850\end{array}$ & $\begin{array}{l}1,810 \\
1,700 \\
1,680 \\
1,600 \\
1,530\end{array}$ & $\begin{array}{l}600 \\
505 \\
474 \\
463 \\
318\end{array}$ & $\begin{array}{l}22 \\
19 \\
19 \\
19 \\
19\end{array}$ & $\begin{array}{l}3.0 \\
3.0 \\
3.2 \\
3.0 \\
2.8\end{array}$ & $\begin{array}{l}1.6 \\
1.5 \\
1.6 \\
1.6 \\
1.6\end{array}$ \\
\hline $\begin{array}{l}11 \\
12 \\
13 \\
14 \\
15\end{array}$ & $\begin{array}{l}40 \\
52 \\
58 \\
62 \\
66\end{array}$ & $\begin{array}{l}78 \\
77 \\
76 \\
75 \\
73\end{array}$ & $\begin{array}{r}1,400 \\
1,000 \\
800 \\
680 \\
560\end{array}$ & $\begin{array}{l}358 \\
463 \\
400 \\
392 \\
326\end{array}$ & $\begin{array}{l}888 \\
810 \\
772 \\
698 \\
648\end{array}$ & $\begin{array}{l}1,340 \\
1,430 \\
1,600 \\
1,260 \\
1,160\end{array}$ & $\begin{array}{l}1,640 \\
1,540 \\
1,530 \\
1,440 \\
1,340\end{array}$ & $\begin{array}{l}1,530 \\
1,520 \\
1,500 \\
1,430 \\
1,340\end{array}$ & $\begin{array}{l}318 \\
276 \\
214 \\
179 \\
165\end{array}$ & $\begin{array}{r}19 \\
18 \\
16 \\
* 14 \\
14\end{array}$ & $\begin{array}{r}2.8 \\
* 2.8 \\
2.8 \\
2.8 \\
2.5\end{array}$ & $\begin{array}{l}1.6 \\
1.6 \\
1.8 \\
1.8 \\
1.8\end{array}$ \\
\hline $\begin{array}{l}16 \\
17 \\
18 \\
19 \\
20\end{array}$ & $\begin{array}{l}69 \\
72 \\
74 \\
76 \\
77\end{array}$ & $\begin{array}{r}70 \\
67 \\
65 \\
64 \\
350 \\
\end{array}$ & $\begin{array}{l}480 \\
420 \\
380 \\
350 \\
330\end{array}$ & $\begin{array}{l}276 \\
228 \\
155 \\
130 \\
221\end{array}$ & $\begin{array}{l}600 \\
578 \\
568 \\
568 \\
568\end{array}$ & $\begin{array}{r}930 \\
848 \\
835 \\
1,000 \\
1,110\end{array}$ & $\begin{array}{l}1,540 \\
1,810 \\
2,010 \\
1,990 \\
1,830\end{array}$ & $\begin{array}{l}1,330 \\
1,330 \\
1,300 \\
1,260 \\
1,220\end{array}$ & $\begin{array}{l}160 \\
155 \\
145 \\
140 \\
125\end{array}$ & $\begin{array}{l}14 \\
14 \\
14 \\
10 \\
11\end{array}$ & $\begin{array}{l}2.1 \\
2.1 \\
2.1 \\
2.1 \\
2.0\end{array}$ & $\begin{array}{l}1.8 \\
1.9 \\
1.9 \\
1.9 \\
2.0\end{array}$ \\
\hline $\begin{array}{l}21 \\
22 \\
23 \\
24 \\
25\end{array}$ & $\begin{array}{r}78 \\
78 \\
78 \\
* 78 \\
76\end{array}$ & $\begin{array}{l}300 \\
280 \\
280 \\
280 \\
290\end{array}$ & $\begin{array}{l}310 \\
300 \\
290 \\
280 \\
270\end{array}$ & $\begin{array}{l}\frac{916}{735} \\
735 \\
668 \\
547\end{array}$ & $\begin{array}{l}547 \\
536 \\
526 \\
526 \\
526\end{array}$ & $\begin{array}{l}1,110 \\
1,110 \\
1,110 \\
1,110 \\
1,110\end{array}$ & $\begin{array}{l}1,810 \\
1,720 \\
1,640 \\
1,480 \\
1,810\end{array}$ & $\begin{array}{r}1,170 \\
1,080 \\
1,030 \\
1,030 \\
860\end{array}$ & $\begin{array}{r}103 \\
99 \\
83 \\
83 \\
83\end{array}$ & $\begin{array}{l}11 \\
9.4 \\
8.8 \\
8.8 \\
7.4\end{array}$ & $\begin{array}{l}2.0 \\
2.1 \\
2.1 \\
2.0 \\
2.0\end{array}$ & $\begin{array}{l}2.0 \\
2.0 \\
2.2 \\
2.2 \\
2.2\end{array}$ \\
\hline $\begin{array}{l}26 \\
27 \\
28 \\
29 \\
30 \\
31\end{array}$ & $\begin{array}{l}72 \\
69 \\
66 \\
64 \\
63 \\
62\end{array}$ & $\begin{array}{c}300 \\
310 \\
320 \\
330 \\
350 \\
-\end{array}$ & $\begin{array}{l}260 \\
250 \\
245 \\
240 \\
235 \\
230 \\
\end{array}$ & $\begin{array}{l}505 \\
558 \\
735 \\
735 \\
432 \\
568\end{array}$ & $\begin{array}{l}526 \\
526 \\
526 \\
494 \\
- \\
-\end{array}$ & $\begin{array}{l}1,110 \\
1,140 \\
4,920 \\
5,850 \\
3,480 \\
3,250\end{array}$ & $\begin{array}{c}1,740 \\
1,800 \\
1,760 \\
1,720 \\
3,480 \\
-\end{array}$ & $\begin{array}{r}1,030 \\
1,030 \\
1,000 \\
1,000 \\
860 \\
785\end{array}$ & $\begin{array}{r}69 \\
54 \\
107 \\
41 \\
33 \\
-\end{array}$ & $\begin{array}{l}5.8 \\
5.2 \\
5.1 \\
5.1 \\
5.0 \\
5.2\end{array}$ & $\begin{array}{l}1.9 \\
1.8 \\
1.7 \\
1.8 \\
1.7 \\
1.6\end{array}$ & $\begin{array}{c}\frac{2.4}{2.3} \\
2.2 \\
2.2 \\
2.2 \\
-\end{array}$ \\
\hline $\begin{array}{l}\text { Total } \\
\text { Mean } \\
\text { Ac-ft }\end{array}$ & $\begin{array}{r}2,109 \\
68.0 \\
4,183 \\
\end{array}$ & $\begin{array}{r}4,744 \\
158 \\
9,410 \\
\end{array}$ & $\begin{array}{r}20,000 \\
645 \\
39,670 \\
\end{array}$ & $\begin{array}{r}11,571 \\
373 \\
22,950 \\
\end{array}$ & $\begin{array}{r}30,829 \\
1,063 \\
61,150 \\
\end{array}$ & $\begin{array}{r}41,205 \\
1,329 \\
81,730 \\
\end{array}$ & $\begin{array}{r}54,780 \\
1,826 \\
108,600 \\
\end{array}$ & $\begin{array}{r}45,795 \\
1,477 \\
90,830 \\
\end{array}$ & $\begin{array}{r}8,580 \\
286 \\
17,020 \\
\end{array}$ & $\begin{array}{c}474.8 \\
15 \\
948 \\
\end{array}$ & $\begin{array}{c}78.2 \\
2.52 \\
155 \\
\end{array}$ & $\begin{array}{c}55.7 \\
1.86 \\
110\end{array}$ \\
\hline
\end{tabular}

Calendar year 1957: Max 40,000 Min 35 Mean 2,184 Ac-ft 1,581,000

water year 1957-58: Max 5,850 Min 1.5 Mean 602 Ac-ft 436,800

* Discharge measurement made on this day.

Nete. - No gage-heights Oct. 1 to Dec. 31; discharge estimated on basis of records for Khash River at Dilaram. 
FARAH RIVER BASIN

Farah River near Farah, Afghanistan

Discharge, in cubic feet per second, water year October 1958 to September 1959

\begin{tabular}{|c|c|c|c|c|c|c|c|c|c|c|c|c|}
\hline Day & Oct. & Nov. & Dec. & Jan. & Feb. & Mar. & Apr。 & May & June & July & Augo & Sept. \\
\hline $\begin{array}{l}1 \\
2 \\
3 \\
4 \\
5\end{array}$ & $\begin{array}{l}2 \\
1\end{array}$ & $\begin{array}{l}2 \\
1\end{array}$ & $\frac{2}{1}$ & $\begin{array}{l}16 \\
16 \\
14 \\
14 \\
13\end{array}$ & $\begin{array}{l}510 \\
480 \\
460 \\
440 \\
450\end{array}$ & $\begin{array}{r}1,290 \\
13,700 \\
9,550 \\
5,560 \\
* 4,340\end{array}$ & $\begin{array}{l}7,710 \\
6,980 \\
7,520 \\
7,650 \\
6,680\end{array}$ & $\begin{array}{l}\frac{3,010}{2,830} \\
2,740 \\
2,560 \\
2,520\end{array}$ & $\begin{array}{l}1,230 \\
1,260 \\
1,200 \\
1,150 \\
1,320\end{array}$ & $\begin{array}{l}\frac{318}{214} \\
49 \\
14 \\
12\end{array}$ & 4 & $\begin{array}{l}3 \\
1\end{array}$ \\
\hline $\begin{array}{r}6 \\
7 \\
8 \\
9 \\
10\end{array}$ & $\begin{array}{l}1 \\
2\end{array}$ & $\frac{1}{2}$ & $\begin{array}{l}1 \\
2\end{array}$ & $\begin{array}{l}16 \\
17 \\
17 \\
15 \\
14\end{array}$ & $\begin{array}{l}480 \\
470 \\
420 \\
370 \\
340 \\
\end{array}$ & $\begin{array}{l}4,200 \\
3,910 \\
3,670 \\
3,420 \\
8,010\end{array}$ & $\begin{array}{l}6,100 \\
5,660 \\
5,380 \\
5,220 \\
5,330\end{array}$ & $\begin{array}{l}2,470 \\
2,380 \\
2,290 \\
2,160 \\
2,060\end{array}$ & $\begin{array}{l}1,430 \\
1,380 \\
1,320 \\
1,200 \\
1,150\end{array}$ & $\begin{array}{l}10 \\
16 \\
14 \\
12 \\
70\end{array}$ & & \\
\hline $\begin{array}{l}17 \\
12 \\
13 \\
14 \\
15\end{array}$ & 2 & 2 & $\begin{array}{r}2 \\
2 \\
960 \\
688 \\
460\end{array}$ & $\begin{array}{l}13 \\
12 \\
10 \\
10 \\
10\end{array}$ & $\begin{array}{l}544 \\
946 \\
791 \\
688 \\
628\end{array}$ & $\begin{array}{l}7,280 \\
5,150 \\
4,820 \\
3,830 \\
3,540\end{array}$ & $\begin{array}{l}5,330 \\
5,220 \\
5,060 \\
4,900 \\
4,600\end{array}$ & $\begin{array}{l}1,990 \\
1,920 \\
1,840 \\
1,880 \\
1,770\end{array}$ & $\begin{array}{r}1,150 \\
1,090 \\
1,010 \\
977 \\
948\end{array}$ & $\begin{array}{r}76 \\
96 \\
122 \\
96 \\
90\end{array}$ & & \\
\hline $\begin{array}{l}16 \\
17 \\
18 \\
19 \\
20\end{array}$ & * & $\frac{1}{2}$ & $\begin{array}{l}360 \\
298 \\
241 \\
183 \\
138\end{array}$ & $\begin{array}{r}10 \\
* 10 \\
13 \\
12 \\
10\end{array}$ & $\begin{array}{r}568 \\
544 \\
520 \\
1,190 \\
994\end{array}$ & $\begin{array}{l}3,380 \\
3,270 \\
3,190 \\
\frac{2,120}{3,120}\end{array}$ & $\begin{array}{l}4,400 \\
4,250 \\
4,150 \\
4,050 \\
3,860\end{array}$ & $\begin{array}{l}1,770 \\
2,060 \\
2,420 \\
2,130 \\
1,950\end{array}$ & $\begin{array}{l}920 \\
977 \\
948 \\
920 \\
948\end{array}$ & $\begin{array}{r}63 \\
14 \\
10 \\
4 \\
8\end{array}$ & & \\
\hline $\begin{array}{l}21 \\
22 \\
23 \\
24 \\
25\end{array}$ & 2 & $\begin{array}{r}2 \\
+\end{array}$ & $\begin{array}{r}104 \\
95 \\
70 \\
60 \\
50\end{array}$ & $\begin{array}{r}18 \\
20 \\
32 \\
1,480 \\
1,830 \\
\end{array}$ & $\begin{array}{l}915 \\
838 \\
822 \\
868 \\
930\end{array}$ & $\begin{array}{l}3,160 \\
3,340 \\
3,630 \\
3,710 \\
3,630\end{array}$ & $\begin{array}{l}4,100 \\
5,600 \\
4,600 \\
4,400 \\
4,100\end{array}$ & $\begin{array}{r}1,840 \\
1,740 \\
1,630 \\
1,560 \\
* 1,460\end{array}$ & $\begin{array}{l}878 \\
815 \\
794 \\
668 \\
521\end{array}$ & $\begin{array}{r}8 \\
* 5 \\
1\end{array}$ & & \\
\hline $\begin{array}{l}26 \\
27 \\
28 \\
29 \\
30 \\
31\end{array}$ & $\begin{array}{l}1 \\
2\end{array}$ & $\begin{array}{l}1 \\
2 \\
-\end{array}$ & $\begin{array}{l}38 \\
32 \\
23 \\
20 \\
18 \\
16\end{array}$ & $\begin{array}{r}1,170 \\
915 \\
776 \\
688 \\
616 \\
556\end{array}$ & $\begin{array}{c}884 \\
868 \\
915 \\
= \\
- \\
-\end{array}$ & $\begin{array}{r}3,630 \\
3,590 \\
15,430 \\
20,790 \\
12,590 \\
* 9,100\end{array}$ & $\begin{array}{l}3,730 \\
3,460 \\
3,280 \\
3,190 \\
3,100 \\
=\end{array}$ & $\begin{array}{l}1,430 \\
1,430 \\
1,380 \\
1,320 \\
1,320 \\
1,290\end{array}$ & $\begin{array}{l}521 \\
416 \\
360 \\
388 \\
332 \\
-\end{array}$ & $\begin{array}{l}1 \\
5\end{array}$ & 4 & $\begin{array}{l}1 \\
3 \\
-\end{array}$ \\
\hline $\begin{array}{l}\text { Total } \\
\text { Moan } \\
\text { Ac-ft }\end{array}$ & $\begin{array}{r}62 \\
2 \\
123\end{array}$ & $\begin{array}{r}60 \\
2 \\
119\end{array}$ & $\begin{array}{r}3,878 \\
125 \\
7,692\end{array}$ & $\begin{array}{r}8,363 \\
270 \\
16,590\end{array}$ & $\begin{array}{r}18,873 \\
674 \\
37,430\end{array}$ & $\begin{array}{r}180,950 \\
5,837 \\
358,900\end{array}$ & $\begin{array}{r}49,610 \\
4,987 \\
296,700\end{array}$ & $\begin{array}{r}61,150 \\
1,973 \\
121,300\end{array}$ & $\begin{array}{r}28,221 \\
941 \\
55,980\end{array}$ & $\begin{array}{r}1,366 \\
44 \\
2,709\end{array}$ & $\begin{array}{r}124 \\
4 \\
246\end{array}$ & $\begin{array}{r}90 \\
3 \\
179\end{array}$ \\
\hline
\end{tabular}

Calendar year 1958: Max 5,850 Min 1.5 Mean 541 Ac- It 391,500 Water year 1958-59: $\operatorname{Max} 20,790$ Min 2 Mean 1,240 Ac-ft 898,000

* Discharge measurement made on this day.

Note.-Discharge for Oct. 1 to Dec. 12, July 22 to Sept. 30 estimated on basis of field estimates and record of stages. 
FARAH RTVER BASTN

Farah River noar Farah, Afghanietan

DHacharge, in cubic feet per second, water year October 1959 to September 1960

\begin{tabular}{|c|c|c|c|c|c|c|c|c|c|c|c|c|}
\hline Daj & oct. & Nov. & Dec. & Jan. & Feb. & Mar. & Apr. & May & June & July & Aug. & Sept. \\
\hline $\begin{array}{l}1 \\
2 \\
3 \\
4 \\
5\end{array}$ & 3 & 4 & $\begin{array}{r}878 \\
2,270 \\
* 3,820 \\
* 1,430 \\
920\end{array}$ & $\begin{array}{l}\frac{899}{780} \\
700 \\
620 \\
520\end{array}$ & $\begin{array}{r}\frac{6}{6} \\
2,400 \\
4,100 \\
2,000\end{array}$ & $\begin{array}{r}542 \\
472 \\
416 \\
360 \\
1,030\end{array}$ & $\begin{array}{l}4,000 \\
3,640 \\
3,370 \\
3,100 \\
3,010\end{array}$ & $\begin{array}{l}7,400 \\
7,400 \\
8,260 \\
\frac{8,500}{8,010}\end{array}$ & $\begin{array}{l}\frac{2,920}{2,830} \\
2,650 \\
2,560 \\
2,560\end{array}$ & $\begin{array}{l}\frac{276}{262} \\
248 \\
220 \\
207\end{array}$ & 2 & $\underline{2}$ \\
\hline $\begin{array}{r}6 \\
7 \\
8 \\
9 \\
10\end{array}$ & & & $\begin{array}{l}752 \\
584 \\
416 \\
346 \\
304\end{array}$ & $\begin{array}{l}450 \\
380 \\
330 \\
280 \\
240\end{array}$ & $\begin{array}{l}1,780 \\
1,650 \\
1,500 \\
1,450 \\
1,400\end{array}$ & $\begin{array}{r}1,490 \\
1,030 \\
878 \\
752 \\
710\end{array}$ & $\begin{array}{l}2,650 \\
2,470 \\
2,380 \\
2,380 \\
2,380\end{array}$ & $\begin{array}{l}7,280 \\
6,810 \\
6,340 \\
5,880 \\
5,660\end{array}$ & $\begin{array}{l}2,420 \\
2,380 \\
2,290 \\
2,200 \\
2,060\end{array}$ & $\begin{array}{l}194 \\
181 \\
168 \\
155 \\
142\end{array}$ & & \\
\hline $\begin{array}{l}11 \\
12 \\
13 \\
14 \\
15\end{array}$ & & 4 & $\begin{array}{l}262 \\
318 \\
304 \\
290 \\
388\end{array}$ & $\begin{array}{l}210 \\
190 \\
170 \\
150 \\
140\end{array}$ & $\begin{array}{l}1,360 \\
1,320 \\
1,280 \\
1,240 \\
1,200\end{array}$ & $\begin{array}{r}626 \\
626 \\
4,900 \\
6,220 \\
* 4,900\end{array}$ & $\begin{array}{r}\frac{2,130}{2,130} \\
14,100 \\
13,800 \\
15,200\end{array}$ & $\begin{array}{l}5,440 \\
5,330 \\
5,220 \\
5,000 \\
5,000\end{array}$ & $\begin{array}{l}1,990 \\
1,920 \\
1,700 \\
1,490 \\
1,320\end{array}$ & $\begin{array}{r}142 \\
129 \\
116 \\
103 \\
90\end{array}$ & & $\frac{1}{2}$ \\
\hline $\begin{array}{l}16 \\
17 \\
18 \\
19 \\
20\end{array}$ & & 5 & $\begin{array}{l}486 \\
626 \\
773 \\
836 \\
977\end{array}$ & $\begin{array}{r}125 \\
110 \\
105 \\
95 \\
90\end{array}$ & $\begin{array}{l}1,160 \\
1,120 \\
1,080 \\
1,040 \\
1,000\end{array}$ & $\begin{array}{l}3,820 \\
3,190 \\
2,470 \\
1,990 \\
1,770\end{array}$ & $\begin{array}{r}12,100 \\
* 28,000 \\
* 26,000 \\
21,200 \\
16,900\end{array}$ & $\begin{array}{l}4,900 \\
4,800 \\
4,800 \\
4,800 \\
4,600\end{array}$ & $\begin{array}{r}1,090 \\
977 \\
878 \\
836 \\
710\end{array}$ & $\begin{array}{l}76 \\
63 \\
49 \\
36 \\
22\end{array}$ & & $\underline{1}$ \\
\hline $\begin{array}{l}21 \\
22 \\
23 \\
24 \\
25\end{array}$ & & $\left.\right|_{* 5}$ & $\begin{array}{l}1,120 \\
1,060 \\
1,060 \\
1,120 \\
1,180\end{array}$ & $\begin{array}{r}83 \\
76 \\
* 76 \\
63 \\
49\end{array}$ & $\begin{array}{r}960 \\
930 \\
900 \\
870 \\
* 836\end{array}$ & $\begin{array}{r}1,490 \\
1,320 \\
1,150 \\
1,030 \\
920\end{array}$ & $\begin{array}{r}13,800 \\
12,700 \\
12,900 \\
11,000 \\
9,700\end{array}$ & $\begin{array}{l}4,500 \\
4,300 \\
4,100 \\
3,960 \\
3,820\end{array}$ & $\begin{array}{l}710 \\
584 \\
584 \\
472 \\
416\end{array}$ & $\begin{array}{r}18 \\
14 \\
10 \\
6 \\
* 2\end{array}$ & & \\
\hline $\begin{array}{l}26 \\
27 \\
28 \\
29 \\
30 \\
31 \\
\end{array}$ & $\begin{array}{l}1 \\
3 \\
\end{array}$ & $\begin{array}{r}5 \\
4,230 \\
3,220 \\
1,460 \\
1,030 \\
3\end{array}$ & $\begin{array}{r}1,180 \\
1,090 \\
1,060 \\
977 \\
977 \\
920\end{array}$ & $\begin{array}{r}10 \\
6 \\
6 \\
6 \\
6 \\
6\end{array}$ & $\begin{array}{r}710 \\
* 668 \\
626 \\
584\end{array}$ & $\begin{array}{r}4,880 \\
19,500 \\
9,700 \\
6,810 \\
5,440 \\
4,700\end{array}$ & $\begin{array}{l}8,800 \\
8,260 \\
7,770 \\
7,520 \\
7,400\end{array}$ & $\begin{array}{l}3,640 \\
3,500 \\
3,370 \\
3,280 \\
3,100 \\
3,010 \\
\end{array}$ & $\begin{array}{r}332 \\
304 \\
* 332 \\
318 \\
304\end{array}$ & $\begin{array}{l}2 \\
2 \\
2 \\
2 \\
2 \\
2\end{array}$ & $\left.\right|_{2} ^{1}$ & 1 \\
\hline $\begin{array}{l}\text { Total } \\
\text { Moan } \\
\text { Ac-ft }\end{array}$ & $\begin{array}{r}93 \\
3 \\
184 \\
\end{array}$ & $\begin{array}{r}10,755 \\
358 \\
21,330 \\
\end{array}$ & $\begin{array}{r}28,724 \\
927 \\
56,970 \\
\end{array}$ & $\begin{array}{r}6,971 \\
225 \\
13,830 \\
\end{array}$ & $\begin{array}{r}35,176 \\
1,213 \\
69,770 \\
\end{array}$ & $\begin{array}{r}95,132 \\
3,069 \\
188,700 \\
\end{array}$ & $\begin{array}{r}280,790 \\
9,360 \\
556,900 \\
\end{array}$ & $\begin{array}{r}162,010 \\
5,226 \\
321,300\end{array}$ & $\begin{array}{r}42,137 \\
1,405 \\
83,580\end{array}$ & $\begin{array}{r}2,947 \\
94.9 \\
5,833 \\
\end{array}$ & $\begin{array}{r}62 \\
2 \\
123 \\
\end{array}$ & $\begin{array}{r}45 \\
1.5 \\
89 \\
\end{array}$ \\
\hline $\begin{array}{l}\text { Calend } \\
\text { Water }\end{array}$ & ar 19 & $\begin{array}{l}959: \\
-60:\end{array}$ & $\begin{array}{l}20,7 \\
28,0\end{array}$ & & $\begin{array}{l}3 \\
1\end{array}$ & $\begin{array}{ll}\text { Mean } 1,3 \\
\text { Mean } 1,8\end{array}$ & & ft 1 . & $\begin{array}{l}\infty \\
100\end{array}$ & & & \\
\hline
\end{tabular}

* Discharge measurament made on this day.

Note. Doupt 1 or to Nov. 26, July 25 to Sept. 30 estimated on basis of measured discharge in irrigation ditch in river channel. 


\section{Baba Wall Canal near Kandahar, Afghanistan}

Location.-Iat $31^{\circ}$ se: N., lang $65^{\circ} 39$ E., at south end of South Canal where South Canal dividesits water between the Tarnak and Baba Wall Camals; about 13 kilometers northwest of Kandahar.

Discharge measurements are those made near point of diversion; some on the South Canal bridge are corrected for diversion into Tamak Canal.

\begin{tabular}{|c|c|c|c|c|c|}
\hline Date & $\begin{array}{l}\text { Width } \\
\text { (feet) }\end{array}$ & $\begin{array}{c}\text { Area } \\
(s q-f t)\end{array}$ & $\begin{array}{l}\text { Velocity } \\
\text { (ft/sec) }\end{array}$ & $\begin{array}{l}\text { Gage Hoight } \\
\text { (meters) }\end{array}$ & $\begin{array}{l}\text { Discharge } \\
\text { (cfs) }\end{array}$ \\
\hline $\begin{array}{l}\text { pr. 1, } 1958 \\
\text { Apr. 1 } \\
\text { pr. } 8 \\
\text { Magy } 12 \\
\text { Nay } 13 \\
\text { Jim } 13 \\
\text { Jume } 5\end{array}$ & $\begin{array}{l}18.0 \\
18.0 \\
20.0 \\
22.0 \\
71.0 \\
74.0 \\
24.0\end{array}$ & $\begin{array}{l}12.8 \\
13.6 \\
179 \\
195 \\
365 \\
395 \\
36.8\end{array}$ & $\begin{array}{l}1.98 \\
1.76 \\
2.22 \\
2.06 \\
1.42 \\
1.62 \\
3.49\end{array}$ & $\begin{array}{l}5.045 \\
5.016 \\
5.80 \\
5.955 \\
6.16 \\
6.35 \\
5.39\end{array}$ & $\begin{array}{l}24.1 \\
24.0 \\
397 \\
402 \\
517 \\
640 \\
128\end{array}$ \\
\hline
\end{tabular}

\section{Boghra Canal near Girishk, Afghanlstan}

Iocation.-Iat. $31^{\circ} 54^{\prime} \mathrm{N}$, , long $64^{\circ} 44^{\prime} \mathrm{E}, 3$ kilometers southwest of Oirishk. Diversion is fram Helmand River about 8 kilaneters upstream from Girishk and wasteway No. 1 is at $10+917$ meters beIow the divorsion.

Discharge measurements are those made above wasteway No. 1 and represent the diversion water.

\begin{tabular}{|c|c|c|c|c|c|}
\hline Date & $\begin{array}{l}\text { Width } \\
\text { (feet) }\end{array}$ & $\begin{array}{c}\text { Area } \\
(s q-f t)\end{array}$ & $\begin{array}{l}\text { Velocity } \\
(\mathrm{ft} / \mathrm{sec})\end{array}$ & $\begin{array}{l}\text { Gage Height } \\
\text { (meters) }\end{array}$ & $\begin{array}{c}\text { Discharge } \\
\text { (cfs) }\end{array}$ \\
\hline 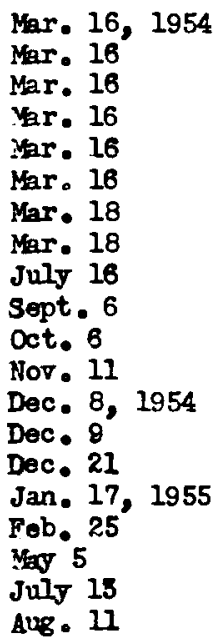 & $\begin{array}{l}68.8 \\
89.9 \\
93.0 \\
92.0 \\
99.0 \\
99.5 \\
98.0 \\
106 \\
98.0 \\
101 \\
98.0 \\
97.0 \\
94.0 \\
92.0 \\
92.0 \\
58.0 \\
91.0 \\
94.0 \\
94.0 \\
91.0\end{array}$ & $\begin{array}{l}74.5 \\
112 \\
158 \\
202 \\
251 \\
313 \\
264 \\
416 \\
238 \\
243 \\
276 \\
253 \\
143 \\
118 \\
218 \\
24 \cdot 2 \\
250 \\
266 \\
259 \\
260\end{array}$ & $\begin{array}{l}1.25 \\
2.02 \\
2.79 \\
3.73 \\
4.10 \\
4.60 \\
4.32 \\
4.99 \\
3.76 \\
3.51 \\
3.49 \\
3.36 \\
3.32 \\
3.02 \\
3.26 \\
0.06 \\
3.75 \\
4.06 \\
3.60 \\
3.56\end{array}$ & $\begin{array}{l}0.13 \\
0.25 \\
0.37 \\
0.56 \\
0.69 \\
0.88 \\
0.78 \\
1.16 \\
0.67 \\
0.77 \\
0.78 \\
0.68 \\
0.44 \\
0.37 \\
0.58 \\
0.0 \\
0.70 \\
0.79 \\
0.78 \\
0.80\end{array}$ & $\begin{array}{c}93.3 \\
228 \\
441 \\
755 \\
1,030 \\
1,420 \\
1,140 \\
2,080 \\
892 \\
833 \\
958 \\
806 \\
472 \\
351 \\
710 \\
15.8 \\
938 \\
1,080 \\
941 \\
928\end{array}$ \\
\hline
\end{tabular}


MISGEYLANEOUS DISCHARGE MEASUREMENTS

Boghra Canal near Girishk, NGhanis tan (Continued)

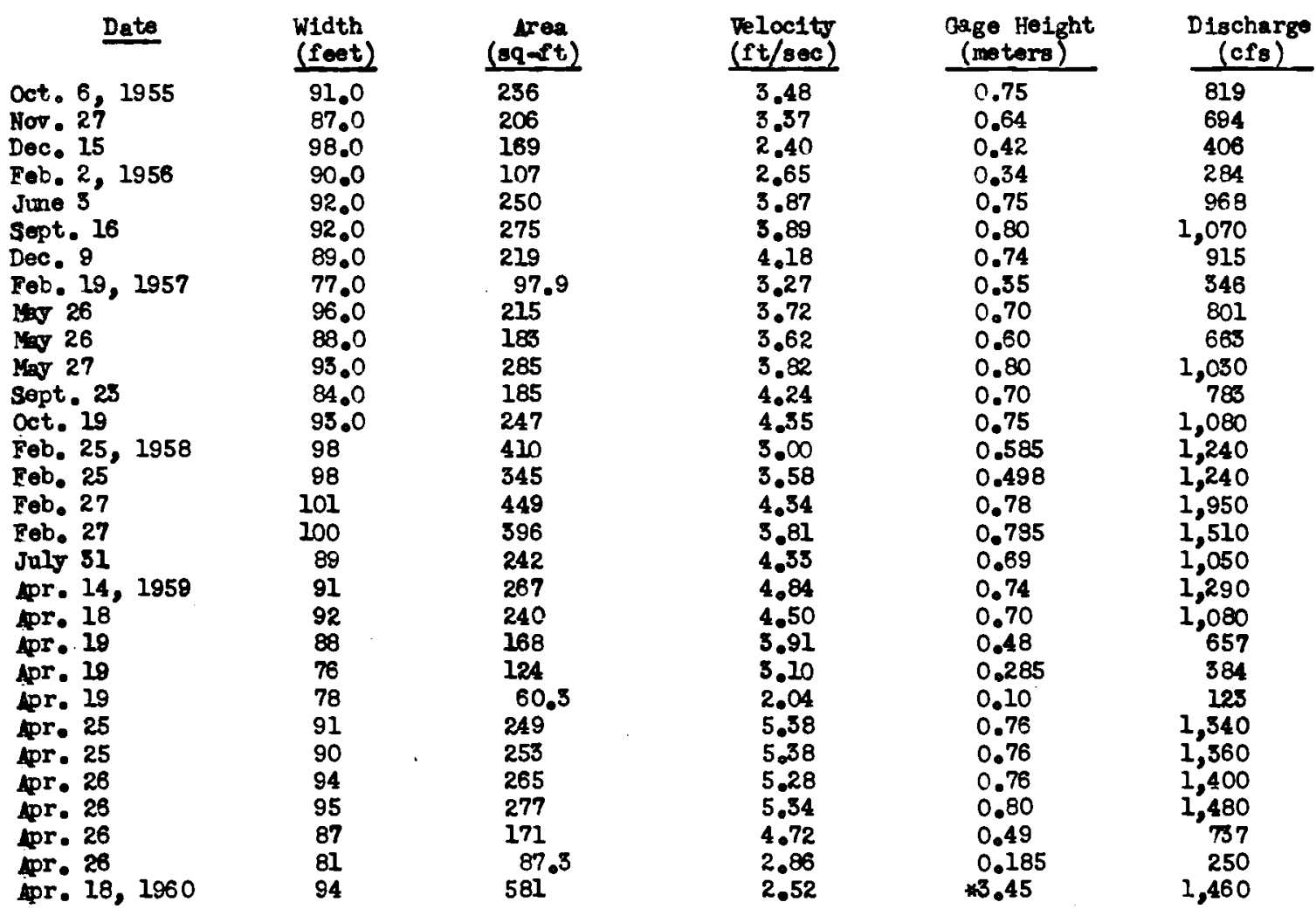

* At Wasteway No. 1.

\section{Boghra Canal below Wasteway No. I near Mrishk, Afghanistan}

Iocation.--Lat $31^{\circ} 481 \mathrm{~N} .$, long $64^{\circ} 31$ : E., about 12 kllometers downstream from diversion dam 3 kflometer: southest of Girishk and below gated control dam at Was teway No. 1.

Discharge measurements were made from a flune over the canal about 300 meters downstream or by wading.

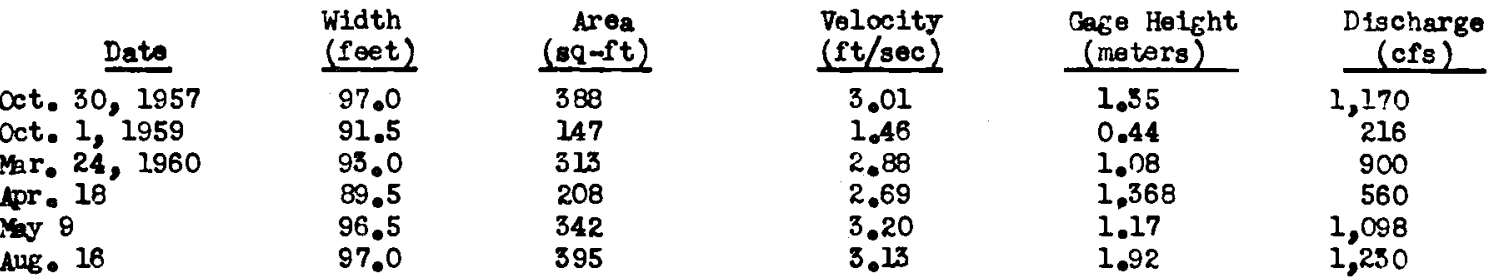


MISCEUTANEOUS DISCHAR CE MEASUREMENTS

\section{Darweshan Canal at Diversicn Iam near Darweshan, Afghanistan}

Location.-Lat $31^{\circ}$ cal N., Iong $64^{\circ} 07^{\prime}$ E., below diversion gates at left side of Helmand River 6.2 Cllometers uptrean from Darweshan bridge.

Discharge measurements made by wading or from bridged gate sectior. 3.5 kiloneters downetream.

\begin{tabular}{|c|c|c|c|c|c|}
\hline Date & $\begin{array}{l}\text { Width } \\
\text { (feet) }\end{array}$ & $\begin{array}{c}\text { Area } \\
\text { (equft) }\end{array}$ & $\begin{array}{l}\text { velocity } \\
\text { (ft/oec) }\end{array}$ & $\begin{array}{c}\text { Gage Height } \\
\text { (meterg) }\end{array}$ & $\begin{array}{l}\text { Discharge } \\
\text { (cfs) }\end{array}$ \\
\hline 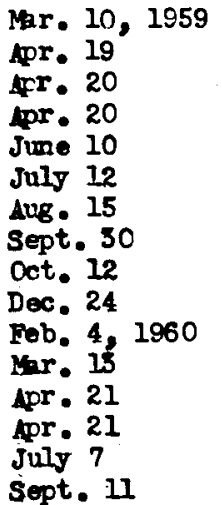 & $\begin{array}{l}34 \\
23.1 \\
23 \cdot 1 \\
23.1 \\
56 \\
23.2 \\
25 \cdot 1 \\
23.1 \\
23.0 \\
23.1 \\
23.1 \\
23.2 \\
23.3 \\
23.5 \\
24.0 \\
24.0\end{array}$ & $\begin{array}{l}93.7 \\
162 \\
150 \\
173 \\
60.6 \\
160 \\
169 \\
161 \\
175 \\
139 \\
159 \\
184 \\
136 \\
136 \\
178 \\
174\end{array}$ & $\begin{array}{l}0.57 \\
2.12 \\
1.60 \\
3.29 \\
1.52 \\
1.65 \\
1.79 \\
1.73 \\
1.71 \\
1.21 \\
0.74 \\
2.01 \\
0.97 \\
0.76 \\
1.39 \\
1.56\end{array}$ & $\begin{array}{l}5.21 \\
6.50 \\
6.30 \\
6.88 \\
5.775 \\
6.42 \\
6.42 \\
6.32 \\
6.35 \\
5.955 \\
6.08 \\
6.495 \\
5.795 \\
5.87 \\
6.29 \\
6.44\end{array}$ & $\begin{array}{l}53.2 \\
344 \\
240 \\
573 \\
74.1 \\
264 \\
302 \\
278 \\
300 \\
168 \\
118 \\
384 \\
152 \\
104 \\
247 \\
271\end{array}$ \\
\hline
\end{tabular}

\section{hazerjuft Cenal near Darweshan, Afghanjstan}

Location.-Iat $31^{\circ}$ o8t N., Iong $64^{\circ} 071 E_{.}$, on left bank of Helmand River about 300 meters upstream fram Darreshan canal and 6,5 kilameters north of Darweshan bridge.

Discharge messuremte are those made by wading near the diversion from the Helmand River.

\begin{tabular}{|c|c|c|c|c|c|}
\hline Dete & $\begin{array}{l}\text { Width } \\
\text { (feet) }\end{array}$ & $\begin{array}{c}\text { Area } \\
(s q-f t)\end{array}$ & $\begin{array}{l}\text { Velocity } \\
\text { (ft/sec) }\end{array}$ & $\begin{array}{c}\text { Gage Height } \\
\text { (meters) }\end{array}$ & $\begin{array}{c}\text { Discharge } \\
\text { (cfs) }\end{array}$ \\
\hline $\begin{array}{l}20,1959 \\
20 \\
21 \\
21 \\
21 \\
10 \\
15 \\
30 \\
-2 \\
4,1960 \\
8 \\
11\end{array}$ & $\begin{array}{c}13.0 \\
11.0 \\
6.00 \\
7.00 \\
9.00 \\
13.0 \\
15.0 \\
20.0 \\
22.0 \\
11.0 \\
18.0 \\
17.0\end{array}$ & $\begin{array}{c}20.3 \\
11.0 \\
4.40 \\
7.10 \\
10.6 \\
13.9 \\
13.5 \\
26.4 \\
35.5 \\
13.4 \\
36.5 \\
28.3\end{array}$ & $\begin{array}{l}1.28 \\
1.15 \\
1.02 \\
1.2 .1 \\
1.04 \\
0.94 \\
1.10 \\
0.47 \\
0.45 \\
0.62 \\
0.16 \\
0.39\end{array}$ & $\begin{array}{l}7.65 \\
7.54 \\
7.135 \\
7.51 \\
7.40 \\
7.495 \\
7.51 \\
7.775 \\
7.89 \\
7.37 \\
6.97 \\
6.92\end{array}$ & $\begin{array}{c}26.0 \\
12.7 \\
4.45 \\
8.66 \\
11.2 \\
13.1 \\
14.9 \\
12.5 \\
15.9 \\
8.26 \\
5.76 \\
11.1\end{array}$ \\
\hline
\end{tabular}


Location.-Iat $31^{\circ} 46$ N., lons $64^{\circ} 22^{\prime}$ E., 8 kilometers north of Chah-i-Anjir and 24 kilameters southwest of Girlohk. Shamalon Canal diverts water from south side of Boghra Canal at station $31+680$ meters from point where Boghra Canal diverts from Helmand River.

Discharge measurements made by wading or by temporary cable across canal.

\begin{tabular}{|c|c|c|c|c|c|}
\hline Date & $\begin{array}{l}\text { W1dth } \\
\text { (feet) }\end{array}$ & $\begin{array}{c}\text { Area } \\
(8 q-f t)\end{array}$ & $\begin{array}{l}\text { Velocity } \\
\text { (ft./sec) }\end{array}$ & $\begin{array}{l}\text { Gage Height } \\
\text { (meters) }\end{array}$ & $\begin{array}{c}\text { Discharge } \\
\text { (cfs) }\end{array}$ \\
\hline 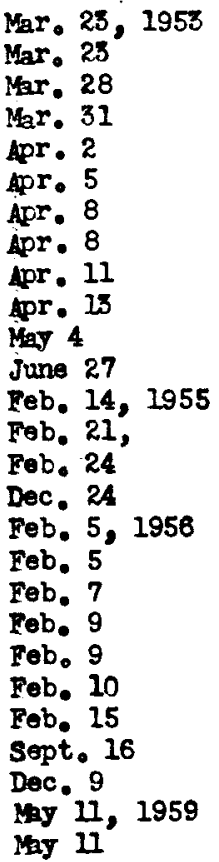 & $\begin{array}{l}30.8 \\
27.6 \\
38.0 \\
40.6 \\
37.5 \\
38.0 \\
48.9 \\
28.0 \\
28.0 \\
42.0 \\
42.5 \\
38.2 \\
29.0 \\
38.0 \\
42.0 \\
38.0 \\
38.0 \\
34.0 \\
32.0 \\
38.0 \\
38.0 \\
39.0 \\
44.0 \\
42.0 \\
44.0 \\
40.0 \\
46.0\end{array}$ & $\begin{array}{l}37.9 \\
15.5 \\
64.3 \\
77.5 \\
80.9 \\
172 \\
103 \\
144 \\
146 \\
155 \\
145 \\
98.1 \\
22.9 \\
90.4 \\
108 \\
61.5 \\
37.5 \\
38.2 \\
32.9 \\
75.7 \\
77.0 \\
72.9 \\
102 \\
175 \\
168 \\
152 \\
154\end{array}$ & $\begin{array}{l}1.71 \\
1.20 \\
3.30 \\
3.69 \\
2.80 \\
1.62 \\
3.46 \\
2.45 \\
3.09 \\
3.43 \\
3.34 \\
3.30 \\
1.10 \\
2.84 \\
3.06 \\
2.19 \\
1.57 \\
1.55 \\
1.30 \\
2.19 \\
2.08 \\
2.44 \\
3.03 \\
3.20 \\
3.44 \\
4.09 \\
4.11\end{array}$ & $\begin{array}{l}0.390 \\
0.174 \\
0.760 \\
0.85 \\
0.82 \\
0.38 \\
0.96 \\
0.96 \\
1.02 \\
1.10 \\
1.08 \\
0.94 \\
0.28 \\
0.85 \\
0.945 \\
0.63 \\
0.49 \\
0.385 \\
0.315 \\
0.64 \\
0.64 \\
0.695 \\
0.58 \\
1.15 \\
1.16 \\
1.15 \\
1.17\end{array}$ & $\begin{array}{l}64.6 \\
18.6 \\
212 \\
286 \\
226 \\
278 \\
356 \\
353 \\
451 \\
531 \\
485 \\
324 \\
25.1 \\
255 \\
331 \\
135 \\
59.0 \\
59.1 \\
42.7 \\
166 \\
160 \\
178 \\
310 \\
560 \\
579 \\
540 \\
633\end{array}$ \\
\hline
\end{tabular}

\section{South Canal near Kandahar, Afghanlatan}

Iocation.-Iat $31^{\circ} 46 \mathrm{r} \mathrm{N}_{\bullet}, \operatorname{long} 65^{\circ} 46^{\prime} \mathrm{E}_{\bullet}$, on left bank of Arghandab River at diversion dara about 18 kilometers north of Kandahar.

Discharge measurements ihade by a temporary cable suspension across the canal.

\begin{tabular}{|c|c|c|c|c|c|}
\hline Data & $\begin{array}{l}\text { Width } \\
\text { (feet) }\end{array}$ & $\begin{array}{c}\text { Area } \\
\text { (sq-ft) }\end{array}$ & $\begin{array}{l}\text { Velocity } \\
\text { (ft/seo) }\end{array}$ & $\begin{array}{c}\text { Gage Height } \\
\text { (meters) }\end{array}$ & $\begin{array}{c}\text { Discharge } \\
\text { (cfs) }\end{array}$ \\
\hline $\begin{array}{l}\text { Apr. } 1,1958 \\
\text { Apr. } 2 \\
\text { May } 12 \\
\text { May } 13\end{array}$ & $\begin{array}{l}30.0 \\
43.0 \\
67.0 \\
67.0\end{array}$ & $\begin{array}{l}53.4 \\
125 \\
308 \\
321\end{array}$ & $\begin{array}{l}0.34 \\
2.44 \\
2.12 \\
2.49\end{array}$ & $\begin{array}{l}0.205 \\
0.935 \\
1.33 \\
1.47\end{array}$ & $\begin{array}{l}18.0 \\
306 \\
652 \\
800\end{array}$ \\
\hline
\end{tabular}


MISCETLANEOUS DISCHARGE MEASUREMEMIS

\section{Tarmak Canal near Kandahar, Afghnistan}

Iocation.-Lat $31^{\circ} 39 \times \mathrm{N}$, , long $65^{\circ} 39 \times \mathrm{E}$, , at South end of South Canal whore the South Canal divide. Its water between Tamak and Baba Wall Canals; about 13 kiloneters northwest of Kandahar.

Discharge measurements are those made near point of diversion or above any turnouts from the Tarnak Canal.

\begin{tabular}{|c|c|c|c|c|c|}
\hline Date & $\begin{array}{l}\text { Width } \\
\text { (feet) }\end{array}$ & $\begin{array}{c}\text { Area } \\
(s q-f t)\end{array}$ & $\begin{array}{l}\text { Velocity } \\
\text { (ft/sec) }\end{array}$ & $\begin{array}{c}\text { Gage Height } \\
\text { (neters) }\end{array}$ & $\begin{array}{l}\text { Discharge } \\
\text { (cfs) }\end{array}$ \\
\hline $\begin{array}{l}\text { Jan. 4, } 1958 \\
\text { Lpr. } 2 \\
\text { Apr. } 8 \\
\text { June } 5\end{array}$ & $\begin{array}{r}26.0 \\
16.0 \\
28.0 \\
7.0\end{array}$ & $\begin{array}{r}38.6 \\
7.6 \\
37.0 \\
2.9\end{array}$ & $\begin{array}{l}0.90 \\
0.81 \\
1.57 \\
0.96\end{array}$ & $\begin{array}{l}2.125 \\
2.06 \\
2.715 \\
1.14\end{array}$ & $\begin{array}{c}34.9 \\
6.19 \\
58.0 \\
2.77\end{array}$ \\
\hline
\end{tabular}




\section{ARGHANDAB RESERVOIR NEAR KANDAHAR, AFGHANISTAN}

Temperature, Precipitation, Evaporation

Location.-Observation station is at north end of radio rocm 5 meters west of gasoline pump about .6 Allaneters west of valve house to reservoir outlet. Lat $31^{\circ} 51^{\mathrm{P}} \mathrm{N}$, , lang $65^{\circ} 54^{\mathrm{*}}$ E., and about 35 kilameters northeast of Kandahar.

Equipment.-Fain gage: A standard 8-inch non-recording U.S. Weather Bureau rain gage can and measuring stiok.

Thermameters: Maximum and minimum thermometers and support in a wooden shelter.

Evaporation Pan: Evaporation pan is of welded steel plate 36 inches square and $19-3 / 4$ inches deep set in the ground. Water surface was read from a raference point at top edge of pan.

\section{iverages and extremes for period of record.-}

January 1952 to Septamber 2960

Temperatures:

Lverage dally mintmum

Meximum

Minimum

Precipitation:

Evaporation:

\section{Average yearly}

Average yearly pan

$$
\begin{aligned}
& 34.2^{\circ} \mathrm{C} \\
& 14.8^{\circ} \mathrm{C} \\
& 47.8^{\circ} \mathrm{C}
\end{aligned}
$$

$-7.8^{\circ} \mathrm{C}$

252.7 millimeters

1799 millinoters 
Wonth $1 y$ and anusl average maximum and minimun and maximurs and minimum Terperatures in degrees centigrade

at Arghandab Reservoir, Afghan is tan

\begin{tabular}{|c|c|c|c|c|c|c|c|c|c|c|c|c|c|c|}
\hline Iea & & oct. & Nov. & Dec. & Jan. & Feb. & Mar. & Apr. & May & $\operatorname{Jun} \theta$ & July & Aug. & Sept. & mual \\
\hline 1952 & $\begin{array}{l}\text { Ave. } \\
\text { Ave. } \\
\text { Max. } \\
\text { Min. }\end{array}$ & & & & & $\begin{array}{r}25.1 \\
4.0 \\
34.5 \\
-1.1\end{array}$ & $\begin{array}{r}26.9 \\
7.6 \\
34.5 \\
-0.6\end{array}$ & $\begin{array}{l}34.1 \\
15.7 \\
41.7 \\
20.6\end{array}$ & $\begin{array}{l}36.5 \\
17.7 \\
42.8 \\
11.7\end{array}$ & $\begin{array}{l}44.4 \\
24.4 \\
47.8 \\
16.1\end{array}$ & $\begin{array}{l}41.5 \\
24.7 \\
44.4 \\
22.2\end{array}$ & $\begin{array}{l}39.4 \\
21.5 \\
42.3 \\
18.3\end{array}$ & $\begin{array}{l}35.4 \\
16.1 \\
40.0 \\
13.4\end{array}$ & $\begin{array}{l}47.8 \\
-1.1\end{array}$ \\
\hline 1953 & $\begin{array}{l}\text { Ave. } \\
\text { Ave. } \\
\text { Max. } \\
\text { Min. }\end{array}$ & $\begin{array}{r}29.3 \\
10.1 \\
33.4 \\
3.3\end{array}$ & $\begin{array}{r}23.2 \\
4.4 \\
28.9 \\
0.6\end{array}$ & $\begin{array}{r}16.3 \\
0.9 \\
22.8 \\
-4.4\end{array}$ & $\begin{array}{r}15.2 \\
1.4 \\
20.0 \\
3.3\end{array}$ & $\begin{array}{r}18.2 \\
6.4 \\
30.0 \\
2.3\end{array}$ & $\begin{array}{r}24.3 \\
9.8 \\
31.7 \\
2.2\end{array}$ & $\begin{array}{r}30.5 \\
14.3 \\
39.5 \\
6.7\end{array}$ & $\begin{array}{l}35.4 \\
19.3 \\
39.5 \\
17.2\end{array}$ & $\begin{array}{l}40.0 \\
22.0 \\
45.0 \\
15.0\end{array}$ & $\begin{array}{l}42.6 \\
25.2 \\
45.6 \\
22.2\end{array}$ & $\begin{array}{l}40.5 \\
21.8 \\
44.4 \\
18.3\end{array}$ & $\begin{array}{l}36.1 \\
17.4 \\
40.0 \\
15.0\end{array}$ & $\begin{array}{l}29.3 \\
12.7 \\
45.6 \\
-4.4\end{array}$ \\
\hline 1954 & $\begin{array}{l}\text { Ave. } \\
\text { dve. } \\
\text { Max. } \\
\text { Min. }\end{array}$ & $\begin{array}{r}28.8 \\
9.4 \\
35.0 \\
1.1\end{array}$ & $\begin{array}{r}22.6 \\
4.5 \\
28.9 \\
-1.1\end{array}$ & $\begin{array}{r}20.3 \\
4.2 \\
26.7 \\
-0.6\end{array}$ & $\begin{array}{r}12.4 \\
1.8 \\
18.9 \\
-6.1\end{array}$ & $\begin{array}{r}14.1 \\
3.6 \\
20.0 \\
-1.1\end{array}$ & $\begin{array}{r}21.7 \\
9.0 \\
31.1 \\
-1.1\end{array}$ & $\begin{array}{r}29.4 \\
15.3 \\
37.8 \\
3.3\end{array}$ & $\begin{array}{l}36.8 \\
19.4 \\
43.3 \\
16.7\end{array}$ & $\begin{array}{c}e 40.7 \\
e 20.9 \\
- \\
-\end{array}$ & $\begin{array}{l}40.0 \\
22.9 \\
42.9 \\
18.9\end{array}$ & $\begin{array}{l}38.0 \\
20.5 \\
42.2 \\
17.2\end{array}$ & $\begin{array}{l}37.3 \\
16.5 \\
41.7 \\
11.1\end{array}$ & $\begin{array}{r}28.5 \\
12.3 \\
43.3 \\
-6.1\end{array}$ \\
\hline 1955 & $\begin{array}{l}\text { Ave. } \\
\text { Ave. } \\
\text { Kax. } \\
\text { Min. }\end{array}$ & $\begin{array}{r}26.5 \\
9.4 \\
28.9 \\
4.4\end{array}$ & $\begin{array}{r}21.4 \\
7.0 \\
28.9 \\
2.8\end{array}$ & $\begin{array}{r}16.8 \\
2.1 \\
20.6 \\
-5.0\end{array}$ & $\begin{array}{c}013.0 \\
01.7 \\
= \\
=\end{array}$ & $\begin{array}{c}e 17.7 \\
e 3.7 \\
= \\
=\end{array}$ & $\begin{array}{c}023.4 \\
08.3 \\
= \\
-\end{array}$ & $\begin{array}{r}28.4 \\
13.4 \\
33.4 \\
3.3\end{array}$ & $\begin{array}{l}32.8 \\
17.2 \\
37.8 \\
11.1\end{array}$ & $\begin{array}{l}39.5 \\
21.7 \\
42.2 \\
16.7\end{array}$ & $\begin{array}{c}041.1 \\
e 24.0 \\
= \\
-\end{array}$ & $\begin{array}{c}e 40.0 \\
e 21.3 \\
- \\
-\end{array}$ & $\begin{array}{c}036.8 \\
016.9 \\
- \\
-\end{array}$ & $\begin{array}{c}28.1 \\
12.2 \\
- \\
-\end{array}$ \\
\hline 1956 & $\begin{array}{l}\text { Ave. } \\
\text { Ave. } \\
\text { Max. } \\
\text { Min. }\end{array}$ & $\begin{array}{c}28.8 \\
\text { ell.4 } \\
= \\
=\end{array}$ & $\begin{array}{r}24.5 \\
6.1 \\
28.9 \\
3.3\end{array}$ & $\begin{array}{c}016.0 \\
03.0 \\
= \\
-\end{array}$ & $\begin{array}{c}013.0 \\
01.7 \\
- \\
-\end{array}$ & $\begin{array}{r}17.2 \\
3.9 \\
25.6 \\
2.2\end{array}$ & $\begin{array}{r}23.9 \\
7.8 \\
26.1 \\
0\end{array}$ & $\begin{array}{r}28.4 \\
13.9 \\
35.6 \\
3.3\end{array}$ & $\begin{array}{l}38.9 \\
19.4 \\
45.6 \\
14.4\end{array}$ & $\begin{array}{l}39.5 \\
21.7 \\
44.4 \\
17.8\end{array}$ & $\begin{array}{l}39.5 \\
25.6 \\
43.3 \\
21.1\end{array}$ & $\begin{array}{l}38.9 \\
22.2 \\
42.8 \\
18.9\end{array}$ & $\begin{array}{c}286.8 \\
016.9 \\
- \\
-\end{array}$ & $\begin{array}{r}28.3 \\
12.3 \\
45.6 \\
2.2\end{array}$ \\
\hline 1957 & $\begin{array}{l}\text { Ave. } \\
\text { Ave. } \\
\text { Max. } \\
\text { Min. }\end{array}$ & $\begin{array}{l}\text { 28.8 } \\
\text { e11.4 } \\
- \\
-\end{array}$ & $\begin{array}{r}22.8 \\
6.1 \\
26.1 \\
3.3\end{array}$ & $\begin{array}{r}16.7 \\
2.8 \\
21.1 \\
2.2\end{array}$ & $\begin{array}{r}6.7 \\
2.2 \\
15.6 \\
-5.0\end{array}$ & $\begin{array}{r}13.4 \\
1.7 \\
21.1 \\
3.3\end{array}$ & $\begin{array}{r}21.1 \\
8.3 \\
29.5 \\
3.3\end{array}$ & $\begin{array}{r}23.9 \\
11.7 \\
28.4 \\
6.1\end{array}$ & $\begin{array}{l}31.7 \\
17.2 \\
40.0 \\
13.4\end{array}$ & $\begin{array}{l}38.9 \\
20.6 \\
41.7 \\
17.8\end{array}$ & $\begin{array}{l}40.6 \\
22.2 \\
43.9 \\
16.7\end{array}$ & $\begin{array}{l}39.5 \\
20.6 \\
43.3 \\
16.7\end{array}$ & $\begin{array}{l}34.5 \\
14.4 \\
36.1 \\
12.2\end{array}$ & $\begin{array}{l}26.5 \\
11.6 \\
43.9 \\
-5.0\end{array}$ \\
\hline 1958 & $\begin{array}{l}\text { Ave. } \\
\text { Ave. } \\
\text { Max. } \\
\text { MUn. }\end{array}$ & $\begin{array}{r}28.9 \\
10.6 \\
35.6 \\
2.2\end{array}$ & $\begin{array}{r}19.4 \\
6.7 \\
27.8 \\
-1.1\end{array}$ & $\begin{array}{r}12.8 \\
4.4 \\
24.5 \\
-1.1\end{array}$ & $\begin{array}{r}14.4 \\
3.9 \\
17.8 \\
-1.1\end{array}$ & $\begin{array}{r}17.2 \\
2.8 \\
22.2 \\
-1.1\end{array}$ & $\begin{array}{r}25.0 \\
10.0 \\
32.2 \\
1.1\end{array}$ & $\begin{array}{r}32.2 \\
16.1 \\
38.3 \\
9.4\end{array}$ & $\begin{array}{l}34.5 \\
17.2 \\
36.7 \\
13.4\end{array}$ & $\begin{array}{l}40.6 \\
22.2 \\
42.8 \\
17.8\end{array}$ & $\begin{array}{l}41.7 \\
25.6 \\
47.2 \\
21.1\end{array}$ & $\begin{array}{l}38.3 \\
21.7 \\
41.7 \\
17.8\end{array}$ & $\begin{array}{l}37.1 \\
17.9 \\
42.2 \\
15.6\end{array}$ & $\begin{array}{l}28.5 \\
13.2 \\
47.2 \\
-1.1\end{array}$ \\
\hline 1959 & $\begin{array}{l}\text { Ave. } \\
\text { Ave. } \\
\text { Max. } \\
\text { Min. }\end{array}$ & $\begin{array}{r}29.1 \\
14.4 \\
32.8 \\
7.8\end{array}$ & $\begin{array}{r}23.9 \\
2.8 \\
27.9 \\
-1.1\end{array}$ & $\begin{array}{r}16.1 \\
5.9 \\
26.7 \\
2.2\end{array}$ & $\begin{array}{r}13.2 \\
1.4 \\
17.2 \\
-3.3\end{array}$ & $\begin{array}{r}13.9 \\
1.6 \\
21.1 \\
-3.3\end{array}$ & $\begin{array}{r}24.0 \\
8.7 \\
32.2 \\
0\end{array}$ & $\begin{array}{r}31.6 \\
13.8 \\
37.2 \\
8.3\end{array}$ & $\begin{array}{l}34.6 \\
17.2 \\
41.2 \\
10.0\end{array}$ & $\begin{array}{l}41.3 \\
20.6 \\
45.6 \\
16.7\end{array}$ & $\begin{array}{l}42.0 \\
24.0 \\
45.6 \\
18.3\end{array}$ & $\begin{array}{l}43.4 \\
23.0 \\
45.0 \\
20.0\end{array}$ & $\begin{array}{l}40.0 \\
18.7 \\
42.9 \\
14.4\end{array}$ & $\begin{array}{l}29.4 \\
12.7 \\
45.6 \\
-3.3\end{array}$ \\
\hline
\end{tabular}

- - Btimated. 
Mathly an anol serage maxinum and minimu and maxinum and minimum temperatures in degrees centigrade

at Arghand ab Reservoir, Afghanistan

\begin{tabular}{|c|c|c|c|c|c|c|c|c|c|c|c|c|c|c|}
\hline I00 & & $\infty$ ct. $_{\text {. }}$ & Y्र. & Dec. & Jan. & Fob. & Par. & spr. & Yy & $\sin \theta$ & Jary & Aug. & Sopt. & Irnual \\
\hline 1960 & 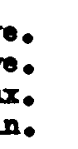 & $\begin{array}{l}30.2 \\
14.7 \\
34.5 \\
11.7\end{array}$ & $\begin{array}{r}24.8 \\
10.1 \\
28.4 \\
3.9\end{array}$ & $\begin{array}{r}13.2 \\
0.9 \\
20.6 \\
-7.8\end{array}$ & $\begin{array}{r}15.9 \\
-0.4 \\
19.4 \\
-3.9\end{array}$ & $\begin{array}{r}22.3 \\
5.5 \\
27.8 \\
-1.1\end{array}$ & $\begin{array}{r}20.0 \\
5.5 \\
30.0 \\
-3.9\end{array}$ & $\begin{array}{r}26.0 \\
10.8 \\
37.2 \\
7.2\end{array}$ & $\begin{array}{l}35.9 \\
16.2 \\
43.5 \\
10.0\end{array}$ & $\begin{array}{r}41.2 \\
13.8 \\
43.9 \\
8.9\end{array}$ & $\begin{array}{l}40.8 \\
21.8 \\
45.0 \\
12.8\end{array}$ & $\begin{array}{l}41.6 \\
19.2 \\
43.3 \\
16.1\end{array}$ & $\begin{array}{l}37.0 \\
17.7 \\
38.9 \\
12.8\end{array}$ & $\begin{array}{l}29.0 \\
11.3 \\
45.0 \\
-7.8\end{array}$ \\
\hline Portad & $\begin{array}{l}\Delta v e . \\
\text { Lre. } \\
\text { Yix. } \\
\text { Yilin. }\end{array}$ & $\begin{array}{r}28.8 \\
11.4 \\
35.6 \\
1.1\end{array}$ & $\begin{array}{r}22.8 \\
6.0 \\
28.9 \\
-1.1\end{array}$ & $\begin{array}{r}16.0 \\
3.0 \\
26.7 \\
-7.8\end{array}$ & $\begin{array}{r}13.0 \\
1.7 \\
20.0 \\
-6.1\end{array}$ & $\begin{array}{r}17.7 \\
3.7 \\
34.5 \\
-3.5\end{array}$ & $\begin{array}{r}23.4 \\
8.3 \\
34.5 \\
-3.9\end{array}$ & $\begin{array}{r}29.4 \\
13.9 \\
41.7 \\
3.3\end{array}$ & $\begin{array}{l}35.2 \\
17.9 \\
45.6 \\
10.0\end{array}$ & $\begin{array}{r}40.7 \\
20.9 \\
47.8 \\
8.8\end{array}$ & $\begin{array}{l}41.1 \\
24.0 \\
47.2 \\
12.2\end{array}$ & $\begin{array}{l}40.0 \\
21.3 \\
45.0 \\
16.1\end{array}$ & $\begin{array}{l}36.8 \\
16.9 \\
42.8 \\
11.1\end{array}$ & $\begin{array}{l}84.2 \\
14.8 \\
47.8 \\
-7.8\end{array}$ \\
\hline
\end{tabular}


PRECIPITATION IN MTLLINETERS AT ARGHANDAB RESERVOIR, AFCHANISTAN

1951
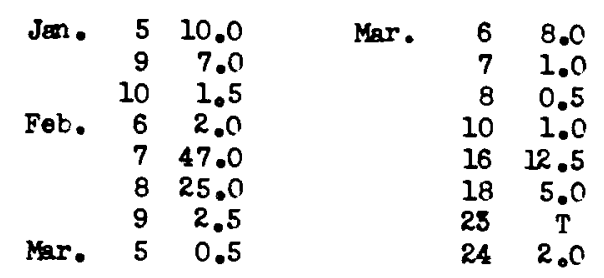

Apr. $\begin{array}{rr}25 & 10.0 \\ 26 & 15.5 \\ 28 & 0.5 \\ 1 & 7.0 \\ 2 & 10.0 \\ 3 & 1.0 \\ 22 & 0.5 \\ 24 & 0.5\end{array}$

$\begin{array}{rr}5 & 0.5 \\ 6 & 4.5 \\ 7 & 10.0 \\ 8 & 3.5 \\ 12 & 1.0\end{array}$

1952

Feb. $\begin{array}{cc}1 & 2.0 \\ 2 & 1.5 \\ 12 & 2.0 \\ 13 & 22.5 \\ 14 & 9.5 \\ 26 & T \\ 28 & T\end{array}$

\begin{tabular}{|c|c|}
\hline Mar. & $\begin{array}{r}3 \\
4 \\
8 \\
12 \\
14 \\
20 \\
25\end{array}$ \\
\hline
\end{tabular}

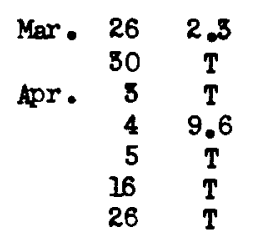

$\begin{array}{lrc}\text { Apr. } & 27 & \text { T } \\ \text { May } & 2 & 2.0 \\ & 7 & T \\ \text { July } & 21 & T\end{array}$

No record for January and June.

1953

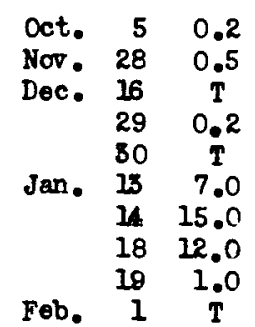
Feb. $\quad \begin{array}{cc}2 & 3.2 \\ 7 & T \\ 8 & 6 \AA\end{array}$
$10 \quad 38.2$
$12 \quad 9.2$
$13 \quad 28.7$
Mar. $\begin{array}{rr}14 & 21.2 \\ & 1.1\end{array}$
$\begin{array}{rr}5 & 21.2 \\ 6 & 1.1 \\ & 0.2\end{array}$

$\begin{array}{crc}\text { Mar. } & 22 & \mathrm{~T} \\ & 28 & 0.9 \\ & 24 & 0.9 \\ \text { Apr. } & 7 & \mathrm{~T} \\ & 11 & 3.1 \\ & 12 & 8.4 \\ & 14 & \mathrm{~T} \\ \text { May } & 10 & \mathrm{~T} \\ \text { rune } & 1 & 0.9 \\ & 7 & T\end{array}$

$\begin{array}{crr}\text { June } & 18 & T \\ & 28 & T \\ \text { July } & 9 & T \\ & 18 & T \\ & 28 & T \\ \text { Aug. } & 7 & T \\ & 19 & T\end{array}$

1854

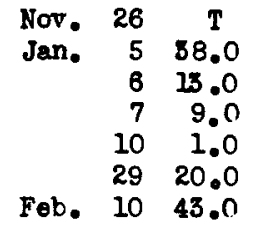
Fob. $11 \quad 10.5$
$\begin{array}{rr}12 & 17.0 \\ 13 & 9.5 \\ 15 & 4.0 \\ 16 & 16.0\end{array}$
1716.0

Fob. $28 \quad 20$

$\begin{array}{rrr}\text { Mar. } 31 & 1.0 \\ \text { Apr. } 16 & 1.0 \\ 22 & 5.0 \\ & 23 & 6.0 \\ \text { May } & 9 & 5.0\end{array}$

No record for Jun*.

$T$ - Trace. 
PRECIPITATI ON II MIILIMETRES AT ARGHANDAB RESERDOIR, AFGHLN ISTAN

1955
$25 \quad 8.0$
m $26 \quad 1.0$

No recard Jenuary, Febriary, March, July, Augue t and September.

1956

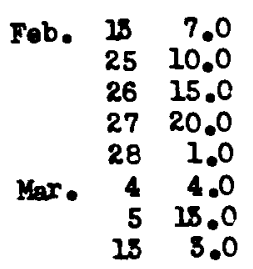

$\begin{array}{rr}14 & 6.0 \\ 15 & 3.0 \\ 18 & 1.0 \\ 19 & 1.0 \\ 20 & 15.0 \\ 24 & 25.0 \\ 29 & 2.0 \\ 30 & 8.0\end{array}$

$\begin{array}{rr}7 & 5.0 \\ 8 & 6.0 \\ 21 & 8.0 \\ 29 & 1.0 \\ 15 & 5.0 \\ 16 & 2.0 \\ 17 & 12.0 \\ 18 & 6.0\end{array}$

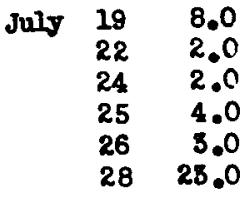

No record October, December, January and september.

1957

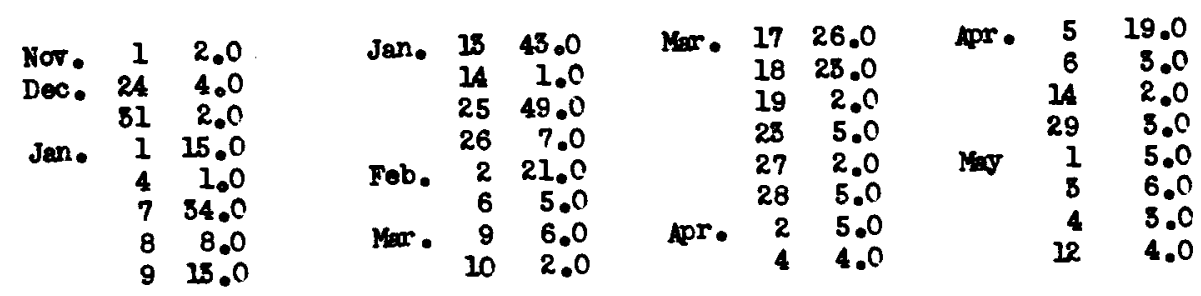

No rocord for october.

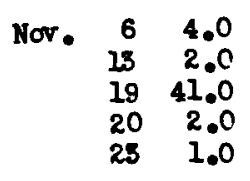

No record for Juno
1958

\begin{tabular}{|c|c|c|c|c|c|c|c|c|}
\hline \multicolumn{9}{|c|}{1958} \\
\hline $\begin{array}{l}\text { Nor. } \\
\text { Dec. }\end{array}$ & $\begin{array}{r}25 \\
3 \\
6 \\
8 \\
10\end{array}$ & $\begin{array}{r}8.0 \\
2.0 \\
26.0 \\
29.0 \\
9.0\end{array}$ & $\begin{array}{l}\text { Dec. } \\
\text { Jan. } \\
\text { Fob. }\end{array}$ & $\begin{array}{l}11 \\
29 \\
30 . \\
21 \\
26\end{array}$ & $\begin{array}{r}20.0 \\
2.0 \\
5.0 \\
8.0 \\
9.0\end{array}$ & $\begin{array}{l}\text { spr. } \\
\text { spr. } \\
\text { July }\end{array}$ & $\begin{array}{r}11 \\
28 \\
2 \\
16 \\
13\end{array}$ & $\begin{array}{r}2.0 \\
11.0 \\
4.0 \\
3.0 \\
2.0\end{array}$ \\
\hline
\end{tabular}

July $13 \quad 2.0$ 
PRECIPITATION IN MILLTMETERS AT ARGHANDAB RESERVOTR, AFGHANISTAN

1959

\begin{tabular}{|c|c|c|c|c|c|c|c|c|c|c|c|}
\hline $\begin{array}{l}\text { Nor. } \\
\text { Dec. } \\
\text { Jan. }\end{array}$ & $\begin{array}{r}10 \\
11 \\
12 \\
20 \\
21 \\
3 \\
4\end{array}$ & $\begin{array}{r}1.0 \\
24.0 \\
43.0 \\
20.0 \\
4.0 \\
20.0 \\
12.0\end{array}$ & Fob. & $\begin{array}{r}15 \\
18 \\
24 \\
5 \\
9 \\
10 \\
18\end{array}$ & $\begin{array}{r}3.0 \\
3.0 \\
14.0 \\
4.0 \\
0.5 \\
16.0 \\
23.0\end{array}$ & $\begin{array}{l}\text { Feb. } \\
\text { Mar. }\end{array}$ & $\begin{array}{r}23 \\
26 \\
1 \\
2 \\
9 \\
28 \\
2\end{array}$ & $\begin{array}{r}7.0 \\
7.0 \\
25.0 \\
6.0 \\
5.5 \\
0.5 \\
20.0\end{array}$ & Apr. & $\begin{array}{r}3 \\
21 \\
22 \\
23 \\
16 \\
17\end{array}$ & $\begin{array}{r}9.0 \\
2.0 \\
6.0 \\
1.5 \\
0.5 \\
15.0\end{array}$ \\
\hline \multicolumn{12}{|c|}{1980} \\
\hline Dec. & $\begin{array}{r}3 \\
4 \\
5 \\
25 \\
27 \\
50 \\
1 \\
2\end{array}$ & $\begin{array}{r}4.0 \\
5.0 \\
15.0 \\
1.5 \\
17.0 \\
2.0 \\
0.5 \\
18.0\end{array}$ & $\begin{array}{l}\text { Dec. } \\
\text { Jen. } \\
\text { Feb. } \\
\text { Mar. }\end{array}$ & $\begin{array}{r}14 \\
15 \\
10 \\
11 \\
1 \\
20 \\
1 \\
5\end{array}$ & $\begin{array}{r}4.0 \\
5.0 \\
11.0 \\
1.0 \\
2.0 \\
1.0 \\
2.0 \\
2.0\end{array}$ & Mar. & $\begin{array}{l}11 \\
12 \\
13 \\
14 \\
16 \\
17 \\
20 \\
22\end{array}$ & $\begin{array}{r}3.0 \\
3.5 \\
8.5 \\
8.0 \\
3.5 \\
1.0 \\
2.0 \\
10.0\end{array}$ & $\begin{array}{l}\text { Mar. } \\
\text { Apr. }\end{array}$ & $\begin{array}{l}27 \\
28 \\
15 \\
16 \\
17 \\
18 \\
19 \\
22\end{array}$ & $\begin{array}{r}3.0 \\
24.0 \\
9.0\end{array}$ \\
\hline
\end{tabular}


Monthly and annual precipitation, in millineters, at Arghand ab Reservoir near Kandahar, Afghanista

\begin{tabular}{|c|c|c|c|c|c|c|c|c|c|c|c|c|c|}
\hline Year & oct. & Hov. & Dec. & $\operatorname{Jan}$. & reb. & Kar. & Apr. & Mny & June & Juy & Aug. & Sept. & Annual \\
\hline Ave. & 0 & 11.8 & 27.2 & 58.9 & 55.3 & 47.5 & 21.4 & 6.9 & 0.1 & 7.7 & 0 & 0 & 252.7 \\
\hline
\end{tabular}

T - Trace

Monthly and ennual evaparati an, in rilllimeters, at Arghandab Reservoir near Kandahar, Afghanisten

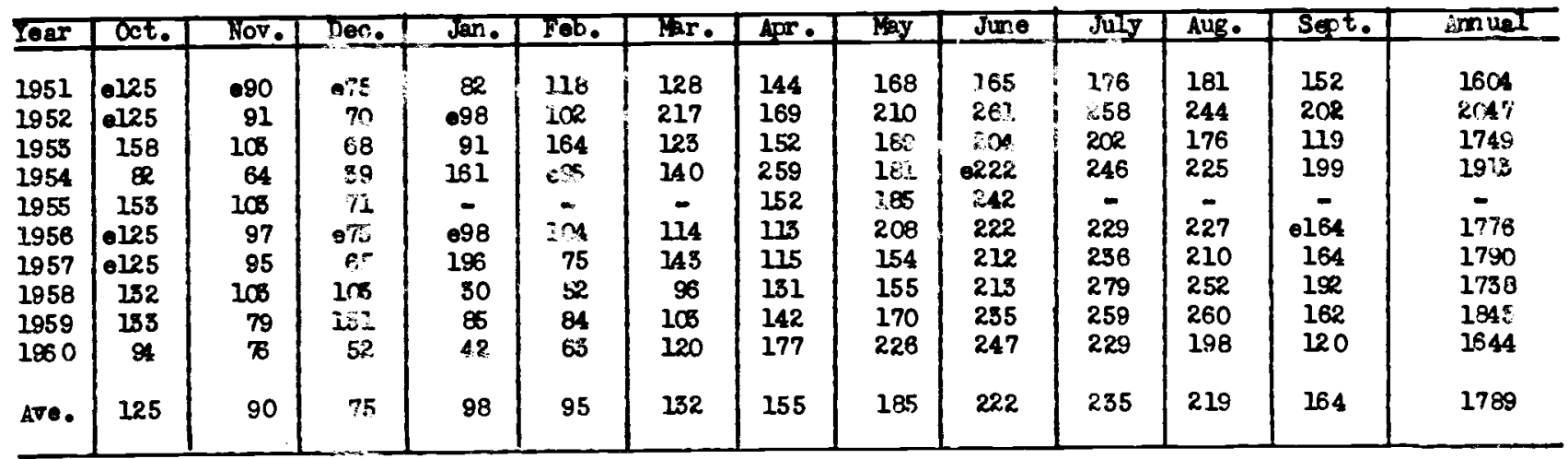

- Estimated. 
BAKWA NEAR FARAH, AFGHANISTAN

Tamperature and Procipitation

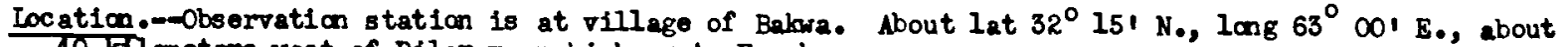
40 Klometer west of Dilaram on highwa to Farah.

Equipment.--Raingages 1 standard non-recording rain gage cen and measuring stick.

Thermanoters: Yaximm and minimu thermometers and support in a standard woodem sheltor.

Averages for period of record.-

February 1944 to September 1946

February 1950 to March 1954

Temperatures: Average dally maximum

Average dally minimum

$$
31.9^{\circ} \mathrm{C}
$$

Preelpitation: Average yearly 7 year

$114.6 \mathrm{millineters}$ 
Manthly and annual average maximum and minimum tamporatures in degrees centigrado

at Bakra near Farah, Afghanistan

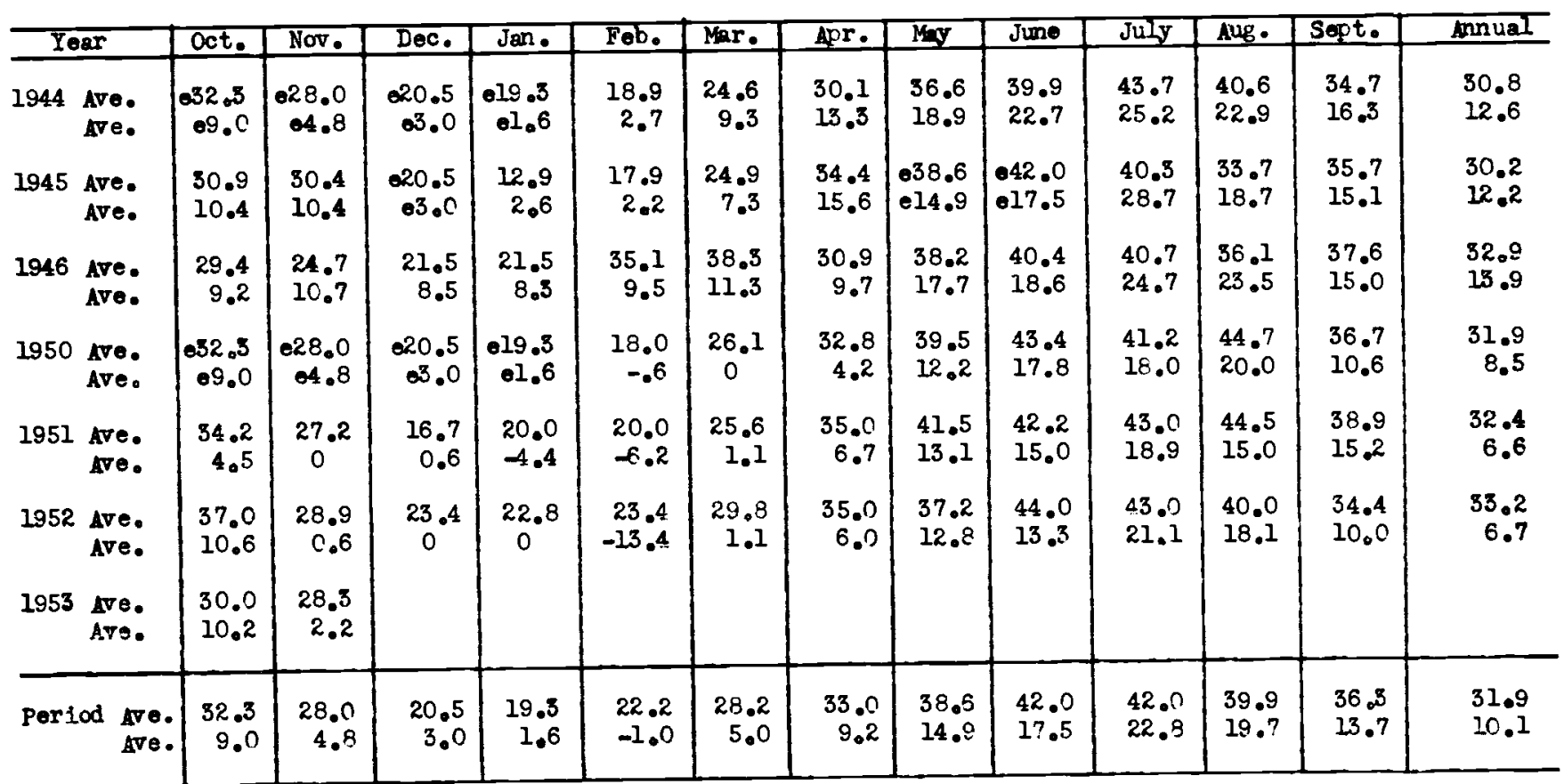

- Estinated. 
Nonthly and annual precipitation, in millimoters, at Bakwa near Farah, Afghanistan

\begin{tabular}{|c|c|c|c|c|c|c|c|c|c|c|c|c|c|}
\hline Yoar & Oct. & Nor . & Dec. & Jan. & Feb. & Kar. & $4 \mathrm{pr}$ & Kay & June & JuY & Aug. & Sept. & Annual \\
\hline $\begin{array}{l}1951 \\
1952 \\
1953 \\
1954\end{array}$ & $\begin{array}{r}0 \\
0 \\
0 \\
0\end{array}$ & $\begin{array}{c}06.2 \\
0 \\
3.4 \\
06.2\end{array}$ & $\begin{array}{r}1.5 \\
11.9 \\
4.2 \\
014.3\end{array}$ & $\begin{array}{r}10.9 \\
29.6 \\
3.0 \\
81.0\end{array}$ & $\begin{array}{r}29.0 \\
5.6 \\
-42.6 \\
151.0\end{array}$ & $\begin{array}{r}17.0 \\
4.8 \\
15.0 \\
7.0\end{array}$ & $\begin{array}{l}13.0 \\
18.0 \\
10.0 \\
88.9\end{array}$ & $\begin{array}{l}6.9 \\
0 \\
1.2 \\
2.2\end{array}$ & $\begin{array}{r}0 \\
0 \\
0 \\
0\end{array}$ & $\begin{array}{r}0 \\
0 \\
0 \\
0\end{array}$ & $\begin{array}{r}0 \\
0 \\
00 \\
0\end{array}$ & $\begin{array}{r}0 \\
0 \\
0 \\
0\end{array}$ & $\begin{array}{r}84.3 \\
69.9 \\
79.4 \\
270.6\end{array}$ \\
\hline sve. & 0 & 6.2 & 14.3 & 29.6 & 42.6 & 10.8 & 8.9 & 2.2 & 0 & 0 & 0 & 0 & 214.6 \\
\hline
\end{tabular}

- Bstimatod 
Location.-Observation station was in MKA operations area in Chah-i-Anjir. Lat $31^{\circ} 37^{1} \mathrm{~N} ., 10 n g 64^{\circ} 161^{2}$., about 37 kllometars sonthwest of Girishk and about 145 kiloneters west of Kandahar.

Equipment.-Rain gage: E. standard 8-tnch non-recording U. S. Weather Bureau rain gage can and me3suring stick。

Thermaneters: Maximum and minimum thermometers mounted in a standard wooden shelter.

Evaporatice pan: Pan was galvanized iron 48-inch diameter, 10-inch deep, with an enamel

gage, calibrated to cartimeters, at one side. The pan set on a brick base on the ground. Pan water was read to millimevers each day and pan was rafilled when level dropped as much as $100 \mathrm{millimeters.}$

Averages and extremes for jeriod of record.--

January 1951 to October 1952

Temperatures: Average daily maximus Average daily minimum

Maximum

Mintum

Precipitation: Average jearly

Evaporation: Average yezily pan

$$
\begin{aligned}
& 29.8^{\circ} \mathrm{C} \\
& 11.0^{\circ} \mathrm{C} \\
& 49.4^{\circ} \mathrm{C} \\
& -8.0^{\circ} \mathrm{C}
\end{aligned}
$$

124.4 millimeters

2994 millimeters 
Monthly and annual average maximum and minimum and maximum and winimum Temperatures in degrees centigrede

at Chah-1-Anjir, Afghanietan

\begin{tabular}{|c|c|c|c|c|c|c|c|c|c|c|c|c|c|c|}
\hline Yea & $a r$ & oct. & Nov. & Dec. & Jan. & Feb. & Mar. & por. & May & Jun $\theta$ & Juy & Aug. & Sept. & Annual \\
\hline 1951 A & $\begin{array}{l}\text { Ave. } \\
\text { Ave. } \\
\text { Minx. } \\
\text { Min. }\end{array}$ & & & & $\begin{array}{r}18.5 \\
1.5 \\
25.0 \\
3.0\end{array}$ & $\begin{array}{r}15.2 \\
2.2 \\
25.6 \\
-7.8\end{array}$ & $\begin{array}{r}11.3 \\
7.1 \\
18.0 \\
0.5\end{array}$ & - & $\begin{array}{l}33.3 \\
24.0 \\
39.5 \\
16.0\end{array}$ & $\begin{array}{l}39.6 \\
24.7 \\
41.5 \\
19.5\end{array}$ & - & $\begin{array}{l}39.0 \\
23.0 \\
41.6 \\
19.7\end{array}$ & $\begin{array}{l}39.1 \\
19.4 \\
40.2 \\
17.6\end{array}$ & $\begin{array}{c}- \\
\overrightarrow{41.6} \\
-7.8\end{array}$ \\
\hline $1952 \mathrm{~A}$ & $\begin{array}{l}\text { Ave. } \\
\text { Lve. } \\
\text { Mox. } \\
\text { Min. }\end{array}$ & $\begin{array}{c}.27 .2 \\
05.7 \\
= \\
-\end{array}$ & $\begin{array}{c}.21 .3 \\
2.6 \\
- \\
-\end{array}$ & $\begin{array}{r}19.3 \\
2.2 \\
24.5 \\
-5.6\end{array}$ & $\begin{array}{r}14.2 \\
0.9 \\
22.2 \\
-7.8\end{array}$ & $\begin{array}{r}19.5 \\
4.5 \\
25.6 \\
2.2\end{array}$ & $\begin{array}{r}24.8 \\
9.1 \\
35.0 \\
-1.7\end{array}$ & $\begin{array}{l}34.9 \\
17.2 \\
40.0 \\
11.0\end{array}$ & $\begin{array}{l}39.5 \\
19.5 \\
45.0 \\
11.0\end{array}$ & $\begin{array}{l}44.0 \\
22.7 \\
47.2 \\
13.0\end{array}$ & $\begin{array}{l}43.7 \\
23.9 \\
47.0 \\
19.0\end{array}$ & $\begin{array}{l}40.2 \\
19.3 \\
42.2 \\
16.0\end{array}$ & $\begin{array}{r}36.1 \\
13.0 \\
41.0 \\
8.0\end{array}$ & $\begin{array}{l}30.4 \\
11.7 \\
47.2 \\
-7.8\end{array}$ \\
\hline 1955 A & $\begin{array}{l}\text { Ave. } \\
\text { ive. } \\
\text { sax. } \\
\text { Min. }\end{array}$ & $\begin{array}{r}28.4 \\
4.9 \\
55.7 \\
-1.6\end{array}$ & $\begin{array}{r}21.3 \\
1.3 \\
29.3 \\
-5.0\end{array}$ & $\begin{array}{l}15.1 \\
-1.7 \\
21.0 \\
-8.0\end{array}$ & $\begin{array}{l}15.7 \\
-1.6 \\
23.5 \\
-7.7\end{array}$ & $\begin{array}{r}20.2 \\
6.9 \\
31.0 \\
0.5\end{array}$ & $\begin{array}{r}26.1 \\
9.1 \\
33.5 \\
0.4\end{array}$ & $\begin{array}{r}34.4 \\
14.6 \\
43.1 \\
8.6\end{array}$ & $\begin{array}{l}38.8 \\
19.6 \\
44.0 \\
15.7\end{array}$ & $\begin{array}{l}43.8 \\
22.6 \\
49.4 \\
15.7\end{array}$ & $\begin{array}{l}44.5 \\
24.2 \\
49.1 \\
16.1\end{array}$ & $\begin{array}{l}38.0 \\
17.1 \\
41.8 \\
11.6\end{array}$ & $\begin{array}{r}32.8 \\
10.4 \\
37.9 \\
4.2\end{array}$ & $\begin{array}{l}29.9 \\
10.6 \\
49.4 \\
-8.0\end{array}$ \\
\hline $1954 \mathrm{~A}$ & $\begin{array}{l}\text { fre. } \\
\text { Ave. } \\
\text { Lex. } \\
\text { Min. }\end{array}$ & $\begin{array}{r}25.5 \\
3.6 \\
30.3 \\
-5.8\end{array}$ & $\begin{array}{r}21.1 \\
1.6 \\
26.8 \\
-5.7\end{array}$ & $\begin{array}{r}18.2 \\
5.2 \\
26.8 \\
-5.3\end{array}$ & $\begin{array}{r}13.5 \\
1.3 \\
18.7 \\
-6.1\end{array}$ & $\begin{array}{r}14.0 \\
4.1 \\
23.0 \\
-5.0\end{array}$ & $\begin{array}{r}23.4 \\
8.1 \\
34.0 \\
2.0\end{array}$ & $\begin{array}{r}31.6 \\
13.6 \\
38.9 \\
6.1\end{array}$ & $\begin{array}{l}39.5 \\
18.9 \\
46.1 \\
12.8\end{array}$ & $\begin{array}{l}41.7 \\
21.7 \\
46.1 \\
17.2\end{array}$ & $\begin{array}{l}42.6 \\
20.4 \\
45.5 \\
18.4\end{array}$ & $\begin{array}{l}40.7 \\
19.0 \\
43.5 \\
15.0\end{array}$ & $\begin{array}{r}36.1 \\
13.4 \\
42.2 \\
6.1\end{array}$ & $\begin{array}{l}29.0 \\
10.7 \\
46.1 \\
-6.1\end{array}$ \\
\hline 1955 & $\begin{array}{l}\text { Ave. } \\
\text { Ave. } \\
\text { Min. } \\
\text { sin. }\end{array}$ & $\begin{array}{r}27.8 \\
8.0 \\
34.5 \\
2.8\end{array}$ & $\begin{array}{c}21.6 \\
4.9 \\
30.0 \\
0\end{array}$ & $\begin{array}{r}16.9 \\
-0.6 \\
20.0 \\
-7.2\end{array}$ & & & & $\begin{array}{l}\text { Start of } \\
\text { Recorder }\end{array}$ & $\begin{array}{l}33.7 \\
18.1 \\
38.0 \\
11.1\end{array}$ & $\begin{array}{l}39.1 \\
21.0 \\
42.0 \\
16.1\end{array}$ & $\begin{array}{l}43.0 \\
24.2 \\
46.2 \\
17.2\end{array}$ & $\begin{array}{l}41.4 \\
21.4 \\
45.7 \\
15.5\end{array}$ & $\begin{array}{r}36.7 \\
14.3 \\
40.3 \\
8.5\end{array}$ & $\begin{array}{l}- \\
- \\
46.2 \\
-7.2\end{array}$ \\
\hline $1956 \mathrm{~A}$ & $\begin{array}{l}\text { Ave. } \\
\text { Ave. } \\
\text { Max. } \\
\text { Min. }\end{array}$ & $\begin{array}{r}27.5 \\
6.4 \\
31.5 \\
3.5\end{array}$ & $\begin{array}{l}\text { Station } \\
\text { Closed }\end{array}$ & & & & & & & & & & & \\
\hline Period & $\begin{array}{l}\text { Ave. } \\
\text { Ave. } \\
\text { Max } \\
\text { Min. }\end{array}$ & $\begin{array}{r}27.2 \\
5.7 \\
34.5 \\
3.8\end{array}$ & $\begin{array}{r}21.3 \\
2.6 \\
30.0 \\
-5.7\end{array}$ & $\begin{array}{r}17.4 \\
0.8 \\
26.8 \\
-8.0\end{array}$ & $\begin{array}{r}15.5 \\
0.5 \\
25.0 \\
-7.8\end{array}$ & $\begin{array}{r}17.2 \\
4.4 \\
31.0 \\
-7.8\end{array}$ & $\begin{array}{r}21.4 \\
8.4 \\
35.0 \\
2.0\end{array}$ & $\begin{array}{r}33.6 \\
15.1 \\
43.1 \\
6.1\end{array}$ & $\begin{array}{l}37.0 \\
20.0 \\
46.1 \\
11.0\end{array}$ & $\begin{array}{l}41.5 \\
22.5 \\
49.4 \\
13.0\end{array}$ & $\begin{array}{l}43.4 \\
23.2 \\
49.1 \\
16.1\end{array}$ & $\begin{array}{l}39.9 \\
20.0 \\
45.7 \\
11.6\end{array}$ & $\begin{array}{r}36.2 \\
14.1 \\
42.2 \\
4.2\end{array}$ & $\begin{array}{l}29.8 \\
11.0 \\
49.4 \\
-8.0\end{array}$ \\
\hline
\end{tabular}

- Es timated. 
PRECIPITATION IN MULLIMETERS AT TASHKAR GAH, AFGHANISTAN

1951

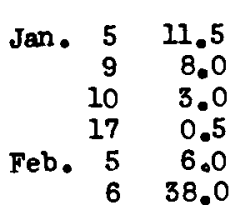

$\begin{array}{rr}\text { Feb. } 7 & 12.0 \\ 8 & 3.0 \\ 13 & 1.5 \\ 17 & 1.5 \\ \operatorname{Mar} .6 & 3.5 \\ 7 & 1.0\end{array}$

Mar. $\begin{array}{rr}8 & 0.5 \\ 9 & 5.0 \\ 14 & 2.0 \\ 15 & 9.0 \\ 17 & 4.0 \\ 22 & 4.5\end{array}$

Mar. 2350.0

$\begin{array}{ll}24 & 1.0 \\ 28 & 7.0 \\ 29 & 1.5 \\ 31 & 4.5\end{array}$

1952

$\begin{array}{rc}\text { Dec. } 24 & 6.4 \\ 25 & \mathrm{~T} \\ 6 & 3.5 \\ 7 & 6.0 \\ 11 & 1.0 \\ 19 & 7.8 \\ 27 & \mathrm{~T} \\ 28 & \mathrm{~T}\end{array}$

$\begin{array}{cc}\text { Jan. } 29 & 4.1 \\ 30 & \mathrm{~T} \\ 2 & 3.7 \\ 12 & 1.0 \\ 14 & 2.7 \\ 15 & 1.5 \\ 25 & \mathrm{~T} \\ 28 & \mathrm{~T}\end{array}$

$\begin{array}{rc}\text { Mar. } 3 & \mathrm{~T} \\ 4 & \mathrm{~T} \\ 9 & 9.9 \\ 20 & 0.1 \\ 24 & 1.2 \\ 25 & 6.7 \\ 26 & 5.5 \\ 29 & 0.2\end{array}$

$\begin{array}{rc}\text { Apr . } 1 & 1.0 \\ 3 & 8.0 \\ 17 & T \\ 18 & T \\ \text { May } 1 & 1.0 \\ 2 & 1.0 \\ 6 & 2.0 \\ 13 & 1.0\end{array}$

1953

$\begin{array}{rc}\text { oct. } & \text { T } \\ 13 & \mathrm{~T} \\ 15 & 1.0 \\ 16 & \mathrm{~T} \\ 30 & \mathrm{~T} \\ 15 & 4.0 \\ 28 & 2.0\end{array}$

$\begin{array}{cc}\text { Dec. } 29 & \mathrm{~T} \\ \text { Jan. } 12 & 5.0 \\ 13 & 3.0 \\ 14 & \mathrm{~T} \\ 17 & \mathrm{~T} \\ 18 & \mathrm{~T} \\ 23 & \mathrm{~T}\end{array}$

Feb. $3 \quad 3.0$

Nar. 236.0

$\begin{array}{rr}11 & 17.0 \\ 12 & 6.0 \\ 13 & 14.0 \\ \operatorname{Mar} .4 & 2.0\end{array}$

Apr. 9.0

MBy $\begin{array}{rr}11 & 6.0 \\ 9 & 1.0\end{array}$

$\begin{array}{ll}27 & \mathrm{~T} \\ 31 & 1.0\end{array}$

1954

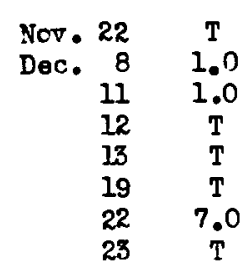

$\begin{array}{rc}\text { Dec. } 29 & 2.0 \\ \text { Jan. } & 2 \\ 3 & 2.0 \\ 4 & 39.0 \\ 5 & 5.0 \\ 6 & 5.0 \\ 7 & T \\ 10 & T\end{array}$

\begin{tabular}{rr} 
Jan. 27 & \multicolumn{1}{c}{$T$} \\
29 & 24.0 \\
Feb. 3 & 2.0 \\
8 & 3.0 \\
9 & 3.0 \\
11 & 10.0 \\
12 & 13.0 \\
16 & 4.0
\end{tabular}

$\begin{array}{cc}\text { Feb. } 27 & T \\ 28 & 2.0 \\ 9 & 4.0 \\ \text { Mar. } & 11.0 \\ 21 & 1.0 \\ 29 & 15.0 \\ 12 & T \\ 22 & 4.0 \\ \text { Apr. } 8 & T\end{array}$

$T$ - Trace 
Monthly and amual precipitation, in millimeters, at Chah-i-Anjir, Afghanjstan

\begin{tabular}{|c|c|c|c|c|c|c|c|c|c|c|c|c|c|}
\hline Year & Oct. & Nor. & Dec. & Jen • & Fob. & Mar. & Apr. & May & June & July & Aug. & Sept. & Annual \\
\hline $\begin{array}{l}1951 \\
1952 \\
1953 \\
1954 \\
1955\end{array}$ & $\begin{array}{r}\circ 0 \\
0 \\
\mathrm{~T} \\
0 \\
0\end{array}$ & $\begin{array}{c}e 0 \\
0 \\
1.0 \\
T \\
0\end{array}$ & $\begin{array}{r}6.0 \\
6.4 \\
6.0 \\
11.0 \\
0\end{array}$ & $\begin{array}{r}23.0 \\
22.4 \\
8.0 \\
75.0\end{array}$ & $\begin{array}{r}62.0 \\
8.9 \\
46.0 \\
37.0\end{array}$ & $\begin{array}{l}93.5 \\
23.6 \\
10.0 \\
20.0\end{array}$ & $\begin{array}{r}88.0 \\
9.0 \\
10.0 \\
4.0\end{array}$ & $\begin{array}{c}0 \\
5.0 \\
2.0 \\
T\end{array}$ & $\begin{array}{l}0 \\
0 \\
0 \\
0\end{array}$ & $\begin{array}{l}0 \\
0 \\
0 \\
0\end{array}$ & $\begin{array}{l}0 \\
0 \\
0 \\
0\end{array}$ & $\begin{array}{l}0 \\
0 \\
0 \\
0\end{array}$ & $\begin{array}{r}192.5 \\
75.3 \\
83.0 \\
147.0\end{array}$ \\
\hline Ave. & 0 & 0.2 & 5.9 & 32.1 & 38.5 & 36.8 & 7.8 & 1.8 & 0 & 0 & 0 & 0 & 124.4 \\
\hline
\end{tabular}

$\mathbf{T}$ - Irace

Nonthly and annual pan exaporation, in millineters, at Chah-1-Anjir, Afghanis tan

\begin{tabular}{|c|c|c|c|c|c|c|c|c|c|c|c|c|c|}
\hline Year & oct. & Nov. & Dec. & $\operatorname{Tan}$. & Feb. & Mar. & Apr. & Mgy & June & July & Aug. & Sept. & Annugl \\
\hline $\begin{array}{l}1951 \\
1952 \\
1953 \\
1954 \\
1955\end{array}$ & $\begin{array}{r}0214 \\
212 \\
245 \\
185\end{array}$ & $\begin{array}{r}e 149 \\
138 \\
204 \\
105\end{array}$ & $\begin{array}{l}47 \\
78 \\
87\end{array}$ & $\begin{array}{l}61 \\
31 \\
79 \\
68\end{array}$ & $\begin{array}{r}67 \\
112 \\
102 \\
73\end{array}$ & $\begin{array}{r}65 \\
125 \\
214 \\
146\end{array}$ & $\begin{array}{l}- \\
268 \\
241 \\
218\end{array}$ & $\begin{array}{l}286 \\
362 \\
351 \\
352\end{array}$ & $\begin{array}{l}340 \\
478 \\
386 \\
692\end{array}$ & $\begin{array}{l}518 \\
378 \\
400\end{array}$ & $\begin{array}{l}-17 \\
372 \\
368\end{array}$ & $\begin{array}{l}30 R \\
286 \\
270\end{array}$ & $\begin{array}{l}3023 \\
2837 \\
3123\end{array}$ \\
\hline are. & 214 & 149 & 71 & 60 & 88 & 138 & 242 & 338 & 474 & 432 & 386 & 286 & 2994 \\
\hline
\end{tabular}

- - Estimetod. 


\section{DARWESHAN, AFGHANISTAN}

Temperature, Precinitation, Evaporation

Iocation.-Observation station is 20 feet west of north end of most westerly building, $05 \mathrm{kilaneter}$

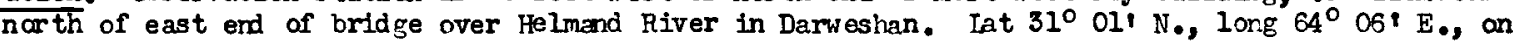
east bank of Helmand River about 53 kllometers domstream from Arghandab River and a bout 175 kllaneters southwest of Kandahar.

Equipment.-Rain gage: A standard 8-inch non-recording U.S. Weather Bureau rain gage can and measuring stick. Centigrade scales.

Evaporation pan: Pan is galvanized iron, round, $125 \mathrm{cms}$ in diameter, $26 \mathrm{cms}$ deep. Pan sets on a gravel base. Depth of water over a reference roint in the pan is $r$ ead to millimeters each day and is $r e f i l l e d$ then level drops as much as 100 militimeters.

located.

An 8-foot high woven wire fence enclnses a $3.35 \times 3.35$ meter area where above equipment is

\section{Averages and extremes for period of record:-}

Decenber 1956 to September 1960 Average daily minimum Meximum

Minimum

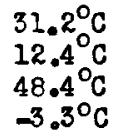

Precipitation: Average yearly

88.6 millimeters

Evaporations Average yearly pan

2816 millimeters 
PRBCIPITATION IN MUITIETEPS AT DARWESHAN, AFGANTSTAN

\section{7}

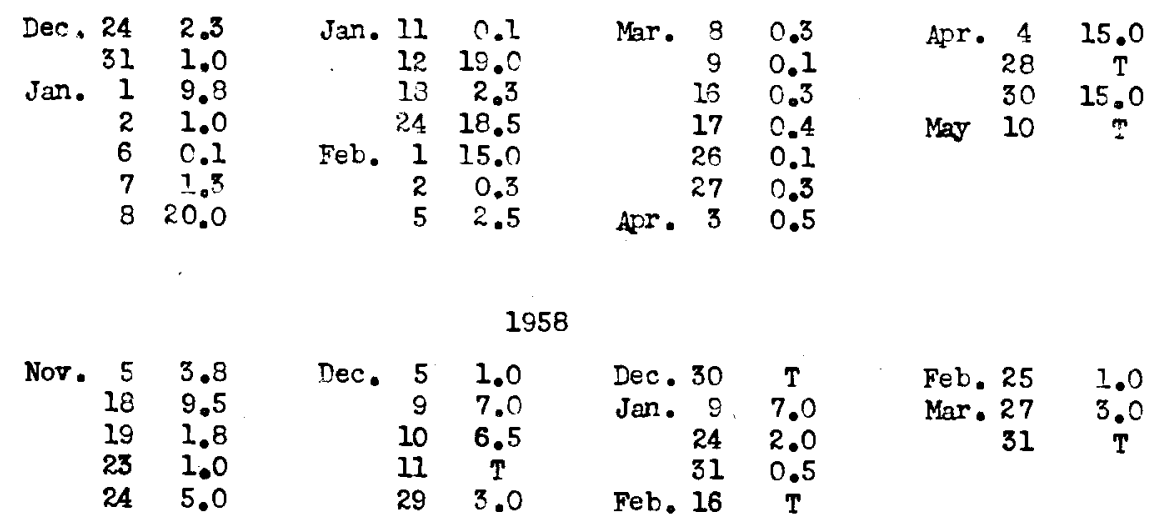


Nonthly average maximum and minimum and maximum and minimum temperatures in degrees cont igrade at

Darweshan, Afghanistan

\begin{tabular}{|c|c|c|c|c|c|c|c|c|c|c|c|c|c|c|}
\hline \multicolumn{2}{|c|}{ Year } & Oct. & Nov. & Dec. & Jan. & Feb. & Mar. & Apr. & May & Jume & July & Aug. & Sept. & Annual \\
\hline 1957 & $\begin{array}{l}\text { Ave. } \\
\text { Ave. } \\
\text { Max. } \\
\text { Min. }\end{array}$ & & & start & $\begin{array}{r}12.8 \\
2.0 \\
24.5 \\
-1.1\end{array}$ & $\begin{array}{r}17.6 \\
2.2 \\
24.5 \\
-3.3\end{array}$ & $\begin{array}{r}25.2 \\
7.8 \\
30.6 \\
1.1\end{array}$ & $\begin{array}{r}28.0 \\
12.0 \\
31.7 \\
6.1\end{array}$ & $\begin{array}{l}34.6 \\
16.4 \\
41.2 \\
12.2\end{array}$ & $\begin{array}{l}39.8 \\
19.8 \\
42.2 \\
17.8\end{array}$ & $\begin{array}{l}44.4 \\
20.6 \\
47.8 \\
16.7\end{array}$ & $\begin{array}{l}42.2 \\
19.2 \\
45.4 \\
14.4\end{array}$ & $\begin{array}{r}34.8 \\
13.0 \\
36.7 \\
0.4\end{array}$ & $\begin{array}{r}48.4 \\
-3.3\end{array}$ \\
\hline 1958 & $\begin{array}{l}\text { Ave. } \\
\text { Are. } \\
\text { Max. } \\
\text { Min. }\end{array}$ & $\begin{array}{r}30.4 \\
8.4 \\
35.7 \\
0.6\end{array}$ & $\begin{array}{r}22.0 \\
5.8 \\
28.4 \\
-1.7\end{array}$ & $\begin{array}{r}17.0 \\
3.4 \\
25.6 \\
3.3\end{array}$ & $\begin{array}{r}18.2 \\
4.0 \\
25.6 \\
2.2\end{array}$ & $\begin{array}{r}20.8 \\
2.4 \\
26.1 \\
-2.8\end{array}$ & $\begin{array}{r}30.3 \\
10.9 \\
34.5 \\
1.1\end{array}$ & $\begin{array}{r}35.6 \\
15.5 \\
43.3 \\
8.9\end{array}$ & $\begin{array}{r}36.7 \\
15.6 \\
40.6 \\
6.7\end{array}$ & $\begin{array}{l}42.2 \\
21.8 \\
45.0 \\
19.4\end{array}$ & $\begin{array}{l}43.2 \\
23.4 \\
46.1 \\
18.9\end{array}$ & $\begin{array}{l}40.7 \\
20.2 \\
44.4 \\
15.6\end{array}$ & $\begin{array}{l}37.4 \\
17.4 \\
41.7 \\
11.1\end{array}$ & $\begin{array}{l}31.2 \\
12.4 \\
45.1 \\
-3.3\end{array}$ \\
\hline 1959 & $\begin{array}{l}\text { Ave. } \\
\text { Ave. } \\
\text { Max. } \\
\text { Min. }\end{array}$ & $\begin{array}{r}30.5 \\
8.6 \\
35.6 \\
0.6\end{array}$ & $\mathrm{Cl} \propto \mathrm{ed}_{\mathrm{d}}$ & & & & & & & & & & & \\
\hline
\end{tabular}

Monthly and annual precipitation in millimsters at Darweshan, Afghanistan

\begin{tabular}{c|c|c|c|c|c|c|c|c|c|c|c|c|c|c|c|}
\hline Year & Oct. & Nov. & Dec. & Jan. & Feb. & Mar. & Apr. & May & June & JuIy & Auz. & Sept. & Annual \\
\hline 1957 & 0 & 0 & 3.3 & 72.1 & 17.8 & 1.5 & 30.5 & $\mathrm{~T}$ & 0 & 0 & 0 & 0 & 125.2 \\
1958 & 0 & 21.1 & 17.5 & 9.5 & 1.0 & 3.0 & 0 & 0 & 0 & 0 & 0 & 0 & 52.1 \\
\hline
\end{tabular}

Monthly evaporation, in millimeters at Darweshan, Afghanistan

\begin{tabular}{|c|c|c|c|c|c|c|c|c|c|c|c|c|c|}
\hline Year & Oct. & Nor. & Dec. & Jan. & Feb. & Mar. & Apr. & May & June & July & Ax & Sert. & Annue 1 \\
\hline $\begin{array}{l}1957 \\
1958 \\
1959\end{array}$ & $\begin{array}{l}212 \\
219\end{array}$ & $\begin{array}{c}101 \\
\text { Closed }\end{array}$ & $\begin{array}{r}\text { Start } \\
52\end{array}$ & $\begin{array}{l}46 \\
55\end{array}$ & $\begin{array}{r}64 \\
\ln 9\end{array}$ & $\begin{array}{r}97 \\
209\end{array}$ & $\begin{array}{l}201 \\
284\end{array}$ & $\begin{array}{l}3 \mathrm{CO} \\
331\end{array}$ & $\begin{array}{l}394 \\
434\end{array}$ & $\begin{array}{l}443 \\
448\end{array}$ & $\begin{array}{l}410 \\
422\end{array}$ & $\begin{array}{l}321 \\
335\end{array}$ & $\begin{array}{r}22641 \\
2591\end{array}$ \\
\hline
\end{tabular}

a Estimated Cctober, November and December thos ame as 1958 
KALA KANG, AFGHANISTAN

Temperatire, Precipitation, Evaporation, Wind Velocity, Humidity

rocation.-Observation station is in military compound in Kala Kang. Lat $31^{\circ} 061^{\mathrm{N} .}$, $1 \mathrm{mg} 61^{\circ} 53{ }^{\prime}$ E., about 9 kllometers north of Shela Charkh, about 18 kilometers southwost of Chakansur, about 27

kflometers dowstream from Khoabgah where Afghanistan-Iran border meets Helmand River and about 110 kilometers downstream from Chahar Burjak.

Equipment.--Rain gage: Standard 8-inch non-recording U.S. Weather Bureau rain gage can and measuring stick.

Thermometers I Maximum and minimum thermometers and support and a Hydrothermograph for recording air temperature and humirity are in a wooden shelter.

Evaporation pan: Pan is galvanized iron $30 " 1 \times 30 "$ square and 12 inches deep set on wooden boards. Pan has $30 \mathrm{~cm}$ enemel iron gage section mounted in orner of $t$ ank. Pan water is read to millimeters each day and has heen refilled when level drops as mush as 100 millimeters.

Note:-This pan replaced a round 48-inch dianeter galvanized 1ran pan one foot deep and set on wonden

boards with an enamel ir on gage mounted on one side on Apr. 24, 1960.

A barbed wire fence encloses the area where the abcve equipment is located.

Anemoreter is located on the rocf of the guard house at the entrance to the military comfound and is read daily in miles.

\section{Averages and extremes for period of record:-}

August 1955 to September 1960

Temperatures: Average daily maximum Average daily ininimum Maximum

Minimum

Precipitation: Arerage yearly

Evaporation: Averare yearly pan

Wind :

Average velocity

Maximum velocity

Maximum velocity Noverber to Apr1l, ivarare

Meximim velocity Hay to Cotober, fverace

Hunidity: Average daily high Average daily low $28.0^{\circ} \mathrm{C}$

$12.2^{\circ} \mathrm{C}$

$47.6^{\circ} \mathrm{C}$

$-9.2^{\circ} \mathrm{C}$

75.6 millimeters

4306 millimeters

305 kilometers cer day

1179 kilometers ner day

207 kilometers per day

407 kilometers per day

$59.2 \%$

$21.3 \%$ 
Monthly and amual average maximum and ninimar and maximur and minimum temreratures in degrees centigrace

at Kala Kang, Afghanistan

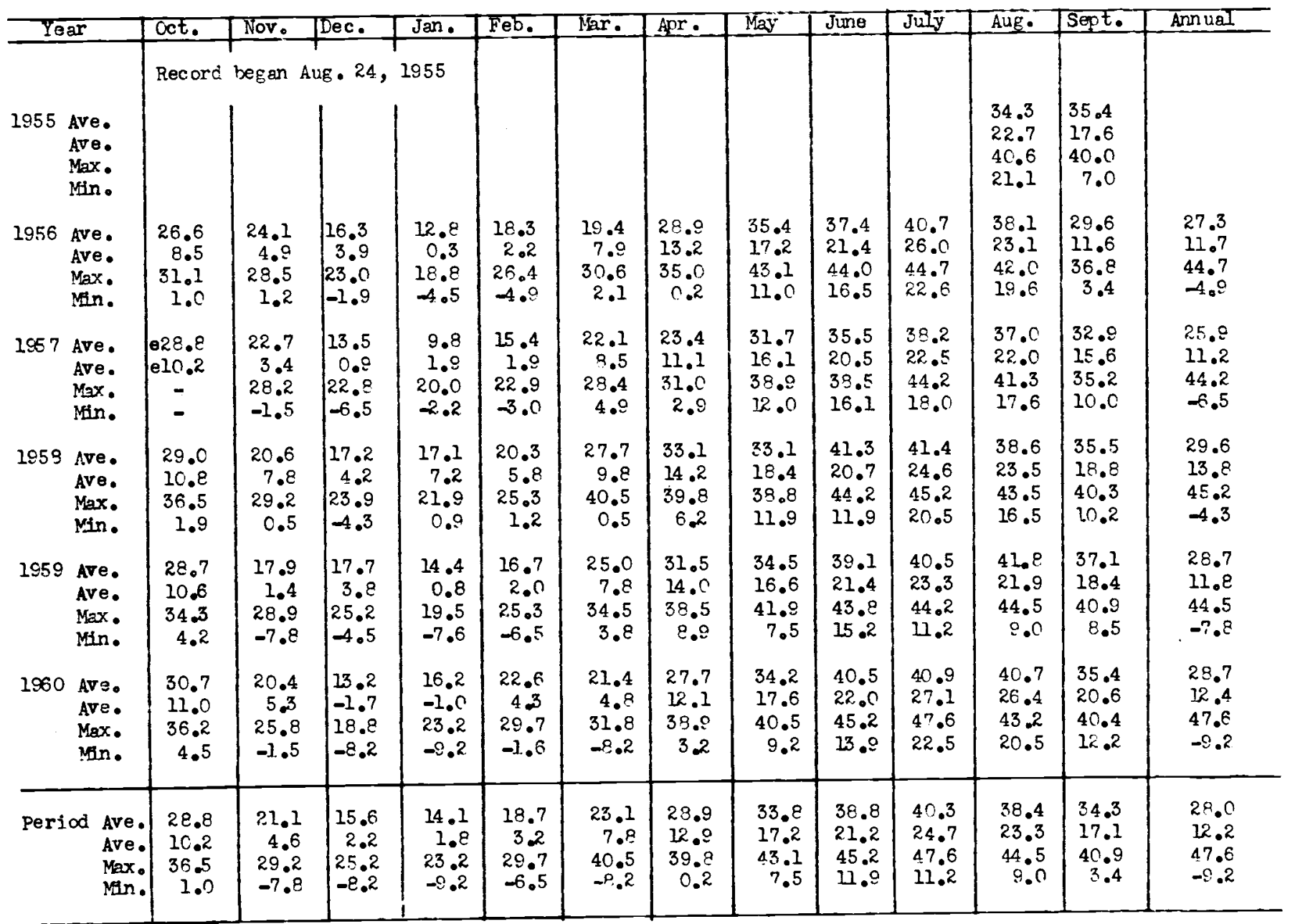

e - estimated 
PRECIPITATION IN MILLTETERS AT KALA KANG, AFGHANIS TAN

1956

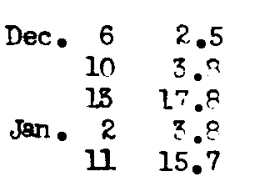

Dec. 223.0

$\begin{array}{ll}30 & 3.8 \\ 31 & 6.6\end{array}$

Jan. 26.6

$\begin{array}{ll}6 & 11.4 \\ 7 & 21.1\end{array}$

Nov. $\begin{array}{rr}6 & 2.5 \\ 11 & 1.8 \\ 24 & 5.3\end{array}$

Dec. $\begin{array}{ll}12 & 1.8 \\ 18 & 3.3 \\ 19 & 8.9\end{array}$

oct. $31 \quad 3.8$

$\begin{array}{ll}\text { Nov. } 26 & 4.1 \\ \text { Dec. } 1 & 3.3\end{array}$

Dec. $\begin{array}{ll}1 & 3.3 \\ 2 & 3.8\end{array}$

$\begin{array}{rr}\text { Jan. } 15 & 5.1 \\ \text { Feb. } 27 & 10.9 \\ \text { Mar. } 4 & 4.7 \\ 5 & 0.8 \\ 14 & 16.5\end{array}$

Mar. 19

$\begin{array}{ll}19 & 7.6 \\ 20 & 3.8 \\ 21 & 1.3 \\ 23 & 8.9 \\ 28 & 1.9\end{array}$

1957

$\begin{array}{rr}\text { Jan. } 10 & 3.8 \\ 11 & 21.6 \\ 21 & 3.8 \\ 23 & 2.5 \\ \text { Feb. } 2 & 5.9\end{array}$

Mar.

$\begin{array}{rl}2 & 1 \\ 2 & 5.6 \\ 17 & 5 \\ 20 & 1.0 \\ 26 & 4.1\end{array}$

1.8
5.6
5.6
1.0
4.1

Mar. 29

Apr. 4

$\begin{array}{ll}5 & 16.5\end{array}$

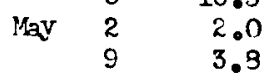

1958

$\begin{array}{rrrrrr}10 & 6.3 & \text { Jan. } 24 & 0.4 & \text { Feb. } 1 & 3.9 \\ 11 & 5.6 & 25 & 5.1 & & \\ \text { Jan. } 10 & 4.6 & 26 & 12.7 & & \end{array}$

1959

$\begin{array}{rrrrrr}15 & 4.3 & \text { Jan. } 25 & 3.0 & \text { Mar. } 27 & 2.0 \\ 18 & 14.4 & \text { Mar. } 8 & 1.5 & & \\ 24 & 1.3 & 26 & 1.8 & & \end{array}$

1960

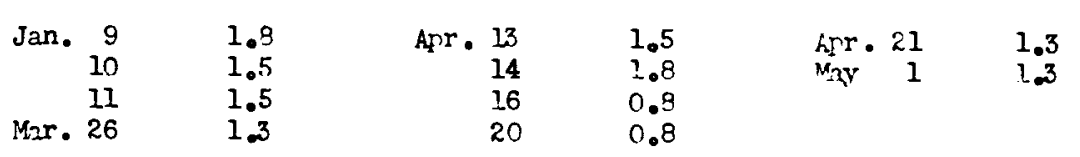


Monthly and Annual Precipitation, in millimeters, at Kala Kang, Afghanlstan

\begin{tabular}{|c|c|c|c|c|c|c|c|c|c|c|c|c|c|}
\hline Year & oct. & Now. & Dec. & Jan. & Feb. & Mar. & Apr. & May & June & July & Aug. & Sept. & Annua I \\
\hline 1955 & & & & & & & & & & & 0 & 0 & \\
\hline 1956 & 0 & 0 & 24.1 & 24.6 & 10.9 & 44.8 & 12.7 & 0 & 0 & 7.6 & 0 & 0 & 124.7 \\
\hline 1957 & 0 & 0 & 13.4 & 70.8 & 5.8 & 18.9 & 19.8 & 5.8 & 0 & 0 & c & 0 & 134.5 \\
\hline 1958 & 0 & 9.6 & 11.9 & 22.8 & 3.8 & 0 & 0 & 0 & 0 & 0 & 0 & 0 & 48.1 \\
\hline 1959 & 0 & 0 & 14.0 & 23.0 & 0 & 5.3 & 0 & 0 & 0 & 0 & 0 & 0 & 42.3 \\
\hline 1960 & 3.8 & 4.1 & 7.1 & 4.8 & 0 & 1.3 & 6.2 & 1.3 & 0 & 0 & 0 & 0 & 28.6 \\
\hline Ave. & 0.8 & 2.7 & 14.1 & 29.2 & 4.1 & 14.1 & 7.7 & 1.4 & 0 & 1.5 & 0 & 0 & 75.6 \\
\hline
\end{tabular}

Monthly and anual evaporation, in millimeters, at Kala Kang, Afgharistan

\begin{tabular}{|c|c|c|c|c|c|c|c|c|c|c|c|c|c|}
\hline Year & Oct. & Nov. & Dec。 & Jan. & Feb. & $\mathrm{Mar}$. & Apr. & May & $\operatorname{Jun} \theta$ & Mily & Aug. & Sept. & Annua 1 \\
\hline & & Note & These & e abse & ad pa? & Vapora & on $\mathrm{re}$ & Igs . & & & & & \\
\hline $\begin{array}{l}1955 \\
1956\end{array}$ & & & & & & & & & & & 785 & 457 & \\
\hline 1956 & 322 & 264 & 98 & 84 & 159 & 156 & 261 & 409 & 657 & 670 & 642 & 356 & 4078 \\
\hline $\begin{array}{l}1957 \\
1958\end{array}$ & 282 & 187 & 171 & 215 & 54 & 198 & 375 & 409 & 569 & 681 & 580 & 459 & 4280 \\
\hline $\begin{array}{l}1958 \\
1958\end{array}$ & 267 & 138 & 152 & 78 & 151 & 310 & 385 & 551 & 628 & 603 & $\begin{array}{r}693 \\
\times \quad 753\end{array}$ & 491 & 4447 \\
\hline $\begin{array}{l}1958 \\
1960\end{array}$ & $\begin{array}{r}434 \\
-\end{array}$ & 231 & - & $\begin{array}{l}117 \\
-\end{array}$ & $\begin{array}{l}130 \\
-\end{array}$ & $\begin{array}{l}155 \\
-\end{array}$ & $\begin{array}{r}239 \\
-\end{array}$ & $\begin{array}{l}406 \\
479\end{array}$ & $\begin{array}{l}670 \\
585\end{array}$ & $\begin{array}{l}732 \\
825\end{array}$ & $\begin{array}{r}+753 \\
869\end{array}$ & $\begin{array}{r}8441 \\
935\end{array}$ & 4419 \\
\hline Ave。 & 326 & 205 & 133 & 124 & 124 & 205 & 315 & 451 & 622 & 702 & 737 & 523 & 4306 \\
\hline
\end{tabular}

* - Based on 12 days record.

- Estimeted. 
Monthily and annual average wind velocity and maximum velocity, in kilometers, cer dąy at Kala Kang, Afghan is tan

\begin{tabular}{|c|c|c|c|c|c|c|c|c|c|c|c|c|c|}
\hline & & & & & & & & & & & & & \\
\hline Year & Uct. & Nor. & Deo: = & Jan. & Fob. & Mar. & Anr. & $\mathrm{Mav}$ & June & Julv & Aug. & set. & Annual \\
\hline 1955 & & & & & & & & & & & 604 & 410 & \\
\hline 1956 & 318 & 210 & 8 & 136 & 201 & 269 & 215 & 357 & 562 & 379 & $\begin{array}{l}506 \\
537\end{array}$ & $\begin{array}{l}845 \\
457\end{array}$ & 321 \\
\hline & 770 & 640 & $4 \div$ & 549 & 329 & 951 & 668 & 914 & 850 & 1179 & 864 & 746 & 1179 \\
\hline 1957 & 257 & 160 & 22.4 & 1.96 & 221 & 185 & 275 & 274 & 475 & 492 & 518 & 397 & 307 \\
\hline & 561 & 606 & $5 \%$ & $5: 3$ & 557 & 432 & 698 & 628 & 660 & 998 & 588 & 664 & 998 \\
\hline 1958 & 293 & 251 & $1 ? 9$ & 186 & 186 & 212 & 220 & 358 & 371 & 458 & 485 & 406 & 296 \\
\hline & 795 & 558 & 71 & $38=$ & 343 & 501 & 489 & 756 & 642 & 902 & 750 & 693 & 902 \\
\hline 1959 & 282 & 234 & 169 & 1.73 & 276 & 208 & 230 & 346 & 388 & 491 & 451 & 366 & 301 \\
\hline & 583 & 797 & 522 & 690 & 636 & 736 & 550 & 602 & 608 & 736 & 652 & 572 & 797 \\
\hline $196 n$ & 279 & 202 & 187 & $17 \%$ & 155 & 257 & 237 & 364 & 383 & 497 & 4.48 & 415 & 300 \\
\hline & 713 & 484 & $56 ?$ & 39 & 392 & 645 & 734 & 913 & 664 & 697 & 648 & 586 & 913 \\
\hline Ave. & 286 & 211 & 184 & 174 & 208 & 228 & 235 & 340 & 436 & 463 & $50 ?$ & 408 & 305 \\
\hline Max. & 795 & 797 & 662 & 590 & 636 & 951 & 734 & 914 & 850 & 1179 & 888 & 845 & 1179 \\
\hline
\end{tabular}

Manthly and enninl average relative humidity, high and low in percent at Kala Kang, Afghan is tan

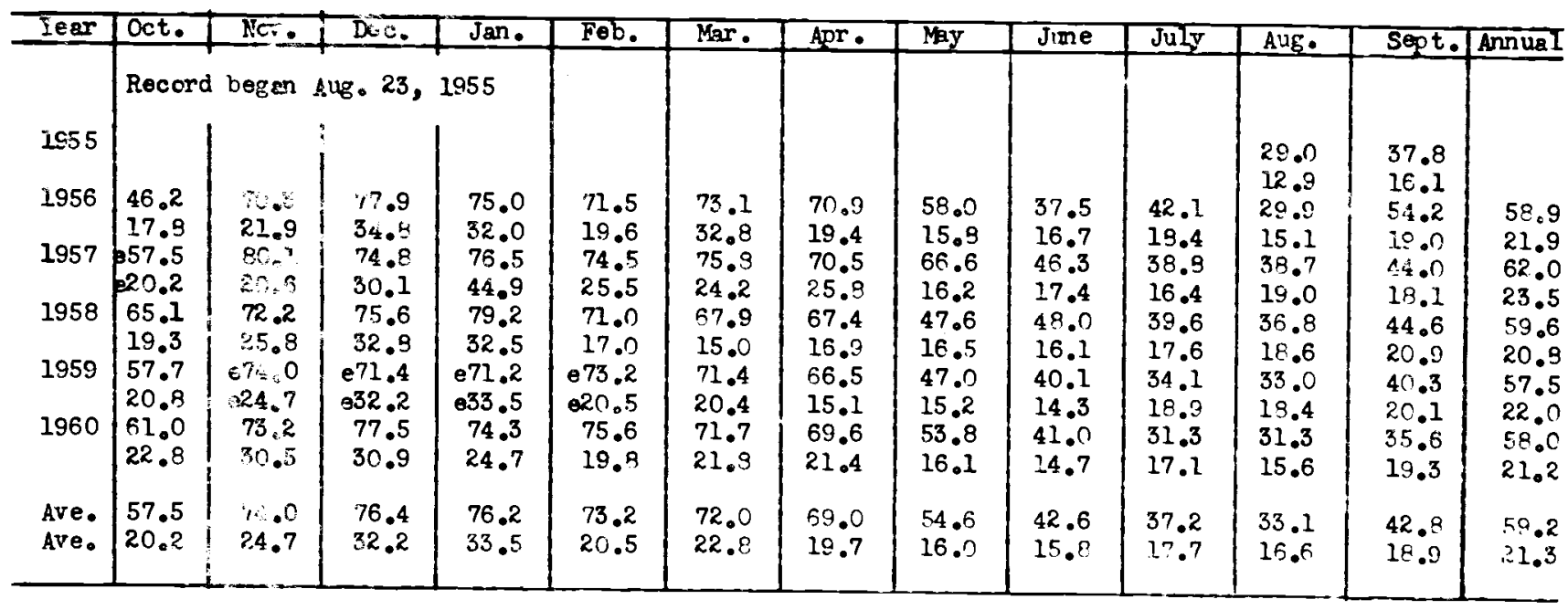

e - Estimzted. 


\section{KANDAHAR, AFGHANISTAN}

Temperature, Precipitation, Evaporation

Location.--Observation statign is 30 meters west of radio room in Manzel Bagh campound on nortineast side of Kandahar. Lat $3 I^{\circ} 371 \mathrm{~N}$, , long $65^{\circ} 431 \mathrm{E}$.

Equipment.-Pain gages A standard B-inch non-recording U.S. Weather Bureau rain gage can and masuring stick.

Thermancters: Maxtmun and ninimum thermometers and suppcrt in a wooden shelter.

Svaporation Pan: Evaporation pan is of welded stel plate 36 inches square and 19-3/4 inches deep set on the ground.

Averages and extremes for period of record.--

\section{Jenuary 1940 to February 1960}

Temperatures: Average daily maximum Average daily minimum

Madimum 1952

Minimum 1951

$$
\begin{array}{r}
27.5^{\circ} \mathrm{C} \\
9.2^{\circ} \mathrm{C} \\
45.6^{\circ} \mathrm{C} \\
-12.20^{\circ} \mathrm{C}
\end{array}
$$

Precipitation: Average yoarly

177.8 millineters

Evqporation:

Arerage yearly pan

1752

miliimeters 
Monthly and annul average maximum and minimum and maxinum and minimum Termeratures in degrees centigrade

at Kandahar, Afghanistan

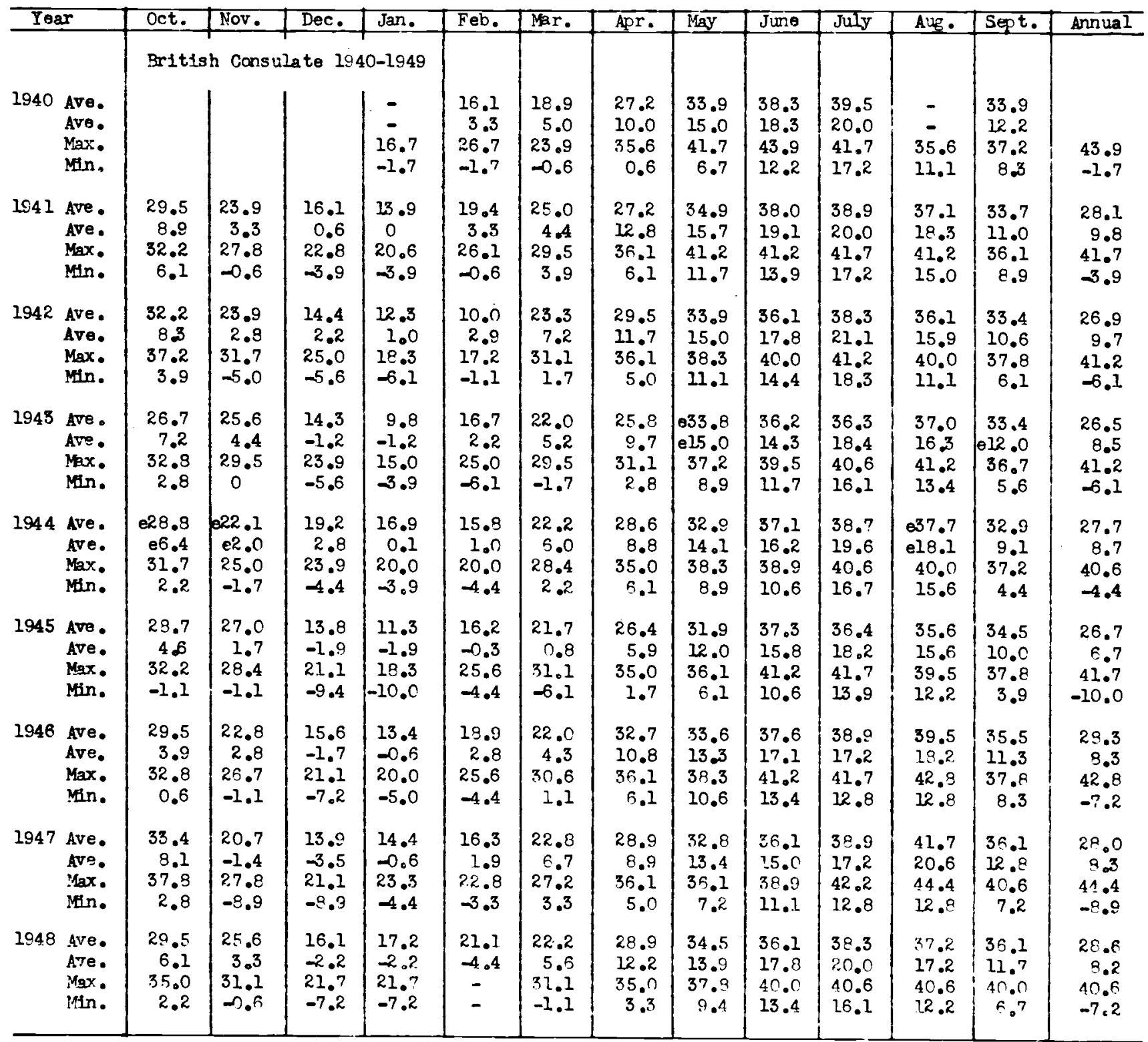

e - Estimated 
at Kandahar, Afghanistan

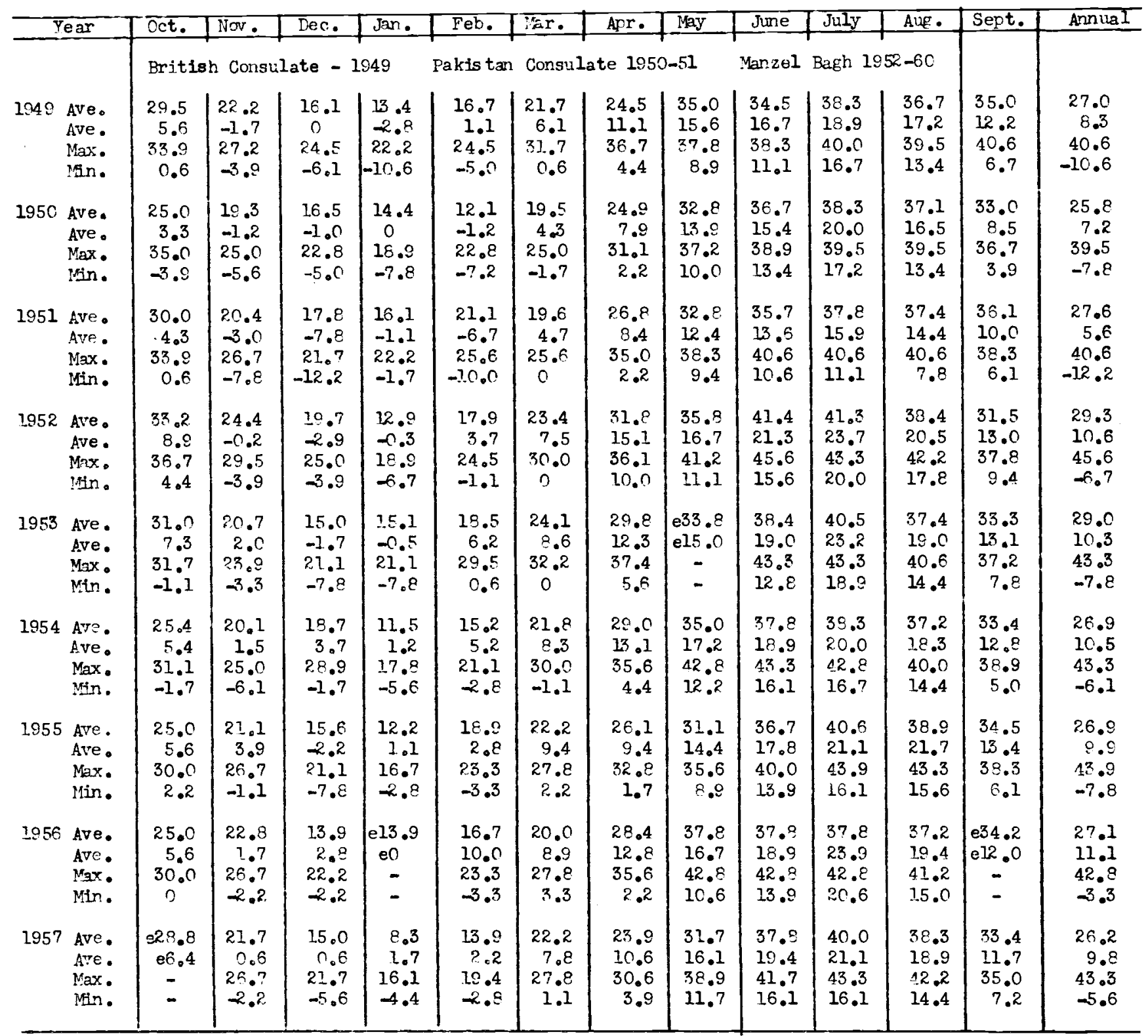


Monthly and mnual average maximim and minimum and maximum and minimum Temperatures in degrees centigrade at Kandahar, Af ghanistan

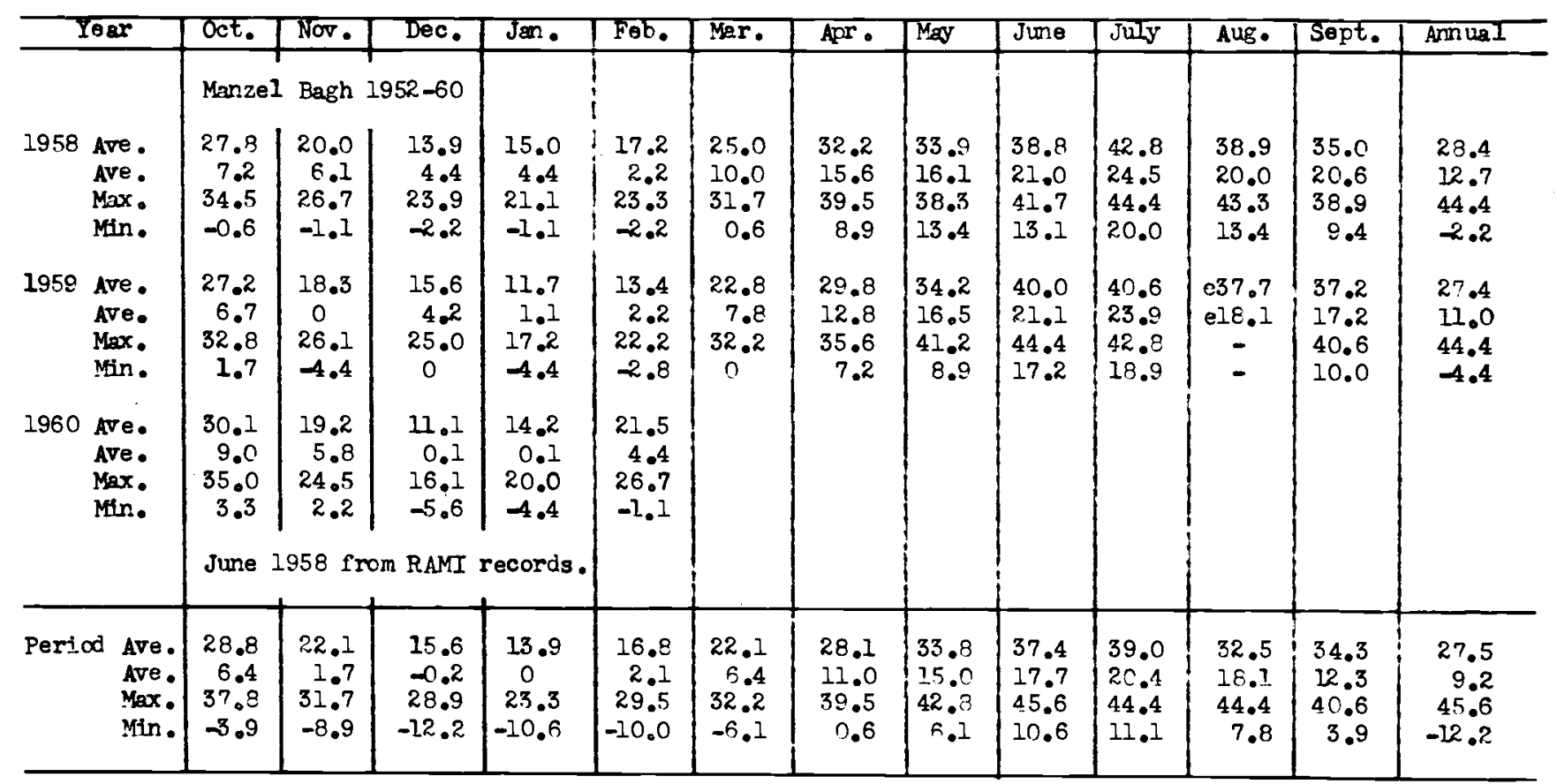

e - Estimted. 
PPECIPITATICN IN MTLUDETERS AT KANDAHAR, AFCHAN IS TAN

1940

\begin{tabular}{|c|c|c|c|c|c|c|c|c|c|c|}
\hline Jan • & $\begin{array}{l}11 \\
14 \\
15 \\
16 \\
17 \\
18\end{array}$ & $\begin{array}{l}0.8 \\
4.3 \\
1.3 \\
3.0 \\
5.8 \\
0.5\end{array}$ & Jan. & $\begin{array}{r}29 \\
30 \\
31 \\
3 \\
8 \\
10\end{array}$ & $\begin{array}{r}30.2 \\
76.2 \\
2.3 \\
4.3 \\
0.5 \\
1.0\end{array}$ & Feb. & $\begin{array}{l}12 \\
13 \\
18 \\
28 \\
13 \\
14\end{array}$ & $\begin{array}{r}0.8 \\
9.6 \\
1.0 \\
8.0 \\
0.8 \\
10.2\end{array}$ & $\begin{array}{l}\text { Mar. } \\
\text { Apr. }\end{array}$ & $\begin{array}{l}15 \\
28 \\
10 \\
30\end{array}$ \\
\hline
\end{tabular}

1941

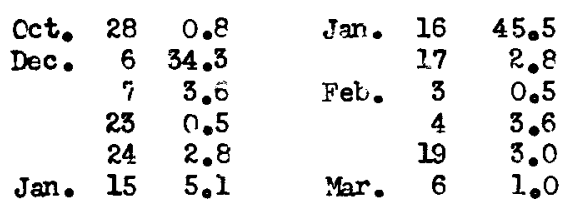

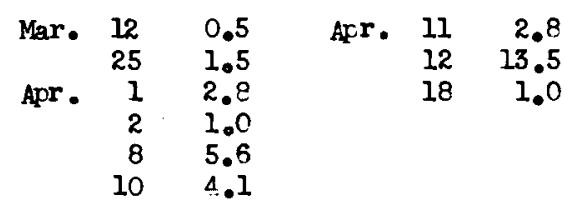

1942

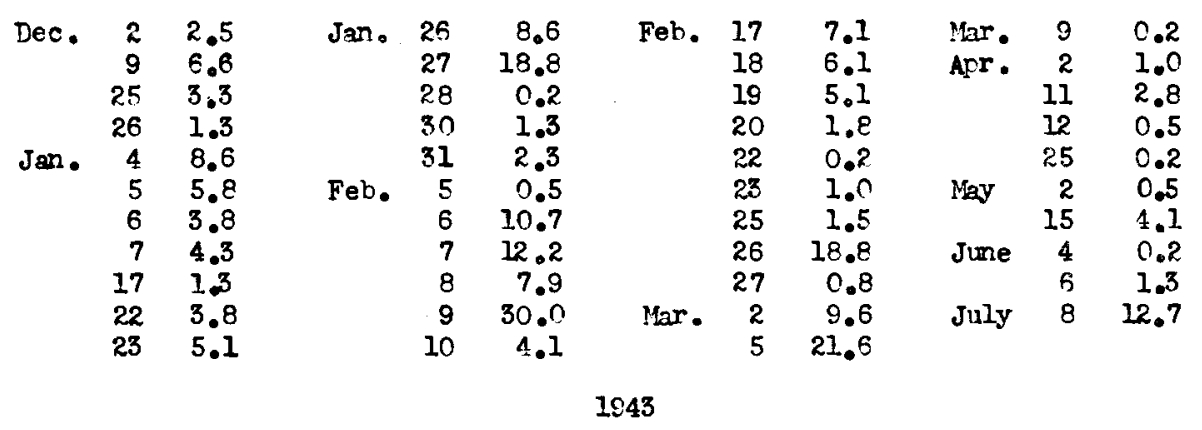

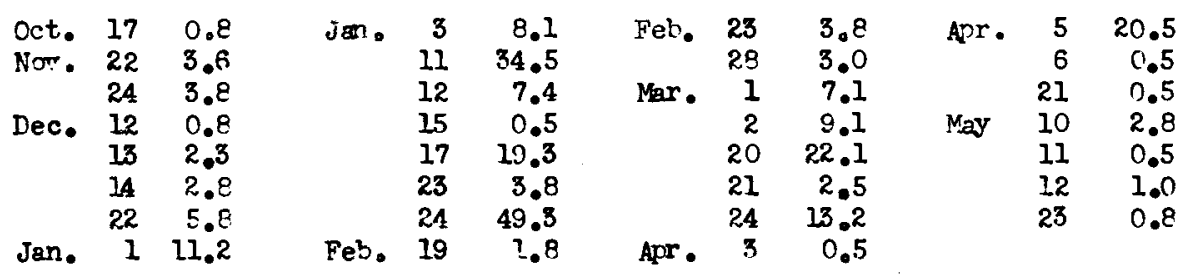


PRECIF ITATION IN NILLTMETERS AT KANE AHAR, AFGHAN ISTAN

1944

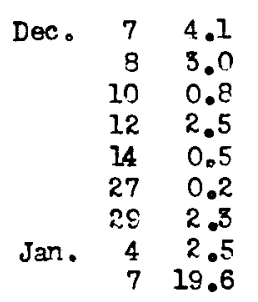

\begin{tabular}{|c|c|c|c|c|c|c|c|c|}
\hline Jan. & $\begin{array}{l}11 \\
21\end{array}$ & $\begin{array}{r}14.2 \\
2.0\end{array}$ & Feb. & $\begin{array}{l}21 \\
24\end{array}$ & $\begin{array}{r}1.0 \\
8.1\end{array}$ & Mar. & $\begin{array}{l}26 \\
30\end{array}$ & $\begin{array}{l}1.0 \\
1.5\end{array}$ \\
\hline & 22 & 12.2 & & 25 & 15.4 & Apr. & 1 & 0.8 \\
\hline & 23 & 2.8 & & 26 & 1.0 & May & 15 & 2.0 \\
\hline & 24 & 13.7 & & 27 & 55.6 & & 16 & C. .5 \\
\hline Feb. & 1 & 1.8 & Mar. & 11 & 0.5 & July & 13 & 4.8 \\
\hline & 2 & 0.5 & & 12 & 2.3 & & 14 & 6.9 \\
\hline & 11 & 5.6 & & 18 & 0.5 & Aug. & 2 & 0.2 \\
\hline & 14 & 7.1 & & 23 & 0.8 & & & \\
\hline
\end{tabular}

1945

$\begin{array}{lrr}\text { Nor. } & 22 & 0.8 \\ 23 & 2.8 \\ \text { Dec. } & 1.0 \\ 4 & 2.5 \\ 8 & 3.0 \\ 21 & 2.0 \\ 26 & 0.5\end{array}$

\begin{tabular}{|c|c|}
\hline Dec. & 27 \\
\hline Jan & $\begin{array}{r}28 \\
3\end{array}$ \\
\hline & 4 \\
\hline & \\
\hline & 8 \\
\hline & 22 \\
\hline
\end{tabular}
Jan. $29 \quad 15.7$
$30 \quad 1.0$
Feb. $\begin{array}{rr}8 & 1.0 \\ 16 & 5.6\end{array}$ $26 \quad 1.0$

Mar. $29 \quad 0.8$

Mar. $27 \quad 0.8$

Apr 50.7

May $\begin{array}{rr}30 & 3.3 \\ 1 & 3.3 \\ 16 & 0.8\end{array}$

1946

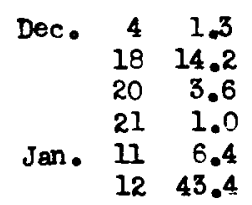

Jan. $\begin{array}{rr}13 & 18.8 \\ 14 & 7.1 \\ 15 & 0.8 \\ 22 & 5.3 \\ 23 & 23.6 \\ 29 & 2.0\end{array}$

Fet. 1

$\begin{array}{ll}7 & 0.8 \\ 10 & 8.6 \\ 11 & 3.0 \\ 19 & 1.5 \\ 21 & 1.0 \\ 26 & 19\end{array}$

$\begin{array}{rrr}\text { Feb. } & 28 & 0.5 \\ \text { Mer. } & 1 & 1.0 \\ 11 & 15.2 \\ & 12 & 3.0 \\ \text { May } & 3 & 0.5\end{array}$

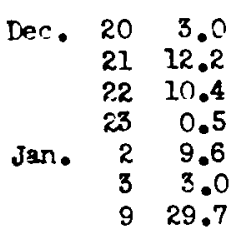

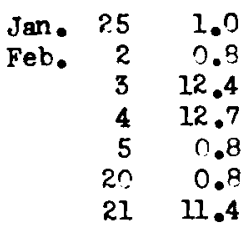

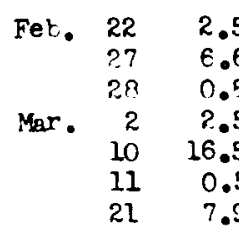

Mar. $22 \quad 4.1$

$\begin{array}{rr}\text { Mar. } & 22 \\ 1 & 4.1 \\ 8 & 5.6 \\ 9 & 9.6\end{array}$ 
FRECIPIT ATION IN! MIILIMETERS AT KANDAHAR, AFGHAN TS TAN

\begin{tabular}{|c|c|c|c|c|c|c|c|c|c|c|c|}
\hline \multirow[b]{2}{*}{$\begin{array}{l}\text { Nov } \\
\text { Dec. } \\
\text { Jan. }\end{array}$} & \multicolumn{11}{|c|}{1948} \\
\hline & $\begin{array}{r}14 \\
4 \\
5 \\
10 \\
27 \\
28\end{array}$ & $\begin{array}{r}3.0 \\
2.3 \\
10.4 \\
21.6 \\
5.3 \\
4.8\end{array}$ & Mar. & $\begin{array}{r}1 \\
13 \\
19 \\
25 \\
2 \\
6\end{array}$ & $\begin{array}{l}9.4 \\
3.3 \\
7.6 \\
4.1 \\
3.0 \\
5.1\end{array}$ & Apr. & $\begin{array}{r}7 \\
10 \\
17 \\
25 \\
8 \\
9\end{array}$ & $\begin{array}{r}7.1 \\
13.0 \\
2.3 \\
2.8 \\
7.6 \\
1.8\end{array}$ & Apr. & $\begin{array}{l}10 \\
17 \\
18 \\
20\end{array}$ & $\begin{array}{l}0.8 \\
0.2 \\
1.0 \\
1.8\end{array}$ \\
\hline
\end{tabular}

$\begin{array}{lll}\text { Dec. } & 19 & 3.0 \\ 26 & 0.2 \\ 30 & 3.0 \\ \operatorname{Jan} . & 14 & 4.1 \\ 24 & 0.8\end{array}$

$\begin{array}{lr}\text { Jan. } 28 & 0.2 \\ \text { Feb. } & 4.6 \\ 2 & 16.5 \\ 3 & 1.0 \\ 21 & 4.8\end{array}$

Mar.

$$
\begin{array}{r}
6 \\
7 \\
8 \\
10
\end{array}
$$$$
\begin{array}{rrrr}
1.8 & \text { Mar. } 11 & 5.3 \\
0.5 & \text { Apr. } & 5 & 3.3 \\
6.9 & & 6 & 4.8 \\
37.3 & & 9 & 1.5 \\
33.0 & \text { Aug. } & 5 & 0.8
\end{array}
$$

1950

\begin{tabular}{|c|c|c|c|c|c|c|c|c|c|c|}
\hline $\begin{array}{l}\text { Nor. } \\
\text { Dec. }\end{array}$ & $\begin{array}{r}25 \\
26 \\
9 \\
12 \\
15 \\
31 \\
11 \\
12\end{array}$ & $\begin{array}{r}0.5 \\
3.8 \\
2.5 \\
3.3 \\
0.5 \\
2.0 \\
1.5 \\
10.2\end{array}$ & Feb. & $\begin{array}{r}13 \\
18 \\
26 \\
28 \\
29 \\
5 \\
6 \\
7\end{array}$ & $\begin{array}{r}1.0 \\
7.9 \\
2.8 \\
46.2 \\
35.6 \\
7.1 \\
7.0 \\
5.8\end{array}$ & $\begin{array}{l}\text { Feb. } \\
\text { Mar. }\end{array}$ & $\begin{array}{r}8 \\
10 \\
13 \\
19 \\
20 \\
28 \\
29\end{array}$ & $\begin{array}{r}9.9 \\
0.5 \\
18.3 \\
3.6 \\
8.4 \\
1.9 \\
13.0\end{array}$ & $\begin{array}{l}\text { Apr. } \\
\text { May }\end{array}$ & $\begin{array}{r}1 \\
2 \\
7 \\
8 \\
9 \\
10\end{array}$ \\
\hline
\end{tabular}

1951

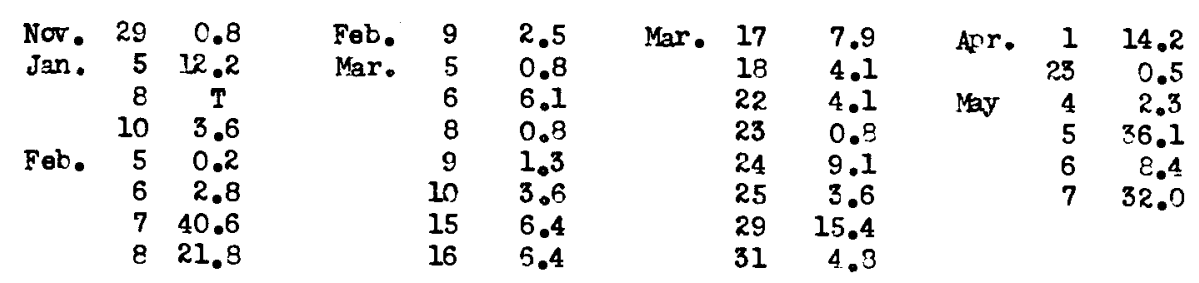


PRECIP TTATION IN MILLTETERS AT KANDAHAR, AFGHANISTAN

1852

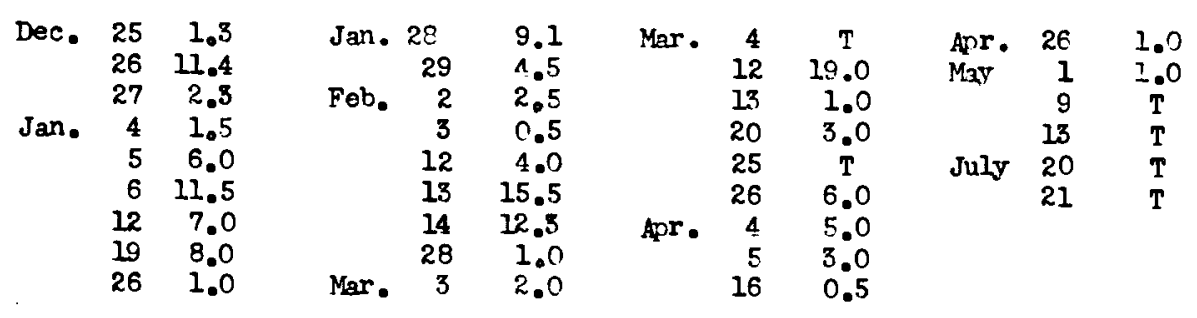

1953

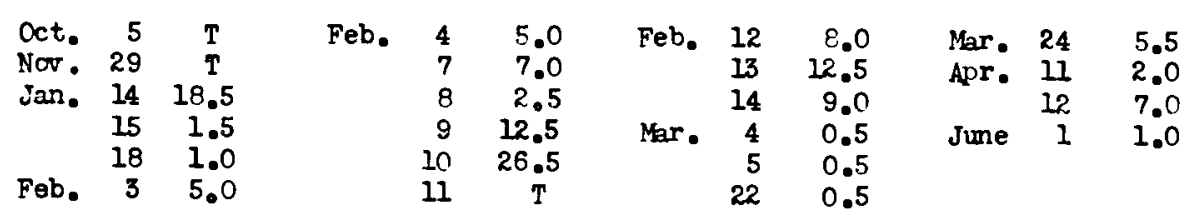

1954

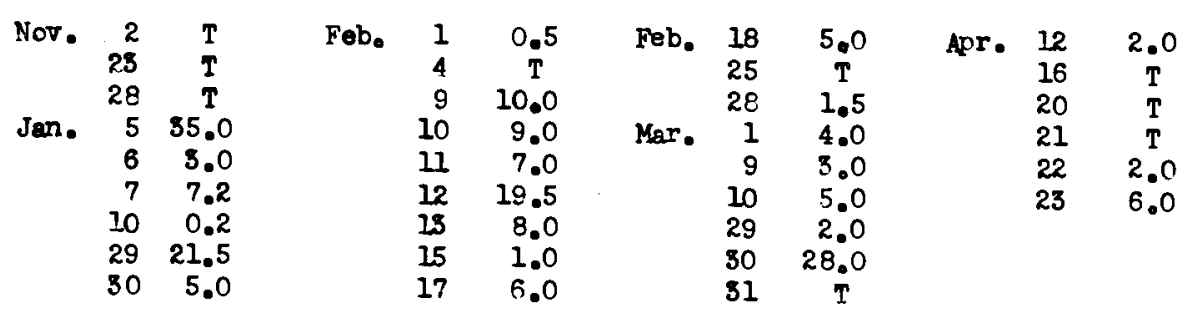

Daily record not available for January 1955 to January 1956.

1956

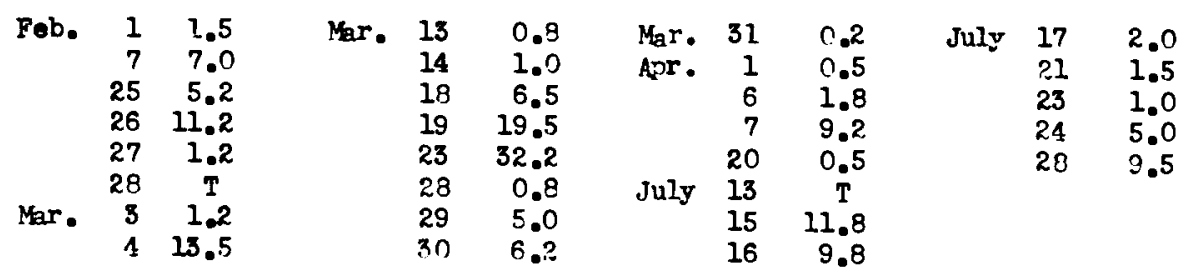

T - Trece 
PRECIPITATION IN MIULINETERS AT KANDAHAR, AFGHANISTAN

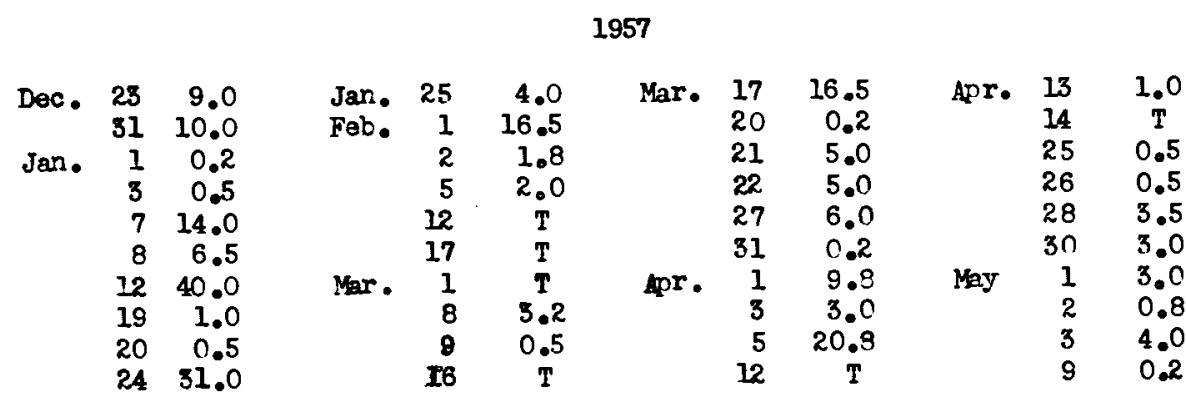

1958
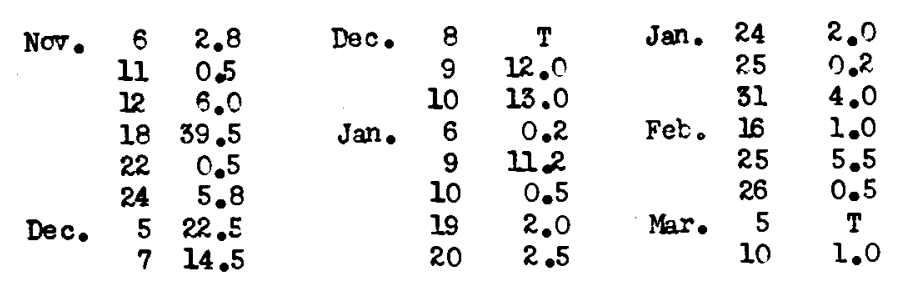

$\begin{array}{lcc}\text { Mar. } & 27 & 3.5 \\ & 31 & 0.2 \\ \text { Apr. } & 14 & \text { T } \\ & 15 & 5.0 \\ \text { May } & 21 & 1.0 \\ \text { July } & 11 & T \\ & 12 & 1.5 \\ & 13 & 0.2\end{array}$

1859
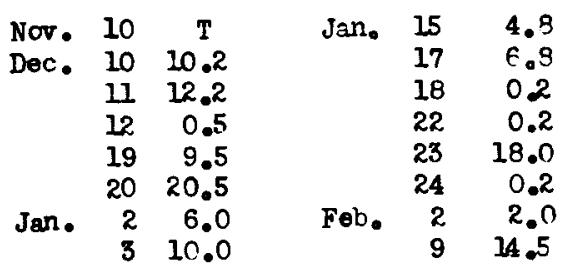

$\begin{array}{rr}\text { Feb. } 17 & 16.0 \\ 22 & 7.2 \\ 26 & 3.5 \\ 28 & 17.8 \\ 1 & 6.5 \\ 8 & 1.5 \\ 9 & 6.5 \\ 24 & 1.0\end{array}$

Mar. 28 T

Apr. 16.0

$\begin{array}{lrr} & 2 & 12.2 \\ & 20 & 1.0 \\ 21 & 0.5 \\ \text { May } & 17 & 0.2 \\ \text { June } & 2 & T \\ \text { July } & 2 & T\end{array}$

1960

Nov. $\begin{array}{rrrrrrrrrr}1 & T & \text { Nov. } 27 & 10.8 & \text { Dec. } 15 & 10.2 & \text { Jan. } 31 & 1.8 \\ 2 & 1.0 & & 30 & 4.5 & & 16 & 3.2 & \text { Feb. } 14 & 0.8 \\ 4 & 9.5 & \text { Dec. } 1 & 1.5 & \text { Jan. } 10 & 3.8 & & 21 & 1.0 \\ 5 & 5.0 & & 2 & 8.0 & & 12 & 0.5 & & \\ 26 & \text { T } & & 14 & 4.0 & & 29 & 1.2 & & \end{array}$


Nonthly and annual. precipltation, in millimeters, at Kandahar, Afghanistan

\begin{tabular}{|c|c|c|c|c|c|c|c|c|c|c|c|c|c|}
\hline Iear & oct. & Nor. & Dec. & $\operatorname{san}$ & Feb. & Mar. & Apr. & May & June & JuIy & Aug. & sept. & smual \\
\hline $\begin{array}{l}1940 \\
1941 \\
1942 \\
1943 \\
1944 \\
1945 \\
1946 \\
1947 \\
1948 \\
1949 \\
1950 \\
1951 \\
1952 \\
1953 \\
1956 \\
1955 \\
1956 \\
1557 \\
1958 \\
1959 \\
1980\end{array}$ & $\begin{array}{l}0.8 \\
0 \\
0.8 \\
0 \\
0 \\
0 \\
0 \\
0 \\
0 \\
0 \\
0 \\
0 \\
T \\
0 \\
0 \\
0 \\
0 \\
0 \\
0 \\
0\end{array}$ & $\begin{array}{l}0 \\
0 \\
7.4 \\
0 \\
3.6 \\
0 \\
0 \\
3.0 \\
0 \\
4.3 \\
0.8 \\
0 \\
T \\
T \\
0 \\
0 \\
0 \\
55.1 \\
T \\
31.8\end{array}$ & $\begin{array}{c}41.2 \\
13.7 \\
11.7 \\
13.4 \\
17.9 \\
20.1 \\
26.1 \\
34.3 \\
6.2 \\
8.3 \\
0 \\
15.0 \\
0 \\
0 \\
0 \\
66.1 \\
19.0 \\
62.0 \\
52.9 \\
28.9\end{array}$ & $\begin{array}{r}124.4 \\
53.4 \\
63.9 \\
134.1 \\
67.0 \\
44.9 \\
107.4 \\
43.3 \\
10.1 \\
5.1 \\
105.2 \\
15.8 \\
48.6 \\
21.0 \\
71.9 \\
48.0 \\
060.0 \\
97.7 \\
22.8 \\
46.2 \\
7.8\end{array}$ & $\begin{array}{r}26.1 \\
7.1 \\
107.8 \\
8.6 \\
96.1 \\
8.1 \\
35.2 \\
48.8 \\
24.4 \\
26.9 \\
30.7 \\
67.9 \\
35.8 \\
88.0 \\
67.5 \\
0 \\
26.1 \\
20.3 \\
7.0 \\
61.0 \\
1.8\end{array}$ & $\begin{array}{r}13.3 \\
3.0 \\
31.4 \\
54.0 \\
6.6 \\
1.6 \\
19.2 \\
31.5 \\
33.5 \\
84.8 \\
46.6 \\
72.2 \\
31.0 \\
7.0 \\
42.0 \\
21.8 \\
86.9 \\
36.6 \\
4.7 \\
15.5\end{array}$ & $\begin{array}{r}1.8 \\
30.8 \\
4.5 \\
22.0 \\
0.8 \\
14.0 \\
0 \\
0 \\
13.2 \\
9.6 \\
15.0 \\
14.7 \\
9.5 \\
9.0 \\
10.0 \\
0 \\
12.0 \\
42.1 \\
5.0 \\
19.7\end{array}$ & $\begin{array}{l}0 \\
0 \\
4.6 \\
5.1 \\
2.5 \\
4.1 \\
0.5 \\
14.2 \\
0 \\
0 \\
7.6 \\
78.8 \\
1.0 \\
0 \\
0 \\
1.5 \\
0 \\
8.0 \\
1.0 \\
0.2\end{array}$ & $\begin{array}{l}0 \\
0 \\
1.5 \\
0 \\
0 \\
0 \\
0 \\
0 \\
0 \\
0 \\
0 \\
0 \\
0 \\
1.0 \\
0 \\
0 \\
0 \\
0 \\
0 \\
T\end{array}$ & $\begin{array}{c}0 \\
0 \\
12.7 \\
0 \\
11.7 \\
0 \\
0 \\
0 \\
0 \\
0 \\
0 \\
0 \\
\mathrm{~T} \\
0 \\
0 \\
0 \\
40.6 \\
0 \\
1.7 \\
\mathrm{~T}\end{array}$ & $\begin{array}{l}0 \\
0 \\
0 \\
0 \\
0.2 \\
0 \\
0 \\
0 \\
0 \\
0.8 \\
0 \\
0 \\
0 \\
0 \\
0 \\
0 \\
0 \\
0 \\
0 \\
0\end{array}$ & $\begin{array}{r}0 \\
0 \\
0 \\
0 \\
0 \\
0 \\
0 \\
0 \\
0 \\
0 \\
0 \\
0 \\
0 \\
0 \\
0 \\
0 \\
0 \\
0 \\
0 \\
0\end{array}$ & $\begin{array}{r}136.3 \\
240.1 \\
243.7 \\
198.3 \\
94.2 \\
182.4 \\
163.9 \\
118.3 \\
133.4 \\
217.7 \\
250.2 \\
140.9 \\
126.0 \\
191.4 \\
71.5 \\
291.7 \\
223.7 \\
159.1 \\
195.5\end{array}$ \\
\hline Ave. & 0.1 & 5.3 & 21.7 & 57.1 & 57.9 & 32.2 & 11.7 & 6.5 & 0.1 & 3.3 & 0.05 & 0 & 177.8 \\
\hline
\end{tabular}

T. Irace

Monthly and anval opeporation, in millimeters, at Kandahar, Afghanistan

\begin{tabular}{|c|c|c|c|c|c|c|c|c|c|c|c|c|c|}
\hline Year & Dct. & Nov. & Dec. & $\operatorname{san}$. & Fob. & 面r. & Apr. & May & June & July & Aug. & Sept. & Innual \\
\hline 1951 & & & & & & 95 & 152 & 192 & 226 & 220 & 200 & 165 & \\
\hline 1952 & 148 & 114 & 53 & 71 & 115 & 146 & 175 & 220 & 251 & 265 & 260 & 137 & 1955 \\
\hline 1953 & 72 & $6 ?$ & 46 & 46 & 48 & 124 & 176 & e226 & e253 & 285 & 277 & 219 & 1839 \\
\hline 1.954 & 137 & 81 & 47 & 59 & 22 & 78 & 155 & 271 & 287 & $30 R$ & 280 & 218 & 1957 \\
\hline 1955 & 123 & 75 & 49 & 25 & 43 & 79 & 150 & 191 & 211 & 229 & 221 & 170 & 1566 \\
\hline 1956 & 63 & 48 & 36 & 237 & 43 & 58 & 139 & 233 & 244 & 204 & 257 & el83 & 1545 \\
\hline 1957 & el12 & 58 & 42 & 24 & 54 & 83 & 118 & 197 & 258 & 259 & 237 & 155 & 1597 \\
\hline 1958 & 117 & 34 & 20 & 26 & 50 & 114 & 180 & 274 & e253 & 278 & 279 & 196 & 1821 \\
\hline 1959 & 118 & 57 & 36 & 19 & 30 & 78 & 144 & 229 & 293 & 292 & e251 & 207 & 1754 \\
\hline 1960 & 119 & 39 & 18 & 28 & 50 & & & & & & & & \\
\hline AVO. & 112 & 64 & 38 & 37 & 51 & 95 & 154 & 226 & 253 & 259 & 251 & 183 & 1752 \\
\hline
\end{tabular}

- timated. 


\section{KAJAKAI RESERVOIR, AFGHANISTAN}

Temperature, Precipitation, Evaporation

Location.-Observation station is on left bank of Helmand River about 100 meters upstream from righway brIdgo about 2 kflameters downstream fram Kajakal dam. Iat $32^{\circ} 18^{\prime} \mathrm{N}$., lang $65^{\circ} 06^{\circ} \mathrm{E}$, abcut 60 kilameters north of airishk and about 90 kilometers northwest of Kand ahar.

Equipment.-Rain gage: A standard 8-inch non-recording U.S. Weather Bureau rain gage can and measuring stick.

Thermoneters: Maximurn and minimum thermometers and support in a standard wooden shel ter. Evaporation pan: Pan is of welaed steel plate 36 inches square and $19-3 / 4$ inches deep set

in ground. Water surface was read from a reference point on the top edge of the par.

\section{Averages and extremes for period of record.-}

\section{February 1956 to september 1960}

$\begin{array}{llc}\text { Temperatures: Average daily maximum } & 34.0^{\circ} \mathrm{C} \\ & 11.6^{\circ} \mathrm{C} \\ & \text { Average daily minimim } & 48.9^{\circ} \mathrm{C} \\ & \text { Minimum } & -12.2^{\circ} \mathrm{C} \\ \text { Precipitation: Average yearly } & 197.8 \text { millimeters } \\ \text { Evaporation: Averace yearly pan } & 2391 \text { millimeters }\end{array}$


Nonthly and amual everage maximum and minimun and maximum and minimum Temperatures in degrees centigrade at Kajakal Reservolr at Kajakai, Afghanistan

\begin{tabular}{|c|c|c|c|c|c|c|c|c|c|c|c|c|c|c|}
\hline les & ar & oct. & Nov. & Dec. & Jan. & Fab. & Mar. & Apr. & May & June & Juy & Aus. & Sept. & Annus I \\
\hline $1956 \mathrm{~A}$ & $\begin{array}{l}\text { lve. } \\
\text { axe. } \\
\text { ain. }\end{array}$ & & & & & $\begin{array}{r}18.3 \\
1.7 \\
23.3 \\
-5.6\end{array}$ & $\begin{array}{r}21.7 \\
8.3 \\
26.1 \\
5.0\end{array}$ & $\begin{array}{r}28.4 \\
12.8 \\
36.7 \\
2.8\end{array}$ & $\begin{array}{l}40.6 \\
14.4 \\
43.9 \\
10.6\end{array}$ & $\begin{array}{l}41.2 \\
18.9 \\
45.0 \\
14.4\end{array}$ & $\begin{array}{l}41.2 \\
23.3 \\
45.6 \\
18.9\end{array}$ & $\begin{array}{l}38.9 \\
18.3 \\
43.3 \\
14.4\end{array}$ & - & \\
\hline $1957 \quad$ & $\begin{array}{l}\text { dve. } \\
\text { dve. } \\
\text { sox. } \\
\text { in. }\end{array}$ & $\begin{array}{l}29.8 \\
7.2 \\
- \\
-\end{array}$ & $\begin{array}{r}23.9 \\
1.7 \\
27.8 \\
-0.6\end{array}$ & $\begin{array}{r}16.1 \\
0.6 \\
23.3 \\
-3.9\end{array}$ & $\begin{array}{r}10.0 \\
1.7 \\
17.8 \\
-1.1\end{array}$ & $\begin{array}{r}15.6 \\
2.2 \\
21.1 \\
2.2\end{array}$ & $\begin{array}{r}21.1 \\
5.0 \\
26.7 \\
3.3\end{array}$ & $\begin{array}{r}22.8 \\
7.8 \\
27.8 \\
6.1\end{array}$ & $\begin{array}{r}31.7 \\
13.9 \\
38.9 \\
9.4\end{array}$ & $\begin{array}{l}38.3 \\
17.8 \\
41.2 \\
15.0\end{array}$ & $\begin{array}{l}41.2 \\
20.0 \\
45.6 \\
16.7\end{array}$ & $\begin{array}{l}40.0 \\
17.2 \\
42.8 \\
12.8\end{array}$ & $\begin{array}{r}82.8 \\
10.6 \\
35.0 \\
8.9\end{array}$ & $\begin{array}{r}26.9 \\
8.8 \\
45.6 \\
-3.9\end{array}$ \\
\hline 1958 A & $\begin{array}{l}\text { Ave. } \\
\text { Ave. } \\
\text { ins. } \\
\text { ins. }\end{array}$ & $\begin{array}{r}28.4 \\
7.2 \\
35.6 \\
1.1\end{array}$ & $\begin{array}{r}20.7 \\
5.6 \\
27.8 \\
-1.1\end{array}$ & $\begin{array}{r}15.2 \\
2.9 \\
21.1 \\
2.2\end{array}$ & $\begin{array}{r}14.2 \\
3.6 \\
18.9 \\
2.2\end{array}$ & $\begin{array}{r}18.0 \\
1.6 \\
22.2 \\
2.2\end{array}$ & $\begin{array}{r}25.5 \\
7.8 \\
32.2 \\
-1.1\end{array}$ & $\begin{array}{r}32.2 \\
12.7 \\
37.8 \\
6.7\end{array}$ & $\begin{array}{l}34.8 \\
13.5 \\
38.9 \\
10.0\end{array}$ & $\begin{array}{l}40.8 \\
17.3 \\
43.3 \\
11.1\end{array}$ & $\begin{array}{l}41.7 \\
20.3 \\
45.6 \\
16.1\end{array}$ & $\begin{array}{l}39.8 \\
16.9 \\
44.4 \\
12.2\end{array}$ & $\begin{array}{r}36.6 \\
11.9 \\
41.2 \\
6.7\end{array}$ & $\begin{array}{l}29.0 \\
10.1 \\
45.6 \\
2.2\end{array}$ \\
\hline $1959 \mathrm{~A}$ & $\begin{array}{l}\text { Ave. } \\
\text { Ave. } \\
\text { vax. } \\
\text { tin. }\end{array}$ & $\begin{array}{r}29.0 \\
6.1 \\
32.2 \\
1.1\end{array}$ & $\begin{array}{l}19.8 \\
-1.3 \\
27.8 \\
-5.6\end{array}$ & $\begin{array}{r}17.3 \\
2.6 \\
24.5 \\
2.2\end{array}$ & $\begin{array}{r}14.5 \\
0.6 \\
17.8 \\
-4.4\end{array}$ & $\begin{array}{r}15.4 \\
0.6 \\
22.2 \\
-5.6\end{array}$ & $\begin{array}{r}21.9 \\
6.2 \\
30.0 \\
0.0\end{array}$ & $\begin{array}{r}30.4 \\
10.4 \\
34.5 \\
5.5\end{array}$ & $\begin{array}{r}34.8 \\
14.5 \\
40.0 \\
8.9\end{array}$ & $\begin{array}{l}39.9 \\
16.9 \\
42.2 \\
12.2\end{array}$ & $\begin{array}{l}41.4 \\
19.2 \\
44.4 \\
15.6\end{array}$ & $\begin{array}{l}42.0 \\
18.1 \\
45.6 \\
15.6\end{array}$ & $\begin{array}{l}37.7 \\
13.2 \\
40.0 \\
10.0\end{array}$ & $\begin{array}{r}28.7 \\
8.9 \\
45.6 \\
-5.6\end{array}$ \\
\hline $1960 \mathrm{~A}$ & $\begin{array}{l}\text { Are. } \\
\text { Ave. } \\
\text { vas. } \\
\text { int. }\end{array}$ & $\begin{array}{r}32.1 \\
8.4 \\
57.8 \\
5.0\end{array}$ & $\begin{array}{r}21.0 \\
3.8 \\
25.6 \\
1.7\end{array}$ & $\begin{array}{r}13.1 \\
-0.8 \\
17.2 \\
-10.0\end{array}$ & $\begin{array}{r}17.1 \\
-1.3 \\
20.6 \\
-12.2\end{array}$ & $\begin{array}{r}26.6 \\
0.4 \\
31.1 \\
-6.1\end{array}$ & $\begin{array}{l}29.0 \\
20.1 \\
36.7 \\
14.4\end{array}$ & $\begin{array}{c}28.4 \\
010.9 \\
- \\
-\end{array}$ & $\begin{array}{c}e 35.5 \\
e 14.1 \\
- \\
-\end{array}$ & $\begin{array}{l}43.7 \\
19.2 \\
46.1 \\
10.6\end{array}$ & $\begin{array}{l}38.0 \\
17.6 \\
48.9 \\
13.4\end{array}$ & $\begin{array}{l}31.9 \\
18.2 \\
37.8 \\
15.0\end{array}$ & $\begin{array}{l}28.5 \\
18.2 \\
33.9 \\
-1.1\end{array}$ & $\begin{array}{r}28.7 \\
10.7 \\
48.9 \\
-12.2\end{array}$ \\
\hline Pariod & $\begin{array}{l}\text { Ave. } \\
\text { Ave. } \\
\text { Kax. } \\
\text { Man. }\end{array}$ & $\begin{array}{r}29.8 \\
7.2 \\
37.8 \\
1.1\end{array}$ & $\begin{array}{r}21.4 \\
2.4 \\
27.8 \\
-5.6\end{array}$ & $\begin{array}{r}15.4 \\
1.3 \\
24.5 \\
-10.0\end{array}$ & $\begin{array}{r}14.0 \\
1.2 \\
20.6 \\
-12.2\end{array}$ & $\begin{array}{r}18.9 \\
1.3 \\
31.1 \\
-6.1\end{array}$ & $\begin{array}{r}23.8 \\
9.5 \\
36.7 \\
-1.1\end{array}$ & $\begin{array}{r}28.4 \\
10.9 \\
37.8 \\
2.8\end{array}$ & $\begin{array}{r}35.5 \\
14.1 \\
43.9 \\
8.9\end{array}$ & $\begin{array}{l}40.8 \\
18.1 \\
46.1 \\
10.6\end{array}$ & $\begin{array}{l}40.7 \\
20.1 \\
48.9 \\
13.4\end{array}$ & $\begin{array}{l}38.5 \\
17.7 \\
45.6 \\
12.2\end{array}$ & $\begin{array}{l}27.1 \\
10.8 \\
41.2 \\
-1.1\end{array}$ & $\begin{array}{r}34.0 \\
11.6 \\
48.9 \\
-12.2\end{array}$ \\
\hline
\end{tabular}

- Batimated. 
PRECIPITATTON IN MILLTEETERS AT KAJAKAI RESERVOIR, AFGHANISTAN

\begin{tabular}{|c|c|c|c|c|c|c|c|c|c|c|c|}
\hline \multicolumn{12}{|c|}{1956} \\
\hline Feb. & 24 & 17. & Mar. & 5 & 23. & Mar. & 24 & 51. & Apr. & 15 & 2. \\
\hline & $\begin{array}{l}25 \\
26\end{array}$ & $\begin{array}{l}1 . \\
2 .\end{array}$ & & $\begin{array}{l}13 \\
15\end{array}$ & $\begin{array}{l}70 . \\
20\end{array}$ & & $\begin{array}{l}28 \\
29\end{array}$ & $\begin{array}{r}5 . \\
20 .\end{array}$ & July & $\begin{array}{l}16 \\
24\end{array}$ & $\begin{array}{r}14 . \\
2 .\end{array}$ \\
\hline Mar. & $\begin{array}{r}27 \\
3\end{array}$ & $\begin{array}{r}3 \\
20\end{array}$ & & $\begin{array}{l}17 \\
18\end{array}$ & $\begin{array}{l}18 . \\
10 .\end{array}$ & Ap. & $\begin{array}{l}7 \\
8\end{array}$ & 6. & & $\begin{array}{l}25 \\
28\end{array}$ & 1 \\
\hline
\end{tabular}

No record october, November, December, January and September.

1957

\begin{tabular}{|c|c|c|c|c|c|c|c|c|c|c|}
\hline $\begin{array}{l}\text { Dec. } \\
\text { Jen. }\end{array}$ & $\begin{array}{r}24 \\
31 \\
5 \\
6 \\
7 \\
8 \\
12 \\
18 \\
19\end{array}$ & $\begin{array}{r}12 . \\
5 \\
3 \\
10 . \\
3 \\
7 \\
40 \\
2 . \\
3 .\end{array}$ & $\operatorname{Jan}$ & $\begin{array}{r}20 \\
23 \\
24 \\
25 \\
1 \\
5 \\
7 \\
8 \\
28\end{array}$ & $\begin{array}{r}11 . \\
2 . \\
53 . \\
6 . \\
8 . \\
5 . \\
2 . \\
3 . \\
3 .\end{array}$ & Mar. & $\begin{array}{r}8 \\
16 \\
17 \\
22 \\
26 \\
27 \\
4 \\
5 \\
10\end{array}$ & $\begin{array}{r}10 . \\
35 \\
13 . \\
2 . \\
13 \\
10 \\
15 \\
8 . \\
1 .\end{array}$ & $\begin{array}{l}\text { Apr. } \\
\text { May }\end{array}$ & $\begin{array}{r}28 \\
29 \\
2 \\
3 \\
4 \\
10\end{array}$ \\
\hline
\end{tabular}

No record for detober.

1958

\begin{tabular}{|c|c|c|c|c|c|c|c|c|c|c|}
\hline Nov. & $\begin{array}{l}18 \\
19 \\
22 \\
23 \\
24\end{array}$ & $\begin{array}{r}24 \\
19 \\
5 \\
7 \\
5\end{array}$ & Dec. & $\begin{array}{r}2 \\
5 \\
7 \\
9 \\
10\end{array}$ & $\begin{array}{r}5 . \\
17 . \\
15 . \\
6 . \\
18\end{array}$ & $\begin{array}{l}\text { Dec. } \\
\text { Jan. }\end{array}$ & $\begin{array}{r}27 \\
29 \\
9 \\
19 \\
24\end{array}$ & $\begin{array}{r}2 . \\
5 \\
20 \\
8 . \\
2 .\end{array}$ & $\begin{array}{l}\text { Jan. } \\
\text { Mar. }\end{array}$ & $\begin{array}{l}25 \\
31 \\
27\end{array}$ \\
\hline
\end{tabular}

1959

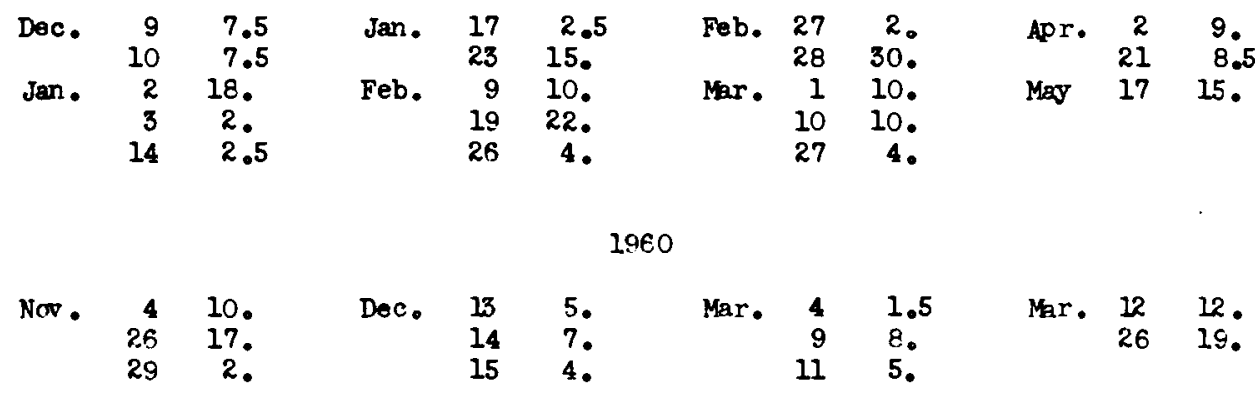

No record for April and May. 
Montbly and annual precipitation, in mill imeters, at Kajakal Reservoir at Kajakai, Afghanistan

\begin{tabular}{|c|c|c|c|c|c|c|c|c|c|c|c|c|c|}
\hline Year & oct. & Nov. & Dec. & Jan. & Feb. & Mar. & Apr. & Mgy & June & July & Aug. & sept. & An uaI \\
\hline $\begin{array}{l}1956 \\
1957 \\
1958 \\
1959 \\
1960\end{array}$ & $\begin{array}{r}00 \\
0 \\
0 \\
0\end{array}$ & $\begin{array}{c}0 \\
60.0 \\
0 \\
29.0\end{array}$ & $\begin{array}{l}17.0 \\
63.0 \\
15.0 \\
16.0\end{array}$ & $\begin{array}{c}140.0 \\
60.0 \\
40.0 \\
0\end{array}$ & $\begin{array}{c}23.0 \\
20.0 \\
0 \\
68.0 \\
0\end{array}$ & $\begin{array}{r}237.0 \\
83.0 \\
8.0 \\
24.0 \\
45.5\end{array}$ & $\begin{array}{c}16.0 \\
32.0 \\
0 \\
17.5 \\
916.0\end{array}$ & $\begin{array}{c}0 \\
14.0 \\
0 \\
15.0 \\
98.0\end{array}$ & $\begin{array}{l}0 \\
0 \\
0 \\
0 \\
0\end{array}$ & $\begin{array}{c}18.0 \\
0 \\
0 \\
0 \\
0\end{array}$ & $\begin{array}{l}0 \\
0 \\
0 \\
0 \\
0\end{array}$ & $\begin{array}{l}0 \\
0 \\
0 \\
0\end{array}$ & $\begin{array}{l}306.0 \\
191.0 \\
179.5 \\
114.5\end{array}$ \\
\hline Ave. & 0 & 22.2 & 27.8 & 60.0 & 22.2 & 79.5 & 16.3 & 7.4 & 0 & 3.6 & 0 & 0 & 197.8 \\
\hline
\end{tabular}

Manthly and amul pan evaporation in millimeters at Kajakal Reservolr, Af ghanistan

\begin{tabular}{|c|c|c|c|c|c|c|c|c|c|c|c|c|c|}
\hline Year & oct. & Nov. & Dec. & $\operatorname{Jan}$. & $\mathrm{Feb}$ & Mar. & Apr. & May & June & July & Auge & Sept. & Annual \\
\hline $\begin{array}{l}1956 \\
1957 \\
1958 \\
1959 \\
1950\end{array}$ & $\begin{array}{r}214 \\
176 \\
202 \\
264\end{array}$ & $\begin{array}{r}162 \\
92 \\
102 \\
166\end{array}$ & $\begin{array}{l}91 \\
46 \\
54 \\
91\end{array}$ & $\begin{array}{l}40 \\
81 \\
47 \\
76\end{array}$ & $\begin{array}{l}86 \\
84 \\
87 \\
67 \\
88\end{array}$ & $\begin{array}{r}71 \\
81 \\
152 \\
122 \\
143\end{array}$ & $\begin{array}{r}181 \\
130 \\
227 \\
221 \\
9190\end{array}$ & $\begin{array}{r}306 \\
197 \\
306 \\
270 \\
\text { e270 }\end{array}$ & $\begin{array}{l}405 \\
280 \\
379 \\
343 \\
347\end{array}$ & $\begin{array}{l}370 \\
330 \\
390 \\
430 \\
387\end{array}$ & $\begin{array}{l}389 \\
331 \\
368 \\
397 \\
103\end{array}$ & $\begin{array}{r}\infty 6 \\
302 \\
300 \\
73\end{array}$ & $\begin{array}{l}2206 \\
2606 \\
2555 \\
2198\end{array}$ \\
\hline Ave. & 214 & 130 & 70 & 61 & 82 & 114 & 190 & 270 & 351 & 381 & 318 & 235 & 2391 \\
\hline
\end{tabular}

- - Estimated. 
Location.-Observation station is 70 feet south of south and of Inited States Operations staff House in Jashiar Geh. Iat. $31^{\circ} 33^{\prime} \mathrm{N}_{0}$, long. $64^{\circ} 18 \mathrm{i}$., on east bank of helmand River about 8 kilometers ups treem from Arghand ab River.

Equipment.-Rain gage: A standard B-irich non-recoriing Us. Weather Bureau rain gage can and measuring stick.

Thermameters: Maximum and minimum Thermometers and support and a hydrothermograch for recording air temperature and humidity are in a wcoden shelter.

Eraporati on pan. $P$ an is ealvanized iron, round $p a n, 10$ inches deep and 48 inches in dlameter with an enamel gage caltbrated to centineters at one side. The pan sets on a brick base an the groind. Pan water is read to millimeters eacin day and has been refilled when level trops as much as $100 \mathrm{millimeters.}$

located.

A five foot high woven wire fence enc loses a $12 \times 14 \mathrm{ft}$. area whe re above equipment is

in kilaneters.

Averages and extremes for periad of record.--

January 1955 to September 1960

Temperatures: Average daily mexinum Average datly minimu

Maximum

Ninimum

$$
\begin{array}{r}
28.9^{\circ} \mathrm{C} \\
11.5^{\circ} \mathrm{C} \\
46.3^{\circ} \mathrm{C} \\
-6.0^{\circ} \mathrm{C}
\end{array}
$$

Preeloltation: Average yearly

$$
135.5 \text { millimeters }
$$

Evaporation: Average yearly pan

\section{2 millimeters}

Wind: Average velocity Maximum velocity

190 kilaneters per day

875 kilometers per day

Humidity: Average daily high Average daily low

$61.8 \%$

$20.7 \%$ 
Monthly and annual average maximum and minimum and maximum and minimum Temperatures in degres centigrade

at Lashkar Gah, Afghanistan

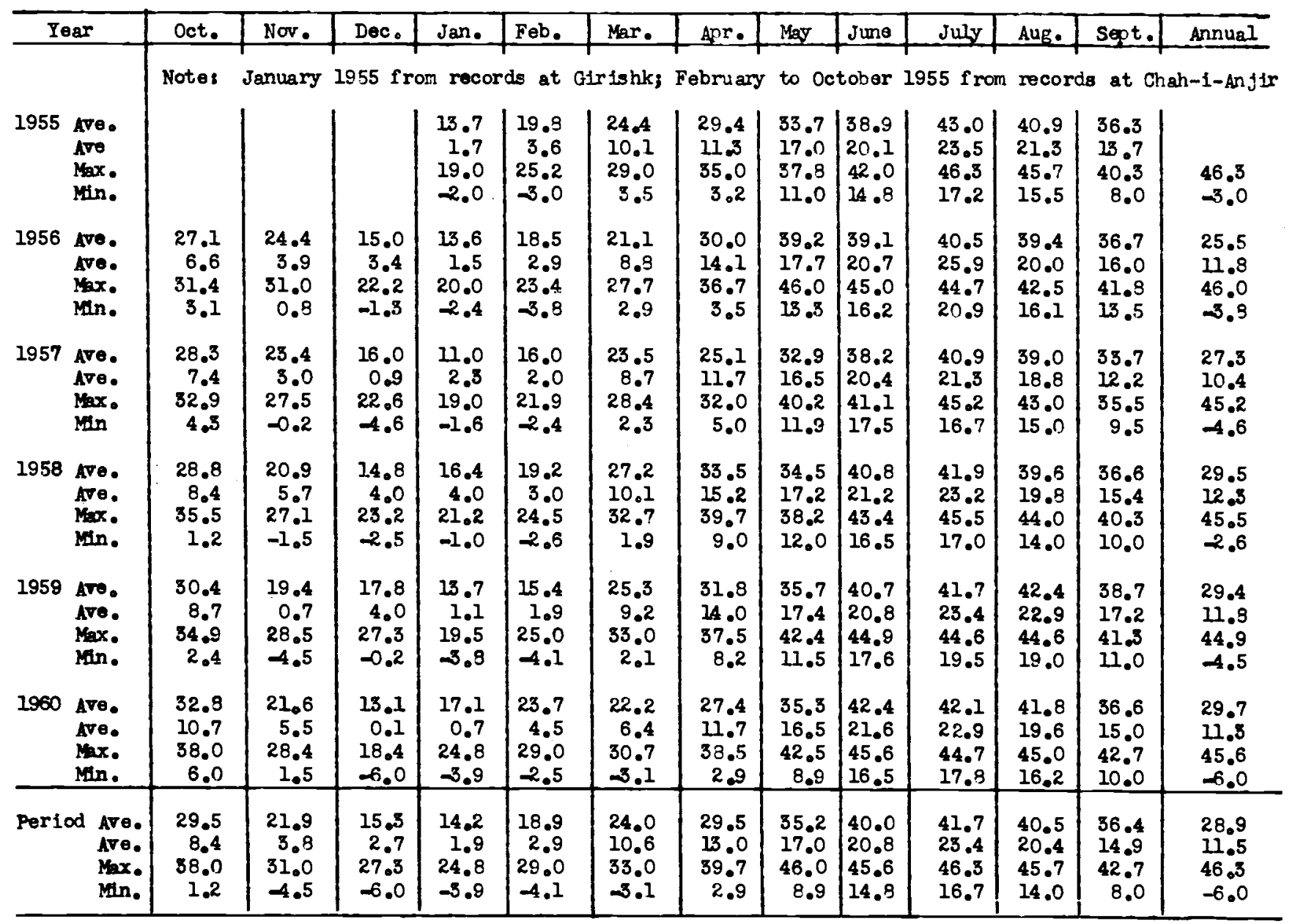


PRECIPITATTON IN MILITMETERS AT LASHKAR GAH, AFGHAN ISTAN

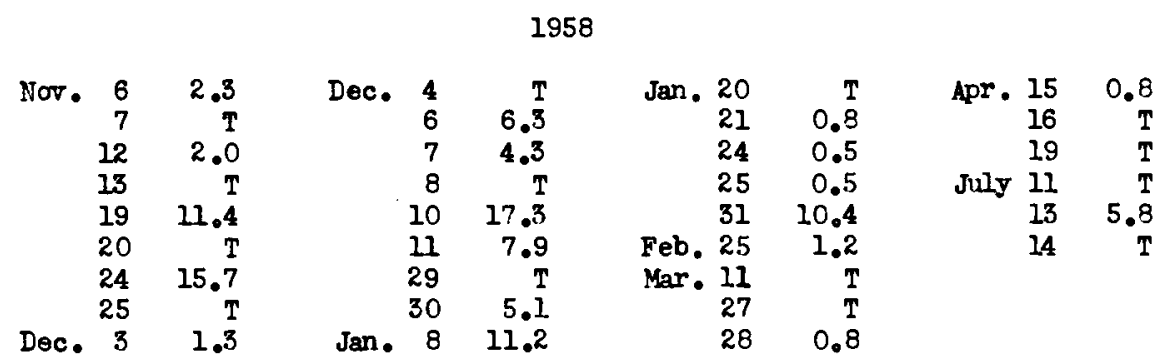

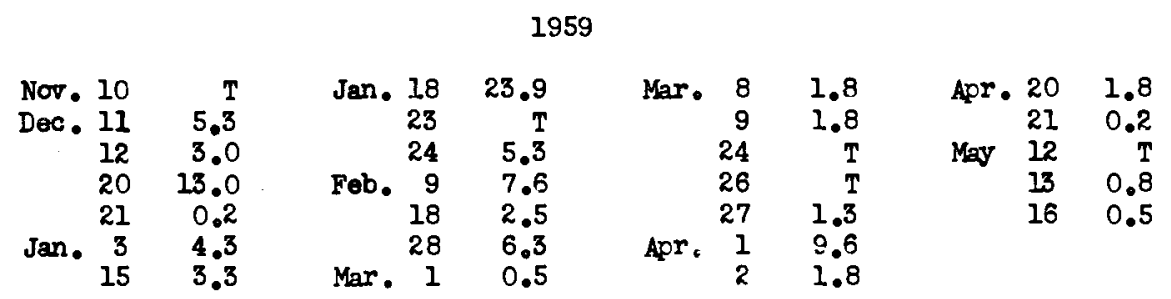

1960

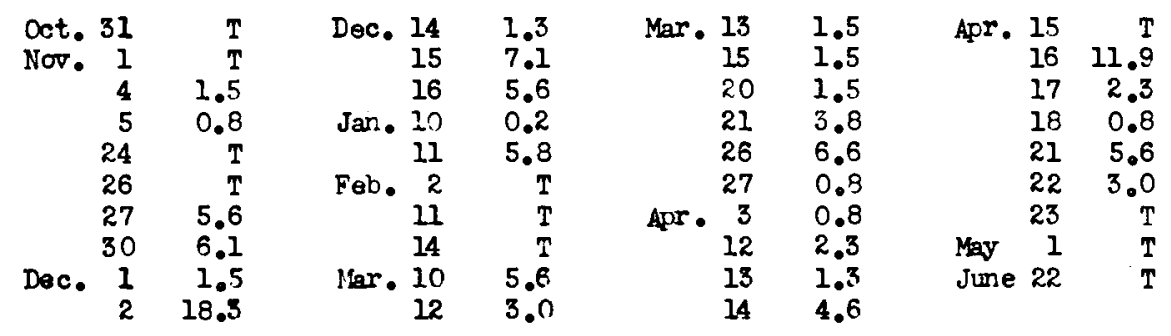

T - Trace 
PRECIPITATION IN MILLTMETERS AT TASHKAR GAH, AFGHANISTAN

1955

\begin{tabular}{|c|c|c|c|c|c|c|c|}
\hline $\begin{array}{r}\operatorname{Jan} . \quad 2 \\
5 \\
6 \\
10 \\
11 \\
20\end{array}$ & $\begin{array}{r}0.8 \\
5.1 \\
16.5 \\
1.5 \\
\mathrm{~T} \\
3.3\end{array}$ & $\begin{array}{r}\text { Jan. } 24 \\
28 \\
29 \\
30 \\
\text { Feb. } 3 \\
\text { Mar. } 10\end{array}$ & $\begin{array}{l}0.8 \\
2.0 \\
2.0 \\
2.3 \\
2.3 \\
T\end{array}$ & 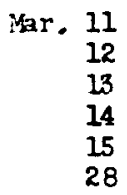 & $\begin{array}{r}1.2 \\
T \\
3.6 \\
12.2 \\
0.2 \\
0.2\end{array}$ & $\begin{array}{l}\text { Apr. } \\
\text { May }\end{array}$ & $\begin{array}{r}11 \\
4 \\
6 \\
2 \\
10\end{array}$ \\
\hline
\end{tabular}

1956

\begin{tabular}{|c|c|c|c|c|c|c|c|c|c|c|}
\hline Jan. & $\begin{array}{r}5 \\
6 \\
7 \\
8 \\
9 \\
13 \\
14 \\
15 \\
18 \\
20 \\
1 \\
2 \\
3\end{array}$ & $\begin{array}{r}6.3 \\
5.3 \\
T \\
8.6 \\
24.1 \\
5.1 \\
0.5 \\
T \\
0.2 \\
7.6 \\
7.1 \\
T\end{array}$ & $\begin{array}{rr}\text { Jan. } 10 \\
12 \\
14 \\
15 \\
16 \\
\\
26 \\
\text { Feb. } 1 \\
12 \\
25 \\
26 \\
27 \\
\\
\\
\text { Mar. } 4\end{array}$ & $\begin{array}{r}0.8 \\
1.8 \\
T \\
0.8 \\
T \\
T \\
T \\
2.0 \\
T \\
1.3 \\
1.5 \\
0.5 \\
2.5 \\
2.8\end{array}$ & $\begin{array}{r}\text { Mar. } \\
1 \\
1 \\
1 \\
1 \\
2 \\
2 \\
2 \\
2 \\
2 \\
2 \\
3 \\
\text { Apr. }\end{array}$ & $\begin{array}{r}5 \\
13 \\
14 \\
18 \\
19 \\
21 \\
22 \\
23 \\
24 \\
28 \\
29 \\
30 \\
1\end{array}$ & $\begin{array}{r}6.9 \\
2.8 \\
10.4 \\
4.6 \\
4.8 \\
3.8 \\
T \\
22.9 \\
T \\
T \\
6.3 \\
6.3 \\
T\end{array}$ & $\begin{array}{l}\text { Apr. } \\
\text { July }\end{array}$ & $\begin{array}{r}6 \\
7 \\
20 \\
23 \\
16 \\
13 \\
23 \\
25 \\
26 \\
29\end{array}$ & $\begin{array}{r}2.8 \\
20.1 \\
\mathrm{~T} \\
\mathrm{~T} \\
4.1 \\
0.8 \\
0.8 \\
19.3 \\
\mathrm{~T} \\
0.2\end{array}$ \\
\hline
\end{tabular}

1957

\begin{tabular}{|c|c|c|c|c|c|c|c|c|c|c|c|}
\hline $\begin{array}{l}\text { Oct. } \\
\text { Dec. } \\
\text { Jan. }\end{array}$ & $\begin{array}{l}30 \\
31 \\
13 \\
23 \\
24 \\
31 \\
1 \\
3 \\
4 \\
7 \\
8\end{array}$ & $\begin{array}{r}\mathrm{T} \\
8.9 \\
\mathrm{~T} \\
0.5 \\
2.0 \\
1.2 \\
9.4 \\
0.8 \\
0.2 \\
13.7 \\
5.3\end{array}$ & Feb. & $\begin{array}{r}9 \\
10 \\
12 \\
13 \\
14 \\
19 \\
20 \\
25 \\
26 \\
2 \\
3\end{array}$ & $\begin{array}{r}4.3 \\
0.2 \\
0.2 \\
21.3 \\
0.5 \\
0.8 \\
1.2 \\
23.4 \\
1.2 \\
8.6 \\
2.8\end{array}$ & $\begin{array}{l}\text { Feb. } \\
\text { Mar. } \\
\text { Apr. }\end{array}$ & $\begin{array}{r}6 \\
9 \\
17 \\
18 \\
21 \\
27 \\
30 \\
31 \\
1 \\
4 \\
5\end{array}$ & $\begin{array}{r}4.6 \\
7.1 \\
9.1 \\
8.4 \\
0.5 \\
5.3 \\
0.2 \\
0.2 \\
T \\
4.8 \\
18.3\end{array}$ & Apr & $\begin{array}{r}6 \\
25 \\
29 \\
30 \\
1 \\
2 \\
4 \\
11\end{array}$ & $\begin{array}{r}0.2 \\
0.2 \\
T \\
3.8 \\
0.5 \\
T \\
T \\
1.2\end{array}$ \\
\hline
\end{tabular}

T - Trace 
Manthly and amual precipitation, in millimeters, at Lashkar Gah, Afghanistan

\begin{tabular}{|c|c|c|c|c|c|c|c|c|c|c|c|c|c|}
\hline Year & oct. & Nov. & Dec. & $\operatorname{Jan}$ & Feb. & Mar. & Apr. & May & June & July & Aug. & Sept. & Amual \\
\hline $\begin{array}{l}1955 \\
1956 \\
1957 \\
1958 \\
1959 \\
1960\end{array}$ & $\begin{array}{c}0 \\
8.9 \\
0 \\
0 \\
T\end{array}$ & $\begin{array}{c}0 \\
0 \\
31.4 \\
0 \\
14.0\end{array}$ & $\begin{array}{r}50.1 \\
3.7 \\
42.2 \\
21.5 \\
33.8\end{array}$ & $\begin{array}{r}34.3 \\
18.1 \\
82.5 \\
23.4 \\
36.8 \\
6.0\end{array}$ & $\begin{array}{r}2.3 \\
7.8 \\
16.0 \\
1.2 \\
16.4 \\
T\end{array}$ & $\begin{array}{r}17.4 \\
71.6 \\
30.8 \\
0.8 \\
5.4 \\
24.3\end{array}$ & $\begin{array}{r}\mathrm{T} \\
22.9 \\
27.3 \\
0.8 \\
13.4 \\
32.6\end{array}$ & $\begin{array}{c}0.2 \\
0 \\
1.7 \\
0 \\
1.3 \\
T\end{array}$ & $\begin{array}{l}0 \\
0 \\
0 \\
0 \\
0 \\
T\end{array}$ & $\begin{array}{c}0 \\
25.2 \\
0 \\
5.8 \\
0 \\
0\end{array}$ & $\begin{array}{l}0 \\
0 \\
0 \\
0 \\
0 \\
0\end{array}$ & $\begin{array}{l}0 \\
0 \\
0 \\
0 \\
0 \\
0\end{array}$ & $\begin{array}{r}195.7 \\
170.9 \\
105.6 \\
94.8 \\
110.7\end{array}$ \\
\hline Ave. & 1.8 & 9.1 & 30.3 & 33.5 & 7.3 & 25.0 & 16.2 & 0.5 & 0 & 5.2 & 0 & 0 & 135.5 \\
\hline
\end{tabular}

Manthly and annual evapcrati on, in millimeters, at Lashkar Cah, Afghan istan

\begin{tabular}{|c|c|c|c|c|c|c|c|c|c|c|c|c|c|}
\hline Year & oct. & Nor. & Dec. & $\operatorname{Jan}$ & Feb. & Mar. & Apr. & Mgy & June & July & Aug. & Sept. & Ann uaI \\
\hline & Note: & $\begin{array}{l}\text { Apr il } \\
\text { These }\end{array}$ & $\begin{array}{l}\text { Octol } \\
\text { obs }\end{array}$ & $\begin{array}{l}1955 \\
d \mathrm{p}\end{array}$ & $\begin{array}{l}\mathrm{re} \\
\mathrm{rapor}\end{array}$ & $\begin{array}{l}\text { is at } \\
\text { on re }\end{array}$ & $h-1-$ & & & & & & \\
\hline $\begin{array}{l}1955 \\
1956\end{array}$ & ו0 & & & & & & 306 & 386 & 467 & $\begin{array}{l}446 \\
49 ?\end{array}$ & $\begin{array}{l}383 \\
392\end{array}$ & $\begin{array}{l}276 \\
295\end{array}$ & \\
\hline 1957 & 185 & 115 & 87 & 130 & 108 & 171 & 196 & 294 & 372 & 379 & 331 & 239 & 2602 \\
\hline 1958 & 191 & 139 & 94 & 77 & 108 & 216 & 274 & 354 & 395 & 405 & 356 & 254 & 2863 \\
\hline 1959 & 185 & 108 & 88 & 103 & 112 & 190 & 252 & 321 & 384 & 406 & 359 & 259 & 2762 \\
\hline 1960 & 186 & 114 & 75 & 80 & 137 & 174 & 224 & 325 & 369 & 376 & 334 & 257 & 2629 \\
\hline Are. & 190 & 118 & 90 & 98 & 120 & 165 & 252 & 350 & 413 & 406 & 360 & 260 & 2772 \\
\hline
\end{tabular}

T - Trace 
lionthly and annual average wind velocity and raximum velocity, in kiloneters, per day at Lashkar Gah Afghan is tan

\begin{tabular}{|c|c|c|c|c|c|c|c|c|c|c|c|c|c|}
\hline Year & oct. & Nov. & Dec. & $\operatorname{San}$. & Feb. & Mar。 & Apr. & May & June & July & Aug. & Sert. & Annual \\
\hline & Notes & July & octobe & 1955 & $\mathbf{r e c}$ & at & $1-1-A n$ & & & & & & \\
\hline 1955 & & & & & & & & & & $\begin{array}{l}164 \\
304\end{array}$ & $\begin{array}{l}116 \\
224\end{array}$ & $\begin{array}{r}76 \\
257\end{array}$ & \\
\hline 1956 & 66 & & 198 & 162 & 218 & 208 & 234 & 190 & 236 & 198 & 169 & 147 & 184 \\
\hline & $10 R$ & & 612 & 359 & 495 & 329 & 739 & 453 & 538 & 397 & 244 & 249 & 739 \\
\hline 1957 & 180 & 145 & 206 & 211 & 219 & 204 & 217 & 235 & 259 & 198 & 183 & 153 & 201 \\
\hline & 319 & 352 & 796 & 413 & 409 & 555 & 465 & 376 & 482 & 367 & 323 & 223 & 796 \\
\hline 1958 & 140 & 202 & 157 & 198 & 193 & 218 & 185 & 243 & 216 & 189 & 203 & 192 & 195 \\
\hline & 571 & 467 & 439 & 521 & 728 & 533 & 402 & 368 & 720 & 331 & 364 & 296 & 728 \\
\hline 1959 & 182 & 176 & 185 & 204 & 285 & 227 & 201 & 210 & 233 & 215 & 185 & 160 & 205 \\
\hline & 351 & 522 & $32 ?$ & 453 & 583 & 456 & 340 & 571 & 496 & 417 & 295 & 267 & 588 \\
\hline 1960 & 143 & 160 & 130 & 169 & 156 & 238 & 220 & 211 & 166 & 129 & 136 & 114 & 164 \\
\hline & 283 & 341 & 341 & 379 & 292 & 503 & 536 & 559 & 340 & 283 & 875 & 237 & 875 \\
\hline $\begin{array}{l}\text { Are. } \\
\text { Max. }\end{array}$ & $\begin{array}{l}142 \\
571\end{array}$ & $\begin{array}{l}171 \\
522\end{array}$ & $\begin{array}{l}175 \\
796\end{array}$ & $\begin{array}{l}189 \\
521\end{array}$ & $\begin{array}{l}214 \\
728\end{array}$ & $\begin{array}{l}21 \varepsilon \\
555\end{array}$ & $\begin{array}{l}211 \\
739\end{array}$ & $\begin{array}{l}218 \\
571\end{array}$ & $\begin{array}{l}222 \\
720\end{array}$ & $\begin{array}{l}182 \\
417\end{array}$ & $\begin{array}{l}165 \\
875\end{array}$ & $\begin{array}{l}140 \\
296\end{array}$ & $\begin{array}{l}190 \\
875\end{array}$ \\
\hline
\end{tabular}

Monthly and annual avorage relative humidity - high and low, in percent, at Iashkar Geh, Afghanistan

\begin{tabular}{|c|c|c|c|c|c|c|c|c|c|c|c|c|c|}
\hline Year & $\cot _{0}$ & Nov. & Dec. & Jan. & Feb. & Mar. & Apr. & May & June & Juty & AUE. & Sept. & Annul \\
\hline & Note: & Januar & 1955 & rece & at G & shk & Febru & to $\alpha$ & ber 10 & are & ords & Chah-1 & jir \\
\hline 1955 & & & & 79.3 & & & 33.0 & 43.3 & 35.7 & 24.4 & 37.4 & 37.5 & \\
\hline 1956 & $\begin{array}{l}47.3 \\
18.2\end{array}$ & $\begin{array}{l}64.6 \\
24.1\end{array}$ & $\begin{array}{l}89.3 \\
40.4\end{array}$ & $\begin{array}{l}50.2 \\
91.7 \\
35.5\end{array}$ & $\begin{array}{l}78.2 \\
21.8\end{array}$ & $\begin{array}{l}92.2 \\
35.2\end{array}$ & $\begin{array}{r}78.7 \\
22.7\end{array}$ & $\begin{array}{l}17.1 \\
50.2 \\
13.3\end{array}$ & $\begin{array}{l}10.0 \\
40.2 \\
16.3\end{array}$ & $\begin{array}{r}9.5 \\
60.9 \\
21.7\end{array}$ & $\begin{array}{l}13.1 \\
41.1 \\
11.8\end{array}$ & $\begin{array}{l}14.1 \\
47.0 \\
15.3\end{array}$ & $\begin{array}{l}65.1 \\
23.1\end{array}$ \\
\hline 1957 & $\begin{array}{l}54.9 \\
19.4\end{array}$ & $\begin{array}{l}56.2 \\
15.5\end{array}$ & $\begin{array}{l}68.0 \\
27.5\end{array}$ & $\begin{array}{l}83.5 \\
43.5\end{array}$ & $\begin{array}{l}77.5 \\
19.5\end{array}$ & $\begin{array}{l}75.4 \\
18.0\end{array}$ & $\begin{array}{l}76.5 \\
21.4\end{array}$ & $\begin{array}{l}59.8 \\
15.2\end{array}$ & $\begin{array}{l}45.1 \\
14.7\end{array}$ & $\begin{array}{l}40.5 \\
16.0\end{array}$ & $\begin{array}{l}47.0 \\
16.8\end{array}$ & $\begin{array}{l}46.9 \\
18.8\end{array}$ & $\begin{array}{l}60.9 \\
20.5\end{array}$ \\
\hline 1958 & $\begin{array}{l}58.1 \\
2.4 .2\end{array}$ & $\begin{array}{l}75.3 \\
28.6\end{array}$ & $\begin{array}{l}86.7 \\
41.0\end{array}$ & $\begin{array}{l}82.1 \\
29.8\end{array}$ & $\begin{array}{l}76.0 \\
14.5\end{array}$ & $\begin{array}{l}54.1 \\
14.0\end{array}$ & $\begin{array}{l}51.8 \\
14.4\end{array}$ & $\begin{array}{l}45.4 \\
16.3\end{array}$ & $\begin{array}{l}37.6 \\
14.4\end{array}$ & $\begin{array}{l}38.5 \\
14.1\end{array}$ & $\begin{array}{l}38.9 \\
13.9\end{array}$ & $\begin{array}{l}46.7 \\
16.6\end{array}$ & $\begin{array}{l}57.6 \\
20.2\end{array}$ \\
\hline 1959 & $\begin{array}{l}46.3 \\
19.0\end{array}$ & $\begin{array}{l}59.9 \\
27.8\end{array}$ & $\begin{array}{l}87.9 \\
41.9\end{array}$ & $\begin{array}{l}94.5 \\
33.0\end{array}$ & $\begin{array}{r}56.4 \\
23.9\end{array}$ & $\begin{array}{l}79.8 \\
19.3\end{array}$ & $\begin{array}{l}68.6 \\
16.9\end{array}$ & $\begin{array}{l}59.4 \\
14.8\end{array}$ & $\begin{array}{l}40.1 \\
12.1\end{array}$ & $\begin{array}{l}40.7 \\
15.9\end{array}$ & $\begin{array}{l}42.8 \\
23.3\end{array}$ & $\begin{array}{l}63.5 \\
15.3\end{array}$ & $\begin{array}{l}64.2 \\
21.9\end{array}$ \\
\hline 1960 & $\begin{array}{l}55.4 \\
17.5\end{array}$ & $\begin{array}{l}76.3 \\
23.1\end{array}$ & $\begin{array}{l}92.7 \\
31.9\end{array}$ & $\begin{array}{l}79.7 \\
19.7\end{array}$ & $\begin{array}{l}69.4 \\
14.2\end{array}$ & $\begin{array}{l}69.9 \\
13.9\end{array}$ & $\begin{array}{l}72.7 \\
16.8\end{array}$ & $\begin{array}{l}50.6 \\
11.8\end{array}$ & $\begin{array}{l}\text { e39.7 } \\
\text { el4.2 }\end{array}$ & $\begin{array}{l}40.0 \\
16 . ?\end{array}$ & $\begin{array}{l}42.0 \\
14.8\end{array}$ & $\begin{array}{l}48.0 \\
16.3\end{array}$ & $\begin{array}{l}61.4 \\
17.6\end{array}$ \\
\hline $\begin{array}{l}\text { Ave. } \\
\text { Ave. }\end{array}$ & $\begin{array}{l}52.4 \\
19.9\end{array}$ & $\begin{array}{l}66.5 \\
23.8\end{array}$ & $\begin{array}{l}34.8 \\
36.5\end{array}$ & $\begin{array}{l}85.1 \\
36.3\end{array}$ & $\begin{array}{l}73.2 \\
20.3\end{array}$ & $\begin{array}{l}71.3 \\
21.7\end{array}$ & $\begin{array}{l}63.6 \\
18.2\end{array}$ & $\begin{array}{l}51.4 \\
14.8\end{array}$ & $\begin{array}{l}39.7 \\
14.2\end{array}$ & $\begin{array}{l}40.8 \\
15.7\end{array}$ & $\begin{array}{l}41.5 \\
15.7\end{array}$ & $\begin{array}{l}48.3 \\
16.1\end{array}$ & $\begin{array}{l}61.8 \\
20.7\end{array}$ \\
\hline
\end{tabular}

e - estimated. 
MARJA, AFGHANIS TAN

Temperatire, Precipitation, Evaporation

Location.-Observation station is at radio tower near office building 200 meters west of east antrance to Marja. Iat $31^{\circ} 30^{\prime} \mathrm{N}$, , long $64^{\circ}$ OR $\mathrm{E}$, , about 25 kilameters west of Lashkar Gah about 48 kilameters southwest of Girishk.

Equipment.-Rain gage: A standard 8-inch non-recording U.S. Weather Bureau rain gage can and measuring stick. Centigrade scales.

Thermometers: Maximum and minimum thermameters and support are in a standard wooden shelter.

Evaporation pan: Pan was iron, $85 \mathrm{~cm}$. in diameter, $30 \mathrm{~cm}$. deep and set in ground. Depth of water over reference point was read to millimeters daily. Pan was refilled when water level dropped as much as 100 millimeters.

Averages and extremes for period of record: -

February 1955 to $\mathrm{May} 1957$

Temperaturess Average daily maximum Average dally minimum

Meximum

Minimum

Precipitation: Average yearly

Evaporation: Average yearly pan

$$
\begin{aligned}
& 28.9^{\circ} \mathrm{C} \\
& 13.0^{\circ} \mathrm{C} \\
& 48.4^{\circ} \mathrm{C} \\
& -3.3^{\circ} \mathrm{C}
\end{aligned}
$$

159.9 millimeters

3145 millimeters 
Manthly and annual averago maximum and minimum and maximum and minimum Temperatures in degrees centigrade at Marja, Afghanistan

\begin{tabular}{|c|c|c|c|c|c|c|c|c|c|c|c|c|c|c|}
\hline Yea & & oct. & Nov. & Dec. & $\operatorname{Jan}$ & Feb. & 潐r。 & Apr. & May & June & JuIy & Aug. & Sept. & Annual \\
\hline 1955 & $\begin{array}{l}\text { Ave. } \\
\text { Ave. } \\
\text { Max. } \\
\text { Min. }\end{array}$ & & & & Started & $\begin{array}{r}22.3 \\
7.7 \\
27.2 \\
-2.2\end{array}$ & $\begin{array}{r}24.8 \\
14.0 \\
27.8 \\
7.2\end{array}$ & $\begin{array}{l}30.0 \\
16.1 \\
35.6 \\
10.0\end{array}$ & $\begin{array}{l}33.9 \\
20.3 \\
37.8 \\
16.1\end{array}$ & $\begin{array}{l}37.8 \\
22.8 \\
41.7 \\
17.2\end{array}$ & $\begin{array}{l}41.7 \\
26.7 \\
46.1 \\
22.2\end{array}$ & $\begin{array}{l}38.9 \\
23.9 \\
44.4 \\
19.4\end{array}$ & $\begin{array}{l}35.2 \\
18.5 \\
38.7 \\
14.0\end{array}$ & \\
\hline 1956 & $\begin{array}{l}\text { Ave. } \\
\text { Ave. } \\
\text { Nax. } \\
\text { Min. }\end{array}$ & $\begin{array}{r}27.2 \\
11.4 \\
32.0 \\
7.2\end{array}$ & $\begin{array}{r}22.5 \\
7.8 \\
28.0 \\
4.4\end{array}$ & $\begin{array}{r}16.5 \\
5.4 \\
22.0 \\
0.0\end{array}$ & $\begin{array}{r}15.2 \\
1.5 \\
21.7 \\
-2.8\end{array}$ & $\begin{array}{r}19.4 \\
3.7 \\
25.6 \\
2.2\end{array}$ & $\begin{array}{r}21.7 \\
9.2 \\
28.9 \\
2.8\end{array}$ & $\begin{array}{r}30.6 \\
14.4 \\
36.7 \\
3.9\end{array}$ & $\begin{array}{l}41.4 \\
19.4 \\
48.4 \\
15.0\end{array}$ & $\begin{array}{l}41.7 \\
21.4 \\
46.7 \\
17.8\end{array}$ & $\begin{array}{l}41.2 \\
26.1 \\
45.0 \\
22.2\end{array}$ & $\begin{array}{l}40.0 \\
20.0 \\
43.3 \\
17.2\end{array}$ & $\begin{array}{l}38.0 \\
16.2 \\
41.2 \\
14.4\end{array}$ & $\begin{array}{l}29.6 \\
13.0 \\
48.4 \\
-2.8\end{array}$ \\
\hline 1957 & $\begin{array}{l}\text { Ave. } \\
\text { Ave. } \\
\text { Max. } \\
\operatorname{Man} .\end{array}$ & $\begin{array}{r}30.2 \\
7.9 \\
37.8 \\
5.0\end{array}$ & $\begin{array}{r}24.4 \\
4.0 \\
28.4 \\
0.6\end{array}$ & $\begin{array}{r}16.1 \\
0.6 \\
22.8 \\
-3.3\end{array}$ & $\begin{array}{r}11.1 \\
2.0 \\
20.0 \\
2.2\end{array}$ & $\begin{array}{r}16.1 \\
1.7 \\
22.8 \\
2.8\end{array}$ & $\begin{array}{r}23.9 \\
8.9 \\
28.4 \\
3.3\end{array}$ & $\begin{array}{r}26.1 \\
12.2 \\
31.1 \\
5.0\end{array}$ & $\begin{array}{l}34.5 \\
16.7 \\
41.2 \\
12.2\end{array}$ & Closed & & & & \\
\hline $\begin{array}{l}\text { Ave. } \\
\text { Ave. }\end{array}$ & $\begin{array}{l}\text { Mex. } \\
\text { Min. } \\
\text { Max. } \\
\text { Min. }\end{array}$ & $\begin{array}{r}28.7 \\
9.6 \\
37.8 \\
5.0\end{array}$ & $\begin{array}{r}23.4 \\
5.3 \\
28.4 \\
0.6\end{array}$ & $\begin{array}{r}16.3 \\
3.0 \\
22.8 \\
-3.3\end{array}$ & $\begin{array}{r}13.2 \\
1.8 \\
21.7 \\
2.8\end{array}$ & $\begin{array}{r}19.3 \\
4.4 \\
25.6 \\
-2.8\end{array}$ & $\begin{array}{r}23.4 \\
10.7 \\
28.9 \\
2.8\end{array}$ & $\begin{array}{r}28.9 \\
14.2 \\
36.7 \\
3.9\end{array}$ & $\begin{array}{l}36.6 \\
18.8 \\
48.4 \\
12.2\end{array}$ & $\begin{array}{l}39.8 \\
22.1 \\
46.7 \\
17.2\end{array}$ & $\begin{array}{l}41.4 \\
26.4 \\
46.1 \\
22.2\end{array}$ & $\begin{array}{l}39.4 \\
22.0 \\
44.4 \\
17.2\end{array}$ & $\begin{array}{l}36.6 \\
17.4 \\
41.2 \\
14.0\end{array}$ & $\begin{array}{l}28.9 \\
13.0 \\
48.4 \\
-3.3\end{array}$ \\
\hline
\end{tabular}


PRECIPITATION IN MULIMETERS AT MAR'A, AFGHANISTAN

1955

$$
\begin{array}{rr}
\operatorname{Jan} . \quad \begin{array}{r}
4 \\
5
\end{array} & 5.0 \\
10 & 19.0
\end{array}
$$

$$
\begin{array}{lr}
\operatorname{Jan} .19 & 2.5 \\
\text { Mar. } 4 & 1.5 \\
11 & 1.1
\end{array}
$$

Mar. 13

$$
\begin{array}{cc}
13 & 1.6 \\
14 & 6.9 \\
23 & T
\end{array}
$$

1956
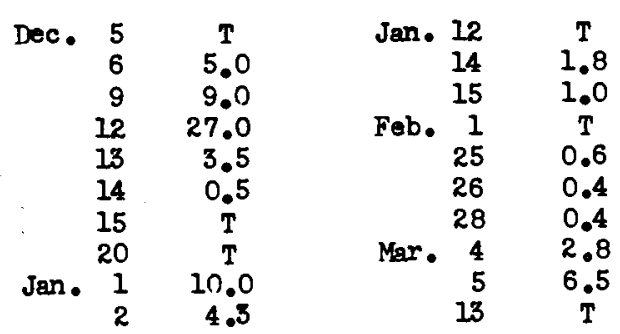

Har. 14

$\begin{array}{ll}14 & 3.5 \\ 15 & 5.6 \\ 19 & 8.3 \\ 20 & 5 . \\ 21 & 5.5\end{array}$

3.5
5.6
8.3
5.4
5.5
0.5

0.5
10.5

$\begin{array}{lc}24 & 19.5 \\ 25 & \mathrm{~T} \\ 29 & \mathrm{~T} \\ 30 & 16.3\end{array}$

1957

$$
\begin{array}{rr}
\text { Det. } 29 & \multicolumn{1}{c}{\text { T }} \\
30 & 12.1 \\
\text { Dec. } 23 & 2.7 \\
30 & 1.1 \\
31 & 6.5 \\
\text { Jan. } 2 & 0.8 \\
6 & 12.5 \\
7 & 5.5 \\
8 & 2.8
\end{array}
$$

$\begin{array}{rc}\text { Jan. } 12 & 23.5 \\ 18 & \mathrm{~T} \\ 19 & \mathrm{~T} \\ 23 & \mathrm{~T} \\ 24 & 23.3 \\ 25 & \mathrm{~T} \\ \text { Fob. } & 8.0 \\ 1 & 2.0 \\ 2 & 1.0 \\ 5 & 1.0\end{array}$

$\begin{array}{rc}\text { Mar. } 8 & 7.4 \\ 9 & T \\ 16 & 13.0 \\ 17 & 11.3 \\ 26 & 4.2 \\ 27 & T \\ 29 & T \\ 3 & 7.7 \\ 4 & 16.4\end{array}$

Apr. $\begin{array}{rc}5 & \mathrm{~T} \\ 13 & 1.0 \\ 14 & 0.5 \\ 30 & \mathrm{~T} \\ 1 & 5.5 \\ 9 & \mathrm{~T} \\ 10 & \mathrm{~T}\end{array}$

T - Trace 
Manthly and annul precipitati on, in millimeters, at $\mathrm{M}_{a} \mathbf{r} \mathbf{j a}$, Afghanistan

\begin{tabular}{|c|c|c|c|c|c|c|c|c|c|c|c|c|c|}
\hline Year & oct. & Nor. & Dec. & Jen. & Feb. & Mar. & Apr. & May & June & July & Aug. & sept. & Annul \\
\hline $\begin{array}{l}1955 \\
1956 \\
1957\end{array}$ & $\begin{array}{c}0 \\
12.1\end{array}$ & $\begin{array}{l}0 \\
0\end{array}$ & $\begin{array}{l}45.0 \\
10.3\end{array}$ & $\begin{array}{l}27.5 \\
17.1 \\
68.4\end{array}$ & $\begin{array}{c}0 \\
1.4 \\
11.0\end{array}$ & $\begin{array}{l}11.1 \\
73.9 \\
35.9\end{array}$ & $\begin{array}{c}0 \\
9.6 \\
25.6\end{array}$ & $\begin{array}{c}T \\
0 \\
5.5\end{array}$ & $\begin{array}{l}0 \\
0\end{array}$ & $\begin{array}{c}0 \\
12.9\end{array}$ & $\begin{array}{l}0 \\
0\end{array}$ & $\begin{array}{l}0 \\
0\end{array}$ & 159.9 \\
\hline Ave. & 6.0 & 0 & 27.6 & 37.7 & 4.1 & 40.3 & 11.7 & 1.8 & 0 & 6.4 & 0 & 0 & \\
\hline
\end{tabular}

T - Trace

Monthly and amual evaporation in millimeters at Marja, Afghan istan

\begin{tabular}{l|c|c|c|c|c|c|c|c|c|c|c|c|c|c|}
\hline Year & Oct. & Nov. & Dec. & Jan. & Feb. & Nar. & Apr. & Mgy & June & July & Aug. & Sept. & Armual \\
1955 & & & & Started & 137 & 176 & 284 & 392 & 421 & 480 & 476 & 357 & 23315 \\
1956 & 240 & 178 & 102 & 108 & 141 & 161 & 249 & 458 & 468 & 446 & 431 & 354 & 3336 \\
1957 & 219 & 155 & 111 & 70 & 111 & 179 & 231 & 392 & closed & & \\
Ave. & 230 & 166 & 106 & 89 & 130 & 172 & 255 & 414 & 444 & 463 & 454 & 356 & 3145 \\
\hline
\end{tabular}

a - Used average of 1956, 1957 for October to January.

b - Used average of 1955, 1956 for hme to september. 


\section{NAD-I-ALI, AFGHANISTAN}

Temperature, Precipitation, Evaporation, Wind Velocity, Hunidity

Iocation.-Observation station was at Nad-i-Al1 Agriculture Training School. Lat $31^{\circ} 38^{\prime} \mathrm{N} \cdot$,

Iong $64^{\circ} 151 \mathrm{Z}$. about 12 kilometers northwest of Lashkar Gah and 36 kilameters southwest of Girishk. Bquipment.-Rain gage: A standard 8-inch non-recording U.S. Weather Bureau rain gage can and measuring

Thermometers: Maxinum and minimum thermaneters and support and a hydrothermograph for recording air temperature and humidity in a standard wooden shelter.

Evaporation pan: Pan was of welded steel plate, 36 inches square and $193 / 4$ inches deep set
an bricks on the ground.

It was calibrated in kilometers and returned to neror the evaporation pan and about 5 feet above it.

Averages and extremes for period of record.-

June 1954 to Merch 1957

February to May 1958

Texperatures, Average datly maximim

Average daily minimum

Maximum

$28.4^{\circ} \mathrm{C}$

$12.00 \mathrm{C}$

Hinimur

$46.0^{\circ} \mathrm{C}$

Presipitation: Average yearly 3 years

213 millimeters

Evaporation: Average yearly pan 2 year:

2298 millimeters

Wind Average velocity 2 years Haximum velocity

180 kilometers per day

997 kilometers per doy

Hundity: Arerage dally high 1956

Average datly low

$74.0 \%$

$24.5 \%$ 
Monthly and annual average maximum and minimun and maximum and minimum temperatures in degrees centigrado at Nad-1-All, Afghanistan

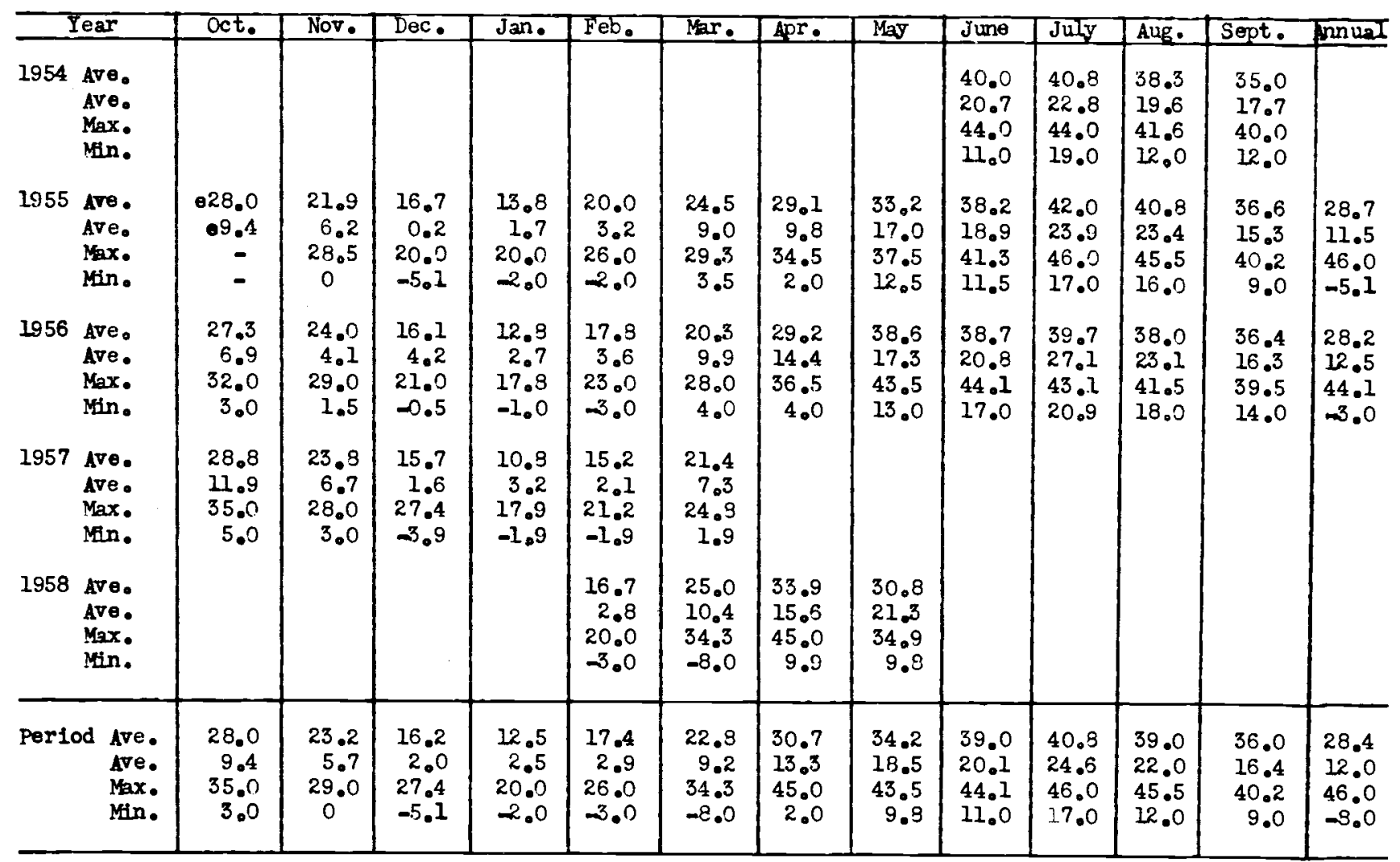

e - Estimated. 
PRECIPITATION IN MILCDMETERS AT NAD-I-AII, AFGHANISTAN

1955

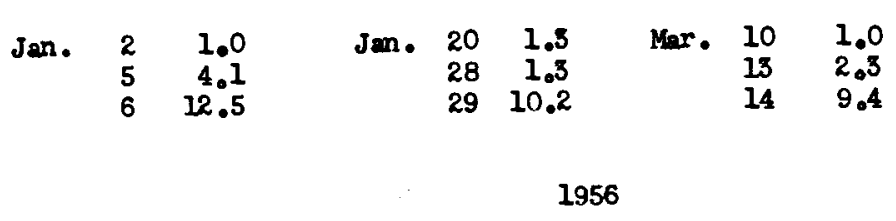

\begin{tabular}{|c|c|c|c|c|c|c|c|c|c|c|}
\hline Dec. & $\begin{array}{r}7 \\
10 \\
13 \\
14 \\
1 \\
2 \\
12\end{array}$ & $\begin{array}{r}3.6 \\
7.6 \\
25.4 \\
5.1 \\
7.1 \\
6.9 \\
1.5\end{array}$ & $\begin{array}{l}\text { Jan. } \\
\text { Feb. }\end{array}$ & $\begin{array}{r}16 \\
2 \\
26 \\
28 \\
4 \\
5 \\
14\end{array}$ & $\begin{array}{l}2.0 \\
2.0 \\
3.0 \\
3.3 \\
3.8 \\
7.6 \\
2.5\end{array}$ & $\operatorname{Mar}$. & $\begin{array}{r}15 \\
19 \\
21 \\
23 \\
30 \\
31 \\
5\end{array}$ & $\begin{array}{r}8.1 \\
6.4 \\
3.6 \\
15.5 \\
10.2 \\
21.9 \\
2.5\end{array}$ & $\begin{array}{l}\text { Apr. } \\
\text { July }\end{array}$ & $\begin{array}{r}6 \\
7 \\
16 \\
24 \\
25 \\
29\end{array}$ \\
\hline
\end{tabular}

\section{7}

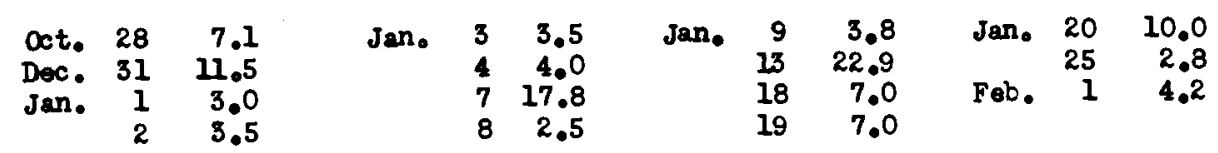


Nonthly and annual precipitation, in millineters, at Nad-i-Al1, Afghanistan

\begin{tabular}{|c|c|c|c|c|c|c|c|c|c|c|c|c|c|}
\hline Year & oct. & Nor. & Dec。 & Jan。 & $\mathrm{Feb}$. & Mar。 & Apr. & May & June & JuLy & Aug. & Sept. & mnual \\
\hline $\begin{array}{l}1955 \\
1956 \\
1957\end{array}$ & $\begin{array}{l}0 \\
0 \\
7.1\end{array}$ & $\begin{array}{l}0 \\
0 \\
0\end{array}$ & $\begin{array}{c}0 \\
41.7 \\
21.5\end{array}$ & $\begin{array}{l}25.5 \\
17.5 \\
87.5\end{array}$ & $\begin{array}{l}0 \\
8.3 \\
4.2\end{array}$ & $\begin{array}{c}12.7 \\
79.6 \\
0\end{array}$ & $\begin{array}{c}0 \\
7.8 \\
e 4.0\end{array}$ & $\begin{array}{c}0 \\
0 \\
e 1.0\end{array}$ & $\begin{array}{r}0 \\
0 \\
0\end{array}$ & $\begin{array}{l}0 \\
30.5 \\
\mathrm{eO}\end{array}$ & $\begin{array}{r}0 \\
0 \\
0\end{array}$ & $\begin{array}{r}0 \\
0 \\
0\end{array}$ & $\begin{array}{r}38.2 \\
185.4 \\
115.6\end{array}$ \\
\hline Ave。 & 2.4 & 0 & 17.7 & 43.6 & 4.2 & 30.8 & 3.9 & 0.3 & 0 & 10.2 & 0 & 0 & 113.1 \\
\hline
\end{tabular}

- Estimated.

Monthly and annual evaporation in millimeters at Nad-1-Al1, Afghanistan

\begin{tabular}{|c|c|c|c|c|c|c|c|c|c|c|c|c|c|}
\hline Year & Oct. & Nov. & Bec o & $\operatorname{Jan}_{0}$ & Feb. & Mar. & Apr. & May & June & July & Aug. & Sept. & Annual \\
\hline $\begin{array}{l}1.954 \\
1.955 \\
1956 \\
1957\end{array}$ & $\begin{array}{l}144 \\
140 \\
117\end{array}$ & $\begin{array}{l}70 \\
89 \\
65\end{array}$ & $\begin{array}{r}52 \\
89 \\
117\end{array}$ & $\begin{array}{l}67 \\
79 \\
88\end{array}$ & $\begin{array}{l}77 \\
46 \\
95\end{array}$ & $\begin{array}{r}182 \\
125 \\
90\end{array}$ & $\begin{array}{l}266 \\
246\end{array}$ & $\begin{array}{r}e 300 \\
285\end{array}$ & $\begin{array}{l}429 \\
330 \\
306\end{array}$ & $\begin{array}{l}388 \\
394 \\
305\end{array}$ & $\begin{array}{l}355 \\
315 \\
290\end{array}$ & $\begin{array}{l}300 \\
210 \\
190\end{array}$ & $\begin{array}{l}2407 \\
2190\end{array}$ \\
\hline Ave. & 134 & 75 & 86 & 78 & 73 & 132 & 256 & 292 & 355 & 362 & 320 & 233 & 2298 \\
\hline
\end{tabular}

e - Estimated. 
Monthly and annual average wind velocity and maximum velocity, in kilometers, per day at Nad-i-hli, Afghanistan

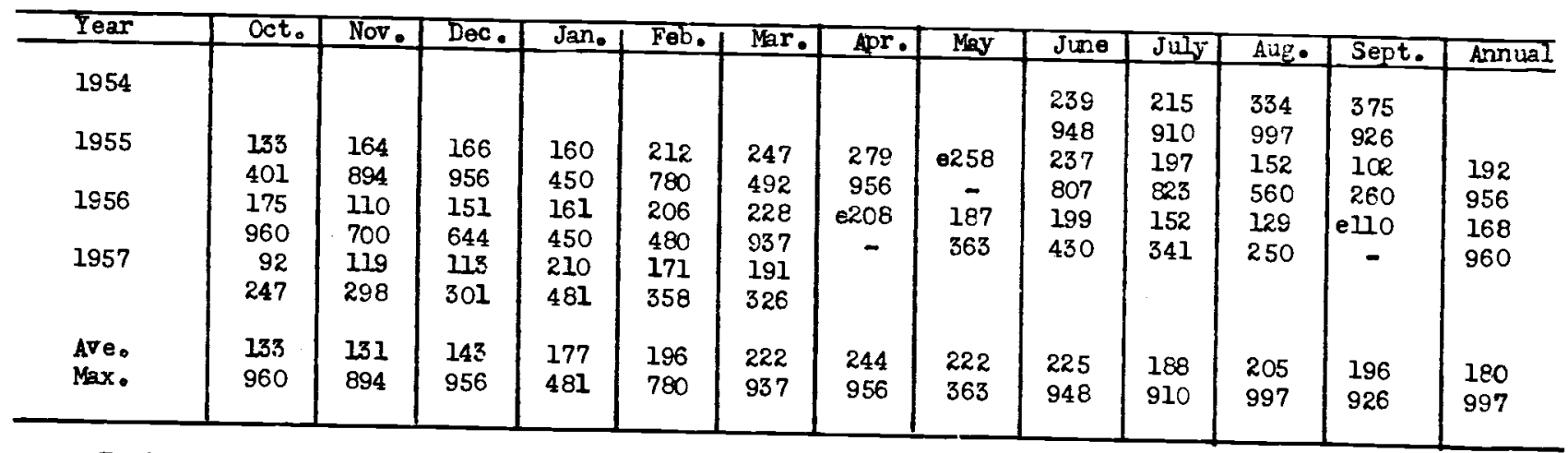

- Estimated.

Monthly and annual average relative humidity - high and low, in percent, at Nad-1-Al1, Afghanis tan

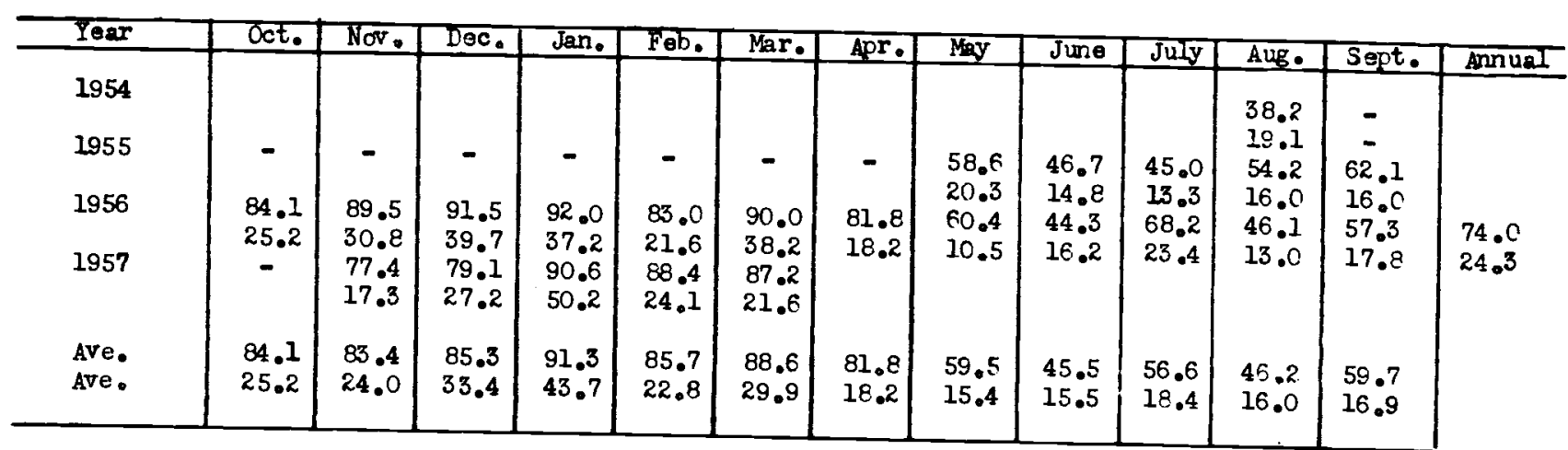


HELMAND RTVER BASIN - AFGHANISTAN

\section{Snow Surveys $1954-1960$}

General.-The snow courses are located along the Kandahar-Kabul highway and ut1lize the 25-foot 8 teel pole, 4-wire telephane line as reference. The poles are about 150 feet 9 art and 10 poles give a
good representative course for the vallog.

Course No. 1.-Aproximately 3 miles north of Makr; elevation 6,800 feet; use 10 poles of line which parallels the highwey and is about 300 feet to the west.

Course No. 2.-Approximately 2 miles north of Mashaki; elevation 7,000 feet; use 10 poles of telephone ine to west of raad. Course extends north from initial pole which is marked No. 1 with red paint and is 4 poles south of guyed pole at change in direction of line.

Course No. 3.- - pproximately 4 miles south of Chami; elevation 7,430 feet; use 10 poles of telephone Iine to outh of reference pole. Reference pole is at change in direction of line and is marked
with a band of red paint 5 feet abore ground.

Course No. 4.-Approximately $17 \mathrm{miles}$ north of Chazni; elevation 8,300 feet; use 10 poles of telephone ine to south of reference pole. Reference pole wh th 5 gujs is marked No. 2230 and is at hange in drection of line just north of the village of Shashgao. Course is on Irrigated land and about in
center of shinitz Rdver valley.

Courses Nos. 1,2 and 3 are at approxinately the same location as those used prior to 1960 ; No. 4 was
established $2 / 16 / 60$.

Equipment.-1 Mt. Rose snow sampler is used.

Cross section of cutter 1.5 inches

Design: che ounce of snow equals 1 inch of water.

Scale is graduated in ounces.

Snow courses were run with 2 samples at each of 10 telephone poles 150 feet apart and

total compite saple at 20 points were welghed and averaged for both snow depth and inches 
HELMAND RIVER BASDN - AFGHANISTAN

Snow Surveys - 1954 to 1960

SUMMARY OF SURVEYS IN TNCHES OF WATER

\begin{tabular}{|c|c|c|c|c|c|c|c|c|c|}
\hline \multirow[t]{2}{*}{ Iear } & \multicolumn{8}{|c|}{ Course No. } & \multirow[t]{2}{*}{ Ave. } \\
\hline & 1 & & & 2 & & 3 & & 4 & \\
\hline 1954 & $\begin{array}{l}\text { Jan. } 28 \\
\text { Feb. } 16 \\
\text { Feb. } 22\end{array}$ & $\begin{array}{l}3.1 \\
5.8 \\
6.7\end{array}$ & Feb. & 16 & 3.8 & Feb. 20 & 5.9 & & 5.47 \\
\hline 1955 & $\begin{array}{ll}\text { Feb. } & 2 \\
\text { Feb. } & 8\end{array}$ & $\begin{array}{l}1.3 \\
1.5\end{array}$ & & & & & & & 1.50 \\
\hline 1956 & Feb. 19 & al. 0 & Feb. & 19 & 0 & Feb. 13 & 2.8 & & 1.27 \\
\hline 1957 & Feb. 17 & 3.7 & Feb. & 17 & 1.4 & Feb. 16 & 2.3 & & 2.47 \\
\hline 1958 & $\begin{array}{l}\text { Jan. } 20 \\
\text { Jan. } 30\end{array}$ & $\begin{array}{l}0 \\
0\end{array}$ & $\begin{array}{l}\text { Jan. } \\
\text { Jan. }\end{array}$ & $\begin{array}{l}20 \\
30\end{array}$ & $\begin{array}{l}0 \\
0\end{array}$ & $\begin{array}{l}\text { Jan. } 20 \\
\text { Jan. } 30\end{array}$ & $\begin{array}{c}0 \\
1.5\end{array}$ & & 0.50 \\
\hline 1959 & $\begin{array}{l}\text { Feb. } 3 \\
\text { Feb. } 17\end{array}$ & $\begin{array}{l}2.4 \\
1.7\end{array}$ & $\begin{array}{l}\text { Feb. } \\
\text { Feb. }\end{array}$ & $\begin{array}{r}5 \\
17\end{array}$ & $\begin{array}{l}0.7 \\
1.1\end{array}$ & $\begin{array}{l}\text { Feb. } 2 \\
\text { Feb. } 17\end{array}$ & $\begin{array}{l}3 \cdot 3 \\
3.3\end{array}$ & & 2.03 \\
\hline 1960 & Feb. 16 & 0 & Feb. & 16 & 0 & Feb. 16 & $\mathrm{a} 0.1$ & Feb. 16 a0.2 & 0.07 \\
\hline
\end{tabular}

a - Estimated.
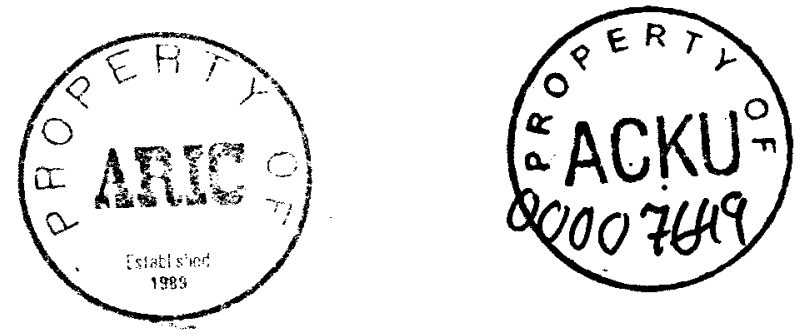